\title{
Measuring the Effects of Light Distribution on Spatial Brightness
}

\author{
James Sullivan
}

\author{
A thesis \\ submitted to Victoria University of Wellington in \\ fulfilment of the requirements for the degree of \\ Doctor of Philosophy \\ in \\ Architecture (Building Science)
}





\section{Abstract}

By changing the light distribution it is possible to double the apparent amount of light in a space without any increase in its overall luminance. If one simply assumes that the apparent amount of light in a space — its spatial brightness — is described by its mean luminance (or similar measures) then substantial errors may be made.

We carried out two experiments, measuring the brightness of 19 different model spaces. Our results demonstrate that making light distributions more non-uniform can make spaces appear both significantly brighter and significantly darker, depending on how the light distribution is changed. This challenges most existing studies in the field that argue that non-uniformity of the luminance distribution simply makes spaces look darker. Indeed, the observed pattern in brightness between our conditions cannot be consistently explained by a simple measure of the uniformity of the luminance distribution. We thus reject all previously proposed models of light distribution and spatial brightness.

The best explanation of this and the apparent disagreements in the literature over the effects of non-uniformity appears to be that spatial brightness is affected by the qualitative appearance of the luminances in the space. Light sources and non-luminous surfaces have different effects. We propose a 'duel'-process model of spatial brightness in which it is the sum of two opposed processes: the effects of the surfaces, and the effects of the light source(s). Non-uniform patterns of surface reflectance and illumination tend to make a space appear brighter. Non-uniformity as a result of a large difference between luminance of the light source(s) and the surfaces makes a space appear darker. If the light source is hidden from direct view its darkening effect is removed, which can make the space appear significantly brighter. Depending on the relative strength of these two processes, a nonuniform luminance distribution may thus appear either brighter or darker than a more uniform distribution.

Additionally, we highlight issues demonstrated in both the failure of models previously proposed by the literature, and our exploration of potential implementations of the 'duel'process model. It is very easy to produce a good correlation with a defensible metric that will not generalise to other data sets. A metric having a good correlation in a study provides very little reason to actually believe it. If we wish to develop a model of the effects of light distribution that we can trust, we need to demonstrate its robustness by testing its underlying assumptions and showing them to be well supported. As we show, there is a large variety of these that need to be worked through. 


\section{Acknowledgements}

Firstly, I would like to thank my supervisors, Michael Donn and Kit Cuttle, for their invaluable support and guidance over a project that grew both longer and infinitely more complicated than I think any of us expected at the beginning. This thesis would have been much the worse without our many discussions.

I would also like to thank Professor Mika Kato who aided my analysis of the literature by answering my questions about their work, and providing me with many valuable papers from Japan that I would have otherwise been unaware of. On a similar note, I thank Professor Keven Mansfield, and Dr. Jennifer Veitch for assistance with my literature review with regards to their research.

I would like to thank my parents for their support throughout these years, and the many stimulating discussions on the subject of research methodology and psychology.

Finally, a special thanks to all my participants. Without your generous contributions, this work would not be possible, and we would still be in the dark about everything we have learnt here. 


\section{Contents}

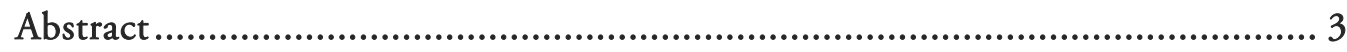

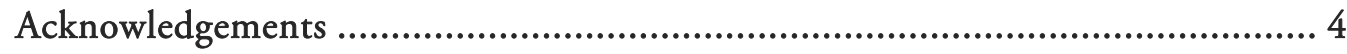

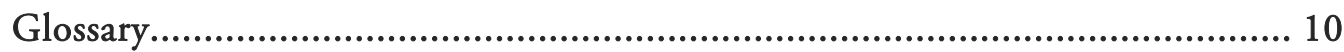

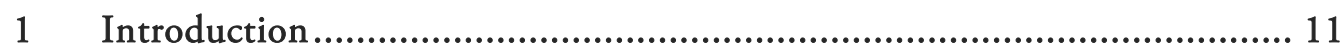

2 Effects of Light Distribution on Spatial Brightness: literature review ......... 15

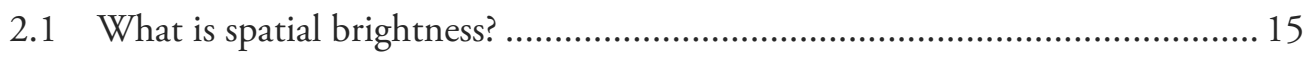

2.2 What do we mean by "light distribution"? ......................................................... 19

2.3 Observed Effects of Light Distribution on Spatial Brightness.......................... 20

2.3.1 Effects of Uniformity .......................................................................... 20

2.3.2 Effects of location: relative importance of the walls, floor, and ceiling .... 29

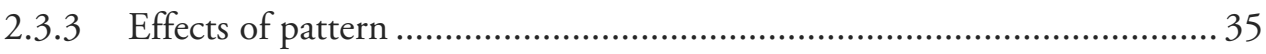

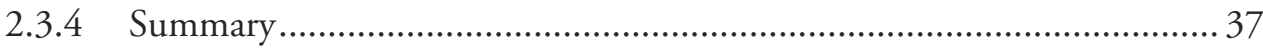

2.4 Models of the relationship between light distribution and spatial brightness .. 38

2.4.1 Kirsch: $\mathrm{max} / \mathrm{min}$ ratio in the $40^{\circ}$ horizontal band ..................................... 38

2.4.2 Hsieh: $\mathrm{min} /$ mean ratio of horizontal work plane illuminance ................... 43

2.4.3 Ko et al.: standard deviation of log luminance ......................................... 43

2.4.4 Kato et al.: Directional Diffusivity ........................................................... 44

2.4.5 Kobayashi et al.: Power Functions .......................................................... 47

2.4.6 The Marsden Equation......................................................................... 49

2.4.7 Comparison of the models' predictions ………………...........................50 


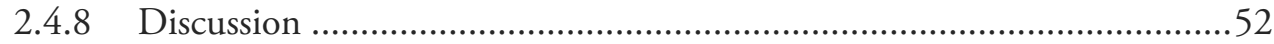

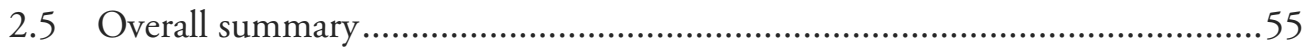

3 Experiment 1: In which we estimate the practical impact of non-uniformity and upend all prior predictions ........................................................... 57

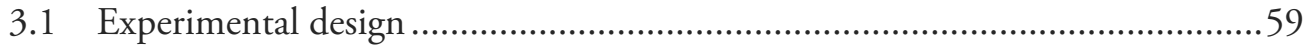

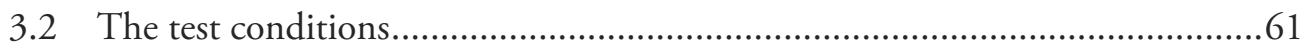

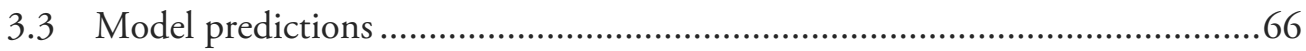

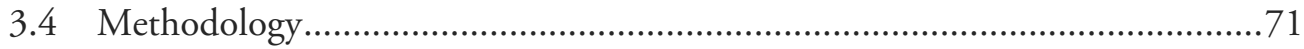

3.4.1 Brightness matching (main experiment) ...........................................73

3.4.2 Magnitude estimation (ME) …........................................................

3.4.3 Counterbalanced brightness matching ............................................... 80

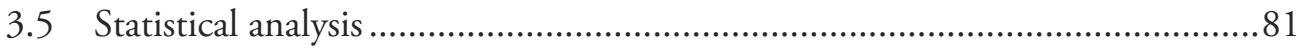

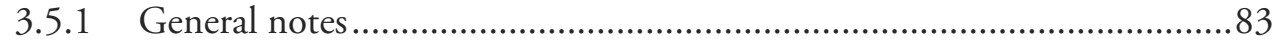

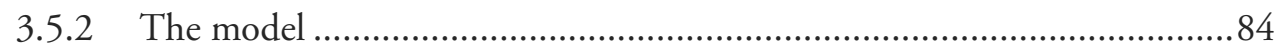

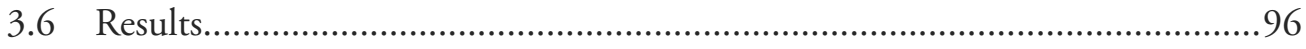

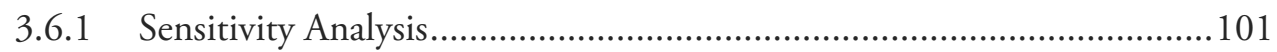

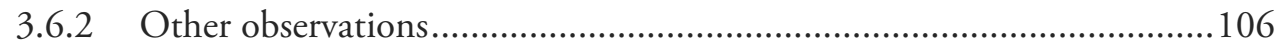

3.6.3 Potential issues with Experiment 1 estimates........................................108

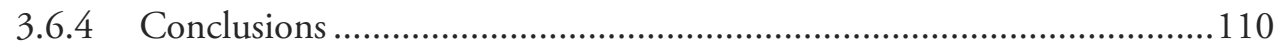

$4 \quad$ Why were our predictions wrong, and what does it mean?..................... 111

1.2 Why contradictions and failure may be unsurprising ..................................112

4.1.1 The problem with talking about the brightness/uniformity relationship 112

4.1.2 It is very easy for fundamentally flawed models to appear to work well .114

4.1.3 Conclusion: why should we believe any model? ..................................121

1.3 Illuminating the way forward: differences in light distribution between studies 123

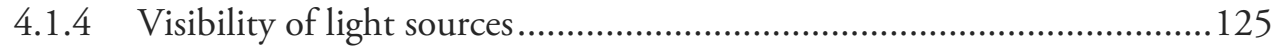

4.1.5 Modes of Appearance used to produce the different light distributions . 125

4.1.6 Orientation of contrast changes ......................................................... 127

4.1.7 Adaptation level ....................................................................... 128 
4.1.8 Summary 128

5 Experiment 2: why non-uniform spaces sometimes appear brighter, and sometimes darker .131

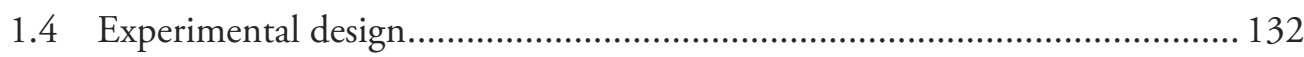

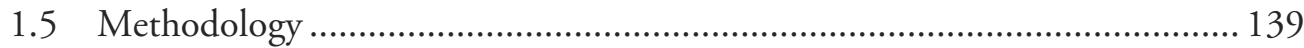

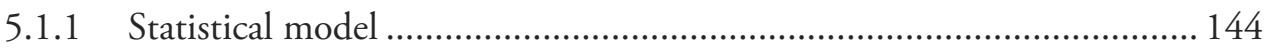

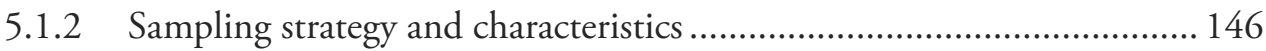

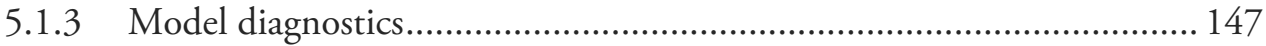

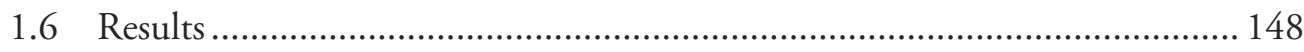

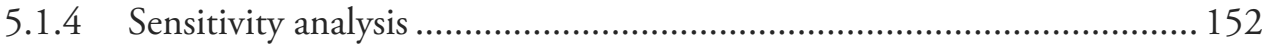

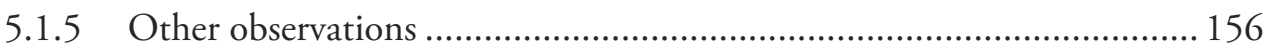

5.1.6 Discussion: The failure of uniformity as a predictor............................. 157

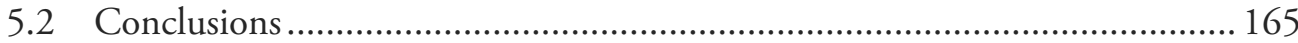

6 Exploration: how might we better describe and model our results? ..........167

6.1 Reformulating the Modes of Appearance hypothesis .................................... 167

6.2 A mathematical model .............................................................................. 171

6.2.1 Modelling the apparent effects of non-uniform surfaces ...................... 172

6.2.2 Modelling the apparent effects of the light source(s) ........................... 179

6.2.3 Potential position weighting models ................................................ 183

6.3 Testing the model ideas ............................................................................ 186

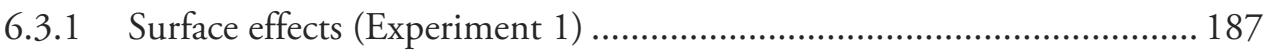

6.3.2 Light source effects (Experiment 1+2) .............................................. 199

6.4 Discussion: More questions than answers ….............................................. 213

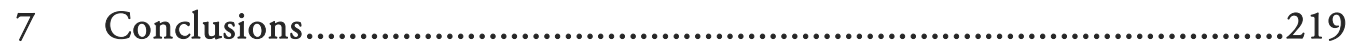

7.1 Implications for lighting design ........................................................... 221

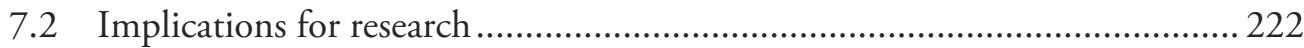

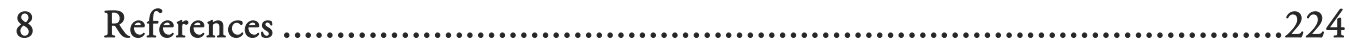

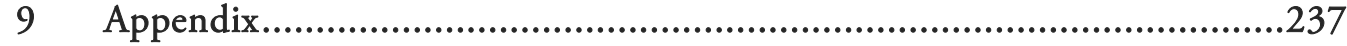

Appendix A Light measurement.....................................................239

A.1 Uncertainty in measurements of the overall light level .................................. 240 
A.1.1 Experiment 1 conditions …...............................................................240

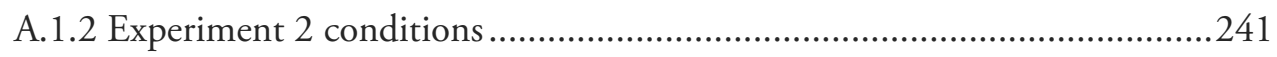

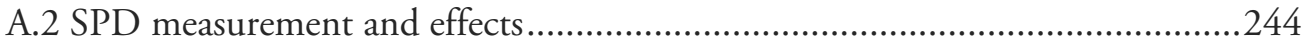

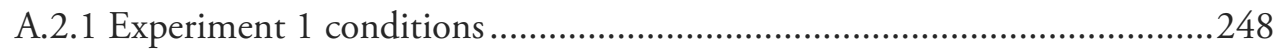

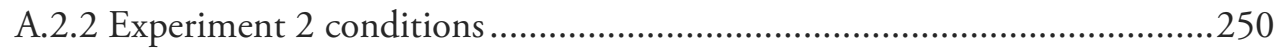

A.3 Measuring the light distribution: HDR luminance mapping...........................253

A.3.1 Measurement Reliability .....................................................................254

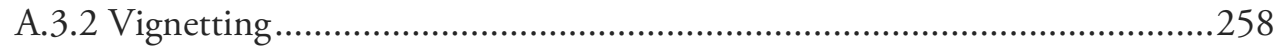

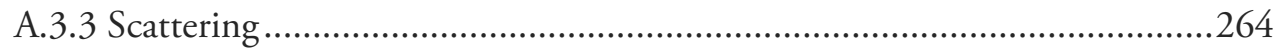

A.3.4 Luminance mapping workflow...........................................................293

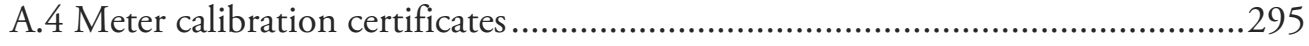

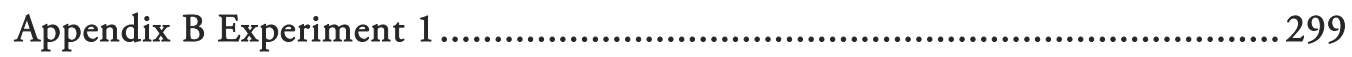

B.1 Experiment instructions.........................................................................29

B.2 Example of semi-randomised condition order ..............................................300

B.3 Measured lamp output after being turned on ................................................300

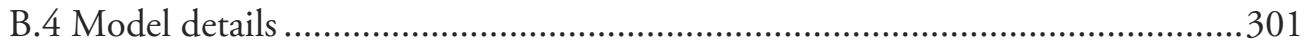

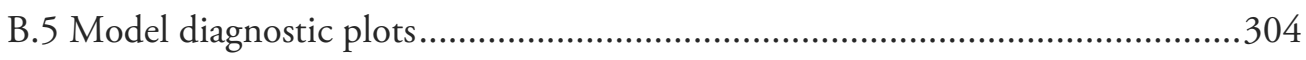

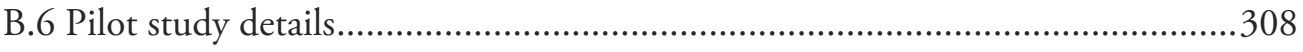

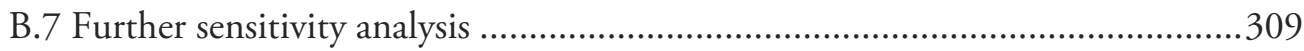

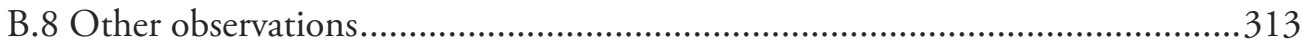

Appendix C Implications of simple models of bias control in brightness matching

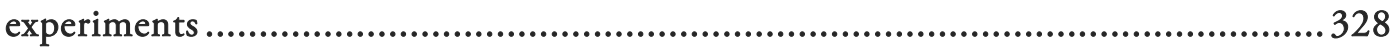

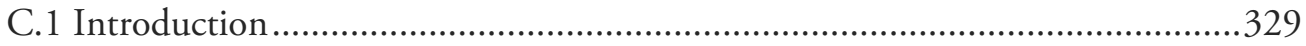

C.2 Fundamentals: the basic brightness matching experiment...............................330

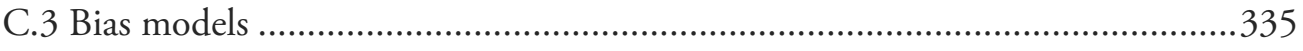

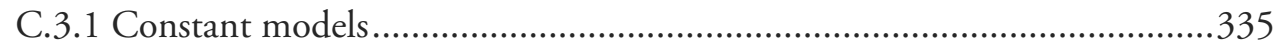

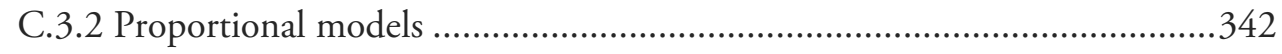

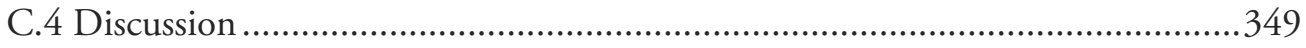

C.4.1 Adjustment range bias and confounding ............................................350

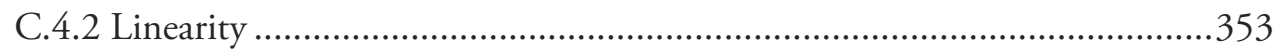


C.4.3 Homogeneity ............................................................................. 353

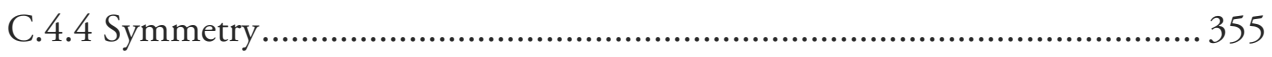

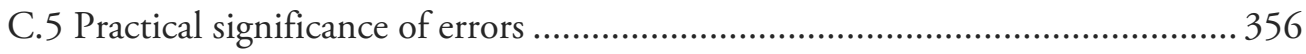

C.6 Postscript: All-possible-pairs design as a means of bias control ....................... 361

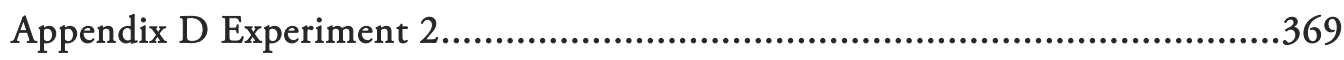

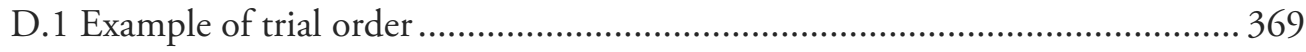

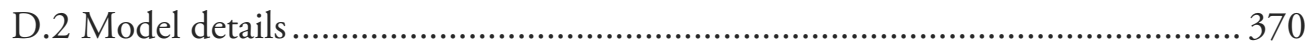

D.3 Model diagnostic plots........................................................................... 372

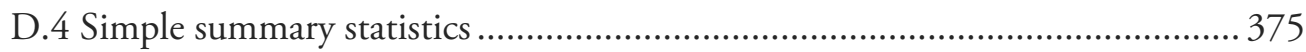

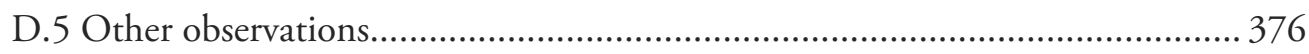




\section{Glossary}

Prior: the probability distributions describing the range of values the Bayesian statistical model considered "plausible" before looking at the data (along with their relative plausibility).

Posterior: the estimated probability of the parameter values after analysing the data i.e. the results.

Null condition trial: a trial wherein both conditions being compared are (theoretically) identical, and so in the absence of bias or measurement error should be matched in brightness at the same light level.

Counterbalancing: a method for controlling bias by causing it to cancel itself out. For example, one could counterbalance position by showing every condition on both the left and on the right, or counterbalance the starting light level by starting half the trials high, and half low.

Position bias: the position a condition is in in a side-by-side comparison may affect judgments of brightness, with people showing a tendency to report one side as brighter than the other.

Dimming bias: a matching bias wherein the condition being adjusted may tend to be set lower than it "should" be (also referred to as "conservative adjustment" bias).

Start-point bias: a matching bias where the initial light level of the condition being adjusted affects the brightness match.

Response contraction bias: a bias where the response range is compressed compared to what it "should" be, with higher magnitudes being underestimated, and lower ones being overestimated. This may occur in brightness matching experiments when multiple reference light levels are used.

Adjustment range bias: the range that a subject can adjust the light level in may affect their brightness matches, with matches tending towards the centre of the adjustment range.

Magnitude Estimation: a method of brightness evaluation wherein participants are asked to give a numerical estimate of the brightness of a stimulus, generally relative to some standard.

Brightness Matching: a method of brightness evaluation wherein participants adjust the light level of a test stimulus to make it match the brightness of a reference. 


\section{Introduction}

Imagine a room.

Imagine it to be a simple cube, the walls an even grey that reflect $50 \%$ of the light that falls on them, and a light source in the centre that evenly illuminates all the surfaces in the room.

Is this room well-lit?

How can we answer this question? What do we mean by "well-lit"? What is the purpose of lighting a room?

At the most fundamental level, light allows us to see and carry out visual tasks. Too little light, and we cannot discriminate details and colours, hindering our ability to operate. Too much light however, and we may suffer from glare. At this most basic level, a well-lit space is one that provides the right amount of light. Not too little, and not too much. The very question of "how much light should we provide" could be considered perhaps the dominant issue facing lighting research for the past century. Visual performance research was based around the question of how much light was required for people to do work ${ }^{1}$. The question of light level is similarly common in studies of people's preferences ${ }^{2}$. Lighting recommendations in various codes (e.g. NZS 1680.1, 2006) are dominated by a focus on light levels (Boyce, 1996; Fischer, 1973; Mills and Borg, 1999), and correspondingly efforts to improve the way we measure and describe lighting also tend to focus on how we measure light levels (e.g. Cuttle, 2010; Rea, 2014).

${ }^{1}$ E.g. (Boyce, 1970, 1973, 1974, 1976, 1979, 1985, 2001; Bullough \& Rea, 1996; Charness \& Dijkstra, 1999; Cundall, 1972; Lindner, Hübmer, Schlote, \& Röhl, 1989; Magister \& Colombo, 2001; McGuiness \& Boyce, 1984; Perry, Campbell, \& Rothwell, 1987; Rea, Boyce, \& Ouellette, 1987; Rea \& Ouellette, 1988, 1991; Saunders, 1969)

${ }^{2}$ E.g. (Bean \& Hopkins, 1980; Boyce et al., 2006; Butler \& Biner, 1987; Cundall, 1972; Escuyer \& Fontoynont, 2001; Fotios \& Cheal, 2010; Juslén, Wouters, \& Tenner, 2005; Moore, Carter, \& Slater, 2002a, 2002b; Tregenza, Romaya, Dawe, Heap, \& Tuck, 1974; Uttley, Fotios, \& Cheal, 2012) 
So what is the "right" light level?

In standard lighting practice, the answer is simple. The appropriate lighting level is determined according to the activities carried out in the space, and the light is distributed over the area where those activities are expected to be carried out. For a typical office in New Zealand, for example, this means an average of roughly 320-400 lux over an imaginary desk referred to as the horizontal work plane. If activities requiring discrimination of finer details were to be required (drafting for instance), then light levels would need to be higher. For areas where visual tasks are less demanding, such as corridors, less light is required. It is a simple, straightforward, and rational approach: work out how much light is required for your task, and apply it to your task area.

Let us return now to our hypothetical room. With these parameters, if our light source outputs 200 lux of direct illumination on the surfaces then the final illumination on all surfaces and the horizontal plane will be -400 lux. According to New Zealand Standard 1680.1 this is an appropriate light level for an office space, and we may thus regard the space as being well-lit. Problem solved.

Or is it?

The reasoning there relies on the assumption that the goal of lighting, at least as it pertains to the light level, is to ensure that we are given enough light to see clearly and carry out our desired tasks. This is, however, not entirely true. In fact, despite the apparent logic of our standards, we don't actually light according to visual performance (Boyce, 1996; Cuttle, 2013).

If we did then, based on our understanding of visual performance (Rea and Ouellette, 1991), for typical office tasks such as reading black print on a white page, a normallysighted person would need somewhere between 20-50 lux to read clearly, and even 6-point font would only require -100 lux (Cuttle, 2013). Not 3-400.

The implication of this, as argued by Cuttle $(2013,2010)$ is that we really light for what people want, and what they will pay for ${ }^{3}$. If we were to merely provide the minimum light levels required for good visual performance, people would complain that spaces are gloomy and under lit (Boyce, 1996; Cuttle, 2010). Indeed, even if the light levels we provide are clearly justified by task performance requirements, if the occupants do not feel the space is adequately lit then the lighting design is arguably a failure. Hence, the goal of standard practice is not lighting for task performance, but lighting for perceived adequacy of

\footnotetext{
${ }^{3}$ The dramatic decline in recommended light levels in North America after the energy crisis is an interesting indication of the role of this (Boyce, 1996; Mills and Borg, 1999). If we actually required over 1000 lux to see clearly, as was recommended in the U.S. before the 1970s, then reducing that to save energy would not have been tenable.
} 
illumination (Cuttle, 2010). In other words, our measurements need to map to people's perceptions of how much light is in the space.

Does the illumination on the horizontal work plane do this?

In short: No (Bean, 1977, 1978; Cuttle, 2010; Duff et al., 2015b, 2015c; Julian, 1987; Longmore, 1969; Lynes, 1970; Waldram, 1969).

To quote Lynes (1970):

"It is now widely accepted that the traditional arbiter of lighting levels - the illumination on a horizontal working plane — cannot be relied upon to tell whether one room will look brighter than another or whether one object will seem to be receiving more light than another."

So, what should we measure instead?

The logical answer is to measure all the light that people see, instead of imagining that they are lying on their backs staring at the ceiling. Indeed, studies have found such metrics (e.g. mean luminance, or Mean Room Surface Exitance (Cuttle, 2013)) to have high correlations with people's perceptions of room brightness, and to perform substantially better than work plane illuminance (Duff et al., 2015b, 2015c; Loe et al., 1994).

And yet... Is that all that is needed?

Let us take our room, with its grey walls and simple uniform lighting, and tweak it a little as designers are prone to (also, because it's terribly dull).

We could try painting the walls red, or blue, or green. We could change the colour of the light to produce a nice warm glow. We could paint the floor black and the ceiling white, or paint the walls in a checkerboard pattern. We could move the light to the edge of the room, or direct it at the walls to create a more interesting and less uniform distribution.

We could do a lot of things.

The question that this brings up, however, is what effect our decisions will have on how much light people perceive as being in the room. Let us assume that the amount of light we were providing to our boring grey room was a "good" amount that people liked, and that we have the same total light in all of our new designs.

Will they appear to be as brightly lit as our original room?

The answer to this question is also: No.

Both the colour of light (Fotios et al., 2015; Rea et al., 2015), and its distribution (Kato and Sekiguchi, 2005; Kirsch, 2014; Tiller and Veitch, 1995) are known to affect how bright a space appears. Simply measuring the overall quantity of light in a space is 
insufficient if we want to understand how it will appear to people. If we want to understand the consequences of lighting design decisions, and predict how bright a given space will appear, then we need models that account for all of these factors - the magnitude of light, its colour, and its distribution. While the effects of the colour of light on brightness have seen significant research (Fotios et al., 2015), and the development of sophisticated models (Rea et al., 2015), the effects of light distribution on spatial brightness are less well understood.

Creating a certain impression of brightness in the viewer is a critical element that drives much of lighting design (Cuttle, 2013). Spaces commonly differ in light distribution, and indeed playing with the arrangement of lights and how the light is distributed throughout a space may be considered a key part of lighting design (Cuttle, 2013; Russell, 2008).

However, while we can look at a lighting design textbook like The Architecture of Light (Russell, 2008) and find a great deal of discussion of the importance of factors like brightness and the arrangement of lighting to manipulate people's perceptions, the advice given is not quantified beyond the most approximate level. Russell (2008) for example, presents a table of typical illuminance levels in different spaces as guidance for ambient illumination (e.g. that a "dim, romantic restaurant" might have -5.0 foot-candles $[-54$ lux], or that an office might have $-50-70$ foot-candles [ $-540-750$ lux]), and the "2-5 times contrast rule" for making objects stand out. But such rules of thumb have very limited precision, and do not account for how lighting design decisions beyond raw light level could affect the impression of brightness. If decisions around the light distribution and colour could make an intended 400 lux space appear 50\% brighter or darker, then this would seem to be important to be able to account for. It may be that with sufficient experience good designers can learn what does and does not work - but how do we predict the appearance of truly novel designs? How are novice designers supposed to anticipate the consequences of their decisions, and accurately link desired appearance to the needed photometric quantities?

To know the consequences of such lighting design decisions we need robust practical measurements of the effects of light distribution. We need to know what effects are important - if the differences were generally only equivalent to around a $5 \%$ change in the light level, then they might not be meaningful. And, finally, we need a detailed understanding of the factors that affect spatial brightness, and quantitative models that allow us to reliably predict the effects of our decisions.

And so we have the question that is the focus of this thesis:

\section{"What is the effect, in quantifiable terms, of light distribution on spatial brightness?"}




\section{Effects of Light Distribution on Spatial Brightness: literature review}

Before examining the known effects of the light distribution on how bright a space appears, it is worth discussing what, exactly, we mean by "spatial brightness" and "light distribution”. We begin with spatial brightness.

\subsection{What is spatial brightness?}

An early attempt to define spatial brightness was given by the IES Visual Effects of Lamp Spectral Distribution Committee as follows:

Spatial brightness describes a visual sensation to the magnitude of the ambient lighting within an environment, such as a room or lighted street. Generally, the ambient lighting creates atmosphere and facilitates larger visual tasks such as safe circulation and visual communication. This brightness percept encompasses the overall sensation based on the response of a large part of the visual field extending beyond the fovea. It may be sensed or perceived while immersed within a space, or when a space is observed remotely but fills a large part of the visual field. Spatial brightness does not necessarily relate to the brightness of any individual objects or surfaces in the environment, but may be influenced by the brightness of these individual items. (Fotios and Atli, 2012)

A more concise definition is: the "attribute of a visual perception according to which a luminous environment appears to contain more or less light" (CIE, 2016). This is also consistent with Ishida and Oguichi (2002), who found that people's assessments of "brightness" lined up best with the perceived "amount of light filling the space" rather than "perceived intensity of the light source".

Henceforth, we shall be using "spatial brightness" to refer to this, following common practice. 
The generally accepted approach for researchers interested in the brightness of a scene or objects is to measure mean luminance, or vertical illuminance at the eye $\mathrm{e}^{4}$ (e.g. Kato and Sekiguchi, 2005; Kirsch, 2014; Loe et al., 2000; Marsden, 1970; Newsham et al., 2010; Rea et al., 2015; Van Den Wymelenberg, 2012). This is entirely logical, as the luminance of a scene is a measurement of the intensity of the light coming to an observer's eye, and thus should describe what they see.

The problem with this is that it does not really describe what we actually perceive. We do not perceive the world as consisting of many fields of light of varying intensity rather, we see illuminated surfaces, we see shadows, we see light sources (Boyce, 2003; Cuttle, 2004; Gilchrist, 2006; Kingdom, 2011; Lynes, 1983). It is our ability to perceptually discriminate between these different kinds of appearance that gives us the problem of lightness constancy ${ }^{5}$ (Kingdom, 2011, 2008).

Modes of Appearance was developed as a way to describe how we perceive the world. It is essentially a framework classifying the various ways in which we perceive the appearance of objects. It originated from Katz (1935), and was based on the idea that "any theory of colour vision must be based on a study of the ways that colours look to the observer in all their complexity" (Thouless, 1936). Over the decades, it was developed by various parties (Jones, 1953, 1943; Judd, 1961) into its modern form (Boyce, 2003; Cuttle, 2004; Lynes, 1983). There are five modes: the surface mode, the volume mode, the aperture mode (what Katz called the film mode), the illuminant mode, and the illumination mode. Importantly, objects seen in the different modes have different properties. The illuminant and illumination modes have the property of brightness, describing the perceived intensity of the light. The surface mode has the property of lightness, describing the perceived reflectance of the surface - whether it is white or black.

So how does spatial brightness fit in to this? The definition quoted above described it as "a visual sensation to the magnitude of the ambient lighting within an environment" and held it distinct from the appearance of other objects in the scene. Hence, while it may be

${ }^{4}$ Briefly, for those unfamiliar with the terms: luminance describes how much light would be seen to be emitted from an object in a particular direction, and is measured in candela per square metre $\left(\mathrm{cd} / \mathrm{m}^{2}\right)$.

Illuminance is a description of the amount of light falling on a surface — the illumination — and is measured in lux.

${ }^{5}$ Lightness constancy regards the basic question of why a piece of coal looks black, and a piece of paper white, even when we dramatically vary the level of illumination? This perceptual stability in appearance is what is known as constancy. Researchers into lightness constancy highlight the problem in relating luminance to the appearance of objects, noting that any luminance can be produced by an almost infinite array of illuminance/reflectance combinations. Thus any given luminance can appear as any shade of grey . For example $100 \mathrm{~cd} / \mathrm{m}^{2}$ of light coming off a wall could be produced by shining -630 lux on a $50 \%$ reflective surface (mid-grey), or -3140 lux on a $10 \%$ reflective surface (near black) and so forth. The question is thus how we correctly identify that the wall is, say, grey and not black or white. 
related to the appearance of surfaces, and the appearance of illuminants, it is not them. Similarly, while it may be considered close to perceived illumination, it is quite distinct. The illumination mode is specifically defined by surfaces, with the illumination itself defined by lighting a surface.

Spatial illumination is distinct in that it is not just defined by the illumination of surfaces. It is instead the ambient lighting, the light filling the space - a concept similar to what Katz (1935) referred to as Erleuchtung, or the illumination of empty space. This distinct appearance is described by Schirillo (2013), discussing what he called "inferred illumination". He noted that we perceive the empty space around us as being filled with light. Space, however, is invisible - it appears to be empty. Thus, as Katz (1935) noted, we do not see the illumination of the space, rather it is "felt" or "apprehended". As it cannot be directly perceived, it must be inferred from the surroundings. We can connect this to Fotios and Atli's (2012) point that spatial brightness is distinct from the brightness of individual elements in the space, even if it is influenced by them.

Cuttle (2004) has suggested that the ambient illumination of a room may also constitute a distinct mode of appearance, and given the phenomenologically distinct descriptions of it in the literature this would appear to be valid.

Table 2.1: Modes of Appearance (following Boyce, 2003; Cuttle, 2004; Lynes, 1983). Spatial illumination mode added following Cuttle (2004).

\begin{tabular}{|l|l|l|}
\hline Modes of Appearance & Examples & Properties \\
\hline Aperture mode & $\begin{array}{l}\text { A clear sky, a colour viewed through } \\
\text { the aperture of a spectroscope }\end{array}$ & Brightness, hue, saturation \\
\hline Illuminant mode & A light source, a luminous object & $\begin{array}{l}\text { Brightness, hue, saturation, } \\
\text { pattern }\end{array}$ \\
\hline Illumination mode & The light falling on a wall & $\begin{array}{l}\text { Brightness, hue, saturation, } \\
\text { pattern }\end{array}$ \\
\hline Surface mode & The colour of the surface of a wall & $\begin{array}{l}\text { Lightness, hue, saturation, } \\
\text { pattern, texture, gloss }\end{array}$ \\
\hline \begin{tabular}{l} 
Volume mode \\
\hline \hline $\begin{array}{l}\text { Spatial illumination } \\
\text { mode }\end{array}$
\end{tabular} & $\begin{array}{l}\text { The inferred illumination of empty } \\
\text { space, ambient illumination }\end{array}$ & $\begin{array}{l}\text { Bightness, hue, saturation, } \\
\text { pattern, transparency }\end{array}$ \\
\hline
\end{tabular}

The importance of distinguishing these different ways that objects and surfaces appear is highlighted by decades of research into the perception of lightness and brightness (Arend \& Spehar, 1993a, 1993b; Arend \& Goldstein, 1990; Blakeslee, Reetz, \& McCourt, 2008; Gilchrist, 2006; Kingdom, 2011, 2011; Zdravković, Economou, \& Gilchrist, 2006, 2012). Kingdom (2011), in his review, discusses studies finding that light sources appear brighter than reflectances of equal luminance, that shadows appear brighter than reflectances of equal luminance, and that objects that fall under shadow or behind a dark transparency 
appear brighter than if presented against a background of equivalent luminance. A study that demonstrates nicely the importance of such distinctions was reported by Gilchrist (2006, p. 256). Subjects were shown a display that was bent down the middle, such that one side was in shadow, while the other was brightly illuminated (Figure 2.1). A patch in the shadowed side had its luminance set high enough for it to appear reliably luminous. Subjects were then asked to adjust the luminance of a patch on the illuminated side to match in brightness. This was found to be impossible because the patch on the shadowed side, while appearing luminous, was also generally of lower luminance than the surfaces on the illuminated side. While the shadowed patch was luminous, and the illuminated patch was not, the shadowed patch appeared brighter. However, if the illuminated patch had its brightness increased enough to appear luminous as well, then it became immediately apparent that it was actually a much higher luminance than the shadowed patch. Because of this, matching the brightness was impossible.

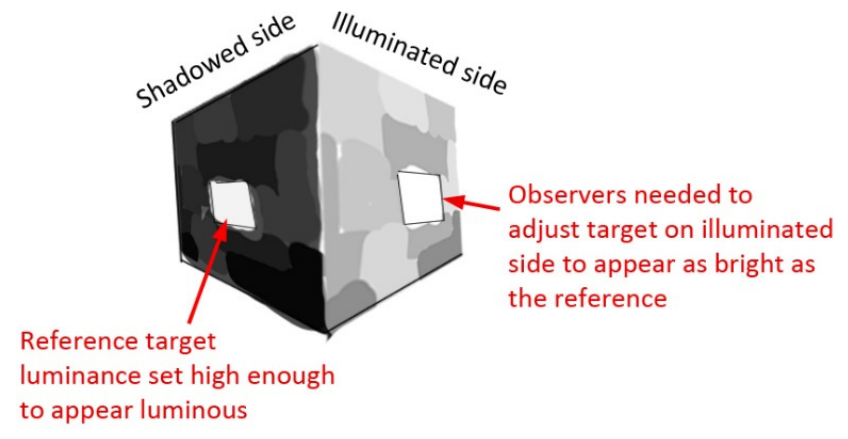

Figure 2.1 Illustrative sketch of the stimulus used by Gilchrist (2006) to examine the effects of perceived luminosity on brightness matching.

As Gilchrist (2006, p. 104) notes, the failure of many of the older theories of brightness perception to distinguish between the different effects of changes in reflectance vs. changes in illumination vs. changes in transparency may be considered a significant flaw. With that in mind, it is concerning to note that there appears to be little consideration of the issue in spatial brightness research. Perhaps the closest to considering this are the studies of Bernecker and Mier (1985) and Akashi et al. (2000), who attempted to study how light source visibility affected spatial brightness. Their results suggested that making light sources visible increased brightness. However, they did not control either overall scene luminance or the light distribution, which could potentially explain such results. Akashi et al. (2000) did, however, suggest that smaller light sources that appeared "sparkling" increased spatial brightness more, which may somewhat support the idea that qualitative elements of object appearance are relevant. That being said, this could also just be that arrays of small light sources are less uniform than large ones, and so we could also argue it just implies 
brightness is affected by the uniformity of the luminance distribution, and reference to qualitative appearance is unneeded.

Other than these though, spatial brightness studies appear to assume that spatial brightness is simply inferred from luminance, and do not consider the possibility that the different Modes of objects in the environment may affect the inference.

To summarise, spatial brightness, as defined as the sense of ambient illumination filling a space, is perceptually distinct from the brightness of luminants, the illumination of surfaces, and the appearance of objects in the space - though it may be related to them. Importantly, the light filling space must be inferred - it cannot be directly seen - and it is possible that the qualitative differences between different elements such as light sources and surfaces may affect this inference beyond simply being an area of luminance.

\subsection{What do we mean by "light distribution"?}

Another point of note is the definition of "light distribution". Most commonly in studies of lighting in this area, this will be framed in terms of "uniformity". This follows logically from the standard lighting metric — the "uniformity ratio" — that is commonly used to measure the variation in the lighting in the space. The uniformity ratio (and other similar metrics $)^{6}$, are just a measure of the range of light levels in the measurement field. They do not account for the spatial arrangement or pattern of the light — which are also an integral part of the light distribution.

Hence, in this thesis, we shall attempt to differentiate between these aspects in the following manner:

- Light distribution refers to the general concept of spatial distribution of the light in a space. It encompasses all aspects of the distribution of the light, including both magnitude and spatial arrangement.

- Uniformity refers to the magnitude of the variation in light levels in the space. This describes the range of light levels in the space, and the difference between the light and dark areas. It does not consider the spatial arrangement of the light.

- Position of light refers to the interaction of luminance with position in the space or view. For example, are the "brightest" regions on the ceiling? Or the floor? In the centre of view? How does this affect overall perception?

- Pattern of light refers to the spatial arrangement of the different light levels in a space, ignoring location. In essence, this is an attempt to describe those aspects of light distribution that are not captured by the simple measure of luminance range

\footnotetext{
${ }^{6}$ E.g. Max/min, max/mean, $\mathrm{min} /$ mean, standard deviation...
} 
that is uniformity metrics. It is somewhat less defined owing to there being very

little research pertaining to it, and most commonly used metrics do not describe it.

Following this, we may rephrase our general question as: "what are the effect(s) of light distribution on spatial brightness?" with the literature on the subject often focussing on the sub-questions of "what is the effect of uniformity on spatial brightness?", "what is the effect of position of light on spatial brightness?", and occasionally "what is the effect of the pattern of light on spatial brightness?"

To describe the current state of knowledge regarding these questions we will discuss the (limited) lighting research on the subject of light distribution and spatial brightness.

\subsection{Observed Effects of Light Distribution on Spatial Brightness}

As noted, what the literature says about the potential effects of light distribution on spatial brightness can be broadly divided into three categories:

- The effects of uniformity/magnitude of variation

- The effects of position/where the light is coming from

- The effects of pattern of light

We will discuss each in turn.

\subsubsection{Effects of Uniformity}

The first point that should be taken from the literature is that uniformity does appear to affect perceived spatial brightness (Aya et al., 2015b; Hsieh, 2012; Kato and Sekiguchi, 2005; Kirsch, 2014; Kobayashi et al., 1998; Tiller and Veitch, 1995). The exact nature of this relationship is somewhat disputed. The majority of studies suggest that more uniform lighting appears brighter than less uniform lighting given the same overall light level (Aya et al., 2015b; Hsieh, 2012; Kato et al., 2016; Kato and Hara, 2011; Kato and Sekiguchi, 2005; Kimura et al., 2004; Kirsch, 2014; Kobayashi et al., 1998). However, a few studies suggest effects in the opposite direction (Newsham et al., 2004; Okajima and Fujimoto, 2008; Tiller et al., 1995; Tiller and Veitch, 1995).

Tiller and Veitch (1995) is perhaps the strongest of these contrary studies. It compared two full scale office spaces using a psychophysical brightness matching task, and found the non-uniformly illuminated space appeared brighter. The non-uniformity was produced by using a luminaire that produced a sharper cut-off of the illumination on the wall. It was also directly replicated in Tiller et al. (1995), adding to its reliability. It had a relatively 
large sample size of $\mathrm{n}=30$, and used both counterbalancing ${ }^{7}$ and null condition trials ${ }^{8}$ to measure and control for potential bias. Unfortunately, it has several limitations that make it difficult to take as strong evidence in favour of non-uniform spaces being brighter. Firstly, no quantifiable measurements of luminance uniformity were reported, making it difficult to be sure how the two spaces actually compared to each other. Secondly, the study only compared two spaces, and the effect found was small $\left(5-10 \%{ }^{9}\right)$. It is thus conceivable that if we knew the "true" way of measuring light distribution (in so far as it affects brightness), and plotted all the different study conditions on it, that we would find that Tiller and Veitch's results were actually consistent with the others' all along.

To elaborate: imagine that brightness is affected by the light distribution, and that this may be predicted by a "truth" metric of uniformity (Figure 2.2 (A)). Now, imagine that we do not know what this "truth" metric is, but we do have some simple metrics that work to approximately describe the uniformity in the space. Because these metrics are imperfect descriptions of the light distribution, they will not perfectly correlate with the perceived brightness - though they will tend to capture the general trend (Figure $2.2(\mathrm{~B})$ ). However, the inaccuracy of our simple uniformity metric(s) mean than in some cases, spaces that "should" be described as more uniform may be described as being less uniform (Figure 2.2 (C)). The result of this is that if you carry out a study that only looks at a pair of conditions like this, then you run the risk of finding an apparent trend in the opposite direction from the "true" trend - purely because your measurement of the uniformity of the conditions is an imperfect reflection of the "true" uniformity. Hence, we cannot confidently say that Tiller and Veitch's (1995) results are truly conflicting with other studies in the literature. Additionally, with only two conditions, it is hard to say whether or not this comparison is representative of a broader pattern of effects or if it is an outlier.

\footnotetext{
${ }^{7}$ For example, having both trials where subjects adjusted the light level of the uniform condition to match the non-uniform, and trials where they adjusted the non-uniform to match the uniform. Similarly, in half the trials the adjusted condition was initially set to a low light level, and in the other half it was set to a high light level. They also counterbalanced order of presentation, with half the participants viewing the nonuniform condition first and half viewing the uniform one first.

${ }^{8} \mathrm{~A}$ null condition trial is one in which both conditions are the same. It can be used to check for biases for example, if people had a tendency to report the condition being dimmed as brighter even if they are the same.

${ }^{9}$ Such magnitudes are roughly around the threshold of what we might expect people to semi-reliably detect as a "Just-Noticeable-Differences", or JND, which refers to "the smallest difference in stimulus magnitude that is just discriminable" (Kingdom and Prins, 2016). For brightness of small spots of light, JNDs are generally on the order of $8 \%$ or more (Teghtsoonian, 1971).
} 


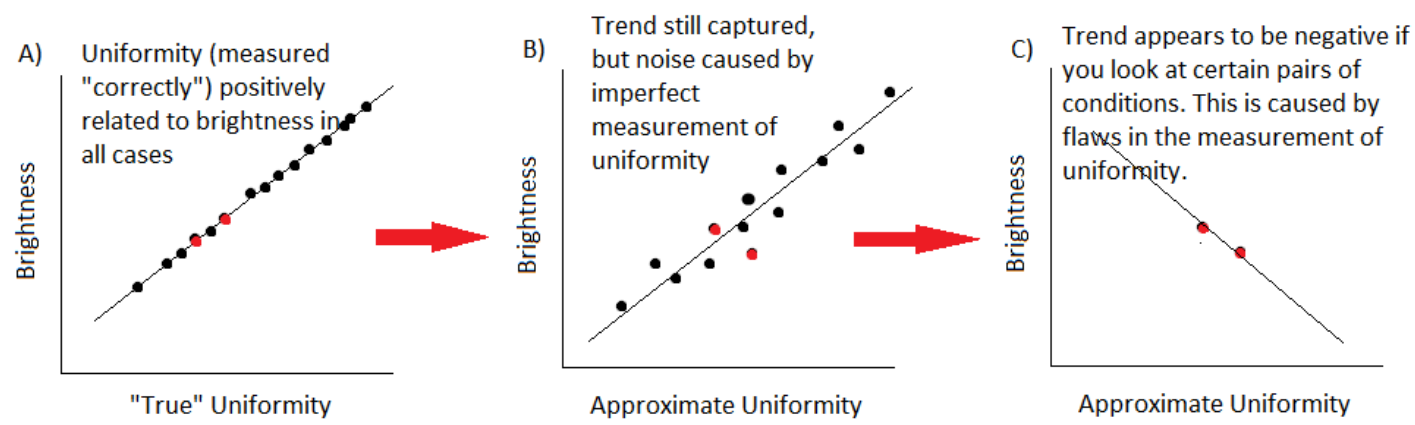

Figure 2.2 - Potential discrepancies caused by imperfect measurement of uniformity.

The other studies suggesting non-uniform spaces may appear brighter are arguably weaker. The Newsham et al. (2004) study found only a small correlation $\left(r^{2}=0.13\right)$ between brightness and uniformity, and this may have at least partially been an artefact of the experimental stimuli. In the follow-up study (Newsham et al., 2005) they noted that uniformity was significantly confounded with mean luminance, and added no explanatory power once that was accounted for. Moreover, the studies were not really designed to look at light distribution and brightness in the first place.

Okajima and Fujimoto (2008) looked at the effect of systematically lowering the contrast of photos of spaces, and found large effects - with the lowest contrast scene requiring about $73 \%$ more light to match the brightness of the original scene. The experiment had very few subjects $(n=2)$ - though there were a substantial number of data points, as the reported trend was derived from the measurements of eight stimulus scenes that each had their contrast reduced nine times. This may produce good measurements of how those individuals respond. At the same time, one may reasonably be concerned about how well the results would generalise, and it may be more accurate to characterise the results as just indicating that some people may perceive distributions with reduced contrast as being substantially darker. They were also only using computer images and not actual rooms. While studies of the validity of using images as proxies for real scenes have suggested that people's responses are generally fairly similar between them (Cauwerts, 2013; Mahdavi and Eissa, 2002), these results should still be interpreted cautiously. Validation studies have typically just examined people's responses to a selection of different spaces, and have not specifically looked at the question of light distribution while controlling for the overall light level. Hence, it is unclear whether or not people interpret changes in contrast in images the same way as they would in the real scene. There is also a lack of any clear information about image position, stimulus adjustment range, or any bias control procedures other than randomisation. However, it is also not easy to simply explain away the results by referring to the matching biases described by Fotios et al. (2008). With most bias estimates being in the single digits (e.g. Boyce, 1977; Fotios and Gado, 2005; Fotios and Cheal, 2011, 2007a; 
Houser et al., 2003), the largest effect ( $\neg 73 \%$ difference) here far exceeds what we might expect matching bias to produce, as does the corresponding systematic trend over 9 contrast levels. While the experiment does not, strictly speaking, rule out the possibility that the observed trend is actually a systematic effect of uniformity on bias rather than brightness, such an explanation would be questionable as the literature has never suggested anything like that. The only systematic matching bias effects that have been suggested are the result of systematically changing the reference light level, but that would have required them to have changed the reference light level as they reduced the contrast. That being said, while it seems hard to say their results are due to bias, their failure to account for it does make the results very uncertain. Overall, the study is weak, perhaps at best just suggesting that if the contrast of photos of spaces is reduced then some people may perceive them to be significantly darker.

Looking now at the studies arguing that uniform distributions appear brighter, the strength of the evidence varies. Hsieh (2012) is the weakest of this group. It has the problem that the light distribution was measured using horizontal work plane illuminance with a low resolution 12 point grid. This raises concerns that their measured uniformity may not reflect how the space appears to people very well (see Duff et al. (2015b) on failures of horizontal illuminance to reflect perceived brightness). The stimuli viewed by subjects was again photos of the scale model used, with the light being shone into the model through different patterns of holes in the ceiling. The procedure used here was the method of constant stimuli. 14 test scenes at five different light levels (100-500 lux) were compared against a standard scene and subjects $(n=50)$ were asked if they were brighter or darker or the same. A null-condition trial was included. However, no attempt was made to address the potential issues of stimulus frequency or range centring bias (Fotios and Houser, 2013), which could affect the results. That being said, these would not produce the observed trend - they could explain the apparent bias observed in the null trial, or could affect the slope of a trend produced by other factors, but would not create a trend on their own.

Kobayashi et al. (1998) used computer images similar to Okajima and Fujimoto (2008). They used magnitude estimation to assess brightness, asking observers $(n=20)$ to rate the brightness of their test scenes relative to a reference that was said to have a brightness of "100". Fourier transforms were used to adjust the intensity of the high or low frequency luminance variations in the images. They found that increasing the intensity, and thus the non-uniformity, of the broad low frequency luminance variations made the spaces appear darker. The high frequency variations - small highlights and shadows - had no significant effect. They only looked at two different room interiors in their main experiment, and adjusted the uniformity in both of them the same way. We could thus 
raise similar concerns here as with Tiller and Veitch - that the results may just be an illusion caused by only comparing a couple of conditions combined with an imperfect understanding of uniformity. We may also note that their use of magnitude estimation may be prone to all the biases identified by Poulton (1979). The degree to which this is a problem depends on the question we are answering. The biases mainly affect the slope of the relationship between stimulus and brightness response. Thus, they would not produce the apparent trend of a systematic change in brightness found. However, they could significantly affect the estimated size of the effects, and the precise parameters of any model produced to predict the results may not generalise to new situations.

The study of Kirsch (2014), and the various studies of Kato et al. (Aya et al., 2015b, 2015a; Kato et al., 2016; Kato and Hara, 2011; Kato and Sekiguchi, 2005; Kimura et al., 2004; Yamaguchi et al., 2016) provide studies with multiple conditions (5-11 conditions in each study), and use real spaces. Kato et al.'s work initially focused on the size and spacing of arrangements of "windows" around the walls of their test space (Kato and Hara, 2011; Kato and Sekiguchi, 2005; Kimura et al., 2004), but later work then extended this to similar arrangements of lights around the walls (Aya et al., 2015b), and then to more natural spaces including both windows and ceiling and floor lamps (Kato et al., 2016). Kirsch (2014) looked at different gradients of light across the walls of their room using LED panels, with both soft and sharp changes in luminance. Their consistent trends across a range of conditions would appear to provide fairly strong evidence in favour of nonuniformity generally making spaces appear darker. That being said, some caveats must still be raised. Kato et al. (2016) does not provide strong evidence here, as the trend in the plot of their luminance $\mathrm{x}$ uniformity metric against brightness appears to be largely driven by changes in luminance between conditions, and it is unclear that their uniformity metric adds anything. Further work on that data, presented by Yamaguchi et al. (2016), provided a newer version of their model that did provide slightly better correlations than just mean luminance ( $r^{2}=0.88$ vs 0.8 ), which could be seen as evidence that uniformity is positively related to brightness. At the same time, it could also just be seen as a demonstration of how flexibility in measurement and model specification can always enable one to find some kind of statistical relationship (see Gelman and Loken, 2013; Simmons et al., 2011). Even removing that study though, we still have five others showing the same trends. The studies use a range of methods: Kirsch had subjects $(\mathrm{n}=34)$ rate brightness on 8-point interval scales (dim $\rightarrow$ bright); Early work from Kato et al. (Kato and Hara, 2011; Kato and Sekiguchi, 2005; Kimura et al., 2004) used pairwise comparisons, where subjects ( $\mathrm{n}=10)$ were shown conditions in sequence and asked to rate how the second compared to the first on a 7-point scale from extremely dark to extremely bright; The more recent studies (Aya et al., 2015a, 2015b; Kato et al., 2016) used magnitude estimation $(\mathrm{n}=20)$. 
In terms of quality, Kirsch followed the recommendations by Poulton to minimise potential bias using randomised order, standard distraction scenes between stimuli, anchoring the scale with high and low luminance scenes at the start, and using a scale without a step change in the number of digits. One potential issue is grouping bias (CIE, 2014), wherein if you have less points on your scale than you have conditions then respondents may not be able to differentiate between all of them - some conditions will be forced into the same interval on the scale even if they are different (Figure 2.3). In this case, Kirsch's experiment technically had more conditions than scale points - 6 light distributions $\mathrm{x} 2$ desk luminances. The findings were a trend following differences in brightness between the light distributions, and no significant effect of the desk luminances. While one could argue the possibility of small differences in the effects of desk luminance being obscured by such grouping bias, it likely would not affect the main finding (the trend with light distribution) that we are interested in here.

Scenario 1

Conditions all line up

with the scale.

Rating is easy

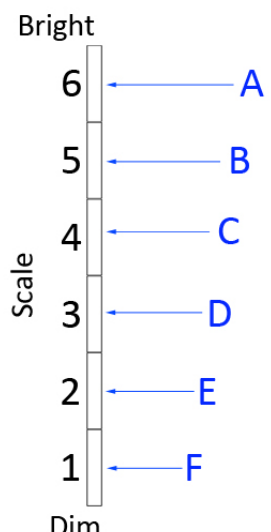

Scenario 2

Too many conditions to

fit neatly on the scale.

Grouping may be a

significant issue

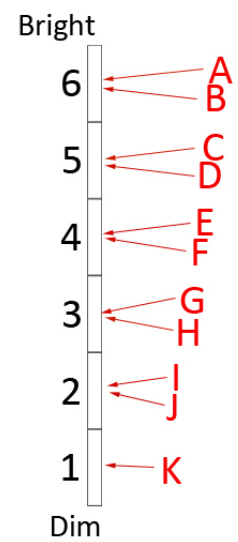

However, major trends may still hold even if smaller differences are lost:

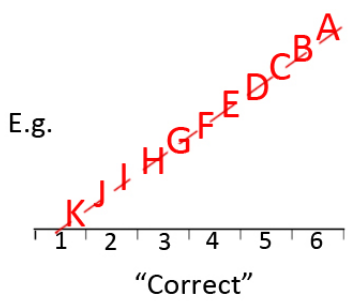

vs.

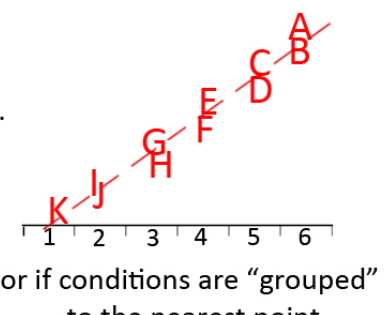

to the nearest point

Figure 2.3 Grouping bias problem in the use of interval scales

The pairwise comparisons in Kato et al.'s work are complicated to assess because they are similar to a sequential brightness discrimination task, but use an interval scale. They used randomisation, counterbalanced order of presentation, and all-possible-pairs design, 
meeting many of the recommendations in CIE 212:2014. However, there are still potential grouping problems as illustrated in Figure 2.4. These could compress some of the observed differences. We could also raise the possibility of some degree of response contraction bias where responses tend to be too close to the centre of the scale, and the far ends are avoided (Poulton, 1989). This could result in the slope of the observed trend being shallower than it "should" be (Figure 2.5). That being said, while these issues could obscure some smaller differences between conditions (e.g. Figure 2.3), or perhaps cause underestimation of the size of some effects, they would seem unlikely to be the cause of the observed trends between uniformity and brightness - indeed quite the opposite. They could hide differences, or produce errors in the estimated magnitude of effects - but would not be expected to produce a trend where none exists. The magnitude estimation experiments are similar to Kobayashi et al.'s (though using real spaces), and thus have similar potential problems with bias. Again, this could affect the accuracy of estimates of the size of effects and any model parameters, but would not be expected to be the cause of the observed trends.

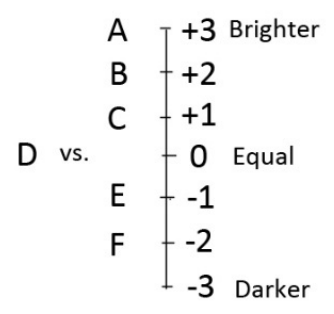

Comparing to middle condition: scale works fine

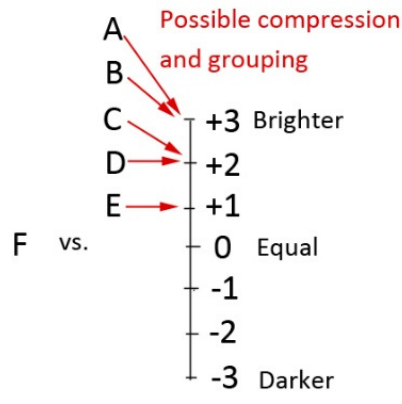

Comparing to end condition:
scale doesn't have room
- grouping will occur

Figure 2.4 Potential grouping bias in the pairwise comparisons of Kimura et al. (2004), Kato and Sekiguchi (2005), and Kato and Hara (2011). With more than four conditions, there may not always be room to differentiate all of them on the scale. Despite this, the broad trend of a progression in brightness from $\mathrm{F}->$ A would still exist, though it may be slightly less clear. 


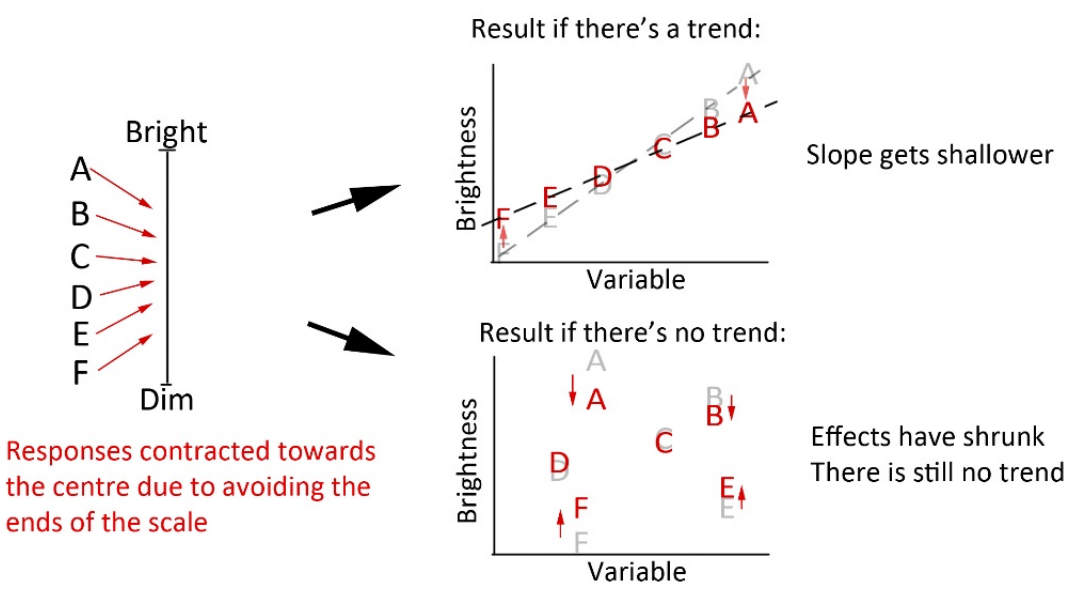

Figure 2.5 Illustration of how possible response contraction bias could affect estimates of effect sizes and slopes. While it could affect the apparent steepness of a slope if there is a trend, if there is not a trend we would not expect it to produce one.

Overall, the evidence in favour of non-uniform spaces appearing brighter is not strong, with only a couple of studies that are limited by low numbers of conditions or subjects and methodological issues. In contrast, the half dozen studies suggesting that uniform spaces appear brighter, while not perfect, overall provide a clear trend across a larger number of light distributions and studies. The weight of the evidence would appear to favour uniform spaces appearing brighter — though we should not dismiss the possibility that the disagreements are the result of a more complex relationship.

\section{Magnitude}

The next question we might ask here is how substantial the effect of light distribution is. This is somewhat difficult to answer due to methodological issues in the literature. Many studies use subjective rating scales to assess brightness. As Tiller and Veitch (1995) discussed, such scales do not really support scaling of brightness-luminance relationships, because they really just give ordinal data. In addition, if we want to attempt to estimate the size of their effects in terms of something meaningful like "equivalent change in luminance" then we need them to have measured the effects of changing light level in their study as well, which many studies do not do.

The most straightforward estimates of magnitude come from brightness matching studies, which directly tell us the effect of the differences in uniformity in terms of the change in light level required to counteract them. Tiller and Veitch (1995) found that the room with non-uniform wall illumination required 5-10\% less light to appear equally as bright as the one with more uniform wall illumination. Unfortunately, as noted, the paper does not report any quantifiable information about the degree of uniformity, making it impossible to scale anything. Okajima and Fujimoto (2008) found that reducing image 
contrast resulted in the scenes requiring up to $76 \%$ more luminance to match the brightness of the original. Again though we cannot apply this anywhere else since we lack clear measurements of the scenes' uniformity.

Studies such as Kato and Hara (2011) provide no way to estimate the practical magnitude of their effects, telling us only that some conditions are brighter than others. Aya et al. (2015b), however, used magnitude estimation and assessed their uniform condition at three different light levels in their experiment. By comparing the resulting changes in brightness due to that to the changes in brightness between the different conditions we can estimate the rough magnitude of their observed effects. Based on this, we might estimate that the effects of increasing uniformity in their experiment were roughly equivalent to increasing the light level by $50 \%$.

Kirsch's (2014) results are also problematic, as all of his conditions in Experiment 2 (the one where he compared different light distributions) had the same mean luminance. However, in his first experiment he did look at different light levels using the same scales. Comparing the results of the two experiments suggests that over the range of uniformity levels he assessed (uniformity ratio increased by $-150 \%$ ), the effect of the increase in nonuniformity was roughly equivalent to a $30-40 \%$ decrease in mean luminance. This estimate should be interpreted cautiously given that we are comparing across separate experiments. Responses on subjective scales are known to be biased by the range of conditions viewed (Poulton, 1989). While the two experiments were using the same experimental laboratory, and were carried out at similar overall light levels to similar responses, combining them in this way is still slightly questionable.

However, despite these limitations this would seem to suggest that effects of uniformity could be very substantial in practical terms. A change in lighting design that makes a space look like it has 50\% more light in it could have substantial implications regarding room appearance and energy efficiency.

Are these suggested differences plausible though? Comparison with the literature on the effects of colour of light suggests that, indeed, they are. Fotios and Levermore (1997) found that incandescent lamps with a blue glass filter required $25 \%$ less illuminance than normal incandescents, while high pressure sodium required $100 \%$ more light than a warm white lamp ${ }^{11}$. Fotios and Gado (2005) found that D65 daylight fluorescent $(6500 \mathrm{~K})$ required $-11 \%$ less light than warm white fluorescent (2950K). Rea et al. (2015) indicate that halogens require $-67 \%$ more light to match $5400 \mathrm{~K}$ LED lights. Precedent thus suggests that spatial brightness can be substantially altered by factors beyond mere luminance. If we

\footnotetext{
${ }^{11}$ Technically these were matched on "Visual Clarity". However, Fotios and Gado (2005) suggest that this gives the same results as matching for brightness.
} 
look at the basic brightness perception literature we can observe even greater effects. Consider the simple case of a patch against a uniform background (Figure 2.6). Increasing the luminance of the background will reduce the apparent brightness of the target (Gilchrist, 2006) - potentially quite substantially (e.g. Diamond, 1955).

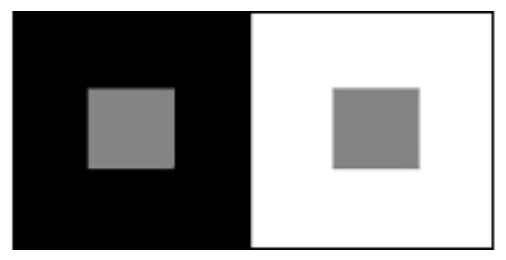

Figure 2.6 Illustration of the basic brightness illusion known as simultaneous brightness contrast. The target on the left looks brighter, though both of them have the same luminance. Visual illusions such as this demonstrate how our perception of brightness can be substantially influenced by factors other than just the raw luminance of the element being observed.

If we model this simple situation using the Haubner Equation (Bodmann et al., 1979; Bodmann and Toison, 1994), then we find that increasing the luminance of the background 50x has roughly the same effect on the target's brightness as decreasing the luminance of the target by a factor of $\sim 16$. While we would probably not expect complex real scenes to show effects as powerful as this, it does again highlight that factors such as contrast can potentially have very large effects on apparent brightness. In this light, there seems little reason to be sceptical of the suggested size of potential uniformity effects.

\subsubsection{Effects of location: relative importance of the walls, floor, and ceiling}

A number of studies have suggested that where the light is coming from/is placed affects the apparent brightness of a space. Most commonly, the suggestion is that the walls are particularly important (Flynn, 1977; Houser et al., 2002; Iwai et al., 2001; Kato and Sekiguchi, 2005; Kirsch, 2014; Loe et al., 1994; van Ooyen et al., 1987). There has also been the occasional suggestion that the ceiling may be more important than the floor or work plane (Houser et al., 2002; Kirsch, 2014). We will discuss the strength of the evidence provided by these different studies in turn. Most of these studies used some form of interval rating scale to assess brightness, and did not attempt to anchor the scale by providing high and low stimuli standards to participants at the start of the experiment. Response contraction bias may be present, but this is unlikely to be critical to the conclusions we are focused on here - the steepness of slopes may be underestimated, but that does not challenge the reports of apparent correlations.

\section{Kato and Sekiguchi (2005)}

Kato and Sekiguchi (2005) examined the perceived brightness of a 2 × 2 × $2 \mathrm{~m}$ space. In their first experiment they assessed spaces lit through either the ceiling or the wall in front of the subject at three different mean luminance levels (the surfaces were sheets through 
which light could be shone). Participants' views were fixed to either a task on a desk or to the wall in front of them. When the participant was facing the wall, it was found that walllit space appeared brighter than the ceiling-lit space at the same overall luminance level, and that vertical illuminance was a much better predictor than horizontal illuminance. When they were looking at the task, the opposite results were found.

In their second experiment, participants' views were not fixed, and they could look around the space. They compared spaces lit through either the walls or the ceiling, at three different mean luminance levels. It was found that the space lit through the walls appeared brighter than that lit through the ceiling at the same overall luminance level, and that vertical illuminance had the same kind of predictive power that it did in the previous experiment when subjects were looking at the wall.

From this, it was concluded that:

a) viewing direction matters

b) under free-viewing conditions, people tend to look in the horizontal direction

c) because of this, in everyday conditions spaces will appear brighter when they are lit from the walls

This is a controlled experiment that would seem to support the hypothesis that the walls (as they are generally what is in the horizontal direction) have a greater impact upon spatial brightness than the ceiling.

There are, however, some challenges to this. In the second experiment the different conditions are confounded with changes to uniformity. In the wall lighting condition all the walls were lit. Compare this to the ceiling lighting condition, and we see that since the walls are in total four times the area of the ceiling, the ceiling condition would have to be less uniform as it focuses the light into a smaller area. As their studies suggest that uniform spaces appear brighter, the apparent brightness of the wall condition could simply be due to the space being more uniform. Because of this, the second experiment's findings cannot be used as strong evidence that people tend to look in the horizontal direction. Even if we agreed that people do tend to look in the horizontal direction (and based on casual observation it is plausible, as well as being supported by eye-tracking studies (Rockcastle et al., 2017)), it does not necessarily follow that light from the horizontal direction is inherently more influential than light from other directions. It may just appear to be important in this experiment because the small size of the experimental space means that horizontal views are strongly dominated by the walls - i.e. the source of "horizontally incident" light. In larger spaces, the ceiling and floor would take up significantly larger proportions of the visual field (Figure 2.7, Figure 2.8), and so would be expected to become 
more important. The apparent importance of light from the walls may simply be an artefact of the specific experimental setup, and not necessarily reflective of anything else.

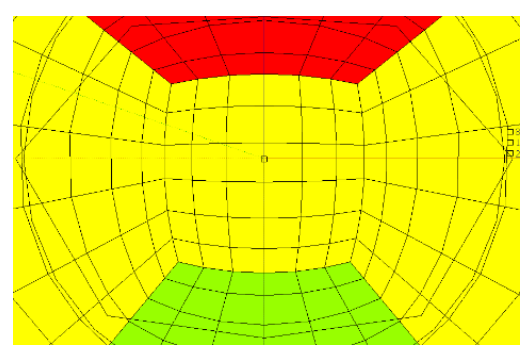

Figure 2.7: View of $2 \times 2 \times 2 \mathrm{~m}$ room

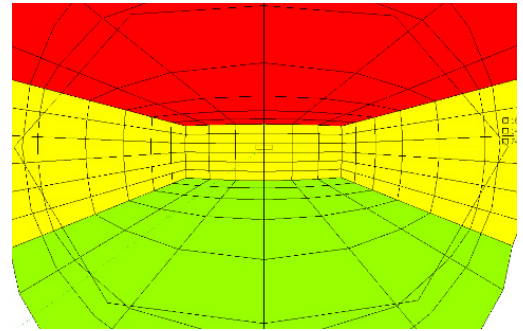

Figure 2.8: View of $6 \times 6 \times 2 \mathrm{~m}$ room

The first experiment did not suffer from these uniformity confounds, since in its wall condition, only one wall was lit, making the lit area in each condition identical. However, as they discussed, the first experiment's results are best explained by perceived brightness being dependent on the light levels in the field of view. When the subjects were facing the wall it would dominate their view, and they would see only a fraction of the ceiling.

In light of this, the vertical weighting function they developed and used in their model to attempt to predict spatial brightness is not well supported. If their second experiment's results can be explained by the wall condition being more uniform then applying a positional weighting function to attempt to fix the failure of unweighted mean luminance is unjustified. The results of their third experiment, which tested their proposed model, say nothing about the value of position weighting as the calculated metric was virtually identical regardless of whether or not luminance was weighted by vertical angle.

From this study we can conclude that when people's views are fixed, their assessments of spatial brightness will be based on the light levels in their field of view - something which is perfectly logical. We cannot, however, conclude that the walls, or light from the horizontal direction, is intrinsically more important than light from other directions or surfaces.

Houser et al. (2002), Flynn et al. $(1973,1977)$

While the studies of Houser et al. (2002), and Flynn et al. (Flynn, 1977; Flynn et al., 1973) may not appear to have much in common, they all suffer from the same basic problem - reliance on horizontal work plane illuminance to control overall light level.

Houser et al. (2002) examined the perceived appearance of a space lit by varying ratios of indirect and direct lighting. They found that the perceived brightness of the walls, and the brightness of the ceiling, had "moderately high" correlations with the overall brightness of the room $(r=0.74$ and 0.68 respectively). They also found that the brightness of the desktop, and the brightness of the floor, had significantly lower correlations $(r=0.49$, 
0.48 ), and so suggested that the floor and the desktop were "less influential on overall room brightness perception" (p. 255).

Flynn et al. (1973) compared lighting arrangements that consisted of downlighting focussed onto a central table, and the same downlighting with low level wall lighting added. They also examined a condition with only wall lighting. "Light level" was controlled by keeping the horizontal illuminance on the table constant. From this study, they suggested that wall lighting seemed to produce more positive impressions, including making the room appear brighter. An additional study (Flynn, 1977) examining the addition of wall lighting to a limited area in the front field of view, found similar results.

The issue with this is that horizontal work plane illuminance is a terrible predictor of spatial brightness (Duff et al., 2015b). If we hold the lighting on the horizontal work plane constant, and add more light to the walls or ceiling, we will, unavoidably, increase the overall light level of the room. It is thus unsurprising if this then raises perceptions of brightness. In the study of Houser et al. they were holding the horizontal illuminance constant and changing the overall light level by changing the wall and ceiling illuminance. In this light we would expect that wall and ceiling brightness would have a stronger relationship with the overall room brightness, as they are the main changes in overall room light level. Because of this, none of these studies provide any evidence towards the walls having any particular importance towards the overall impression of the space.

\section{Van Ooyen et al. (1987)}

Another old paper that is occasionally referenced in discussions of the value of wall luminance is van Ooyen et al. (1987), who claimed (somewhat vaguely) that: "wall luminance contributes most to the way the room is experienced" (p. 155). However, the article is short, with limited detail on their methodology or analysis. For example, they report using 38 semantic scales to assess the lit environments, but do not actually say what the specific questions were. Basic summary statistics are likewise unreported. Furthermore, their reported results are about preferred luminances, rather than brightness, and judging by the reported methodology, the statement about the importance of wall luminance is most likely in comparison to desk luminance. It would hardly be surprising if the luminance of the walls was more important to the overall impressions of a room than the luminances of a few tables with substantially less surface area. And finally, it is not readily apparent that the claim about the importance of wall luminance is actually supported by the data presented in the article. The article should not be considered to provide any evidence for the hypothesis that wall luminance has particular importance to the impressions of a space. 


\section{Kirsch (2014)}

Kirsch (2014), in his first experiment, assessed the perceived brightness of a mock office space, comparing the effect of increasing the wall/ceiling luminances to increasing the surrounding area illuminance. Surrounding area illuminance varied from 100-300 lux, while wall/ceiling luminance ranged from $11-75 \mathrm{~cd} / \mathrm{m}^{2}$. As predicted, changing wall/ceiling luminance had substantially greater impact on overall room brightness than changing surrounding area illuminance. This does not, however, indicate that the walls or ceiling are of special importance to spatial brightness impression. The results are simply due to the fact that changing the level of illumination falling on a dark floor has very little impact on the overall light luminance level in the space. As he demonstrated, mean luminance provides a sound explanation for his results.

His, and a couple of other studies also suggest that taking the mean luminance from areas focussed around the walls provides slightly better predictions of brightness than the mean luminance of the broader field (the $40^{\circ}$ band for Kirsch (2014) and Van Den Wymelenberg (2012), the wall in front of the viewer for Iwai et al. (2001)). The way this effect appears to pop up in multiple studies may be indicative of something meaningful. At the same time, it is worth noting that the paper that originally proposed focusing on the central $40^{\circ}$ horizontal band (Loe et al., (1994) never actually had any evidence supporting that band in particular. They calculated the average luminance of multiple fields including the whole measurement area, the ceiling, the $20^{\circ}$ horizontal band, and the table top, and noted that "the correlation coefficients obtained were generally fairly similar (p123)". However, they focused on the $40^{\circ}$ horizontal band because they felt that it was "the most logical and influential because it encompasses the main area viewed when a person looks around the space (p123)". This despite the fact its correlation coefficient of 0.72 was not meaningfully different from that of the whole measurement area (0.71), or even the ceiling (0.70). More recently, Oi and Mansfield (2015), carried out further reanalysis of that data looking at $30^{\circ}, 40^{\circ}, 50^{\circ}$, and $60^{\circ}$ bands as well as the full $78^{\circ}$ image., They again showed that the correlation coefficients varied little, ranging from 0.7448 to $0.7577^{12}$, suggesting that the width of band had limited significance.

\section{Miscellaneous}

A recent study examining the effects of lighting the walls is de Vries et al. (2015). Unfortunately, in their study wall luminance co-varied with overall luminance, so the fact

\footnotetext{
${ }^{12}$ These correlation coefficients differ slightly from those originally reported in Loe et al. (1994). This appears to be because Oi and Mansfield chose to focus on just the responses on the bright-dim scale, while Loe et al. were looking at a composite measure of "visual lightness" based off the average of the bright-dim, radiant-gloomy, and spacious-confined scales.
} 
that adding wall lighting made the space brighter says nothing about the relative importance of the walls.

Loe et al. (1991) is also sometimes cited as saying that the floor is of relatively lesser importance. While we have been unable to track down the original paper in question, Mansfield (pers. comm (2016)) noted that in that specific study they did not consider the luminance of the floor because the participants could not see it - the tables blocked their view. In this light, it seems unlikely that the study could say anything about the general importance of the floor.

\section{Discussion}

In general, there is a lack of good evidence that the walls are of particular importance to the brightness impression of a space. The strongest evidence comes from a few studies finding slightly better correlations when they use luminances calculated from areas that prioritise the walls - which cannot be considered particularly strong evidence.

Nevertheless, the hypothesis may still be considered plausible. The role of position in the effects of glare is well established (Boyce, 2014; Hirning, 2014; Kim and Kim, 2010), and it would not be unreasonable to suggest that brightness could be similarly affected with the walls tending to appear brighter because they tend to lie in the centre of vision. Similarly, some studies looking at small flashed targets indicated that they appeared brightest when in the fovea (Greenstein and Hood, 1981; Osaka, 1980), though other studies did not find any effects (Pöppel and Harvey Jr, 1973; Zihl et al., 1980), and a recent review noted that there had been limited research on the subject (Strasburger et al., 2011).

Only a few studies have suggested that the ceiling may have more importance than the floor. Houser et al. (2002) and Kirsch (2014) do not, however, as previously discussed, provide any evidence that could support such claims.

Part of the problem here is that the idea that the walls are important actually has different meanings in different studies. The most pure form of the "wall importance" hypothesis - what we are interested in - would be to suggest that, all else being equal, the appearance of the walls (for example, their luminance) has a greater influence on the overall impression of the lighting in the space than the ceiling or the floor. Collins et al. (1990) speculated at this hypothesis, though they noted that their study did not provide any evidence on the subject. Many studies, however, when they make statements about the importance of walls, are looking at the issue from the perspective of horizontal work plane illuminance and standard lighting practice. Effectively, what they mean by "the walls are important" is that the walls can have significant effects on the overall impression of the space in ways not reflected by horizontal work plane illuminance. Others effectively just say 
that when the walls are the dominant part of the field of view, lighting them will be the most effective way to raise the mean light levels people observe. While these are valid design observations, they are not particularly pertinent to the question of whether or not the walls or luminances in the central field of view are more intrinsically more important to people's perceptions.

\subsubsection{Effects of pattern}

A few studies have suggested that the way the light is distributed throughout the space its pattern - may affect perceived brightness (Kato and Sekiguchi, 2005; Kimura et al., 2004; Kirsch, 2014; Moore et al., 2003).

Moore et al. (2003) in a field study of offices, found a correlation between brightness (5 point scale: "too dim" - "a bit dim" - "OK" - "a bit bright" - "too bright") and the "skew" of the luminance distribution (which they defined as the ratio between median and mean). Scenes with a positive skew had larger darker areas, and were perceived as dimmer, while the opposite was true for negatively skewed scenes. This could suggest that brightness is affected not only by the overall light level, and the overall amount of variance, but also by the pattern of the distribution. That being said, as an uncontrolled field study this should be considered at best "possibly indicative of something interesting". Due to its nature, it is hard to be sure that the results are not confounded with another unknown factor.

Additionally, we must note that the ratio between median and mean does not, technically, care how the luminance pattern is arranged (Figure 2.9). The extent to which this actually describes pattern effects is thus unclear.

\begin{tabular}{|lllll|lllll|}
\hline 1 & 1 & 1 & 1 & 1 & 10 & 10 & 10 & 10 & 10 \\
\hline
\end{tabular}

b)

\begin{tabular}{|l|l|l|l|l|l|l|l|l|l|}
\hline 1 & 10 & 1 & 10 & 1 & 10 & 1 & 10 & 1 & 10 \\
\hline
\end{tabular}

Figure 2.9 Illustration of pattern problems. Both of the "luminance" distributions shown above have the same mean, median, and skew, and uniformity ratio. Despite this, they are quite distinct. Does this matter for spatial brightness?

The question posed in Figure 2.9 was tackled by Kimura et al. (2004). They carried out an experimental study of how different arrangements of "windows" (fluorescent lights behind a white sheet) affected relative brightness, varying the spread and size of the "window" openings. Their first experiment looked at 8 different conditions divided into two sets: one with windows filling a $90^{\circ}$ horizontal field, and one with them filling a $180^{\circ}$ field. Windows were placed filling $2 / 3$ of the area, and the size spacing, and number of openings were varied. Their second experiment was focused specifically on the $180^{\circ}$ field, as that had shown a possible effect of window spacing in the first experiment. They had 10 conditions, with the windows covering between 1/2 - 5/6 of the area. Again, the size, 
spacing, and number of openings was varied. Their results suggested a trend where having large numbers of small windows spread around a room would make a space appear brighter compared to having one or two large windows of the same total area - at least if the "windows" are spanning a large fraction of the room (though it must be noted that due to them focusing on the $180^{\circ}$ field results, the $90^{\circ}$ lack of effect was not replicated).

The practical magnitude of the suggested effect is not assessable due to the study methodology (conditions were compared in brightness using a subjective scale of - 3 [very dark] to +3 [very bright]).

Later studies also pointed to spread out light sources making rooms appear brighter than equivalent condensed light sources. Kato and Sekiguchi (2005), in their 3rd experiment, examined a room lit through different combinations of the walls (for example, one wall lit, two walls lit etc.). Of particular interest here is the difference they found between the two "two wall" conditions - one lit from two adjacent walls, and the other lit from two opposite walls. Despite their mean luminance being the same, the room lit from two opposite walls appeared brighter. This again suggests that the pattern of light is also important to brightness. They demonstrated that the difference could be accounted for using their metric of directional diffusivity, if you smoothed the distribution over roughly $100^{\circ}$ before calculating uniformity. Under their model, this also implies that a large number of small light sources spread around a room is effectively more uniform than condensing all that light into one part of the room. This pattern was again supported by Kato and Hara (2011), comparing four different conditions with the light source spread out over progressively more openings (for the same total area and luminance). It was also more loosely supported by later work in Aya et al. (2015a), where they found that smoothing in this way improved the fit of their model (though they did adjust the area that they smoothed over).

Broadly, while not $100 \%$ supported by all the data (which is normal) the research of Kato et al. does seem to point, overall, to a pattern where the pattern of light sources in a space can affect how bright it appears, as illustrated in Figure 2.10 (though it should be noted that their work focused only on horizontal spread of the light around the walls of a room, and ignored vertical variation up and down the walls). 
Brighter

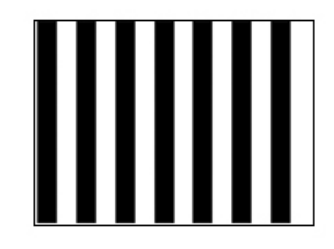

More uniform
Darker

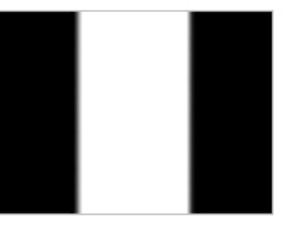

Less uniform

Figure 2.10 Illustration of the effects of pattern of light suggested in studies by Kato et al.

\subsubsection{Summary}

To sum up, based on the literature we can say the following:

- How uniform the light distribution in a space affects its brightness.

- The trend generally appears to be one where more uniform spaces appear brighter. However there is some disagreement in the literature, and the relationship may be more complicated than this.

- The magnitude of the effect may potentially be quite substantial, and may be able to have an impact equivalent to changing the luminance by as much as $50 \%$.

- The hypothesis that light in different areas may have different levels of effect in particular that the walls may have greater influence than other surfaces - is plausible. However, the literature does not provide sufficient evidence to support such claims. Further study specifically targeted at this area is needed.

- There is reason to suggest that the pattern of light distribution may affect brightness, however there are only a couple of studies pertaining to this. The main experimental finding here is that an array of light sources spread out around a room makes the room appear brighter (and in the relevant study effectively more uniform) than if the same area and intensity of light is focused into a single part of the room.

An additional point that should be made is that the various studies on the effects of light distribution use a wide range of metrics to quantify elements like uniformity - and in some (e.g. Tiller and Veitch (1995)) the change is not quantified at all. Given that these metrics do not always agree on whether or not a space is more or less uniform than another, this potentially creates problems for comparing results. 


\subsection{Models of the relationship between light distribution and spatial brightness}

With the evidence suggesting that light distribution can significantly affect spatial brightness, the next question is of prediction. What models have been proposed to predict the effects? What observations do they describe? How well do they work?

Several studies have proposed models of the relationship ${ }^{13}$. These range from simple linear correlations against convenience metrics (e.g. Hsieh, 2012; Kirsch, 2014; Ko et al., 2013), to more sophisticated attempts to construct new metrics (Kato and Sekiguchi, 2005), to attempts to apply psychophysical brightness models to spatial brightness (e.g. Kobayashi et al., 1998). We discuss these different models below.

\subsubsection{Kirsch: $\max / \mathrm{min}$ ratio in the $40^{\circ}$ horizontal band}

In two separate experiments, Kirsch (2014) presented two equations for predicting spatial brightness $(B)$. The first one simply predicted it from the mean luminance in the $40^{\circ}$ band $\left(L_{40^{\circ}}\right)$ :

$$
B=0.35 L_{40^{\circ}}^{0.63}
$$

The second predicted it from the uniformity ratio in the $40^{\circ}$ band:

$$
B=13.888 \cdot\left(L_{\max 40^{\circ}} / L_{\min 40^{\circ}}\right)^{-0.306}
$$

The problem we face in attempting to apply these is twofold: firstly, the equations are both from separate experiments. There is no combined equation that predicts brightness in terms of the combination of light level and uniformity. Secondly, his study measured brightness using 8 point semantic scales, with "visual lightness" defined as the average of three scales (bright-dim, radiant-gloomy, vague-distinct) following Veitch et al. (2010). This makes translating the "brightness" predicted by his equations into something concrete difficult - a brightness of " 5 " has no objective meaning, and the interpretation of such scales can vary significantly between different people and contexts (Tiller, 1990). An issue with rating scales is that people tend to adjust their judgements to fit the scale they are provided with. Hence, the range of stimuli they are exposed to affects their responses, with smaller ranges producing steeper slopes (Poulton, 1989). Indeed, following recommended practice (see Poulton, 1989), Kirsch deliberately showed his participants a high and a low anchor scene before the experiment proper in order to minimise response contraction bias. However, since the two different equations come from two separate experiments (and

\footnotetext{
${ }^{13}$ We restrict this discussion to studies that have actually proposed concrete equations to predict spatial brightness. Observations such as Moore et al.'s (2003) note that "skewness" appeared to have a statistically significant correlation with perceived brightness do not count, as they were not presenting it as any kind of usable model.
} 
indeed had different anchor scenes - the brightest and darkest in the first, and the most and least uniform in the second), we cannot be sure that participants were using the scales in the same way in both experiments. Kirsch $(2014$, p48) argues that by leaving participants free to decide how much of the response range they would use range equalising biases may be avoided. However, this may be a misreading of Poulton (1989). Poulton notes that: "In category rating, the [stimulus/response] range equalising bias is unavoidable (p226, 231)". The suggested strategy of allowing participants to freely choose their response range is for numerical magnitude judgements (p231). The 8 point semantic scales being used by Kirsch (2014) are a form of category rating, and provide a fixed response range to the participants. We may thus be concerned about the possibility of bias. The brightness ratings given may not necessarily be directly comparable across the two experiments.

With these caveats in mind, the best attempt we can make to translate his brightness model into something more objective is to try to predict the equivalent luminance shift caused by the different light distributions. To do this we need to equate the two equations. If we do so (Table 2.2) we find that the relative change in luminance required can be related to an equivalent change in uniformity ratio by the function:

$$
\text { Change in uniformity }=\text { change in luminance } e^{0.63 /-0.306}
$$

So, for example, if we had an arbitrary space with a brightness of " 5 " then we could increase its brightness to " 6 " by either:

a) Increasing the mean luminance (in the $40^{\circ}$ band) to $134 \%$ of its current level

b) Reducing the uniformity ratio in the $40^{\circ}$ band to $55 \%$ of its current level ${ }^{14}$

Table 2.2 Mean luminances and uniformity ratios in the $40^{\circ}$ band required for different room brightnesses according to Kirsch (2014). The percentages show the relative change in the metric for each scale point increase in brightness.

\begin{tabular}{|r|r|c|r|c|}
\cline { 2 - 5 } \multicolumn{1}{c|}{} & \multicolumn{2}{c|}{ Mean Luminance } & \multicolumn{2}{c|}{ Uniformity Ratio } \\
\hline Brightness & Value & Change & Value & Change \\
\hline 7 & 116 & $128 \%$ & 9.4 & $60 \%$ \\
\hline 6 & 91 & $134 \%$ & 16 & $55 \%$ \\
\hline 5 & 68 & $143 \%$ & 28 & $48 \%$ \\
\hline 4 & 48 & $158 \%$ & 58 & $39 \%$ \\
\hline 3 & 30 & $190 \%$ & 150 & $27 \%$ \\
\hline 2 & 16 & $300 \%$ & 563 & $10 \%$ \\
\hline 1 & 5.3 & - & 5421 & - \\
\hline
\end{tabular}

Similarly, if you double the uniformity ratio (making it more non-uniform) then this predicts that it should have an effect on brightness equivalent to reducing the light level by

\footnotetext{
${ }^{14} 134 \% 0^{0.63 /-0.306}=55 \%$
} 
$-29 \%$. Increasing the uniformity ratio by $900 \%$ has an effect like reducing the light level by $-67 \%$. Decreasing the uniformity ratio by $90 \%$ is like increasing the light level by $-200 \%$

(Figure 2.11).

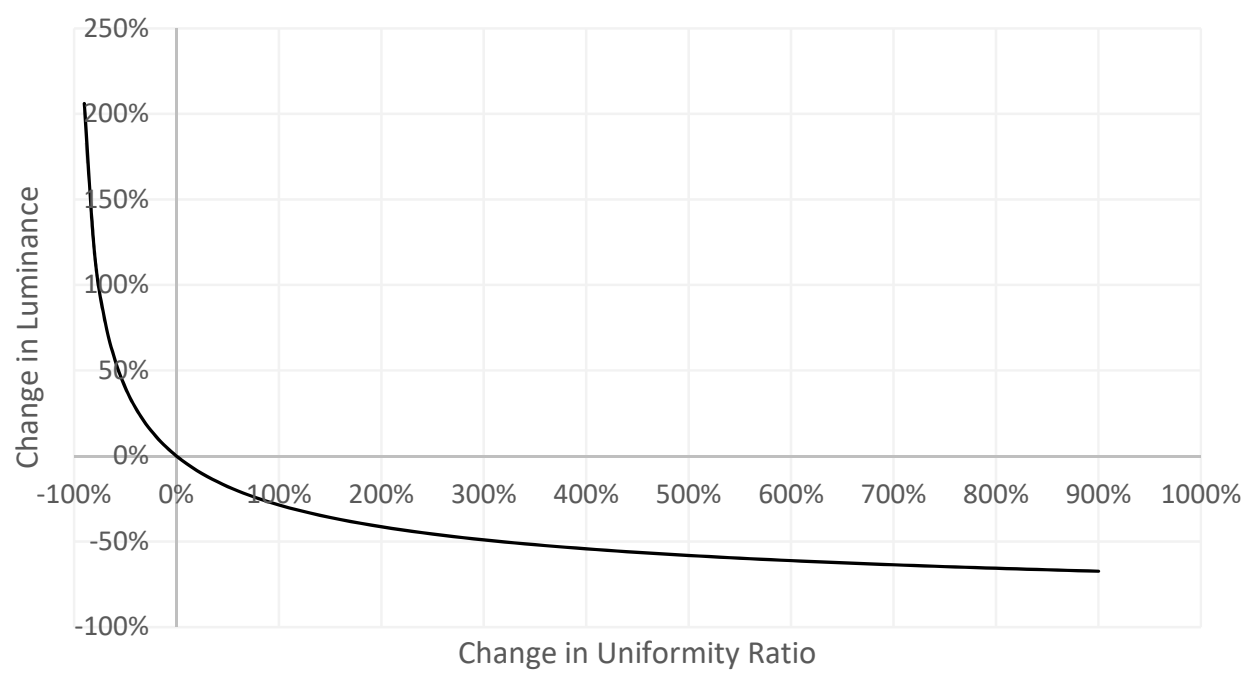

Figure 2.11 Effect of changes to uniformity ratio on spatial brightness in terms of equivalent changes in luminance according to Kirsch's model

To compare two conditions one can, thus, theoretically, use the following equation:

$$
\frac{L_{e f f 1}}{L_{e f f 2}}=\left(\frac{\text { uni }_{1}}{\text { unif }_{2}}\right)^{-\frac{0.306}{0.63}}
$$

Where $L_{\text {eff }}$ is the effective luminance, and unif is the uniformity ratio. The result of the equation tells us how much we need to increase the light level of condition 2 to match the apparent light level of condition 1.

In practice, we would also need to consider more than just the differences in uniformity. Kirsch's equations are based entirely off the $40^{\circ}$ horizontal band, and effectively assume that it is all that matters. When we equated the two equations above, we were actually describing the effect of uniformity in units of equivalent shift in the mean luminance of the $40^{\circ}$ band, rather than the mean luminance. The light distribution in any real space will vary vertically, and so the mean luminance in the $40^{\circ}$ band will not be the same as the overall mean luminance. Because of this, we need to also account for differences in the mean luminance in the $40^{\circ}$ band. To this this, we simply need to apply the principle that the luminance in the $40^{\circ}$ band is all that matters. Thus, if we compare two light distributions at the same overall mean luminance, and see that the mean luminance in the $40^{\circ}$ band $\left(\mathrm{L}_{40}\right)$ is twice as high in condition 1 as in condition 2 , that tells us that condition 
2 "should" need to have its light level doubled in order to match condition 1 in brightness. Hence, to compare the effective light level of two conditions we calculate:

$$
\frac{L_{e f f 1}}{L_{e f f 2}}=\frac{L_{40^{\circ}, 1}}{L_{40^{\circ}, 2}}
$$

To combine this with the previous uniformity effect calculation (Eq.2.4) to calculate the overall effect, we simply multiply the two effects together:

$$
\frac{L_{e f f 1}}{L_{e f f 2}}=\frac{L_{40^{\circ}, 1}}{L_{40^{\circ}, 2}} \cdot\left(\frac{\text { unif }_{1}}{u_{\text {unif }}}\right)^{-\frac{0.306}{0.63}}
$$

This makes the model theoretically something we could use to predict the effect of changing uniformity. However, there are issues. Kirsch's model has not, to our knowledge, been cross-checked in other spatial brightness studies. An older study, Moore et al. (2003), noted that the ratios of $\max / \mathrm{min}$ and $90^{\text {th }} / 10^{\text {th }}$ percentiles were not related to brightness in their study. As this was an uncontrolled field study of a range of office environments though this could be due to many reasons, and does not necessarily imply the model is wrong. Ko et al. (2013) did check the max/min ratio, and found it to have a poor correlation with their results, but they were examining the brightness of abstract patterns viewed on screens. The extent to which these challenge the use of the max/min ratio for spatial brightness is minor, but may be worth noting.

Perhaps more significant are the theoretical concerns about the robustness of the chosen metrics here (beyond the issues with equating the equations in the first place). While the maximum/minimum ratio is popular as a uniformity index in lighting, its validity as a theoretical model of human perception is doubtful. Formally, its use implies that we believe that that only the maximum and minimum luminance value matter, and that the arrangement of all the other luminances between these values is irrelevant. The max/min ratio model makes the strong prediction that, if we take a uniformly lit grey room, and paint a small white dot and a small black dot on the wall, that this will significantly change the appearance of the space (Figure 2.12). Unfortunately, this prediction has not, to our knowledge, been tested. 

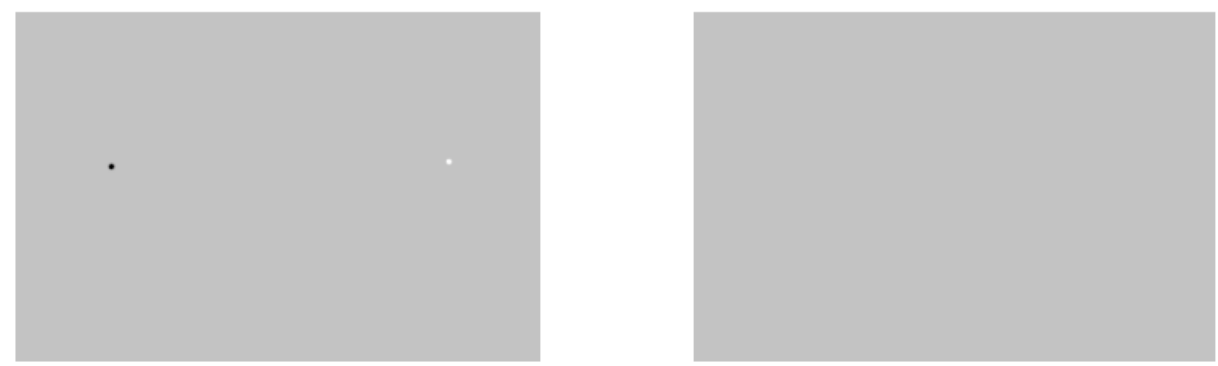

Figure 2.12 If these two rectangles were spaces, then according to the $\mathrm{max} / \mathrm{min}$ model of spatial brightness they should look dramatically different.

This is compounded by this particular model restricting assessment to the central $40^{\circ}$ horizontal band. The core assumption this makes is that any light outside of the $40^{\circ}$ band has no effect on people's perception of brightness (see Figure 2.13). This is a strong theoretical claim, and one that seems rather implausible given that a) we can clearly see things outside of that band, and b) glare can be produced by light sources in all parts of the visual field — and even potentially outside it (Boyce et al., 2003). As before, this assumption has not, to our knowledge, been directly tested.

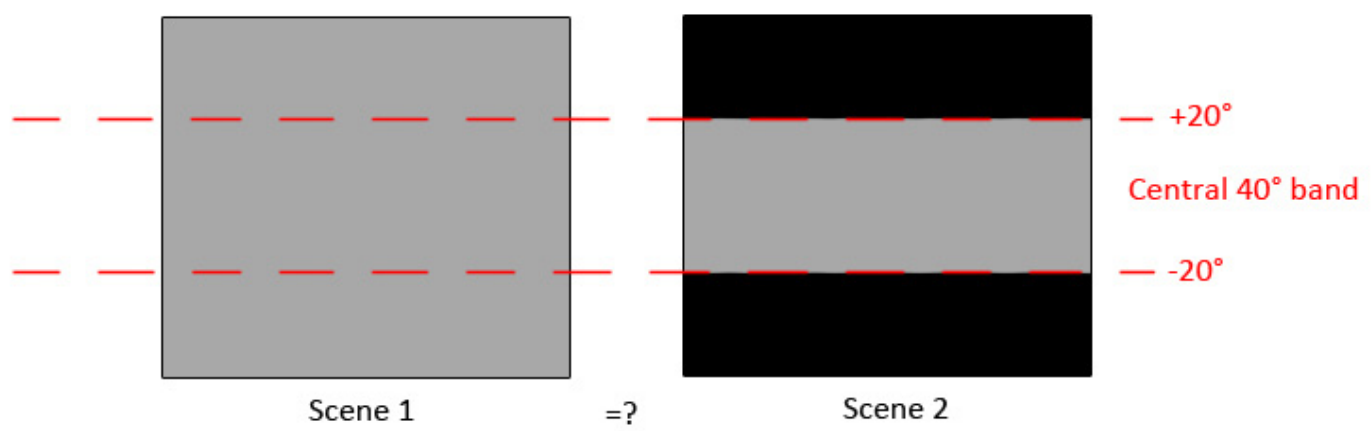

Figure 2.13 The use of the $40^{\circ}$ horizontal band assumes that these two "scenes" should be perceived as identical in terms of brightness as long as the luminances in the central $40^{\circ}$ band are the same.

Perhaps more plausible would be the idea that different parts of the visual field should be weighted differently (e.g. Kato and Sekiguchi, 2005). This would have precedent in the position indices of glare metrics (Boyce et al., 2003), and is more theoretically plausible than the notion that visual judgement has a sharp cut-off on the information it uses $20^{\circ}$ above the centre of vision.

These issues do not, of course, rule out the notion that the model may provide a reasonable approximation of the effects of light distribution in most real life scenarios. To prove this though would require us to either a) know what the true model is and establish that any errors caused by approximation are generally rare or minor, or b) to carry out a 
sufficiently large scale study to establish that this model works to predict most scenarios in practice. Neither of these have been done - the 6 different lighting arrangements in Kirsch (2014) are a good start, but are not enough on their own.

\subsubsection{Hsieh: $\mathrm{min} / \mathrm{mean}$ ratio of horizontal work plane illuminance}

Hsieh (2012) compared 14 different top lit distributions and assessed the horizontal illuminance levels required for them to appear equally bright to people. From this, they used linear regression to predict relative illuminance in terms of the sum of the uniformity ratio, front-rear symmetry, and left-right symmetry. This model attempts to account for both uniformity and, in some simple way, the pattern of light in descriptions of symmetry. They tried both the $\mathrm{min} / \mathrm{max}$ ratio and the $\mathrm{min} /$ mean ratio, and chose the min/mean ratio because it had a slightly better correlation $\left(\mathrm{R}^{2}=0.72 \mathrm{vs} 0.67\right)$ - though they did not report whether or not this difference was statistically meaningful. Cohen et al. (2003, p. 88) provide a formula for the confidence interval on the $\mathrm{R}^{2}$ value from a multiple regression, however it is only sound for samples of at least $n=60$ - much larger than the 14 conditions in this study. If there were 60 conditions then the confidence intervals would be $-0.54-$ 0.79 and 0.61 to 0.83 , which overlap substantially. Given that the lower sample size should have larger uncertainty, this suggests it would be hard to say there is any statistical difference between the two models. That being said, given that the set of conditions in the study is hardly a random sample, it may be questioned whether or not any of this statistical analysis is relevant. Comparison of the size of the actual errors in the models may be more useful, however such information is not provided.

Unfortunately, as the whole study is based off horizontal work plane illuminance its model is largely useless for spatial brightness prediction, as horizontal work plane illuminance is a terrible predictor of people's perceptions of spaces (Duff et al., 2015b), to say nothing of the low resolution of their measurements (a 12 point measurement grid). It may have worked in this specific situation as their conditions were all generated by punching holes in a replaceable ceiling panel that let light down into a model space. This arrangement directs light down towards the horizontal plane and will tend to have less of an effect on aspects like the balance of wall and ceiling illumination. Because of this, it would be unsurprising if the changes in light distribution in this particular experiment were reasonably well captured by the horizontal illuminance. However, we would expect it to fail if it was applied more broadly.

\subsubsection{Ko et al.: standard deviation of log luminance}

Ko et al. (2013) carried out several experiments looking at various gradient patterns presented on a computer screen using Magnitude Estimation. Their patterns varied in 
contrast, overall luminance, and the orientation of the gradient. Their results showed that the higher contrast (i.e. more non-uniform) patterns appeared darker. They regressed this against the standard deviation, coefficient of variation ${ }^{15}$, Michelson Contrast $^{16}, \max / \mathrm{min}$ ratio, and max-min difference. They found the best performance with the standard deviation of the log luminance $\left(\mathrm{R}^{2}=0.94\right)$, and proposed that the following equation could be used to predict brightness in rooms, accounting for uniformity:

$$
B=\left(21.5-8.4 S D_{\log }\right) * L^{0.33}
$$

Where $\mathrm{B}$ is brightness, $\mathrm{SD}_{\log }$ is the standard deviation of the $\log$ luminance, and $\mathrm{L}$ is the mean luminance. Luminance here is related to brightness by a power function following Stevens (1960).

This is a practical equation that directly combines uniformity and luminance to predict relative brightness. Moreover, we can calculate the equivalent change in luminance as a result of a difference in uniformity by rearranging the equation:

$$
\Delta L=\left(\frac{21.5-8.4 * S D_{\log 1}}{21.5-8.4 * S D_{\log 2}}\right)^{3}
$$

Where $\Delta L$ is the equivalent change in luminance, and $\mathrm{SD}_{\log 1}$ and $\mathrm{SD}_{\log 2}$ are the standard deviations of the two distributions being compared. For example, if $\mathrm{SD}_{\log 1}=0.2$, and $\mathrm{SD}_{\log 2}$ $=0.6$, then the equation tells us that the first distribution should appear to have $-175 \%$ as much light as the second.

The model implies that if the $\mathrm{SD}_{\log }$ exceeds 2.559 then the room/object will appear to have zero luminance. On one hand, this is an incredible claim. On the other hand, outside of highly shadowed brightly daylit spaces such conditions are unlikely to occur, as it requires the scene to have extremely substantial variation in luminance, spanning multiple orders of magnitude in both directions. Hence, even if it is formally wrong it may not necessarily stop the model from being a reasonable approximation in many circumstances.

In terms of evidence, the main limitation here is that the model was derived from a purely screen-based study using abstract patterns. How well it performs on real spaces is an open question.

\subsubsection{Kato et al.: Directional Diffusivity}

Kato and Sekiguchi (2005) developed a metric they called "directional diffusivity" as an attempt to describe their experimental results, which consisted of $360^{\circ}$ observations of spatial brightness in various rooms.

\footnotetext{
${ }^{15}$ Normalised standard deviation (SD/mean)

${ }^{16}(\max -\min ) /(\max +\min )$
} 
Calculating Directional Diffusivity involves several steps (Kato and Sekiguchi, 2005):

1) Collapsing the luminance map down to a 1-dimensional horizontal distribution by averaging over the vertical angles (weighted by angle $\left(\Theta_{v}\right)$ using the function $\left.\frac{1+\cos \left(\Theta_{v}\right)}{2}\right)$.

2) Smoothing the distribution over an assumed field of view $\left(100^{\circ}\right.$ wide)

3) Calculating the normalised mean absolute deviation $(m)$

4) Repeating (3) as if all the light came from one direction $\left(1^{\circ}\right)\left(m_{0}\right)$

5) Calculating Directional Diffusivity $(d)$ as $d=1-\frac{m}{m_{0}}$

The resulting metric is thus on a scale of $0-1$, where 1 is a completely uniform distribution and 0 is one where all the light comes from a single direction.

This model has then been found to provide good correlations with spatial brightness in the different studies they have carried out (Aya et al., 2015b; Kato et al., 2016; Kato and Hara, 2011).

Making predictions using predictions is relatively straightforward (at least once you've calculated Directional Diffusivity. The basic model they proposed (as of Kato et al., 2016) is:

\section{Brightness $\propto$ Directional Diffusivity $\times$ Mean Luminance}

Where brightness and the transformed luminance are on the log scale.

This means that any relative change in Directional Diffusivity is equivalent to the same change in mean luminance. Multiplying the Directional Diffusivity by $50 \%$ is the same as multiplying the luminance by $50 \%$.

There are a couple of theoretical questions about the assumptions of Directional Diffusivity. The big one regards the vertical averaging. Collapsing the vertical distribution to a simple horizontal distribution does greatly simplify its description, and allows for nice $2 \mathrm{D} 360^{\circ}$ plots of the horizontal variation around a room. However, the problem with this is that it implicitly assumes that vertical variation in luminance is largely unimportant (outside of differences created by the weighting function). The result of this is illustrated below, in Figure 2.14. The metric predicts that light distributions along these lines, where the contrast is heavily vertical, are, in fact, uniform distributions. Given that brightness contrast effects work perfectly well when the contrast is along the vertical (e.g. Diamond, 1955), and there is no evidence that luminance changes are only perceived horizontally, this assumption is doubtful. 


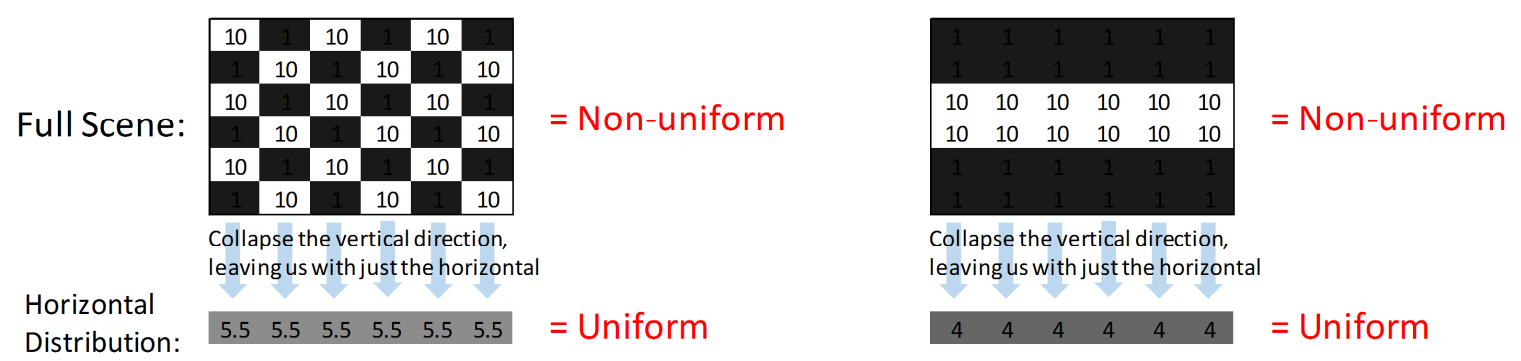

Figure 2.14 Effect of vertical averaging on light distributions with vertical non-uniformity

While their model has been successful in their various studies, its good performance can, perhaps, be largely explained by the conditions they have tested. The studies by Kato et al. have generally looked at varying the arrangement of lights and windows around the walls - i.e. horizontal variation - and in those situations the approach works adequately (Aya et al., 2015b; Kato et al., 2016; Kato and Hara, 2011; Kato and Sekiguchi, 2005). Situations where the differences in light distribution are primarily vertically oriented, such as those in Figure 2.14, or perhaps comparisons of uplighting versus downlighting, do not appear to have been tested.

The weighting of the luminances by vertical angle, while theoretically plausible (as discussed in Section 2.3.2), is also not clearly supported by the evidence. The weighting function derived in Kato and Sekiguchi gives a weight of 0.5 to the luminances at $90^{\circ}$ above or below the horizontal line of sight. While this did technically work, as discussed before (Section 2.3.2) their second experiment's results could be explained by differences in uniformity ${ }^{17}$ rather than needing a weighting function and their third experiment's results were the same regardless of whether or not the weighting function was used. As the rest of the studies were not designed to look at how luminances at different vertical angles affected spatial brightness, it is unclear that the function is supported. More recently (Yamaguchi et al., 2016), they proposed a weighting function of $m+(1-m) \cos ^{n}\left(\Theta_{v}\right)$ where they set $\mathrm{m}=0.1$ and $\mathrm{n}=1$. The use of this weighting function, along with other changes to how they combined mean luminance and directional diffusivity to estimate brightness, provided improved performance on their 2016 results. However, it is unclear how well it works on earlier data, and it still is not derived from a focused study of the effects of light at different angles.

Lastly, there is the use of smoothing. Smoothing the distribution over an assumed field of view the way they suggest is a reasonable way to try to account for the patterns they observed in their results. The one concern here is undiscussed variation in the angle smoothed over. In Kato and Sekiguchi they smoothed over $100^{\circ}$. In Aya et al. (2015a) they

\footnotetext{
${ }^{17}$ At least, if you don't ignore vertical variation
} 
then explored smoothing over $15^{\circ}, 30^{\circ}, 60^{\circ}, 90^{\circ}$, and $120^{\circ}$, and appeared to go with $30^{\circ}$ as the best result. Then, in Kato et al. (2016) they returned to $100^{\circ}$. This lack of consistency raises concerns about the reliability of the approach.

Overall, the directional diffusivity metric is an interesting one, and is the only one to see investigation in multiple studies. It attempts to account for uniformity, location, and pattern effects of light distribution. However, questions about some of the assumptions underlying the model and the degree to which some of the decisions are supported by the evidence may reduce confidence.

\subsubsection{Kobayashi et al.: Power Functions}

Kobayashi et al. (1998) attempt to apply psychophysical power functions following Stevens and Stevens (1963) to predict the effects of different light distributions on spatial brightness. Stevens' power function is:

$$
\text { Brightness }=k\left(L-L_{0}\right)^{\beta}
$$

Where $k$ is a constant, $L$ is luminance, $L_{0}$ is threshold luminance, and $\beta$ is the exponent of the power function, equalling roughly 0.33 for the dark-adapted eye. It should be noted here that $k, L_{0}$, and $\beta$ all vary depending on adaptation level.

To apply this, Kobayashi et al. assume the adaptation level is equal to the average luminance of the scene. They then divide the scene up into areas of different luminance, calculate the brightness of each area, and then calculate the overall average brightness.

The result of this is a function that predicts that increasing non-uniformity will, in general, result in a decrease in overall brightness for the same luminance. This is simply due to the nature of the power function. For example, consider a uniform distribution with a luminance of $100 \mathrm{~cd} / \mathrm{m}^{2}$. We make the distribution non-uniform by increasing half of it by $50 \mathrm{~cd} / \mathrm{m}^{2}$, and lower the other half by the same. The mean luminance is thus still 100 $\mathrm{cd} / \mathrm{m}^{2}$. The brightness of the uniform space will, according to Stevens, be 11.38 brightness units. The brightness of the non-uniform space will be $(13.35+8.66) / 2=11.01$ brightness units - lower than before. The power function means that the relationship between brightness and luminance is non-linear (Figure 2.15). In this case, because the exponent is less than 1 it means that the higher the luminance is the more you need to get the same gains to brightness. Thus, increasing the luminance by $\mathrm{X}$ has a smaller effect on brightness than decreasing it by $\mathrm{X}$, and so the aggregate effect of non-uniformity tends towards reduced brightness. 


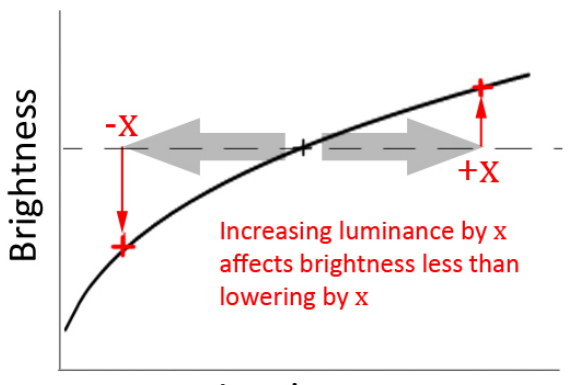

Luminance

Figure 2.15 Illustration of the non-linear Brightness/Luminance relationship, and what it means

Attempting to estimate brightness in this way has a strong theoretical appeal, in that it is based on applying known relationships between brightness and luminance rather than just grabbing a convenient uniformity metric and hoping it happens to approximate human perception. A limitation of this most simple approach, however, is that it fails to account for the more complex contextual effects of human brightness perception - specifically, the effects of contrast.

Contrast effects are well established (Gilchrist, 2006), with perhaps the simplest illustration being the classic simultaneous brightness contrast display (Figure 2.16). Perception of brightness does not simply depend on the luminance of the object in question, but also on how it relates to the surrounding luminances. Simply applying the power function to a luminance map as in this model fails to account for this at all. Given that such effects could potentially be highly relevant to the effect of non-uniformity, this could be a significant limitation. That being said, one could also, perhaps, suggest that in aggregate such effects will tend to cancel out over a whole scene, and thus the average brightness can be reasonably estimated via this approach.

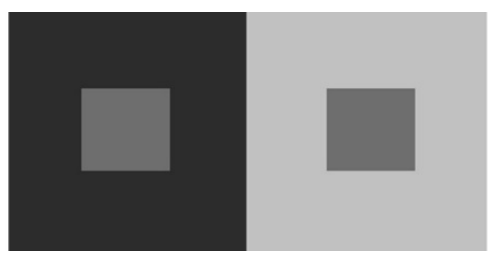

Figure 2.16 Simultaneous brightness contrast. The target on the left looks brighter, though both of them have the same luminance.

The evidence presented by Kobayashi et al. (1998) for the success of their model is also not as strong as it may appear at first glance. After their initial screen based experiment which we have already discussed (Section 2.3.1) they attempted to check their proposed brightness model on a wider selection of real spaces where it performed slightly better than $\log$ mean luminance (raising $\mathrm{R}^{2}$ from 0.48 to 0.55 ). However, much of the same effect can 
be achieved by simply using a power function of mean luminance to predict brightness instead of the logarithm (Figure 2.17).
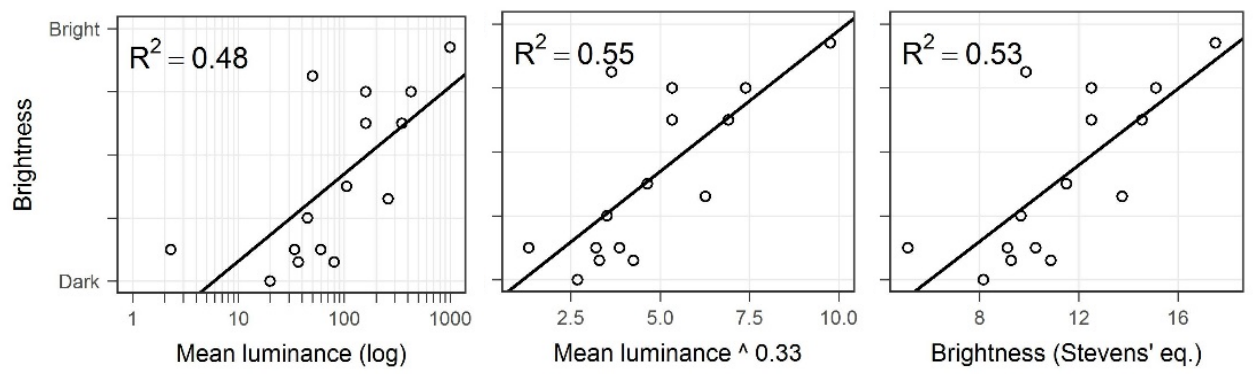

Figure 2.17 Visual reproduction of Kobayashi et al.'s results (left), illustrating how merely transforming mean luminance via a basic power function (mid) or Stevens' equation (right) can provide nearly all of the reported gains that their model did - without having to account for light distribution.

The model's apparent improvement over the use of mean luminance in their field study may simply be because a power function works better to describe the relationship between mean luminance and brightness here, and not because their model has captured the effects of light distribution. This makes it questionable whether or not their second experiment provides meaningful evidence that their model has captured light distribution effects. Without the second experiment, the evidence relies on a screen-based study with a very limited range of conditions.

\subsubsection{The Marsden Equation}

The Marsden Equation (Marsden, 1970) was derived from a lighting study that looked at how we could predict the brightnesses of different surfaces in a room, following the psychophysical relationships that had been discovered by researchers such as S.S. Stevens (e.g. Stevens, 1961). The rough relationship that was suggested was a two part equation. Firstly, the brightness of the brightest surface in the room could be predicted with a simple power function of its luminance:

$$
B_{\max }=L_{\max } 0.35
$$

And following from that, the brightness of the other surfaces would be calculated relative to the brightest surface:

$$
B=\frac{B_{\max }}{L_{\max }{ }^{0.6}} \cdot L^{0.6}
$$

Technically, it is not a model of spatial brightness, and it is questionable whether or not it should be discussed here. However, Zhang and Julian (2012) did use it to calculate spatial brightness in their study, and its simple framework can be adapted to calculate average brightness of a scene fairly easily. All one needs to do is define the "brightest 
surface", using perhaps the maximum or $99^{\text {th }}$ percentile luminance region, and then the brightness of everything else can readily be calculated relative to that using Eq.2.11. The resulting brightness map can then be averaged to estimate the overall brightness.

In general, the Marsden Equation predicts that more non-uniform spaces should appear darker. This follows simply from a) the power function effects discussed previously; and b) its modelling of most of the brightnesses as being relative to the brightest area. Under these equations, the brighter the brightest area is, the darker the rest of the space will appear. A possible issue here is that the equations ignore the opposing effects - that the darker areas will make the brighter regions look brighter. Such a bias may be justified by the known asymmetry in the strength of contrast effects (Rudd, 2013). That being said, ignoring such effects completely could be going too far.

The model does not account for the different local contrast effects that individual surfaces may be influenced by. It may be argued, however, that these local deviations will tend to cancel out over the whole space. While some areas might be underestimated, and others overestimated, on average, the brightness calculated may be roughly correct which is all we need to estimate spatial brightness.

The biggest issue here is that the Marsden Equation has not, to our knowledge, ever been tested as a way of assessing spatial brightness of rooms with different light distributions ${ }^{18}$. Thus, its performance in this regard is unknown.

\subsubsection{Comparison of the models' predictions}

All the models we have discussed predict that non-uniformity of the light distribution should make spaces appear darker. This does not mean, however, that they all agree on the effects beyond that. To explore this, and to examine how confident we could be in any prediction, we compared the predictions of the different models. To do this, we used $R(\mathrm{R}$ Core Team, 2016) to generate random "light distributions" — sequences of 10 numbers constrained to have a maximum $\mathrm{max} / \mathrm{min}$ ratio of 60 , and a mean of $10 \mathrm{~cd} / \mathrm{m}^{2}$. The models were then used to predict how much light each "distribution" would need to match the brightness of a uniform distribution of the same mean luminance ${ }^{19}$.

\footnotetext{
${ }^{18}$ Zhang and Julian (2012), in a study of gloom, do attempt to calculate mean brightness using the Marsden Equation, however their 4 conditions all differed significantly in mean luminance. Hence, their reported results just say that, in general, spaces with significantly more light tend to appear brighter, and it tells us nothing useful about the validity of applying the Marsden Equation in this way.

${ }^{19}$ Note that we are ignoring position effects in these simple distributions, essentially assuming that there is no vertical variation. Elements such as the focus on the $40^{\circ}$ band in Kirsch's model, and the vertical weighting in the Kato et al. model are thus not considered.
} 
Despite generally correlating with each other, the models vary wildly in their predictions (Figure 2.18). Directional Diffusivity and the Kobayashi et al. application of the Stevens' equation predict the smallest effects, predicting that up to around $+75 \%$ more light would be needed for the most non-uniform distributions generated. The Ko et al. log SD model and the Marsden equation trend larger, predicting up to around 300\% more light could be needed. The Kirsch max/min model makes extreme predictions compared to the other models, predicting that in some cases the distributions could require up to $1500 \%$ more light for equal brightness. This may be an indication that relying entirely on the maximum and minimum values of a distribution is risky.
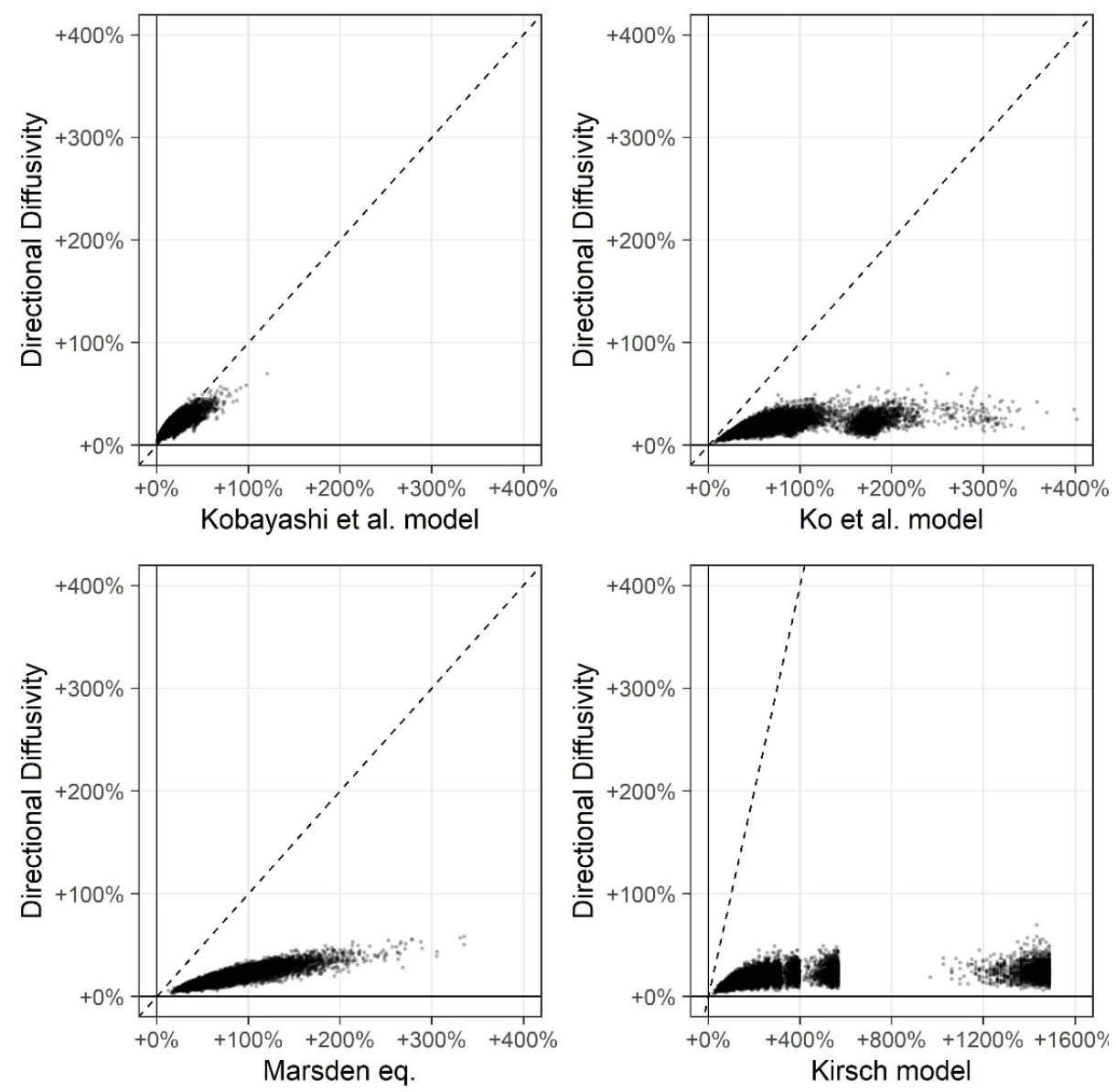

Figure 2.18 Comparison of the light required for random luminance distributions to match the brightness of a uniform distribution of the same luminance according to different models. The Directional Diffusivity model was used as the arbitrary baseline to compare the others to on the grounds that it was the one that had been used in the most studies.

Even if we zoom in on the models that are closest to each other in magnitude (Figure 2.19), we still see substantial disagreement of up to 50 or even $200 \%$. This makes it difficult to have confidence in any individual prediction. 
This comparison, simple though it is, demonstrates our current inability to make confident predictions of the effects of light distribution. When the estimated effect of a given distribution could range from anywhere between 20 to $1500 \%$ more light being required to match another's brightness, it is rather difficult to say that we have a good understanding of the matter.
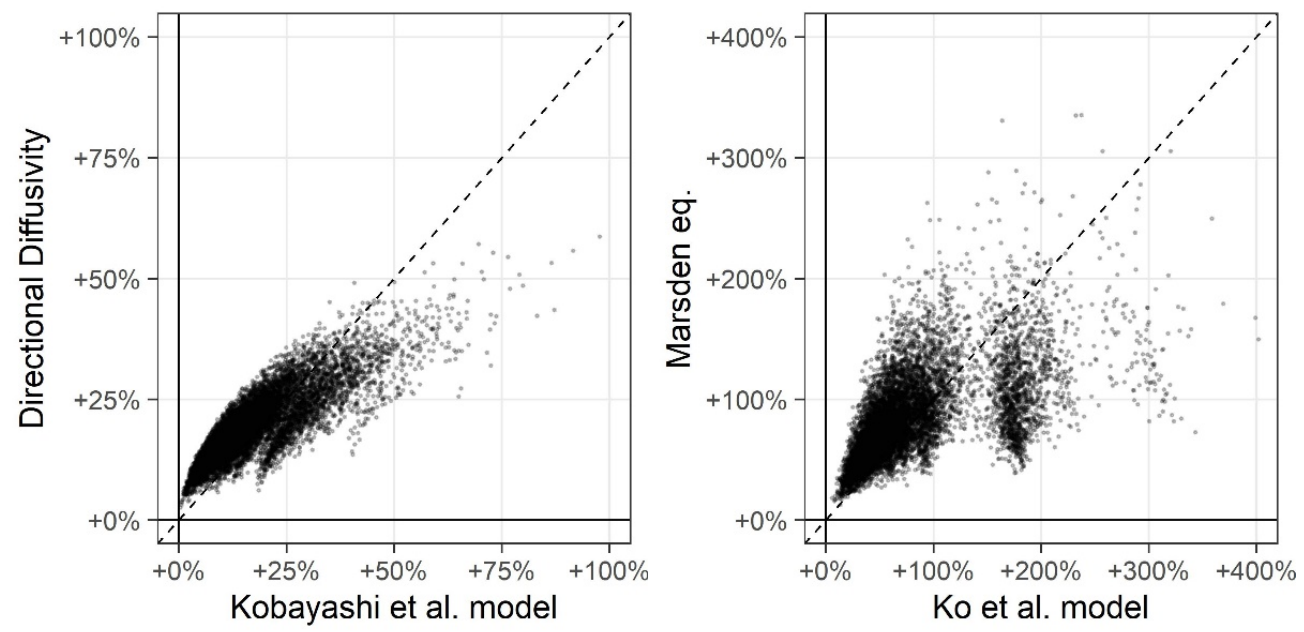

Figure 2.19 Close-up comparison of selected models with predictions of similar magnitudes.

\subsubsection{Discussion}

Overall, while a number of interesting ideas and possibilities have been proposed, it is difficult to say that any model is strongly supported. Models are largely based on single studies, and often make questionable assumptions. In part, this may be blamed on the small size of the field and the limited amount of research that has been done. However, it is not helped by the fact that the studies that have been done generally do not consider other models in the literature. Studies simply present statistical results for whatever their favoured metric is, without examining the performance of alternative models (or at least if they do, they do not report it e.g. (Hsieh, 2012; Kato and Sekiguchi, 2005; Kirsch, 2014; Kobayashi et al., 1998)). Thus, there is a lack of cumulative development and testing. This also makes it difficult to say that any individual study's results could not also be explained by alternative models. The only study here that does report multiple metrics is Ko et al. (2013), and the only metric that they have in common with the other studies here is $\max /$ min luminance ratio. It performed relatively poorly in their study, with a correlation coefficient of 0.31 and $\mathrm{R}^{2}$ of $0.096(\mathrm{~N}=51, \mathrm{p}<0.05)^{20}$, explaining only $-10 \%$ of the

${ }^{20}$ Unfortunately, Ko et al. did not plot the metrics they rejected, or present statistical information beyond correlation coefficients, making it hard to evaluate how large the errors were in practical terms. That being said, we may note that the plot of their chosen model, with a correlation of 0.89 , showed residuals of around $10-20 \%$ in predicted brightness. Converting that to equivalent luminance shifts using their exponent 
variation in brightness. This could be said to be a challenge to Kirsch's use of the metric, though this must be tempered with the fact that Ko et al. were not actually assessing spatial brightness.

Model development would be assisted if researchers tested each other's models in their papers, as well as if they made their results and light distribution measurements openly available to allow new models to be tested on them. It is difficult to say that any one model here is clearly "correct", and predictions vary wildly. Correspondingly, any attempt to estimate the likely effect of a given light distribution on spatial brightness will be highly uncertain.

We may also note some other common limitations here. From the observed effects of light distribution discussed in Section 2.3, the models are largely focused on describing uniformity. Potential effects of location and pattern are less developed. The Kirsch model could said to approximate possible effects of location, by placing less (read no) importance on the higher and lower angles. The most sophisticated model in this regard is Kato et al.'s Directional Diffusivity model which actually weights the luminances by vertical angle (though the degree to which this is supported is questionable). It is also the only one to actually attempt to address the question of pattern, in this case by smoothing the distribution before calculating uniformity. There is clearly a need for more studies to examine these questions, and develop well founded models. The various biases the studies' methods are prone to (see Poulton (1989) and previous discussion) could also affect the apparent size of the estimated effects, and thus the reported model coefficients. Hence, even if one of these models is "correct", it may not accurately predict the size of the differences between light distributions in other situations.

The final point to be made is that all the models that have been proposed here say that uniform spaces should appear brighter than non-uniform ones. This is, it should be noted, also consistent with our knowledge of basic brightness perception as shown through the power function, and the asymmetrical effects of contrast (dark areas have their brightness reduced by brighter surrounds to a greater degree than the bright areas are enhanced (Rudd, 2013)). This correspondence provides further support to the position that uniform light distributions should appear brighter.

of 0.33 , it suggests errors equivalent to $-+33 \%$ or more change in overall light level. This is arguably already substantial, and the max/min uniformity ratio had a substantially worse fit than that model, so its errors would be expected to be even worse. 
Table 2.3 Summary of models for light distribution and spatial brightness proposed by the literature and the strength of the evidence behind them

\begin{tabular}{|c|c|c|c|c|}
\hline Model & \# studies & Describes & 3D spaces? & Questionable assumptions \\
\hline $\begin{array}{l}\text { Kirsch: } \max / \min \\
\text { in } 40^{\circ} \text { band }\end{array}$ & 1 & $\begin{array}{l}\text { Uniformity } \\
\text { Location }\end{array}$ & Yes & $\begin{array}{l}\text { Only the maximum and } \\
\text { minimum luminance are } \\
\text { needed to describe a light } \\
\text { distribution. People do not } \\
\text { attend to luminances outside } \\
\text { the } 40^{\circ} \text { horizontal band. }\end{array}$ \\
\hline $\begin{array}{l}\text { Hsieh: } \\
\text { min/mean } \\
\text { illuminance } \\
\text { ratio }\end{array}$ & 1 & $\begin{array}{l}\text { Uniformity } \\
\text { Pattern? }\end{array}$ & $\begin{array}{l}\text { Yes (though } \\
\text { models) }\end{array}$ & $\begin{array}{l}\text { Assumes horizontal work plane } \\
\text { illuminance adequately } \\
\text { describes the perceived lit } \\
\text { environment. }\end{array}$ \\
\hline $\begin{array}{l}\text { Ko et al.: } \\
\text { standard } \\
\text { deviation of log } \\
\text { luminance }\end{array}$ & 1 & Uniformity & $\begin{array}{l}\text { No (abstract } \\
\text { patterns on } \\
\text { screens) }\end{array}$ & \\
\hline $\begin{array}{l}\text { Kato et al.: } \\
\text { Directional } \\
\text { Diffusivity }\end{array}$ & 4 & $\begin{array}{l}\text { Uniformity } \\
\text { Location } \\
\text { Pattern }\end{array}$ & Yes & $\begin{array}{l}\text { Assumes vertical luminance } \\
\text { variation is unimportant. }\end{array}$ \\
\hline $\begin{array}{l}\text { Kobayashi et al.: } \\
\text { power functions }\end{array}$ & 1 & Uniformity & $\begin{array}{l}\text { Maybe (the } \\
\text { field study } \\
\text { may not } \\
\text { provide } \\
\text { much } \\
\text { evidence) }\end{array}$ & $\begin{array}{l}\text { Ignores the existence of } \\
\text { contrast effects, and the way } \\
\text { that the brightness of objects is } \\
\text { affected by the relative } \\
\text { brightness of surrounding } \\
\text { elements. }\end{array}$ \\
\hline $\begin{array}{l}\text { Marsden } \\
\text { Equation }\end{array}$ & 1 & Uniformity & $\begin{array}{l}\text { No (for } \\
\text { spatial } \\
\text { brightness) }\end{array}$ & $\begin{array}{l}\text { Does not specifically account } \\
\text { for local contrast effects, } \\
\text { though overall effects may be } \\
\text { approximated as being mainly } \\
\text { relative to the brightest } \\
\text { element in the room. }\end{array}$ \\
\hline
\end{tabular}




\subsection{Overall summary}

To conclude: What do we know about the relationship of light distribution and spatial brightness?

In brief:

- The light distribution appears to affect spatial brightness - with effects potentially equivalent to a $50 \%$ change in light level. That being said, studies have been focused more on correlations than practical quantification of differences, and more work looking at magnitude could be helpful.

- Spatial brightness is (probably) strongly affected by uniformity, may be affected by position of light, and is probably affected by the pattern of light.

- The most studied effect is that of uniformity. Most studies suggest that non-uniform spaces should appear darker than uniform ones. There are some exceptions, but their evidence is relatively weak.

- It is commonly argued that light on the walls, or near the line of sight, is more important to people's perceptions of the space. However, while this is theoretically plausible, the evidence for it is lacking.

- A few studies suggest that arrangements of lights that spread them around the space appear brighter than ones that condense the same light into a single area.

- Our ability to model and predict the effects of light distribution is questionable. While a number of models have been proposed, they are largely based on single studies with no further testing. Most are focused on uniformity, with only one (directional diffusivity) addressing all three aspects of light distribution. Unfortunately, that model, while arguably the best supported, also makes significant assumptions that have not been rigorously tested. The models also disagree substantially in their predictions, leaving us with no good estimate of the effect of any given distribution.

Research into the effects of light distribution largely views it, and attempts to model it, through the lens of luminance. Qualitative elements of appearance, such as whether or not the luminance is a light source, a dark wall, or a shadow for instance are treated as irrelevant. Overall, the question of light distribution is clearly important, with it having significant ability to shape how bright a space appears. Despite this, the literature on the topic is relatively small, and there is a clear need for more research. In particular, there is a need to develop and refine our ability to predict the likely outcome of changing the light distribution, and to test the different models that have been proposed in the literature. 



\section{Experiment 1: In which we estimate the practical impact of non-uniformity and upend all prior predictions}

The literature presents us with a problem. While we have an idea of the likely direction of uniformity effects (more uniform = brighter), we have a very poor understanding of the magnitude beyond "probably significant". Most studies, with the exception of Tiller and Veitch's (1995) brightness matching one, fail to even engage with the issue of magnitude at all. While we can, as demonstrated, convert the various models in the literature to provide us with estimates in units of equivalent luminance, these can vary dramatically. Moreover, as the different models have not been compared against each other or tested in new studies we have no ability to refine our estimates any further.

In simple terms: we cannot usefully estimate the likely effect of any novel light distribution. Indeed, even many of the distributions that have already been studied would be difficult to assess because their effects were not really measured in those terms (e.g. Kato and Hara, 2011; Kato and Sekiguchi, 2005).

With this in mind, the original goal of this first experiment was to acquire a better understanding of the magnitude of the effects of a range of light distributions. Following Tiller and Veitch (1995), this was to be done using a brightness matching task so as to avoid the issues around the subjective interpretations of scales and their biases, and to focus on the practical effects of light distribution in units of equivalent luminance. Combined with detailed measurements of the light distribution using HDR luminance mapping (Inanici, 2006), these could then also be used to compare the performance of different uniformity metrics and the models from the literature. Additionally, due to the discussion in the literature about the relative importance of the walls, ceiling, and floor, it was 
considered desirable to create the non-uniform distributions by varying the relative luminance values of those surfaces.

This goal, however, changed as a result of the small pilot study reported in Sullivan and Donn $(2016)^{21}$. Unexpectedly, analysis of the pilot indicated that the effects of the conditions might be both substantial and in the opposite direction to what would predicted from the bulk of the literature. This supported the minority viewpoint presented by the findings of Tiller and Veitch (1995) - that non-uniform spaces appeared brighter — and added emphasis to what had the potential to be a significant controversy in the literature.

In light of this, the design of Experiment 1 was altered. The major concern was why the pilot results were so at odds with what would be predicted from the majority of the literature. One possibility was the notion that it could be due to methodological issues. A notable difference between the various studies was how they measured spatial brightness. The studies arguing that non-uniform spaces appear brighter — ours, Tiller and Veitch $(1995)^{22}$, and Okajima and Fujimoto (2008) all used brightness matching. In contrast, the studies arguing that uniform spaces appear brighter all used various forms of subjective rating scale (Aya et al., 2015b; Kato et al., 2016; Kato and Hara, 2011; Kato and Sekiguchi, 2005; Kobayashi et al., 1998).

With that in mind, it was felt that it would be useful to check if the results of the pilot would replicate using multiple methods. Thus, the main experiment was carried out using brightness matching as before, as well as adding Magnitude Estimation. Magnitude Estimation was chosen as it was the most common form of subjective brightness scale used in these studies (e.g. Aya et al., 2015; Kato et al., 2016; Kobayashi et al., 1998), and because it produces a readily interpretable quantitative ratio scale whose estimates could be compared with the results of the brightness matching study.

Thus, the focus of Experiment 1 shifted from the initial exploratory goals to testing two specific hypotheses:

1) That the pilot study's results are able to be reliably produced, and thus that the apparent increased brightness of the non-uniform conditions are not happenstance.

2) That we would get the same effects using both brightness matching (following our pilot study and Tiller and Veitch (1995)) and magnitude estimation (following

${ }^{21}$ For more details on the pilot study, see Appendix B.6

22 Tiller et al. (1995), in their replication of Tiller and Veitch also cross-checked their results using forced-choice as well as brightness matching, and their results were consistent across both methods. Unfortunately, the study was only ever reported in a CIE conference paper, and a copy had not been located at the time this experiment was being designed. 
Kato et al.'s studies) and so reject the hypothesis that the apparent disagreements between studies are due to that difference in methodology.

\subsection{Experimental design}

The basic framework set out by a spatial brightness matching experiment is the comparison of two different spaces, with participants required to adjust the light level in one of the spaces (the "Test" space) until it appears to have the same overall brightness as the other "Reference" space. Within this framework, the design of the experimental conditions was a product of a number of constraints:

- Different light distributions needed to be able to be swapped out and viewed by participants in a timely manner

- Dimensions needed to allow participants to place themselves "inside" the room, with their eyes at a normal seeming height (i.e. not right by the floor or ceiling)

- Participants needed to be able to comfortably observe and compare the different spaces

- As electronic dimming of lamps is known to result in colour shifts (which could affect apparent brightness), physical dimming was to be used instead following Fotios and Cheal (2011)

- In order to allow "fair" comparisons between the effects of the walls, floor, and ceiling the different surfaces needed to be of the same area

The resulting set-up is illustrated below (Figure 3.1). Models were made 300mm high in order to fit a person's head. This then defined the remaining dimensions of the spaces. Made out of foamboard, the models, while large, were still light enough to move around easily. The angled openings allowed the participants to easily view and compare both spaces sitting side by side. Light to each space was provided by two compact fluorescent lamps ${ }^{23}$, diffused through a polycarbonate sheet and white diffusing box before being released into the space. Light level was controlled using a shutter system to change the amount of light being released into the diffusing box. Participants controlled the shutters for the Test condition using a dial connected by a gear and chain-drive system (Figure 3.2, right). Diffusers ensured that the changing shutter positions did not change the distribution of the illumination viewed by the participants.

\footnotetext{
${ }^{23}$ Specifically Philips Tornado "Cool daylight" ( $\left.5500 \mathrm{~K}\right)$ 24W 1450 Lumens
} 


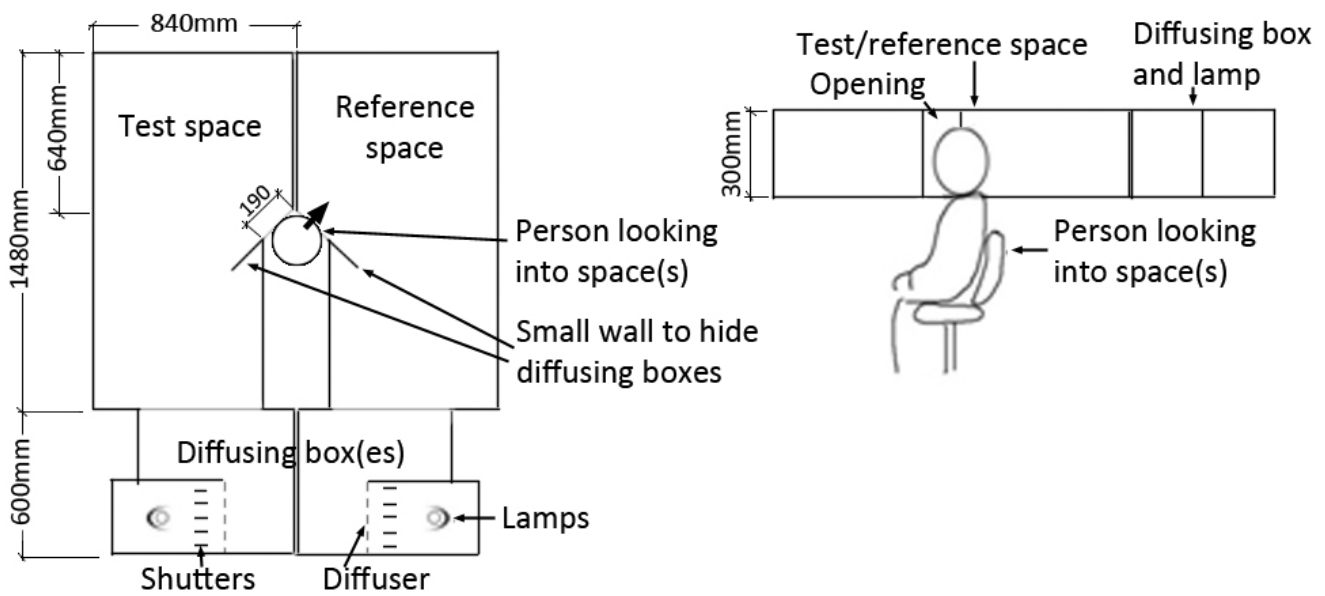

Figure 3.1 Plan and side view diagrams illustrating the experimental set-up

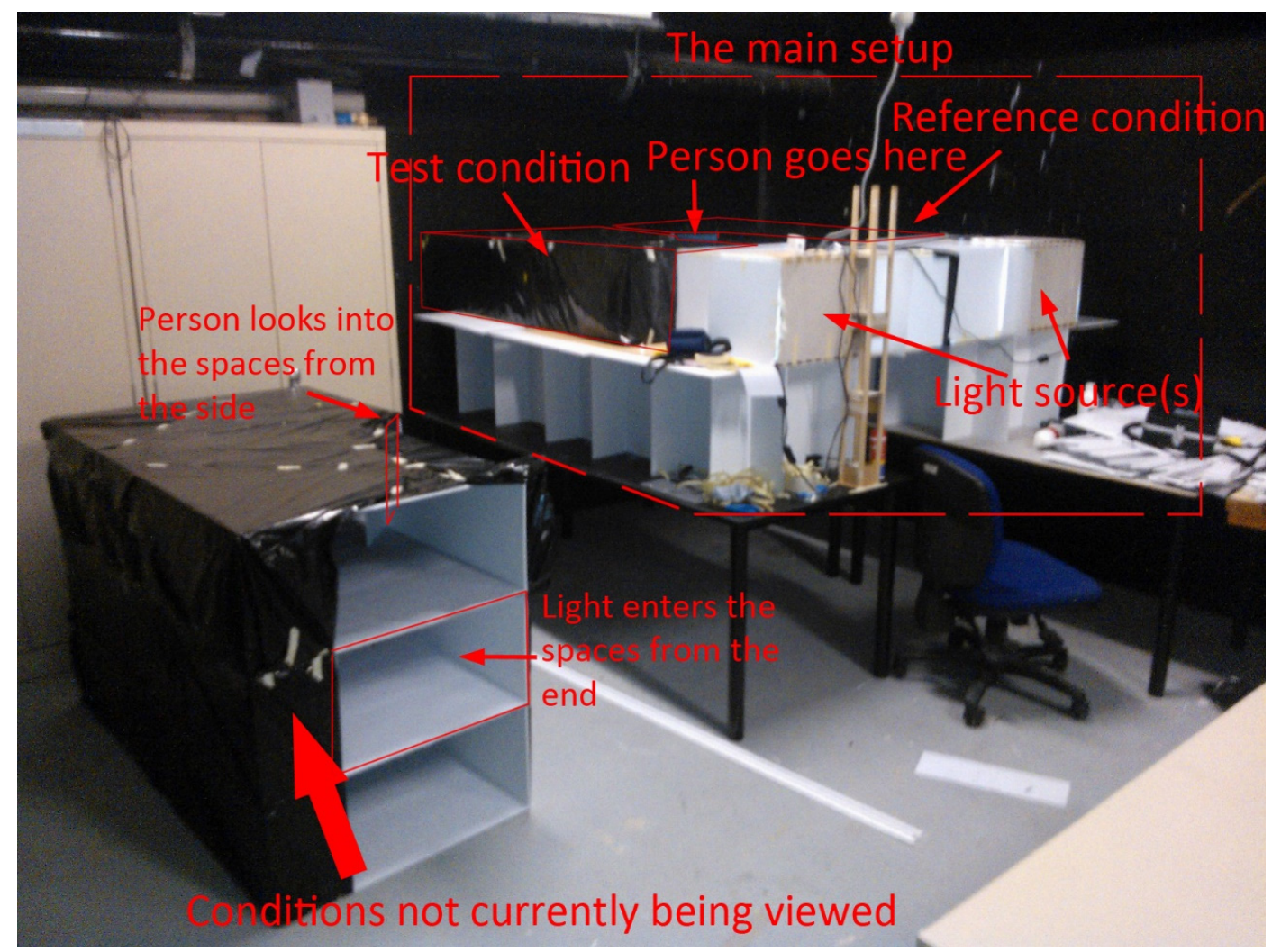

Figure 3.2 Photo taken during construction of the setup described in Figure 3.1 above. 

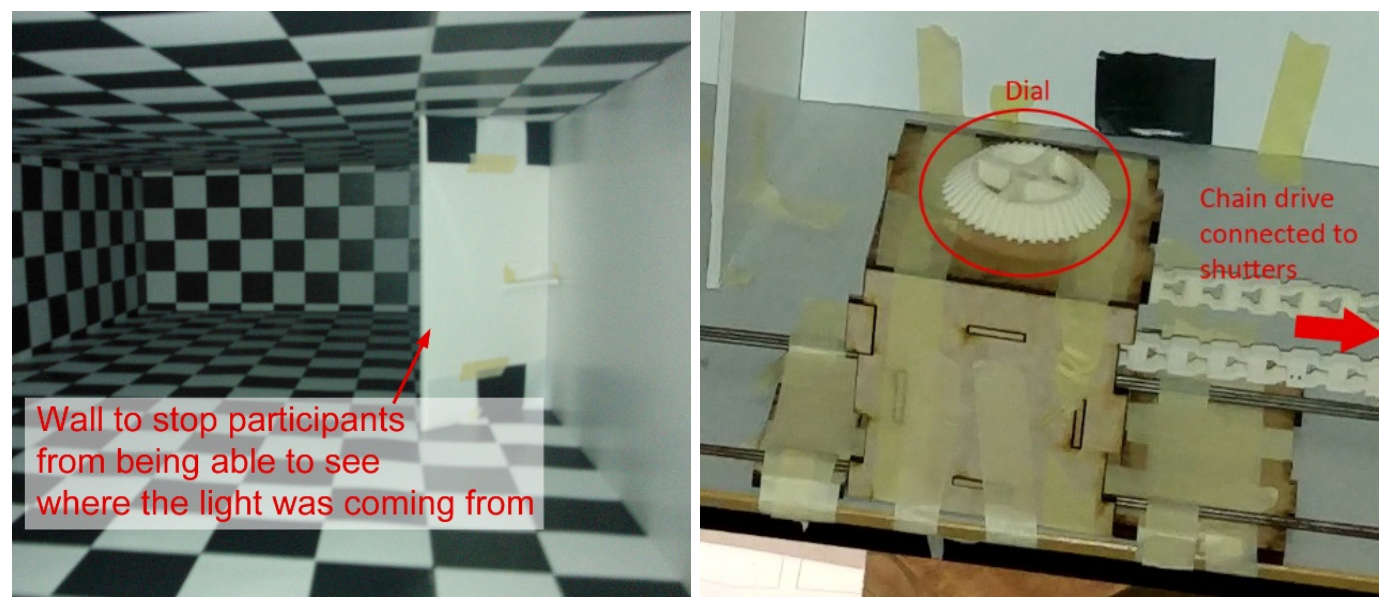

Figure 3.3 Left: photo looking inside the checkerboard condition from the back where the light is coming in to illustrate the walls placed next to the viewport to prevent participants from seeing the diffusing box. Right: the dial the participants used to control the light level

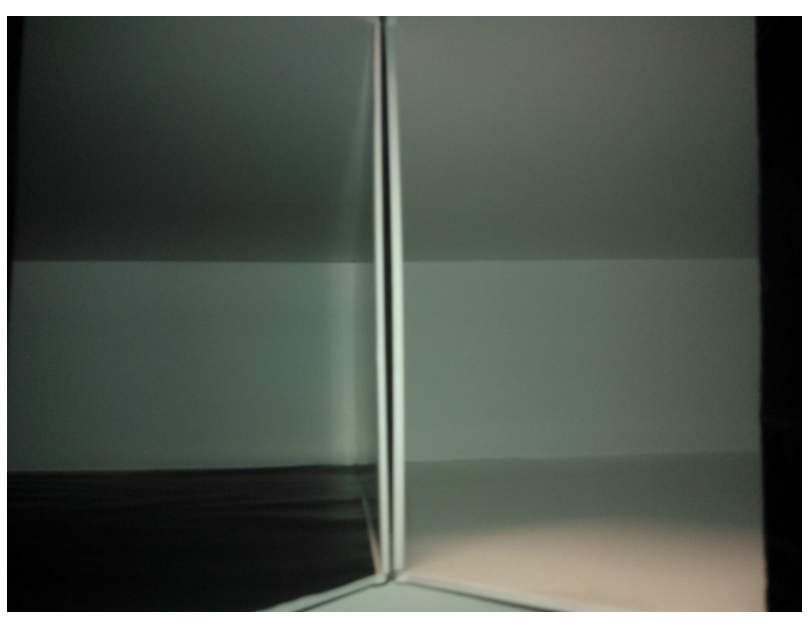

Figure 3.4 Photo of the viewports of two side-by-side conditions. Participants were asked to place their head inside the conditions so it filled their whole field of view and move back and forth.

\subsection{The test conditions}

Eight test conditions were created in total for the original exploratory study (Figure 3.5). These were not changed after the pilot study as the goal was then to see if the pilot study's surprising results were reliable, and if the hypothesis raised by the pilot study held true it would suggest significant issues with existing models of uniformity effects. The following original explanation of their design, however, is given to provide background context as to how the conditions were designed. As the illumination distribution could not be changed (due to the limitations of the dimming system and resulting lamp set-up), the light distributions were varied by changing the surface reflectances. To do this, the interiors of the foamboard models were covered in paper printed in various shades of grey ${ }^{24}$. This

\footnotetext{
${ }^{24} \mathrm{An}$ attempt at using paint ran into the problem that the drying paint warped the foamboard
} 
allowed strong contrasts and patterns such as checkerboards to be readily created. In order to focus on just the differences between the conditions, a short angled wall was installed in the models to the side of the viewing opening to block their sight of the diffusing box(es).
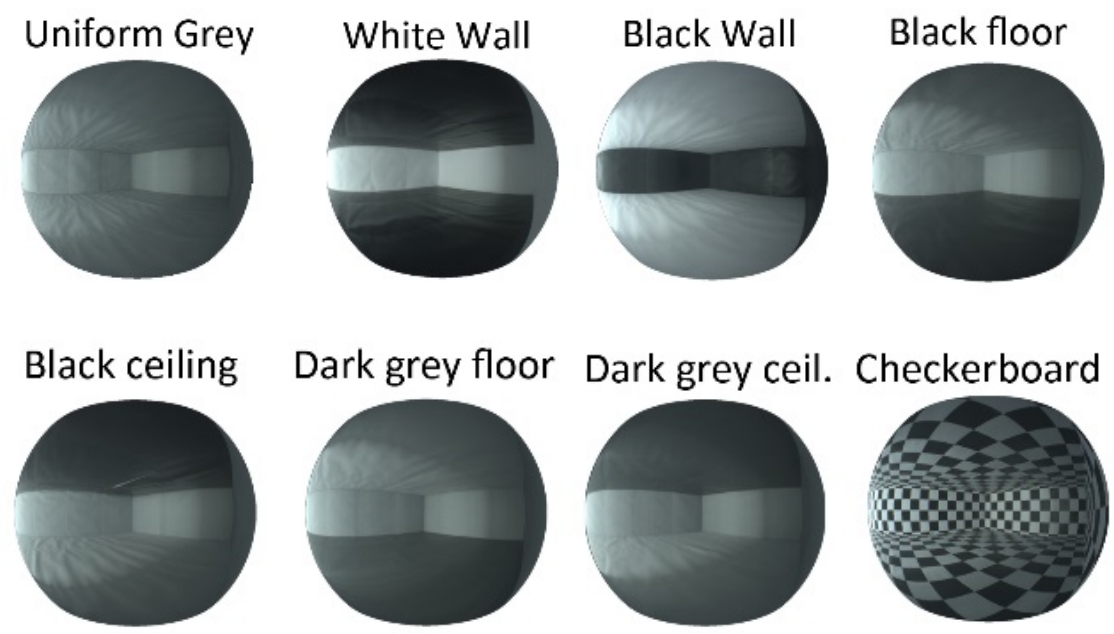

Figure 3.5 The different conditions used in Experiment 1. Photometric properties are described in Table 3.1 and Table 3.2 on the following page.

The general concept was to have a "uniform" baseline reference condition, and then a series of highly non-uniform conditions focused on different balances of wall, floor, and ceiling luminance. The baseline condition was a uniform grey condition, made by covering the interior in a mid-grey tone. Due to the geometry of the set-up, more illumination fell on the walls than the floor and ceiling, so to compensate and get a more uniform balance, the reflectance of the walls was lowered. A mid-grey was used for the baseline because this would allow the non-uniformity to be increased by both increasing and decreasing the luminances of different surfaces. If, for example, the baseline was a uniform white condition then we would only be able to make areas darker to change the light distribution.

Combinations of black and white thus provided the maximum potential change to light distribution. This was used to create condition 2, the White Wall condition, with bright walls and dark floors and ceiling, and its opposite: the Black Wall condition. These aimed to look at the relative effects of the walls against the floor and ceiling. Then, to examine the relative effects of the floor against the ceiling, the same was done with the Black Floor and Black Ceiling conditions. The Checkerboard condition then provided a position-agnostic non-uniform condition, with high contrast checks distributed evenly over the entire space. Rounding out these conditions was then the Dark Grey Floor and Dark Grey Ceiling conditions, which were less non-uniform versions of the Black Floor and Black Ceiling conditions. Compared with their more non-uniform counterparts, these were intended to provide another comparison to measure uniformity variation without position changes. 
Thus, there were two conditions each for wall, floor, and ceiling changes and three comparisons looking at simple non-uniformity increases without changing position.

Table 3.1 Summary of the properties of the different conditions. Relative luminance is the mean luminance of the different surfaces relative to the walls. Surface reflectances were measured using an X-Rite Colormunki Spectrometer. Luminance distribution is measured using HDR luminance mapping as described in the following section.

\begin{tabular}{|c|c|c|c|c|c|c|c|}
\hline \multirow{2}{*}{\multicolumn{2}{|c|}{ Condition }} & \multicolumn{3}{|c|}{ Reflectance } & \multicolumn{3}{|c|}{ Relative luminance } \\
\hline & & Wall & Ceiling & Floor & Wall & Ceiling & Floor \\
\hline 1 & Uniform Grey & $27 \%$ & $52 \%$ & $52 \%$ & 1.0 & 0.64 & 0.67 \\
\hline 2 & White Wall & $78 \%$ & $3 \%$ & $3 \%$ & 1.0 & 0.09 & 0.08 \\
\hline 3 & Black Wall & $3 \%$ & $78 \%$ & $78 \%$ & 1.0 & 6.0 & 6.3 \\
\hline 4 & Black Floor & $40 \%$ & $78 \%$ & $3 \%$ & 1.0 & 0.55 & 0.11 \\
\hline 5 & Black Ceiling & $40 \%$ & $3 \%$ & $78 \%$ & 1.0 & 0.11 & 0.53 \\
\hline 6 & Dark grey floor & $30 \%$ & $78 \%$ & $15 \%$ & 1.0 & 0.70 & 0.24 \\
\hline 7 & Dark grey ceiling & $30 \%$ & $15 \%$ & $78 \%$ & 1.0 & 0.21 & 0.66 \\
\hline 8 & Checkerboard & \multicolumn{3}{|c|}{ Checks: 3\% / 78\% } & 1.0 & 0.44 & 0.44 \\
\hline
\end{tabular}

Table 3.2 Arbitrary uniformity measurements of the different conditions (based off metrics used in the literature)

\begin{tabular}{|c|c|c|c|c|c|c|c|c|c|}
\hline & Condition & $\begin{array}{l}\text { Unif. } \\
\text { grey }\end{array}$ & $\begin{array}{l}\text { White } \\
\text { wall }\end{array}$ & $\begin{array}{l}\text { Black } \\
\text { wall }\end{array}$ & $\begin{array}{l}\text { Black } \\
\text { floor }\end{array}$ & $\begin{array}{l}\text { Black } \\
\text { ceil. }\end{array}$ & $\begin{array}{l}\text { Dk gry } \\
\text { floor }\end{array}$ & $\begin{array}{l}\text { Dk gry } \\
\text { ceil. }\end{array}$ & $\begin{array}{c}\text { Checker- } \\
\text { board }\end{array}$ \\
\hline \multirow{8}{*}{$\begin{array}{l}\frac{\overline{0}}{0} \\
\stackrel{\underline{\underline{T}}}{\bar{J}}\end{array}$} & SD/Mean & 1.00 & 1.79 & 1.01 & 1.35 & 1.41 & 1.24 & 1.34 & 1.44 \\
\hline & SD of log L. & 0.33 & 0.74 & 0.52 & 0.55 & 0.59 & 0.43 & 0.46 & 0.58 \\
\hline & Max/Min & 169 & 1090 & 352 & 589 & 685 & 181 & 277 & 670 \\
\hline & 99th/1st \%ile & 25 & 386 & 139 & 203 & 236 & 69 & 91 & 190 \\
\hline & 99th/Mean & 5.0 & 8.3 & 4.9 & 6.6 & 6.8 & 6.3 & 6.8 & 7.1 \\
\hline & Mean/1st & 5.0 & 46.5 & 28.3 & 31.0 & 34.7 & 11.1 & 13.3 & 26.9 \\
\hline & $\begin{array}{l}\text { Dir.diffusivity } \\
\text { (unsmoothed) }\end{array}$ & 0.61 & 0.69 & 0.62 & 0.62 & 0.61 & 0.60 & 0.61 & 0.65 \\
\hline & $\begin{array}{l}\text { Dir.diffusivity } \\
\text { (smooth } 100^{\circ} \text { ) }\end{array}$ & 0.74 & 0.83 & 0.73 & 0.76 & 0.76 & 0.74 & 0.74 & 0.78 \\
\hline \multirow{5}{*}{ 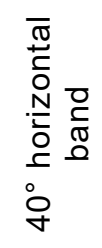 } & SD/Mean & 0.97 & 1.06 & 1.48 & 0.98 & 1.05 & 1.03 & 1.08 & 1.26 \\
\hline & Max/Min & 84 & 179 & 352 & 217 & 249 & 162 & 205 & 419 \\
\hline & 99th/1st \%ile & 42 & 95 & 160 & 86 & 102 & 65 & 87 & 144 \\
\hline & 99th/Mean & 5.8 & 5.5 & 7.1 & 5.3 & 5.7 & 5.4 & 5.5 & 6.2 \\
\hline & Mean/1st & 7.3 & 17.2 & 22.6 & 16.3 & 18.0 & 12.1 & 16.0 & 23.1 \\
\hline
\end{tabular}

The light distributions were measured using High-Dynamic-Range (HDR)

photography to produce luminance maps following Inanici (2006) (see also Hirning, 2014; Van Den Wymelenberg, 2012). The HDR images were produced from exposure-bracketed photographs of each space taken using an LG-G4 smartphone camera with a $180^{\circ}$ fisheye lens attachment to capture the luminances of the full field of view. The multiple exposures 
were then combined into HDR images using hdrgen (Ward, 2006). The HDR images were corrected for vignetting (Van Den Wymelenberg, 2012), and converted to a hemispherical projection ${ }^{25}$ using Radiance. The luminance maps were analysed using R (R Core Team, 2016). An example of this is shown below (Figure 3.6):

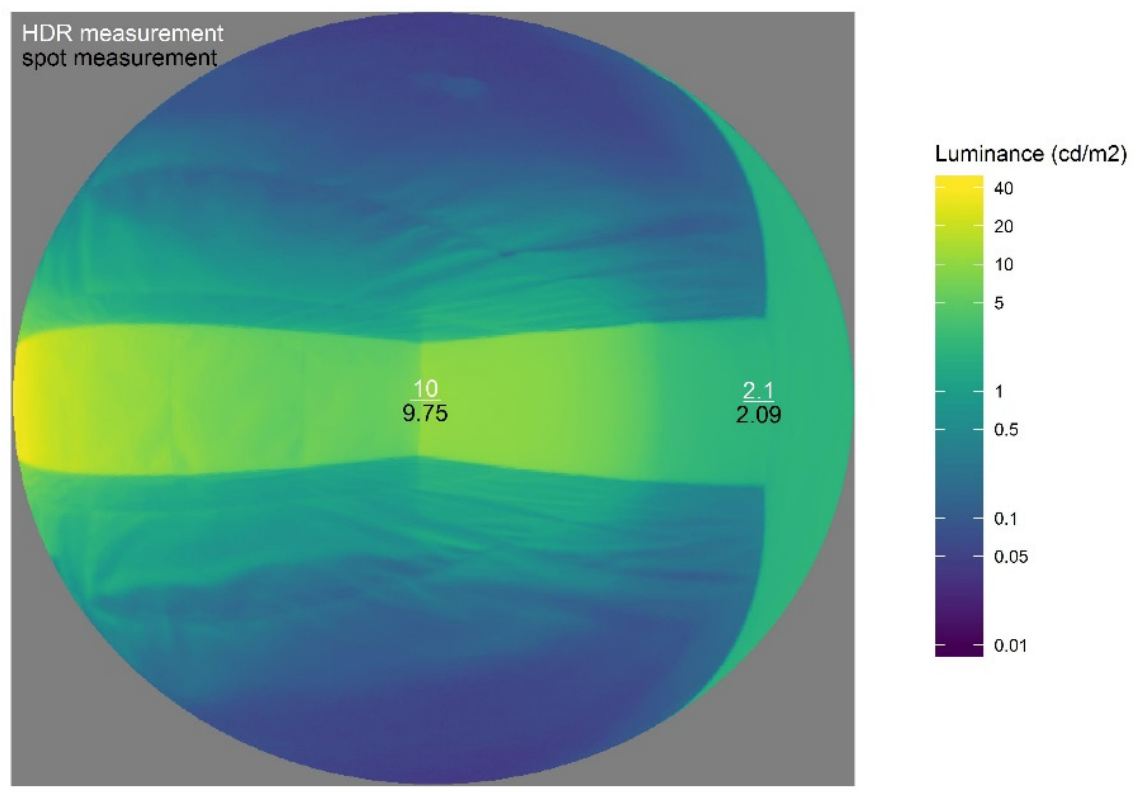

Figure 3.6 Example of a luminance map produced using HDR photography (White wall condition).

In addition to the standard HDR analysis process, it was identified that light scattering in the lens appeared to potentially have significant effects on the measurement of high contrast scenes. A luminance map of a checkerboard, for example, suggested that the black checks were being overestimated by about $20 \%$ on average. We attempted to correct for this by developing a novel technique to generate "scattering error maps" that could be used to roughly correct the images in the same manner as vignetting correction. In brief, by photographing images of the luminance maps of the conditions displayed on a screen, the measured luminances in different regions could be compared to what the camera would measure when photographing a uniform scene of the same luminance. As the uniform scene should have negligible scattering, the difference between the two readings can — in theory — give us an indication of how much scattering is in the scene, and how it is

\footnotetext{
${ }^{25}$ The images produced by the fisheye lens are in the form of an equidistant projection. This means that angular distance is accurately described in the map, but angular area is not. It was judged that capturing the angular area of different parts of the room was more important to describing things like the average light level and the relative importance of different surfaces than mapping angular distance. Similarly, hemispherical projection is needed to calibrate HDR images against vertical illuminance as it allows one to take advantage of the relationship Mean Luminance $=$ Vertical Illuminance $/ \pi$ (Hirning, 2014). Because of this, it was necessary to convert it.
} 
distributed. This was then checked with spot luminance measurements. For more details of the measurement process, see Appendix A.3.

Within the conditions, the light levels were monitored via illuminance meters placed in the corner of the diffusing box(es) (Figure 3.7). To translate this into the overall light level at the participants' eye, we took measurements of the vertical illuminance at the expected eye position using a Minolta T-1H illuminance meter. The relationship between these two readings - the calibration factor - was what was used to estimate the mean luminance of the condition(s) for any given match setting made by a participant (Table 3.3).

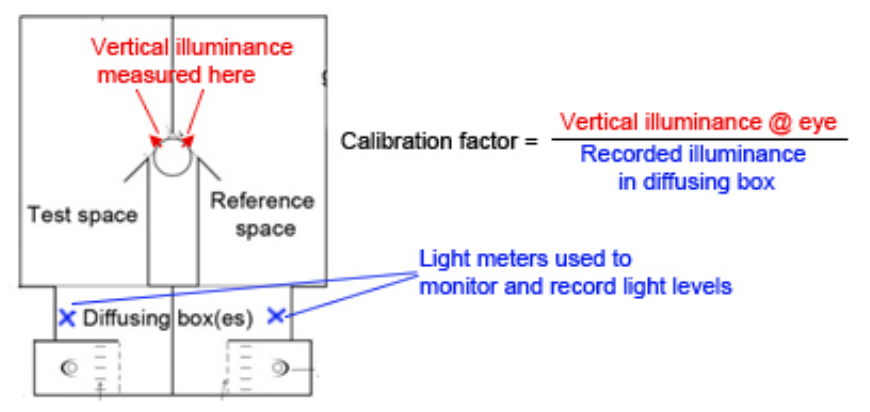

Figure 3.7 Illustration of meter placement for measuring light levels in the model spaces

It should be noted that repeated measurement of the calibration factors indicated an uncertainty of $- \pm 5 \%$ (Appendix A.1). In order to get a better estimate, the calibration factors were measured five times and the results averaged to calculate the mean light level for the conditions in the analysis.

Table 3.3 Calibration factors and adjustment ranges of conditions in Experiment 1. Note that these are the average of five separate measurement sessions.

\begin{tabular}{|l|l|c|l|}
\hline \multicolumn{2}{|c|}{ Condition } & $\begin{array}{c}\text { Calibration } \\
\text { factor }\end{array}$ & $\begin{array}{c}\text { Range } \\
\text { (vertical lux) }\end{array}$ \\
\hline 1 & Uniform Grey & 0.021 & $0.22-6.2$ \\
\hline 2 & White Wall & 0.028 & $0.25-6.9$ \\
\hline 3 & Black Wall & 0.04 & $0.48-13.4$ \\
\hline 4 & Black Floor & 0.02 & $0.20-5.6$ \\
\hline 5 & Black Ceiling & 0.02 & $0.21-5.8$ \\
\hline 6 & Dark grey floor & 0.019 & $0.20-5.5$ \\
\hline 7 & Dark grey ceiling & 0.019 & $0.20-5.4$ \\
\hline 8 & Checkerboard & 0.028 & $0.28-7.7$ \\
\hline
\end{tabular}

In addition to the above measurements, a Minolta CL-70F CRI illuminance meter (calibration certificate in Appendix A.4) was used to measure the Spectral Power Distribution (SPD) of the light at the participants' eye in the different conditions. With the focus of the experiment being on effects of light distribution rather than colour it was important to make sure that colour differences were minimised, and to control for their 
effects if present. To that end, the potential effect of SPD was estimated using the provisional model of spectral sensitivity for scene brightness from Rea et al. (2015). Overall, the colour of the light was very similar across the different conditions, and any effects could be expected to be minor (Table 3.4).

An additional note: later checks of the SPD at different light levels suggested that the light shifted to a "warmer" colour temperature when the shutters were almost closed, though this was not visually noticeable. The differences in SPD, however, were estimated to be equivalent to around a 10\% drop in light level using Rea et al.'s model, which could significantly affect brightness matches that set the light level very low. To account for this, a polynomial function was fit to approximate how the SPD effect became darker at low shutter settings, and used to adjust the SPD effects calculated for the matches made at low light levels (Appendix A.2). As most matches were not at the lowest light levels, the aggregate effect on the test conditions would only be expected to be on the order of a $1-2 \%$ shift in the effective light level.

Table 3.4 Measured light colour and estimated effect of SPD on apparent light level of Experiment 1 test conditions relative to the baseline uniform grey condition.

\begin{tabular}{|c|c|c|c|c|c|}
\hline \multirow{2}{*}{\multicolumn{2}{|c|}{ Conditions }} & \multirow{2}{*}{$\begin{array}{l}\text { Colour } \\
\text { temp. }\end{array}$} & \multicolumn{2}{|c|}{$\begin{array}{l}\text { Chromaticity } \\
\text { (CIE1964) }\end{array}$} & \multirow{2}{*}{$\begin{array}{l}\text { Effect on } \\
\text { apparent } \\
\text { light level }\end{array}$} \\
\hline & & & $x_{10}$ & $\mathrm{y}_{10}$ & \\
\hline 1 & Uniform grey (null) & $6250 \mathrm{~K}$ & 0.317 & 0.358 & - \\
\hline 2 & White wall & $6013 \mathrm{~K}$ & 0.322 & 0.358 & $-1 \%$ \\
\hline 3 & Black wall & $6145 K$ & 0.319 & 0.356 & $-0.8 \%$ \\
\hline 4 & Black floor/white ceiling & $6072 \mathrm{~K}$ & 0.321 & 0.360 & $-1.1 \%$ \\
\hline 5 & White floor/black ceiling & $6054 \mathrm{~K}$ & 0.321 & 0.360 & $-1.4 \%$ \\
\hline 6 & Dark grey floor/light grey ceiling & $6032 \mathrm{~K}$ & 0.322 & 0.362 & $-1.5 \%$ \\
\hline 7 & Dark grey ceiling/light grey floor & $6057 \mathrm{~K}$ & 0.321 & 0.361 & $-1.4 \%$ \\
\hline 8 & Checkerboard & $6238 \mathrm{~K}$ & 0.317 & 0.356 & $+0.6 \%$ \\
\hline
\end{tabular}

\subsection{Model predictions}

As noted, the focus of experiment 1 is on a potentially significant discrepancy between what most of the literature would have us expect, and what was found in the initial pilot study. In light of that, it may be useful to examine what we might predict the experimental results to be based off past research and compare this to the pilot study observations. As discussed in Section 2.4 we can attempt to apply the different light distribution models to predict the effects of our conditions on the effective light level ${ }^{26}$ (Figure 3.9). Applying

\footnotetext{
${ }^{26}$ A note: by "effective light level" we do not mean "brightness". Brightness is related to luminance by a power function (Stevens, 1966), such that, for example, a 25\% increase in brightness requires perhaps a $100 \%$ increase in luminance. While this is valuable to be aware of, in terms of describing the effect of light distribution it is more useful to frame it in terms of "equivalent change in luminance/light level". Hence, by "effective light level" we mean, essentially, the difference in brightness in units of the change in light level that
} 
these models is simple in some cases (e.g. Ko et al.'s (2013) log SD model just requires us to $\log$ transform our luminance map then calculate the standard deviation), but others require additional assumptions that should be noted.

In the case of the Marsden equation, we defined the "brightest surface" as the region of $99^{\text {th }}$ percentile luminance our luminance maps. From there the equation could simply be applied to the map as usual, with the pixels below the $99^{\text {th }}$ percentile having their brightness calculated relative to that.

Kato et al.'s directional diffusivity metric is slightly complicated to calculate here because it was designed for full $360^{\circ}$ views of spaces, while we have only a $180^{\circ}$ view from an opening. The main issue this causes is with the application of smoothing over $100^{\circ}$, and what to do at the edges (Figure 3.8). In this case, we cut off the smoothing region at the ends of the distribution, so, for example, the luminances at the end would just be smoothed with those within $50^{\circ}$ on one side, and nothing on the other. Alternatively, we could perhaps choose to wrap the distribution and imagine it as being connected around the viewing position. If we do that, the resulting uniformity measurements are "more uniform", and predicted effects are smaller. We chose not to wrap here because it was felt that with the design of the spaces clearly indicating further space outside the field of view, wrapping the ends of the visible area around and ignoring the gap between them would conflict with the appearance of the space. With that said, however, the effect is minor overall, with wrapping merely changing the predicted effect of the White Wall condition from $+12 \%$ to $+8 \%$.

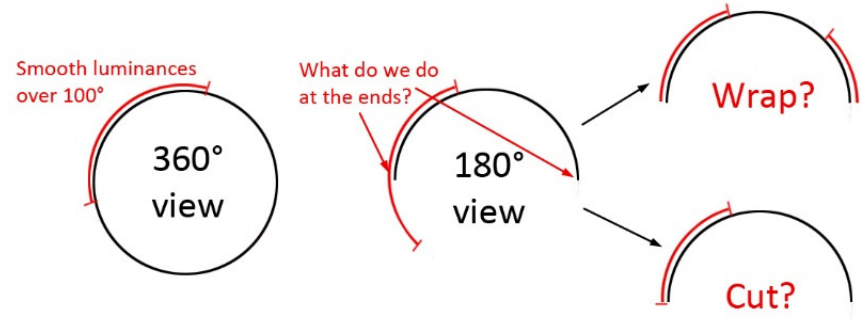

Figure 3.8 Illustration of the smoothing problem that happens when our view of the space has defined edges. With a $360^{\circ}$ view we can readily go around the whole distribution smoothing the luminances. With a $180^{\circ}$ view, however, we run into the ends of the distribution, and need to decide what to do about the smoothing calculation.

Looking at the model predictions (Figure 3.9), we can see that:

would be required to produce such a difference in brightness. For example, a space may appear to have a brightness as if its luminance was increased by $20 \%$ (which would translate into an increase in brightness of -6$7 \%$ ). We may thus say that the effective light level of the space is $120 \%$ of what it is physically measured to be. 
a) The general trend is one where the less uniform spaces are expected to appear darker.

b) The effects of light distribution could potentially be very large, with even some of the more modest effects being predicted to be equivalent to around a $20-40 \%$ difference in light level.

c) While Floor/Ceiling conditions have relatively consistent predictions, the White Wall, Black Wall, and Checkerboard conditions show substantial variation.

d) The Directional Diffusivity model from Kato and Sekiguchi (2005) stands out from the others by predicting relatively small differences between conditions. Conversely, Kirsch's max/min model stands out by predicting some effects that are much larger than the others.

e) The Marsden equation, Ko et al. model, and Kobayashi et al. model are fairly consistent, with predictions generally only differing by $-5-10$ percentage points.

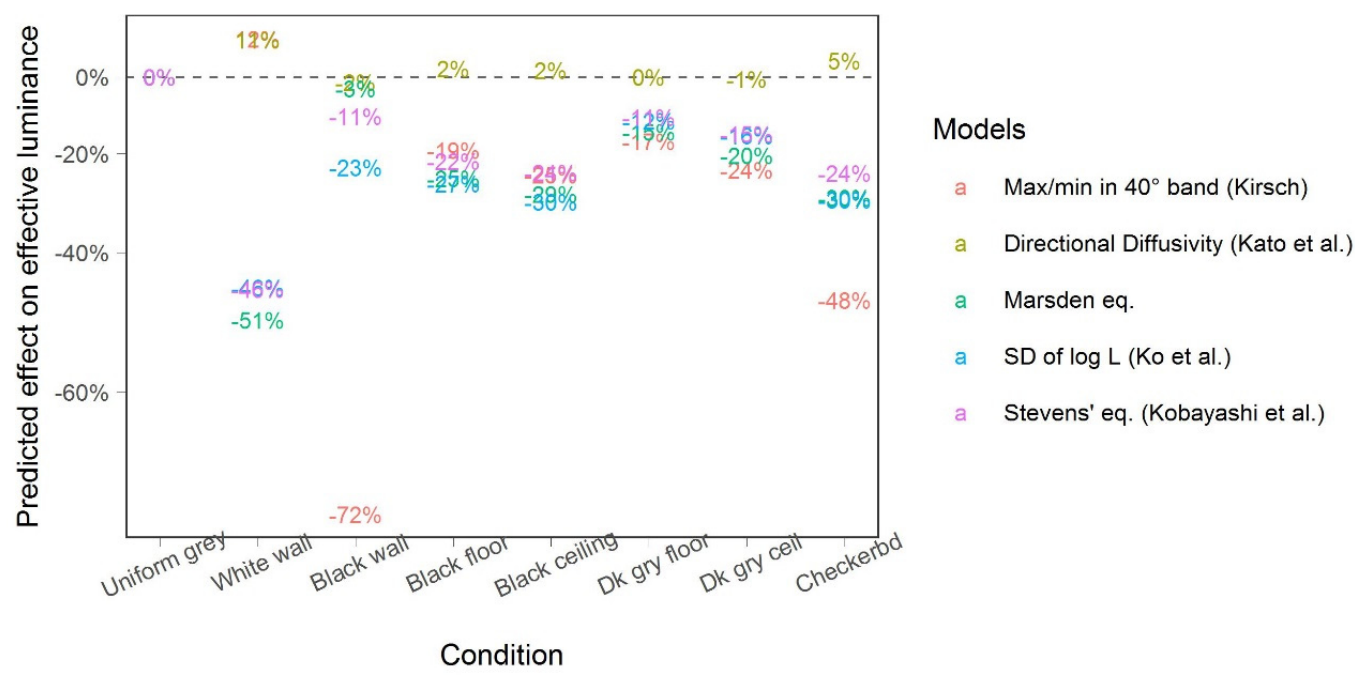

Figure 3.9 Predicted effects of Experiment 1 conditions on effective luminance relative to the baseline Uniform Grey condition according to different models

If we examine these observations more closely, the predictions of the Directional Diffusivity model here seem particularly questionable. For example, the idea that the Checkerboard condition is slightly more uniform than the neutral grey baseline condition (directional diffusivity of 0.78 vs 0.74 ) seems questionable. Overall, the metric argues that the conditions are all generally very similar in uniformity, despite their differences. This can be explained by the fact that in the design of these conditions we largely created nonuniformity through vertical variation. As previously discussed (Section 2.4.4), the calculation of Directional Diffusivity involves averaging over the vertical angles, and the effect of this is that most of the differences in contrast we have created here are effectively eliminated. This may highlight a fundamental flaw in the assumptions of the model. 
However, if the results of the experiment align with these predictions, then it could provide strong evidence for the validity of the model.

Consistent with its previously noted propensity for predicting dramatically large effects, the Kirsch model predicts significantly larger effects than the others on a couple of conditions (Checkerboard and Black Wall). Interestingly, however, the White Wall condition is actually predicted to be slightly brighter, despite the fact that on the model's chosen uniformity metric it is solidly less uniform ( $\mathrm{max} / \mathrm{min}$ ratio in the $40^{\circ}$ band $=179$ vs. 84 for the uniform grey baseline). This is due to the assumptions that the $40^{\circ}$ band is all that matters (see Figure 2.13, Section 2.4.1). The White Wall condition concentrates most of its luminance in the central band, and the model holds that that effect is stronger than the effect of its non-uniformity (Table 3.5). In contrast, the Black Wall condition appears dark in the central horizontal band, and so combined with its high non-uniformity the model predicts that it should appear drastically darker than the other conditions.

Table 3.5 Uniformity ratio ( $\mathrm{max} / \mathrm{min}$ ) and mean in the $40^{\circ}$ band for each condition and the associated effects on effective light levels according to Kirsch (2014). The breakdown illustrates how the effect of the mean luminance in the $40^{\circ}$ can fight against, and even potentially outweigh, the effect of uniformity. See Section 2.4.1 for an explanation of the equations.

\begin{tabular}{|l|l|l|}
\cline { 2 - 3 } \multicolumn{1}{c|}{} & $\begin{array}{l}\text { Unif. } \\
\text { Ratio }\end{array}$ & $\begin{array}{l}\text { Equivalent } \\
\text { to x\% of } \\
\text { baseline }\end{array}$ \\
\hline Uniform grey & 84 & $100 \%$ \\
\hline White wall & 179 & $69 \%$ \\
\hline Black wall & 352 & $50 \%$ \\
\hline Black floor & 217 & $63 \%$ \\
\hline Black ceiling & 249 & $59 \%$ \\
\hline Dk gry floor & 162 & $73 \%$ \\
\hline Dk gry ceil & 205 & $65 \%$ \\
\hline Checkerbd & 419 & $46 \%$ \\
\hline
\end{tabular}

\begin{tabular}{|l|l|}
\hline $\begin{array}{l}\text { Mean } \\
\text { of } \mathbf{4 0}^{\circ} \\
\text { band }\end{array}$ & $\begin{array}{l}\text { Equivalent } \\
\text { to } \mathbf{x} \% \text { of } \\
\text { baseline }\end{array}$ \\
\hline 1.33 & $100 \%$ \\
\hline 2.15 & $161 \%$ \\
\hline 0.75 & $56 \%$ \\
\hline 1.71 & $128 \%$ \\
\hline 1.69 & $127 \%$ \\
\hline 1.52 & $114 \%$ \\
\hline 1.56 & $118 \%$ \\
\hline 1.52 & $114 \%$ \\
\hline
\end{tabular}

\begin{tabular}{|l|}
\hline $\begin{array}{l}\text { Com- } \\
\text { bined }\end{array}$ \\
\hline $100 \%$ \\
\hline $112 \%$ \\
\hline $28 \%$ \\
\hline $81 \%$ \\
\hline $75 \%$ \\
\hline $83 \%$ \\
\hline $76 \%$ \\
\hline $52 \%$ \\
\hline
\end{tabular}

The assumption that only the $40^{\circ}$ band matters is, as previously noted, fairly questionable. However, its use here has produced some novel predictions that diverge from the other models. If supported by the results, this could provide a strong case for focusing more on luminances in the central band.

The general consistency of the other model predictions is also worth noting. Despite their potential for a high degree of disagreement (as the theoretical illustration in Section 2.4.7 showed), they can also potentially agree about many real spaces. This highlights how the correlations between metrics means experimental results can often be consistent with multiple models. Without the models actually being compared, we have no way of knowing that, for example, Kirsch's (2014) results aren't also consistent with, say, 
Kobayashi et al. or Ko et al.'s models. That being said, even these models aren't entirely consistent. We see some non-trivial disagreement around the effects of the Black Wall condition, where, even ignoring the Kato et al. and Kirsch model predictions as outliers, the predictions range from trivial $(-3 \%)$ to substantial $(-23 \%)$.

While these disagreements between models are interesting, and highlight opportunities for experimental data to differentiate between models, they are also ultimately minor compared to the larger potential discrepancy here with the pilot study results (Figure 3.10). The pilot, while having considerable uncertainty due to its small size, suggested that the effects could potentially be substantial and in the opposite direction to the predictions in Figure 3.9. If these results could be reliably reproduced, then it would suggest that none of the existing models can be relied upon to provide reliable predictions of the effects of light distribution on spatial brightness. Hence the focus of Experiment 1 on testing whether or not these conditions do, indeed, produce an apparent effect of non-uniformity increasing brightness, and why these conditions were kept. An experiment designed specifically to test and compare the different model predictions without the information from the pilot would probably have different conditions - conditions designed to produce greater disagreements between models and to deliberately test key model assumptions. In this case, however, if these conditions do have the effects suggested by the pilot then they are already a powerful test of all the existing models.

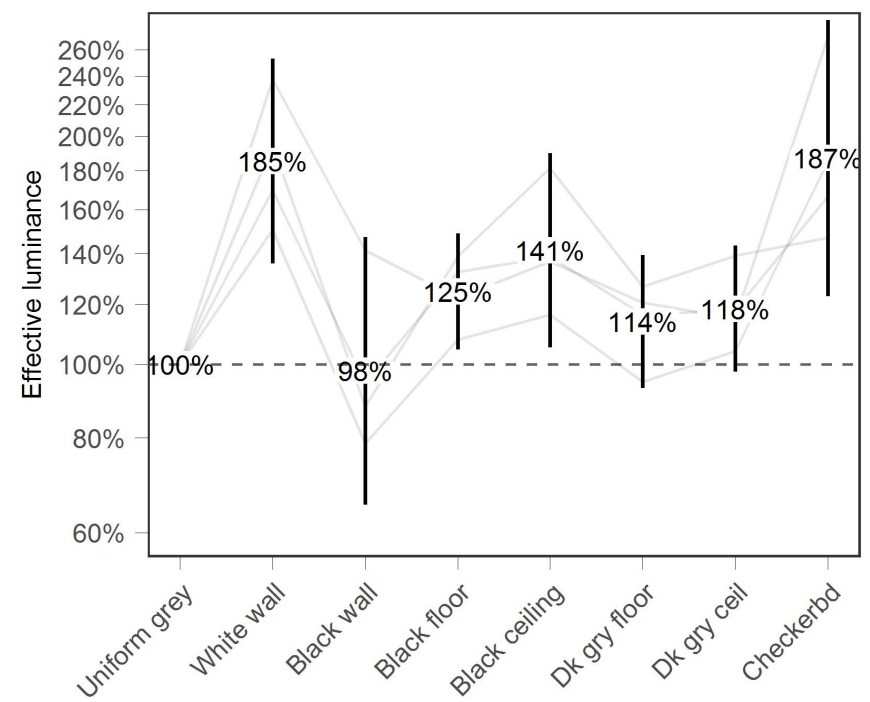

Figure 3.10 Pilot study results showing means and 95\% confidence intervals. Results are normalised to the baseline uniform grey condition. Grey lines show the means of the individual participants. 


\subsection{Methodology}

The main method chosen for this study was brightness matching, which involves participants comparing two different stimuli and then adjusting the test stimulus until they think it matches the brightness of the reference. Magnitude estimation was then chosen to cross-check the results from brightness matching as one of the most commonly used methods in the studies that suggested that uniform distributions should appear brighter.

These are, of course, not the only methods that could have been used. There is a wide range of valid methods that could be used to measure spatial brightness - forced-choice discrimination or any of the various forms of brightness rating scale are other options for example. All have their advantages and disadvantages, and may be prone to different forms of bias (CIE 212:2014 provides an overview of the methodological issues here). Choices may depend on both the practical constraints of a given experiment and ultimately experimenter preference. For example, in a field study a method such as brightness matching or discrimination may not be possible, as one may be unable to set up easy comparisons for participants. Rating scales may be the most practical option there. In this case, forced-choice discrimination was rejected due to impracticality as Fotios and Houser (2013) recommend an all-possible-pairs design to control for bias, which can massively increase the number of comparisons to be made. With eight conditions, for example, one would need 28 different pairs. This is multiplied even further if one is carrying out comparisons at multiple light levels in order to measure the magnitude of any difference in brightness - five light levels for instance would result in 820 different pairs. As Fotios and Houser note:

A problem with all-possible-pairs is that there is an increase in the number of lamp pairs to be examined, increasing from four to ten pairs when comparing five lamps, and this has implications for experimentation resources. There may be occasions when an all-possiblepairs approach is not practical or possible due to limitations in time or nuances of the apparatus. In these cases where the experimenter wishes to proceed with a fixed reference, we recommend that the discrimination procedure is either avoided, or complemented with alternative procedures (for example, matching and rating). (Fotios and Houser, 2013, p255)

Based on this, discrimination tasks were avoided. In the case of subjective rating scales, there are several problems. Some of their biases are functionally intractable. Stimulus and response equalizing biases, which involve observers essentially mapping the response scale to the stimulus range (and so a different stimulus range could produce different apparent effect sizes using the same scale) are said to be unavoidable for instance (Poulton, 1979). Indeed, one of the suggested strategies for avoiding it is to instead use a matching task (Poulton, 1989, p227). Others are not necessarily practical to avoid - the suggested methods for avoiding the stimulus spacing bias, for example, are to either ensure the stimuli 
are equally spaced on the scale (not practical if the point of our experiment is to find out where they lie) or have participants only make a single judgement (which would massively inflate the required number of participants we need to recruit) (Poulton, 1989). If our goal is estimation then the limited granularity of, say, an 8-point scale may also be problematic. Even if we are providing as many scale points as we have conditions (as recommended by CIE 212:2014) the roughness of the scale may still be problematic if the differences between conditions do not conveniently align with it. A further problem with simple interval rating scales (e.g. how bright is this on a scale of 1-8?) is interpretability: what does a 1 scale-point difference in brightness actually mean? One can potentially manage this by taking measurements at multiple light levels, and then using the measured brightness/luminance relationship to scale the estimates of other effects (as we did to translate Kirsch's (2014) results), but this is arguably just a more complicated way to get to what brightness matching provides. These issues may not stop us from identifying rough trends with such methods - but they are less satisfactory if we are trying to make practical estimates of the size of the effects. This is why Brightness Matching was originally chosen: because it directly measures relative brightness in practical terms - i.e. "this condition needs $20 \%$ more light to match the brightness of that condition”. Moreover, it does so without the need to translate it or worry about how people are using your scale. While it too may have some biases, as discussed by Fotios et al. (2008), they are relatively straightforward to manage through null-condition trials and counterbalancing (Fotios et al., 2008), and its use has strong precedent in spatial brightness research (e.g. Fotios and Gado, 2005; Fotios and Cheal, 2011, 2007b; Tiller and Veitch, 1995). Thus, it was seen as the best choice for an experiment trying to measure the practical effects of light distribution.

Finally, after the first two experiments were completed, a third replication was done with brightness matching using a pair-wise counterbalanced design to control the biases, rather than the null-condition based design used in the pilot and main brightness matching experiment. This was done because both the main brightness matching experiment and the Magnitude Estimation experiment used the same basic setup of a constant reference condition that all the test conditions were compared to. The small counterbalanced experiment was run to see if swapping around which condition was the reference would significantly change the results in a way that would not have been picked up in the Magnitude Estimation experiment.

Ethical approval for this study was obtained from the Victoria University of Wellington Human Ethics Committee (\#0000022479). 


\subsubsection{Brightness matching (main experiment)}

In the brightness matching task, participants were instructed to adjust the light level in the test space until it appeared to them to be as bright as a reference space (the Uniform Grey condition). Participants were given written instructions based off those described in Tiller and Veitch (1995) and Fotios and Cheal (2011) to read at the start of the test (Appendix B.1). Brightness was defined as the amount of light in the space. Participants adjusted their seat height until their eyes were half way up the height of the space. They were instructed to adopt a viewing position such that their chin was just over the edge of the spaces' opening, in order to make sure that it filled their field of view. They then looked back and forth between the spaces to compare them. While spaces in other brightness matching experiments have often used models viewed at a distance, it was felt that in the case of a light distribution experiment it would be important to have people "in" the space. This was because of the location effects that have been proposed by people, and the fact that a "background" being visible could affect perception of the spaces via contrast.

While brightness matching tasks are held to avoid the biases of rating scales identified by Poulton (1989), they can be prone to other biases (Fotios et al., 2008). Briefly, the potential biases are (Fotios et al., 2008):

a) A position bias, wherein the condition on one side may tend to be reported as brighter than the one on the other, whether due to psychological biases (such as those discussed in McCourt et al. (2013)) or simply slight physical differences between the two sides that are not perfectly reflected by the measurements.

b) A bias associated with the application of dimming, wherein the condition being adjusted would tend to be set lower than it "should" be. For example, if we had two identical conditions we might find that the dimmed one tended to be set $5 \%$ lower than the light level of the reference, despite the fact that they should be the same.

c) Anchoring bias associated with the initial light level of the test condition (start point bias), where, for instance, if the test condition was initially set to a high light level then the resulting matches would tend to be higher than if it were initially set to a low light level.

d) A response contraction bias, where if the experiment involved references at a range of light levels the matches would tend to be biased towards the middle of the reference range. So, for example, if we ran null condition trials at a low, medium, and high light level we might find that the resulting matches were higher than the reference at the low light level, about the same at the medium light level, and lower than the reference at the high light level. 
The two strategies recommended for dealing with these biases are null condition trials ${ }^{29}$ and/or counterbalancing ${ }^{30}$ (Fotios et al., 2008). In this experiment, anchoring bias was addressed by counterbalancing high and low initial light levels, while the other biases were addressed by use of a null condition trial ${ }^{31}$. Null trials were used in this case as having to swap both conditions in each trial to counterbalance position/dimming would have significantly increased the time needed for the experiment, and the burden on the participants. By also using the Uniform Grey condition as a test condition as well as the reference, we can measure the size of potential bias and control for it. Since, in this null condition trial, the conditions are the same, any difference between them should logically be due to bias. Test condition results are discussed relative to this rather than the reference condition in order to control for the bias. Note here that this design confounds position and application of dimming, so the resulting measured bias will be the combination of the two of them. This does not prevent bias control, as subtracting the measured total bias is no different from measuring each individually and then subtracting them from the results, but it should be noted if one is interested in using the estimates from this study to talk about bias $^{32}$.

${ }^{29}$ Null condition trial: a trial wherein both conditions are identical, and so theoretically in the absence of bias or measurement error should be matched in brightness at the same light level. Deviations from equality may be taken as measurements of bias, and one may subtract this from one's measurements in order to try to control the bias (e.g. Fotios and Cheal (2007a, 2011))

${ }^{30}$ Counterbalancing: evenly applying biases to a comparison such that when trials are averaged the biases may cancel out and thus produce an unbiased estimate of the difference between the test and reference conditions. For example, one might place the test condition on the left in one trial, and then on the right on the other. Or, for start point bias, one may start the test condition at a high light level in one trial, and then a low light level in the other.

${ }^{31}$ In retrospect a mistake (Appendix C). The problem with null-condition trials is that their usage assumes that bias is the same across all pairs of conditions. If this is not the case then the null-condition trial will not be a good measure of the bias in the other trials. That being said, as will be seen, the effects found in this experiment are so large that any plausible degree of variation in bias between conditions would not meaningfully alter the conclusions.

32 We may consider this mathematically. When we calculate the difference between match and reference light levels, the observed difference, $o b s_{\text {diff }}$, is equal to $-\operatorname{dif} f_{A B}+$ bias where $\operatorname{dif} f_{A B}$ is the difference in effect between the two conditions (denoted A and B). In the case of the null condition trial, wherein the two conditions are the same, then $\operatorname{dif} f_{A B}=0$ and so the observed result $\left(o b s_{n u l l}\right)$ is assumed to be the bias. If we assume that we can use the null condition trial as a useful measure of potential bias in the actual test condition matches, and use it to control for it (as done in, for example, Fotios and Cheal (2007a, 2011)) then we may calculate the "unbiased" test effect by simply subtracting the null trial result from our main results. If the total bias is the combination of both position and dimming bias, then:

$$
\begin{aligned}
o b s_{\text {diff }}-o b s_{\text {null }} & =\left(-\operatorname{diff}_{A B}+\text { bias }_{\text {pos }}+\text { bias }_{\text {dim }}\right)-\left(0+\text { bias }_{\text {pos }}+\text { bias }_{\text {dim }}\right) \\
& =-\operatorname{diff}_{A B}
\end{aligned}
$$

This may be similarly applied to counterbalancing. There we may swap the positions of the two conditions to control position bias, and swap the application of dimming between the two conditions to 
The reference condition was held constant at $2.72^{33}$ vertical lux $\left(0.87 \mathrm{~cd} / \mathrm{m}^{2}\right)$ in order to avoid the response contraction bias (Fotios and Cheal, 2007b) and to allow room for both high and low match settings. The starting setting was varied in order to prevent participants from learning how far to turn the dial, and turning it the same distance each time. The start levels $\left(0.28,0.33,0.39,1.26,1.5,1.75 \mathrm{~cd} / \mathrm{m}^{2}\right)$ were set to be evenly spaced around the average expected match level (on a log scale) in order to provide better counterbalancing. With two trials for each start setting, there were a total of six trials per condition. The 48 trials total took up to two hours to complete, and this was spread over two sessions in order to minimise participant fatigue. At the start of each session, participants were given several practice matches while they spent 10 minutes adapting (assuming needed adaptation is within $-2-3$ orders of magnitude — reasonable given participants were coming from indoor light levels - then it should be complete within seconds to minutes (Boyce, 2014;

Hopkinson and Collins, 1970)). Practice matches were done in random order with random initial light levels, and always included the null trial and one of the most extreme differences (either White Wall or Checkerboard condition) in order to show participants the range of differences they would be dealing with. Anything about the procedure they were unsure about was clarified during this time. This also served to ensure the lamps had time to warm up and stabilise their output (measurements in Appendix B.3). Test condition order was varied randomly between each session. The order within each session however was only semi-random. Given the size of the models, laying them all out in the laboratory was not practical. Hence, the order was designed to allow them to be stacked on top of each other in two stacks. Essentially, the order was designed in three blocks. Within the first block, all eight conditions were viewed in a random order. In the second block, they were viewed in reverse order. In the final block, they were viewed alternating between

control dimming bias. Fotios and Cheal (2008) recommend doing this for all combinations of the biases, which if we have both position and dimming bias would produce four trials as follows (noting that when we switch the application of dimming, the direction of the observed effect will change, and to counterbalance we need to take the inverse of it):

$$
\begin{aligned}
\text { Trial1 }_{\text {left,dim }} & =-\operatorname{diff}_{A B}+\text { bias }_{\text {pos }}+\text { bias }_{\text {dim }} \\
\text { Trial }_{\text {right }, \text { dim }} & =-\operatorname{diff}_{A B}-\text { bias }_{\text {pos }}+\text { bias }_{\text {dim }} \\
\text { Trial3 }_{\text {left,fixed }} & =-\left(\operatorname{diff}_{A B}-\text { bias }_{\text {pos }}+\text { bias }_{\text {dim }}\right) \\
\text { Trial4 }_{\text {right,fixed }} & =-\left(\operatorname{diff}_{A B}+\text { bias }_{\text {pos }}+\text { bias }_{\text {dim }}\right)
\end{aligned}
$$

If we tally up the positive and negative signs on the biases, we can see that averaging all of the trials will counterbalance and eliminate them. Furthermore, we can also see that doing all four trials is unnecessary. If we were to only run trials 1 and 4 (corresponding to the dimmed condition always being on the left), then they would still counterbalance the same way. In short, if you believe that the four way counterbalancing works to eliminate the bias, then you must also believe that it still works if we confound the biases. For further discussion of the mathematics of bias control see Appendix C.

${ }^{33}$ It was intended to be 2.75 lux using the original calibration factors. 2.72 was what it is estimated as after we average the estimates of all the calibration measurements. 
the two stacks. Thus, the order was varied, with conditions never being viewed after the same condition twice, but not completely randomly (Appendix B.2).

\section{Participants and Sampling}

The sampling strategy used was, following Kruschke (2015), to sample until the desired level of precision in statistical estimates was reached. Note that by using precision as the target the estimates of the effects are not biased (Kruschke, 2015) ${ }^{34}$. The chosen target was to achieve $95 \%$ intervals on those estimates of $- \pm 10 \%$ (or more specifically, \pm 0.043 on the $\log 10$ scale). This level of precision was estimated to require approximately $\mathrm{N}=10$, and should be roughly equivalent to \pm 1 Just-Noticeable-Difference (or slightly higher) ${ }^{35}$. More precise estimates (e.g. $\pm 5 \%$ ), would arguably be of questionable utility, since at that point people are unlikely to notice any error. Moreover, in the absence of hypotheses that require extremely precise estimates to test we do not really have a need for them. The goal was achieved after 11 participants had been assessed ${ }^{36}$.

The participants were post-graduate students in their 20s and 30s. 6/11 participants wore visual correction, and the gender ratio $(\mathrm{M}: \mathrm{F})$ was 8:3.

${ }^{34}$ The classic example of what is known as "optional stopping" is to sample until you get $\mathrm{p}<0.05$, which is forbidden because by doing that you can essentially ensure that you will get a "statistically significant" result even if there is no effect. A similarly problematic procedure from a Bayesian standpoint would be if we sampled until our 95\% intervals excluded 0, which would bias our estimates of the condition effects and inflate the error rate (Kruschke, 2015; Kruschke and Liddell, 2017). By instead targeting a desired level of precision, we avoid this problem, as our stopping rule is not dependent on the size of the condition effects or whether or not a difference is "significant" (Kruschke, 2015). This kind of design is not restricted to Bayesian statistics. Lakens (2014) has written on sequential design for Frequentist (or "standard") statistics, wherein one's alpha-level is divided over several possible predetermined stopping points providing a chance to end an experiment early if the evidence is strong enough. Similarly, in the 1990s, Frick (1998) noted that if one sampled until the p-value either dropped below 0.01 , or rose above 0.36 then one could maintain an overall alpha of 0.05 .

${ }^{35}$ A "Just-Noticeable-Differences", or JND, refers to "the smallest difference in stimulus magnitude that is just discriminable" (Kingdom and Prins, 2016). For brightness of small spots of light, JNDs are generally on the order of $8 \%$ or more (Teghtsoonian, 1971), and the wide variance in individuals' matches in this study (around $\pm 20 \%$ or more) would suggest that people cannot reliably discriminate between small changes in overall luminance with regards to spatial brightness.

${ }^{36}$ For those who are used to a more traditional power analysis, and would like to know what it would say, the key point to note here is how large the effects suggested in our pilot are. The largest effects were around 2.5-3 times the standard deviation. The mid-range conditions are around 1-2 times the standard deviation. A standardised effect size (d) of 2 has $-80 \%$ power in a two-sided t-test at $\mathrm{N}=4$, and $-90 \%$ in a one-sided test (which would be appropriate here as we expect the effect to be in a specific direction). A sample size of 8-10 would give us $80 \%$ power for even the lower mid-range effects we have of $\sim d=1$, and would have $>99 \%$ power for the largest effects. Alternatively, estimating the power based off confidence interval targets (ala. Cumming (2014, p. 24)) we might estimate that, taking the average sd of 0.13 from the pilot, we would have $-80 \%$ power to achieve a confidence interval of $\pm 10 \%$ or less with $\mathrm{N}=11$. 


\subsubsection{Magnitude estimation (ME)}

The basic setup of the magnitude estimation version of the experiment was essentially the same as the brightness matching experiment. The key difference here is that instead of the participants adjusting the light level of the Test condition to match the reference, the reference is instead set to a specific light level and they are then required to report how bright it appears relative to the reference.

Following Aya et al. (2015b), participants were told that the reference had a brightness of "100", and that they should report the brightness of the other conditions relative to that. For example, if they thought the test condition was twice as bright as the reference, they should say " 200 ". The practice trials done during the adaptation period were selected to cover the expected range of brightest to darkest conditions and light levels based off the pilot. Poulton (1989) identified difficult to avoid response contraction bias when people are aware of the range of stimuli they are judging, wherein stimuli above the centre of the range would be underestimated, and stimuli below the centre overestimated. Thus, on the grounds that this would be unavoidable we attempted to show participants the stimulus range during the practice trials so that the bias would at least be consistent throughout the experiment.

Each condition was assessed at three different light levels $(0.54,0.88,1.46 \mathrm{~cd} / \mathrm{m} 2)$, for a total of 24 trials, presented in random order as described previously. The high and low light levels were chosen based on the general principle of them being $\sim \pm$ the largest effect observed in the pilot. The uniform grey reference condition was again always on the right, and set to a vertical illuminance of $-2.72 \operatorname{lux}^{37}\left(0.87 \mathrm{~cd} / \mathrm{m}^{2}\right)$. Repetitions of the same stimulus at the same light level were avoided due to the risk that participants could remember the number they reported previously. Multiple light levels also allows us to account for the light level/brightness relationship, and convert the ME results into equivalent luminances that can be compared to the brightness matching results.

It should be noted that Magnitude Estimation is known to suffer from a number of biases, as noted by Poulton (1989). These may, however, be less problematic in our experiment. The biases he was concerned with primarily affect factors such as the observed slope of the brightness/luminance relationship — the relationship between how much increasing the light level would increase apparent brightness. This is obviously a significant problem for psychophysics research aiming to precisely quantify the brightness/luminance relationship. That, though, is not what we are interested in here, and our main effects the effects of light distribution on effective light level — may plausibly be largely unaffected

\footnotetext{
${ }^{37}$ It was intended to be 2.75 lux. 2.72 was what it is estimated as after we average the estimates of all the calibration measurements.
} 
by these biases once we have converted them into units of equivalent light level. To explain: imagine we want to describe the effect that a difference in light levels will have on brightness. For example, the mean luminance of our test condition may be twice as high as that of our reference. What magnitude would we expect a participant to report to us when they compare the two conditions?

The relationship between luminance and brightness can be described by a power function, thus (in this instance):

$$
B=100\left(\frac{L}{r e f}\right)^{k}
$$

where $B$ is the reported brightness of the test condition, $L$ is the mean luminance of the test condition, ref is the luminance of the reference condition, and $k$ is the slope of the brightness/luminance relationship - and the exponent of the power function. If brightness and luminance were simply directly proportional to each other $(\mathrm{k}=1)$, then if the luminance of the test condition was twice that of the reference, $\frac{L}{r e f}$ would equal 2 , and so we would expect the reported brightness to be $2 \times 100=200$. If $k=0.33$, as it is for the dark adapted eye (Stevens and Stevens, 1963), then we would expect a reported brightness of $\sim 126$.

It is more useful, however, to express this on a log scale, where it becomes a simple linear model:

$$
\log (B)=\log (100)+k \cdot \log \left(\frac{L}{r e f}\right)
$$

We may now add in the effect of the condition(s) on brightness:

$$
\log (B)=\log (100)+k \cdot \log \left(\frac{L}{\text { ref }}\right)+\log \left(\frac{E f f_{\text {test }}}{E f f_{\text {ref }}}\right)
$$

Where Eff test is the effect of the test condition on apparent brightness, and Effre is the effect of the reference. As with light level, the reported brightness will be a product of the relative difference between the conditions.

Expressing the equation this way, however, has a flaw: the effects of the conditions will be expressed in units of "brightness". This is not the same as our brightness matching study, which expresses its results in units of "light level" (which is also more practical). This inhibits comparisons. Moreover, when expressed in units of brightness, we would expect the observed differences between the conditions to be biased by the biases identified by Poulton (1989) that affect such measurements of brightness. However, we can convert brightness differences into luminance differences by means of our measured brightness luminance relationship. Just as multiplying $\log (L)$ by $k$ tells us $\log (B)$, so dividing $\log (B)$ by $k$ will give us $\log (L)$. Or, more efficiently, we may simply rearrange our model as follows: 


$$
\log (B)=\log (100)+k\left(\log \left(\frac{L}{\text { ref }}\right)+\log \left(\frac{E f f_{\text {test }}}{E f f_{\text {ref }}}\right)\right)
$$

Expressed this way, the effect of condition is now in units of light level. This, along with the effect of light level, is translated into observed brightness by means of the brightness/luminance relationship described by $k$.

The theoretical implications of this are interesting. The difference in light level is not biased - it is simply the difference in the physical light level. The bias lies in people's brightness estimates made in response to that difference in light level. Hence, the brightness/luminance relationship may vary between studies due to elements such as the stimulus range used (Poulton, 1989). So, while in one study we might observe that increasing the light level by $40 \%$ increased brightness by $18 \%(k=0.5)$, in another we might observe that it increases brightness by $29 \%(k=0.75)$. In both cases, however, when we divide the biased measurement (brightness) by the coefficient we get the unbiased measurement of the underlying change in light level. Logically then, when we translate our biased observations of brightness differences between conditions into their effective changes in light level, the resulting measurement of the effects should be unbiased in the same way $^{38}$.

\section{Participants and Sampling}

Participants were post-graduate students and lecturers at the university. 20 were between 20-30 years old, 2 were between 30-40, 1 was between 40-50, and 1 was 60+. As before, the sampling strategy was to sample until the desired level of precision in statistical estimates was reached. In this case, because the goal of the ME experiment was to check whether or not the direction of the effects would flip around and go in the opposite direction with another method, we aimed to get a useful degree of precision on the overall trend of the brightness/uniformity relationship. Specifically, we used a linear model predicting brightness in terms of the normalised standard deviation. The choice of this metric should not be taken as an indication of broader support for it as a metric - it is just that it happened to correlate well with our results in the pilot, so it seemed reasonable to use it as a measure of the overall trend for these specific conditions. The goal was to get the $95 \% \mathrm{HDI}$ of the slope of the relationship to be within \pm 0.1 . As the pilot results indicated the slope was on the order of 0.3-0.4, and most likely above 0.2 , this seemed a reasonable level of accuracy to be able to clearly describe the direction of the trend ${ }^{39}$. Simulations with

\footnotetext{
${ }^{38}$ This is just referring to the biases that affect the brightness/luminance relationship of course. If one is concerned that, for example, there could be a position bias, such would need to be accounted for as well.

${ }^{39}$ In retrospect, it probably would have been better to target $95 \%$ intervals of $\pm 10 \%$ on the estimates of the individual condition effects rather than assuming that the relationships suggested by the pilot study would
} 
variance based off the literature (SD likely between -0.06-0.2 (Aya et al., 2015b; Rea et al., 2015)) suggested that this would probably be achieved somewhere between $\mathrm{N}=15-25$, which was considered achievable, and the target was reached with $\mathrm{N}=24$.

9/24 participants wore visual correction, and the gender ratio $(\mathrm{M}: \mathrm{F})$ was 14:10.

\subsubsection{Counterbalanced brightness matching}

After the main experiments described above, the question arose as to whether or not the fact that both the ME and brightness matching experiments essentially worked off the same null-condition constant reference basis could be affecting their results. To check this, a brief pairwise counterbalanced experiment was run to check whether or not swapping around the test and reference conditions would result in the effects changing dramatically. The conditions that were chosen to be compared were the baseline uniform grey condition, and the two conditions with the largest effects (White Wall and Checkerboard). This produced three pairs (Uniform Grey vs. White Wall, Uniform Grey vs. Checkerboard, and White Wall vs. Checkerboard). Each condition in a pair spent half their time on either the left or the right, with dimming always being applied to the left condition. Each pair had 8 trials, with 4 initial light levels $\left(0.25,0.29,1.68,1.96 \mathrm{~cd} / \mathrm{m}^{2}\right)$, for a total of 24 trials.

Of note here is that the counterbalanced design meant that more space needed to be given to allow adjustments to high light levels. For example, if the White Wall condition needs half the light of the Uniform Grey condition to match it that means that when the White Wall condition is being used as a reference the Uniform Grey condition will need to be set to twice its light level. To accommodate this, the reference light level was reduced by roughly $30 \%$ to 1.95 lux.

The only goal of this experiment was to provide a quick check that the non-uniform conditions would not have their very large effects flip around and go in the opposite direction. Given their large effects (2-3 times the standard deviation), it was calculated that 4 participants should be enough to check that the effects are in the same direction as our other experiments say, and not $10-20 \%$ in the opposite direction (for example, $\mathrm{d}=2$ has $90 \%$ power for a one-sided test at $\mathrm{N}=4$, and our effects are expected to be larger than that).

Of the four participants, all were in their 20s, 2 wore visual correction, and the gender ratio (M:F) was 3:1.

continue to describe the overall trend well. They did, and it was not a problem, but it was not something that should have been relied upon. 


\subsection{Statistical analysis}

The basic goal of our statistical analysis here is an attempt to estimate the effects of the different conditions accounting for uncertainty. For this, we are using Bayesian statistical modelling following Kruschke (2015) (see also Gelman et al., 2013; McElreath, 2016).

The basic framework of Bayesian statistics is based around the idea of allocation of probability based on our observations and prior knowledge (Gelman et al., 2013; Kruschke, 2015; Kruschke and Liddell, 2015; McElreath, 2016). In the context of scientific research and statistics, the possibilities that we allocate probability to can be described as parameters in a statistical model. For example, the size of position bias in a brightness matching experiment might be $0 \%$, or $+1 \%$, or $-5 \%$ or many other possibilities. By examining how likely such parameter values would be to generate our observed data, we can compute the likelihood of these values. For example, our data might say that the most likely value is 5\%, but that given the uncertainty in our results could plausibly be anywhere from $10 \%$ to $0 \%$. To then produce our final estimate, however, we should also account for prior information. For example, if prior research had found that bias in such studies was typically about $10 \%$, then we would be sceptical of the idea that the bias was actually $0 \%$, and be inclined towards larger effects. If prior evidence very strongly suggested that the bias should be $10 \%$, then we could infer that the best inference to draw from the data is that the bias in our experiment was probably also $10 \%$, and evidence to the contrary was probably just sample error. If the prior and our data disagreed strongly, then this could also be an indication that our model is failing to describe all the relevant factors - for example, it could be that there are crucial differences between experiments that affect bias strength that we haven't accounted for, or that there is something wrong with our methodology.

This explicit application of existing knowledge to allocate probability is one of the key distinguishing features of Bayesian inference (Gelman et al., 2013; Kruschke, 2015; McElreath, 2016). Essentially, it is a mathematical formulation of the idea that we would require stronger evidence to convince us of something that our existing knowledge suggests is unlikely, while the converse is true for something that is already well supported.

The other key distinguishing feature of Bayesian statistics that we will note here is its philosophical position on probability. Specifically, Bayesianism sees probability as describing uncertainty - for example, the probability that the average effect of a condition on brightness is $+20 \%$ (Gelman et al., 2013; Kruschke, 2015; McElreath, 2016). This may be contrasted with Frequentism, the basis for "standard" statistical tests such as ANOVAs, which would hold that the probability of a condition having an effect of $20 \%$ is either 0 or 1 (Cumming and Finch, 2005). In this view, a 95\% confidence interval of [10\%, 30\%] does not say that there is a $95 \%$ probability that the mean is between $10-30 \%$. Rather, it says that you can be 95\% "confident" that whatever interval you have constructed (whose 
size can and will vary between experiments) will overlap with the true mean (Cumming and Finch, 2005). This is not particularly intuitive, and the idea that the Bayesian framework aligns better with how people naturally interpret statistics is a common argument by Bayesians (Gelman et al., 2013; Kruschke and Liddell, 2015; Morey et al., 2015).

Ultimately, much ink has been spilled on the philosophical reasons for preferring one statistical framework over another (Dongen et al., 2019; Gelman and Hennig, 2017; Kruschke, 2015; Kruschke and Liddell, 2015; Lakens, 2019; Mayo, 2018; Mayo and Spanos, 2010; Morey et al., 2015; Rouder et al., 2009; Wagenmakers et al., 2017), and litigating that here would be outside the scope of this work. Suffice to say that a) all the frameworks have validity, and can readily be justified for studies, b) different frameworks and statistical tests answer different questions (Lakens, 2019), and c) for the goal of estimating effects with uncertainty, the Bayesian framework is arguably the most appropriate as the probability distribution of the estimated effects is what it is designed to produce (Gelman et al., 2013; Kruschke, 2015; Kruschke and Liddell, 2015; McElreath, 2016).

Beyond this, we note certain advantages of the Bayesian method in the context of this thesis, in particular their flexibility and the use of priors. The emphasis that Bayesian statistics puts on the need to account for our prior knowledge, and the framework it puts in place, encourages us to quantify the extent of what the literature can tell us. This is useful for experimental planning (where it improves upon the typical power analysis by pushing us to describe and account for our uncertainty instead of assuming a single value), but is also valuable in that it provides another, more quantitative, perspective on the literature. Indeed, the model comparisons in Section 2.4.7 and the predictions in Section 3.3 were prompted by this. Perhaps more valuable though is the flexibility of the Bayesian process and statistical modelling tools such as Stan (Carpenter et al., 2017; Gelman et al., 2015, 2013; Kruschke, 2015). At the most basic level, models such as that shown in Eq. 3.3 can be directly modelled, and we can estimate the most likely values of all the parameters at once while also allowing that elements such as bias or the brightness/luminance relationship may vary between people and conditions. For brightness matching, as we will demonstrate, it is particularly useful as it allows us to better model and control for certain biases (for example, it makes sense to model biases such as start point bias as being proportional to how far away the start point is instead of just assuming it will counterbalance). It also allows us to make use of all the data in the counterbalanced experiment and combine it with the data from our main brightness matching experiment.

To elaborate: in the counterbalanced experiment we make three paired comparisons uniform v. white wall, uniform v. checkerboard, and white wall v. checkerboard. The standard analytical approach using counterbalancing would just be to calculate the mean 
and confidence interval of each of those differences, and report all of them individually. The problem with this is that it does not make full use of the data. The data about the difference between the white wall and checkerboard conditions is highly relevant to our estimates of relative difference between the uniform/white wall comparison, and the uniform/checkerboard comparison. And, of course, the opposite is also true. The Bayesian modelling method used here on the other hand does account for this, making use of all the data at once to determine the best estimates of the various condition effects.

\subsubsection{General notes}

Before we discuss the analysis in more detail there are a couple of general methodological points that should be mentioned.

Firstly, statistical analysis was carried out using $R$ (R Core Team, 2016). The Markov Chain Monte Carlo sampling process that underpins the calculation of the Bayesian statistics here was done using Stan (Carpenter et al., 2017), accessed through the RStan package (Stan Development Team, 2018).

Secondly, the analysis was carried out with the data transformed to the $\log _{10}$ scale. This is because it makes more sense to frame the effects we are analysing here as a multiplicative process. For example, to say that checkerboard condition requires $40 \%$ less light than the reference condition to match its brightness is more generally useful than to say that the absolute difference in match levels in this experiment was $0.2 \mathrm{~cd} / \mathrm{m}^{2}$ (which is why spatial brightness matching studies like (Fotios and Cheal, 2008) report ratios). Similarly, we describe biases as relative changes (e.g. Fotios et al. (2008)). This makes sense when we consider that brightness perception is relative. A difference of, say, 10 lux may be a major change if we started at 5 lux, but would be undetectable if we started at 1000 lux (a JustNoticeable-Difference for brightness is $\sim 8 \%$ (Teghtsoonian, 1971)). If we consider the model as multiplicative, working on a log scale is mathematically convenient. On a log scale, multiplication becomes simple addition, allowing us to frame the process in the form of a standard linear model. It is similarly convenient for modelling and plotting the relationship between brightness and luminance in the magnitude estimation experiment, as illustrated earlier (Section 3.4.2).

Finally, a log scale makes sense for presenting the results. If we are describing the effects of the conditions as relative differences then a linear scale can be highly misleading. It would seem odd to say that a doubling of light level is twice as great a change as a halving it is (perceptually at least), yet that is what is implied when you compare $100 \%->50 \%$ to $100 \%->200 \%$. On a log scale however the differences are the same, and this asymmetry is removed. 


\subsubsection{The model}

The core of our brightness matching model is the following equation:

$$
\begin{aligned}
\mu=r e f & -\left(E f f_{\text {test }}-E f f_{\text {ref }}\right)-\left(S P D_{\text {test }}-S P D_{\text {ref }}\right) \\
& + \text { bias }_{\text {pos }+ \text { dim }} \\
& + \text { bias }_{\text {start }}
\end{aligned}
$$

Or in other words: an average match $(\mu)$, for a given person and pair of conditions, is a product of the reference light level $(r e f)$, the difference in effective light level between the two conditions $\left(E f f_{\text {test }}, E f f_{\text {ref }}\right)^{40}$, the difference in SPD between the conditions (SPD test, SPD $D_{\text {ref, }}$ effects pre-calculated using the Rea et al. (2015) model), the combined position and dimming bias (bias postdim $_{\text {) }}$, and the start bias $\left(\right.$ bias $\left._{\text {start }}\right)$.

We then assume that the variation in matches made for a given condition and participant is normally distributed, as the residuals from the pilot study and the main experiment look normal (Figure 3.11).

Pilot study

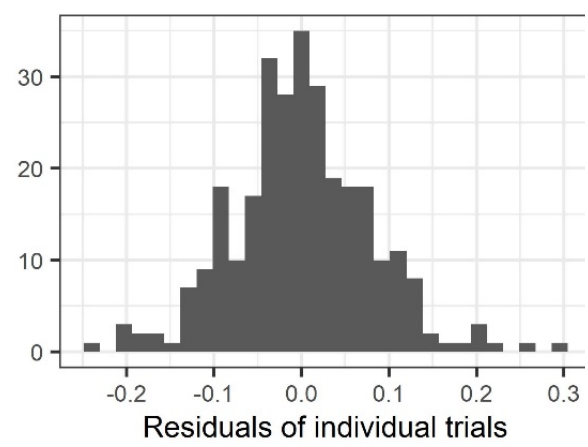

Main experiment

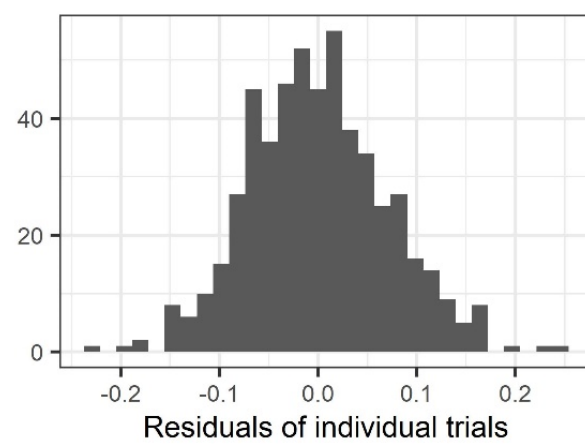

Figure 3.11 Histograms of the data from the pilot study and main experiment

Hence, we can model each individual match as being randomly drawn from a normal distribution with a mean as described above, and standard deviation $(\sigma)$ describing the reliability of their matches (within-person variance):

$$
\text { match } \sim \operatorname{Normal}(\mu, \sigma)
$$

To this we then add the following assumptions:

1. The effect of each individual condition may vary between different people. This is the between-person variance, and this variance may itself vary between conditions. This too is assumed to be normally distributed. When looking at

\footnotetext{
${ }^{40}$ Note that we subtract the difference between conditions from the reference light level here. This is due to how brightness matching works. If the test condition appears to have twice as much light as the reference at equal luminance, then to make it match it will have to be set to have half as much light as the reference. To make a match you must negate the difference in brightness between conditions.
} 
the variation in the average results for participants we have fewer data points and plotted distributions can be too noisy to read clearly (Figure 3.12). ShapiroWilk tests did not indicate deviation from normality in the pilot $(\mathrm{p}=0.2)$, but did in the main experiment $(\mathrm{p}=0.004)$ - which appears to be driven by two outliers in the white wall condition's results ( $\mathrm{p}=0.14$ with it removed). The impact of this is examined in sensitivity analysis (Section 3.6.1).

Pilot study

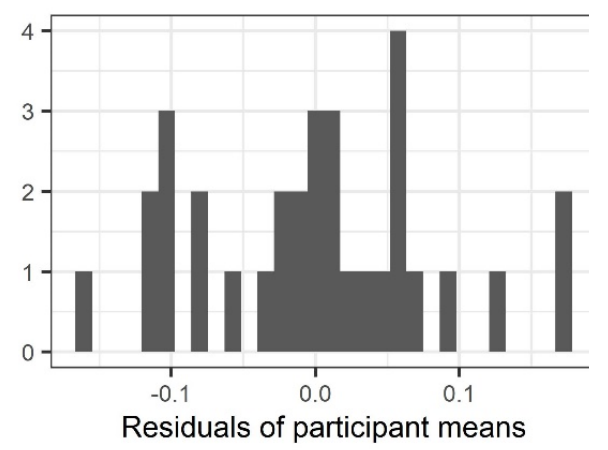

Main experiment

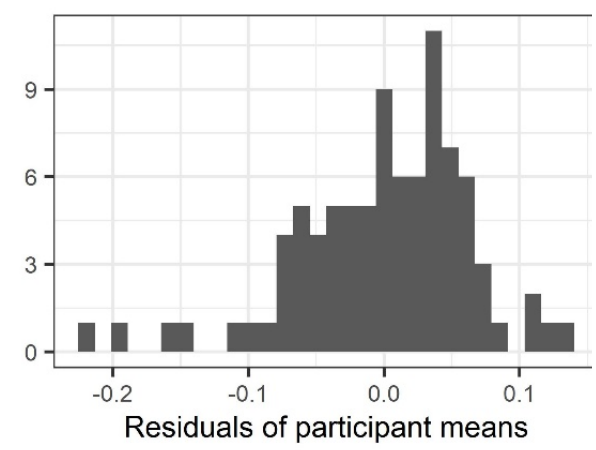

Figure 3.12 Histograms of participant means (normalised to the overall condition means)

2. Position+dimming bias is simply a constant, though it also may vary between individuals.

3. Start-point bias is assumed to be proportional to the difference between the initial light level and the match level and may vary between individuals and conditions. This is calculated as follows:

$$
\begin{aligned}
\text { bias }_{\text {start }} & =k_{\text {start }}\left(\text { match }_{\text {intended }}-\text { start }\right) \\
& =k_{\text {start }}\left(\left(\text { ref }-\left(E f f_{\text {test }}-E f f_{\text {ref }}\right)-\left(S P D_{\text {test }}-S P D_{\text {ref }}\right)\right)-\text { start }\right)
\end{aligned}
$$

... where $k_{\text {start }}$ is the coefficient describing the strength of the bias, and start is the initial light level.

4. The standard deviation describing the reliability of the matches may also vary between people and pairs of conditions. Some pairs of conditions might be harder to match, for example, while some individuals may be more or less reliable (in the pilot study for example one participant had roughly twice as much variance as the others).

From this, we can estimate the underlying mean effect of each of the conditions, as well as the biases and the various sources of error. Note that this is a multi-level or hierarchical model (Gelman et al., 2013), and our estimates of the individual biases and variances are made using partial-pooling. We assume that the bias and/or variance of a given person is not unrelated to that of others. While it would be unreasonable to assume that every 
individual is identical (homogeneity), it would likewise be unreasonable to say that they are all completely independent. We would expect them to have similarities and for the observations of one person or condition to give us an indication of the approximate magnitude of bias or variance that we may expect to see from others. Just as we describe the individual matches as random draws from a normal distribution, we may similarly conceive of the individual biases (for example) as being a random sample drawn from a broader population. The result of this, in practical terms, is that the estimates of parameters such as "the bias of participant 4" or "the variance of condition 2" are a compromise between assuming homogeneity and assuming independence (Figure 3.13). They are allowed to vary, but are also informed by the estimates of the other participants, and thus are effectively "shrunk" towards the mean bias/variance, with the degree of shrinkage dependent upon the data (Kruschke, 2015).

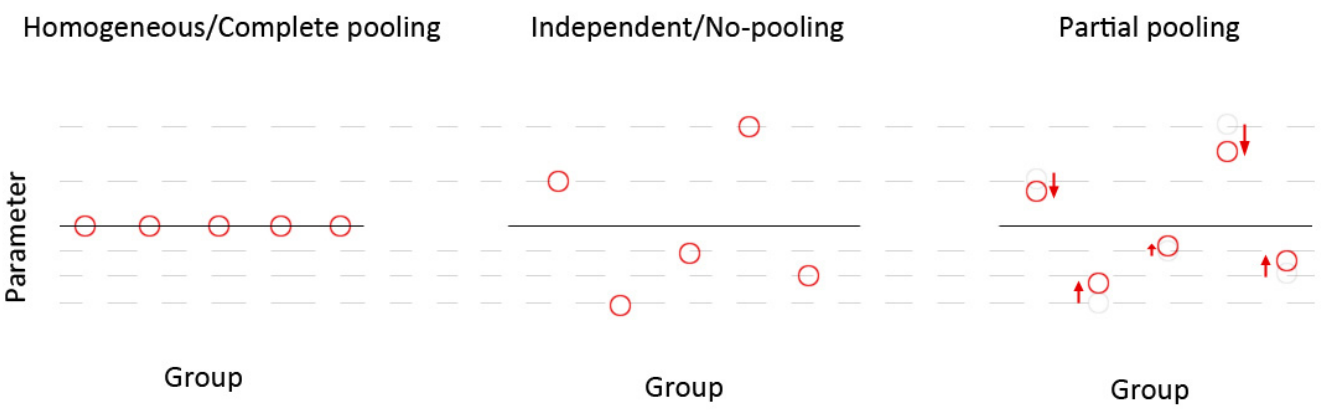

Figure 3.13 Illustration of different model assumptions. On the left we have the assumption of homogeneity - we assume that a parameter (such as the variance) is the same for all groups, and would estimate it as the overall value across all groups. In the middle we have the assumption of independence - that all the groups are different, and have no relation. Here, the value for each group would be calculated separately. Finally, on the right we have partial pooling, where it is assumed that the groups may vary (if the data supports such), but that they may also be the same, and frames them as being a sample from a population. The estimates for each group are essentially an average of the left and middle estimates, with the individual estimates being "shrunk" towards the overall mean.

Note, however, that there are still some instances where we assume independence or homogeneity across conditions. Position+dimming bias, in this model, is allowed to vary between people, but not conditions. This is because of the use of null condition trials to control for the bias. For the null condition trial to work as a useful way of measuring and controlling bias (as used, for instance, in Fotios et al. (2008)) we must assume that the bias in the null condition is the same as in the other conditions. At the other end, we are assuming independence between the mean effects of the different conditions. This is partly for the practical reason that if we want to put individual priors on each condition (based on, perhaps, the model predictions), then we cannot model them as draws from a higher level distribution. However, the main reason is that based on the literature we expect them to vary systematically — indeed they have been specifically designed to do so. In that light, 
modelling them as random draws representing some overarching group that can be approximated as something like a normal distribution seems a little odd. Compare that to, for example, the variation in effects between people, where we have no particular expectations of systematic variation, and treating them as if they were random samples from a broader population that is roughly normally distributed seems plausible. That being said, we do allow the variance to vary between conditions, and do apply partial pooling there. This is because we do not have any particular expectation that the light distribution will systematically affect the variance. It may, but it also may not. Assuming homogeneity and ignoring the possibility of differences, however, would seem unjustified, as would assuming independence and setting up the model to potentially jump at noise. Hence, we go for the middle ground, and allow the data to inform us about how much variation there is, if there is any.

A more detailed description of the mathematical model can be found in the Appendix (B.4).

\subsubsection{Model priors}

As noted earlier ( $\mathrm{p} 81$ ), prior information about the likely values forms an important part of Bayesian analysis. These may be described using mathematical distributions. For example, one could describe the prior on the mean effect of a condition using a normal distribution with the mean centred over the most likely values, and a standard deviation describing the range of plausible values. An illustration of such a prior is shown below (Figure 3.14). Setting such a prior on a hypothesised effect would suggest that we think the effect is likely around $+10 \%$, but could plausibly range from $+0 \%$ to around $+20 \%$.

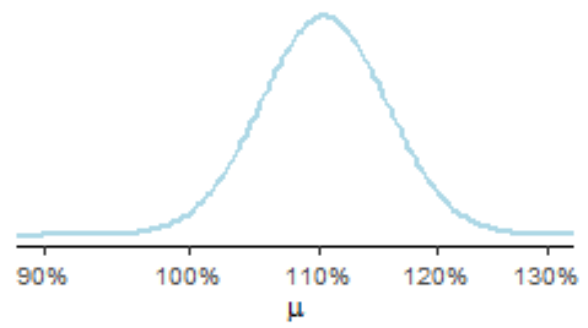

Figure 3.14 Illustration of a simple prior on a mean $(\mu)$, using a normal distribution with a mean of 0.043 and standard deviation of 0.02 on the $\log 10$ scale

This serves a few purposes. Firstly, it can provide better inferences by taking account of existing knowledge (Kruschke and Liddell, 2017; Lee and Vanpaemel, 2017). Secondly, and in as many ways just as importantly, it can serve as a way to help the model run more efficiently and actually fit the data. To estimate the probability distributions, tools such as Stan use Markov Chain Monte Carlo methods, which essentially work out the best fitting probability distributions via a random walk process (Gelman et al., 2013). If the chains 
have to spend time wandering through areas that are clearly ridiculous in light of our knowledge — such as exploring the possibility that the SD is $10000 \%$ of the mean — it can make it hard to fit the model (Gelman and Hennig, 2017).

A common concern and criticism is that Bayesian priors are "subjective" (Gelman and Hennig, 2017). From a practical standpoint, it is worth noting two points: 1) that the priors are only part of the model, and unless we have very strong knowledge about what a parameter should be, our conclusions will tend to be dominated by the data; and 2) that model structure and assumptions are often far more critical - and are equally "subjective" (Gelman and Hennig, 2017).

The priors used in our analysis were informed by the literature, and are summarised below $^{41}$. Note that ultimately our inferences are not overly sensitive to the precise scale of most of these (see sensitivity analysis later), so a certain level of approximation is not a problem.

- Condition effects

- Prior 1: "non-committal prior" - $\operatorname{Normal}(0,0.4)$ (Figure 3.16)

- An example of the kind of vague prior that would be commonly used in studies if one is trying to avoid having the prior "bias" the results in a specific direction. Useful in this situation because while we might have predictions, it may be useful to have this as a comparison to see the effect using a more informed prior has on the results. It should also be noted that there is significant uncertainty in the literature and in the strength of the evidence behind the model predictions we have. Also the pilot study suggested they do not work well on our conditions, so basing our analysis purely on the premise that the predictions were sound in the first place may be a mistake.

- While this prior has no directional preference (mean=0), it may be considered semi-informed with the scale here being informed by our predictions and the literature. We allow that effects of even up to a factor of 3-4 may be possible, but that going beyond that seems unlikely.

○ Prior 2: "Informed prior" - Normal(varies, varies) (Figure 3.15)

- Priors are based on the model predictions from Section 3.3. There is significant uncertainty based on both the discrepancies demonstrated between some of the models, as well as the limitations of the evidence (see Section 2.4.8) and the possibility held out by

\footnotetext{
${ }^{41}$ Beginning with the brightness matching model.
} 
the results of Tiller and Veitch (1995) that they might actually go in the opposite direction.

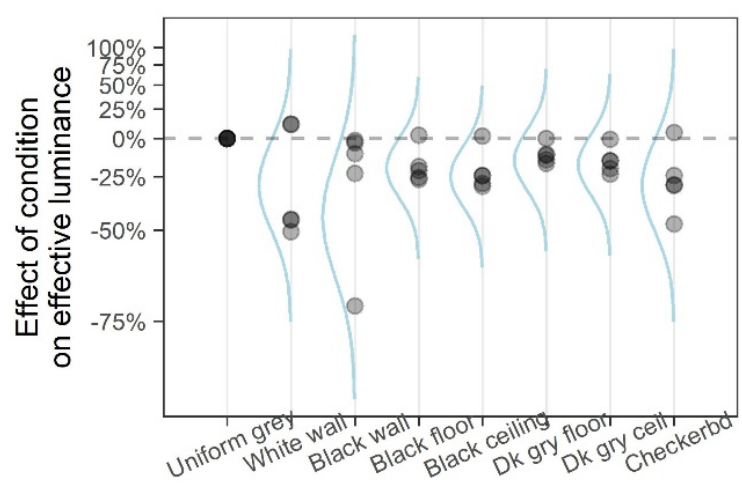

Condition

Figure 3.15 Informed priors on mean effects of conditions based off predictions in Section 3.3.

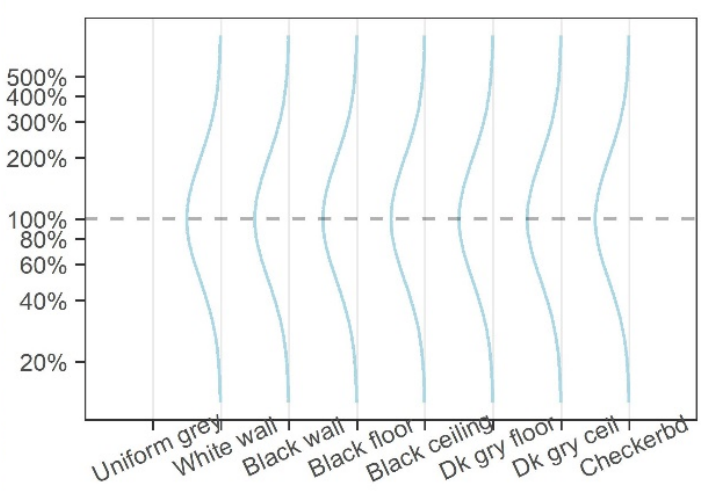

\section{Condition}

Figure 3.16 Broad non-committal priors on effects of conditions.

\section{- Standard Deviation}

- Between-person variance in condition effects: gamma(mode $=0.09, \mathrm{sd}=0.06$ )

- Examination of the distribution of standard deviations found in a range of spatial brightness matching studies suggests that we should probably expect something around 0.09 on the log scale, though there is significant uncertainty (Figure 3.17).
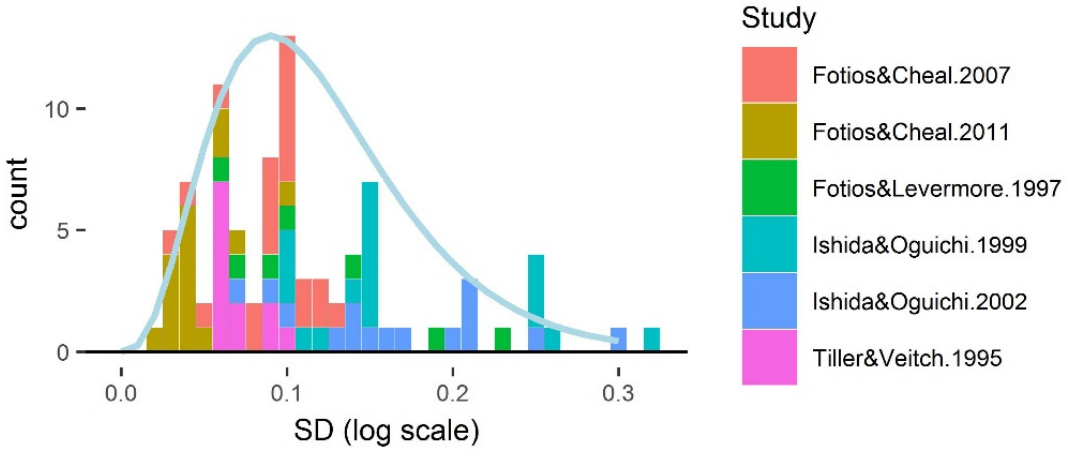

Figure 3.17 Histogram of the reported standard deviations in various spatial brightness matching studies. SDs have been adjusted to the log scale to fit our analysis. The blue line shows the shape of our suggested gamma prior with mode of 0.09 and sd of 0.06 .

$\circ$ Within-person variance in matches $^{42}$ : gamma(mode $=0.08, s \mathrm{~d}=0.06$ )

- Hard to find reports of the variance of individuals' matches, since most studies just give aggregate results. Set slightly lower than the between-person variance because Ishida and Oguichi's (1999) data

\footnotetext{
${ }^{42}$ Because each participant makes multiple matches for each condition, we may differentiate between the variance between participants in how they see each condition, and the variance in each individuals' matches.
} 
suggested that the individual variance seems to be lower than the overall variance (Figure 3.18), though as that study was only $\mathrm{N}=5$ this should not be over-interpreted.

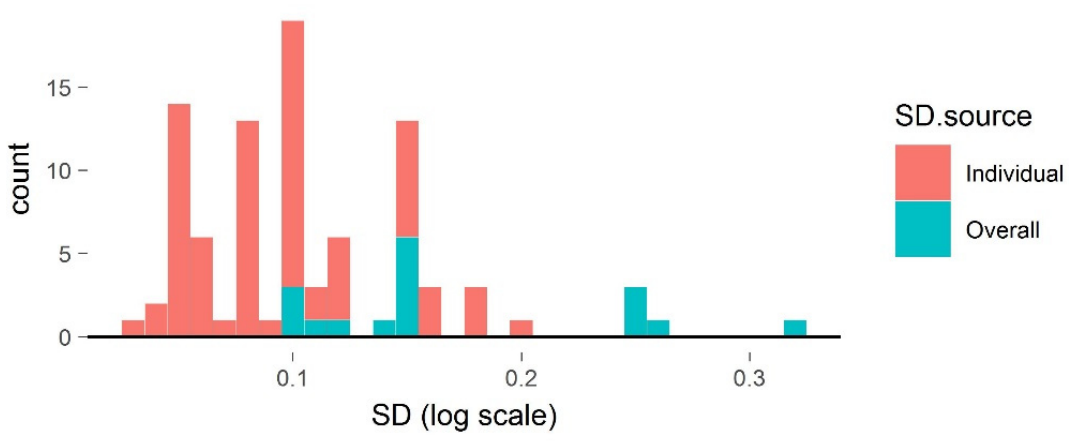

Figure 3.18 Comparison of individual and overall SD estimates in Ishida and Oguichi (1999)

○ Between-condition variance in between-person SD: half-normal (0, 0.04)

- Reported SDs for individual conditions in different studies vary, but we would expect significant variation due to noise even if there were actually no real differences. The standard deviations of the standard deviations vary from $\sim 0.02$ to 0.06 (Fotios and Levermore, 1997; Fotios and Cheal, 2011, 2007b; Ishida and Ogiuchi, 2002, 1999; Tiller and Veitch, 1995). Allowing the possibility of it being zero, and with variance constrained to be positive, we set the prior as a half-normal with standard deviation of 0.04 .

- Between-condition variance in within-person SD: half-normal $(0,0.4)$

- The within-person SD is parameterised differently due to the need to handle both between-condition and between-person variation (see Appendix B.4). SDs seem to vary by a factor of up to two in past studies, and while this is overall SD rather than within-person $\mathrm{SD}$, it is the best guide we have (Figure 3.19). Taking this potential level of variance as a rough guide, we set the prior to a reasonably broad half-normal with an SD of 0.4 on the log scale. 


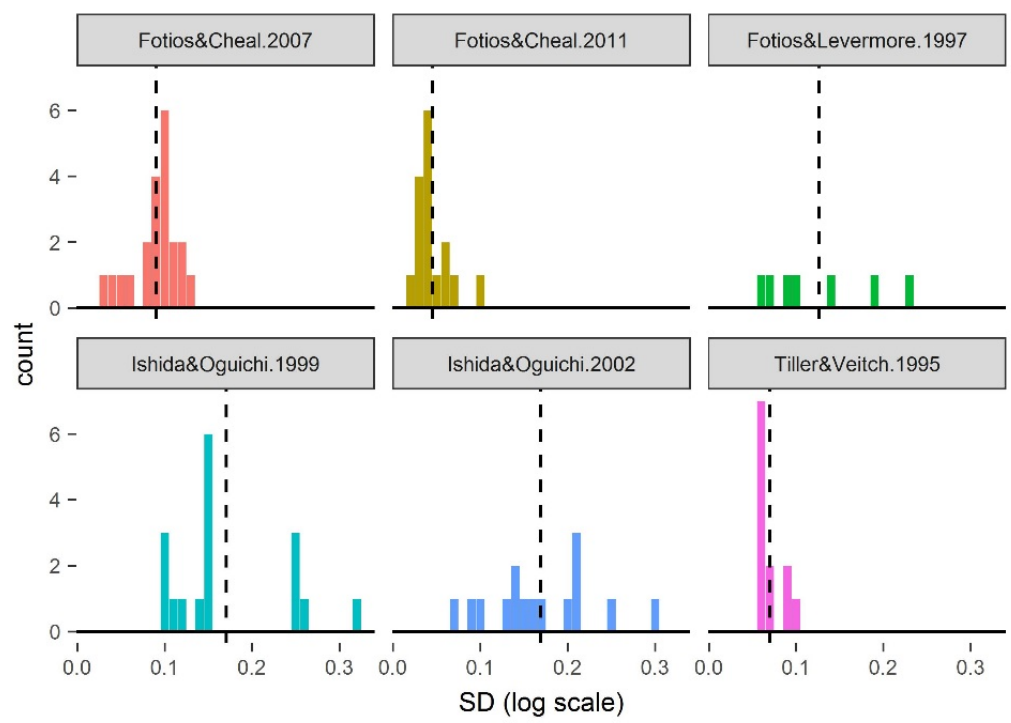

Figure 3.19 Distributions of individual condition SDs found in different spatial brightness matching studies. Individual conditions varying by a factor of up to around two, though this could be being exaggerated by noise.

○ Between-condition variance in within-person SD: half-normal $(0,0.3)$

- Between-subject variation in within-subject SD is harder to estimate, as we only really have a single informative study (Ishida and Ogiuchi, 1999), and it only had 5 participants. If we calculate the average SD for each participant, we get a tight range of $\sim 0.08$ to 0.11 , which would suggest that people don't vary that much in this regard. At the same time, the limited number of participants here makes it hard to be too confident in any assertion, and ruling out the possibility of significant variation would seem premature. We place a broad uncertain prior that is somewhat smaller than the one on the between-condition variance: a half-normal with an SD of 0.3 .

\section{- Position+dimming bias}

- Mean bias: $\operatorname{Normal}\left(0,0.05^{43}\right)$

- The brightness matching bias literature has results ranging from nothing, to around $10 \%$ or more (Fotios et al., 2008; Fotios and Cheal, 2011). This is complicated here by the fact that we have confounded position and adjustment bias in this study, and they could easily enhance or counteract each other. Most of the estimates in the literature are around 5\% or less (Boyce, 1977; Fotios and

\footnotetext{
${ }^{43}$ Or roughly $12 \%$ if we move off the log scale
} 
Gado, 2005; Fotios and Cheal, 2011, 2007a; Houser et al., 2003), a few more are between 5-10\% (Fotios and Gado, 2005; Fotios, 2001), and some occasional estimates larger than 10\% (Thornton and Chen, 1978). Based on this, small biases of $5 \%$ or less seem fairly likely, as much as $10 \%$ is plausible, but moving beyond that starts to become less likely. The confounding of the position and dimming biases increases the uncertainty - they could counteract each other, or they could add together. Based on this, we used a fairly broad prior with a standard deviation of $\sim 12 \%$.

○ Between-person SD: Gamma(mode=0.08, sd=0.06)

- The SD in estimates of bias in null condition trials has tended to be about as large as the SDs of regular trials, or perhaps somewhat lower (e.g. Fotios and Cheal, 2011). Following this, we suggest that the $\mathrm{SD}$ of the between-person variation in bias strength is probably going to be roughly similar to the between-person variation in other condition effects, and we use the same prior.

- (Counterbalanced experiment only) Between-condition SD: half$\operatorname{normal}(\mathrm{m}=0, \mathrm{sd}=0.04)$

- The counterbalanced design makes it (potentially) possible to estimate variation in bias between pairs of conditions. We suggest a half-normal prior with an SD of 0.04 here. This reflects the idea that variation of $\pm 5 \%$ or so is plausible, while variation of more than $\pm 10 \%$ seems less plausible (based on the comparison of nullcondition trials in Fotios and Cheal (2011) and the general scale of position bias estimates).

\section{- Start point bias}

- Mean bias: $\operatorname{Normal}(0,0.08)$

- The effect of start point bias is somewhat unclear, with Fotios et al. (2008) noting that effects were observed in both directions, and that effects were also often not significant. Estimating the observed slopes of bias strength/light level range gives us estimates of -0.076 for Houser et al. (2003), and 0.016 for the data of Fotios and Cheal (2007b). Given this, we set a prior that did not favour a direction and had a fairly broad width with an sd of 0.06.

$\circ$ Between-condition and between-person SDs: half-normal $(0,0.1)$

- Variance in start point bias is not clear at all (given that past studies were not trying to estimate it in this way), and we do not have any data to estimate it from. Given this, we put priors on the SD that 
are relatively broad over the scale we expect the bias to be on (less than 0.1 ).

\subsubsection{Model differences: Magnitude Estimation}

The Magnitude Estimation model differs from the matching task model in that the participants' responses are in units of "brightness", and not "luminance". Thus, the model needs to account for a) the light level, and b) the brightness/luminance relationship. It also, of course, lacks start point bias, while we cannot rule out the existence of position bias. We thus adjust Eq. 3.4 (p84) to the previously discussed Eq. 3.3 (p79) as follows:

$$
\begin{aligned}
\mu=\log (100) & +k\left(\left(L_{\text {test }}-L_{\text {ref }}\right)+\left(E f f_{\text {test }}-E f f_{\text {ref }}\right)\right. \\
& +\left(S P D_{\text {test }}-S P D_{\text {ref }}\right) \\
& \left.+ \text { bias }_{\text {pos }}\right)
\end{aligned}
$$

... where $k$ is the slope of the brightness/luminance relationship, and $L_{\text {test }}$ and $L_{r e f}$ are the light levels of the test and reference conditions.

Just as in the model of the matching task, effects and biases may vary between people and conditions, so may the slope of the brightness/luminance relationship.

If the conditions are the same and are the same light level then the differences would be zero, and the average magnitude estimate would be expected to simply be the reference magnitude of 100, plus any bias. All the effects are measured in terms of the light level before being adjusted into "brightness" by the slope of the brightness luminance relationship. This slope can be estimated here because all the conditions were assessed at multiple light levels.

The priors are also adjusted, as parameters such as $k$ are new, and variance is different as the method is different:

- Effects of condition: unchanged

- Position bias: may or may not be an issue. We use the same prior as we did before: a normal with mean of 0 and sd of 0.05 .

- Brightness/luminance factor, $k$

○ Mean slope: $\operatorname{Normal}(\mathrm{m}=0.5, \mathrm{sd}=0.3)$

- looking at spatial brightness studies using ME, slopes range from 0.32 (Aya et al., 2015b) to $\sim 0.5$ (Rea et al., 2015). Historically it has also been argued that exponents found in such studies are strongly dependent on range effects (Poulton, 1977; Teghtsoonian, 1973), with tighter stimulus ranges leading to steeper slopes. The other studies had larger stimulus ranges than in this study, $\sim 0.7$ 
compared to our $\sim 0.43$. Based off Poulton's model ${ }^{44}$, we might expect our stimulus range to lead to exponents $\sim 162 \%$ the size of those in the other experiments. Therefore, the slope in this experiment might be expected to be somewhere around $-0.5-0.8$. There is, of course, a fair degree of uncertainty here, and to capture this uncertainty we use a normal distribution with a mean of 0.5 , and an SD of 0.3, giving a fairly broad probability over the plausible range here.

Between-person SD in $k$ : Gamma (mode $=0.07, s d=0.07)$

- Variation in slope between people has been found in psychophysics research (Fagot and Stewart, 1969; Marks and Stevens, 1966). SDs here range from $\sim 0.05-0.1$. These are of course only a few studies, and they were based on simple "bright patch" stimuli rather than our more complex spaces. It does however seem reasonable to assume that there will likely be some variation between people along these lines. Accounting for this uncertainty we use a fairly broad semi-informed prior centred over this range - a gamma distribution with mode of 0.07 and sd of 0.07 .

○ Between-condition SD in $k$ : half-normal $(\mathrm{m}=0, \mathrm{sd}=0.2)$

- Variation of brightness/luminance relationship between different spaces is not something that has seen any research to our knowledge. We can say, however, that slopes have tended to vary significantly between different studies, so we should be open to the idea that there may be significant variance. We place a broad halfnormal prior on the between-condition SD, with an SD of 0.2.

- SD

○ Between-person SD in condition effect: gamma(mode=0.08, $s \mathrm{~d}=0.06)$

- Examples of spatial brightness ME studies can be found in Aya et al. (2015b) and Rea et al. (2015). Estimating SDs from graphs gives a range of values from $\sim 0.06$ to 0.2 (log units). Based on this, we use a gamma prior with a mode of 0.08 , and sd of 0.06 on the likely values of the SD. We use the same prior for both the betweenperson variation in condition effects and the within-person variation in responses as the two are indistinguishable in the other $\mathrm{ME}$ studies $^{45}$.

\footnotetext{
44 exponent $=1.43 / \log \otimes$ range

${ }^{45}$ If one has 20 responses from 20 different people, then the variation in the responses could be due to a) variation in the effect of the stimuli on different people, or b) variation caused by people not being perfectly
} 
- Between-condition SD in between-person SD: half-normal $(\mathrm{m}=0, \mathrm{sd}=0.1)$

- Between-condition variation in the between-person variance is uncertain, just as in the matching tasks. On one hand, examination of the range of SDs in different studies suggests variation is possible. On the other hand, that could be largely a factor of noise, and between-experiment variation isn't necessarily the same as betweencondition variation. Given this, we put a half-normal prior with an $\mathrm{SD}$ of 0.1 on this, which is fairly broad given the expected size of the $\mathrm{SD}$, and allows for potentially significant variation.

- Within-person SD in matches: gamma( mode $=0.08, \mathrm{sd}=0.06$ )

- As noted above

$\circ$ Between-condition variance in within-person SD: half-normal $(0,0.4)$.

- No particular information for this. Used the same prior as the brightness matching analysis, allowing for the possibility of significant variation up to a factor of $\sim 2$ or more.

○ Between-person variance in within-person SD: half-normal $(0,0.7)$

- Possibility for larger variation with ME due to the additional source of variance in how different people use numbers. Marsden (1970) in his ME study reported that individuals could vary by a factor of up to three in the range of numbers they would use, and we observed similarly large variation in our experiment.

\subsubsection{Model diagnostics}

The statistical model was run for 5000 iterations per chain and a warmup of 1000 iterations. Four chains were run, and all converged and were well-mixed. Standard Stan model diagnostics (Betancourt, 2017a) indicated no problems: no divergences occurred, effective sample size / iterations was well above 0.1 , split- $\hat{R}$ was well below 1.1 for all parameters (indeed, mostly below 1.01), no parameters had Monte Carlo standard error above $10 \%$ of the posterior standard deviation (most were below 1.5\%), and the energy Bayesian Fraction of Missing Information indicated no pathological behaviour.

reliable in their responses. In reality it is of course due to the combination of both factors, but without multiple responses from each person to measure their reliability, it is impossible to disentangle the two. 


\subsection{Results}

We introduce our results with Figure 3.20, summarising the estimated effects of the different conditions in each of the four experiments. The graphs show the posterior probability distributions of the estimated parameter values given our model, data, and priors. The posterior probability distribution describes what we should conclude are the plausible values for the parameter in question based on the data and our model assumptions. This not only shows us the range of plausible values, but also shows us their relative probabilities. Values where the probability density is higher (i.e. where the blue histograms are larger) are more plausible. The calculation of this probability distribution is one of the benefits of Bayesian statistical methods (Kruschke and Liddell, 2015).

For example, if we look at the effect of the White Wall condition as estimated by the main brightness matching experiment, the model suggests that our best guess should be that the single most likely estimate is that it appears to have about 60\% more light than the Uniform Grey baseline condition when both are at the same mean luminance - it appears brighter. However, it could quite plausibly be anywhere from around $-45 \%$ to $-85 \%$.

The white bars overlaid over the histograms are the 95\% Highest Density Intervals (HDI). The 95\% Highest Density Interval (HDI) is the range covered by the $95 \%$ most probable values, given the data and our model. This may be thought of as analogous to a 95\% confidence interval in common use (Kruschke and Liddell, 2015). The text labels describe the mode of the posterior. This describes the value that has the highest probability in the distribution (Kruschke, 2015).

The results of experiment 1 replicate the broad pattern we observed in the pilot, with the non-uniform conditions appearing significantly brighter than the uniform grey reference (Figure 3.20). This is true regardless of the method used, a check that reinforces the strength of our findings and repeats the similar findings of Tiller et al. (1995) who also found that different methods gave them the same results.

The one exception to the overall pattern is the Black Wall condition, with our improved estimates pointing to it definitely appearing darker than the reference. This may be related to the fact that according to some metrics, such as the standard deviation, it may be actually described as relatively uniform. It may also be an indication that, as has been oft suggested, luminances near the central band and around the walls are more important to people's perceptions. The Black Wall condition, being relatively dark along its walls compared to the others, would be expected to appear darker if such was true. 


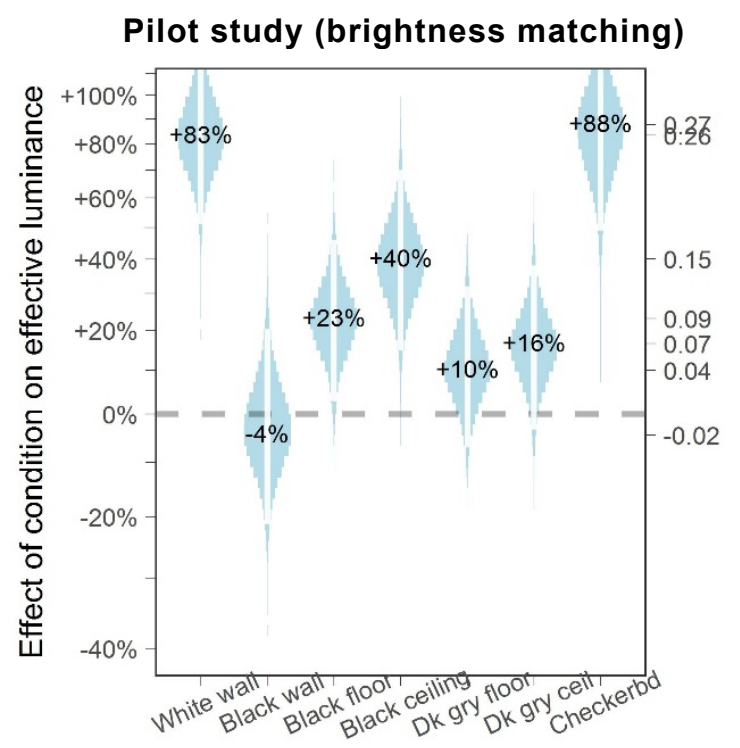

Condition

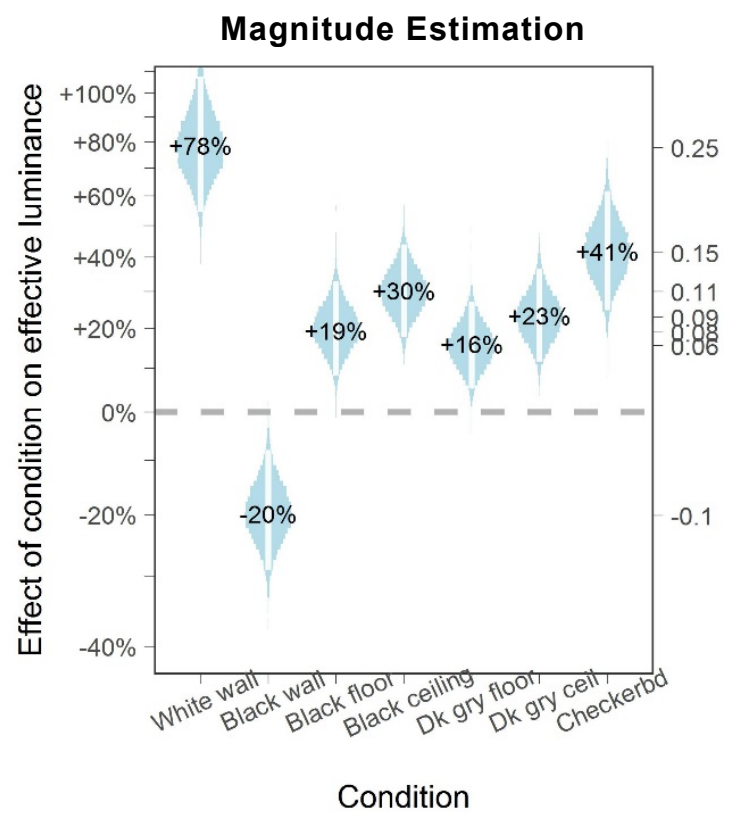

Main Brightness matching w. Null

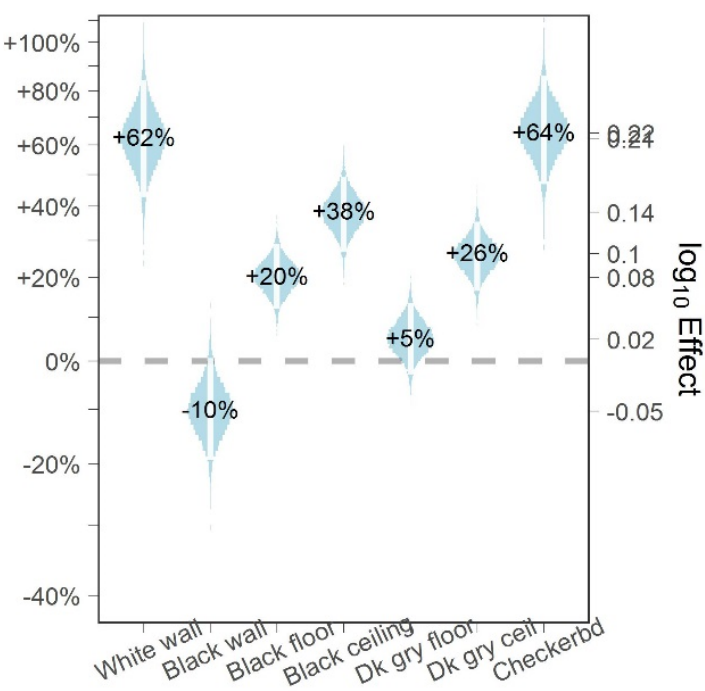

Condition

Pairwise counterbalanced matching

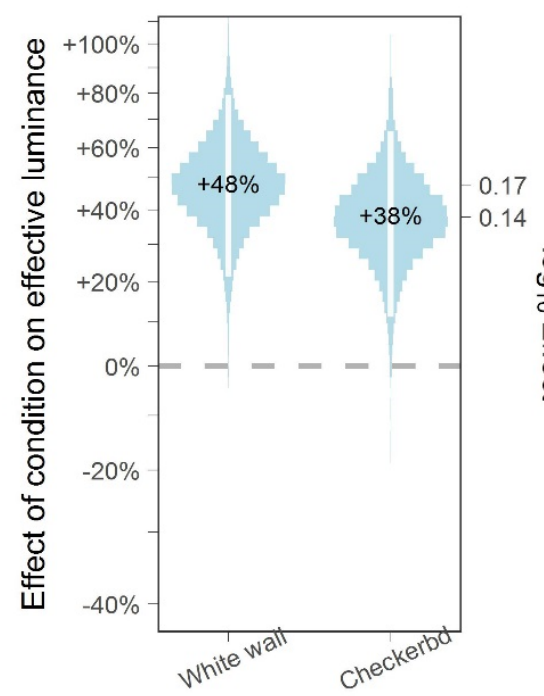

Condition

Figure 3.20 Estimated effects of condition on effective light level from different experiments relative to the uniform grey baseline condition. Estimates here are using our main models described previously with broad non-committal priors on the mean effects.

With all our different methods broadly agreeing, we combined their results into one overall model that assumes that both the brightness matching and magnitude estimation results derive from the same condition effects ${ }^{46}$. The total $\mathrm{N}$ is 35 (or 39 for the White Wall and Checkerboard conditions). Variance and biases were left distinct between the

${ }^{46}$ Similar to a fixed effects meta-analysis 
methods, as it would not make sense to assume that magnitude estimation and brightness matching would have the same properties in those areas. The resulting estimates are shown in Figure 3.21 below:

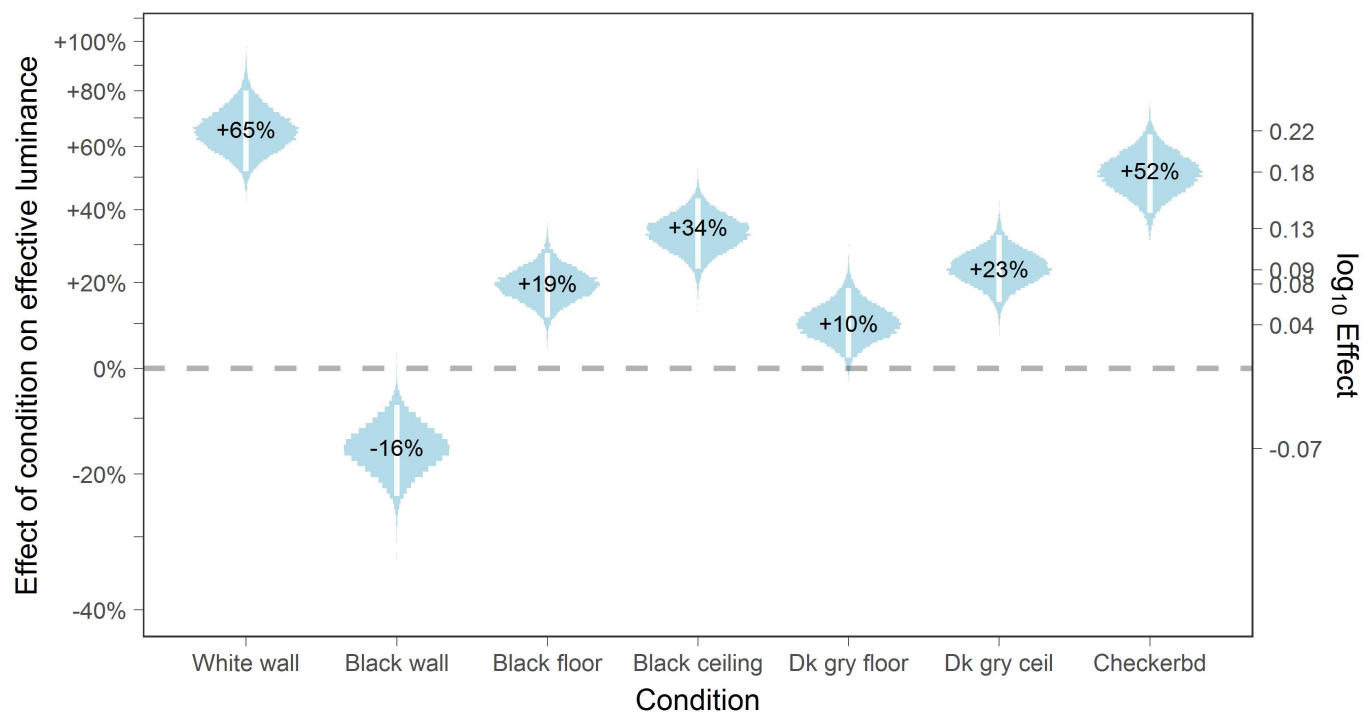

Figure 3.21 Estimated effects of conditions combining all the Experiment 1 data. Estimates here are using our main models described previously with broad non-committal priors on the mean effects.

If we compare this to the predictions of our existing models (see Figure 3.22 below), the scale of the disagreement is readily apparent.

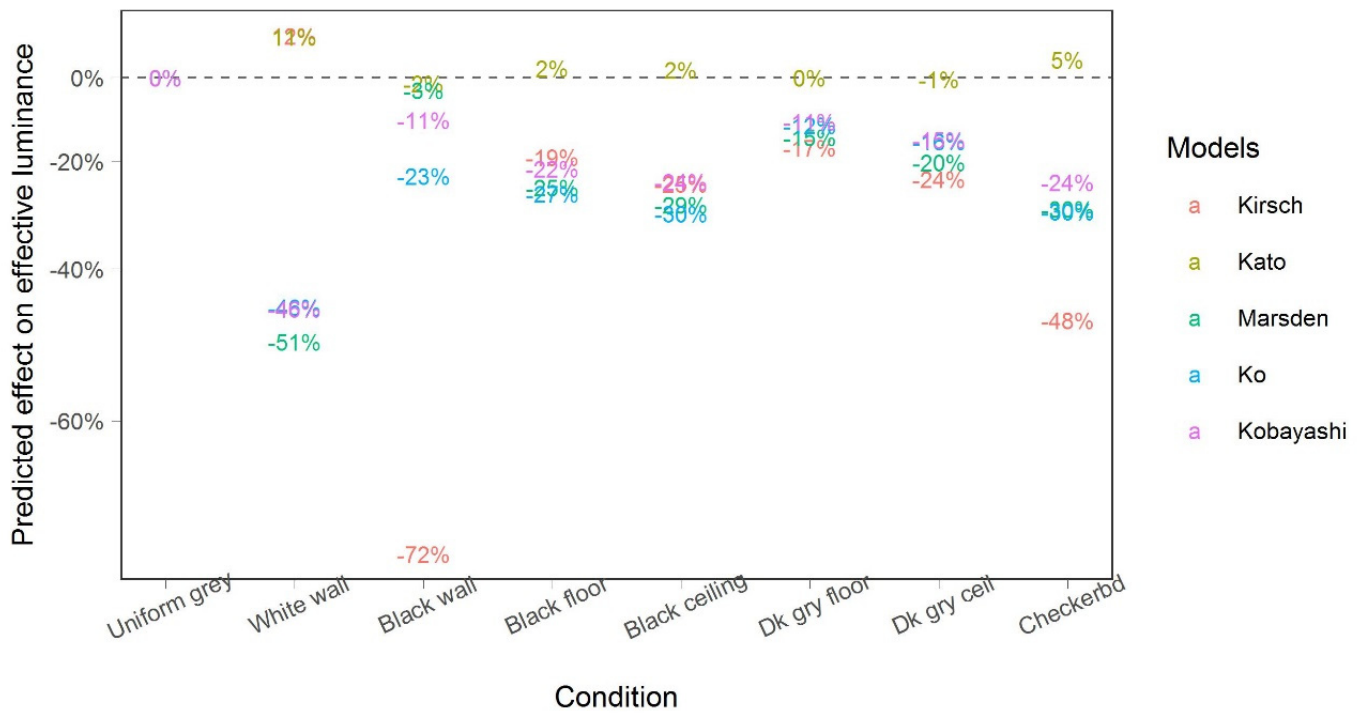

Figure 3.22 Predicted effects of Experiment 1 conditions on effective luminance relative to the baseline Uniform Grey condition according to different models 
We may also look at these estimates using the informed priors ${ }^{47}$ on the effects based on those predictions (Figure 3.23). As can be seen, using these priors pulls the estimates down slightly, reflecting our prior expectation that the effects would be in the opposite direction. However, with this much data the effects of our relatively uncertain priors are minor, with our results dominated by the data. Effect estimates shift by only a few percentage points, and the results and conclusions do not meaningfully change. The experimental results would seem to be providing strong evidence that the predictions of the literature were very wrong, and the level of discrepancy is such that we question the assumption that the predictions (and thus these priors) were ever correct or relevant to this study's conditions to begin with.

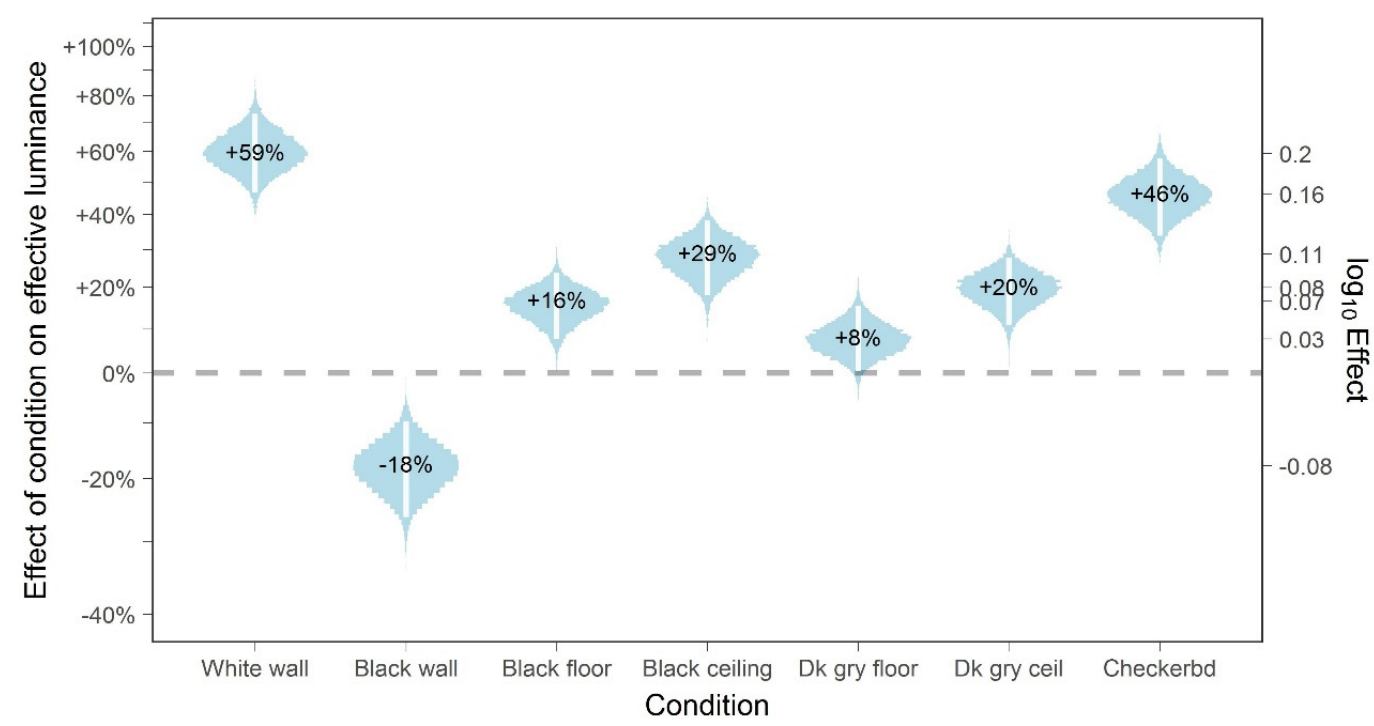

Figure 3.23 Estimated effects of conditions combining all the Experiment 1 data using the informed priors on the mean effects

We may also plot these results against various uniformity metrics that could be calculated from our HDR luminance maps (Figure 3.24). The best performing metrics are the normalised standard deviation, and the uniformity ratio of the $99^{\text {th }}$ percentile/mean (which correlates very closely to the standard deviation). The standard deviation of the $40^{\circ}$ band also performs excellently, which could possibly be another vague indication that focusing on the luminances around the centre of vision is useful, or could just be a coincidence. The Maximum/Minimum ratio for the whole field is relatively weak, and the Max/Min luminance ratio in the $40^{\circ}$ band (suggested by Kirsch, 2014) performs

\footnotetext{
${ }^{47}$ Prior(s): the probability distributions describing the range of values the Bayesian statistical model considered "plausible" before looking at the data (along with their relative plausibility). These may be informed by existing knowledge or past data. Depending on how much data we have, they may have significant effects on our resulting inferences.
} 
particularly poorly here - as do similar metrics. SD of log luminance (as in Ko et al., 2013) performs substantially worse than the normal SD due in large part, it appears, to its failure to capture the effect of the Black Wall condition.

While most of our measurements of uniformity point towards a trend of "non-uniform = brighter", Directional diffusivity (Kato and Sekiguchi, 2005) actually suggests the opposite. Consistent with their results, it suggests a trend of "uniform = brighter".

However, its predictive strength is relatively poor and to get this trend it defines the White Wall and Checkerboard conditions as some of the most uniform spaces. Moreover, as noted when discussing the model predictions (Section 3.3), the model predicts that the conditions should actually be very similar with no significant differences in brightness - a notion clearly in error.

Finally, the average brightness predicted by the Marsden equation actually correlates remarkably well with our results. However, the observed trend is that brighter conditions appear darker, which raises questions about the theoretical validity of the model here.
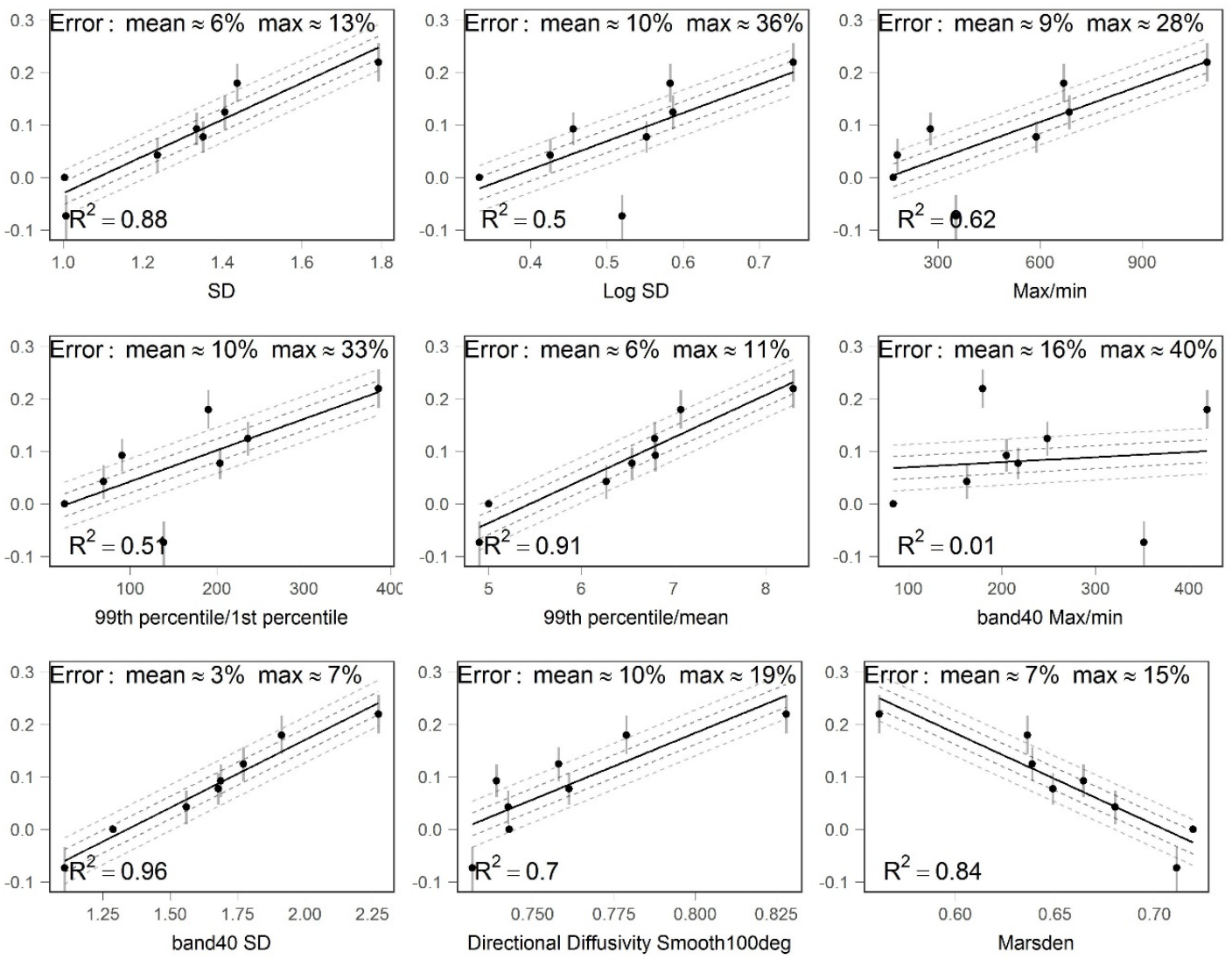

Figure 3.24 Plotting effective luminance (mean estimates from analysis in Figure 3.21) against various uniformity metrics. Black lines are lines of best fit as calculated from a simple linear regression. Dashed lines show 5 and 10\% error in "prediction" 


\subsubsection{Sensitivity Analysis}

Modelling and analysis decisions can potentially have substantial effects on our results. With this in mind, it may be useful to look at several notable decisions that were made, and see how sensitive our results are to them.

In particular:

- The effects of controlling for differences in spectral power distribution (SPD) using the Rea et al. model

- The effects of how we modelled start bias (we assumed that the bias is a linear function of the difference between the match setting and the initial light level, the traditional counterbalancing approach implicitly describes it as being a binary function of whether or not the start point is simply high or low $)^{48}$

- How sensitive our results are to our priors

- Whether or not we model the variance in effects as being a function of the brightness of the individual conditions, or a function of the difference between the pairs of conditions being matched

- The presence of some outliers in the matching data may challenge the assumption of normality. One way to make estimates that are more robust to the presence of outliers may be to use a T-distribution with thicker tails (Kruschke, 2013). Alternatively, we might imagine that a skew-normal distribution could be a better description of the data (Martin and Williams, 2017).

Overall, the results of our analysis are not significantly altered by the various modelling assumptions (Figure 3.25). The largest shifts in the estimates come from accounting for SPD effects, and changing how we model start point bias — and even those are only a few percent at most. Relative to the overall magnitude of the effects demonstrated here, this is trivial — and would likely be imperceptible besides.

\footnotetext{
${ }^{48}$ If we assume that start point bias is, say, just $-3 \%$ with a low start point, and $+3 \%$ with a high one, then we can just counterbalance high/low and it eliminates itself. This is the implicit position held when the counterbalancing approach is used. If, however, we consider the bias magnitude to be proportional to the distance between start and match points, then we would not expect the high and low biases to be equal - and if they are not equal, then they will not perfectly counterbalance. There will be a residual (for more on the subject, see Appendix C.3).
} 


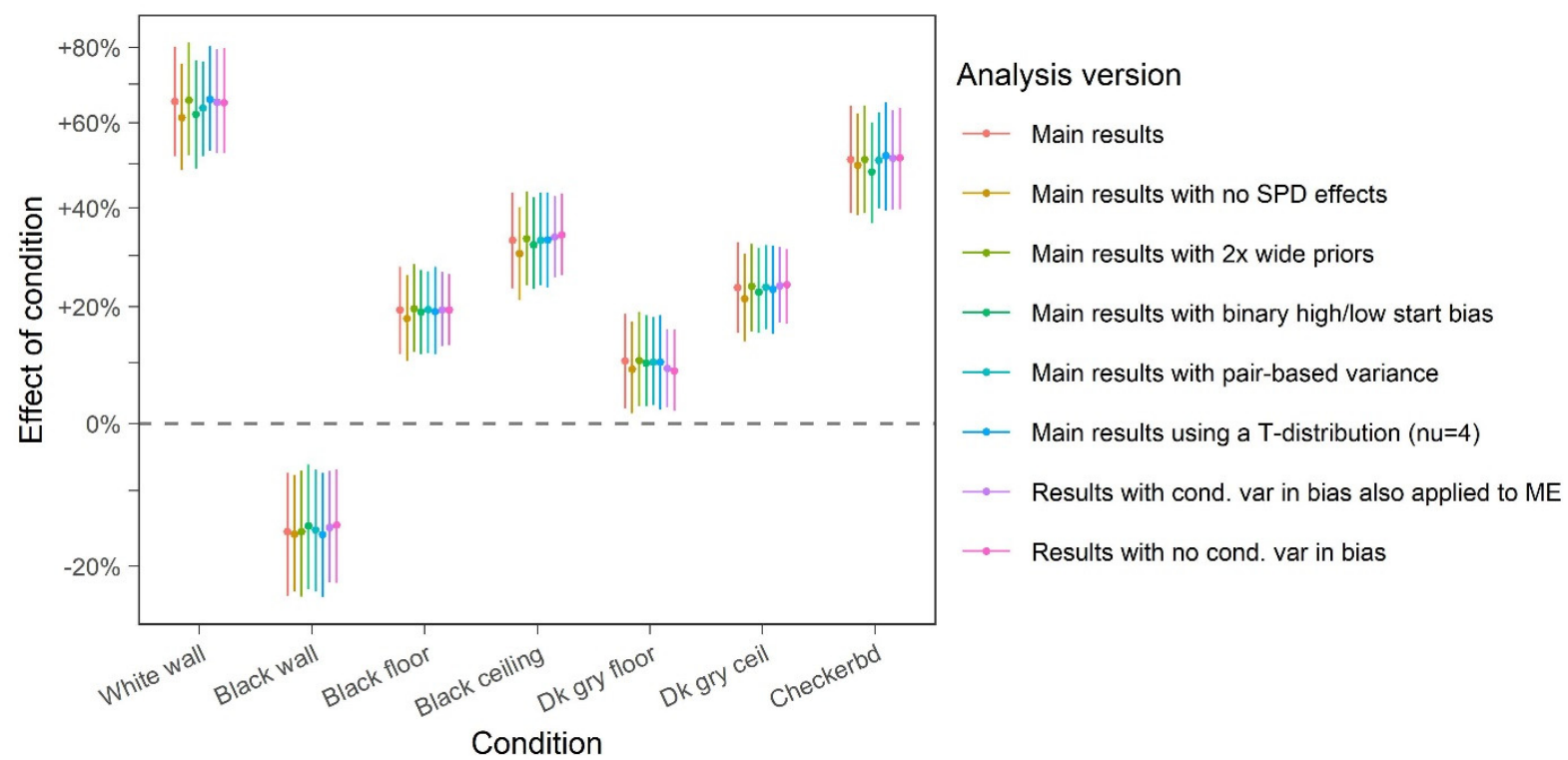

Figure 3.25 Sensitivity analysis of overall results combining all experiments: Effects of different measurement and statistical modelling assumptions on estimated effects of conditions on effective luminance. Error bars show 95\% HDI's.

The limited effect of accounting for differences in SPD is reassuring, given that there is an unknown level of uncertainty in the use of the Rea et al. (2015) brightness model. It suggests that error in the SPD model's predictions is unlikely to change the results significantly unless the model is extremely wrong in some area. Similarly, focusing on the brightness matching results (Figure 3.26), if there are concerns about whether or not the variable SPD adjustment we applied as a result of the apparent shift in colour at low light levels is accurate, it implies that it does not really matter that much. If we just accounted for SPD by using our measurements of the average SPD for each condition and did not adjust the low light level matches further, it would not meaningfully change our conclusions.

Using a T-distribution with thick tails $(v=4)$ instead of a normal distribution does have a noticeable effect on the estimated effects of the white wall and checkerboard conditions in the brightness matching experiment, raising them by several percentage points (Figure 3.26). That being said, this does not change the overall results (Figure 3.25) significantly, as only these brightness matching results have problems with outliers - the other measurements are unaffected. And, of course, increasing the size of the largest effects slightly does not change our conclusions. If we try a skew-normal distribution as perhaps more appropriate for these brightness matching results, we see no meaningful change in the estimates of the mean effects compared to using a normal distribution. 


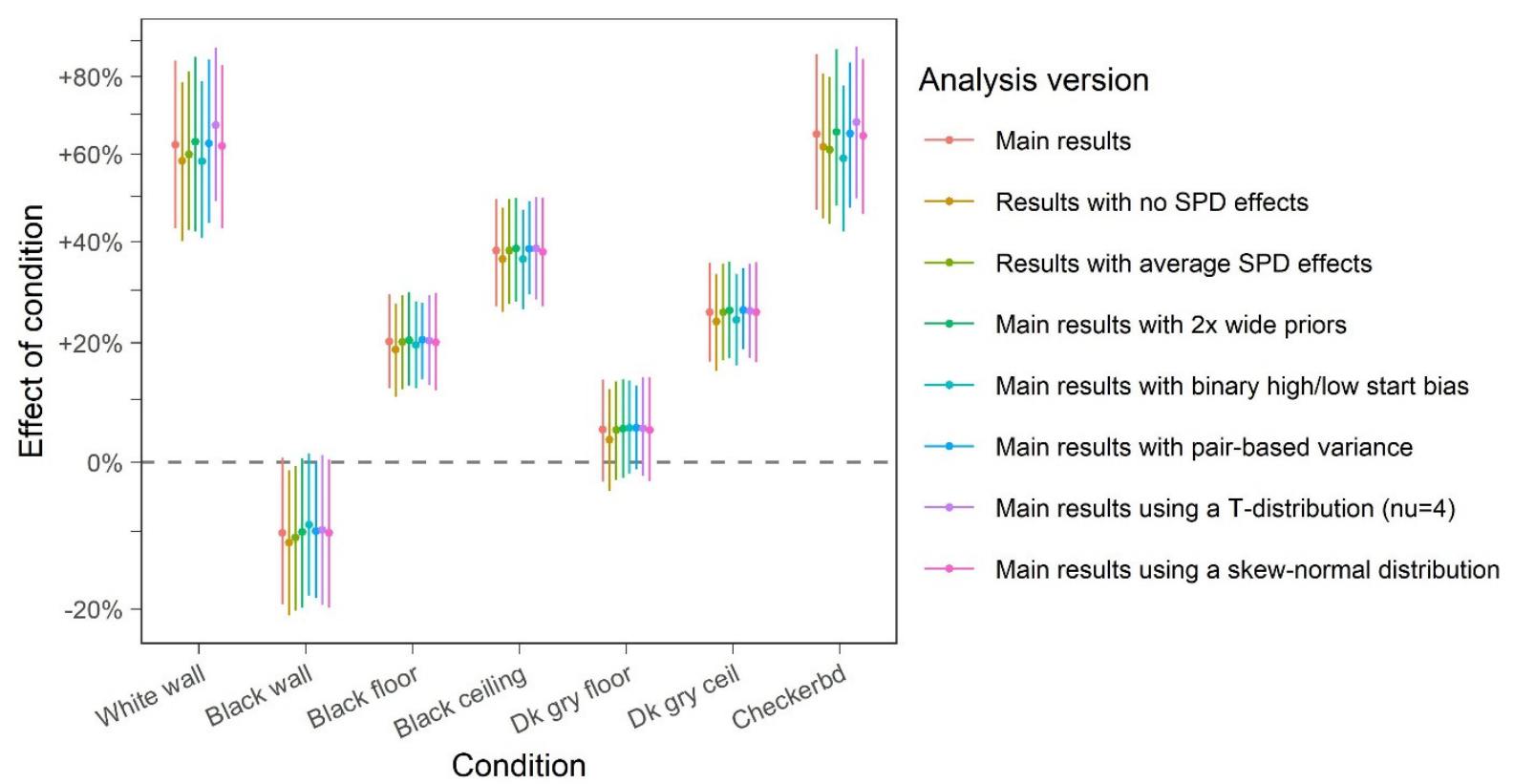

Figure 3.26 Sensitivity analysis of the main brightness matching experiment: Effects of different measurement and statistical modelling assumptions on estimated effects of conditions on effective luminance. Error bars show 95\% HDI's.

The effects of the start bias model are primarily a function of the overall magnitude of the effect. The conditions with the largest effects (the White Wall and Checkerboard conditions) see the most notable differences when we swap between modelling start bias as a proportional function, and modelling it as a simple binary factor that is counterbalanced. In other words, the conditions that it has the largest effect on (in absolute terms) are also the ones where it would have the least ability to change the conclusions.

Our estimates of the main effects are also not particularly sensitive to our priors despite the work put in to working out the best informed priors (Section 3.5.2.1), we can double their width ${ }^{49}$ with no significant effect on our main results.

Perhaps a more interesting question is that of how we model the between-person variance in condition effect. Broadly, there are two different ways we could conceptualise it in the design of this experiment. Firstly, we might imagine that there is variation between people in how they perceive the brightness of each individual condition. This is the position favoured as the default in our model so far. Alternatively, however, we might argue that the data in our experiment — the matches — is actually a report of the difference between the test and reference conditions. Following this, we might suggest that the

\footnotetext{
${ }^{49}$ For example, our resulting priors on the mean effects would be suggesting that a 6 -fold change in light level is very plausible. Similarly, our priors on the mean bias would be suggesting that it being as high as $25 \%$ is quite possible. Thus, such priors would be very broad over the likely scale of any effects we would observe — indeed, arguably too broad and open to unlikely effect sizes.
} 
variance between people in this is a variance in what they think the difference between the conditions is. Modelling the variance this way, as a function of the pairs of conditions being compared, may give different results to modelling it as a function of the individual conditions, and it is not clear which model is "correct". Philosophically, we favour the model where variance is a function of the individual conditions. The rationale here is that while, yes, a brightness match is a report of a difference between two conditions, that difference is because the two conditions are seen as having different effects on brightness. We argue that disagreement between people on the difference between two conditions is a result of them disagreeing on the effects of each individual condition.

That being said, while we have a philosophical preference here, we cannot say that either model is clearly more "correct" than the other. Both are reasonable models of how the data was generated and may provide defensible interpretations of our results. Thus, we consider both of them. In this case, modelling variance as a function of the difference between pairs of conditions has very little effect on the main brightness matching (Figure 3.26) and magnitude estimation (Figure 3.27) results — and thus overall (Figure 3.25) as well. It does, however, make the posteriors significantly narrower in the small $\mathrm{N}=4$ counterbalanced experiment (Figure 3.28). It may continue to be interesting to check this assumption in future studies.

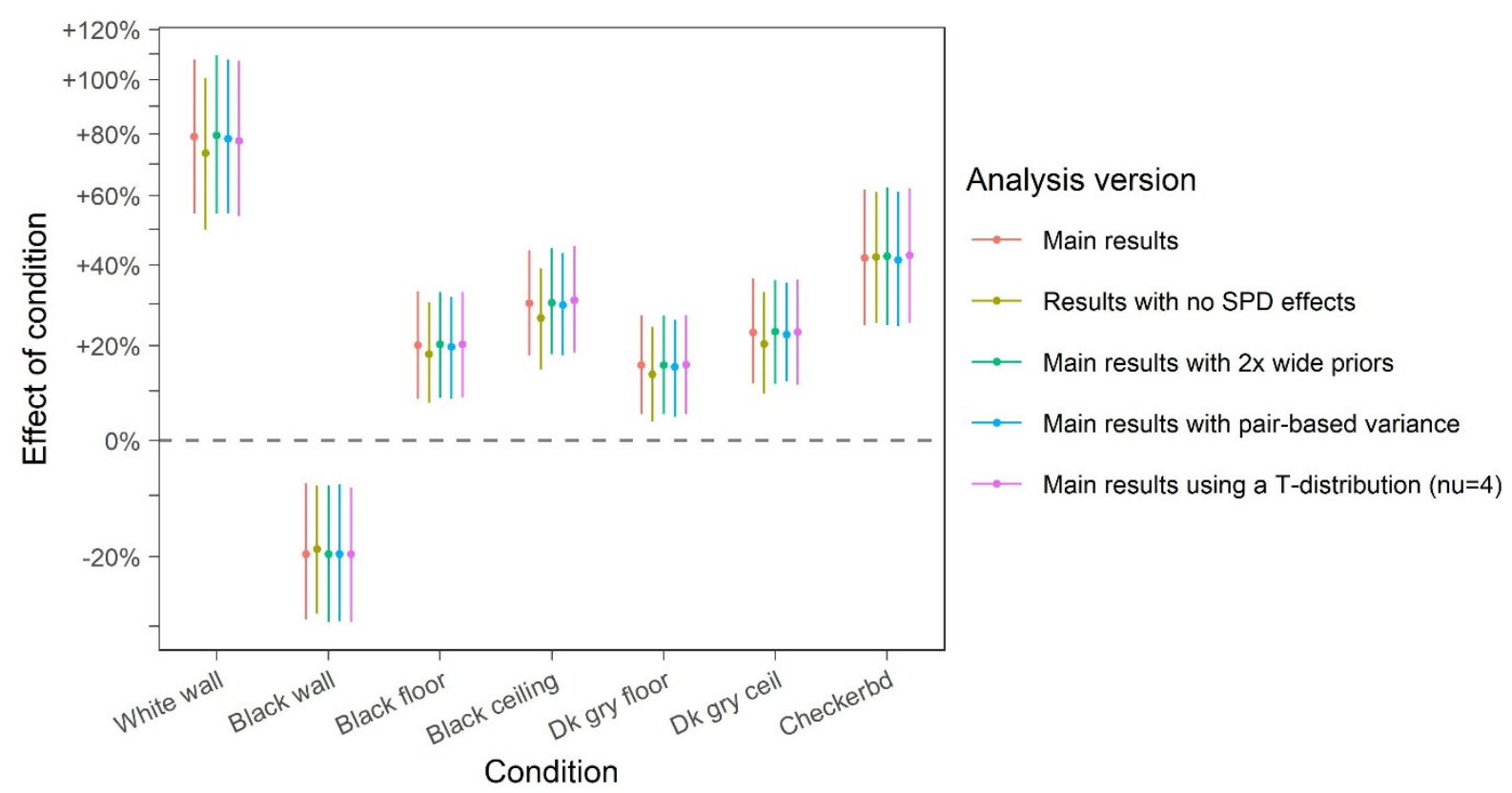

Figure 3.27 Sensitivity analysis of the magnitude estimation experiment: Effects of different measurement and statistical modelling assumptions on estimated effects of conditions on effective luminance. Error bars show 95\% HDI's. 


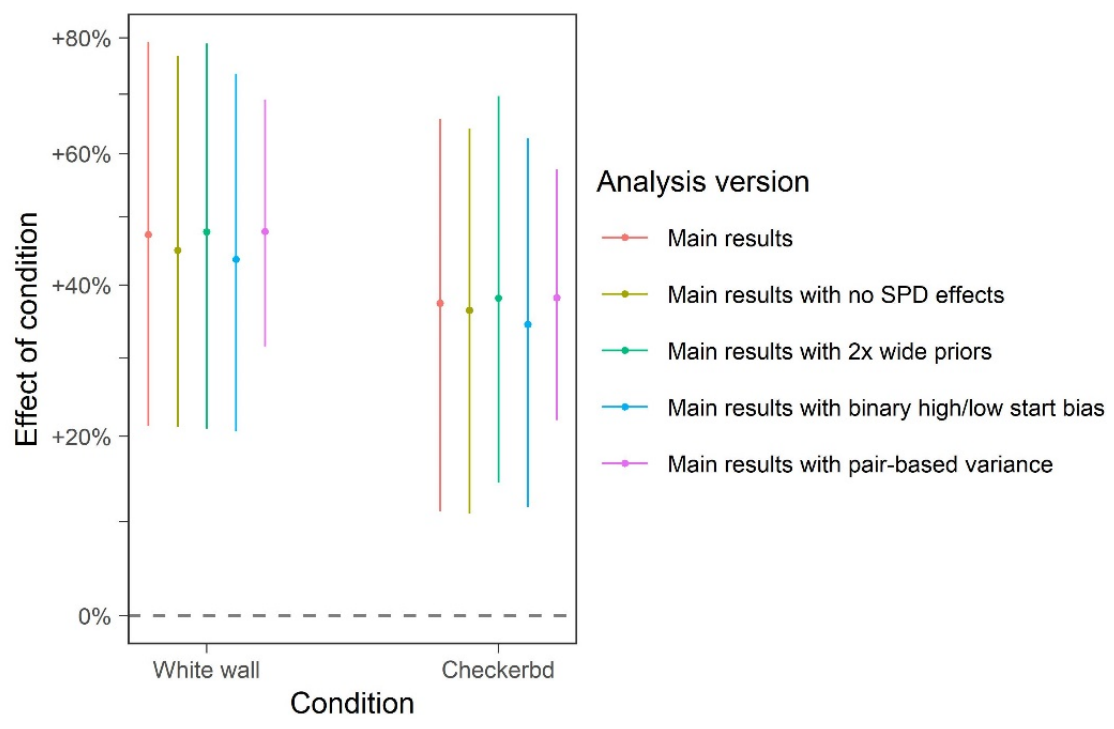

Figure 3.28 Sensitivity analysis of the pairwise counterbalanced brightness matching experiment: Effects of different measurement and statistical modelling assumptions on estimated effects of conditions on effective luminance. Error bars show 95\% HDI's.

An additional question that came up in the calculation of the overall results (Figure 3.25) was whether or not to model between-condition variance in position bias. This was not done in the main brightness matching and magnitude estimation experiments because, relying entirely on a null condition trial to measure the bias, they did not have the information to do it. The counterbalanced experiment did, but was limited to only a couple of conditions and four participants. Indeed, the concern there is that if we tried to put it in the model with only null condition data, and allowed it to vary widely, then the model would end up suggesting that maybe the entire difference between, say, the White Wall and Uniform Grey baseline was just because the White Wall condition has a massive position bias. This would, of course, be highly implausible based on what we know about such biases, but the data in this experiment cannot speak to such. Moreover, the potential correlation between varying position biases and the observed effects of condition could make it very difficult for the model to converge. An observed effect of $+40 \%$ for example "could" potentially be due to a condition difference of $+40 \%$, and a bias difference of $0 \%$, or a condition difference of $20 \%$ and a bias difference of $20 \%$ and so forth. This can be controlled to some degree by setting tight priors that limit how large any position bias can be, but this would then require careful justification.

In this light, we checked what happens if we a) used the estimates of the variance from the counterbalanced experiment to estimate the variation for the Magnitude Estimation experiment as well, or b) just not modelling potential between-condition variation at all (as the counterbalanced experiment really was not large enough to be informative). Neither of 
these meaningfully change our results, with the main effect being that they make our posteriors somewhat narrower.

Overall, our results appear to be robust to these assumptions and decisions. Simple means and confidence intervals also produce the same conclusions (though not precisely the same point estimates) (Appendix B.7).

\subsubsection{Other observations}

A virtue of the Bayesian modelling approach is that it can provide much more than just the estimates of the main effects we have been discussing (Kruschke, 2013). We also have estimates of all the other parameters in our model — the strength of the biases (e.g. Figure 3.29, Figure 3.30), the variance, the variance in the biases and the variance...

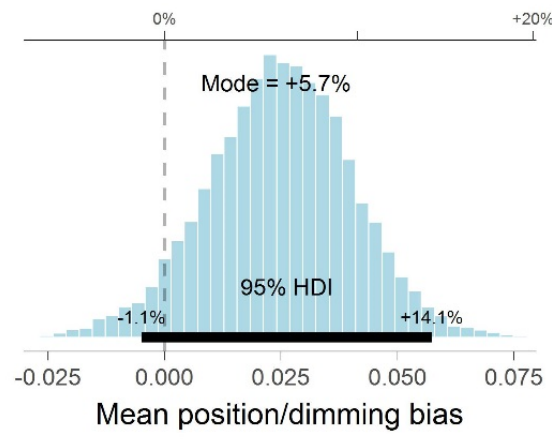

Figure 3.29 Posterior estimate of position+dimming bias in the brightness matching experiments

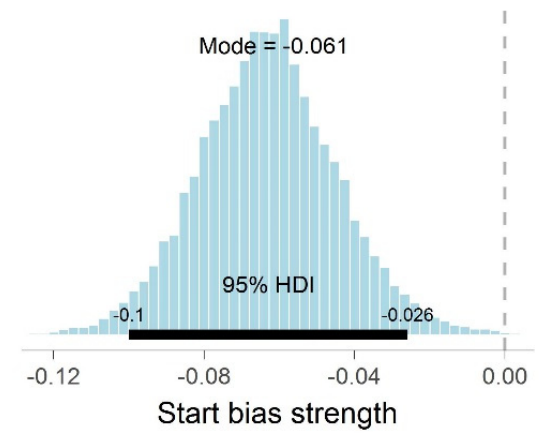

Figure 3.30 Posterior estimate of the strength of the start point bias assuming it is a linear function of the difference between the match setting and the start point. This parameter is essentially the slope of bias against starting light level (log scale).

These are not the focus of this study so we will not spend too much time on them. In brief:

- As we would expect, increasing luminance in the ME study resulted in participants reporting higher brightnesses, and the observed slope of the brightness/luminance relationship $(-0.5-0.6)$ is similar to that found in other spatial brightness studies (e.g. Rea et al., 2015).

- It is unclear if the overall position+dimming bias in the experiments is significant on average (Figure 3.29).

- In the brightness matching experiment the matches of the null condition trials were, on average, close to the light level of the reference, with (perhaps) a small bias of $\sim_{-1} \% 5 \%$. ${ }_{14 \%}$. Similarly, the null trials in the ME experiment gave no particular sign of bias, with an estimate of $-9 \%-1 \%{ }_{7 \%}$. 
- There is solid evidence for start point bias, with matches being biased towards the initial light level.

- We consistently (and unsurprisingly) see significant variance between people. This is true for the effects of conditions, position bias, start bias, and within-person variance.

$\circ$ The average between-person SD is on the low end for the literature (similar to Fotios and Cheal (2011)) at $\sim 0.020 .040 .08$, though still within reasonable bounds.

- There is evidence for significant variation between individuals in position+dimming bias. An estimate for one of the participants in the counterbalanced study suggested a combined position+dimming bias equal to as much as $30 \%$ !

- Variance in start point bias is similarly large (relative to the size of the bias). With the strength of the bias being around 0.06 on average, individuals potentially varying by around $\sim \pm 0.07$ is quite dramatic.

- Evidence for variation between conditions is more mixed. There is evidence for significant variation in between-person variance in the effects of conditions, with the White Wall, Black Wall, and Checkerboard conditions appearing to have significantly higher variance than the others. There is some evidence for variation in within-person SD - however it is unclear if it is significant. There is no meaningful evidence of significant variation in start bias or the brightness/luminance relationship — though we also cannot rule it out.

Overall, our model would appear to be working well, and continuing to account for all these factors in our next experiment would seem sensible. The general alignment of results across the different methods may grant us a certain degree of confidence in the validity of the results, as does the alignment with how we would expect basic brightness assessments to work (i.e. luminance increases brightness to a degree similar to other studies, null trial results generally close to the reference). The large amount of variation between people suggests that at some point it may be desirable to understand this better. The first question it raises is to what degree these apparently strong individual differences actually represent stable differences between people that can be repeatedly shown over time (similar to Teghtsoonian and Teghtsoonian's (1971) study of individuals' brightness/luminance relationships), though this is outside the scope of the current thesis.

For a full discussion of these parameters, see Appendix B.8. 


\subsubsection{Potential issues with Experiment 1 estimates}

Later work on bias control during Experiment 2 (see Appendix C) raised potential concerns about whether or not the methodology of Experiment 1 would, in fact, completely control the bias. In particular:

1) That null condition trials are flawed as a means of bias control as they cannot deal with the possibility of the bias varying between different conditions (Appendix C.3.1)

2) Fotios (personal communication) has suggested that all-possible-pairs should be used in brightness matching as well as forced-choice discrimination experiments, and that the recommendation in Fotios and Houser (2013) to use brightness matching where that was not practical was incorrect

3) The absence of any attempt to account for potential adjustment range centring bias (see Appendix C.3.2 for discussion) could skew the results

Broadly, these issues do not change our conclusions - though they are still worth noting in terms of their effects on the estimates. Perhaps the largest potential issue is the limitations of using null condition trials for managing position+dimming bias instead of counterbalancing (as uncovered in Appendix C.3.1). While no studies have been carried out looking at between-condition variance in position/dimming bias in particular ${ }^{50}$, measurements in different studies and Experiment 2 suggest it could potentially be as large as 5-10\% (Section 5.1.5, Appendix C.4.3). Erring on the side of caution, we might account for this by expanding the error bars of our estimates by $\sim \pm 10 \%$. Fortunately, because the effects observed across Experiment 1 were so large, this does not meaningfully change our overall conclusions.

The question about all-possible-pairs is a complex one, and a more detailed discussion may be found in Appendix C.6. In brief, however, Fotios and Houser (2013) highlighted possible stimulus range centring and stimulus frequency biases as concerns in forced-choice discrimination experiments ${ }^{51}$. These biases were observed when the test condition was

\footnotetext{
${ }^{50}$ And there are some technical challenges there, as apparent differences between, say, the left and right could be caused by subtle physical differences and measurement error as much as psychological bias. Differences caused by physical differences in conditions (exactly mirroring a condition for example is hard, and it is readily conceivable that the left version of one condition might look brighter, while the left version of another might look darker) could be said to be "legitimate" differences that do not actually need to be controlled for unlike variation in psychological bias.

${ }^{51}$ Forced choice discrimination differs from matching in that instead of adjusting the light level of a test condition to match a given reference, participants are instead asked whether or not the test condition is brighter or darker (Teller et al., 2003), or just which condition is brighter (Fotios and Cheal, 2008). If this is done at multiple light levels, then the point of subjective equal brightness (equivalent to our match light level) can be identified as the point at which there is a $50 \%$ chance of a given condition being said to be brighter.
} 
shown at a range of different light levels with a constant reference condition, and involve the point of equal brightness being drawn towards the centre of the test stimulus range.

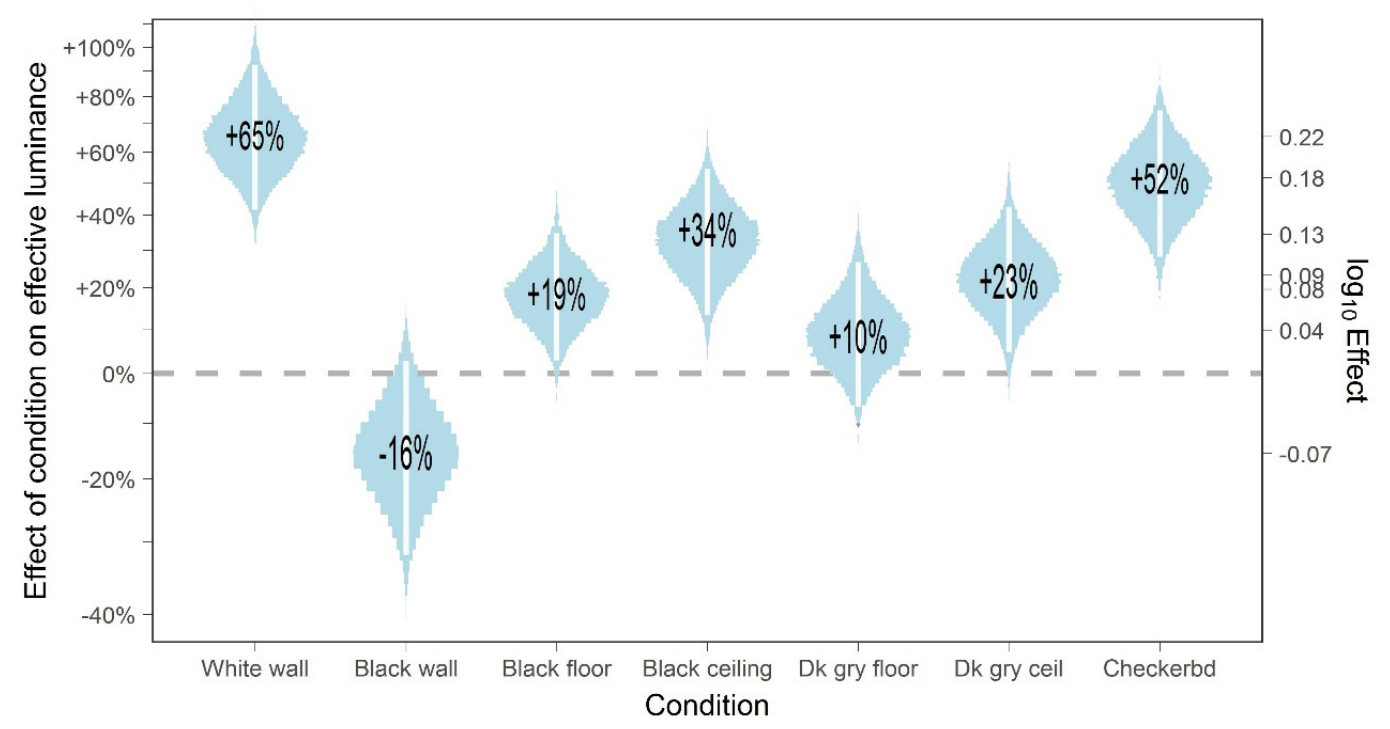

Figure 3.31 Effect of crudely increasing the uncertainty in Figure 3.21 by $\pm 10 \%$ to account for the possibility of significant variance in position/dimming bias between conditions. Despite this, we still see repeated, large, and statistically significant increases in brightness for our non-uniform test conditions.

One may note here that this sounds very similar to the adjustment range centring bias in point (3) above. This is because it is essentially the same effect - indeed, the stimulus range and stimulus frequency bias in the presentation of Fotios and Houser are just two different explanations for the same observations. In that light, we may note that the best way to deal with such a bias in a brightness matching experiment, as explained in Appendix C.3.2.2, is to counterbalance application of dimming and to run trials at multiple adjustment ranges in order to measure the bias. All-possible-pairs is unnecessary. It may also be completely ineffectual, because in brightness matching the test condition and its associated adjustment range will inherently be clearly distinguishable from the reference condition, and an all-possible-pairs design will do nothing to disrupt that.

With regards to potential adjustment range bias the best thing we can do is try to estimate the potential impact of an adjustment range centring bias on our results. At a basic level, it should be noted that the matches made in Experiment 1 were generally relatively low, as most of the test conditions appeared brighter than the reference. If we assume that the matches were influenced by this bias, then they would have been drawn up towards the centre of their adjustment ranges. This means that the theoretical unbiased matches would actually be lower, and the observed effects would be larger. This would reinforce our conclusion that non-uniform distributions could appear brighter. To estimate the potential error here, we may take the estimate of the bias strength computed in Experiment 2, and 
apply it to the Experiment 1 data as a crude estimate. Using an estimate from a separate experiment is not ideal, but it is the best that can be done (and has precedent in the brightness matching literature (e.g. Fotios and Gado, 2005; Fotios and Cheal, 2007b)). The effect is minor, generally only a few percent at most (Table 3.6):

Table 3.6 Effect of controlling for possible adjustment range bias in the brightness matching data using the estimate of bias strength (-0.03) from Experiment 2. Bias for each condition was calculated as a function of the distance from the match point to the centre of the adjustment range following the model described in Experiment 2's methodology.

\begin{tabular}{|l|l|l|}
\hline Condition & $\begin{array}{l}\text { Estimate of } \\
\text { effects from } \\
\text { brightness } \\
\text { matching exp. }\end{array}$ & $\begin{array}{l}\text { Controlling for } \\
\text { possible } \\
\text { adjustment } \\
\text { range bias }\end{array}$ \\
\hline 1 & - & - \\
\hline 2 & $+62 \%$ & $+65 \%$ \\
\hline 3 & $-10 \%$ & $-8.5 \%$ \\
\hline 4 & $+20 \%$ & $+20 \%$ \\
\hline 5 & $+38 \%$ & $+39 \%$ \\
\hline 6 & $+4.9 \%$ & $+4.6 \%$ \\
\hline 7 & $+26 \%$ & $+27 \%$ \\
\hline 8 & $+64 \%$ & $+67 \%$ \\
\hline
\end{tabular}

\subsubsection{Conclusions}

To summarise: the results of this experiment make two key points:

1. Our non-uniform conditions appear brighter — in many cases substantially so — than our more uniform conditions

2. All predictions from existing models of light distribution and spatial brightness failed to predict our results, largely pointing in the opposite direction

The effects appear to be large, repeatable, and robust to both methodology and statistical modelling assumptions. Indeed, given the size of the effects, even substantial error would not likely change the overall pattern.

This reignites the disagreement in the literature over the direction of the spatial brightness/uniformity relationship by reinforcing the findings of Tiller and Veitch (1995). While there are still more studies arguing that uniform spaces appear brighter, it is difficult to argue that this is a reliable trend. The result: we find that not only can we not predict the magnitude of the effects of light distribution, but we also cannot even predict the direction.

The question now is what we can learn from our failures, and where we should go from here. 


\section{Why were our predictions wrong, and what does it mean?}

With the results replicating across both the larger brightness matching and magnitude estimation experiments, we can say that a) the apparent disagreement between this study and others in the literature does not appear to be due to the method of brightness assessment; and b) that, at least in these cases, spaces with light distributions that would be described as more non-uniform can appear significantly brighter than more uniform spaces. These apparent non-uniform = brighter effects were able to be reliably produced across multiple conditions using multiple methods. The idea that non-uniformity can increase spatial brightness in some situations, previously suggested by Tiller and Veitch (1995) and Okajima and Fujimoto (2008), cannot just be dismissed in favour of the position presented by the rest of the literature. Before carrying out our experiment our position, based on the literature, was that while we could not precisely predict the magnitude of the effects, we could at least predict their likely direction. However, we failed to do even that. Not only can we not predict the magnitude of the effects, but we also cannot reliably predict their direction. We now, in a sense, "know" even less than we did before.

If we are to ever understand the relationship of light distribution and spatial brightness and usefully predict its effects, it will be critical to determine why we are getting these apparently divergent results. To begin the examination of this question, let us discuss two interrelated issues:

1) Why the metrics that had been (apparently) supported by previous research failed here

2) How limitations in our descriptions of light distribution could give apparently strong yet fragile and occasionally contradictory results. 


\subsection{Why contradictions and failure may be unsurprising}

To understand these apparent contradictions between studies, we must understand how our theoretical assumptions and methodology affect our results. We focus here on the assumptions underlying the design of our experimental conditions and metrics/models. For the sake of argument, we will assume that all the results in the literature are "true" observations, and would replicate. The idea that some results may be incorrect due to sampling error is not the focus here.

Implicit in the idea that there are contradictions between different studies on the nature of the relationship between uniformity and spatial brightness are several assumptions:

a) That we know what non-uniformity is, and are applying it consistently across studies

b) That uniformity actually drives changes in spatial brightness

c) That the studies actually provided meaningful evidence for their conclusions about the brightness/uniformity relationship

If uniformity is not related to spatial brightness, or the different studies' conclusions were not well founded, it would be unsurprising if their observations were not consistent. To begin, let us examine the question of what non-uniformity actually is.

\subsubsection{The problem with talking about the brightness/uniformity relationship}

Whether or not a given light distribution is more or less "uniform" than another is not actually a simple question - yet it is clearly one whose answer is critical to saying whether or not increasing non-uniformity makes a space appear brighter or darker.

A wide variety of metrics can be used to describe the "uniformity" of a light distribution. Indeed, as noted in the literature review, one of the problems in comparing the different studies is that most of them have tended to use different measurements to describe the light distribution. This matters because, while most of these metrics are correlated with each other to varying degrees (see e.g. Section 2.4.7), they do not always agree.

For example, if we were to use the standard deviation to define "uniformity" of our spaces then we would conclude that that the Black Wall condition is about the same uniformity as the baseline Uniform Grey condition, while the White Wall condition is clearly the most non-uniform. If, however, we used the max/min ratio in the $40^{\circ}$ band (a la Kirsche (2014)) then we would conclude that the Black Wall condition is, in fact, the most non-uniform, while the White Wall condition is relatively uniform. If we are comparing two different spaces that have been observed to have significantly different brightness, we 
could potentially use them to argue that a) non-uniform spaces are brighter, or b) uniform spaces are brighter, simply by changing how we define "uniformity".

Indeed, while we have framed our results as showing that "non-uniform spaces appear brighter (at least in this instance) this is not an unarguable interpretation. If we were to look at the correlation with Directional Diffusivity, it actually suggests the opposite - that spaces that have higher Diffusivity and are thus "more uniform" appear brighter (Figure 4.1). Of course, as discussed earlier, it does this by defining conditions such as our checkerboard one as "relatively uniform", and the model greatly underestimates the size of our effects (Section 3.3), which is why we do not believe it. However, it does illustrate the principle.
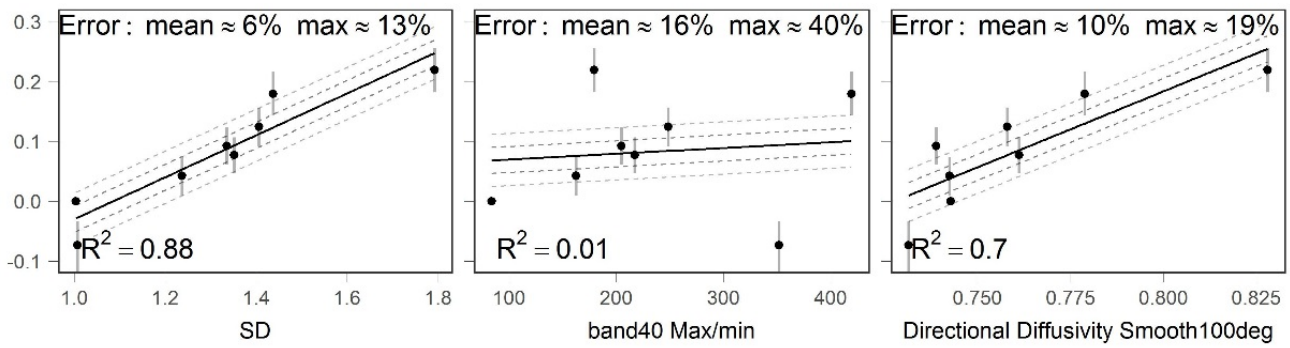

Figure 4.1 Plots of selected uniformity metrics against the estimated effective luminance of the Experiment 1 conditions. Depending on the choice of metric, the relationship of "uniformity" to spatial brightness changes significantly.

This highlights several points:

- Uniformity is actually somewhat vaguely defined. Different studies define it in different ways, and this could potentially produce the appearance of disagreement where none actually exists.

- Generalising from any single correlation to uniformity in general having a clear relationship with spatial brightness is highly questionable.

- Examination of model assumptions can be a useful tool to rule out some results, as illustrated by how Directional Diffusivity does a poor job at describing our conditions.

That being said, despite this vagueness — which is a major issue for attempting to make any strong claim about the relationship between "uniformity" and spatial brightness - it does not actually seem that likely that we would be able to reconcile the different findings in the literature by simply finding the "right" uniformity metric. While the potential for trend reversal we have demonstrated above is undeniable, in practice it only occurs for certain sets of conditions (such as our White Wall and Black Wall conditions), and becomes harder to produce the more conditions one has. Many comparisons simply cannot be reasonably reversed - it makes no sense, for example, to define a checkerboard as more 
uniform than a uniform grey plane. Even though other studies do not report all the different ways one could measure uniformity, when we look at their non-uniform distributions it is hard to argue that we could easily and defensibly reverse their apparent trends. In this light, a more reasonable position may be:

1) Some non-uniform light distributions can make spaces appear brighter

2) Some non-uniform light distributions can make spaces appear darker

3) Therefore, uniformity itself does not affect spatial brightness.

Indeed, the idea that changing uniformity changes spatial brightness could be a complete misreading of the situation. It is hard to think of a reason why, for example, the maximum/minimum ratio would be a good description of how the light distribution is processed and turned into the percept of spatial brightness. However, models such as the simple brightness equations (Kobayashi et al., 1998; Marsden, 1970) that we attempted to apply here illustrate how a theoretical process that is in no way based on uniformity may, nevertheless, have a tendency to produce apparent uniformity effects (Section 2.4.5, $2.4 .6)^{52}$.

In this framing, the apparent contradictions in the literature are due to the fact that our attempts to use uniformity to explain changes in spatial brightness as a result of light distribution was fundamentally flawed from the start. Instead, we are just observing the results of a system that generally perceives different light distributions as differing in brightness, but which is not actually driven by changing uniformity. The apparent correlations are just happening because changes in light distribution in this manner also often produce changes in our uniformity metrics. Indeed, it is hard to produce systematically different light distributions that do not vary in uniformity. In this way, it may be argued that apparent relationship(s) between uniformity and spatial brightness is largely spurious, and the apparent disagreements between studies are a reflection of the fact that spatial brightness is actually driven by some other factor.

\subsubsection{It is very easy for fundamentally flawed models to appear to work well}

The question that may occur at this point is how easy is it for studies like ours and the others in the literature to give highly misleading results in the way that we are suggesting? Surely, if these metrics were so wrong, they should not give such good correlations (statistical error aside)? Surely we should not have multiple studies with different conditions pointing to the same trends?

Unfortunately, it is indeed rather easy to get highly misleading results.

\footnotetext{
52 Though not these specific models obviously, since they completely failed here.
} 
The problems of comparing pairs of conditions we have already discussed (Section 2.3.1, also highlighted by the earlier discussion of how changing uniformity metrics can change which of the white wall or black wall conditions is "most non-uniform"), but most studies looking at these kinds of correlations examine multiple conditions. Should this not address the problem?

Let us look at some examples. In our experiment, the $\max / \mathrm{min}$ ratio in the $40^{\circ}$ band had no meaningful correlation with people's brightness matches. However, we could imagine a parallel universe in which our experiment did not include the White Wall and Black Wall conditions. In that universe, we would find a very strong statistically significant correlation with an $\mathrm{R}^{2}$ of 0.9 (Figure 4.2)! The experiment itself would be perfectly unobjectionable. Six different conditions - just like Kirsch (2014). A baseline uniform condition compared to four different conditions examining varying floor/ceiling contrasts, and then a Checkerboard condition that appears to simply confirm the overall trend that the other conditions suggest, despite its differences. The evidence would arguably appear quite convincing.
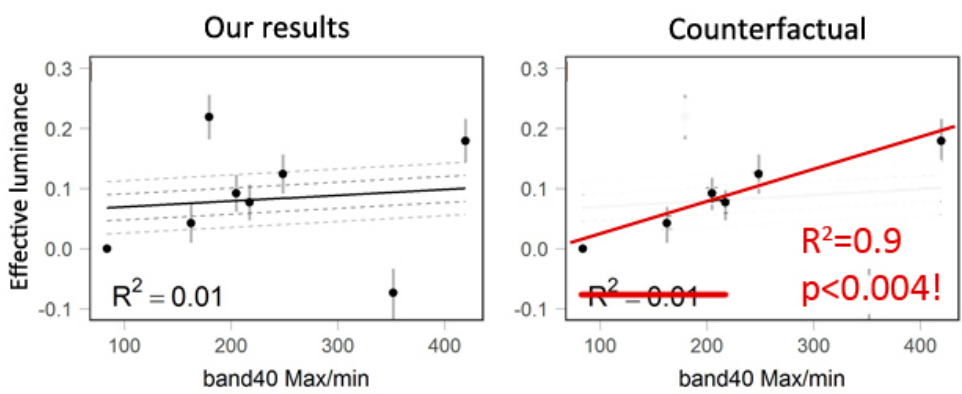

Figure 4.2 Illustration of the strong relationship we would find with the $\mathrm{max} / \mathrm{min}$ ratio in the $40^{\circ}$ band in a counter-factual universe where we did not make the White Wall and Black Wall conditions

It would also happen to be completely wrong. However, until we look at conditions like, in this case, the White Wall and Black Wall ones, we would have no way of knowing that, and the data from such an experiment would look perfectly reasonable.

How likely is this kind of situation though? Is the example above just a narratively convenient coincidence that of course we would be able to find if we plotted enough different uniformity metrics?

One way of exploring the question could be to look at what would happen if we ran simulated experiments looking at the relationship of a uniformity ratio that "really" only has a weak correlation with brightness. For example, let us use the Marsden Equation as a description of the "True Brightness" for the sake of illustration, and plot it against the maximum/minimum ratio for thousands of randomly generated "light distributions" as 
done earlier when we compared the different brightness models (Section 2.4.7) (Figure 4.3).

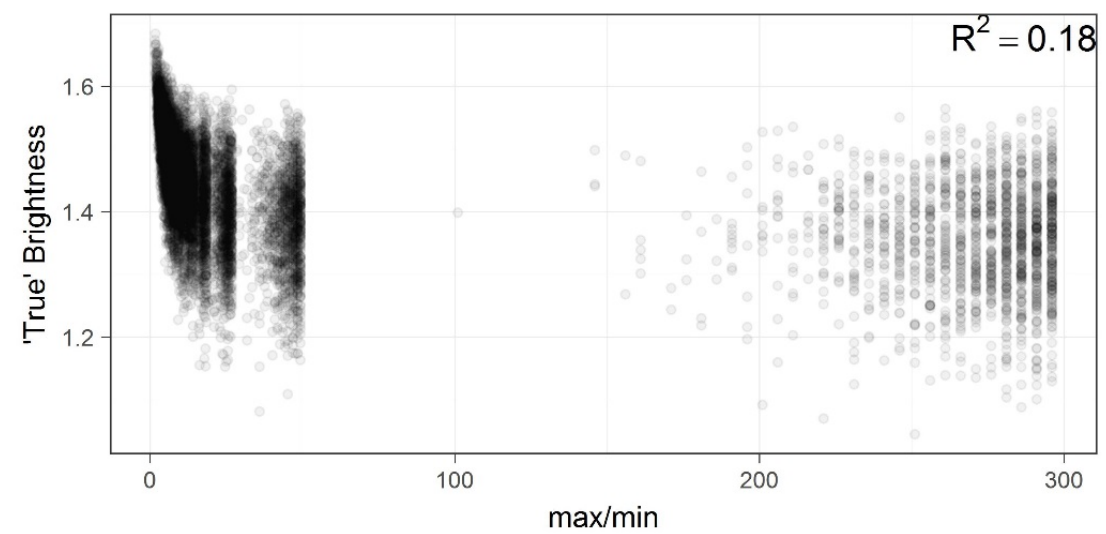

Figure 4.3 Illustration of a hypothetical relationship between a chosen uniformity metric, and the "true" effect on brightness of different light distributions, as approximated through 10,000 randomly generated patterns.

Each "experiment" could be viewed as being a study of a random selection of light distributions from this space of possible light distributions. For example, we might examine six conditions as shown below:

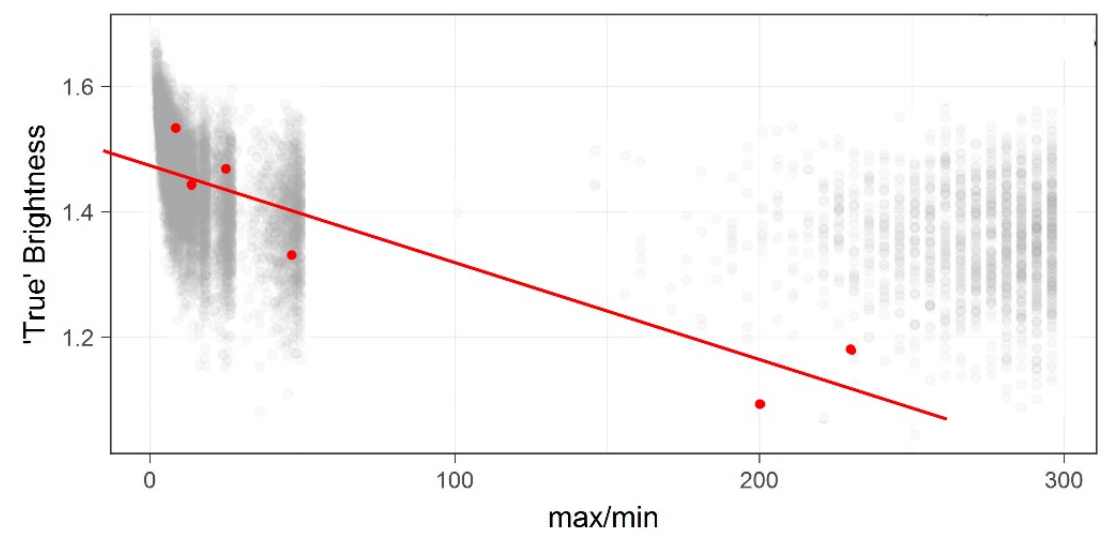

Figure 4.4 Illustration of a hypothetical experiment trying to determine the relationship between a chosen uniformity metric and brightness by looking at six conditions from the theoretical pool of possible conditions. The red points show the conditions that happened to be examined in the hypothetical "experiment".

If we simulate this process thousands of times we find that while we can often observe correlations that are far higher than the "true" one, the odds of us observing a "high" one over, say, 0.75, are "only" $-12 \%$ (Figure 4.5). Admittedly, this is not high. On the other hand, it is also an error statistic that would generally not be considered to meet traditional statistical thresholds (i.e. <5\%). Of course, if we had multiple studies that had all independently found that this metric had a high correlation with brightness, we would 
arguably have strong reason to believe in it. It is merely unfortunate that this particular field lacks such studies.

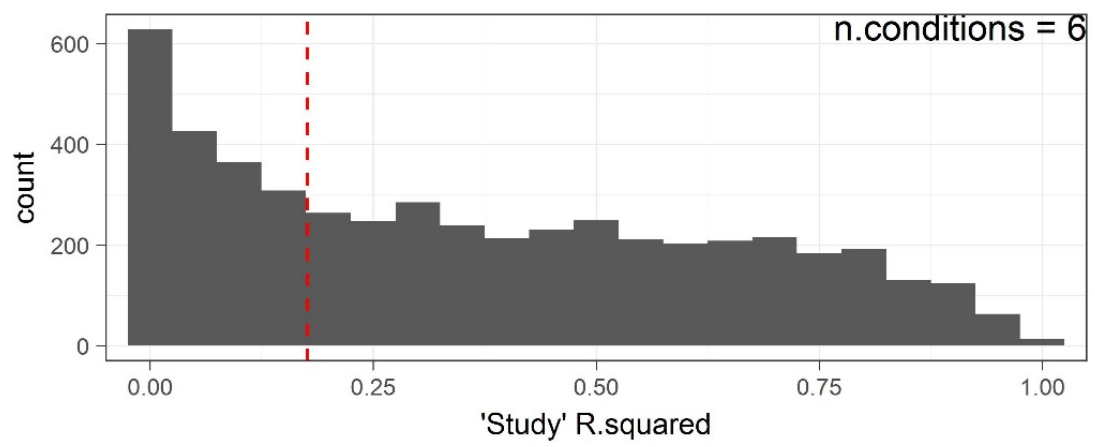

Figure 4.5 Distribution of observed $R^{2}$ values from simulated studies of the relationship between uniformity and brightness with 6 conditions. The "true" $R^{2}$ that would be found if a sufficiently large number of conditions were studied is shown by the red dashed line.

The problem gets worse when we consider that the researcher does not only have a single uniformity metric to try. Instead, they have many options, on top of the ability to apply factors such as position weighting or arbitrarily cutting out whole swathes of the field of view in order to get a good "fit" 53 . As we have discussed, these metrics, while loosely correlated, can also disagree significantly and potentially produce very different patterns. This flexibility in defining uniformity offers many opportunities for a researcher to "find" a relationship between brightness and uniformity ${ }^{54}$.

We may add to this that in reality, the conditions or light distributions examined in a study are not randomly selected from the pool of all possible light distributions. In real experiments they are generally systematically designed to examine a range of different scenarios and (hopefully) test different issues. However, this can also include redundancy (for example, 6 light distributions at 3 light levels is not 18 conditions, it is just 6), and offers great scope for inadvertently introducing systematic bias. If a study has not been designed to specifically test a given question or metric, it is very easy for it to give misleading results.

To illustrate, let us consider some simple examples of the study of brightness. Horizontal work plane illuminance is well known to be a poor metric to describe how

\footnotetext{
${ }^{53}$ It is, admittedly, unclear how much this has occurred in the literature, given that most studies do not report multiple metrics (not that there are very many studies). This could be because they did not look at a lot, but could also be because they felt that reporting their "failures" was not important. It could also be that they just happened to get a good result on the first metric they tested — which would not erase these issues. If, in an alternative universe their response to finding a "poor" result was to try another metric, then the problems remain.

${ }^{54}$ And is arguably just a demonstration of the problems of "fishing expeditions" and "researcher degrees of freedom” (Gelman and Loken, 2013; Simmons et al., 2011)
} 
bright a room appears to people (Cuttle, 2010; Duff et al., 2015a, 2015c; Julian, 1987; Lynes, 1971; Waldram, 1969). Despite this, it would be very easy for a study to show an excellent correlation between it and spatial brightness. For example, a simple way to look at the effect of changing the light level on spatial brightness would be to simply dim the lights to produce multiple light levels, and then have people assess their brightness. Such a study would inevitably find that horizontal work plane illuminance works perfectly well to predict brightness because in this case it (and any other light measurement you take) correlates perfectly with the overall light level in the room. It is not that the study is necessarily a bad study — it's just not designed to test Horizontal Illuminance.

Correlations like this can often drive misleading results. A study that looks at brightness over a range of conditions covering a wide range of light levels could similarly find that horizontal work plane illuminance correlates well with brightness for the simple reason that the conditions that have the highest mean luminance (a better metric) will also often have higher horizontal illuminance. It is not always the case, and the limitations of horizontal illuminance can cause it to fail dramatically when examined closely, but they are related enough that it often can give good looking results. If one does not deliberately try to test the metric by examining conditions that, for example, change the overall light level while keeping horizontal illuminance constant (e.g. Duff et al., 2015a), one can easily get misleadingly positive results. This can be a particular problem when a wide range of light levels are examined. The reason for this is that if we compare spaces that differ in mean luminance by, say, an order of magnitude, we will inevitably find that the effect of mean luminance is far larger than anything else. We will be able to plot mean luminance against brightness, as is done in many studies, and find an excellent correlation. This can obscure other factors that are significant. For example, light distribution or lamp colour might have effects equivalent to a 20 or $50 \%$ change in mean luminance, which would have great practical significance. However, they are still much smaller than a 10 -fold change in luminance, and if that is being plotted on a graph it will overwhelm everything else, and it will appear that mean luminance explains all the changes in brightness very well. The same is true if one has a model of spatial brightness that describes it as a function of light level and some other factor (such as uniformity). If one's experimental conditions cover a wide range of light levels then the model may inevitably "appear" to perform well — at least in terms of having a good correlation coefficient and plotting well. However, the positive result may be entirely driven by the fact that the model accounts for light level, and so such simple analysis would not actually be a useful test (as we know light level is strongly related to brightness).

Issues along these lines can be found throughout the literature. In Kato et al. (2016), their model predictions appear to be mostly explained by variation in mean luminance, 
making it unclear what their model actually adds. Kobayashi et al. (1998) had the problem that their field tests of their model do not actually seem to perform meaningfully better than what would be expected from just plotting brightness against a log or power function of luminance (Section 2.4.5). Houser et al. (2002) observed that overall brightness in their experiment seemed to be more related to the brightness of the walls and ceiling than the floor, but this is entirely explained by the fact that they were holding horizontal illuminance constant. The same was true for Flynn (1977).

In this light, that previously identified trends were not reproduced here may not be surprising. It is not hard to "find" a good correlation in an individual experiment, and the flexibility in how uniformity is defined provides substantial opportunity to do this. Confounding of variables is also common in the literature, and studies often fail to consider alternative explanations for their results. Given this, a positive correlation provides surprisingly little evidence for the generalisability of a given spatial brightness model. To establish a model is robust then we need more. Alternative explanations need to be ruled out. It needs to be shown that the model does not fail easily. The best way to do this, we suggest, is to test the model assumptions. For example, we may identify a range of assumptions embedded in the metrics we have examined here:

- The maximum/minimum (or other arbitrary percentile) is critically influential to human perception

- Only what is in the $40^{\circ}$ horizontal band matters

- Vertical luminance variation is generally unimportant

- Luminances need to be weighted by visual angle

- Effects of variation are symmetrical

- Deviations from the mean have a greater than linear effect

- Pattern is irrelevant

- We should be measuring the uniformity or distribution of luminance rather than brightness

- Brightness is a function (at least in part) of uniformity

- Spatial brightness is just the average brightness of the room

- All luminance is the same - modes of appearance are irrelevant

Some of these assumptions are clearly questionable when highlighted in this way — we have already noted, for example, the problems with basing everything on the maximum/minimum luminance (Section 2.4.1, Figure 2.12), or ignoring everything outside the $40^{\circ}$ horizontal band (Section 2.4.1), or ignoring vertical luminance variation as in Kato et al.'s directional diffusivity model (Section 2.4.4, 3.3). Whether or not we should be weighting luminance according to its position, as described by vertical angle, is, as we have noted, plausible - yet we lack useful data on the subject (Section 2.3.2). Many 
metrics, such as uniformity ratios, the standard deviation, or the simple applications of brightness models such as Stevens' equation ignore pattern entirely. One could take a luminance map and randomly rearrange the XY coordinates of all of the pixels, and such metrics would be entirely unaffected - they are structure-blind. Similarly gradients or sharp edges are assumed to be irrelevant to human perception. There are also more subtle assumptions buried in certain metrics. The standard deviation, for instance, is derived from the mean squared deviation. It assumes that the effect of deviations from the mean is nonlinear, and a space with a large deviation in luminance over a small area is less uniform than a space with a more moderate deviation in luminance over a larger area (Figure 4.6). It also assumes that effects of deviations are symmetrical - that increasing an area by $\mathrm{X}$ has the same effect as decreasing it by $\mathrm{X}$ (Figure 4.7).

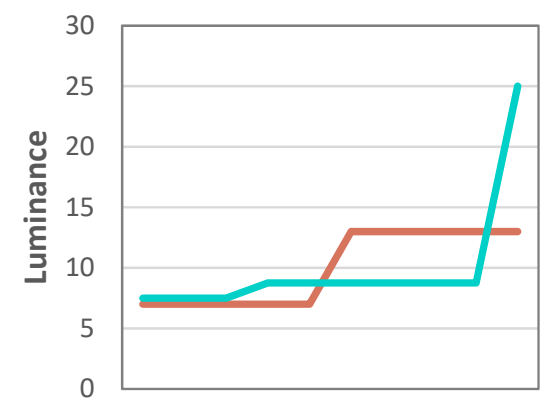

Figure 4.6 Example of the cross-sections of two light distributions that compare the assumptions of the mean absolute deviation (MAD) vs. that of the standard deviation (SD). If the effects of deviations are a constant linear factor as MAD assumes, both should have the same brightness. If the effects are non-linear, with larger deviations having disproportionately larger effects, then the blue distributions should effectively be more nonuniform.

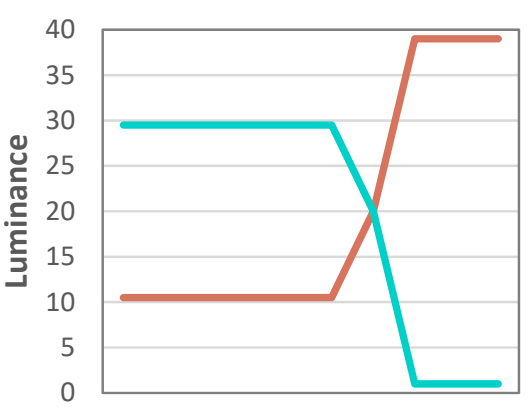

Figure 4.7 Plot of the cross-section of two hypothetical luminance distributions that have the same mean and standard deviation, but are skewed in opposite directions. Would they have the same brightness? This could be a critical test of the validity of the use of the SD as a predictor.

Other assumptions are more fundamental. Should we be building our models off luminance, or should we be building them off brightness — off power-transformed luminance? The use of Stevens' equation or the Marsden equation here is based off the assumption that spatial brightness is just the average brightness of the elements in a room. Their failure here thus gives us strong reason to doubt that, and grants credence to the position that spatial brightness is a distinct percept (see Section 2.1). Perhaps most fundamental is the assumption that luminance is merely luminance, regardless of its provenance. That we need not consider the qualitative appearance of elements in the room when we assess spatial brightness (Modes of Appearance - Section 2.1). 
Such questions are what need to be tested if we want to establish the validity of the various models of spatial brightness that have been proposed. For the same reasons, the theoretical plausibility of such assumptions can also be a useful way to critique the plausibility of the models. Unfortunately, such considerations have been absent from past work. The importance of this is illustrated by the performance of the Directional Diffusivity metric. Despite being the model with the most evidence backing it, with "good" results in multiple studies (Aya et al., 2015a; Kato et al., 2016; Kato and Hara, 2011; Kato and Sekiguchi, 2005; Kimura et al., 2004), it failed to describe our results and conditions. This appears to be due to its assumption about vertical variation being unimportant. The studies it worked on largely looked at very similar sets of light distributions (horizontal arrangements of light sources around the walls), and did not test this core assumption. Our light distributions however were mostly varied through vertical changes, and this broke the model. Their most recent work (Kato et al., 2016) looks at a wider range of light distributions, but suffers from confounding with the overall light level. As noted earlier (p24), it is unclear that Directional Diffusivity is adding anything to the prediction of brightness in that study not already accounted for by mean luminance. Their attempts to then adjust the model further to improve it (Yamaguchi et al., 2016) - while reasonable do not provide strong evidence in favour of the model as the flexibility available in model design can allow researchers to fit almost anything (Gelman and Loken, 2013).

\subsubsection{Conclusion: why should we believe any model?}

The concepts discussed here are not novel within the broader sphere of science and philosophy. The limited ability of studies to provide positive evidence in favour of specific models (as opposed to disproving them, which can be done much more easily) is essentially just an echo of Falsificationism (Popper, 1963). Similarly, we may see, in our discussion of how easy it can be to find convincing yet effectively spurious correlations, an echo of the issues of "fishing expeditions", "HARKing" (Hypothesising After the Results are Known), and "the garden of forking paths" (Gelman and Loken, 2013; Mayo, 2018; Simmons et al., 2011). What is important is that these issues are clearly highly pertinent to the field of spatial brightness research, and should be given more attention.

It is also important to emphasise that these issues are not ones solved by statistics, at least not with respect to the concerns about how variance between people produces uncertainty in our estimates of effects. Even if we had an infinite sample size and no statistical error, our ability to draw generalizable conclusions from these experiments is still substantially limited. The conditions in a typical experiment can be viewed as a small nonrepresentative sample of a broader population of lighting designs. This population has a massive variance - which is also unknown. Due to this, while such experiments can be a 
valuable tool to probe specific assumptions, they are an exceedingly poor tool for making broader assertions about general trend $s^{55}$.

None of this, it should be said, should be taken to say that looking at correlations with uniformity metrics is pointless. Indeed, it is entirely possible that we could find that the right uniformity metric correlates well enough in practice that we could use it as a simple model of spatial brightness without believing that it is the "true" description of the underlying mechanisms at work. If there were multiple research groups all carrying out lots of studies of light distribution and spatial brightness, and they all kept checking all the different metrics and we found one that did consistently correlate well, this could be good evidence!

Unfortunately, that is not the world we live in. Instead we have a small number of studies over the past two decades, most of which only test or report the results of a single model. As we noted, it is very easy to get misleadingly convincing results in this way, and so this approach gives us minimal reason to have faith in any of the metrics that have been proposed. This reason is why we do not consider metrics such as the standard deviation that performed well on our data to be well supported either. Our study was not designed to test their underlying assumptions, and we see no reason to believe that they would not fail in future studies the same way as the others have here ${ }^{56}$.

Despite this negative assessment, it should be said that the studies of light distribution and brightness have done a good job at establishing that light distribution matters, and giving us examples of the kinds of changes that produce large effects on spatial brightness. The patterns of results that have been accumulated through this process show us what we need to explain in any model that wants to be successful. The hypotheses they have produced provide a fruitful source of ideas about how we might take the field further, and what could be useful to test. Their main limitation is that they are not very good at establishing strong evidence for their models of these relationships. This is true for both ours and the other studies in the literature. If we are to move forward, and acquire an understanding of light distribution and spatial brightness that allows us to actually construct useful models, we need to determine what assumptions are valid, and what forms of distributional variation need to be accounted for. The first step on that process, we suggest, should be to work out what the difference is between the non-uniform distributions that increase brightness, and the non-uniform distributions that decrease it.

\footnotetext{
55 Well, positive assertions. They can show that specific trends do not generalise perfectly well.

${ }^{56}$ Moreover, a number of metrics are heavily confounded with each other. For example, the standard deviation and the max/mean ratio are indistinguishable in our results.
} 


\subsection{Illuminating the way forward: differences in light distribution between studies}

Perhaps the best place to start for trying to work out why different studies give us different results is to examine the differences in the kinds of light distributions used in different studies. Reviewing them, we identify four key qualitative differences between our study's conditions and those used elsewhere.

These are:

1. Visibility of light sources

2. Modes of Appearance used to produce the different light distributions

3. Orientation of the light distribution changes

4. Adaptation level

Their presence in the different studies is summarised on the following page: 
Table 4.1 Characteristics of the light distributions compared in studies providing evidence towards the effects of uniformity on spatial brightness

\begin{tabular}{|c|c|c|c|c|c|}
\hline Study & $\mathrm{X}$ is brighter & $\begin{array}{c}\text { Light } \\
\text { sources? }\end{array}$ & $\begin{array}{c}\text { Modes of } \\
\text { Appearance }\end{array}$ & $\begin{array}{l}\text { Orientation of } \\
\text { distribution } \\
\text { changes }\end{array}$ & $\begin{array}{l}\text { Light } \\
\text { level }\end{array}$ \\
\hline $\begin{array}{l}\text { Kobayashi et } \\
\text { al. (1998) }\end{array}$ & Uniform & $\begin{array}{l}\text { Visible } \\
\text { and } \\
\text { hidden }\end{array}$ & $\begin{array}{l}\text { Luminous/ } \\
\text { illumination }\end{array}$ & Unclear/variable & Photopic \\
\hline Hsieh (2012) & Uniform & Visible & $\begin{array}{l}\text { Luminous/ } \\
\text { illumination }\end{array}$ & Lateral/depth & Photopic \\
\hline $\begin{array}{l}\text { Kirsch } \\
(2014)\end{array}$ & Uniform & Visible & $\begin{array}{l}\text { Luminous/ } \\
\text { illumination }\end{array}$ & Mostly lateral & Photopic \\
\hline $\begin{array}{l}\text { Kimura et al. } \\
(2004)\end{array}$ & Uniform & Visible & Luminous & Lateral & Photopic \\
\hline $\begin{array}{l}\text { Kato and } \\
\text { Sekiguchi } \\
(2005)\end{array}$ & Uniform & Visible & Luminous & Lateral & Photopic \\
\hline $\begin{array}{l}\text { Kato and } \\
\text { Hara }(2011)\end{array}$ & Uniform & Visible & Luminous & Lateral & Photopic \\
\hline $\begin{array}{l}\text { Aya et al. } \\
\text { (2015); Kato } \\
\text { et al. (2015) }\end{array}$ & Uniform & Visible & $\begin{array}{l}\text { Luminous/ } \\
\text { illumination }\end{array}$ & Lateral & Photopic \\
\hline $\begin{array}{l}\text { Kato et al. } \\
(2016) ; \text { Lu et } \\
\text { al. (2016) }\end{array}$ & $\begin{array}{l}\text { Uniform? } \\
\text { (confounded } \\
\text { with light } \\
\text { level) }\end{array}$ & Visible & $\begin{array}{l}\text { Luminous/ } \\
\text { illumination/ } \\
\text { Surface }\end{array}$ & variable & Photopic \\
\hline $\begin{array}{l}\text { Tiller \& } \\
\text { Veitch } \\
\text { (1995); } \\
\text { Tiller, Veitch } \\
\text { \& Pasini } \\
\text { (1995) }\end{array}$ & Non-uniform & Hidden & Illumination & Vertical & Photopic \\
\hline $\begin{array}{l}\text { Okajima and } \\
\text { Fujimoto } \\
(2008)\end{array}$ & Non-uniform & $\begin{array}{l}\text { Mainly } \\
\text { hidden }\end{array}$ & $\begin{array}{l}\text { Luminous/ } \\
\text { Illumination/ } \\
\text { Surface }\end{array}$ & Uniform & Photopic \\
\hline $\begin{array}{l}\text { Sullivan and } \\
\text { Donn (2016, } \\
2017, \text { this } \\
\text { thesis) }\end{array}$ & Non-uniform & Hidden & Surface & Mostly vertical & Mesopic \\
\hline
\end{tabular}

We discuss each of these in turn. 


\subsubsection{Visibility of light sources}

Perhaps the simplest and most overt difference between the different studies is visibility of the light source. The studies favouring non-uniform conditions (Tiller and Veitch, 1995; this thesis) kept the light sources out of view of participants. In contrast, in the studies favouring uniform conditions the light sources were visible and often the main source of changes in the light distributions - for example Kato and Sekiguchi (2005) created luminous walls with fluorescent lights behind fabric sheets, Kimura et al. (2004) as well as Kato and Hara (2011) examined different arrangements of artificial windows, and Kirsch (2014) created their different light distributions using a room covered in LED panels.

This suggests that the mere presence of visible light sources in a space could change the apparent effect of changing the light distribution. It is, however, difficult to come up with strong theoretical reasons for this idea. It is true that visible light sources will have a strong effect on the light distribution by creating areas of high intensity luminance. Luminous objects also provide a qualitatively different impression than non-luminous objects - i.e. they appear luminous (Cuttle, 2004; Gilchrist, 2006). In this sense, the concept that luminous light sources might contribute to the impression of spatial brightness in a different fashion than non-luminous surfaces is plausible. It is, however, somewhat of a stretch to say that the mere presence of visible light sources would completely change how non-uniformity in the rest of the space is interpreted.

The two studies that used images, rather than real rooms (Kobayashi et al., 1998; Okajima and Fujimoto, 2008), also do not fit this hypothesis perfectly. Both studies appear to have included both images with visible light sources and images without, yet their results were diametrically opposed. This could be a result of the way that they were adjusting uniformity of their images, or perhaps "light sources" in images are not always interpreted the same way. It is hard to say.

\subsubsection{Modes of Appearance used to produce the different light distributions}

A more plausible way for visible light sources to have such an effect on spatial brightness would be if their distribution affected spatial brightness differently than the other surfaces. Under this hypothesis, we would say that non-uniform arrangements of light sources makes spaces look darker, but non-uniform patterns of surface reflectance or illumination make spaces look brighter.

In the studies favouring uniform spaces, the different light distributions were, as discussed, generally produced using different arrangements of the visible light sources. Differences between conditions were thus based largely on non-uniform distributions of luminosity - of elements whose qualitative appearance is luminous. In contrast, in the 
studies favouring non-uniform spaces the light sources were hidden, and so the differences in uniformity were produced by changing the distribution of surface lightness (this thesis), or the distribution of illumination (Tiller and Veitch, 1995). This hypothesis could serve to explain the apparent differences between the main spatial brightness studies here. That being said, there are issues. Different patterns of light sources will inevitably produce different patterns of illumination, so effects in such studies would not just be a product of different luminosity distributions. Aya et al. (2015b) used spotlights arranged around the walls of their room to produce their different light distributions, and so their changes in light distribution would have been as much a matter of varying illumination as varying luminosity. The image-based studies (Kobayashi et al., 1998; Okajima and Fujimoto, 2008), using images both with and without visible light sources, again do not fit this hypothesis clearly. The most recent study from Kato et al. (Kato et al., 2016; Lu et al., 2016) used a wider range of conditions, varying both lighting and surface reflectance, and reported results consistent with their uniformity-based model. This may challenge the credibility of the hypothesis. That being said, as discussed previously (Section 2.3.1), their results provided only questionable support for their actual uniformity model, as the correlations in this experiment appear to be largely driven by differences in mean luminance. It is thus unclear that "uniformity" even clearly relates to the differences observed in this experiment - which could potentially be a result of them mixing so many elements in their conditions. For studies that have changes in both illumination and light source patterns, one possibility is that the effects of the light sources are just much stronger than those of illumination. Given how much higher the luminance of light sources is compared to everything else in a scene, this is not implausible.

Overall, while not necessarily fitting everything perfectly, this hypothesis at least has theoretical plausibility. It is plausible for elements with qualitatively different appearances to have different effects, and it may be consistent with the way that spatial brightness seems to be built off more than just the brightness of the elements in a room. If a) "spatial brightness" is a distinct Mode of Appearance that is in some way inferred from the elements in the room, and b) people are not just basing it directly off the average brightness of elements (as our results would suggest), then we might expect them to be working off some sense of the interaction of the different elements. To be deriving their impressions from a sense of how the lighting and surface reflectances come together produce the ambient illumination "field" filling the space. Hence, we might expect them to be considering different Modes in some way. 


\subsubsection{Orientation of contrast changes}

Another difference between the various studies is the orientation of the changes in light distribution. The various studies by Kato et al. (with the exception of Kato et al. (2016)) focused on lateral arrangements of lighting around the walls (Aya et al., 2015b; Kato and Hara, 2011; Kato and Sekiguchi, 2005; Kimura et al., 2004). Similarly, all but one of the conditions studied by Kirsch (2014) were forms of horizontal gradient or step change along the walls. In contrast, Tiller and Veitch (1995) adjusted the light distribution on the walls by essentially shifting the cut-angle of the illumination - a vertical change. Similarly, most of our conditions involved vertical changes of the reflectances on the walls, ceiling, and floor of their spaces. This raises the question of whether or not vertical changes in light distribution have different effects than horizontal changes. It has been suggested that orientation of light direction may affect apparent brightness (McCourt et al., 2013), and perceived contrast (Chacón et al., 2015), which gives some grounding for the hypothesis though a complete reversal of effect would be far larger than the effects observed in those studies.

There, however, are a number of challenges to this hypothesis. As noted, Kirsch did include one vertical non-uniform distribution whose results aligned with the others. Similarly, our checkerboard distribution showed effects comparable to the similarly nonuniform white wall condition - despite the fact that the checkerboard is as much horizontal variation as vertical. Adding on to this are the image-based studies, which had both horizontal and vertical changes in their conditions (Kobayashi et al., 1998), or simply adjusted the contrast uniformly across the whole image with no particular orientation in their conditions (Okajima and Fujimoto, 2008). We may also note that contrast effects do not suddenly reverse themselves when you rotate the image $90^{\circ}$ (Figure 4.8). Overall, this hypothesis has some justification, but is questionable.
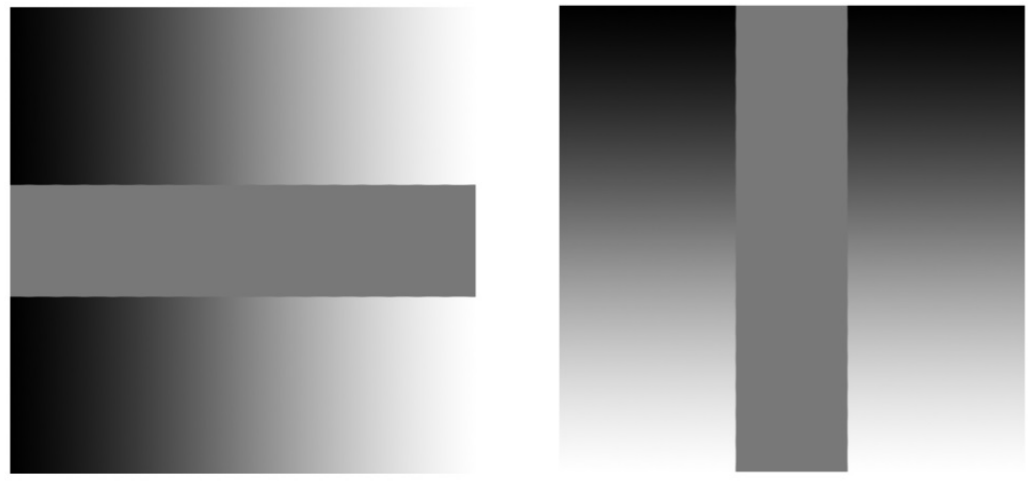

Figure 4.8 Gradient induction illusion rotated $90^{\circ}$. The grey bar down the middle of each image is a uniform grey, but the surrounding gradient makes it look non-uniform via contrast. This occurs regardless of the orientation of the image. 


\subsubsection{Adaptation level}

Lastly, our study was carried out at mesopic light levels ${ }^{57}$. In isolation, this may stand out as anomalous, and could be suggested as an explanation for its apparent differences. However, the other studies suggesting non-uniformity to appear brighter were in the photopic ${ }^{58}$ range (Okajima and Fujimoto, 2008; Tiller and Veitch, 1995). If this hypothesis was the reason for our results, then alternative explanations would have to be found for their results. That being said, Tiller and Veitch only compared two light distributions which can easily be misleading (Section 2.3.1), while Okajima and Fujimoto was a screen based study that adjusted image contrast in a very different manner to the other experiments. An argument could be made for discounting their results here.

Perhaps more importantly, the known effects of light level and adaptation on brightness perception do not lead us to expect this kind of effect. Adaptation level affects the slope of the brightness/luminance relationship, as well as contrast sensitivity (Stevens and Stevens, 1963). Based on this we would only expect some small shifts in the magnitude of the effects - not a complete inversion of the apparent relationship. Taking the estimated effects from Stevens and Stevens (1963), for example, increasing luminance by $-50 \%$ would increase brightness by $\sim 14 \%$ at dark adaptation, and $\sim 19 \%$ at an adaption level of $\sim 3,180 \mathrm{~cd} / \mathrm{m}^{2}$. Overall, this hypothesis seems a poor explanation for the disagreements here - though we cannot rule it out given that light distribution and spatial brightness does not seem to be behaving the way that we would expect from what we know about brightness perception.

\subsubsection{Summary}

Comparison of the different studies raises several possible hypotheses for the discrepancies in the observed trends of the brightness/uniformity relationship. The most likely is arguably that of Modes of Appearance, as it ties into well-established theory on human perception and if true, would undermine the assumptions of our uniformity assessments to a massive degree that could explain the apparent contradictions in our studies. The others are less plausible. Mere light source visibility changing everything has the problem of there being no good reason for it to have such an effect. Orientation of light distribution has the problem that it fits the study patterns relatively poorly, and is arguably already disproved by our checkerboard condition. What we do know about how adaptation level affects brightness and contrast perception says that it should not produce effects like this.

\footnotetext{
${ }^{57} 0.001$ to $3 \mathrm{~cd} / \mathrm{m}^{2}$, typically found outdoors at night (Roufs, 1978)

${ }^{58}$ The normal light levels one experiences in most lit rooms and during the day
} 
None of these hypothesises perfectly explain all the results on their own. This is not necessarily a problem. Simply assuming that all the study's results are "correct" may not be sound. Statistical noise means that some studies may be in error. Similarly, not all of the studies necessarily provide strong evidence in favour of their "side" of the uniformity/nonuniformity debate. For such reasons, perfectly fitting the observed range of studies is not necessary for a reasonable hypothesis. Indeed, one that did could even be argued to be too good to be true. 



\section{Experiment 2: why non-uniform spaces sometimes appear brighter, and sometimes darker}

Our previous experiment has shown large disagreements with others in the literature on the subject of the relationship between uniformity of light distribution and spatial brightness. Some studies suggest that non-uniform spaces should appear darker, others, such as ours, say that they should appear brighter. As individual studies suggest that the effects of light distribution on brightness are large (equivalent to as much as a $30 \%$ or more change in the overall light level), this is a significant disagreement. Indeed, whatever is causing this disagreement between studies is arguably more powerful than most of the main effects found by spatial brightness researchers. It is thus critical that we determine what is causing these discrepancies if the field is to progress.

Comparison of the kinds of conditions used in different studies suggest several possible hypothesises for these differences, of varying levels of plausibility:

1. Modes of Appearance Hypothesis: Compared against more uniform light distributions, non-uniform distributions of light sources will make spaces look darker, while non-uniform distributions of surface reflectance will make spaces look brighter

2. Mere Light Source Visibility Hypothesis: The direction of the brightness/uniformity relationship depends purely on whether or not the light sources are visible. Non-uniformity makes spaces appear darker if light sources are visible and brighter if they are not visible

3. Orientation Hypothesis: Compared against more uniform light distributions, non-uniform light distributions make spaces appear darker if the distribution is horizontally oriented and brighter if vertically oriented 
4. Adaptation Level Hypothesis: Compared against more uniform light distributions, non-uniform light distributions make spaces appear darker when at photopic levels, but brighter when at mesopic levels

More broadly, the goal of this experiment is to reproduce the effects of both our previous study and of opposing studies (e.g. Kato et al) using conditions that are as comparable as possible and that test the hypotheses described above. We need to produce non-uniform conditions that are clearly much less uniform than the baseline conditions, but which differ in how that non-uniformity is produced (e.g. via surface reflectance or via the light source). We do not know the "correct" way to measure uniformity, and different studies all use different metrics. Thus, to ensure that these conditions are indisputably nonuniform the only option is to make sure they are clearly non-uniform on as many metrics as possible - especially the main ones used in these studies. Additionally, because all of the metrics are probably flawed in some way we also want the conditions to be qualitatively similar to the conditions used in the studies whose effects we are trying to replicate.

\subsection{Experimental design}

The basic form used for the conditions was a $530 \mathrm{~mm} \times 530 \mathrm{~mm} \times 300 \mathrm{~mm}$ box with an opening in one corner (Figure 5.1). The walls on one side of the room are covered by diffuse "window" openings from which it is lit, while the other side simply consists of plain walls. By arranging the room in this way, similar non-uniformities can be produced either though altering the "windows" on one half of the room, or the surface reflectances of the walls on the other half. This facilitates our first set of comparisons looking at comparing the effects of non-uniform light distributions generated by changing reflectance, and nonuniform light distributions generated by rearranging the light sources

The setup, as shown in Figure 5.1, was similar to that in the previous experiment with light being diffused before being released into the experimental conditions. Light to the test space was provided by two to five compact fluorescent lamps ${ }^{59}$, while the reference space used a consistent three — the number being increased here in order to move the light levels in this experiment up into the photopic range. The light level was controlled using the same shutter system as before. Due to the position of the light sources/"windows" there was a risk of excessive light spilling over from one condition into the other if they were positioned the way they were previously. Due to this, the models were splayed out to a wider angle, with a wedge placed between them to block any excess light.

\footnotetext{
59 The number was varied according to the condition. Elaborated on in Section 1.5.
} 


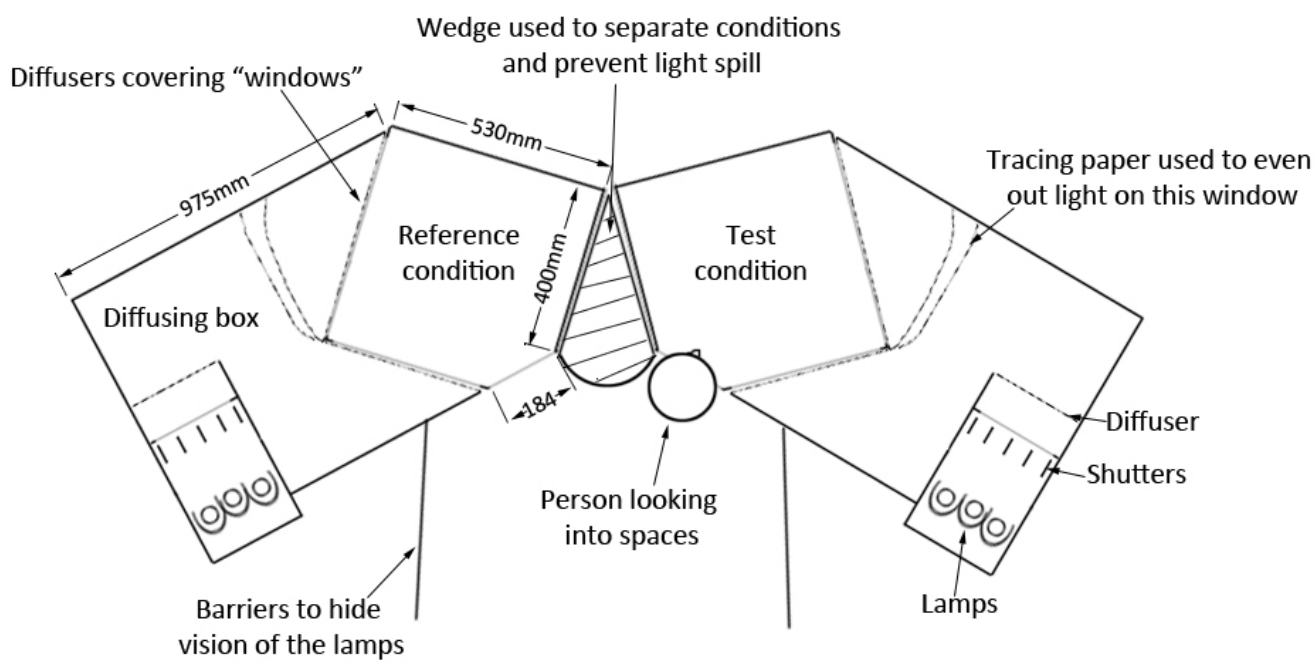

Figure 5.1 Plan of experimental setup for experiment 2

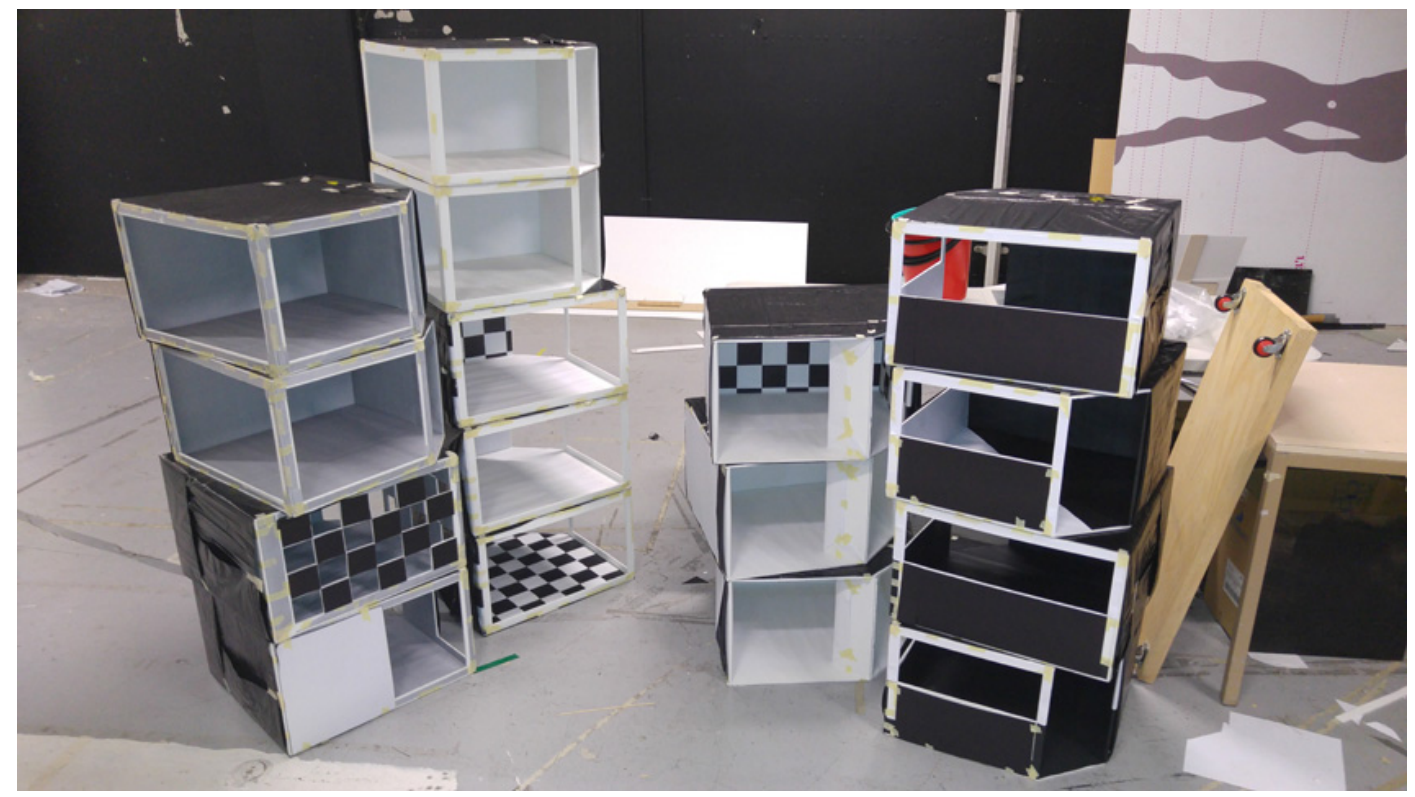

Figure 5.2 The model spaces for Experiment 2 


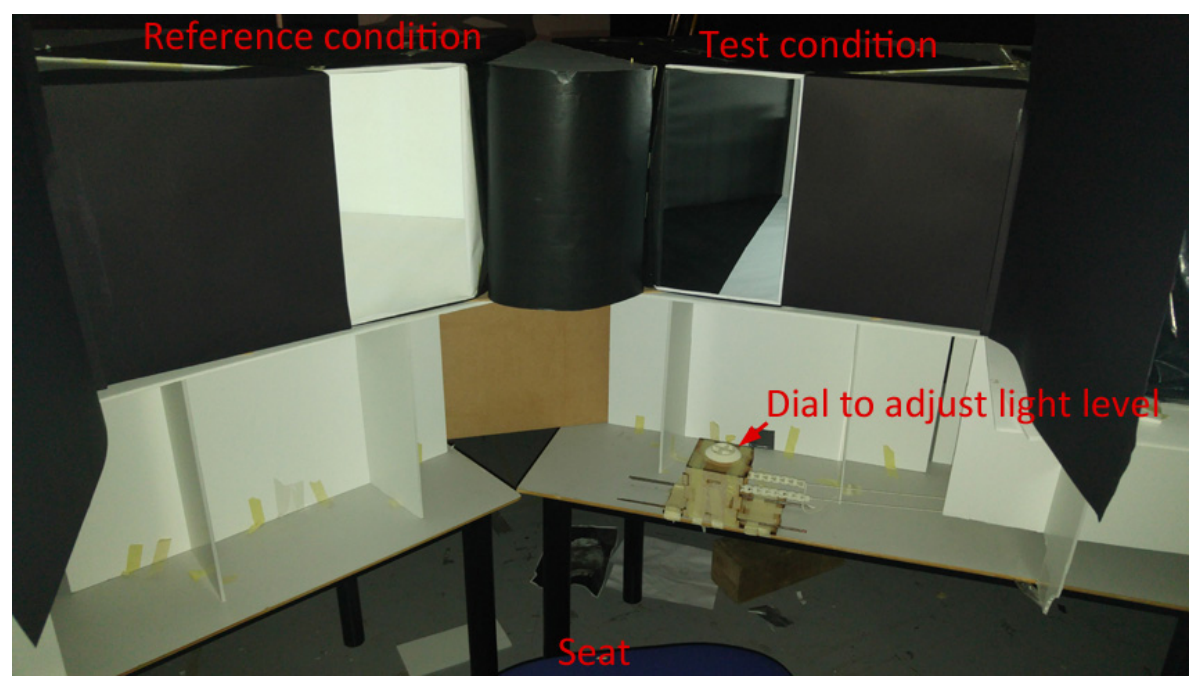

Figure 5.3 The experimental setup from (approximately) the view of the participant

To test the first hypothesis, we set up two sets of conditions based off previous experiments (both ours, and those of Kato et al.). The basic concept was to take a prototypical strongly non-uniform condition from our experiment (in this case, the checkerboard), and to transform it into a similarly prototypical non-uniform condition representing the other studies. The first two sets of comparisons were reflectance conditions (Figure 5.4) and light source based conditions (Figure 5.5):

\section{Uniform White Reference}

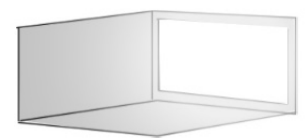

Designed to be clearly more uniform than the test conditions it is compared to

\section{Full checkerboard}

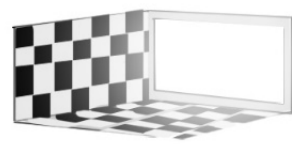

Reproduces the strong non-uniform condition of the previous experiment in the new setup
Wall checkerboard

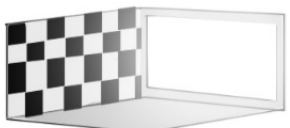

Checkerboard shifted to only being on the wall like the focus of Kato's and Kirsch's experiments

Figure 5.4 Conditions using surface reflectance to change uniformity

Uniform Grey Reference

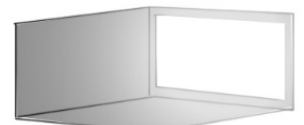

Designed to be clearly more uniform than the test conditions it is compared to.

"Windows" fill nearly the whole wall.
Light narrow

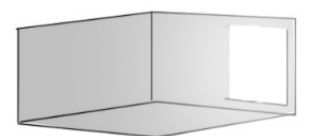

Opening is reduced to half the size of the uniform condition. Designed to produce the most non-uniform space following Kato et al's model and examples.
Light check

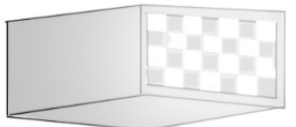

Opening further broken up into a checkerboard pattern like that used in the previous experiment - but here is a light source rather than surface reflectance.

\section{Wall 'narrow'}

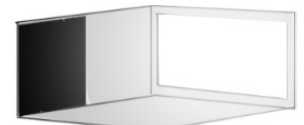

To provide a similar effect to the "narrow window" condition, the dark checks are merged into a single patch covering half of the wall. According to Kato et al's model, this should produce a larger effect

Figure 5.5 Conditions using the light source to change uniformity 
If hypothesis 1 (Modes of Appearance) is true, then the non-uniform reflectance conditions should appear brighter than the Uniform White Reference condition, while the non-uniform light source conditions should appear darker than the Uniform Grey Reference. Note that each "set" of conditions is compared to different reference conditions. This is in order to make sure that the reference in each comparison is clearly more uniform than the test conditions. The Uniform Grey reference condition, for example, actually has roughly the same standard deviation of the luminance as the checkerboard condition, so it would not be a good comparison if we wish to make sure that the non-uniform condition is "clearly" more non-uniform than its reference.

The next condition set (Figure 5.6) was designed to test the second hypothesis: that mere visibility of the light source changes the brightness/uniformity relationship. For this, we created a version of the reflectance condition with the light source hidden. If the mere visibility of the light source is what causes the differences, then this hidden light source comparison should show the non-uniform condition to appear brighter, while all the other comparisons should show them to appear darker.

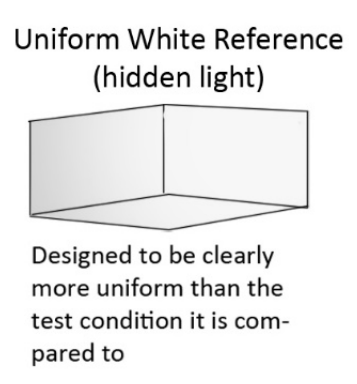

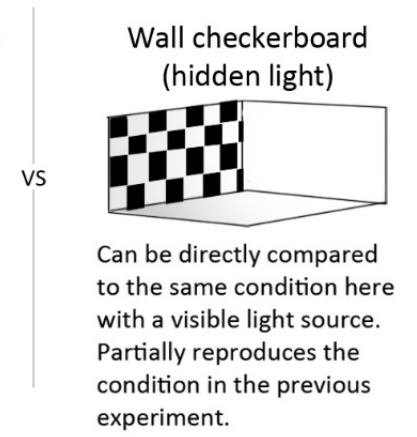

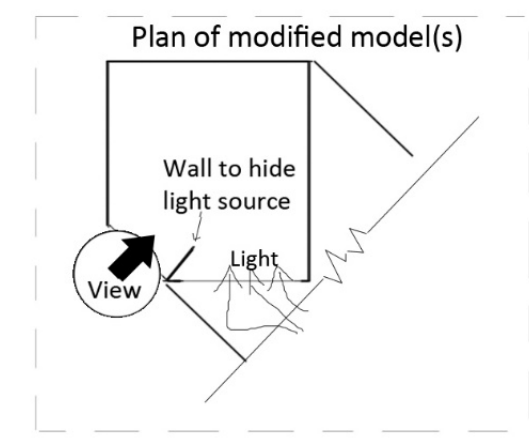

Figure 5.6 Hidden light source conditions. Note that the wall which was a window in the normal checkerboard condition here is left a blank white and is not a checkerboard, in order to keep them as similar as possible. The Wall Checkerboard condition was used rather than the full checkerboard one because a full checkerboard condition with the light source hidden ended up being too dark, making it difficult to keep in the photopic range while matching the Uniform White condition.

The last set of conditions (Figure 5.7) is for examining the effect of different orientations of light distribution (Hypothesis 3). Here, if it is orientation that matters we would expect the horizontal distribution to appear darker than the reference, and the vertical distribution to appear brighter than the reference. 


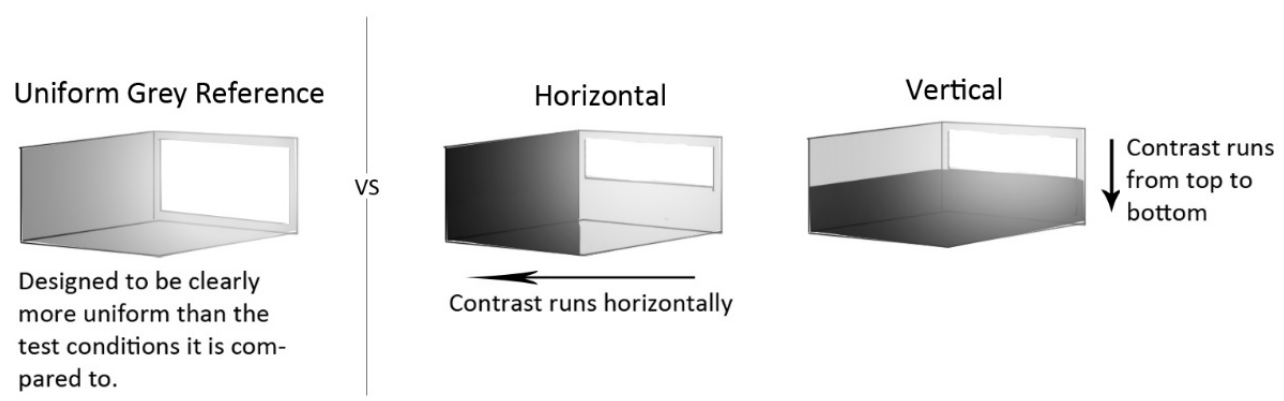

Figure 5.7 Conditions based on changing orientation of uniformity change

Potential effects of adaptation level are not fully tested in this experiment, in that we are not running it at both photopic and mesopic levels. However, if adaptation level was the factor causing the differences between studies then we would expect all the comparisons here at photopic levels to show that the non-uniform conditions appear darker, contradicting our previous results (which were at mesopic levels).

The patterns of results predicted by these hypotheses are summarised in Figure 5.8 below. If, for example, the Modes of Appearance hypothesis is true then we would expect the reflectance-based non-uniform conditions in the "reflectance" and "hidden light" sets to appear brighter, while the light-source based non-uniform conditions should appear darker. The effects of the conditions in the orientation set are unclear for that hypothesis, as they have both light source and reflectance changes to their distribution.

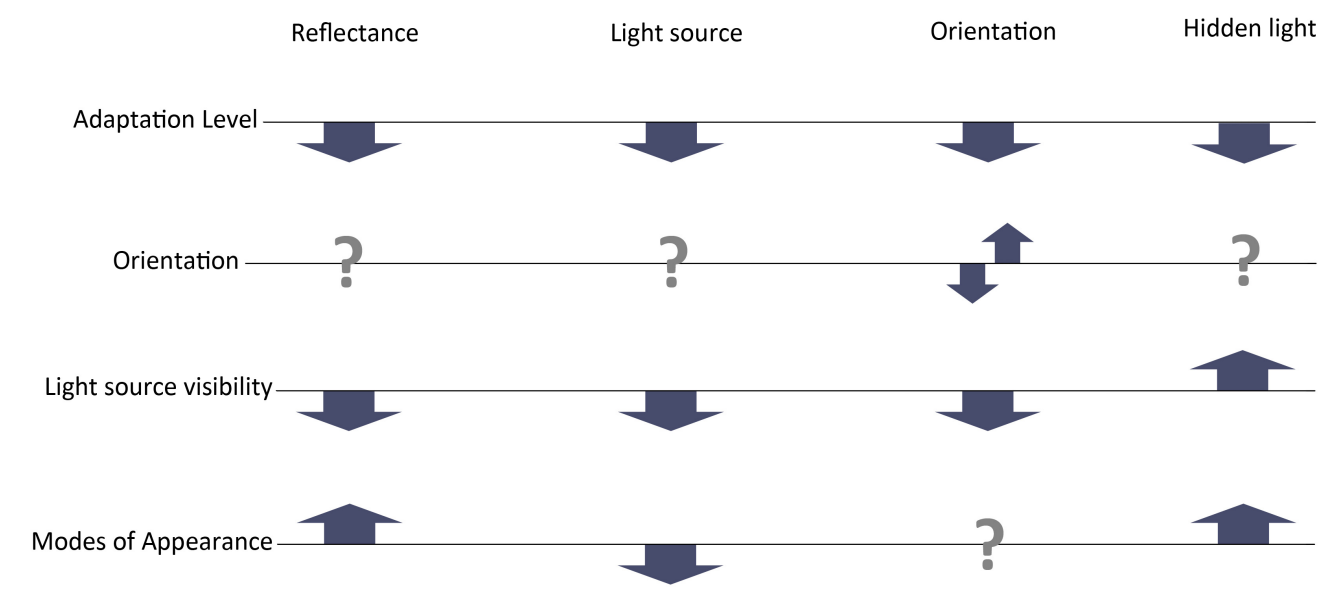

Figure 5.8 Illustration of the pattern of brightness effects predicted by each of the different hypothesises for the non-uniform conditions. 
A key challenge here was ensuring that the non-uniform test spaces were all clearly less uniform than the uniform reference conditions they were being matched against. This is difficult to do when non-uniformity can be described in so many different ways and we do not know what the correct way is. For example, qualitatively one might say that the checkerboard room is clearly far less uniform than the uniform grey room. However, if we use the standard deviation as a measure of uniformity, both spaces would be described as having roughly the same uniformity. However, making the surfaces grey to increase contrast with the light source was necessary for the light source conditions to produce large enough differences in uniformity.

Overall, the non-uniform spaces are more non-uniform on most key metrics from prior studies (Table 5.1, Table 5.2, Table 5.3). The standard deviation is clearly far higher on all of them - and the relative difference is deliberately greater than what we had in the last experiment in order to try and ensure large and easily detectable effects. The $\mathrm{max} / \mathrm{min}$ uniformity ratio (and $\mathrm{max} / \mathrm{min}$ in the $40^{\circ}$ band) is also clearly far higher. Directional diffusivity is generally lower, though the size of the difference is not always large, in large part due to the effects of smoothing. There are also some problem points. The Vertical and Checkerboard Window conditions do not show up as more non-uniform using the directional diffusivity metric. This is an unavoidable problem given the design of those conditions and the way that the metric averages over the vertical angles. Given the failure of directional diffusivity as a metric in the last experiment for exactly that reason, we have chosen to ignore this as simply reflecting a failure of the metric. The Checkerboard Window condition also fails to show non-uniformity using the standard deviation of the $\log$ luminance. This was not something that was considered at the time the experiment was being designed, as the Ko et al. (2013) study proposing the metric was not encountered until after Experiment 2 had been completed. Overall, the Light Check condition was the most questionable in terms of whether or not it would show a significant effect, with the work of Kato et al. (Kato and Hara, 2011; Kato and Sekiguchi, 2005) suggesting that its effects should be markedly attenuated by the small spread out apertures. In spite of this, it was included for the sake of the qualitative comparison with our checkerboard conditions. Even if it failed, however, the Light Narrow condition was expected to provide strong effects for the non-uniform light source condition set. 
Table 5.1 Uniformity measurements for conditions compared to the Uniform White reference

\begin{tabular}{|ll|c|r|r|r|r|}
\hline & Condition & SD & SD (log) & $\begin{array}{c}\text { Directional } \\
\text { Diffusivity }\end{array}$ & $\begin{array}{c}\text { Max/Min } \\
\text { (40deg) }\end{array}$ \\
\hline 1 & Uniform White 1 & 0.53 & 0.17 & 0.92 & 28.0 & 7.7 \\
\hline 1 & Uniform White 2 & 0.53 & 0.17 & 0.92 & 28.6 & 7.7 \\
\hline 2 & Full Checkerboard & 1.27 & 0.57 & 0.82 & 194.7 & 180.1 \\
\hline 3 & Wall Check & 0.79 & 0.39 & 0.86 & 109.7 & 109.7 \\
\hline 4 & Wall Narrow & 0.76 & 0.46 & 0.86 & 165.6 & 165.6 \\
\hline
\end{tabular}

Table 5.2 Uniformity measurements for conditions compared to the Uniform Grey reference

\begin{tabular}{|ll|r|r|r|r|r|}
\hline & Condition & SD & SD (log) & $\begin{array}{c}\text { Directional } \\
\text { Diffusivity }\end{array}$ & Max/Min & $\begin{array}{c}\text { Max/Min } \\
\text { (40deg) }\end{array}$ \\
\hline 5 & Uniform Grey 1 & 1.28 & 0.36 & 0.82 & 69.4 & 44.6 \\
\hline 5 & Uniform Grey 2 & 1.26 & 0.36 & 0.82 & 69.6 & 41.2 \\
\hline 6 & Light Check & 2.10 & 0.37 & 0.81 & 184.4 & 102.2 \\
\hline 7 & Light Narrow & 2.14 & 0.42 & 0.75 & 164.7 & 128.4 \\
\hline 8 & Horizontal & 2.06 & 0.88 & 0.74 & 2280.7 & 1920.4 \\
\hline 9 & Vertical & 2.03 & 0.80 & 0.82 & 836.2 & 736.4 \\
\hline
\end{tabular}

Table 5.3 Uniformity measurements for hidden light source conditions

\begin{tabular}{|ll|r|r|r|r|r|}
\hline & Condition & SD & SD (log) & $\begin{array}{c}\text { Directional } \\
\text { Diffusivity }\end{array}$ & Max/Min & $\begin{array}{c}\text { Max/Min } \\
\text { (40deg) }\end{array}$ \\
\hline 10 & Hidden White 1 & 0.34 & 0.17 & 0.94 & 26.8 & 12.7 \\
\hline 10 & Hidden White 2 & 0.34 & 0.17 & 0.94 & 26.2 & 12.6 \\
\hline 11 & Hidden Checkrbd & 0.61 & 0.36 & 0.89 & 62.0 & 53.4 \\
\hline
\end{tabular}

Colour differences between the light of the different conditions was measured using a Minolta CL-70F illuminance meter placed at the rough position of the participants' eyes.

The effects of SPD were estimated using the Rea et al. (2015) model (assuming an adaptation level of 60 lux — the average in the second experiment) (Table 5.4). The reference conditions on the left were, according to this, $2 \%$ darker than when they were on the right. These effects were included in our statistical model to control for them. Note here that the "white" surfaces in the different conditions were a very light yellow (CIELab $(96,-1,5))$, as it was found that plain white paper reflected too much bluespectrum light, creating excessively large differences in SPD compared to the other conditions. 
Table 5.4 Effects of SPD on apparent light level according to the Rea et al. (2015) model. Effects are normalised to the uniform white condition. Key comparison groups are shaded. The key comparisons within each set of conditions have only minor differences of $\sim 1.5 \%$. Differences between the reference conditions, however, are larger. While such comparisons are not the main focus of this experiment, they should be noted.

\begin{tabular}{|c|c|c|c|c|c|}
\hline \multirow{2}{*}{\multicolumn{2}{|c|}{ Conditions }} & \multirow{2}{*}{$\begin{array}{l}\text { Colour } \\
\text { temp. }\end{array}$} & \multicolumn{2}{|c|}{$\begin{array}{c}\text { Chromaticity } \\
\text { (CIE1964) }\end{array}$} & \multirow{2}{*}{$\begin{array}{l}\text { Effect on } \\
\text { apparent } \\
\text { light level }\end{array}$} \\
\hline & & & $\mathbf{x}_{10}$ & $\mathbf{Y}_{10}$ & \\
\hline 1 & Uniform White & $5500 \mathrm{~K}$ & 0.335 & 0.377 & - \\
\hline 2 & Full checkerboard & $5521 \mathrm{~K}$ & 0.335 & 0.372 & $+1.6 \%$ \\
\hline 3 & Wall checkerboard & $5535 \mathrm{~K}$ & 0.334 & 0.374 & $+1.1 \%$ \\
\hline 4 & Wall narrow & $5538 \mathrm{~K}$ & 0.334 & 0.374 & $+1.1 \%$ \\
\hline 5 & Uniform Grey & $5680 \mathrm{~K}$ & 0.33 & 0.367 & $+4 \%$ \\
\hline 6 & Light Check & $5651 \mathrm{~K}$ & 0.331 & 0.368 & $+4 \%$ \\
\hline 7 & Light Narrow & $5656 \mathrm{~K}$ & 0.331 & 0.367 & $+3.5 \%$ \\
\hline 8 & Horizontal & $5720 \mathrm{~K}$ & 0.329 & 0.366 & $+4.6 \%$ \\
\hline 9 & Vertical & $5685 \mathrm{~K}$ & 0.33 & 0.367 & $+4.2 \%$ \\
\hline 10 & Hidden White & $5573 \mathrm{~K}$ & 0.334 & 0.385 & $-1.5 \%$ \\
\hline & Hidden Check & $5605 \mathrm{~K}$ & 0.333 & 0.381 & $0 \%$ \\
\hline
\end{tabular}

Measurements also suggested that at very low settings ( $-<10 \%$ of the maximum), the colour temperature of the light became warmer, and that this could significantly affect the SPD effects. Fortunately, the vast majority of the matches made in this experiment did not reach those levels, and thus the effect of this is minor $(-1 \%)$. Regardless, the effect of this shift was approximated through a polynomial function, and applied as an adjustment factor to our results in order to account for it (Appendix A.2).

\subsection{Methodology}

The experiment uses a side-by-side brightness matching task as in the previous experiment. Participants were instructed to adjust the light level in the test space until it appeared to them to be as bright as a reference space. Brightness was defined as the amount of light in the space, and the instructions were the same as in the previous experiment. Participants looked back and forth between the reference and test spaces and were instructed to only place their head into one space at a time so that it filled their whole field of view. Participants were given three practice matches while they spent the first 10 minutes adapting (Uniform grey null trial, Hidden light source null trial, Uniform white vs. full checkerboard). These trials were selected to cover the range of the different kinds of comparison they would seeing in the full experiment - comparisons of the same conditions, different conditions, changes of light source visibility, changes of surface reflectance and pattern, and changes in reference light level. The reference light levels $(100 / 35 / 50$ lux) were set so that the average for the practice trials was the same as the 
average of the overall experiment ( -61 lux), while the initial light levels of the practice test conditions were random. The key change in this experiment was that we had moved to a completely counterbalanced design instead of relying on a single null condition trial to measure the position+dimming bias as in the previous experiment. The reason for this was to address the concern that position bias could potentially vary between conditions, and to provide estimates that are generally more robust to failures in our assumptions about bias (see Appendix $\mathrm{C}$ for an in-depth discussion of bias models).

Based on this, all conditions were run counterbalancing both high and low initial start points, as well as position/application of dimming. As before, position and application of dimming were confounded, with the Test condition always being on the right. Thus we counterbalanced [right + dimmed] vs. [left + fixed]. Each pair of conditions was hence compared four times (high/low light levels x position/application of dimming).

The use of counterbalancing and the design of the conditions created several other issues that needed to be addressed. We note that a flaw of counterbalancing is that, if your conditions affect brightness, then swapping which condition is the reference will mean that your reference conditions are varying in brightness, even if the light level is the same. We may thus be concerned about the potential for counterbalancing to induce response contraction bias (see Appendix C.3.2). Moreover, because the different conditions have different overall reflectance levels and "window" sizes they also have significantly different average luminances for a given light level. For example, when four lamps are on, and the shutters are fully open, the Uniform Grey condition may have -206 vertical lux at eye level. In contrast, the Checkerboard Window condition, with its smaller "windows", has only -114 vertical lux at this setting. This raises two issues:

1) That the differences in the adjustment range for the different conditions could bias people's matches ${ }^{61}$.

2) That the variability in usable adjustment ranges may make it hard to ensure that participants have adequate room to make their matches without running into ceiling or floor effects (Figure 5.9).

\footnotetext{
${ }^{61}$ Much of the evidence and references presented in Fotios et al. (2008) for the existence of response contraction due to varying reference light level could also be read as evidence for an adjustment range bias (see Appendix C.4.1).
} 


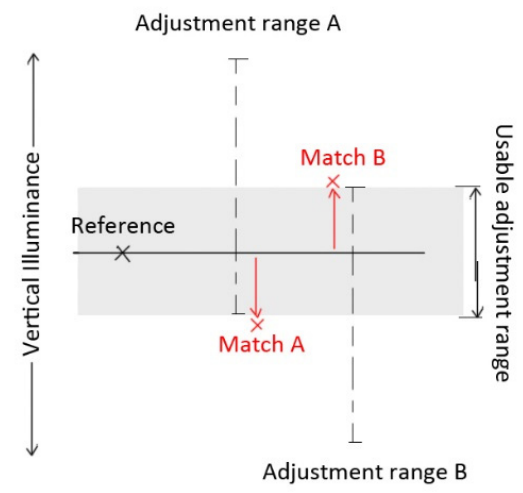

Figure 5.9 Illustration of how different adjustment ranges on different conditions can restrict your ability to make correct matches. In this example, condition A is brighter than condition B, so it requires less light than the reference level to match it. In a counterbalanced experiment, Condition A will be matched to a light level of [ref - effect] while Condition B will be matched in the opposite direction to [ref + effect]. In this case, however, there is not enough room in the adjustment ranges allowed for each condition for the correct matches to be made.

To address these issues, the number of lamps was varied between conditions in order to align the possible adjustment ranges of the different comparisons as much as possible (Table 5.5). Then, in order to measure possible response contraction bias due to varying references and varying adjustment ranges null condition trials were run at different reference light levels and with different numbers of lamps. This allowed the biases to be estimated and accounted for in our statistical analysis.

Varying the number of lamps lighting the test conditions places some restrictions on the sequence the conditions are viewed in. Due to the need for the lamps to warm up and stabilise when first turned on, they cannot be turned on mid-experiment. Hence, while the conditions were randomised, the randomisation was constrained to ensure that the number of lamps would always progress down, from the highest number to the lowest (example in Appendix D.1). 
Table 5.5 Adjustment ranges for the different sets of conditions. Ranges were designed to be as consistent as possible within the main comparisons. Number of lamps ranged from two to six.

\begin{tabular}{|c|c|c|c|c|c|}
\hline \multicolumn{3}{|c|}{ Reflectance conditions } & \multicolumn{3}{|c|}{ Light source/orientation conditions } \\
\hline Condition & Lamps & $\begin{array}{c}\text { Range } \\
\text { (lux) }\end{array}$ & Condition & Lamps & $\begin{array}{c}\text { Range } \\
\text { (Iux) }\end{array}$ \\
\hline Uniform White & 2 & $8-222$ & Uniform Grey & 3 & $5-138$ \\
\hline Full checkerbd & 5 & $8-229$ & Light check & 6 & $4-109$ \\
\hline Wall checkerbd & 3 & $8-220$ & Light narrow & 4 & $4-112$ \\
\hline \multirow{2}{*}{\multicolumn{3}{|c|}{ Wall narrow }} & Horizontal & 6 & $5-125$ \\
\hline & & & Vertical & 6 & $5-123$ \\
\hline \multicolumn{3}{|c|}{ Hidden light source conditions } & \multicolumn{3}{|c|}{ Reference link comparisons } \\
\hline Condition & Lamps & $\begin{array}{l}\text { Range } \\
\text { (lux) }\end{array}$ & Condition & Lamps & $\begin{array}{c}\text { Range } \\
\text { (lux) }\end{array}$ \\
\hline Hidden white & 3 & $3-79$ & Uniform White & 2 & $8-222$ \\
\hline \multirow[t]{3}{*}{ Hidden check } & 6 & $3-86$ & Uniform Grey & 5 & $8-213$ \\
\hline & & & Hidden White & 5 & $5-121$ \\
\hline & & & Uniform Grey & 3 & $5-138$ \\
\hline \multicolumn{6}{|c|}{ Null trials (to observe range effects) } \\
\hline Condition & Lamps & $\begin{array}{c}\text { Range } \\
\text { (lux) }\end{array}$ & Condition & Lamps & $\begin{array}{c}\text { Range } \\
\text { (lux) }\end{array}$ \\
\hline Hidden white & 6 & $5-139$ & Uniform White & 5 & $17-461$ \\
\hline \multirow[t]{3}{*}{ Hidden white } & 3 & $3-79$ & Uniform White & 2 & $8-222$ \\
\hline & & & Uniform Grey & 5 & $8-213$ \\
\hline & & & Uniform Grey & 3 & $5-138$ \\
\hline
\end{tabular}

The reference light level was chosen to make sure that there would be room for significant adjustments of a factor of at least two in either direction while avoiding potential ceiling effects and staying in the photopic range as much as possible (Table 5.6). For the reflectance set of conditions, the reference light level was 80 vertical lux. The light source and orientation sets had a lower range, and so used a reference of 50 lux, while the hidden light source conditions used a reference of 40 lux. 
Table 5.6 Summary of reference light levels used for the different sets of trials. Note that in the null condition trials different reference levels were used for different needs - one for the null trials used to measure the effects of adjustment range, and then a high and low reference to measure response contraction bias.

\begin{tabular}{|l|l|}
\hline Conditions & Reference light level (vertical lux) \\
\hline Reflectance set & 80 \\
\hline Light source set & 50 \\
\hline Orientation set & 50 \\
\hline Hidden light source set & 40 \\
\hline Reference link trials & 60 \\
\hline Null trial (uniform white) & 80 (adj. range trials); 35, 100 (reference trials) \\
\hline Null trial (uniform grey) & 60 (adj. range trials); 35, 100 (reference trials) \\
\hline Null trial (hidden white) & 40 (adj. range trials); 35, 79 (reference trials) \\
\hline
\end{tabular}

In total, each participant carried out 52 trials across 13 comparisons (Figure 5.10). As in the first experiment, participants carried them out over two sessions, with a typical session time of approximately 70 minutes. As before, in each session they spent the first 10 minutes adapting and carrying out practice trials. As well as the main test condition sets, the baseline conditions are also matched against each other in order to link the different sets and allow the relative brightnesses of the different sets of conditions to be assessed ("Link" trials). Carrying out counterbalanced null trials for each of our three reference conditions at both high and low references and high and low adjustment ranges would have increased the length of the experimental sessions excessively beyond what could be achieved in two $\sim 1$ hour sessions. Instead, to manage the burden on participants, we carried out a more limited number of assessments by having each participant carry out two sets of high/low reference trials and one set of high/low adjustment range trials, and randomly assigning which conditions were being used for each set of trials. For example, one participant might be estimating the difference between high and low adjustment ranges using the uniform white conditions, while another would be using the uniform grey conditions. Overall, the effect of response contraction bias on the overall estimates after counterbalancing is expected to be minor anyway ( $-1 \%$ or so, Appendix C.5), so having some uncertainty in those estimates is not considered to be a significant issue. Regardless, simulations suggested that the model should still be capable of estimating the size of the bias(es) to within the right ballpark $( - \pm 0.06)^{62}$, and hence able to tell us if the biases are very large or not and control for them.

\footnotetext{
${ }^{62}$ Which may correspond to an uncertainty in the main effects of around $\pm 0.5-1.0 \%$ for effects of $-20 \%$ (lower or higher depending on how large the effects are)
} 


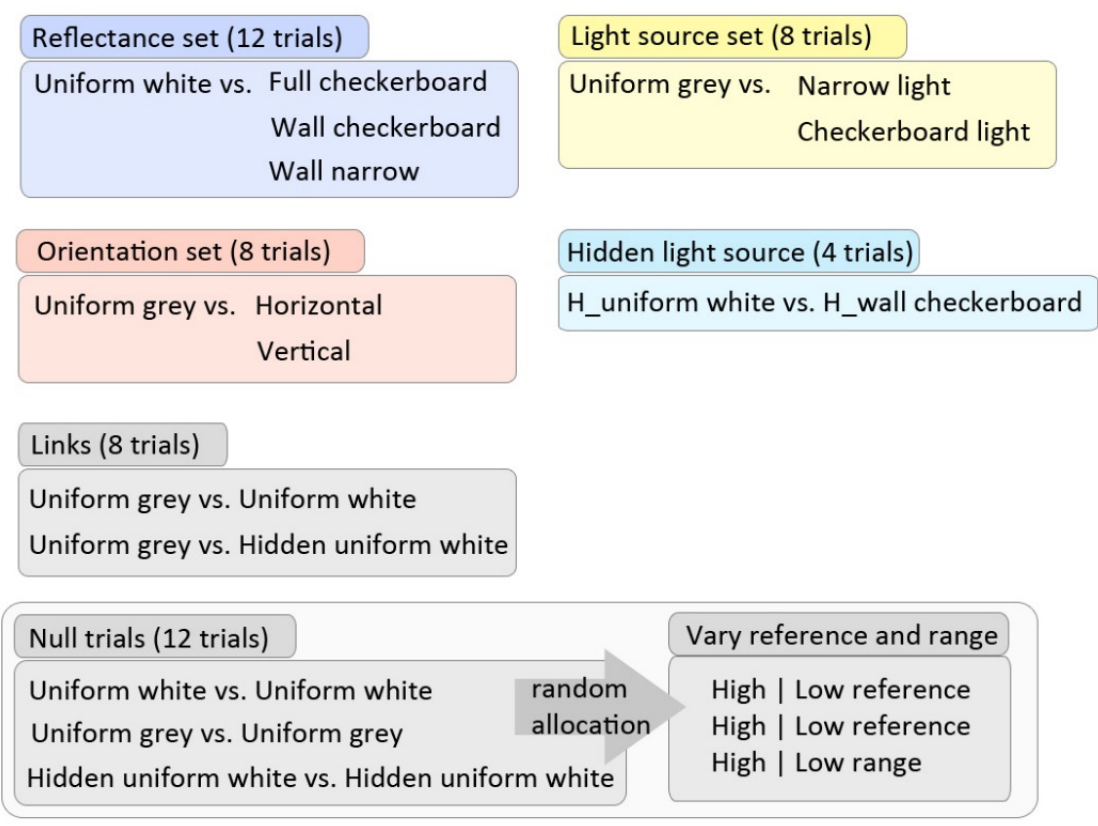

Figure 5.10 Outline of the different matches to be made in Experiment 2

\subsubsection{Statistical model}

The statistical model used for the second experiment was largely the same as that used in the first experiment, but with the addition of response contraction bias due to reference light level and adjustment range.

As before, the mean match is described as the sum of the reference light level, the difference in effect between the conditions, the bias, and the effect of the SPD difference between the conditions (according to the Rea et al. model):

$$
\mu=r e f-\left(\text { Effect }_{\text {test }}-\text { Effect }_{\text {ref }}\right)-\left(S P D_{\text {test }}-S P D_{\text {ref }}\right)+\text { bias }
$$

The major change here is the addition of new biases: on top of the previous position+dimming and start point bias, we add reference response contraction bias and adjustment range bias. Position+dimming bias and start point bias are modelled as before, with start point bias being assumed to be proportional to the distance between the start point and the intended match. Position+dimming bias is, however, allowed to potentially vary between pairs of conditions, as the counterbalanced design here allows us to measure that.

The reference response contraction bias and potential adjustment range bias, are similarly assumed to be linear functions. Reference bias is assumed to be proportional to the distance between the apparent light level of the reference condition and the average 
reference, and adjustment range bias is modelled as being proportional to the difference between the match setting and the centre of the adjustment range (log scale):

$$
\begin{gathered}
\text { bias }_{\text {ref }}=k_{\text {ref }} *\left(r e f+E f f_{\text {ref }}+S P D_{\text {ref }}-\overline{r e f}\right) \\
\text { bias }_{r n g}=k_{r n g}\left(r e f-\left(E f f e c t_{\text {test }}-E f f e c t_{r e f}\right)-\left(S P D_{\text {test }}-S P D_{\text {ref }}\right)-\text { centre }\right)
\end{gathered}
$$

Where $\overline{r e f}$ is the mean reference (accounting for both the different light levels and the differences between conditions in apparent light level due to light distribution and SPD), and $k_{r e f}$ and $k_{r n g}$ are the bias strength factors (essentially the slope of bias against range/reference light level).

As before, parameters may vary between conditions and people - with the exception of the adjustment range and reference contraction biases which would not have the data to estimate possible differences in bias between conditions, and so were only allowed to vary between people. Given that we still lack evidence that these biases do significantly vary between conditions, and that after counterbalancing their net effect is expected to be minor anyway, this is not considered a major concern.

Priors were largely derived from the posteriors of experiment 1 (see Appendix B.8), though with added uncertainty in a number of cases (particularly regarding between condition variance) on the grounds that the conditions in this experiment are different to those in the previous experiment. Briefly, the priors were:

- Effects of condition(s)

$\circ \quad$ mean effect(s): normal $(m=0, s d=0.3)$ : non-committal prior suggesting that differences of a factor of two are quite plausible, but that ones beyond three to four are less so

- mean between-person $s d$ : gamma(mode $=0.04$, $s \mathrm{~d}=0.06)$ : based on previous experiment, but more uncertain as evidence suggests sd may vary between conditions and we have different conditions

○ between-condition sd of between-person sd: gamma(mode=0.03, $\mathrm{sd}=0.04$ )

- Position bias

○ mean bias: $\operatorname{normal}(\mathrm{m}=0, \mathrm{sd}=0.05)$ : same prior as previous experiment, as the reference and test conditions are now on opposite sides, so position+dimming bias may interact differently.

○ between-condition $s \mathrm{~d}$ : half- $\mathrm{t}(\mathrm{m}=0, \mathrm{sd}=0.02, \mathrm{df}=4)$ : based off previous experiment

○ between-person sd: gamma(mode $=0.045$, $s d=0.02)$ : based off previous experiment

- Start point bias (proportional model): based on posteriors of the previous study, erring on the side of being slightly more uncertain 
- mean bias: $\operatorname{normal}(\mathrm{m}=-0.06, \mathrm{sd}=0.03)$

○ between-condition sd: half-normal $(\mathrm{m}=0, \mathrm{sd}=0.04)$

$\circ$ between-person $s \mathrm{~d}$ : gamma(mode $=0.05, \mathrm{sd}=0.02)$

- Start point bias (simple high/low model)

○ mean bias: $\operatorname{normal}(\mathrm{m}=0.024, \mathrm{sd}=0.02)$

○ between-condition sd: half-normal $(\mathrm{m}=0, \mathrm{sd}=0.02)$

$\bigcirc$ between-person $\mathrm{sd}$ : gamma(mode $=0.017, \mathrm{sd}=0.015)$

- Reference response contraction bias

- mean bias: $\operatorname{normal}(\mathrm{m}=-0.06, \mathrm{sd}=0.1)$ : based on previous studies, could be somewhere between 0 (Fotios et al.) to -0.12 (Tiller and Veitch, 1995)

○ between-person sd: half-normal $(\mathrm{m}=0, \mathrm{sd}=0.1)$ : no particular information about what is likely here, this prior is designed to cover a fairly broad range based on the expected size of the bias

- Adjustment range bias

○ mean bias: $\operatorname{normal}(\mathrm{m}=0, \mathrm{sd}=0.1)$ : similar to reference bias, and could actually be based off much of the same evidence - however, as we are suggesting it here for the first time we centre it on 0

○ between-person sd: half-normal $(m=0, s d=0.1)$

- Within-person sd

○ mean sd: gamma(mode $=0.07, \mathrm{sd}=0.02)$

○ between-condition sd in sd: half-t $(\mathrm{m}=0, \mathrm{sd}=0.3, \mathrm{df}=4)$ : narrower than what we used in the previous experiment, as it suggested that variance was unlikely to be that high - but still a relatively diffuse uncertain prior based on the fact that the conditions here are different

○ between-person $s d$ in $s d$ : gamma(mode $=0.16, s d=0.2)$

These priors, and the overall analysis plan and hypotheses were preregistered (Nosek et al., 2018) and the preregistration can be found at https://aspredicted.org/u7mw4.pdf.

\subsubsection{Sampling strategy and characteristics}

The sampling strategy, following Kruschke (2015), was to sample until the desired level of precision was reached. In this case, the target was an average precision of approximately $\pm 10 \%$ in our estimates of the mean condition effects — or more precisely for the mean width of the 95\% HDI's to be less than 0.088 ( $\log _{10}$ scale).

This target precision in the estimates of the effects of the conditions was achieved after 19 participants had been recruited, with 5 half-complete. Recruitment ceased at that point. Note, however, that the final result was affected by some issues that were identified after the completion of the experiment: the apparent change in light SPD at low shutter settings, 
and an error in the original Stan model wherein the SPD effects had only been applied to the overall match calculation, and not the calculation of the start/reference/range biases. After these corrections were applied, the final estimate of the average precision was \pm 0.084 , or approximately $\pm 9.7 \%$.

During the first test session of the sixth participant, it was identified that part of the diffusing screens in the light box for the reference condition had collapsed. Further investigation revealed that it was likely that this had been true for the initial trials of the first six participants. Analysis of the potential effects of this indicated that while this did not meaningfully change the SPD of the light, or the measured uniformity metrics, it did have a substantial impact on the calibration factor. To err on the side of caution, and ensure consistency in the conditions used, it was decided that the data from the first sessions of the first six subjects would be excluded from analysis ${ }^{63}$. This decision was made prior to statistical analysis of the results, and is acknowledged as an unanticipated departure from the preregistered plan.

Participants were students at the university, with 16/19 being in their 20s, 2 in their 30 s, and 1 in their 40s. 6 were female, 13 were male, and 13 wore visual correction.

\subsubsection{Model diagnostics}

The statistical model was run for 5000 iterations per chain and a warmup of 1000 iterations. Four chains were run, and all converged and were well-mixed. Standard Stan model diagnostics (Betancourt, 2017a) indicated no problems: no divergences occurred, effective sample size / iterations was well above 0.1 , split- $\hat{R}$ was well below 1.1 for all parameters (indeed, mostly below 1.01), no parameters had Monte Carlo standard error above $10 \%$ of the posterior standard deviation (most were below 1.5\%), and the energy Bayesian Fraction of Missing Information indicated no pathological behaviour.

\footnotetext{
${ }^{63}$ Excluding half the trials of those six participants raises obvious questions about the impact of incomplete counterbalancing. Fortunately, this is not an issue due to the statistical analysis approach used here. Rather than averaging, for example, left and right trials, the statistical model estimates the result of an individual match as the sum of the effect and the various biases that are affecting it. Hence, if we already know the size of the bias from other trials, we can predict that match $\mathrm{Z}$ is likely to be $\mathrm{X} \%$ higher because it is on the left. Thus, bias is still being accounted for in the model estimates even if some conditions for some participants are not fully counterbalanced. Furthermore, note that the excluded trials were effectively random, and thus there is no reason to be concerned about systematic bias in the exclusions.
} 


\subsection{Results}

The basic pattern shown in the results is that non-uniform spaces appear brighter than more uniform ones - except when the non-uniformity is produced by changing the arrangement of the visible light sources (Figure 5.11).

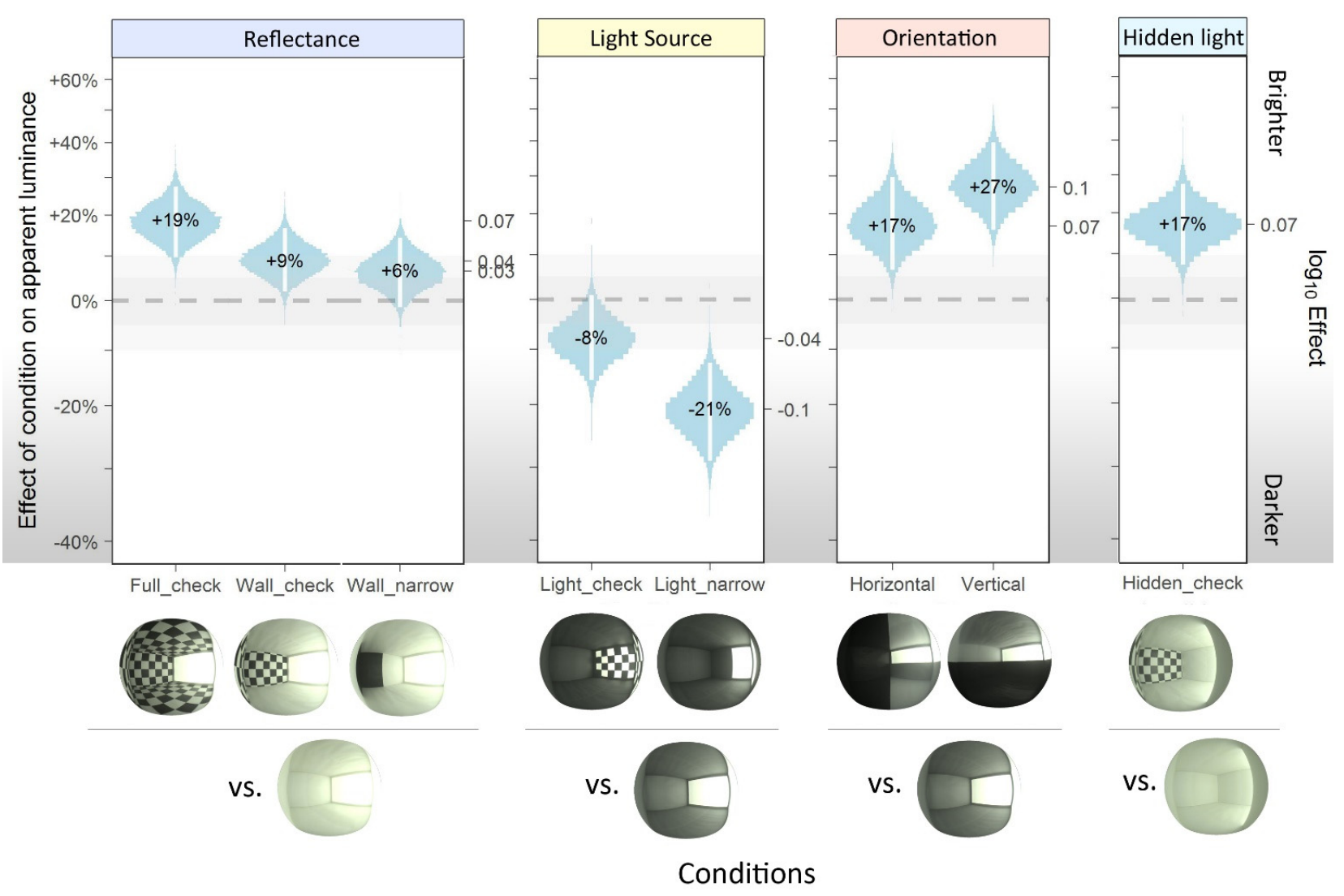

Figure 5.11 Posterior estimates of the mean effects of non-uniform conditions on effective light level compared to their respective uniform conditions.

Comparing these results against our four hypothesises (in reverse order) (Figure 5.12):

1. Adaptation level: if mesopic adaptation levels were causing non-uniform spaces to appear brighter in the first experiment then they should all appear darker in this experiment. This is clearly not the case, and the checkerboard conditions (both with and without visible light sources) replicate the results of the previous experiment by again showing that such patterns increase spatial brightness.

2. Orientation: both the vertically and horizontally oriented non-uniform conditions are significantly brighter than the uniform condition. The hypothesis that orientation of light distribution changes whether or not non-uniformity makes spaces appear brighter or darker is unsupported. Whether or not there is a 
meaningful difference in effect between the two conditions is unclear, with the posterior probability distributions suggesting $\sim 8 \%$ probability that the Horizontal condition is actually brighter than the Vertical, and $-25 \%$ probability that the difference is less than $5 \%$.

3. Visibility of light source: the hidden light source condition and the orientation and reflectance conditions all show non-uniform spaces to appear brighter. If this hypothesis was true then all the conditions with visible light sources should show the opposite effect.

4. Modes of Appearance: the non-uniform reflectance conditions (as well as the hidden light source ones that also used reflectance) appeared brighter. The conditions where non-uniformity was produced by changing the arrangement of light sources appeared darker. This is what was predicted by the hypothesis.

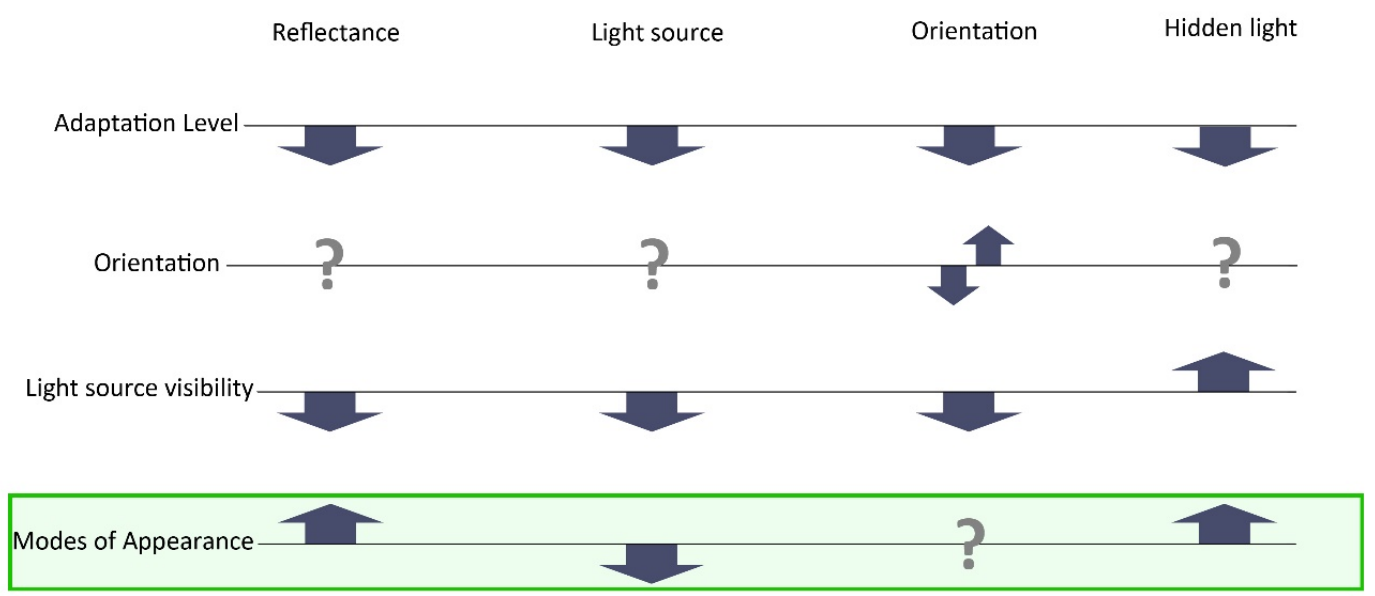

Figure 5.12 Illustration of the pattern of brightness effects predicted by each of the different hypothesises for the non-uniform conditions. If we compare to the results shown in Figure 5.11, we see that the pattern that fits is the Modes of Appearance hypothesis

Thus, the basic reading of the results suggests the disagreements between studies of the spatial brightness/uniformity relationship can be explained by the way in which uniformity was adjusted. The results of our first experiment can be explained by the fact that we were changing the pattern of the surface reflectances, in contrast to the other studies that rearranged the light sources.

There are, however, some complications to this story. Firstly, it must be acknowledged that the effects were not as large as was hoped for based off our previous work. Based off the differences in uniformity between the uniform and non-uniform conditions (as measured by commonly used metrics), it was anticipated that all the conditions should produce effects at least as large as the strongest effects in the previous experiment $-40 \%$ or more. Instead, effects have been significantly smaller, and the Wall_check, Wall_narrow, 
and Light_check conditions showed effects that may not be particularly significant in practical terms ${ }^{64}$. Indeed, the Light_check condition is not even necessarily "statistically significant" from one point of view - the $95 \%$ interval overlaps with zero. That being said, from the point of view of a directional hypothesis the analysis does suggest that the probability of it being darker than the Uniform Grey condition is $-96.5 \%$. One may also, in a meta-analytic sense, argue that each set of conditions shows a clear trend, and that the overall results for the light source conditions are clearly negative and in the opposite direction to the other sets of conditions. However, that does require making the assumption that grouping the conditions like that is "correct", which is not unquestionable. Certainly, we would be leery of calculating an overall meta-analytic effect for the light source conditions by averaging them, as that would imply that they are not significantly different conditions.

The weakness of the Light_check condition was not unanticipated. Based on the observations of Kato and Sekiguchi (2005) and Kato and Hara (2011), it was quite possible that a condition where the "non-uniform" pattern of light sources was comprised of many small light sources spread out over the walls would appear relatively uniform. This was one of the reasons why we made sure to also test the Light_narrow condition, which was supposed to (and did) provide a much stronger effect. In that sense, the failure of the Light_check condition to demonstrate a strong effect here is still consistent with the effects observed in the literature that we were trying to reproduce and explain here. Moreover, despite its weakness, it is not inconsistent with the Light_narrow condition, and does tend towards the same direction.

The second complication is the orientation set. In that, the non-uniform conditions had light sources that were halved in size, and the spaces were dramatically split into black and white halves. Thus, the non-uniformity was produced through a combination of both light source and surface reflectance changes. The resulting conditions appeared significantly brighter, with magnitudes consistent with — indeed even larger than — the other strong effects shown by the reflectance conditions. The way that the hypothesised effects of the reflectance changes appear to have completely overwhelmed the hypothesised effects of the light source changes raises questions about the simple model, and how the proposed effects interact.

\footnotetext{
${ }^{64}$ i.e. may be less than 1 just-noticeable-difference $(-8 \%$ (Teghtsoonian and Teghtsoonian, 1971)) in terms of the effective change in light level and so may not even be noticed by occupants.
} 
Thirdly is the differences between the different uniform conditions. Hiding the light source makes the Hidden_white condition significantly more uniform in its luminance distribution than the Uniform White condition. Darkening the surfaces to produce the Uniform Grey condition makes a condition significantly less uniform than the Uniform White condition.

Hiding the light source is a change in light source, and so under this hypothesis the Hidden_white condition should appear brighter because it is more uniform — which the results agree with (Figure 5.13).

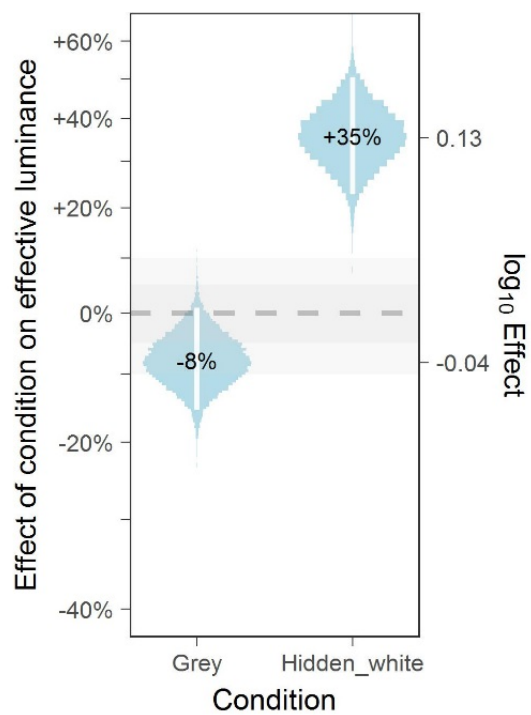

Figure 5.13 Posterior estimates of the differences between the uniform conditions compared with the uniform white condition

In contrast, the Uniform Grey condition is a change in surface reflectance relative to the Uniform White condition. According to the model then, as it is less uniform, it should appear significantly brighter (if we were to use SD as a rough guide, following its excellent correlations in the previous study, it should appear to have a similar brightness to the full checkerboard condition). However, this is not what we see. Instead, we see that the data is trending towards it appearing darker - the opposite of what the hypothesis predicts (though the result is not statistically strong). The comparison here thus challenges the simple hypothesis that increased non-uniformity caused by changing surface reflectance makes nonuniform spaces appear brighter — and this would be true even if there were really no significant difference between them. 


\subsubsection{Sensitivity analysis}

Before continuing any further with discussion of the results, we should examine their robustness. Pre-planned checks were to check alternative versions of the model with variance modelled as being a function of the difference between the pairs of conditions rather than the individual conditions, and with the start bias being modelled as a binary high/low function. We add to this:

- Checks of the effects of our various measurement and SPD adjustments

- Check of the sensitivity of our results to our priors (by doubling their widths)

- Check of the robustness of the estimates to possible outliers and the assumption of normality by using a thick tailed T-distribution or skew-normal distribution, as examination of the between-participant residuals suggests possible deviations from normality (Figure 5.14, Figure 5.15)

- Exploration of alternative bias model assumptions (see Appendix C):

○ Varying start point bias by individual condition

- Modelling position/dimming biases as separate biases that may both vary between individual conditions and be asymmetrical (i.e. the effect of being on the left may not be the inverse of being on the right)

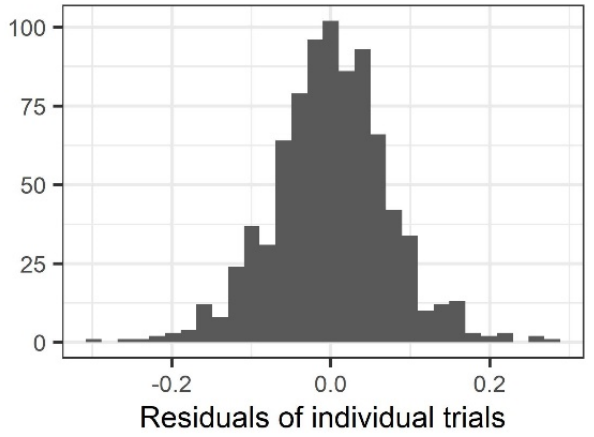

Figure 5.14 Residuals of individual trials for Experiment 2. Distribution looks normal.

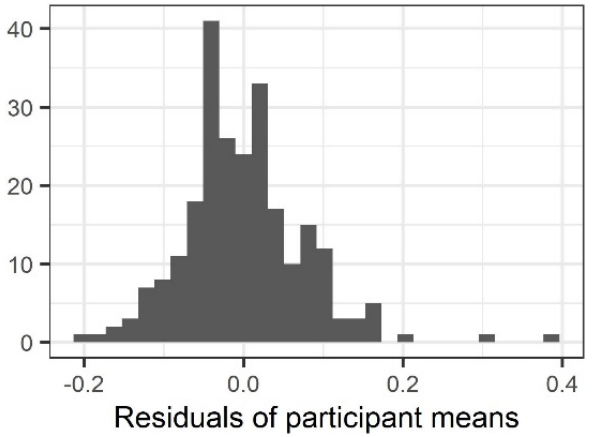

Figure 5.15 Residuals of the betweenparticipant variation in matches. Possible deviations from normality (Shapiro-Wilk $\mathrm{p}=2.35 \mathrm{e}-06$ )

The adjustments made to the measured calibration factors due to post-experiment cross-checking (Appendix A.1.2) largely have only minor effects, perhaps shifting some results like the Vertical and Horizontal conditions by a few percent, but not really changing the overall picture (Figure 5.16). The uncertainty in our estimates it indicates is worth being aware of, but does not seem to be a significant issue. What effect there is indicates that our adjustments, if anything, actually reduced the apparent difference between the Uniform Grey and light source conditions, making our estimates more conservative. 
Doubling the width of our priors also does not seem to significantly alter our results, suggesting our inferences are not overly sensitive to the precision of the prior specification. Neither does using a thicker-tailed T or skew-normal distribution - there is perhaps a small shift of a percentage point in one or two places, but nothing that would change the conclusions. Outliers or thick tails do not appear to cause any problems here. The most visible effect here is the SPD effects, primarily for the Light Source and Orientation sets (conditions through Grey to Vertical). As would be expected, with those conditions appearing about $4 \%$ brighter relative to the Uniform White condition according to the Rea et al. model, the effect of SPD is noticeable. That being said, the effect is largely the same for all those conditions, so for the main effects we care about - the difference of those non-uniform conditions with the uniform grey condition - the effect is largely trivial.

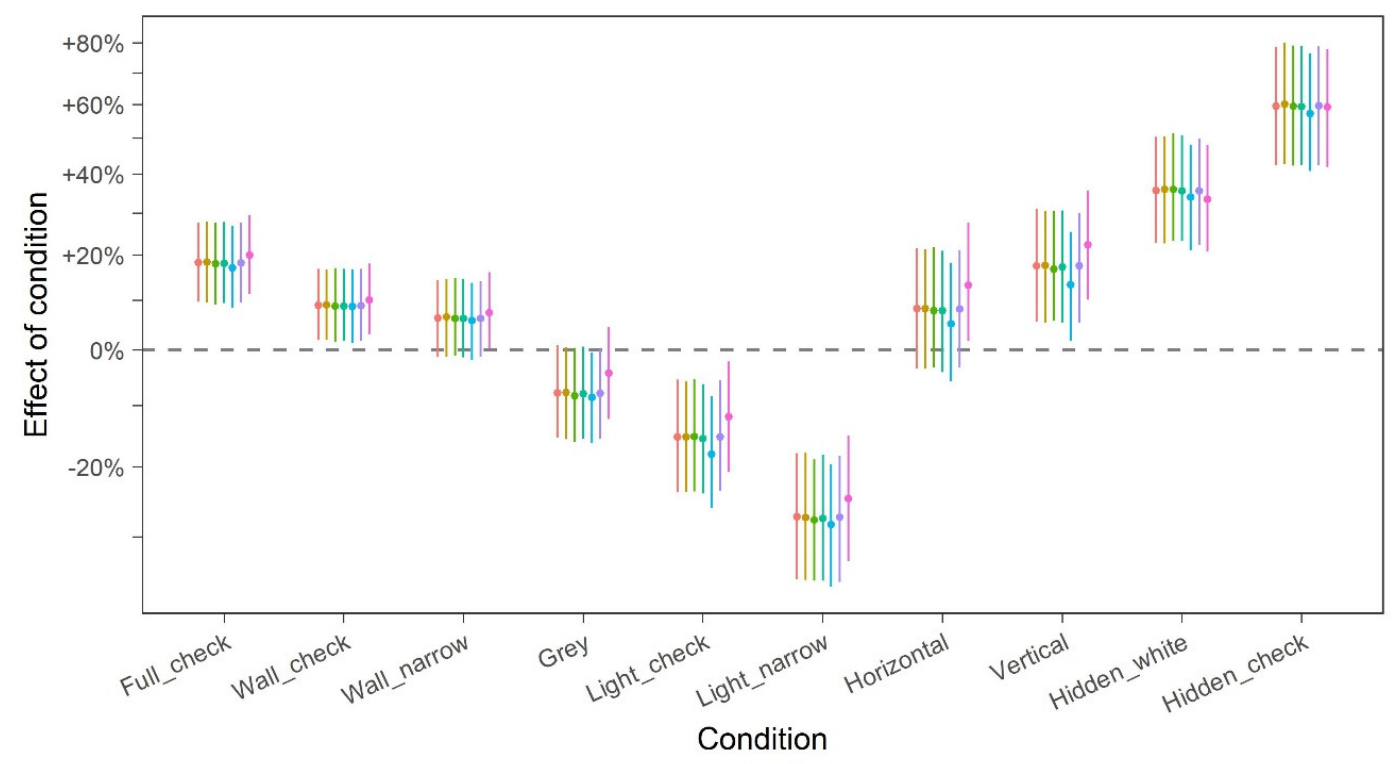

Analysis version

$\ldots$ Main results (SPD, cal. factor adjustment)

$\rightarrow$ Main results with $2 x$ wide priors

$\multimap$ Main results using a T-distribution (nu=4)

$\ldots$ Main results using a skew-normal distribution

$\ldots$ Results with no cal. factor adjustment and mean SPD effects

$\ldots$ Results with cal. factor adjust and mean SPD effects

$\longrightarrow$ Results with cal. factor adjust and no SPD effects

Figure 5.16 Sensitivity of estimated effects of conditions to priors, measurement adjustments, and SPD effects. These are the estimates of the mean effects relative to the Uniform White condition as a baseline. 
Perhaps more interesting are the effects of our modelling assumptions on the results (Figure 5.17). Overall, the conclusion is that most of the assumptions do not noticeably impact our inferences. How we model start bias has even less effect here than in the previous experiment because the overall effects are smaller and counterbalancing mitigates it. Modelling position and dimming bias with potential asymmetry increases the uncertainty slightly, but only by $-1 \%$. The exception, however, is the way we model the variance - which does make a notable difference. Looking at the main effects of the nonuniform conditions, we see that it assuming variance is a function of the pair of conditions results in tighter estimates of the effects in most cases, as well as a few small shifts in their estimated magnitude. This generally does not change our conclusions, though it does argue more strongly that the Light_check condition is indeed darker than the Uniform Grey. The most notable difference though is in the estimates of the differences between the Uniform reference conditions. Modelled this way, the estimates of the differences become substantially more uncertain, and the difference between the Uniform Grey and Uniform White conditions trends smaller. These results thus argue that we cannot say if there is a significant difference between the conditions. Other than this though, the results would not appear to change significantly. 

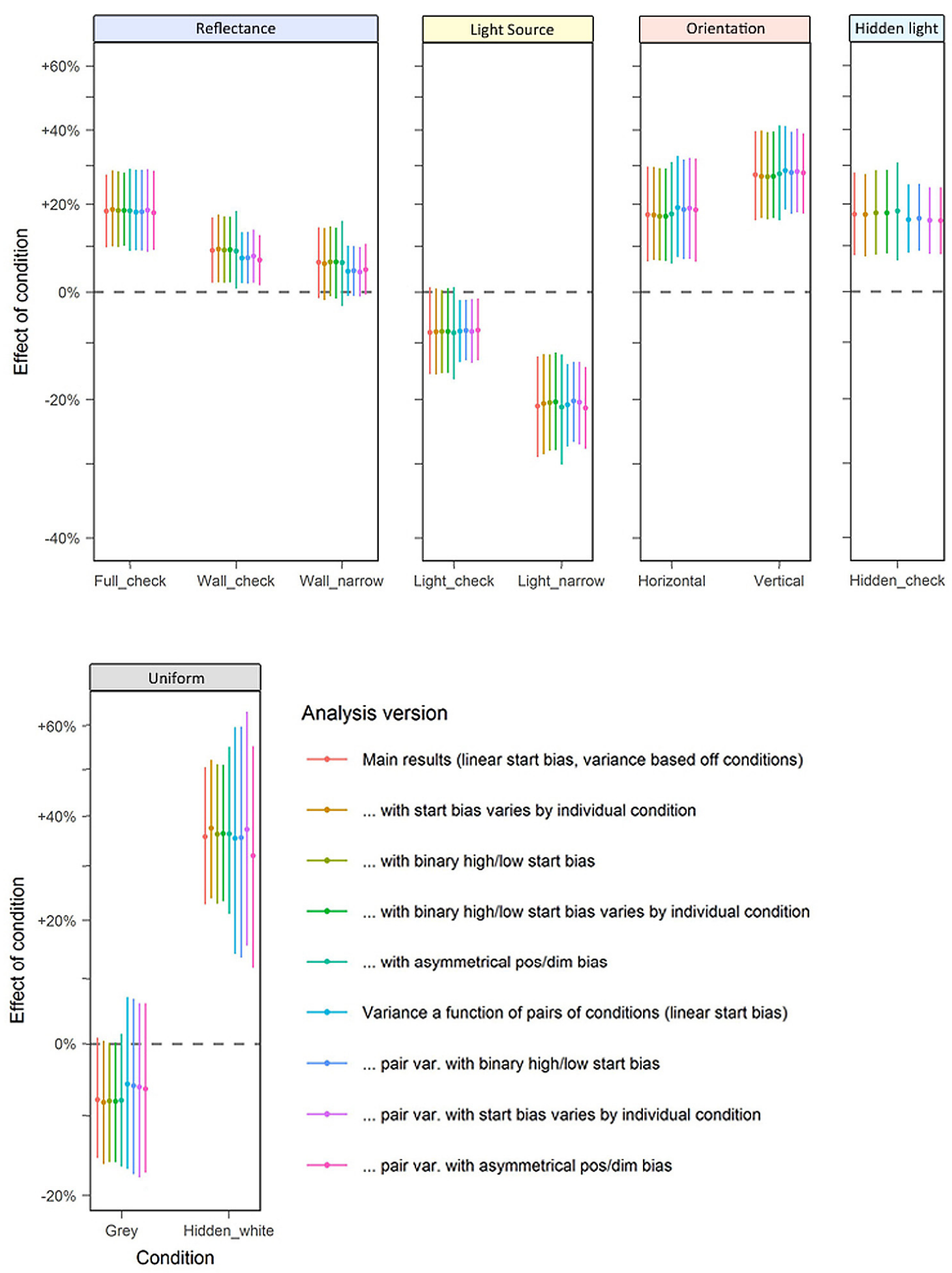

Figure 5.17 Sensitivity of main effects discussed in Figure 5.11 and Figure 5.13 to different modelling assumptions. Error bars show means and 95\% HDI's of the posterior estimates of the mean differences between conditions. 


\subsubsection{Other observations}

Posterior estimates of the other parameters in our models (Appendix D.5) suggest that, as before, the variance in our experiments was in line with other studies - though perhaps slightly on the lower end (mean between-S SD: ${ }_{0.03} 0.05$ 0.08, mean within-S SD: 0.060 .07 0.08). Similarly, there was significant variation between people in effects and biases. Perhaps more interesting here is that with our larger sample size and counterbalanced design, we can observe what appears to be statistical variation between condition pairs in position+dimming and start bias strength (e.g. Figure 5.18, Figure 5.19). This variation, along with similar variation in within-person variance, is relatively small compared to the variation between people, being perhaps half as large at best (Figure 5.19, Figure 5.20). While we would be leery of concluding that a specific pair of conditions here were definitely more or less bias prone without replication ${ }^{65}$, this does provide further weight to the suggestion that we should err on the side of counterbalancing rather than the use of null condition trials to control bias ${ }^{66}$. That being said, the estimated differences here appear to be mostly on the order of a few percent, so as long as one is examining strong effects it should not substantially change one's results. Such errors would not change the conclusions of our first experiment for example.

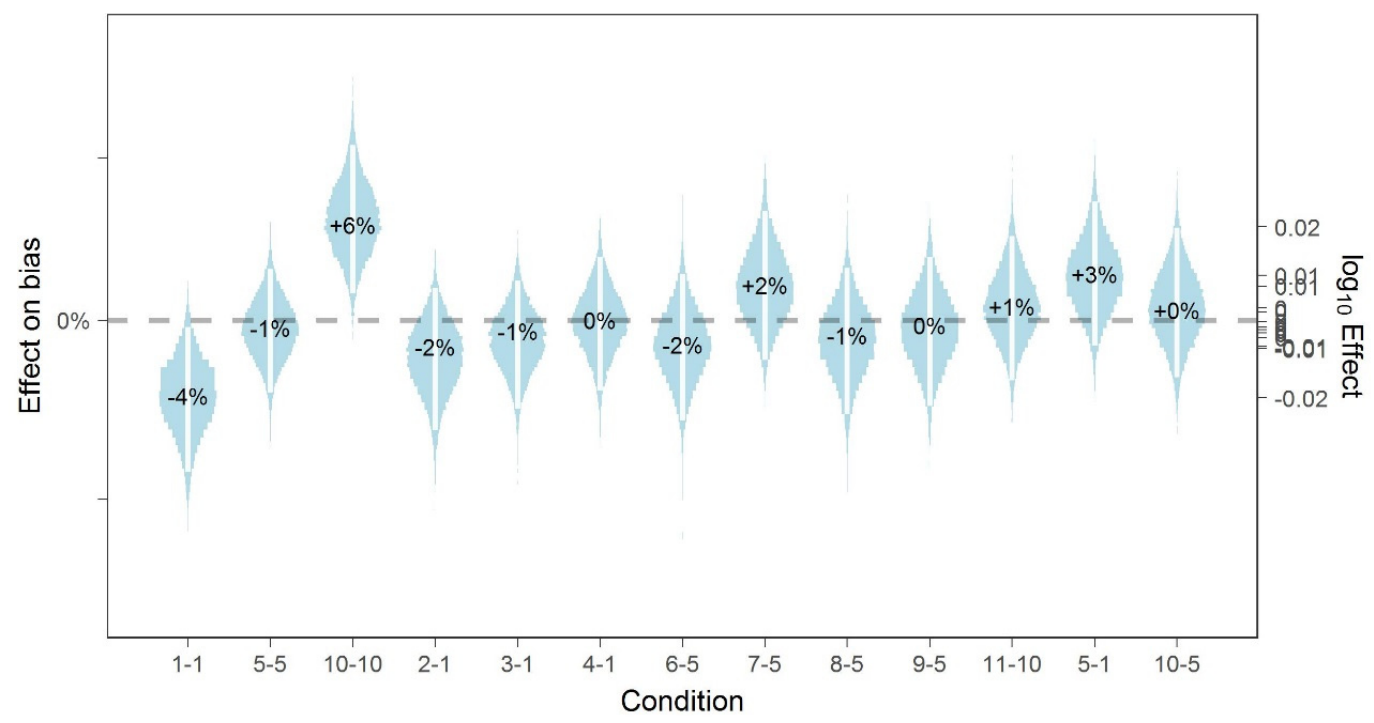

Figure 5.18 Estimated differences in position+dimming bias for different pairs of conditions in Experiment 2.

\footnotetext{
${ }^{65}$ And as an inference is highly model dependent — if we model position/dimming bias separately with potential asymmetry, it becomes much more uncertain and we are unable to infer anything about their variance one way or the other.

${ }^{66}$ If we just use null condition trials, as in the first experiment, we are reliant on the assumption that the bias measured in the null condition trial is the same as that of the other pairs of conditions that are compared. Counterbalancing all the comparisons, as discussed in Appendix $\mathrm{C}$ is (mostly) robust to this.
} 


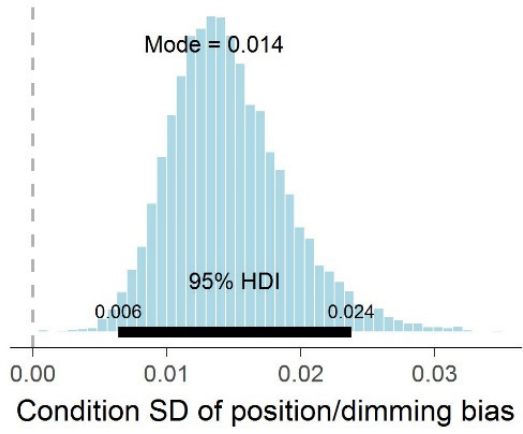

Figure 5.19 Estimated SD of the variation between conditions in position+dimming bias.

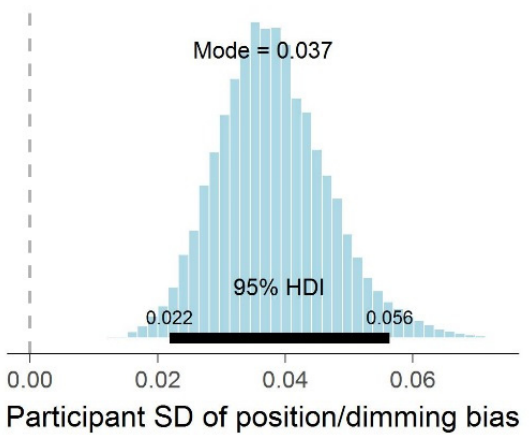

Figure 5.20 Estimated SD of variation in position+dimming bias between participants.

With regard to the biases, it is again unclear whether or not there is significant position+dimming bias (mean bias: $-4 \%+2 \%+7 \%$ ), though the estimates are pulling in towards smaller values. The start bias estimate is tending smaller than in the previous experiment (mean: $-0.05-0.03-0.01$ ), which may be partially due to our addition of adjustment range bias to the model. Response contraction due to reference and adjustment range bias are both uncertain (Reference bias: ${ }_{-0.09}-0.04{ }_{0.03}$; Range bias: $-0.08-0.02_{0.03}$ ), and we cannot draw any conclusions beyond that if there is bias it is likely roughly in the range we would expect.

\subsubsection{Discussion: The failure of uniformity as a predictor}

The flipside of our main findings — the apparent need to account for qualitative differences in how the luminances in a distribution were produced - is the damning failure of uniformity as a correlate with spatial brightness. All the key luminance-based metrics from the literature failed because they predicted that the brightness/uniformity relationship should point in a single clear direction. The non-uniform spaces were deliberately designed to be quantitatively (and indeed qualitatively) more non-uniform on most metrics, and so they should theoretically appear consistently brighter/darker than the uniform spaces. As we successfully produced both non-uniform spaces that appeared brighter, and non-uniform spaces that appeared darker, this basic hypothesis is rejected ${ }^{67}$.

Looking at various arbitrary uniformity metrics that have been explored in this study and others: the normalised standard deviation (coefficient of variation), $\mathrm{max} / \mathrm{min}$ ratio, $99^{\text {th }} / 1^{\text {st }}$ percentile ratio, $95^{\text {th }} / 5^{\text {th }}$ percentile ratio, directional diffusivity, Marsden Equation, $\max / \mathrm{min}$ ratio in the $40^{\circ}$ band, $99^{\text {th }} / 1^{\text {st }}$ percentile ratio in the $40^{\circ}$ band, and standard deviation of the log luminance all failed here. We discuss their failures here in more detail

\footnotetext{
${ }^{67}$ And, of course, producing even more "non-uniform = brighter" results provides further evidence for the existence of such effects, which cannot easily be reconciled with the "uniform = brighter" results in the rest of the literature if we are restricted to trying to model them all with simple uniformity metrics.
} 
both to gain an understanding of how they failed beyond the main story presented above, but also to highlight why using them to attempt to predict spatial brightness may lead one to highly erroneous conclusions.

\section{Directional Diffusivity}

The Directional Diffusivity metric failed in a couple of additional ways. Firstly, it predicts that non-uniform spaces should appear darker. As we saw in the reflectance, orientation, and hidden light source conditions non-uniform spaces can, in fact, appear brighter. This replicates the findings of our previous study and again rejects this simple model.

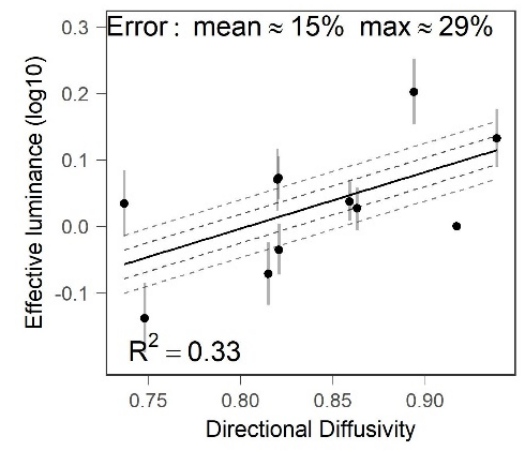

Figure 5.21 Simple linear regression of directional diffusivity against effective light level. Dashed lines show error of $\pm 5 \%$ and $\pm 10 \%$ around the regression line.
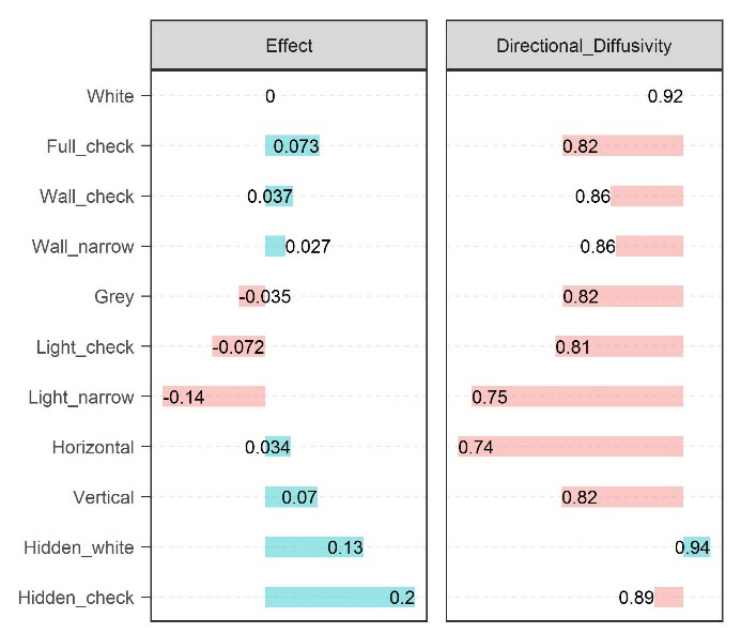

Figure 5.22 Comparison of the observed patterns of changes in uniformity and changes in effective light level across conditions. On the left is the pattern of the estimated differences in apparent light level. On the right is the pattern of differences in "uniformity". If the metric was a good model, these patterns should align.

Secondly, the practice of averaging over the vertical is again challenged. The directional diffusivity model, because it averaged luminances over the vertical axis, predicts that the non-uniform Vertical orientation condition should appear similar in uniformity the uniform grey condition (Figure 5.22). Similarly, that it should be close to the Light_check condition. This is clearly not the case. We may similarly question the difference between the Hidden_check and Hidden_white conditions, which on this metric is smaller than the difference between the Uniform White and Wall_check/Wall_narrow conditions, while our results suggest it should be larger, and comparable to the Full_check condition.

Consistent with the Kato et al studies the Light_check condition did have a significantly smaller effect than the Light_narrow condition. However, this finding is of 
limited significance as in this particular case the Light_check condition was also more uniform than the Light_narrow condition on most metrics.

\section{Standard Deviation (normalised)}

The standard deviation, despite its "strong" performance in the previous experiment does rather poorly here with a mean error of $-15 \%$ (Figure 5.23 ). One failure of the SD is its prediction of the relative size of effects. In particular, it says that the Full Checkerboard condition is roughly $140 \%$ more "non-uniform" than the uniform white condition, compared to the other non-uniform conditions which are only around 40-50\% more nonuniform than their uniform conditions. Following this, we would "expect" the full checkerboard vs. uniform white comparison to be by far the largest effect - however, this is not the case.

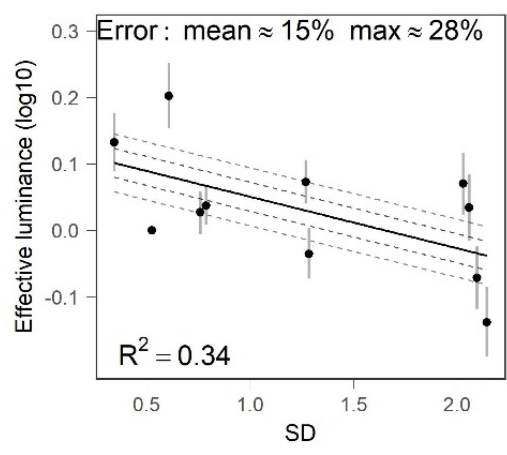

Figure 5.23 Simple linear regression of SD against effective light level. Dashed lines show error of $\pm 5 \%$ and $\pm 10 \%$ around the regression line.

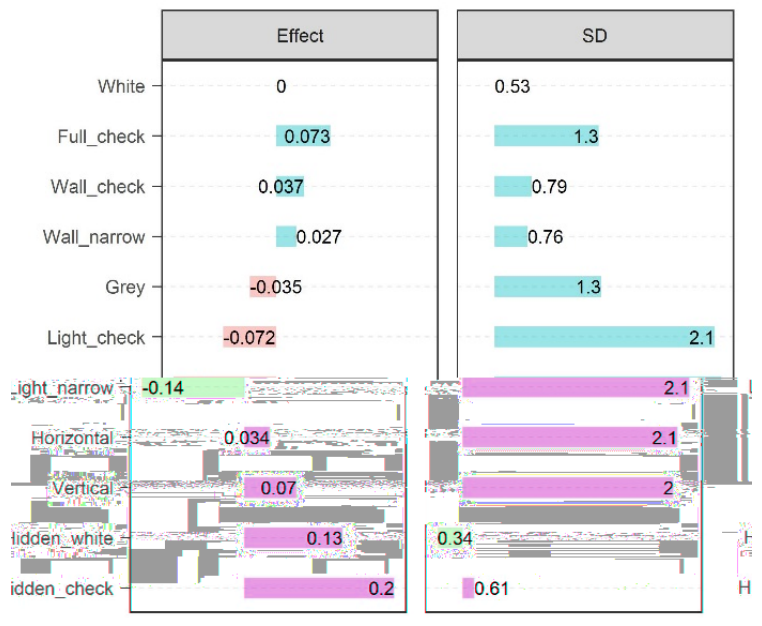

Figure 5.24 Comparison of the observed patterns of changes in SD and changes in effective light level across conditions. On the left is the pattern of the estimated differences in apparent light level. On the right is the pattern of differences in "uniformity". If the metric was a good model, these patterns should align.

Perhaps the most interesting failure though comes when we compare the full checkerboard condition to the uniform grey condition. Both these spaces have the same standard deviation, the average of the checks being roughly the same luminance as the surface of the grey space. This is why the reflectance conditions were compared to the uniform white condition instead of the uniform grey one in contrast to the previous experiment. However, instead of appearing to have the same brightness the full checkerboard condition appears substantially brighter than the uniform grey condition.

On the same note, the SD of the Hidden_check condition is perhaps 10\% larger than that of the Uniform White condition — relatively minor (the non-uniform conditions have 
SDs at least $40 \%$ larger than the uniform conditions they were matched against in Figure 5.11). In contrast to the minor difference in SD though the difference in brightness between the Hidden_check and uniform white conditions - roughly $+70 \%$ — is massive, dwarfing the differences between most of the other conditions. Again, this illustrates a clear failure of the standard deviation to capture the observed brightness effects. This is further reflected in the failure of the conditions in this experiment to produce effects as large as expected from the previous experiment. Despite the differences in SD between the conditions here being as large or larger than those produced by the most non-uniform condition in the first experiment, the brightness effects observed here are much smaller.

\section{Standard Deviation of Log Luminance}

By log transforming the luminances before calculating the SD the impact of the low reflectance surfaces is greatly magnified. On a linear scale, the difference between, for example, a $100 \mathrm{~cd} / \mathrm{m}^{2}$ light source and the $20 \mathrm{~cd} / \mathrm{m}^{2}$ wall next to it is much larger than the difference between a $20 \mathrm{~cd} / \mathrm{m}^{2}$ surface and a $1 \mathrm{~cd} / \mathrm{m}^{2}$ surface. On a logarithmic scale, the opposite is true. The result of this is that the various changes to surface reflectance have a larger effect on the SD of the log luminance. In contrast, the light source changes have much less of an effect, with the Light_narrow condition having an $\mathrm{SD}_{\log }$ only $\sim 10 \%$ higher than that of the uniform grey condition, and the Light_check condition predicted to be the same as the uniform condition (compare this to the other conditions, which have SDs roughly twice that of their uniform comparisons) (Figure 5.26).

While the Light_check condition may have had a relatively weak effect, the Light_narrow condition was clearly as strong as any of the other effects in Figure 5.11. The failure to capture this is glaring. The metric also argues that hiding the light source should have no effect on uniformity and thus brightness, a notion which is clearly rejected by how much brighter the hidden light source conditions are compared to the basic uniform white condition. 


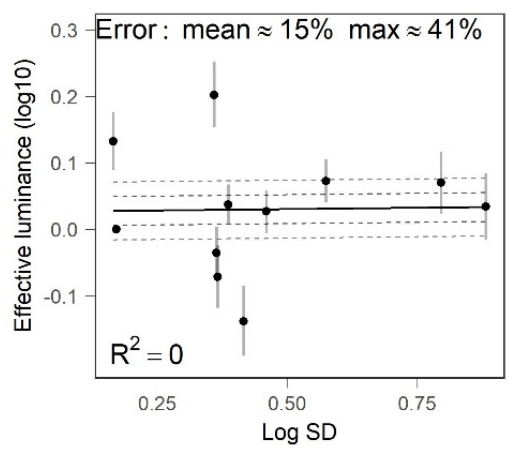

Figure 5.25 Simple linear regression of $\mathrm{SD}_{\text {log }}$ against effective light level. Dashed lines show error of $\pm 5 \%$ and $\pm 10 \%$ around the regression line.

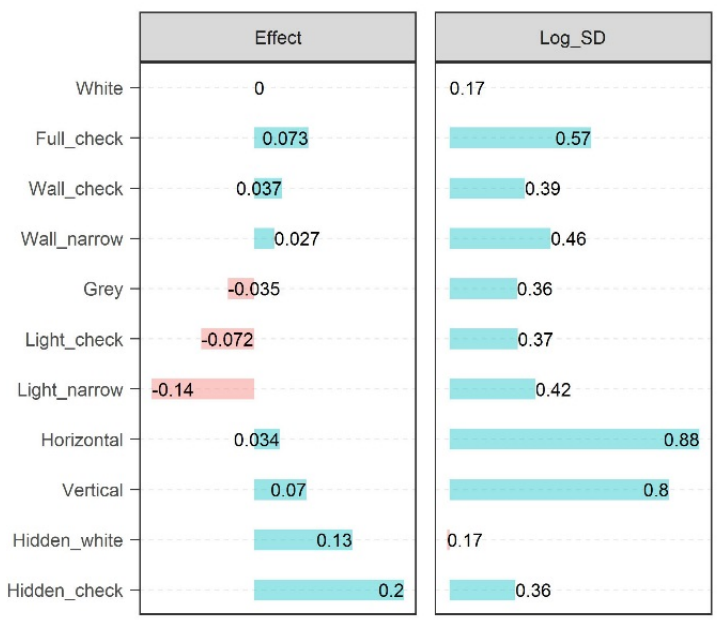

Figure 5.26 Comparison of the observed patterns of changes in SD of log luminance and changes in effective light level across conditions. On the left is the pattern of the estimated differences in apparent light level. On the right is the pattern of differences in "uniformity". If the metric was a good model, these patterns should align.

\section{Max/min uniformity ratio}

The max/min uniformity ratio manages to achieve an $\mathrm{R}^{2}$ of 0 (Figure 5.27). Even by the standards of all the uniformity metrics performing poorly, the max/min uniformity ratio performs particularly badly here - in large part due to how much lower the minimum is in the two orientation conditions. Unfortunately for it, however, the horizontal and vertical conditions did not have effects -5 times as large as the others. We may also note that from its perspective the Light_narrow condition should be the same as the Light_check condition, the orientation conditions should have effects massively larger than anything else, and the hidden light source conditions should be relatively uniform, and closer in brightness to the uniform white condition than anything else. 


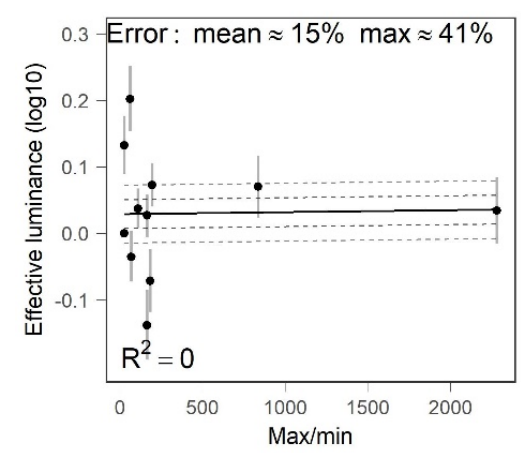

Figure 5.27 Simple linear regression of uniformity ratio against effective light level. Dashed lines show error of $\pm 5 \%$ and $\pm 10 \%$ around the regression line.

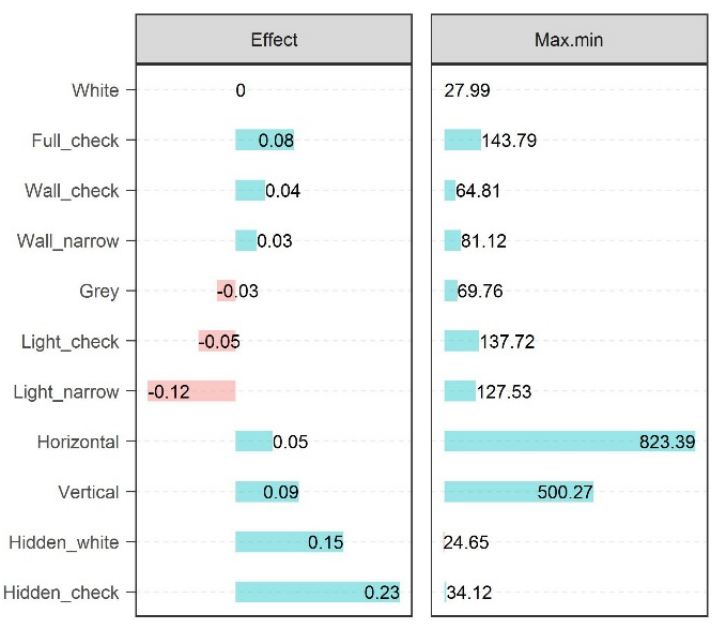

Figure 5.28 Comparison of the observed patterns of changes in uniformity ratio and changes in effective light level across conditions. On the left is the pattern of the estimated differences in apparent light level. On the right is the pattern of differences in "uniformity". If the metric was a good model, these patterns should align.

Needless to say, this pattern is very much not what we observe (Figure 5.28).

Focusing on the $40^{\circ}$ horizontal band does not help either (Figure 5.29, Figure 5.30). Neither does attempting to explain the mismatches in fit by adding in the effects of focusing on the mean luminance in the $40^{\circ}$ band as well (Figure 5.31). Indeed, if anything, that just makes the fit worse, as looking at things through that lens makes the hidden light source conditions appear relatively darker - the exact opposite of what we observe while doing nothing to address any of the issues.

Using the $99^{\text {th }} / 1^{\text {st }}$ percentiles shows essentially the same patterns, as do the $95^{\text {th }} / 5^{\text {th }}$ percentiles (Figure 5.32, Figure 5.33). 


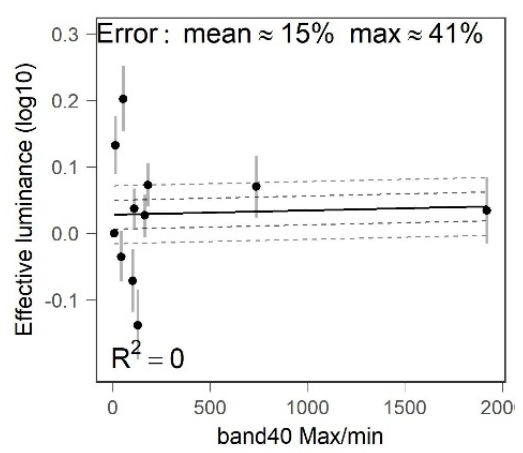

Figure 5.29 Simple linear regression of uniformity ratio in the $40^{\circ}$ band against effective light level

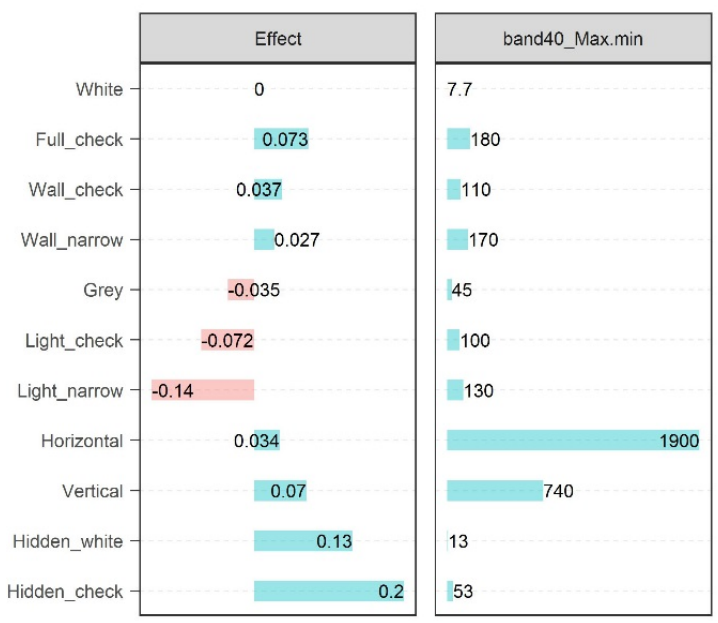

Figure 5.30 Comparison of the observed patterns of changes in uniformity ratio in the $40^{\circ}$ band and changes in effective light level across conditions
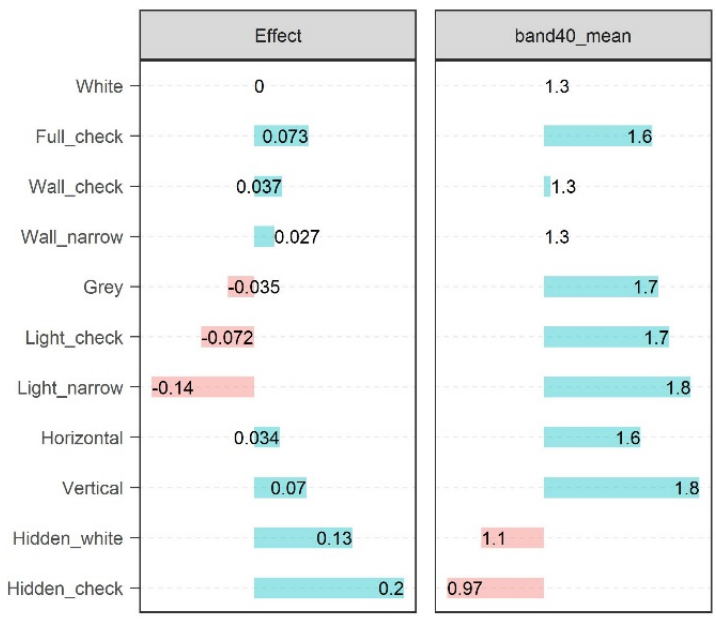

Figure 5.31 Comparison of the observed patterns of changes in the mean luminance of the $40^{\circ}$ band and changes in effective light level across conditions. Note here that the mean luminance of the $40^{\circ}$ band has been calculated from luminance maps that had been normalised to the same overall mean luminance, in this case $1 \mathrm{~cd} / \mathrm{m}^{2}$. Thus, they are effectively the effect of focusing on the $40^{\circ}$ band relative to the whole field of view. 


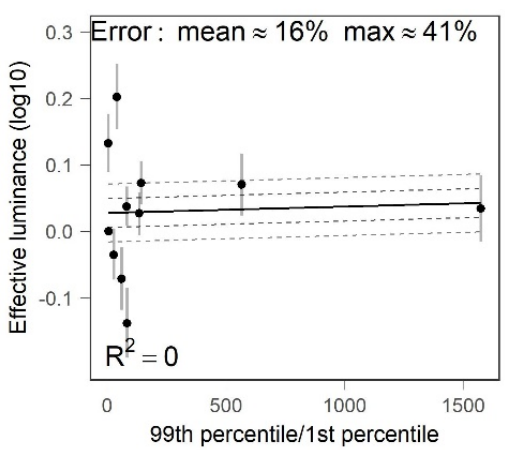

Figure 5.32 Simple linear regression of uniformity ratio against effective light level

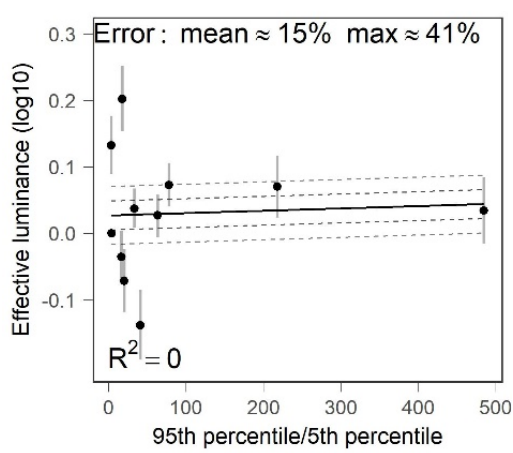

Figure 5.33 Simple linear regression of uniformity ratio against effective light level

\section{Summary}

Uniformity, however one describes it, does not work well to describe the pattern of results that has been observed here. That the Narrow Light condition appears darker, while others such as the Full Checkerboard condition appear brighter, is hard to explain via uniformity. That the hidden light source conditions were the brightest, despite being far closer in uniformity to the uniform white condition, is similarly hard to explain. Indeed, the uniformity metrics commonly pointed to conditions such as the orientation or full checkerboard as being the most different, which was far from the case. While we have not necessarily covered all the possible ways that one could quantify uniformity, it does not seem likely that alternative formulations would resolve these issues. It is difficult to see, for example, how any uniformity metric would be able to describe both the Vertical condition and the Hidden White condition as being similar in uniformity. Similarly, given their physical properties, it is hard to see how any credible uniformity metric could place, for example, the Narrow Light, Uniform White, and Vertical conditions on a straight line. Which condition would one describe as more uniform than the Uniform White one? Simply put, our results defy any simple uniformity-based explanation (Figure 5.34).

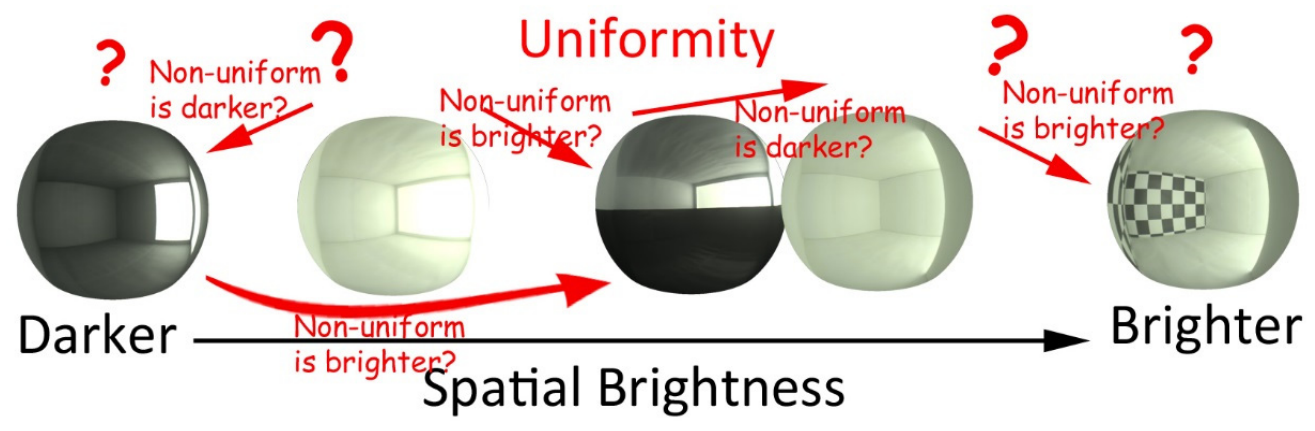

Figure 5.34 These five selected conditions range from the darkest to the brightest we observed. We see no credible way of defining a uniformity scale that would consistently relate to the observed brightness of these conditions. 


\subsection{Conclusions}

The results found reject the hypothesis(es) that the differences between studies can be explained by adaptation level, orientation of light distribution, or simple visibility of the light sources. The basic pattern of results shown - with opposite trends found between the reflectance set and light source set - matches the prediction made by the Modes of Appearance hypothesis. This would seem to suggest that we can explain differences between studies by saying that non-uniformity as a result of changing the surface reflectances makes spaces brighter, but non-uniformity as a result of changing the arrangement of the light sources makes spaces darker. Correspondingly, the overall uniformity of the luminance distribution does a very poor job at explaining the pattern of results. The notion that we can simply say that "uniform/non-uniform light distributions appear brighter" would seem, at best, highly questionable. Continuing to attempt to describe the effects of the light distribution by simply regressing brightness against favoured uniformity metrics seems unlikely to produce generalizable models.

However, the Modes of Appearance hypothesis (as presented here) is challenged by secondary comparisons, in particular the difference between the uniform white and uniform grey conditions. Despite being a more non-uniform space produced by changing the surface reflectances, the Uniform Grey condition does not appear substantially brighter than the Uniform White condition. Indeed, the results suggest the opposite is more likely. This suggests that while the Modes of Appearance hypothesis seems to be getting at something important, it is not quite right. 



\section{Exploration: how might we better describe and model our results?}

The Modes of Appearance hypothesis, as described in the previous chapter, was somewhat vague in its precise definition. Key questions left unaddressed at the time of formulation were a) how the hypothesised effects of light sources and effects of surface reflectance would interact, b) how the uniform reference conditions would be expected to differ, and c) how exactly the effects "work". In many ways, it could be said to be a description of some of the rough trends that we expect to observe, rather than a detailed explanation of why and how light distribution actually affects spatial brightness.

This was deliberate as there was arguably limited value in attempting to refine the model in detail before we had even established that it was, indeed, what we should be focusing on. Now, however, with our results suggesting that the hypothesis has at least roughly identified the key elements behind the apparent disagreements in the literature, we may attempt to develop the model in more detail.

Before we continue it should be noted that the following discussion and analysis is the result of post-hoc discussion and analysis of the results. Given the amount of flexibility we have in such matters, and the fact that this analysis would likely have been different had different results been observed (Gelman and Loken, 2013), the work here should be treated as exploratory rather than confirmatory.

\subsection{Reformulating the Modes of Appearance hypothesis}

To explain the failure of the Uniform Grey/Uniform White comparison to align with the Modes of Appearance hypothesis, we suggest a simple reformulation. Let us say that:

a) Non-uniform luminance distributions over the non-luminous surfaces increase spatial brightness. 
b) Increasing the difference between the luminous (i.e. light sources) and nonluminous elements in a space (thus increasing non-uniformity) decreases spatial brightness.

c) The overall effect is the sum of the two preceding processes.

This reframing implies a model of two opposing processes that happen to, roughly, align with the division we set up to test our Modes of Appearance hypothesis. In the reflectance conditions, the effect is largely one wherein the overall effect is dominated by the effect of the change in surface pattern. However, the reduction in average reflectance also increases the difference between the luminous and non-luminous elements in the room - an effect which decreases the brightness and thus attenuates the overall effect (Figure $6.1)$.

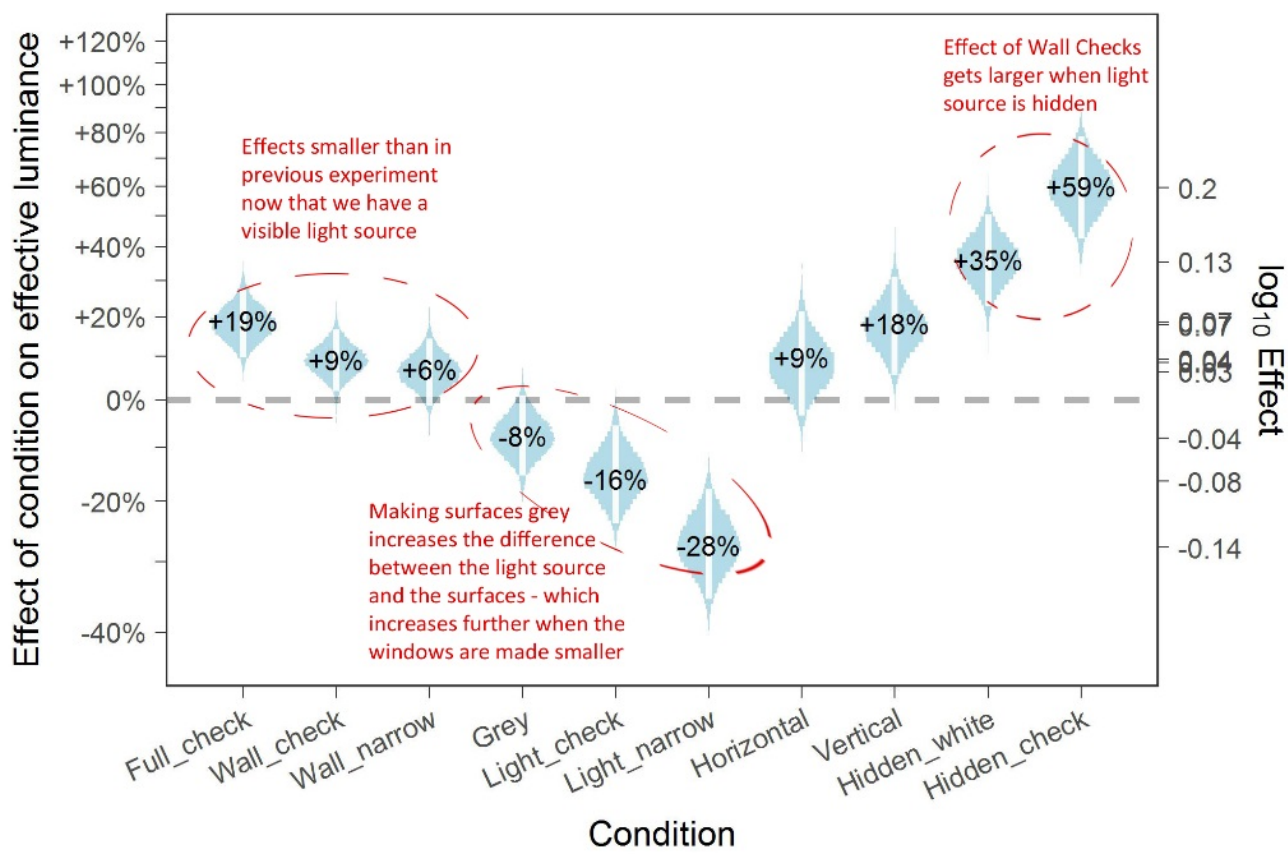

Figure 6.1 Notes on the observed effects in the second experiment

A fight between the two processes can explain why, for example, the full checkerboard condition had so much less of an effect here than it did in the previous experiment. In the previous experiment the light source was not visible, so there were no luminous elements. The effect of the light balance in the different conditions was thus zero, and could not attenuate the effects of changing the surface patterns. Such an explanation may be supported by the effects of hiding the light source that we see in this experiment. The "brightest" conditions here are the ones with the light source hidden. Indeed, the difference between the Uniform White conditions with and without the light source hidden is larger than the effects of the main non-uniform test conditions. When the light source is hidden 
the Wall Checkerboard condition goes from appearing $-10 \%$ brighter than the comparable Uniform White condition to appearing $-20 \%$ brighter — a result consistent with the idea that removing the light source removes an attenuating effect.

However, it would seem to be challenged by the orientation conditions. Logically, with their reduced window size their "light source/room imbalance" effect should be significantly greater than that of the reflectance conditions (Figure 6.2). Yet, in spite of this, the overall effect(s) of the orientation conditions is roughly the same as the reflectance conditions.

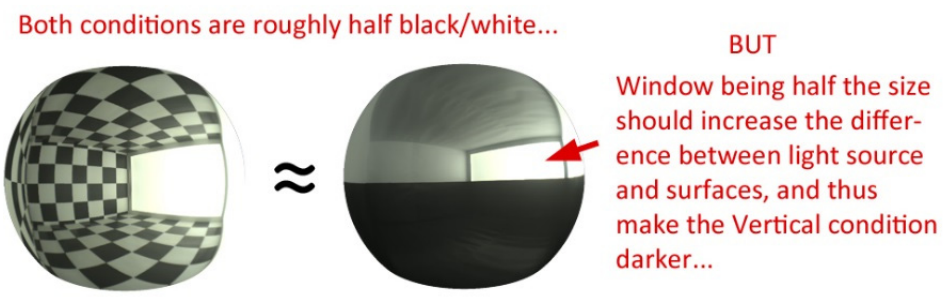

Both conditions similar in brightness

Figure 6.2 The orientation condition challenge

If we start to look at some rough measurements of the light balance and surface uniformity though we can see some reasons why this might be the case (Table 6.1). While the difference between the luminous and non-luminous regions of the orientation conditions is much larger than it is for the Full Checkerboard condition, this is countered by the fact that the surface luminances in the orientation conditions are also significantly more non-uniform (at least as measured by the standard deviation). The relative lack of a significant difference between the Uniform Grey and White conditions can be explained the same way. The Uniform Grey condition might have a significantly more non-uniform light balance, but lower surface reflectances mean a more non-uniform illumination distribution, which mitigates the overall effects. At the same time, its surfaces are still far more uniform than the Full Checkerboard condition, explaining why that condition appears much brighter. 
Table 6.1 Measured "Light balance" and Uniformity of the non-luminous surfaces of the Experiment 2 conditions

\begin{tabular}{|l|l|l|}
\hline Conditions & $\begin{array}{l}\text { Light balance } \\
\text { (luminous/non- } \\
\text { luminous) }\end{array}$ & $\begin{array}{l}\text { SD of non- } \\
\text { luminous } \\
\text { surfaces }\end{array}$ \\
\hline Uniform White & 2.5 & 0.20 \\
\hline Full Checkerboard & 6.7 & 0.81 \\
\hline Wall Check & 3.5 & 0.45 \\
\hline Wall Narrow & 3.3 & 0.47 \\
\hline Uniform Grey & 7.1 & 0.40 \\
\hline Light Check & 15.5 & 0.41 \\
\hline Light Narrow & 20.2 & 0.58 \\
\hline Horizontal & 15.2 & 1.22 \\
\hline Vertical & 14.3 & 1.19 \\
\hline Hidden White & 0 & 0.34 \\
\hline Hidden Check & 0 & 0.61 \\
\hline
\end{tabular}

A rough illustration of the concept based on these crude measures is shown below (Figure 6.3):

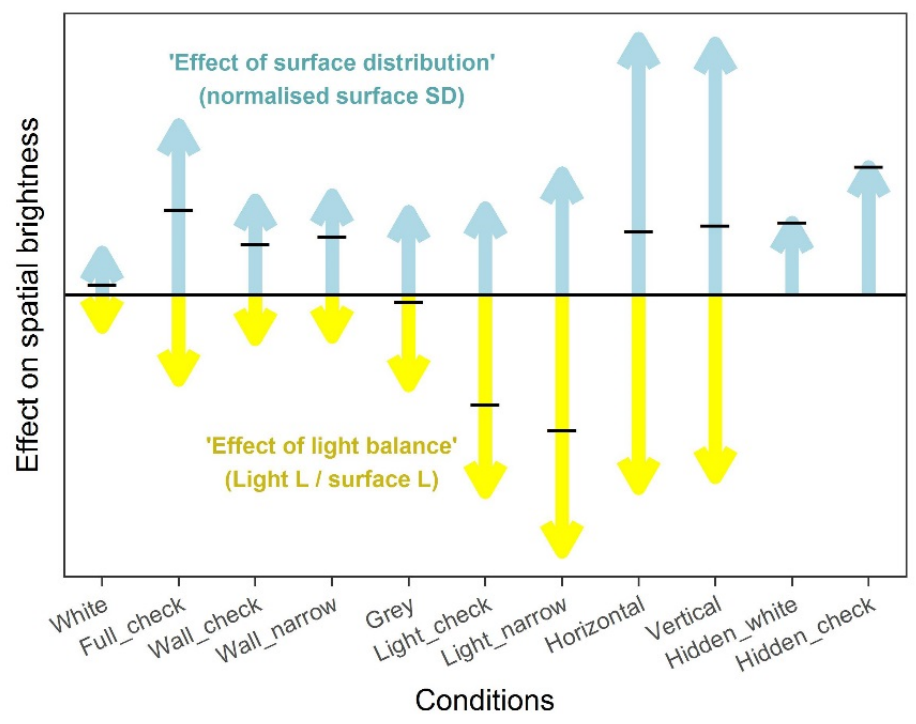

Figure 6.3 Illustration of the concept of how surface uniformity and light balance might work together to produce a pattern of results like what we observe in Experiment 2. The blue arrows use the normalised standard deviation of the surface luminance as a rough measure of uniformity. The yellow arrows are based off the light balance (ratio of mean luminance between luminous/nonluminous) applying a negative effect. Both measures are normalised to the maximum here to put them on comparable scales. Black bars then show the average of the two as the overall effect on brightness. 
These specific measures are only used here to illustrate the basic concept and its potential viability. It should not be taken as a suggestion that they would necessarily comprise a good model of the effects. While the standard deviation may, for instance, be a reasonable description of the overall uniformity, it does not provide a very good explanation or a reason for using it to describe human perception. It does, however, provide a starting point. The question we might ask here then is: how might we construct a model of spatial brightness based on these ideas?

\subsection{A mathematical model}

The basic concept of the dual process model of light distribution that we will explore here is that the overall effective luminance is the result of the combined effects of the pattern of the non-luminous surfaces, and the light balance between the light source and the rest of the room:

$$
\log \left(L_{\text {eff }}\right)=c_{1} E_{\text {surf }}+c_{2} E_{\text {light }}+\log (L)
$$

... where $L_{\text {eff }}$ is the effective luminance, $E_{\text {suf }}$ is the effect of the surface pattern, $E_{\text {light }}$ is the effect of the light balance, $\mathrm{L}$ is the mean luminance, and $c_{1}$ and $c_{2}$ are coefficients controlling the relative strength of the effects.

For a theoretical perfectly uniform condition, $E_{\text {suf }}$ and $E_{\text {light }}$ should equal 0 , so the effective luminance should simply equal the mean luminance. This provides a natural and logical baseline from which to measure the effects of the light distribution.

To implement this model, however, we must address several questions: a) how to model the effects of surface pattern, and b) how to model the effect of the light balance. To this list we may also add a third question: c) whether or not we should also consider position weighting (Figure 6.4). While, as noted previously (Section 2.3.2), the empirical evidence for luminances in different parts of the room having different levels of influence is lacking, the idea is both popular and theoretically plausible - even likely. We may also note that when exploring correlations with the results of Experiment 1, focusing down on the central band in the field of view/the walls did seem to help - particularly with the case of the Black Wall condition.

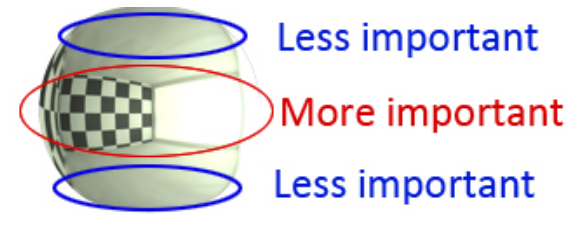

Figure 6.4 The general idea expressed by position weighting based on the literature

We discuss how we might attempt to construct models based off existing ideas and theory for each of these elements below. 


\subsubsection{Modelling the apparent effects of non-uniform surfaces}

Perhaps the simplest option here would be to use the standard deviation of the surface luminance distribution as a predictor of the effects here. It did, after-all, correlate well with the results of Experiment 1 - especially when we weighted the luminances to focus on the central horizontal band. We are, however, not positively disposed towards this metric. While it may potentially be a good correlate, it does not (at least to our eyes) provide a meaningful description of how non-uniform surfaces might be influencing spatial brightness. We would prefer, if possible, to develop a theoretically meaningful model that could actually enhance our understanding.

The first thing to note here is that we cannot use existing brightness models, because as we saw with the Marsden Equation and Stevens' Power Law (Experiment 1: Section 3.6), they predict that the effects should be in the opposite direction from what we observed. So what might explain our observations?

The first hypothesis we explored was related to perceptions of illumination. Kardos (1934, in Gilchrist, 2006, p. 217), in his studies of brightness and lightness perception noted that observers did not mention shadows in their descriptions of a room and, even after being prompted, did not mention shadows attached to objects. While illumination perception has been much less studied than lightness and brightness (Gilchrist, 2006), what studies that have been done on the subject suggest that it is most related to the highest luminance viewed (Beck, 1961, 1959; Kozaki, 1973; Noguchi and Masuda, 1971). Similar to the Anchoring Theory of Lightness, Soranzo and Gilchrist (2019) suggest that illumination too is anchored to the highest luminance.

A simple way of modelling this general idea would be to calculate the effect of surface non-uniformity as a weighted average, with the highest luminances given more weight than the lower luminances. This would, conceptually, represent the idea that when people attempt to determine the contribution of the surfaces to the overall spatial brightness they tend to be more influenced by the brighter surfaces, and discount the darker areas. The resulting mean surface luminance would thus tend to be brighter when the luminance distribution is non-uniform (Table 6.2). 
Table 6.2 Simple illustration of how a less uniform distribution would appear "brighter" if the higher luminances are weighted more heavily in our overall assessment. While both the distributions here have the same mean luminance, by weighting the higher luminance as twice the lower one in the distribution we get a higher weighted mean luminance for the less uniform distribution.

\begin{tabular}{|l|c|c|}
\cline { 2 - 3 } \multicolumn{1}{c|}{} & More uniform & Less uniform \\
\hline Mean Luminance & $\frac{20+10}{2}=15 \mathrm{~cd} / \mathrm{m}^{2}$ & $\frac{25+5}{2}=15 \mathrm{~cd} / \mathrm{m}^{2}$ \\
\hline $\begin{array}{l}\text { Weighted Mean } \\
\text { Luminance }\end{array}$ & $\frac{2 \times 20+1 \times 10}{2+1}=16.7 \mathrm{~cd} / \mathrm{m}^{2}$ & $\frac{2 \times 25+1 \times 5}{2+1}=20 \mathrm{~cd} / \mathrm{m}^{2}$ \\
\hline
\end{tabular}

In this case, to produce the weights we first calculated the percentile rank of every point in each luminance map. This was then combined with a weighting function to produce a weight map, with more weight placed on, for example, the $99^{\text {th }}$ percentile than the $5^{\text {th }}$. From this, we could then calculate the weighted mean luminance, which was then normalised against an equivalent uniform distribution (by simply subtracting the unweighted mean).

The weighting functions we explored were designed to put more weight on the highest luminances. We explored a power function of the percentile rank (Figure 6.5), and a normal distribution centred at 1 (Figure 6.6).

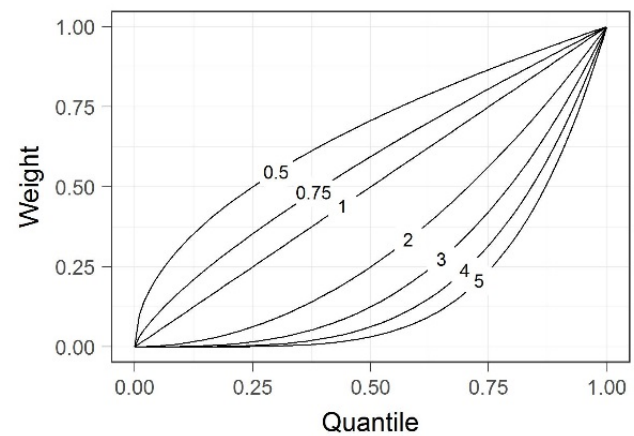

Figure 6.5 Example of power-based weighting functions in the form $x^{\wedge} q$ ( $q$ is the label on each line)

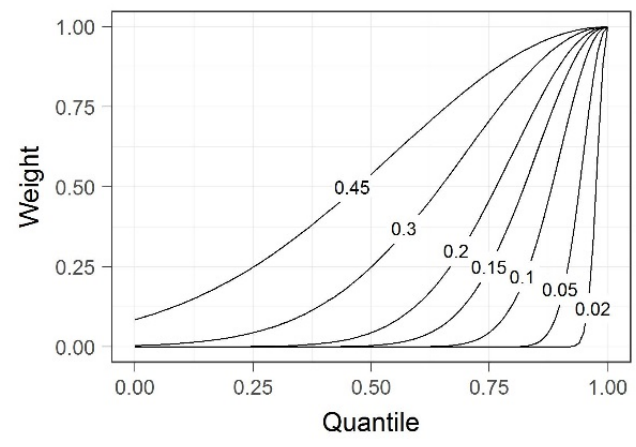

Figure 6.6 Examples of weighting functions based off a normal distribution centred on 1. Each line is based off a different sd.

These could be implemented in a number of different ways. The obvious approach is to rank and calculate the weighted average of the luminances. Alternatively, if we see the perception of brightness as a power function of luminance (Stevens, 1961) we might first convert the luminances into "brightnesses" by applying a power function with an exponent of $\sim 0.35^{68}$. We also have the question of how we integrate this with our potential location

${ }^{68}$ Used by Marsden (1970) in his equation, and within the standard range of exponents typically estimated for brightness (Bauer, 2009; Stevens and Stevens, 1963). If one was interested in refining this specific model, then it would make sense to explore a range of values. 
weights. Intuitively, if we believe that some parts of the scene appear "brighter" or more important than others based off their position then we would want to factor that into our weighted average. However, before that stage we have the question of whether or not we should apply position weighting when we are calculating the percentiles of the luminance distribution. We may interpret these different approaches as the difference between brightness weighting and importance weighting (Figure 6.7). If some parts of the scene are seen as being brighter based on their location then we would want to factor this in to our percentile weighting. If the model is that people pay more attention to the brighter surfaces when estimating spatial brightness, and location affects brightness, then accounting for this is only logical. However, if instead it is just that luminances in some locations are more important than others then it would seem unnecessary to factor this into the calculation of our percentile weights. Indeed, it could be seen as doubling up on the effect of the location weighting. Combining these two "importance weighting functions" in the calculation of the weighted average should be sufficient.

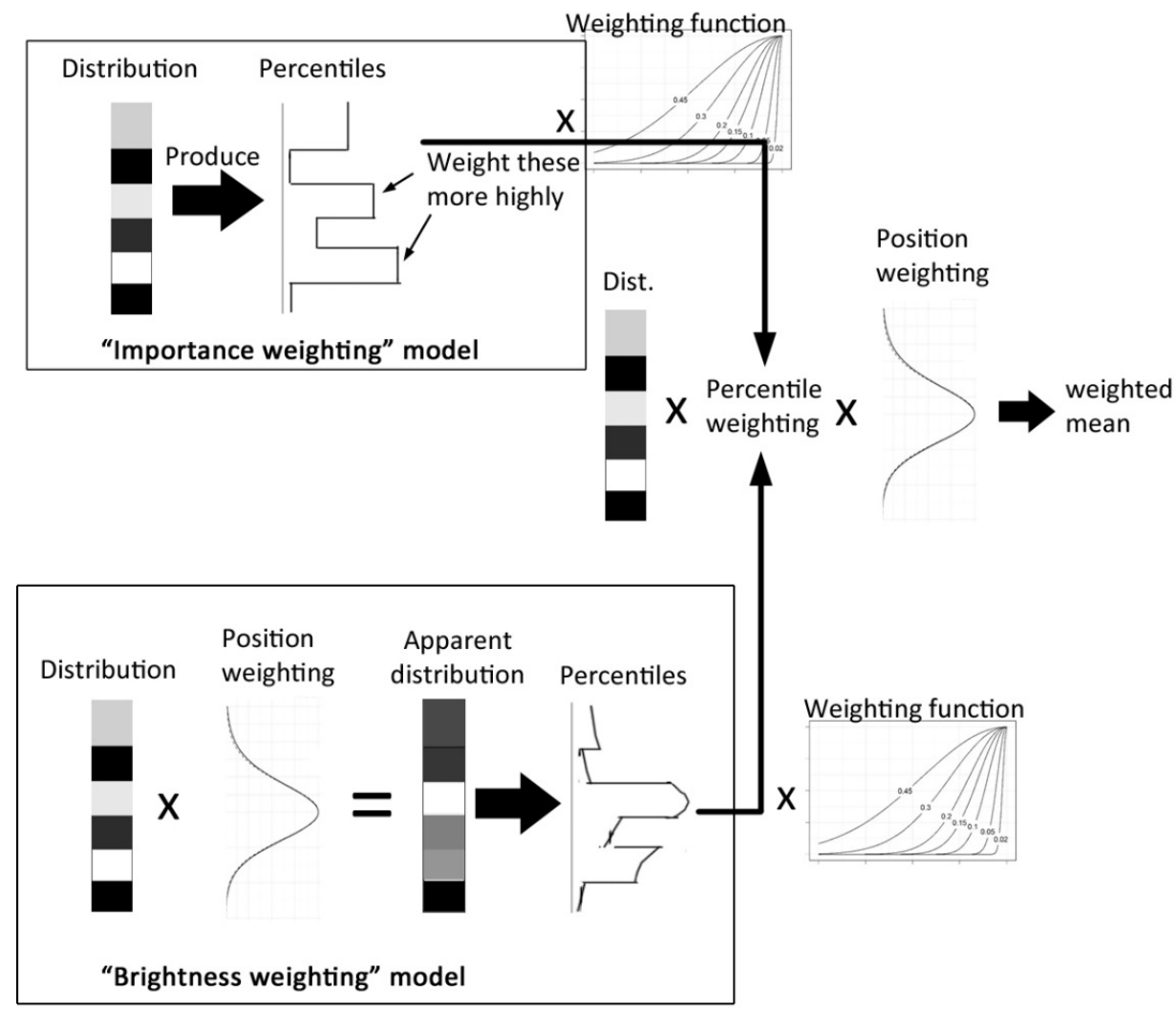

Figure 6.7 Illustration of the basic structure of the percentile weighting model. The overall goal is to compute the weighted average luminance, with the brighter parts of the distribution weighted more highly according to our weighting function. The "brighter areas" are identified here by calculating the percentile rank of every luminance value. The key difference between the "importance weighting" and "brightness weighting" approaches is in the calculation of the percentile ranks, and whether or not they account for the position weighting function. 
Combined, this gives us eight different implementations of the "brighter/higher luminances are more important" model — two weighting functions x [raw luminance vs brightness] x [importance vs brightness position weighting].

A problem with that model, however, is that it is inspired by observations of illumination perception, while we have been changing surface reflectance. Another possibility lies in what Gilchrist (2006) referred to as "scale normalisation". Essentially, studies of perceived lightness have found that log luminance differences do not map directly onto log reflectance differences (Soranzo and Gilchrist, 2019). This he explains as a tendency for the range of perceived greys to normalise to the effective range of white to black - about 30:1. The result of this is stimuli showing a more limited luminance range see an expansion in their perceived range, while those spanning a larger range see a compression (Soranzo and Gilchrist, 2019). On its own, this would not provide any meaningful way to explain our results - expanding or compressing the perceived luminances does not change the overall average. However, in conjunction with the Anchoring Rule (Gilchrist, 2006), which says that the highest luminance surface is seen as white and everything else is relative to that, it can. Broadly, if we hold the highest luminance constant, and then compress the range of the other luminances in the image relative to that, the result is that the scenes with more highly compressed ranges - the most non-uniform ones - will have a higher mean. Hence, they will appear brighter.
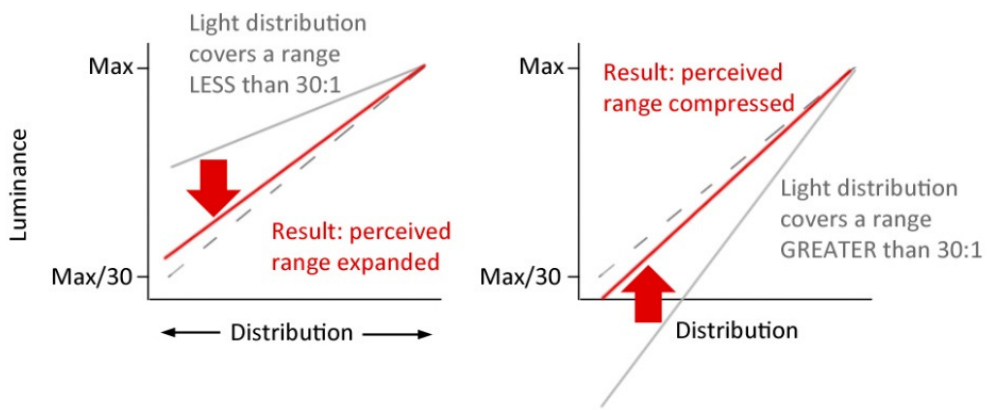

Figure 6.8 Illustration of basic idea of the scale normalisation rule. Light distributions which have a luminance range < 30:1 (more uniform) will have their perceived range expanded towards that. Light distributions which have a luminance range $>$ 30:1 (less uniform) will have their perceived range compressed. If we were to then calculate the perceived average luminance based off that perceived range, we would find that it has gotten darker for the more uniform conditions, and gotten brighter for the less uniform ones.

To apply the idea of scale normalisation, we first looked at the equation presented by Gilchrist (2006), who suggested that perceived range was related to the actual range by the equation:

$$
\text { Percieved Range }=(1+(0.56 *(\log (30)-\log (\text { Range }))) * \text { Range }
$$


This was based off observations of simple two-part stimuli, covering a range from $-1.5: 1$ to 30:1. To apply this, we could take our actual luminance range, calculate the perceived range, and then renormalize our luminances to that perceived range (keeping the maximum the same). Unfortunately, when we examined the implications of this different luminance ranges we found that this equation would blow up at very high ranges (>1000:1) (Figure 6.9). This suggested a fundamental flaw in the model, and so we attempted an alternative scale normalisation method by simply calculating the "perceived minimum" as a weighted average of the log of the true minimum and what the minimum would be if the range was to be 30:1. Following this, we could simply normalise the rest of the luminances as before.

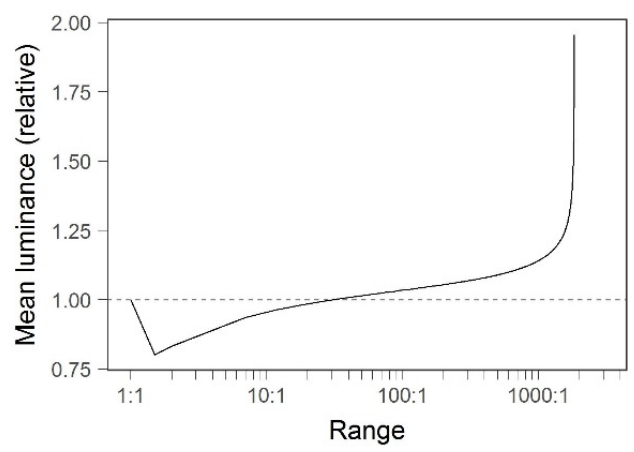

Figure 6.9 Illustration of how normalising the luminance range according to Gilchrist's (2006) equation would affect the apparent mean. As can be seen, at ranges around 2000:1 the model blows up.

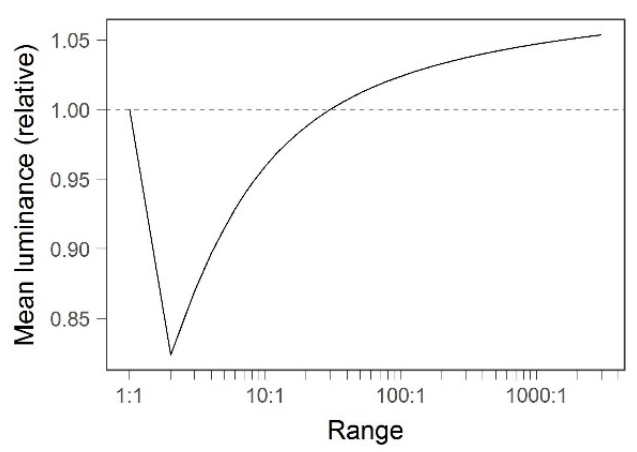

Figure 6.10 Illustration of the effects of our suggested alternative normalisation method

Hence, the process of normalising the range is to first calculate the minimum if the range were 30:1, and then calculate the weighted average of the two:

$$
\begin{gathered}
\min _{30}=\max (L) / 30 \\
\text { perceived } \min =\frac{f * \log 10(\min (L))+\log 10\left(\min _{30}\right)}{f+1}
\end{gathered}
$$

The luminance range is then renormalized to the new minimum (all on the log scale):

$$
L_{i}=(\max (L)-\text { perceived } \min ) * \frac{L_{i}-\min (L)}{\max (L)-\min (L)}+\text { perceived min }
$$

where $L_{i}$ is the luminance of a given pixel, and $f$ is a weighting factor which controls the degree to which the perceived minimum tends towards either the original minimum or the calculated $\min _{30}$.

When the "true minimum" is given a relative weight of $f=4$, the resulting "perceived ranges" over the ranges Gilchrist's equation was based off line up well with his observations. 
An interesting note here is that using scale normalisation like this predicts that luminance ranges below 30:1 would actually appear darker than a theoretical perfectly uniform condition (Figure 6.10). This is due to the fact that they see an expansion of their perceived range. The overall trend, however, is one where increasing range - and thus nonuniformity — results in a "brighter" scene. A perfectly uniform space would likely never be experienced in reality anyway, so this prediction is largely academic.

We may also note that it predicts that the largest differences occur at relatively low ranges, and that the effect slows significantly at higher levels. With a number of our conditions spanning ranges $>100: 1$, this may result in it not working well. We may also note that, at least with the weight used here, the differences in mean luminance it predicts are relatively small compared to what we have observed. That being said, Soranzo and Gilchrist (2019) did note larger normalisation effects in other studies, so the size of the effect here may be flexible.

The "area rule" - the observation that area influences lightness when the darker region is larger (Gilchrist, 2006) may also be applicable here. Such effects are typically studied using simple stimuli with only two regions, which makes translating to our more realistic scenes somewhat challenging ${ }^{69}$. As a simple proxy, however, we could look at the distribution of our luminances. In our experimental conditions, the median luminance is generally significantly lower than the mean. This means that, if we divide our scenes into "darker" and "brighter" areas around the mean luminance, the majority of the distribution falls into the "darker" category (Figure 6.11). The reported effect of area on lightness is that the larger the darker area of the stimuli, the lighter it appears, as well as increasing the apparent lightness of the brighter area (Gilchrist, 2006)(Figure 6.12). The effect this implies is clear - it would predict that increasing non-uniformity in such a way that the surface distribution is skewed towards darkness will, somewhat counterintuitively, make it appear lighter.

\footnotetext{
${ }^{69}$ And, admittedly, questionable.
} 


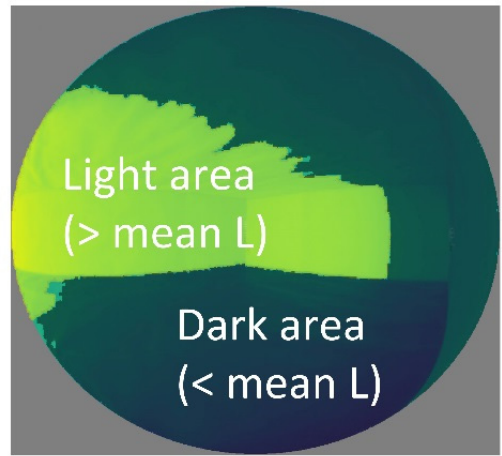

Figure 6.11 Illustration of how breaking down our luminance maps into light and dark areas around the mean luminance works. Example here is the black floor condition in Experiment 1.

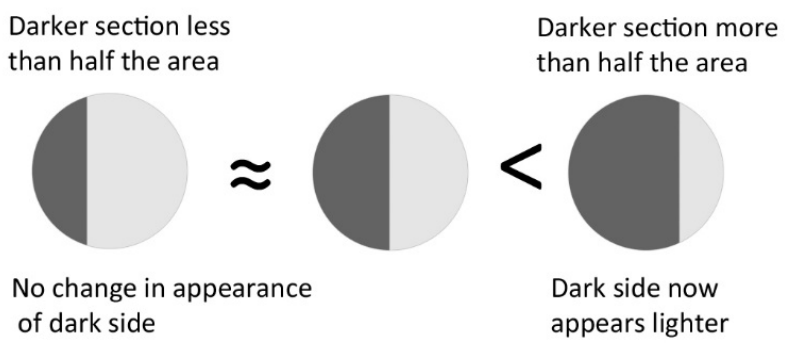

Figure 6.12 Illustration of the idea represented in the Area Rule. The dark side appears lighter when it is more than half the Area, which also pushes up the apparent lightness of the lighter area. The net result should be that the apparent average would increase.

To apply the "area rule" we attempted to adapt the equation Gilchrist (2006) presented for a simple two-part display. To do so, we calculate the area of the "light" and "dark" regions of the space according to whether or not the luminances were above or below the mean. The luminance map of the light distribution is turned into a ratio map, plotting everything in terms of the maximum luminance ${ }^{70}$. We then adjust these ratios according to the formula:

$$
\text { Adj.ratio }=\frac{\left(100-A_{d}\right)}{50} * L_{\text {ratio }}+\frac{A_{d}-50}{50}
$$

where $L_{\text {ratio }}$ is the relative luminance of a given pixel and $A_{d}$ is the percentage area of the darker region.

These adjusted ratio maps can then be multiplied back by the maximum in order to get the adjusted luminance maps, from which we can calculate the mean "apparent" luminance. The area and scale normalisation methods can also (and arguably should) be combined together to produce an overall effect. As with the percentile weighting model, we may also conceive of various different ways of applying this. In this case, one question for applying the area rule is how we should define the "brighter" and "darker" regions. We have done so using the mean, but the mean can be calculated in different ways. Given the non-linear relationship of human perception to luminance, it may be better, for example, to use the mean of the log luminance as our dividing line. We explore both options here.

${ }^{70}$ Due to the unreliability of measurements of the maximum luminance, and the fact that the single brightest pixel is unlikely to be particularly noticeable to a person, we instead calculated the mean of the luminances above the $99^{\text {th }}$ percentile 


\subsubsection{Modelling the apparent effects of the light source(s)}

How we might describe the effects of the light source(s)? Why might a large difference between light source and the rest of the room make spaces appear darker? In contrast to the purported surface effects, this apparent effect is, in fact, in line with what we would expect from the basic brightness models we discussed in our literature review.

In this light, the most basic option would be to simply apply Stevens' power law (Stevens, 1961), as suggested by Kobayashi et al. (1998)(see Section 2.4.5 for previous discussion). The idea here is that if brightness is a power function of luminance with an exponent of $-0.33-0.4$, the average brightness of a non-uniform distribution will be lower than what would be expected from the mean luminance. The larger the difference between light source and the rest of the room, the larger the effect.

To apply the equation to measure the light balance in this way, we have a couple of options. We could:

- Divide the room into surfaces and light sources, calculate their mean luminances and then their respective brightnesses using the equation, then average them for the overall effect (basic light balance approach)

- Like the above, but for the mean surface luminance we instead use the weighted mean "luminance" derived from the surface effect calculated previously (combined model). This may imply we should also change the general form of our model away from our $E_{\text {surf }}+E_{\text {light }}$ one and instead just use the overall effect from Stevens' equation (because we've accounted for the surface effects already) (Figure 6.13).

The effect, as normalised to the brightness of an equivalent uniform condition, can be converted into units of equivalent luminance change by the equation:

$$
E_{\text {light }}=\frac{1}{\beta} \cdot \log \left(\frac{B}{B_{\text {unif }}}\right)
$$

where $B$ is the mean brightness of the room according to Stevens' equation, $B_{\text {unif }}$ is the brightness of an equivalent uniform condition (calculated as a simple power function of the mean luminance), and $\beta$ is the exponent of the power function. 


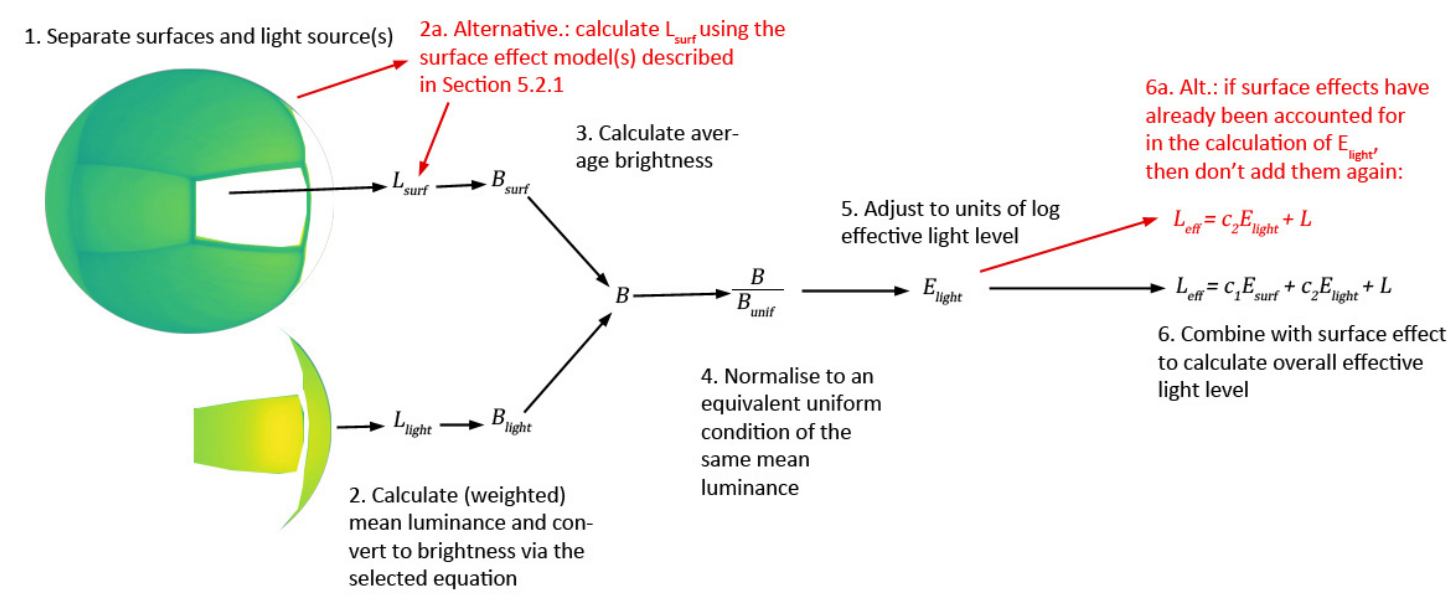

Figure 6.13 The basic structure of the how the Stevens'/Marsden equations were applied to calculate the light source effects, and the alternative approaches tried.

A possible issue with this simple approach is that it does not really get at the idea of contrast effects ${ }^{71}$ at all, which would also provide a solid theoretical reason for high contrasts between light source and surfaces to make rooms appear darker.

A possibility to approximate that may lie in the Marsden Equation (Marsden, 1970) (see Section 2.4.6). It would explain the observed effects by saying that brightness is a power function, with the brightness of the surfaces in the room being relative to the brightest element (which would be the light source). The brighter the light source is relative to the other surfaces, the darker the other surfaces will appear. The light sources will also, in general, comprise a relatively small part of the overall room, and hence when they make the rest of the room appear darker, the overall effect will be a decrease in spatial brightness. It should be noted, however, that the Marsden Equation was not, it must be said, originally produced using the light sources as the maximum luminance - Marsden calculated them as relative to the brightest surface - despite some of the light sources being visible on at least some occasions. Adapting it in this manner may thus be questioned. Regardless, it may be applied using the same methods that we have already described for applying Stevens' equation.

Another possibility to describe the apparent effects of the light balance could just be to measure the light balance as the relative difference between light source and surface luminance (Figure 6.14). The problem here is that such a measure would really be more of a correlate rather than an actual model of the effects of the light source. Even if it fit the data well, it does not really provide an explanation for why the light source is affecting spatial brightness in such a way.

\footnotetext{
${ }^{71}$ How the apparent brightness of surfaces are affected by the brightness of adjacent surfaces.
} 


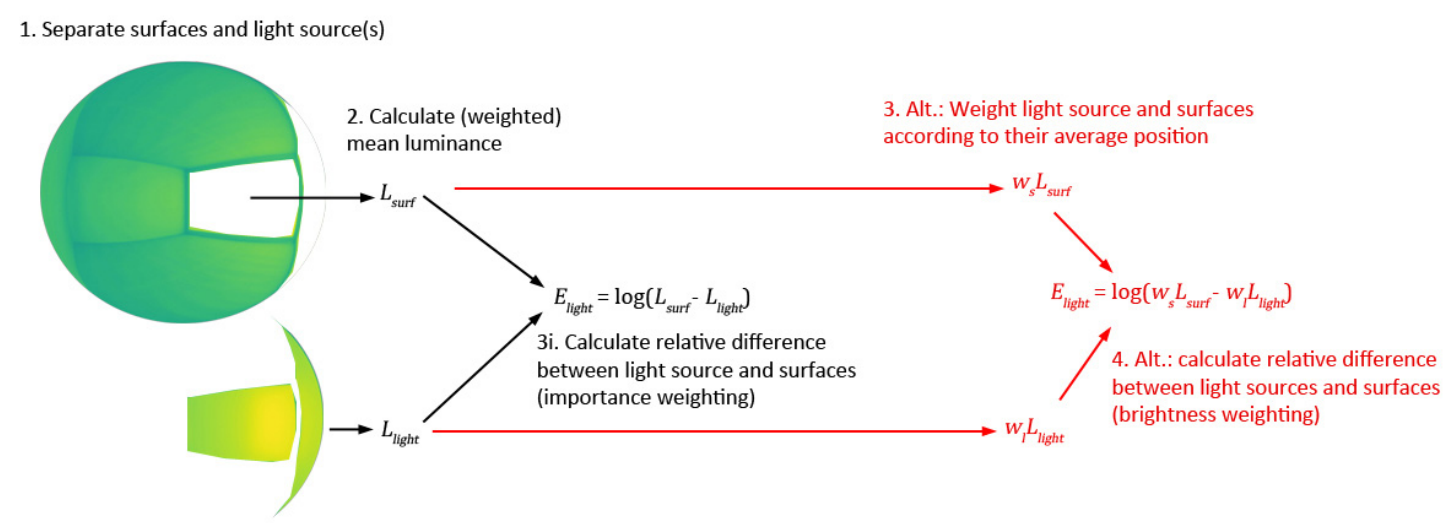

Figure 6.14 Outline of the basic light balance model and its variants.

A potential variation on this idea, however, may be better founded. We could calculate the effect as the difference between the brightness of the surfaces, and the brightness of the light source:

$$
E_{\text {light }}=\log \left(L_{\text {surf }}^{\beta}-a L_{\text {light }}^{\beta}\right)
$$

Where $L_{\text {sur }}$ and $L_{\text {light }}$ are the mean luminances of the surfaces and light source respectively, $\beta$ is the exponent of the power function describing brightness, and $a$ is a variable controlling the relative strength of the brightness reduction imposed by the light source $^{72}$.

This would be similar to the Haubner Equation (Bodmann et al., 1979; Bodmann and Toison, 1994), which states that the brightness of a target is reduced by the brightness of its surround, both being measured as a power function of their luminance. We could try to apply the same idea here, but with the light source reducing the brightness of the surfaces. It would then be logical to, similar to our application of the Marsden Equation, use this model to estimate the brightness of the surfaces, and then average it with the brightness of the light source to calculate the overall brightness. This produces three different versions of this model: the basic light balance model, the brightness difference, and the averaged brightness difference model. This is then multiplied further by the different ways that we can apply position weighting (Figure 6.15).

\footnotetext{
${ }^{72}$ Given that the luminance of the light source will be significantly higher than that of the surfaces, this must be significantly less than one if we are to avoid saying that the brightness is a negative number, which makes no sense (absolute brightness cannot be less than 0 by definition).
} 


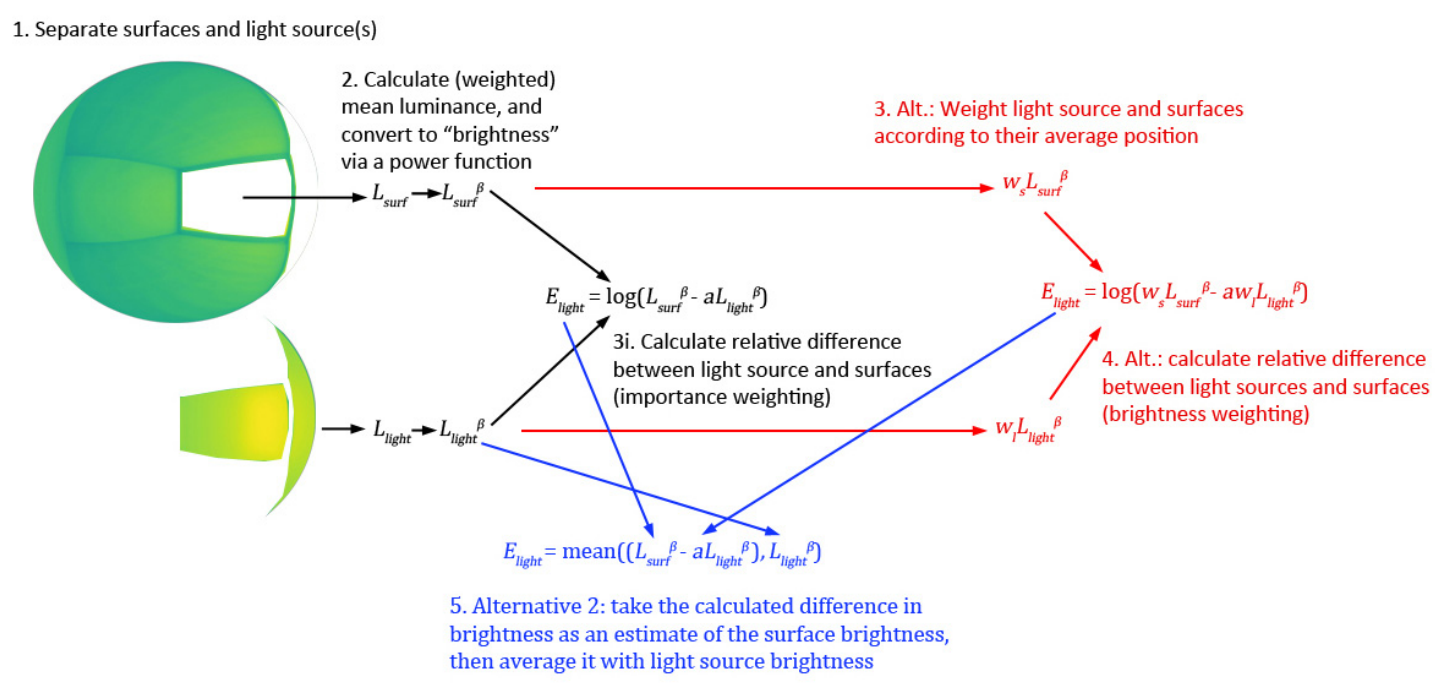

Figure 6.15 Outline of the "brightness difference" model, and the various ways it could be applied.

Similar to the percentile weighting model of surface effects (p174), there are multiple ways in which we could apply the effects of position weighting. Consider the situation in which we are looking at the difference in brightness between the surfaces and the light source. In calculating the average luminance of each element, we apply any position weighting functions we are using. However, we may also consider that the positions of the surfaces and the light source may result in each of them having different overall position weights. For example, if the light source was on the ceiling it may have a different weight relative to the other surfaces than if it were on the walls. We could potentially account for this by multiplying the luminances by their relative weights, for example (modifying eq. $6.4)$ :

$$
E_{\text {light }}=\log \left(w_{s} L_{\text {surf }}^{\beta}-a w_{l} L_{\text {light }}^{\beta}\right)
$$

where $w_{s}$ and $w_{l}$ are the average position weights of the surfaces and light source respectively.

The question is: should we?

The difference, we argue, can again be framed as a question of whether or not position affects brightness, or whether or not it affects importance. The basic form of the equation is that it is calculating the difference between the brightness of the light source, and the brightness of the surfaces. If position affects brightness, then we should account for this effect in our calculation. If it affects importance though then it does not really make sense. For example, say the light source has a relatively high average position weight. If we apply it then it will appear "brighter" and the calculated difference between it and the surfaces will be increased. If position is just affecting relative "importance" though then there is no 
reason for such a change. The light source having, for instance, greater importance to the overall evaluation of the space would justify it being weighted in the calculation of something like the overall average. It would not, however, seem to have any bearing on the apparent difference between it and the surfaces. In this framing then, it would be better to use the original equation, and not add in the weights again.

Finally, we suggest a simple model prompted by Cuttle's $(2013,2010)$ argument that the surfaces are the most important element in assessment of how well lit a space is: that maybe people just discount the light sources when they assess spatial brightness. While light sources generally cover only a small part of the total surface area of a room, they are also much higher in luminance, and the direct light from them has a large effect on measurements of the overall light level. Cuttle argues, however, that it is the surfaces that actually define the room being illuminated - if the surfaces absorbed all the light, we would be unable to see anything, and the room would appear completely dark, no matter how much light the light sources emitted. As an absolute stance, this thought experiment may be challenged: would not people be able to get some sense of a luminous field from being able to see and interact with the light emitted from the light source? However, it is a reasonable argument for why we might consider surface illumination to be more important to the overall impression of the ambient illumination than the direct light. Following this, we could simply calculate the effect as the weighted average luminance of the light source and the surfaces, with the light source being weighted less (and then normalising to an equivalent uniform distribution as always):

$$
E_{\text {light }}=\log \left(\frac{L_{\text {surf }}+w L_{\text {light }}}{1+w}\right)-\log (L)
$$

where $w$ is the weight of the light source $(<1)$, and $L$ is the overall mean luminance.

The resulting weighted mean should always be lower than the "actual" mean, because the light source is the brightest element in the room. The brighter the light source is relative to the surfaces, the darker our weighted mean would be.

As with the previous suggestions, we could also implement this differently: we could instead use our effective surface luminance calculated from our previous methods as the surface luminance in this calculation rather than just adding $\mathrm{E}_{\text {surf }}$ and $\mathrm{E}_{\text {light }}$ together.

\subsubsection{Potential position weighting models}

If we were to weight the light distribution according to position, how would we do it? The general idea presented in the literature is that the luminances in the middle of the field of view, around the walls, are more important. As we have noted (Section 2.3.2), the actual evidence for this in terms of effects on spatial brightness is weak, and we lack any specific 
measurements that we could use. While it is popular, we do not consider the $40^{\circ}$ horizontal band to be defensible as there is no credible reason to imagine that human perception suddenly cuts off $20^{\circ}$ above the horizontal plane. One possibility, however, could be to look at the related field of glare, where they do have models for how glare strength changes according to the angular position of the light.

To explore this, we adapted the position index model presented by Kim and Kim (2011). The formula in that paper is put in terms of "amount of light required to produce as much glare as a reference light at centre of vision”, so to convert it into a weighting function we normalised and inverted it:

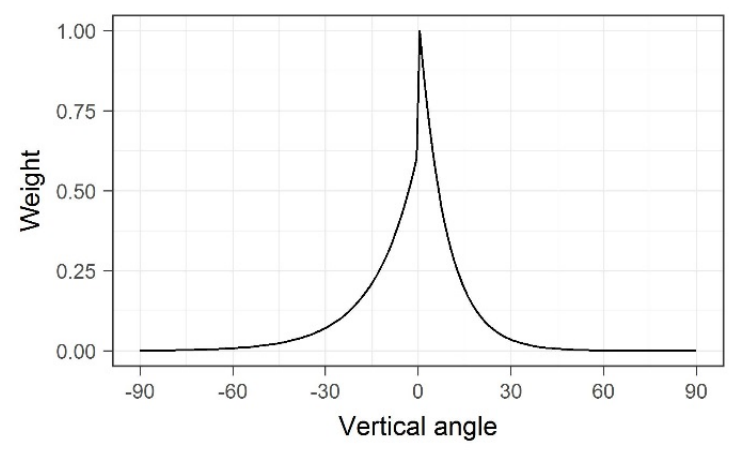

Figure 6.16 Weighting function derived from the glare position index formula in Kim and Kim (2011) (glare_vert)

The formula is two dimensional - glare intensity changes with both horizontal and vertical angle. However, if we consider that in a typical room people would be making an assessment of the whole room by looking around it, we might consider that horizontal variation in any individual view will essentially average out, and so we may instead focus on the vertical weighting only.

Of course, the same point also applies to the vertical angles. People may look up and down to some degree in order to get an impression of the room. The question is how much they do so, and whether or not they tend to focus on any particular area. Kato and Sekiguchi (2005) argued that people primarily look in the horizontal direction, and while, as we noted in our literature review, we are not entirely convinced by their evidence, we also do not disagree with the assertion. Based on our experience with everyday life, we would expect people to largely look horizontally around a room when assessing it rather than at the ceiling or the floor. Rockcastle et al. (2017), in a study of gaze direction, observed that people spent about $75 \%$ of the time looking within the central $-45^{\circ}$ horizontal band, and $-95 \%$ of the time within $\sim 90^{\circ}$. This distribution is roughly equivalent to a normal distribution with an $\mathrm{SD}$ of $20^{\circ}$, and so we used this as a guide to roughly smooth the simple glare weighting function over the vertical angles. This forms our main "theory-driven" position weighting function (gaze_smooth_v1). 


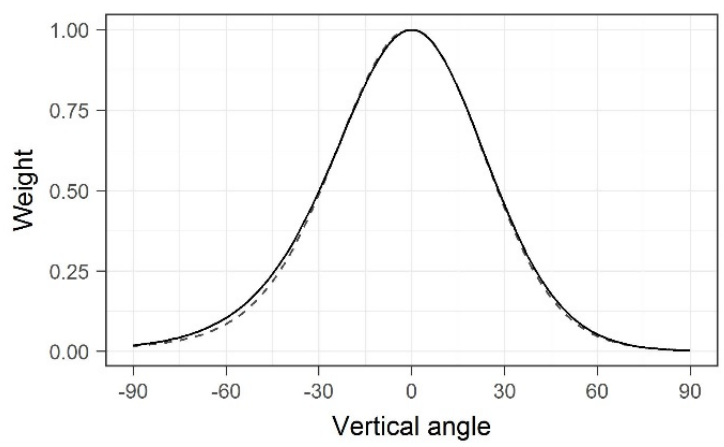

Figure 6.17 Weighting function based off smoothing the glare position index. The dashed line is the smoothed function. The solid line is an approximation using two T-distributions used to actually apply the function to our luminance maps.

We may, however, question the idea that we do not need to worry about horizontal weighting. Observationally, the viewpoint occupied by the participants in our experiments induces a tendency towards looking straight ahead at the corner of the room opposite the opening. While people can and do look around from side to side, they may still primarily look towards the centre of the "view". To explore this idea, we thus created an alternative version of our smoothed vertical function that also incorporated horizontal weighting (glare_smooth_vh).

There are also some other options we can explore if we move away from basing our weighting on glare position formulae. An alternative could simply be that the importance of different parts of the space depends on how much people look at that area. Following this, we could just use a normal distribution as before as a rough estimate of relative gaze frequency according to vertical angle (gaze_norm). Finally, taking the lead from the common suggestion in the literature that "the walls are more important than the floor/ceiling", we apply the idea literally and simply weight the floor and ceiling as less than the walls (example of 0.3 weighting shown below). A question this brings up, however, is whether or not such an approach can be justified. One way of framing it could be to argue that the volume of the space that we are assessing — the "light volume" — is inherently given shape by the key bounding surfaces - the walls, floor, and ceiling. In that sense, they have a role as distinct elements in defining the light distribution that we are responding to. We may also note that perceptual grouping factors and depth planes are acknowledged to be important to brightness and lightness perception (Gilchrist, 2006; Zdravković et al., 2012). The idea then that observers may be grouping the luminances of the walls, floor, and ceiling as distinct elements thus has some plausibility.

Combined with the option of "no position weighting", these six models defined the position weighting options explored in our model tests (Figure 6.18). 


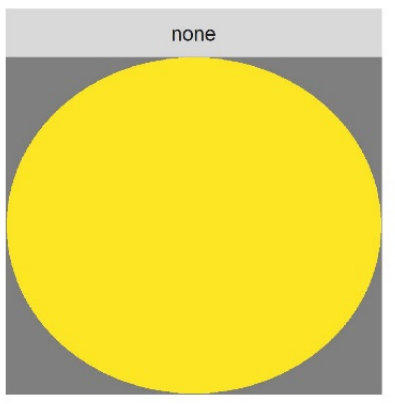

glare_smooth_v1

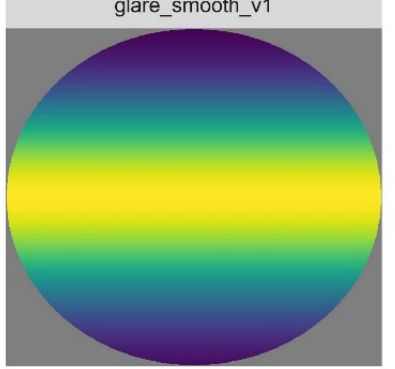

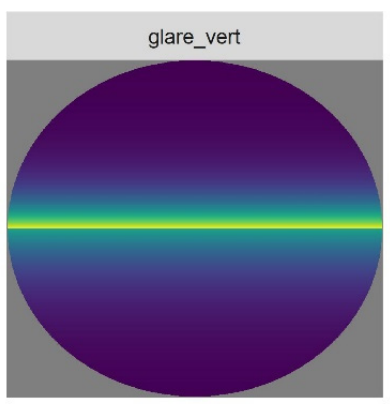

gaze_norm

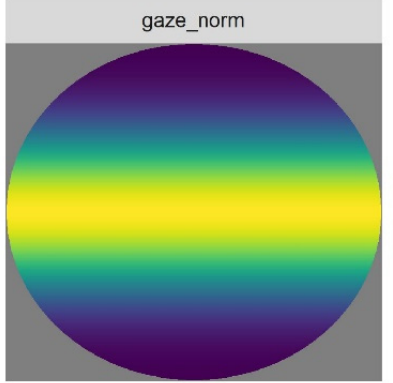

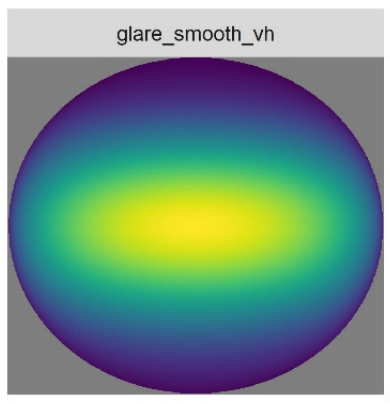
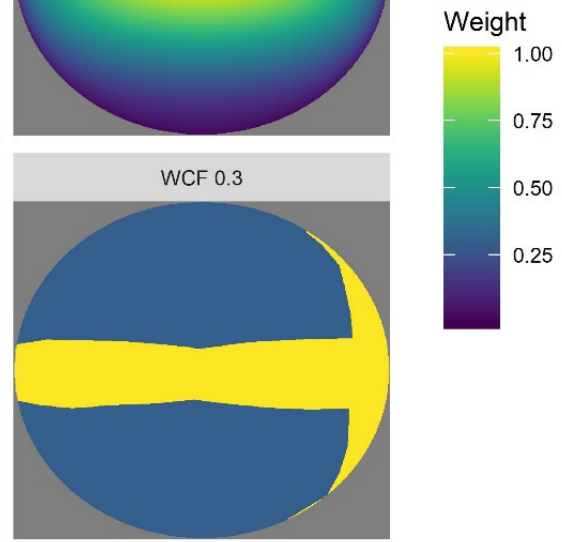

Figure 6.18 Position weighting maps explored in our model tests. These describe the weights of every pixel in the measured luminance maps.

\subsection{Testing the model ideas}

The challenge with testing these different models is the sheer number of potential combinations. With 21 surface effect models x 18 light source models x 6 position weighting functions we have over two thousand possible combinations. This is further expanded by variable parameter values. Applying any of these models requires processing the luminance maps - a non-trivial computational task given the hundreds of thousands of data points that comprise each of them. Because of this, we cannot, for example, merely run a simple linear regression to find the best fitting parameter value for the wall/floor/ceiling weights, or the percentile weighting function. Instead, we have to iterate through the possible parameter values, reprocessing all the luminance maps each time.

To make this procedure more tractable, the model testing was divided into two stages. Firstly, the results of the first experiment were used to explore the models of surface effects. In Experiment 1, the light source was hidden. Theoretically, this means that it should be free of light source related effects, and so we can use it to focus on the question of which surface effect models and position weighting functions may be viable.

In the second stage, the successful surface effect/position weighting model combinations were taken forward to Experiment 2 to explore the addition of models of the light source effects. This approach allows us to limit the number of possible model combinations that need to be tested. 


\subsubsection{Surface effects (Experiment 1)}

To test the different model variants proposed here the previously described models were used to calculate the "effect of the surface distribution", and then simple linear regression applied to find the best fitting values for the coefficient, $c_{1}$ in the equation:

$$
L_{e f f}=c_{1} E_{\text {surf }}+L
$$

where $L_{\text {eff }}$ is the effective luminance observed in our experimental results, and $E_{\text {sur }}$ is the effect of the surfaces according to our model, and $L$ is the mean luminance (all on the log scale).

This model itself can further be approached in multiple ways with regards to the use of location weighting. The question here is whether or not the mean luminance, $L$, should be calculated as the weighted mean luminance, or just left as the baseline mean. On one hand, if we are weighting luminances according to location then it would seem logical to include that in our calculation of the mean luminance. On the other hand, the role of the mean luminance in this equation is that it provides the baseline light level that may then be modified by the light distribution effects - which are already accounting for any location weighting in their calculations. We can thus argue the matter either way, and so chose to test both formulations of the model.

With regards to the percentile weighting functions, the inherent problem was that they could be implemented with any of a wide range of possible parameters, as shown in the examples of Figure 6.9 and Figure 6.10. To account for this, we ran the analysis repeatedly using different parameter values in order to see which ones (if any) could well predict our observed results. The exponent of the power weighting function was varied from 0.5 to 6.5 , in increments of 0.25 . The sd of the normal distribution weighting function was varied from 0.02 to 0.5 , in increments of 0.02 . Similarly, the wall/floor/ceiling weighting function (see Figure 6.18) could have different weights applied to the floor and ceiling. Assuming the walls had a relative weight of " 1 ", the floor/ceiling weights were varied from 0.1 to 0.9 in intervals of 0.1 (the general point being that they should be less important than the walls). In total we explored 546 model combinations and 23,076 variations.

Table 6.3 Summary of different models of surface distribution effects that were tested

\begin{tabular}{|c|c|c|}
\hline \multicolumn{2}{|l|}{ Model } & Description \\
\hline \multirow[t]{2}{*}{$\begin{array}{l}\text { Percentile } \\
\text { weighting } \\
\text { (position affects } \\
\text { importance) }\end{array}$} & $x^{\wedge} q($ raw $)$ & $\begin{array}{l}\text { Calculate the weighted mean luminance using a } \\
\text { weighting function that places more weight on } \\
\text { the brightest areas. In this case, the weighting } \\
\text { function is a power function as shown earlier in } \\
\text { Figure } 6.5 \text {. }\end{array}$ \\
\hline & $\operatorname{Normal}(1, q)$ (raw) & $\begin{array}{l}\text { As above, but using a weighting function based } \\
\text { on the standard deviation as shown in Figure } \\
6.6 \text {. }\end{array}$ \\
\hline
\end{tabular}




\begin{tabular}{|c|c|c|}
\hline & $\begin{array}{l}x^{\wedge} q(p w r), \\
\operatorname{Normal}(1, q)(p w r)\end{array}$ & $\begin{array}{l}\text { As the above two options, but the luminances } \\
\text { are first transformed into "brightness" via a } \\
\text { power function of } \mathrm{L}^{0.35} \text {. }\end{array}$ \\
\hline $\begin{array}{l}\text { Percentile } \\
\text { weighting } \\
\text { (position affects } \\
\text { brightness) }\end{array}$ & $\begin{array}{l}x^{\wedge} q(\operatorname{raw}), \\
\operatorname{Normal}(1, q)(\text { raw }) \\
x^{\wedge} q(p w r) \\
\operatorname{Normal}(1, q)(p w r)\end{array}$ & $\begin{array}{l}\text { As above, but position weighting is applied } \\
\text { before the percentile weights are calculated, as } \\
\text { in Figure } 6.7 \text {. }\end{array}$ \\
\hline $\begin{array}{l}\text { Percentile } \\
\text { weighting: } \\
\text { wall/floor/ceiling } \\
\text { grouped } \\
\text { (brightness, } \\
\text { importance) }\end{array}$ & $\begin{array}{l}x^{\wedge} q(\text { raw) } \\
\text { Normal }(1, q)(\text { raw }) \\
x^{\wedge} q(p w r) \\
\operatorname{Normal}(1, q)(p w r)\end{array}$ & $\begin{array}{l}\text { As the above eight options, but with the } \\
\text { percentile weighting applied differently. Instead } \\
\text { of applying the percentile weighting over the } \\
\text { whole scene, the walls, floor, and ceiling were } \\
\text { instead separated and had their surface effects } \\
\text { calculated individually. These were then } \\
\text { averaged to produce the overall effect. }\end{array}$ \\
\hline \multirow[t]{4}{*}{$\begin{array}{l}\text { Anchoring Rule } \\
\text { models }\end{array}$} & Area Rule & $\begin{array}{l}\text { Basic-divide the light distribution into "light" } \\
\text { and "dark" areas based on whether or not the } \\
\text { luminance is above the mean, and calculate the } \\
\text { shift in apparent luminance based on the } \\
\text { relative size of the dark area according to Eq. } \\
6.2 \text {. }\end{array}$ \\
\hline & Area Rule (log m) & $\begin{array}{l}\text { Alternative - divide the light distribution into } \\
\text { "light" and "dark" areas using the mean of the } \\
\text { log luminance as the divider, then calculate the } \\
\text { effect as before. }\end{array}$ \\
\hline & Scale Normalisation & $\begin{array}{l}\text { The luminance range is renormalized to its } \\
\text { "perceived" range closer to } 30: 1 \text {, keeping the } \\
\text { maximum the same. The new mean luminance } \\
\text { is calculated, and the difference between that } \\
\text { and the "true" mean is the effect. }\end{array}$ \\
\hline & Combined & $\begin{array}{l}\text { Combined both the Area Rule and Scale } \\
\text { Normalisation effects into one. }\end{array}$ \\
\hline
\end{tabular}

Assessment of model performance was done via inspection of plots comparing predicted and observed effects, with mean squared squared error being used as a crude index to rank model performance and filter out the models that were worth looking at (Figure 6.19).
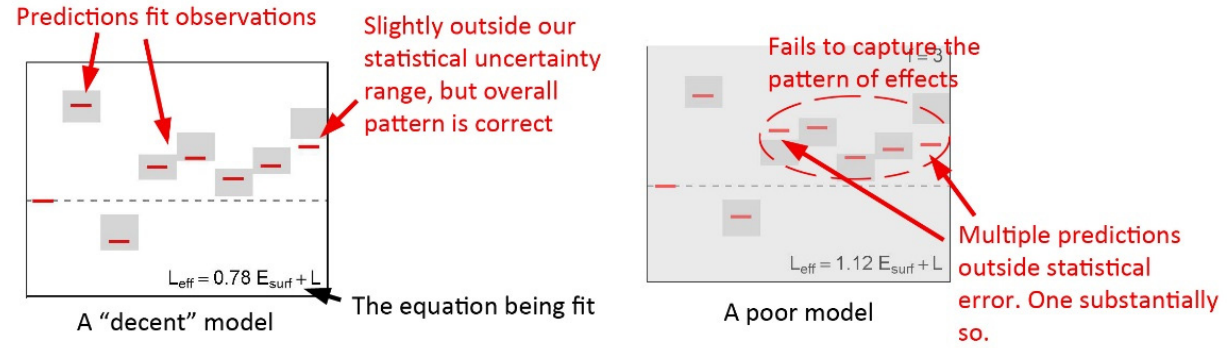

Figure 6.19 Example of how the model predictions were assessed. Ideally the predictions (red bars) would fall within the uncertainty range (95\% interval) of our experimental observations (grey blocks). Conditions are presented in standard order, as shown in Figure 6.20. 


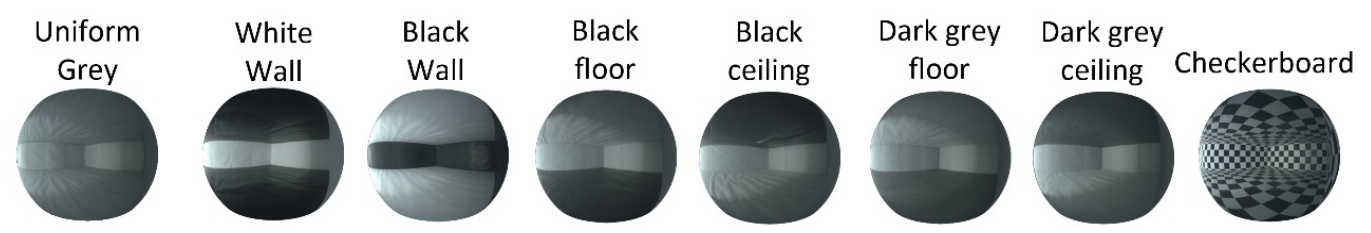

Figure 6.20 Experiment 1 conditions. Results (such as those above) are plotted in the same order from left to right, relative to the Uniform Grey baseline condition.

Firstly, let us examine the performance of the five Anchoring Rule based models (Figure 6.21). The plots shown are the best fitting versions of each surface effect model and position weight combination. Each plot is a surface effect model / position weighting function combination. Red bars show the model predictions, grey blocks show the 95\% intervals from the experiment. The models were filtered by mean squared squared error to identify which ones had potentially reasonable fits — these are the highlighted plots.

The basic Area Rule model in the top row seems to capture the overall pattern of results reasonably well — but seems to have a problem with underestimating the Checkerboard condition. Interestingly, it appears to be largely unaffected by the position weighting function. Technically, the basic unsmoothed vertical glare weighting function performs the worst, falling just outside the limit set on our mean squared error filter (which is why it is greyed out). However, the different weighting functions do not appear to differ significantly in a practical sense.

Looking at the next row and the alternative version of the model, if we instead define the darker area using the mean of the $\log$ luminance, it performs very poorly, suggesting this is a bad idea. The scale normalisation model also does not perform very well. It significantly underestimates the checkerboard condition and predicts that it should perhaps even be darker than the black floor/ceiling conditions - which is not in line with what we observed. Combining the successful area rule model and scale normalisation models into one though does appear to be somewhat better than either individually. 


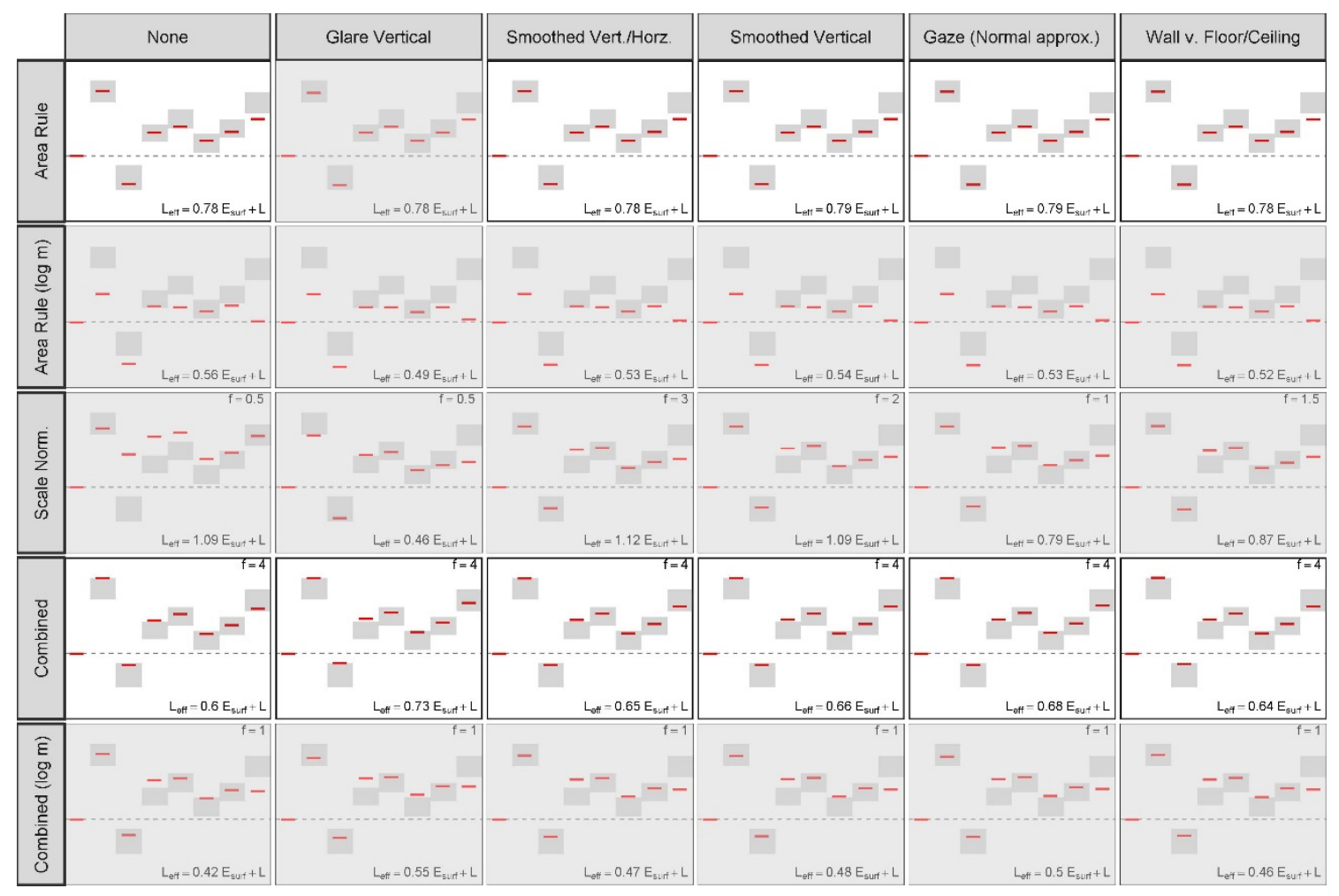

Figure 6.21 Best fits of attempts to apply the anchoring rule based surface effect models to the results of Experiment 1. ' $f$ ', in the upper right corner of the lower three rows is the weight applied to $\min (\mathrm{L})$ when calculating the perceived minimum in the scale normalisation calculation. The results for the Wall v. Floor/Ceiling weighting function shown here are with the floor and ceiling weighted at $40 \%$, however these particular models seem to be largely unaffected by this choice.

The next set is the percentile weighting model (Figure 6.22). Here we see much more substantial differences between different position weighting functions. The strongest are the basic vertical glare weighting function and the wall/floor/ceiling weighting function. The smoothed functions, or no weighting at all, works significantly worse - though we can see a couple of instances with the base gaze direction model where it seems to work.

The weighting function used in those instances, however, is somewhat questionable. If we look at the "successful" gaze direction models, we see that the percentile weighting function that fit best was a normal distribution with an sd of 0.02 . This would place nearly all the weight on the top few percentiles in the image, which raises the question of robustness issues similar to our earlier criticisms of the max/min ratio. Indeed, the $\mathrm{max} /$ mean ratio did in fact correlate quite well with Experiment 1's data (though not, it should be noted, Experiment 2's), so it is possible that the apparent success here is just an artefact of the specific set of conditions. This may also be why the vertical glare model seems to be working reasonably well - it focuses most of its weight on the central horizontal line, which is also where most of the highest luminances are in this instance. 


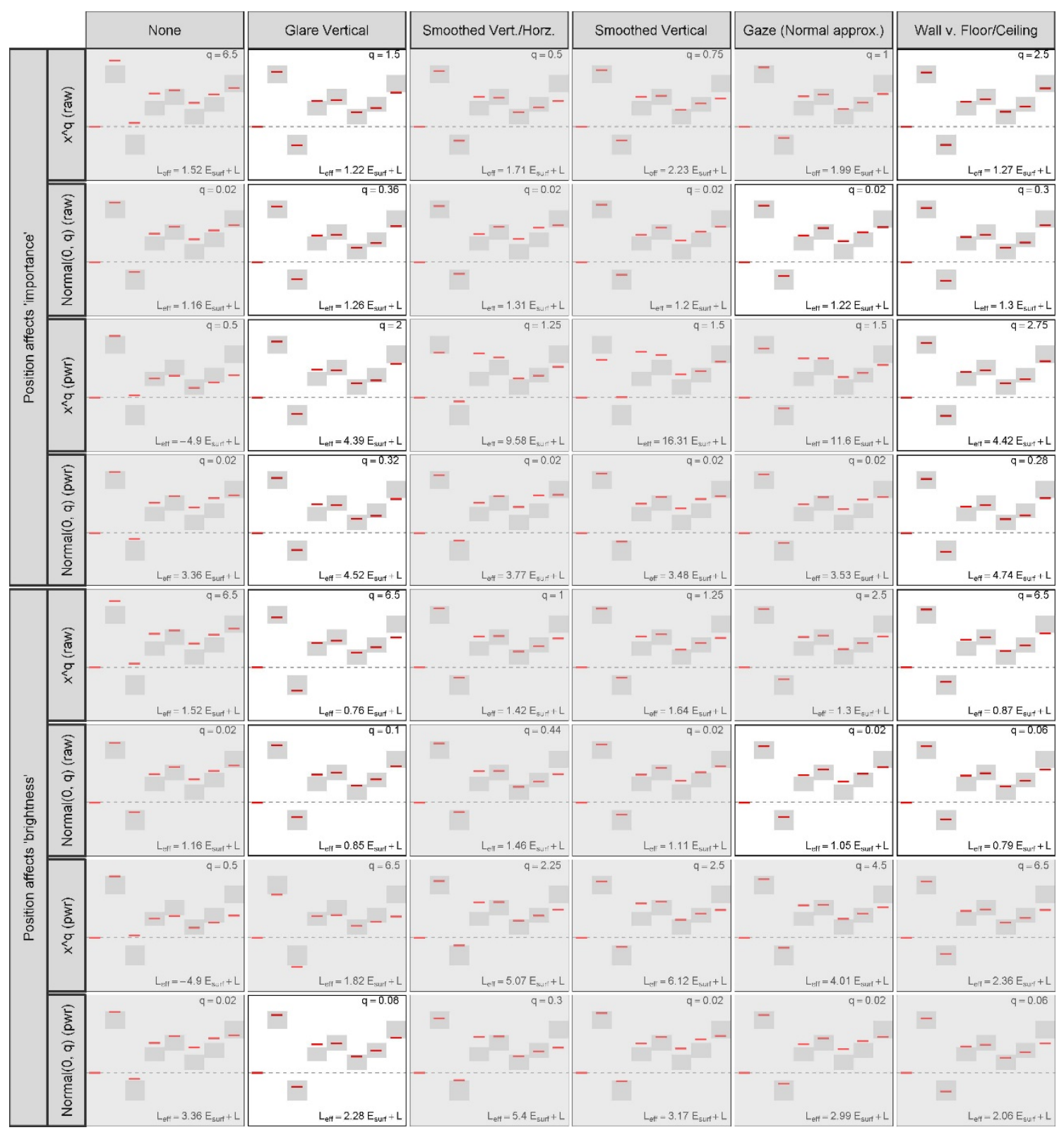

Figure 6.22 Best fits of the basic attempts to apply the percentile weighting based surface effect models to the results of Experiment 1. ' $q$ ', in the upper right corner of the lower three rows is the factor used in the quantile weighting function, either the exponent or SD. The results for the Wall v. Floor/Ceiling weighting function shown here are with the floor and ceiling weighted at $20 \%$, though 10 or $30 \%$ can also work.

Inspired by the apparent strength of simply weighting the floor/ceiling less than the walls, we also explored another way of applying the percentile weighting model — the "wall/floor/ceiling grouped" approach briefly described in Table 6.3. Instead of calculating the weights over all the surfaces at once, and saying that people are most influenced by the brightest areas, we instead calculate this separately for the walls, ceiling, and floor (Figure 6.23). The resulting mean luminances are then combined into the overall average, 
accounting for the relative weights and areas of the different surfaces ${ }^{73}$. The idea here would be that, as with our justification for placing distinct weights on the walls, floor, and ceiling, those different surfaces may operate as distinct elements that define the form of the light volume. Thus, when a person attempts to infer the light volume, they consider how those boundary elements come together to define the light filling the space. Applied to the percentile weighting model, the idea would then be that people look at the combined effect of the walls, floor, and ceiling, with a tendency towards basing their inferences on the brightest parts of each of those separate elements.

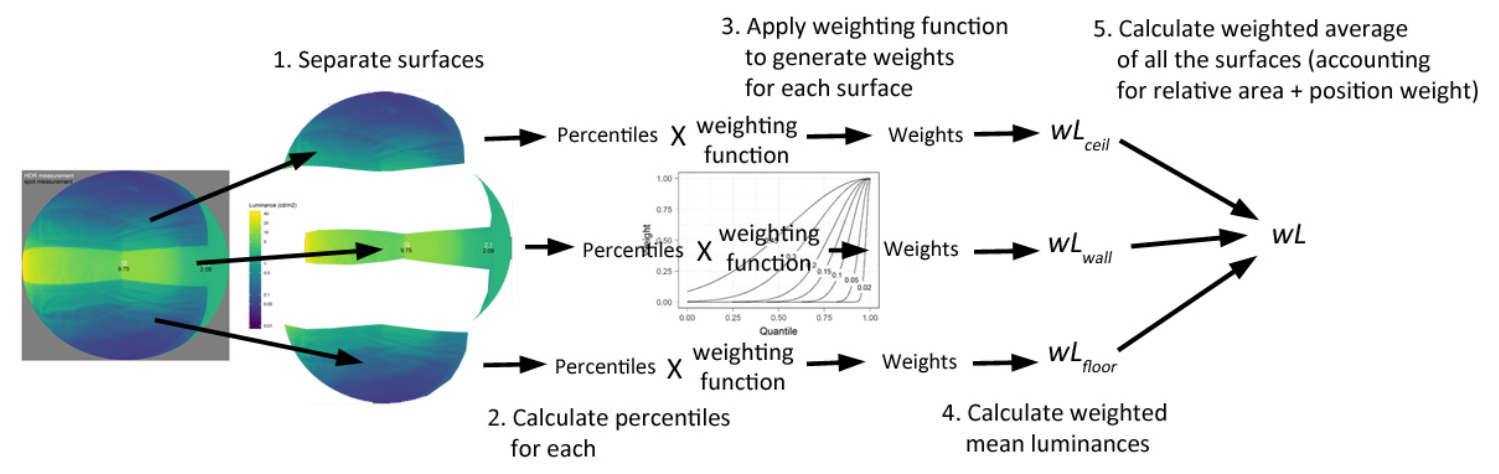

Figure 6.23 The general process used in the "wall/floor/ceiling grouped" application of the percentile weighting model. The process described earlier in Figure 6.7 is instead carried out separately for each surface, before combining them all into one overall effect.

This alternative implementation of the percentile weighting model shows a very different pattern of results (Figure 6.24). Here, the smoothed glare functions are much more successful, while the wall/floor/ceiling weighting function is less so. The application of the model in which position is held to affect brightness seems to have more successes than when it is held to affect importance, but there are plenty of successes in both. Interestingly, this form of the model seems to have problems with overestimating the effect of the Checkerboard condition, in contrast to the previous attempts where it was underestimated.

\footnotetext{
${ }^{73}$ Differences in overall position between surfaces were accounted for by weighting them according to their average position weight.
} 


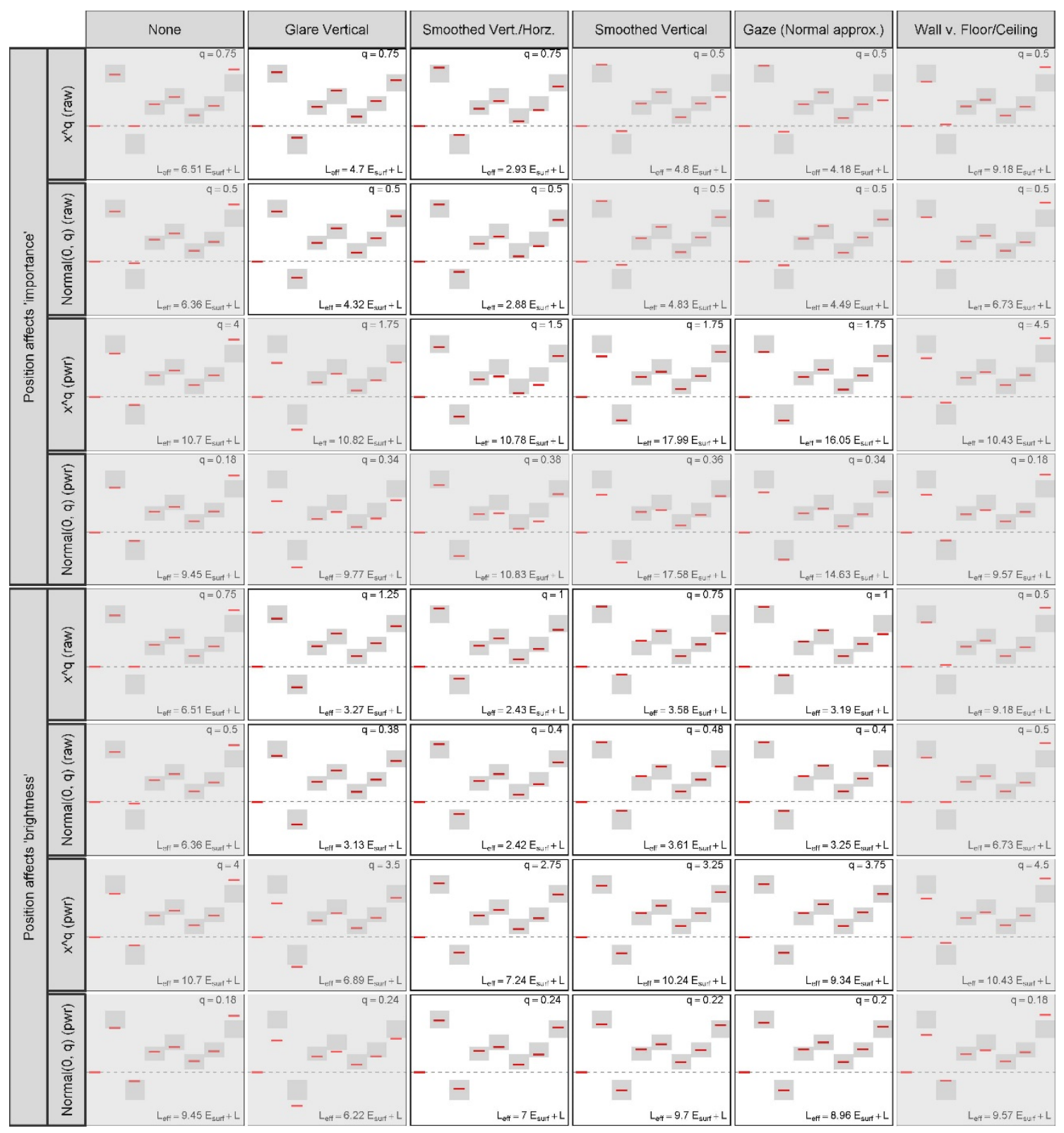

Figure 6.24 Best fits of the "wall/floor/ceiling grouped" version of the percentile weighting based surface effect models to the results of Experiment 1. The results for the Wall v. Floor/Ceiling weighting function shown here are with the floor and ceiling weighted at $70 \%$, though 50 to $80 \%$ can also work.

We now move to the alternate implementation of all of these models where instead of fitting the equation $L_{\text {eff }}=c_{1} E_{\text {surf }}+L$ we instead use the weighted mean luminance $L_{\text {eff }}=c_{1} E_{\text {surf }}+w L$ (where $w L$ is the weighted mean luminance calculated from the position weighting function). This changes our results substantially. For the anchoring rule based models (Figure 6.26) or the basic percentile weighting models (Figure 6.27), it works much worse. The anchoring rule models applied this way have very poor fits, and there are perhaps only a few weak fits for the percentile weighting function models — and these are 
still fairly bad due to their failure to really capture the way the checkerboard condition is brighter than the dark floor/ceiling conditions.

For example, let us look closely at the most successful model highlighted in Figure 6.27 (shown below). It underestimates the checkerboard condition (relative to the uniform grey) fractionally, by $-0.001 \log$ units, while it overestimates the dark grey floor condition to a somewhat greater degree (prediction of 0.0885 compared to the estimate of our statistical analysis of 0.010 .0420 .074$)$. Such errors may be considered acceptable given the known sources of error, and the fact that we are making multiple comparisons here so one or two of the values being outside our intervals may not be unexpected. However, we need also consider the comparisons between conditions, and not just their effect relative to the baseline. In this case, the dark grey floor/ceiling conditions are up at the limit of their intervals, while the checkerboard is at the lower limit of its. Thus, the difference between these conditions is being significantly underestimated - the prediction is that the checkerboard condition appears to have $\sim 0.017$ log units more light than the dark grey ceiling condition, and $0.052 \mathrm{log}$ units more than the dark grey floor condition. If we extract the same comparison from our statistical analysis, we get estimates of $f_{0.048} 0.087_{0.128}$ and ${ }_{0.096} 0.1370 .179$ respectively. The predictions are more than $0.04 \log$ units less than the lower bound of our uncertainty interval - a substantial difference. This is because the estimates as described by our probability distributions are not independent of each other. While, for simplicity's sake, the results are only displayed relative to the baseline, it is important that we are also cognisant of the broader network of comparisons that make up the observed pattern of results. All the comparisons between conditions are important.

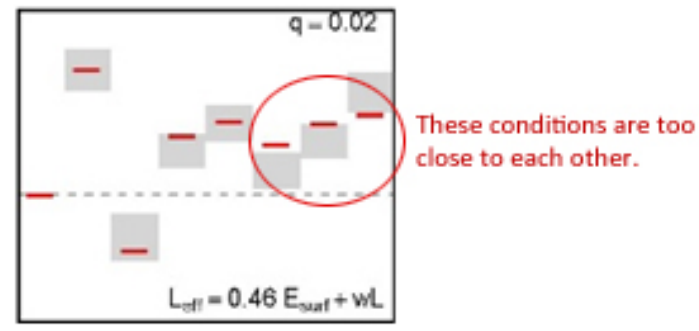

Figure 6.25 The best performing model in Figure 6.27 and its problem.

With the wall/floor/ceiling grouped percentile weighting models, however, we see substantially more success (Figure 6.28). Most of the models see some success, with many being very strong fits (though it must be said that a too good fit is also questionable, as statistical error should result in a certain degree of variation). The basic glare weighting model is the worst performing there, while the smoothed ones perform very well. 


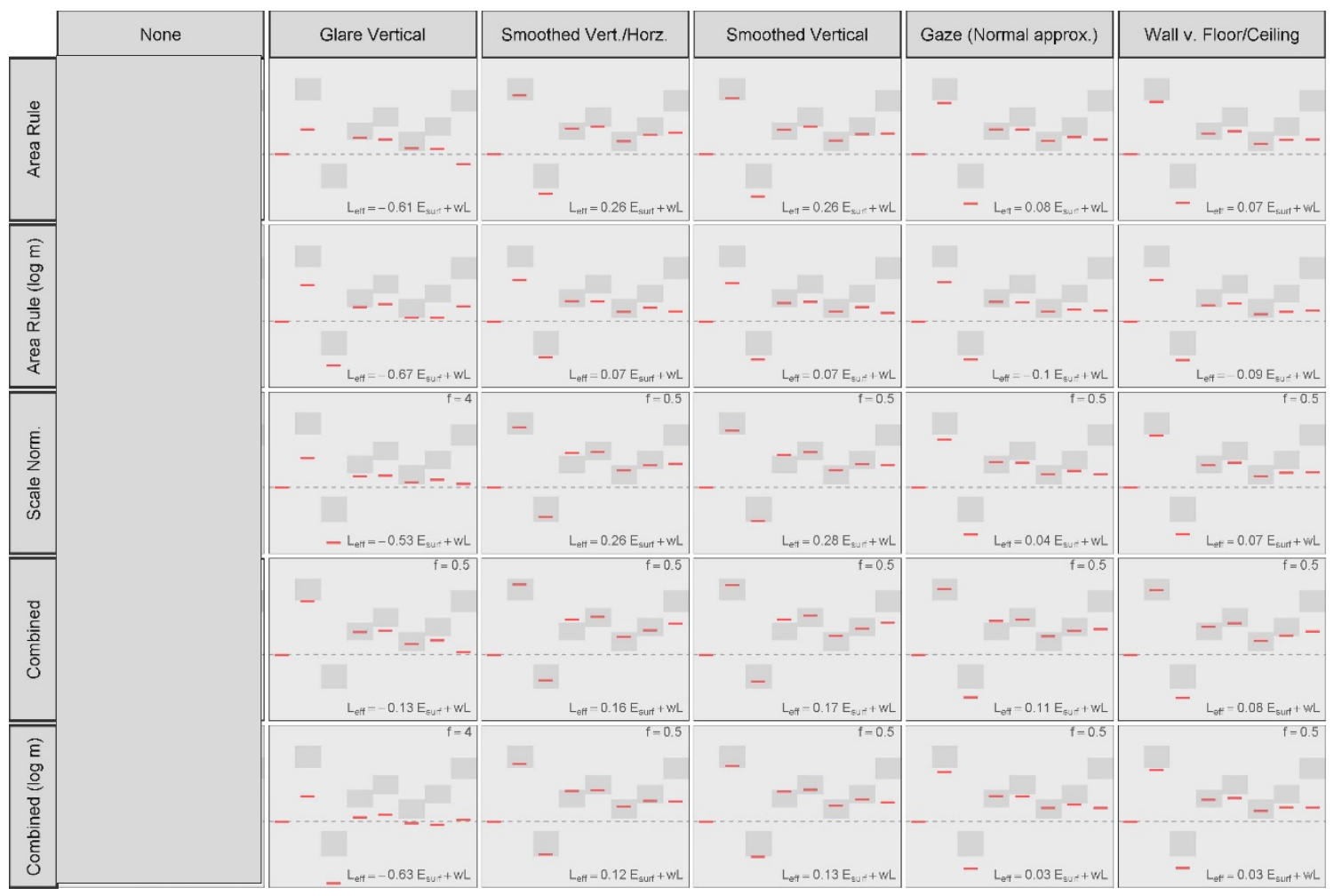

Figure 6.26 Best fits of attempts to apply the anchoring rule based surface effect models to the results of Experiment 1 using the equation with weighted mean luminance, $w L$. The "None" column is blanked out because if there is no position weighting the weighted mean luminance is just the same as the mean luminance. ' $f$ ', in the upper right corner of the lower three rows is the weight applied to $\min (L)$ when calculating the perceived minimum in the scale normalisation calculation. The results for the Wall v. Floor/Ceiling weighting function shown here are with the floor and ceiling weighted at $40 \%$, however these particular models seem to be largely unaffected by this choice. 


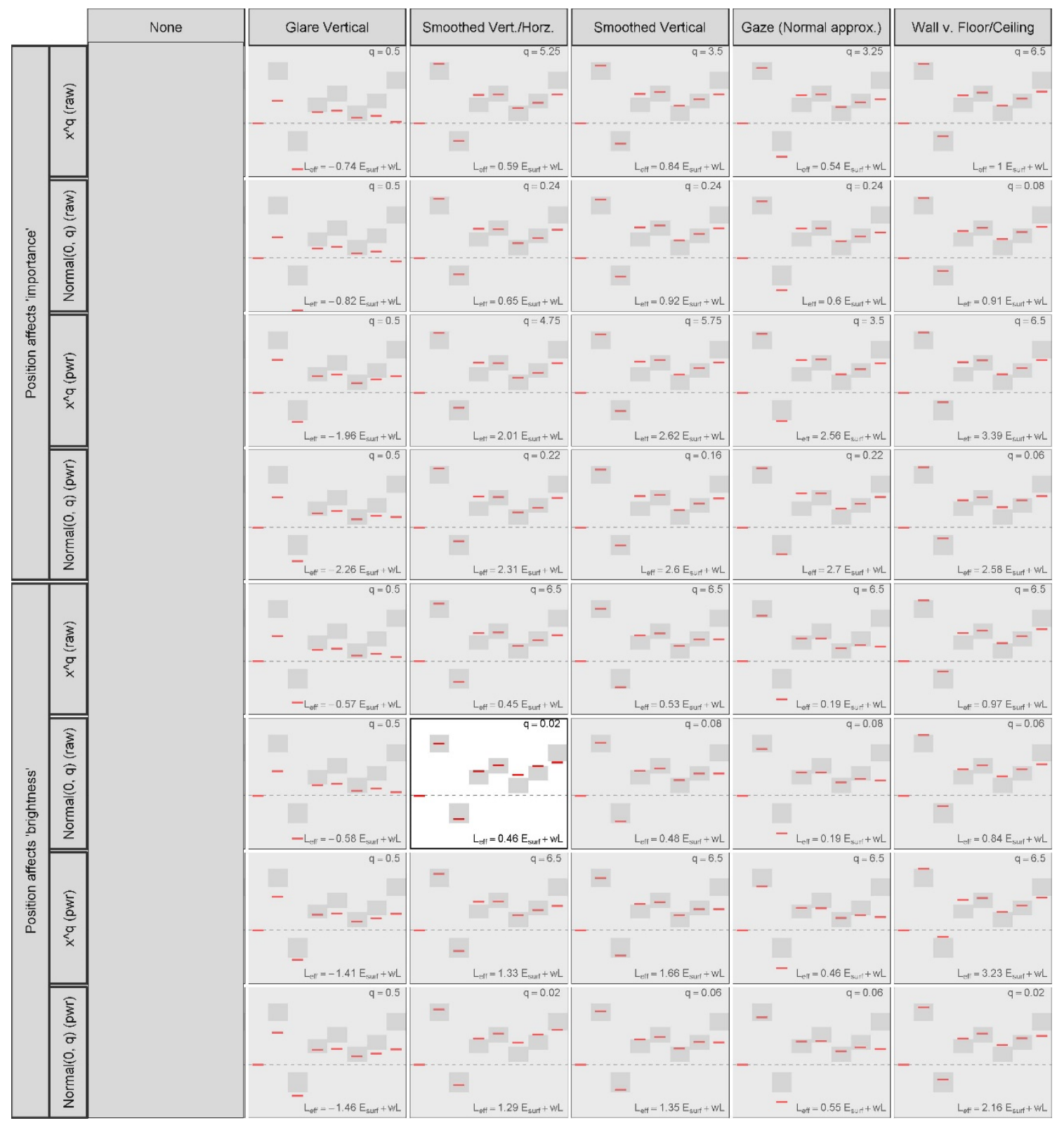

Figure 6.27 Best fits of the basic attempts to apply the percentile weighting based surface effect models to the results of Experiment 1 using the equation with weighted mean luminance, wL. The results for the Wall v. Floor/Ceiling weighting function shown here are with the floor and ceiling weighted at $70 \%$. 


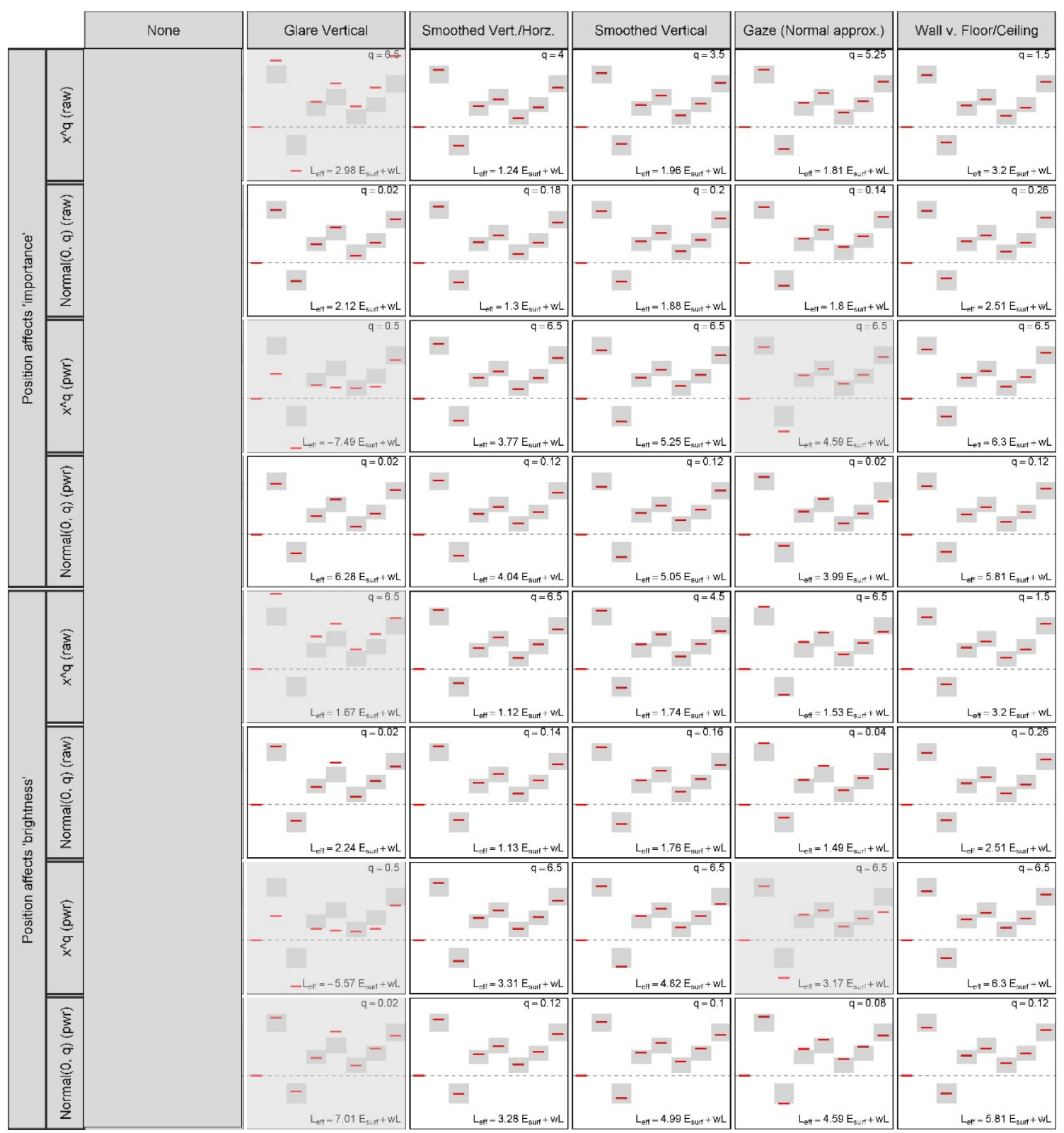

Figure 6.28 Best fits of the "wall/floor/ceiling grouped" version of the percentile weighting based surface effect models to the results of Experiment 1 using the equation with weighted mean luminance, $\mathrm{wL}$. The results for the Wall $\mathrm{v}$. Floor/Ceiling weighting function shown here are with the floor and ceiling weighted at $60 \%$, though $\sim 40$ to $70 \%$ can also work.

A summary of all the different models is shown in Figure 6.29 below. Out of the 546 model combinations tested, around 131 were successful enough (being somewhat generous) to merit further testing and development attempts using the Experiment 2 data. Notably, we can see that not using position weighting had very limited success. In contrast, the position weighting schemes focusing on the walls/centre of view see more successes. This could be an indication that we need to be providing some such weighting scheme - 
though it could also just be a coincidence, or an artefact of our set of experimental conditions.

\begin{tabular}{|c|c|c|c|c|c|c|c|c|c|c|c|c|c|c|c|}
\hline & & & & & Glare base & & Gaze & & & Wall & v. Floc & or/ceil & & & \\
\hline & & Models & None & $\begin{array}{l}\text { Glare } \\
\text { vertical }\end{array}$ & $\begin{array}{l}\text { Smoothed } \\
\text { Vert/Horz }\end{array}$ & $\begin{array}{l}\text { Smoothed } \\
\text { vertical }\end{array}$ & $\begin{array}{l}\text { Normal } \\
\text { approx. }\end{array}$ & 0.1 & 0.2 & 0.3 & 0.4 & 0.5 & 0.6 & 0.7 & 0.8 \\
\hline & & Area Rule & & & & & & & & & & & & & \\
\hline & & Area Rule $(\log m)$ & & & & & & & & & & & & & \\
\hline & Anchoring & Scale Normalisation & & & & & & & & & & & & & \\
\hline & & Area Rule + Scale Norm. & & & & & & 1 & & - & & & & & \\
\hline & & Area Rule + Scale Norm. $(\log m)$ & & & & & & & & & & & & & \\
\hline & & $x^{\wedge} q(\operatorname{raw} L)$ & & & & & & & & & & & & & \\
\hline & affects & $\operatorname{Normal}(1, q)(\operatorname{raw} L)$ & & & & & & & & & & & & & \\
\hline Basic & 'importance' & $x^{\wedge} q($ power transformed $L$ ) & & & & & & 1 & & & & & & & \\
\hline Percentile & & $\operatorname{Normal}(1, q)$ (power transf. L) & & & & & & & & & & & & & \\
\hline Weighting & & $x^{\wedge} q(\operatorname{raw} L)$ & & & & & & 1 & & & & & & & \\
\hline & affects & $\operatorname{Normal}(1, q)(\operatorname{raw} L)$ & & & & & & & & & & & & & \\
\hline & & $x^{\wedge} q($ power transformed $L)$ & & & & & & & & & & & & & \\
\hline & & Normal $(1, q)$ (power transf. L) & & & & & & & & & & & & & \\
\hline & & $x^{\wedge} q(\operatorname{raw} L)$ & & & & & & & & & & & & & \\
\hline Wall/floor & affects & $\operatorname{Normal}(1, q)(\operatorname{raw} L)$ & & & & & & & & & & & & & \\
\hline /ceiling & 'importance' & $x^{\wedge} q($ power transformed $L)$ & & & & & & & & & & & & & \\
\hline grouped & & Normal $(1, q)$ (power transf. L) & & & & & & & & & & & & & \\
\hline Percentile & & $x^{\wedge} q(\operatorname{raw} L)$ & & & & & & & & & & & & & \\
\hline & affects & $\operatorname{Normal}(1, q)(\operatorname{raw} L)$ & & & & & & & & & & & & & \\
\hline & & $x^{\wedge} \mathrm{q}($ power transformed $L)$ & & & & & & & & & & & & & \\
\hline & & Normal $(1, q)$ (power transf. L) & & & & & & & & & & & & & \\
\hline & & Area Rule & & & & & & & & & & & & & \\
\hline & & Area Rule $(\log \mathrm{m})$ & & & & & & & & & & & & & \\
\hline$+w L$ & $\begin{array}{l}\text { Anctorning } \\
\text { Rule. }\end{array}$ & Scale Normalisation & & & & & & & & & & & & & \\
\hline & & Area Rule + Scale Norm. & & & & & & & & & & & & & \\
\hline & & Area Rule + Scale Norm. $(\log \mathrm{m})$ & & & & & & & & & & & & & \\
\hline & & $x^{\wedge} q($ raw L $)$ & & & & & & & & & & & & & \\
\hline & affects & $\operatorname{Normal}(1, q)(\operatorname{raw} L)$ & & & & & & & & & & & & & \\
\hline & 'importance' & $x^{\wedge} q($ power transformed $L$ ) & & & & & & & & & & & & & \\
\hline Percentile & & Normal $(1, q)$ (power transf. L) & & & & & & & & & & & & & \\
\hline Weighting & & $x^{\wedge} q(\operatorname{raw} L)$ & & & & & & & & & & & & & \\
\hline & affects & $\operatorname{Normal}(1, q)(\operatorname{raw} L)$ & & & & & & & & & & & & & \\
\hline & & $x^{\wedge} q$ (power transformed $L$ ) & & & & & & & & & & & & & \\
\hline & & Normal $(1, q)$ (power transf. L) & & & & & & & & & & & & & \\
\hline & & $x^{\wedge} q(\operatorname{raw} L)$ & & & & & & & & & & & & & \\
\hline & affects & $\operatorname{Normal}(1, q)(\operatorname{raw} L)$ & & & & & & & & & & & & & \\
\hline /ceiling & 'importance' & $x^{\wedge} q($ power transformed $L$ ) & & & & & & & & & & & & & \\
\hline grouped & & Normal $(1, q)$ (power transf. L) & & & & & & & & & & 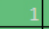 & & & \\
\hline Percentile & & $x^{\wedge} q(\operatorname{raw} L)$ & & & & & & & & & & & & & \\
\hline Weighting & affects & $\operatorname{Normal}(1, q)(\operatorname{raw} L)$ & & & & & & & & & & 1 & & & \\
\hline & & $x^{\wedge} q($ power transformed $L)$ & & & & & & & & & & 1 & & & \\
\hline & & Normal $(1, q)$ (power transf. L) & & & & & & & & & & & & & \\
\hline
\end{tabular}

Figure 6.29 Overview of the success of surface effect model/position weighting combinations. Green squares highlight combinations that saw enough success to be considered worth attempting to develop further with the Experiment 2 data. Top half is the model using the basic equation, bottom half is using the alternative equation using the weighted mean luminance.

Observationally, the conditions that caused the most problems were the Checkerboard and Black Wall condition. The Checkerboard condition was observed to be the second brightest condition in Experiment 1, with a large effect. However, on most uniformity metrics it is closer to the mid-range conditions than the most non-uniform White Wall condition. Correspondingly, many of the models tended to underestimate its brightness. An open question here is perhaps whether or not the pattern of the Checkerboard, with its high frequency high contrast luminance changes, is having a distinct effect that we have not 
accounted for in our models. If this is the case, then not accounting for it would throw predictions out. Such effects, however, need more research to suggest anything concrete ${ }^{74}$.

The Black Wall condition has the problem that it appears darker than the baseline uniform grey condition, yet on most uniformity metrics it is substantially more nonuniform, as well as having large white surfaces in its floor and ceiling. Many of the models thus expected it to appear brighter, rather than darker, and this is arguably one of the key reasons for the poor performance we see if we do not use a position weighting function. Focusing on the central band or the walls makes the Black Wall condition, unsurprisingly, appear darker, and so using weighting functions that do this can help to fit the Black Wall condition's results.

\subsubsection{Light source effects (Experiment 1+2)}

To test the light source models in Experiment 2, we took the surface effect models that were successful at describing the Experiment 1 results and combined them with our potential models of the light source effects. Parameter values for, for example, the percentile weighting functions were the approximate best fitting values from the Experiment 1 tests. To allow some flexibility, as values around the "best" ones could also be viable and could fit the Experiment 2 data better, we also calculated the surface effects for parameter values \pm 2 increments around the selected values. Increments were 0.25 for the power weighting function and the weightings used in the scale normalisation model, and 0.02 for the normal distribution weighting function. The resulting surface effects were then combined with the 18 light source models summarised below:

Table 6.4 Summary of different models of light source effects that were tested

\begin{tabular}{|c|c|c|}
\hline \multicolumn{2}{|l|}{ Model } & Description \\
\hline \multirow[t]{3}{*}{ Stevens } & 1 & $\begin{array}{l}\text { Basic - just calculate brightness from mean luminance of each of } \\
\text { surfaces and light source using Stevens' power function with an } \\
\text { exponent of } \sim 0.37 \text { (based on adaptation level), then average, and } \\
\text { normalise }\end{array}$ \\
\hline & $2 a$ & $\begin{array}{l}\text { Use the mean surface "luminance" from the previous surface } \\
\text { effect calculations as the surface luminance, before calculating } \\
\text { the average and normalising }\end{array}$ \\
\hline & $2 b$ & $\begin{array}{l}\text { Alternative: as } 2 a \text {, but instead of adding } E_{\text {surf }}+E_{\text {light, }} \text { just take the } \\
\text { effect calculated here as the overall effect (as it has already } \\
\text { included the "surface effects") }\end{array}$ \\
\hline Marsden & $\begin{array}{l}1,2 a, \\
2 b\end{array}$ & Same as above, but using Marsden eq. instead \\
\hline
\end{tabular}

\footnotetext{
${ }^{74}$ The smoothing suggested by Kato and Sekiguchi (2005) does not mesh well with our checkerboard conditions for example, and in any case saying that the checkerboard condition should be more "uniform" is the opposite of what we want here.
} 


\begin{tabular}{|l|l|l|}
\hline & $\begin{array}{l}\text { 1i, 2ai, } \\
\text { 2bi }\end{array}$ & $\begin{array}{l}\text { Importance weighting variant: do not apply relative position } \\
\text { weights to mean luminances of surface/light source when using } \\
\text { Marsden eq. to calculate brightness of surfaces }\end{array}$ \\
\hline $\begin{array}{l}\text { Discounted } \\
\text { light source }\end{array}$ & 1 & $\begin{array}{l}\text { Calculate weighted average of the mean light source and surface } \\
\text { luminances }\end{array}$ \\
\cline { 2 - 3 } & 2a, 2b & $\begin{array}{l}\text { As above, but using the mean surface "luminance" as calculated } \\
\text { from the surface effect calculations as before }\end{array}$ \\
\cline { 2 - 4 } & $1 \mathrm{i}$ & $\begin{array}{l}\text { Basic: Simply the log of the ratio between surface and light } \\
\text { source }\end{array}$ \\
\hline \multirow{2}{*}{$\begin{array}{l}\text { Brightness } \\
\text { difference }\end{array}$} & p & $\begin{array}{l}\text { Importance weighting: Do not apply relative position weights to } \\
\text { the surface/light source mean luminances when calculating the } \\
\text { difference between them }\end{array}$ \\
\cline { 2 - 4 } & p_av & $\begin{array}{l}\text { Variant of light balance model where we instead calculate } \\
\text { difference in brightness (as power function of mean luminance } \\
\text { with exponent of } ~ 0.37) \text { between surfaces and light source }\end{array}$ \\
\cline { 2 - 4 } & $\begin{array}{l}\text { Take the result of p, above, and use as the estimate of surface } \\
\text { brightness - then average that with the light source brightness to } \\
\text { get the overall effect of the light source }\end{array}$ \\
\cline { 2 - 3 } & p_avi & $\begin{array}{l}\text { Importance weighting: Do not apply relative position weights to } \\
\text { the surface/light source mean luminances when calculating the } \\
\text { difference between them }\end{array}$ \\
\hline
\end{tabular}

As in Experiment 1, some of the models had a range of potential weightings that could be used. For the discounted light source model we tested potential light source weights from 0.0 to 0.9 , in increments of 0.05 . For the "brightness difference" model wherein the brightness of the light source reduces the brightness of the surface, the result should not imply a negative brightness. Hence, the brightness of the light source, being higher than the brightness of the surfaces, must needs be multiplied by some factor significantly less than 1 . We tested values ranging from 0.05 to 0.5 , in increments of 0.025 .

The following figure shows a subset of the data, showing the success of attempts to predict the Experiment 2 results using the Area Rule to model the surface effects and applying no location weighting function (Figure 6.31). While most of the light source effect models appear to fail, the simple brightness difference one appears to provide a good fit to our results.

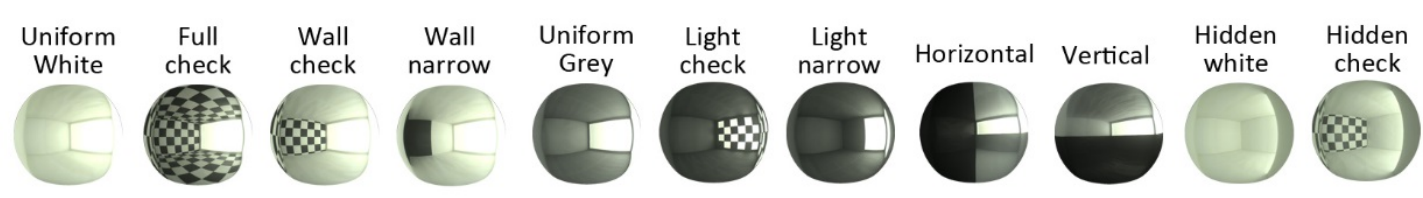

Figure 6.30 Experiment 2 conditions in order. 


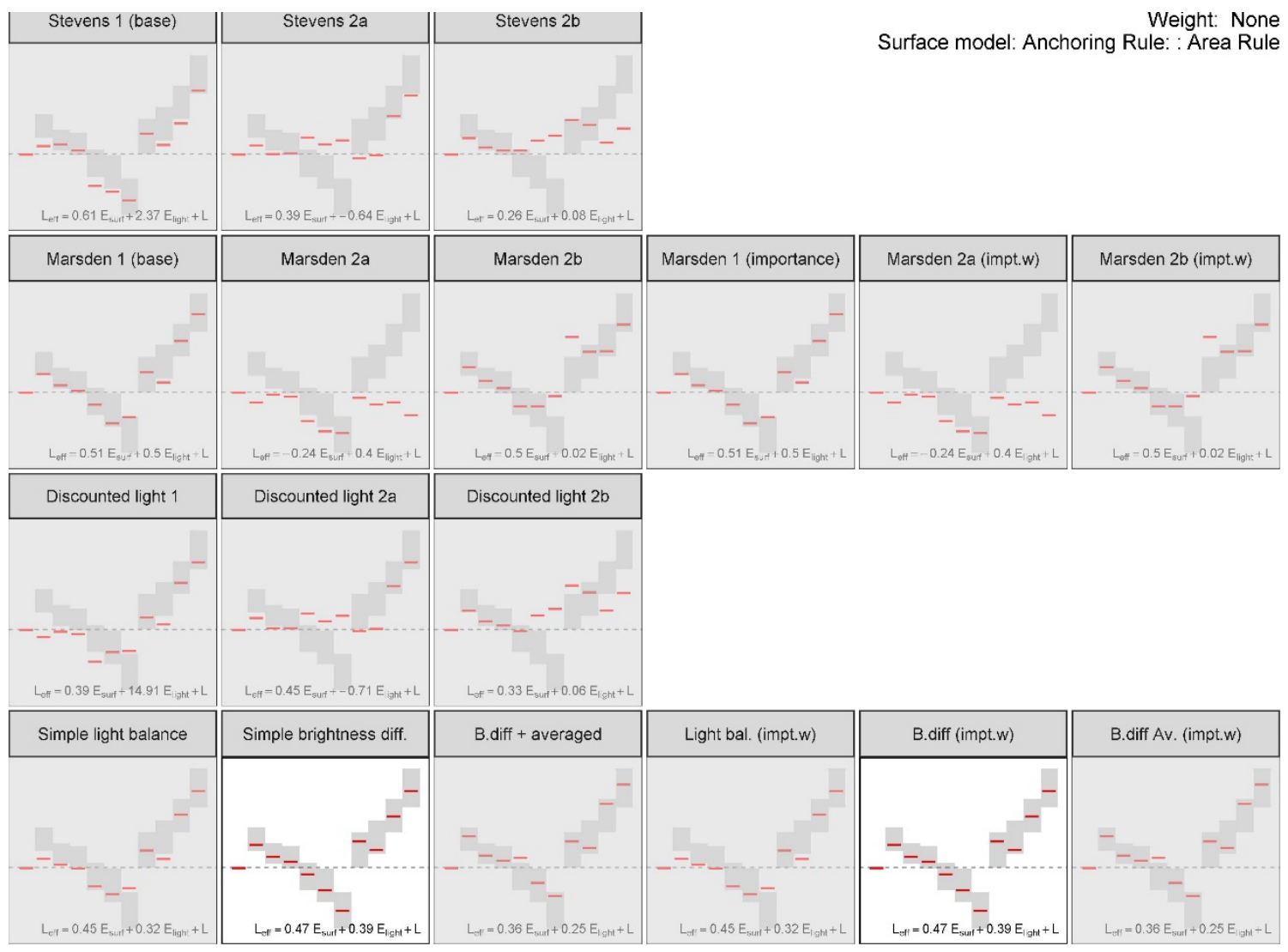

Figure 6.31 Best fits of different light source effect models in predicting the results of Experiment 2 , with no position weighting and the Area Rule used to predict the surface effects. Models are those described in Table 6.3. Points are in the order displayed in Figure 6.30 above.

There were roughly 131 different position weight/surface effect model combinations that were deemed to be worth testing further based on the results of our tests using the Experiment 1 data. Given this, rather than presenting 131 versions of Figure 6.31, we summarise the success of the different models on the following page (Figure 6.32). Each of the grey squares is a position weight/surface effect model combination. Within each, the white outlined grid maps the same light source models shown in Figure 6.31. "Successful" models (identified here by filtering according to mean squared squared error $^{75}$ ) are highlighted in green, while unsuccessful ones are left blank. Lighter green indicates a quantitatively stronger fit. There are a large number of what appear to be successful models.

\footnotetext{
${ }^{75}$ Ultimately we need to visually inspect the fits to check that they are working well, but simple quantitative measures are used here for an initial filter for practical reasons. While working on the experiment 1 data, we initially used squared error as a filter, but found it was not punishing single large errors enough. Thus, we squared the error again in an attempt to filter those out more effectively. We also filtered out all models which had negative coefficients on the grounds that those make no sense theoretically (as they mean that when the model thinks it's brighter it's darker).
} 
The light balance/brightness difference models seem to be most successful, while there are nearly no successful applications of the Stevens' or Marsden equation based models. 


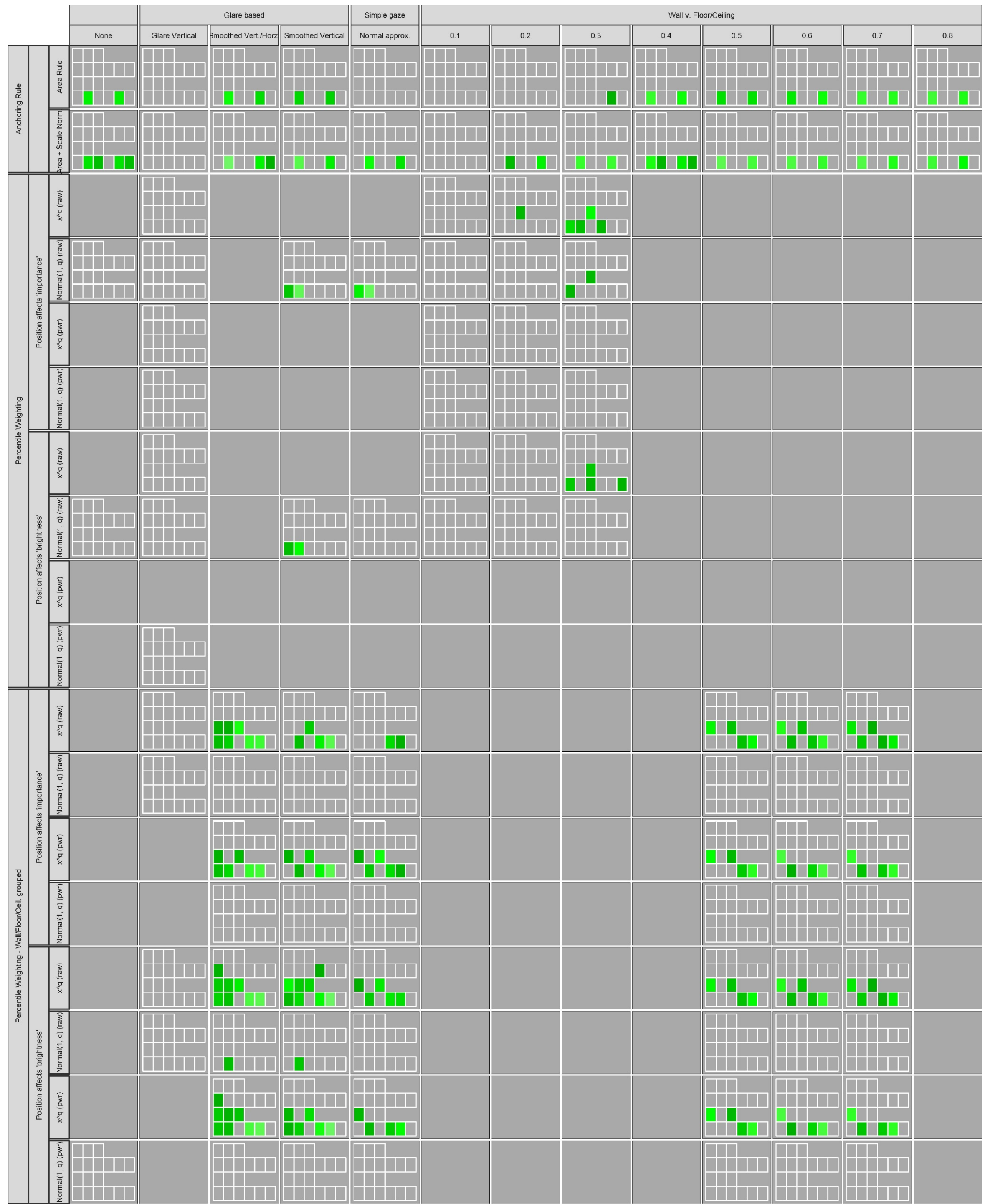

Figure 6.32 Success of different models in predicting the results of Experiment 2. Each grey square is a different location weight/surface effect model combination. The empty squares were model combinations that failed to show any promise in the previous tests on Experiment 1's data, and so were not continued here. The white grids in the squares outline the same light source models shown in Figure 6.31. Potentially "Successful" models, here roughly assessed by filtering according to mean squared squared error, are highlighted in green. The lighter the green, the stronger the fit. 


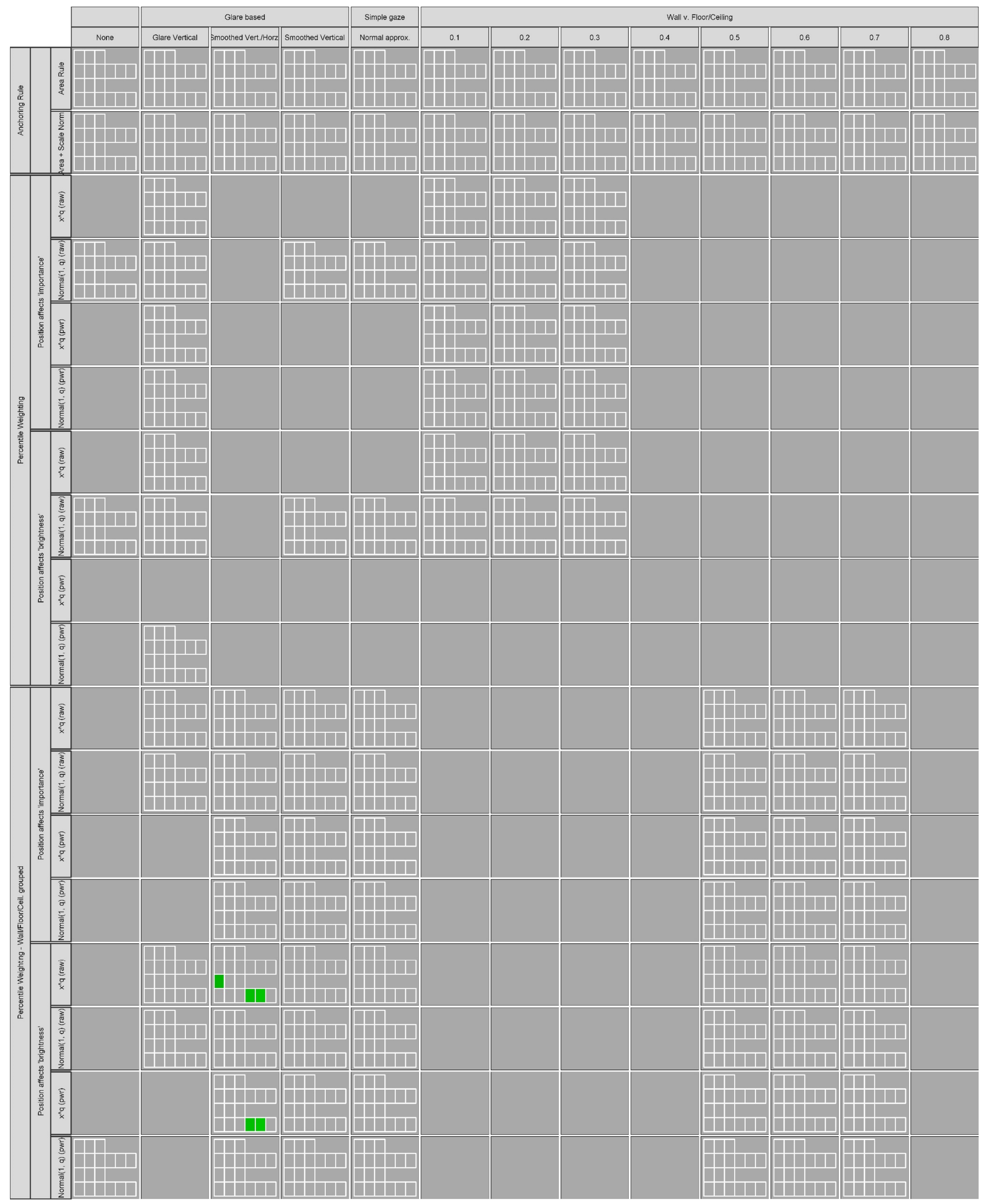

Figure 6.33 Success of different models in predicting both the results of Experiment 2 AND the results of Experiment 1. 
There is, however, an issue here. The preceding analysis was done by just fitting the Experiment 2 data. If we look at the successful models in Figure 6.31, their equation is:

$$
L_{\text {eff }}=0.47 E_{\text {surf }}+0.39 E_{\text {light }}+L
$$

When the same model was fit to the Experiment 1 data, the best fit equation was ${ }^{76}$ :

$$
L_{\text {eff }}=0.78 E_{\text {surf }}+L
$$

In other words, while the same general models can successfully be fit to both data sets separately, they require substantially different coefficients on the strength of the surface effects. The extent to which this is a problem is something that can be debated. For example, it is quite plausible that the strength of the surface effects could change depending on adaptation level - which was, after all, very different between the experiments. At the same time, we have no evidence of this, and no information about how it might be expected to vary if it does. Given this, it might be a better idea to attempt to fit all the experimental data at once, and see what models can provide good fits assuming the coefficients are constants (Figure 6.33).

Doing this dramatically reduces the number of models that provide reasonable fits from 217 to 5 . These are all using the horizontally and vertically smoothed glare function and the wall/floor/ceiling grouped application of the percentile weighting model $^{77}$. The attempts to either simply apply Stevens' power law or the Marsden equation to describe the light source effects did not work well, while the idea of discounting the light source ran into problems with how it interacted with the position weighting functions.

Among the "successful" light source effect models was the basic application of the discounted light source model, and four versions of the light balance model. We may note that only the "importance weighting" based light source models worked here — when calculating the differences we did not apply position weights to the mean light source or surface luminances.

Examining these five models we see that they are not all equal, and there are still issues (Figure 6.34). Most obviously, there is a tendency towards underestimation of the checkerboard condition (Exp. 1 \#8) relative to our observations - though given that we have examined 19 different conditions here, one of our estimates being out by this much may not be surprising. We may also note a slight tendency for the vertical condition to be estimated high and the Hidden White condition to be estimated low in some of the models

\footnotetext{
${ }^{76}$ Light source effects being assumed to be 0 in Experiment 1

${ }^{77}$ In which rather than calculating the surface effects as the percentile weighted average of all the surface luminances, we first divide the surfaces up into the walls, floor, and ceiling and individually calculate the percentile weighted averages of each of those. They are then combined (weighted by area and relative position weight) to produce the overall surface effect.
} 
(the "brightness difference" and "discounted light source"). While on their own this is not necessarily a problem, it does result in the difference between them being underestimated - particularly in the discounted light source model where it predicts that the vertical condition is actually slightly brighter. Similarly, in the "light balance" models there is a slight tendency towards overestimation of the difference between the Uniform Grey and Checkerboard Light conditions. Again though, we may note that we would expect some of the comparisons, statistically, to be out to some degree (given 83 potential comparisons across the two experiments). Overall, the discounted light source model is the weakest with the worst overall fit (in terms of mean squared error), and the most discrepancies.
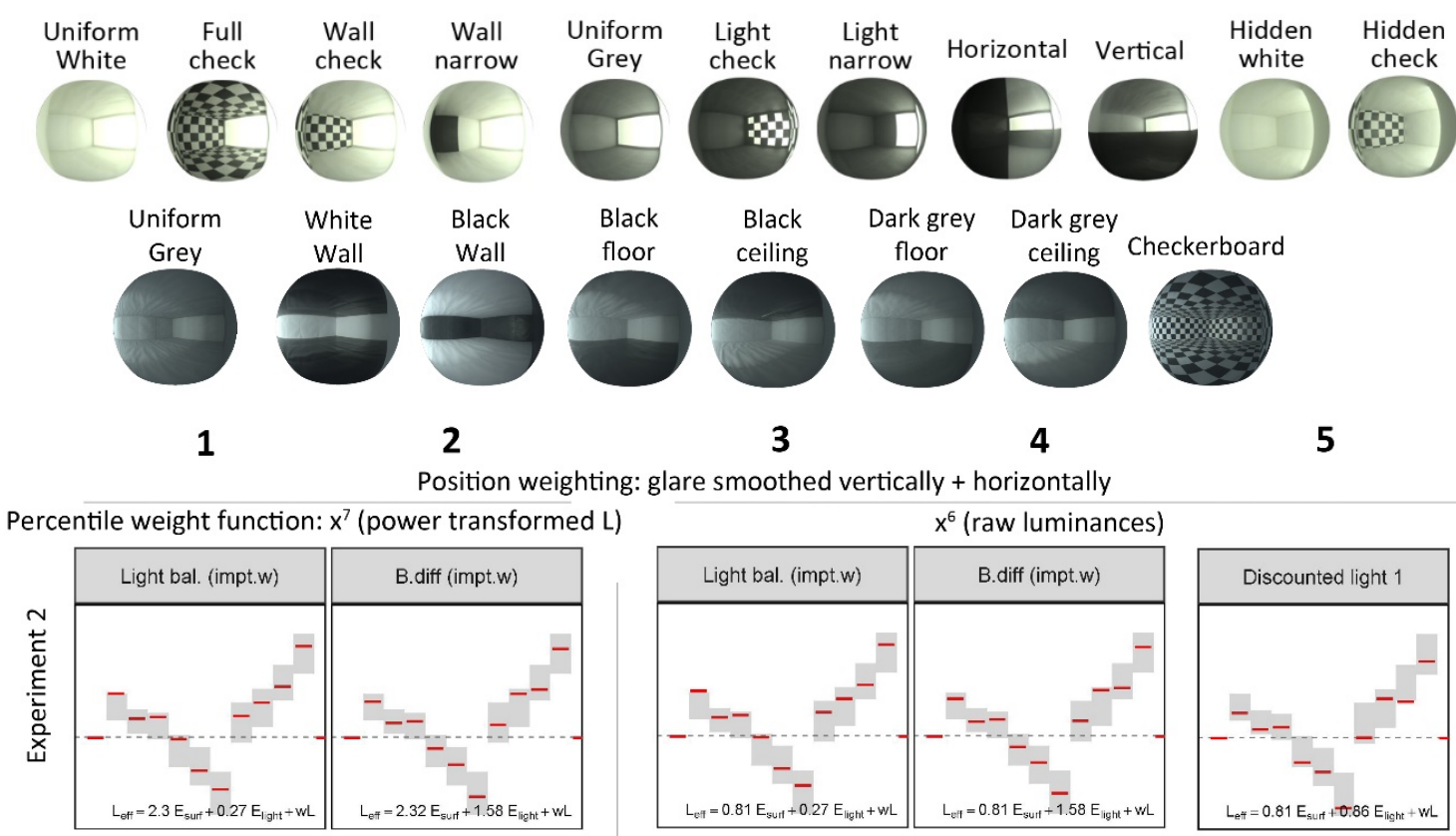
$x^{6}$ (raw luminances)
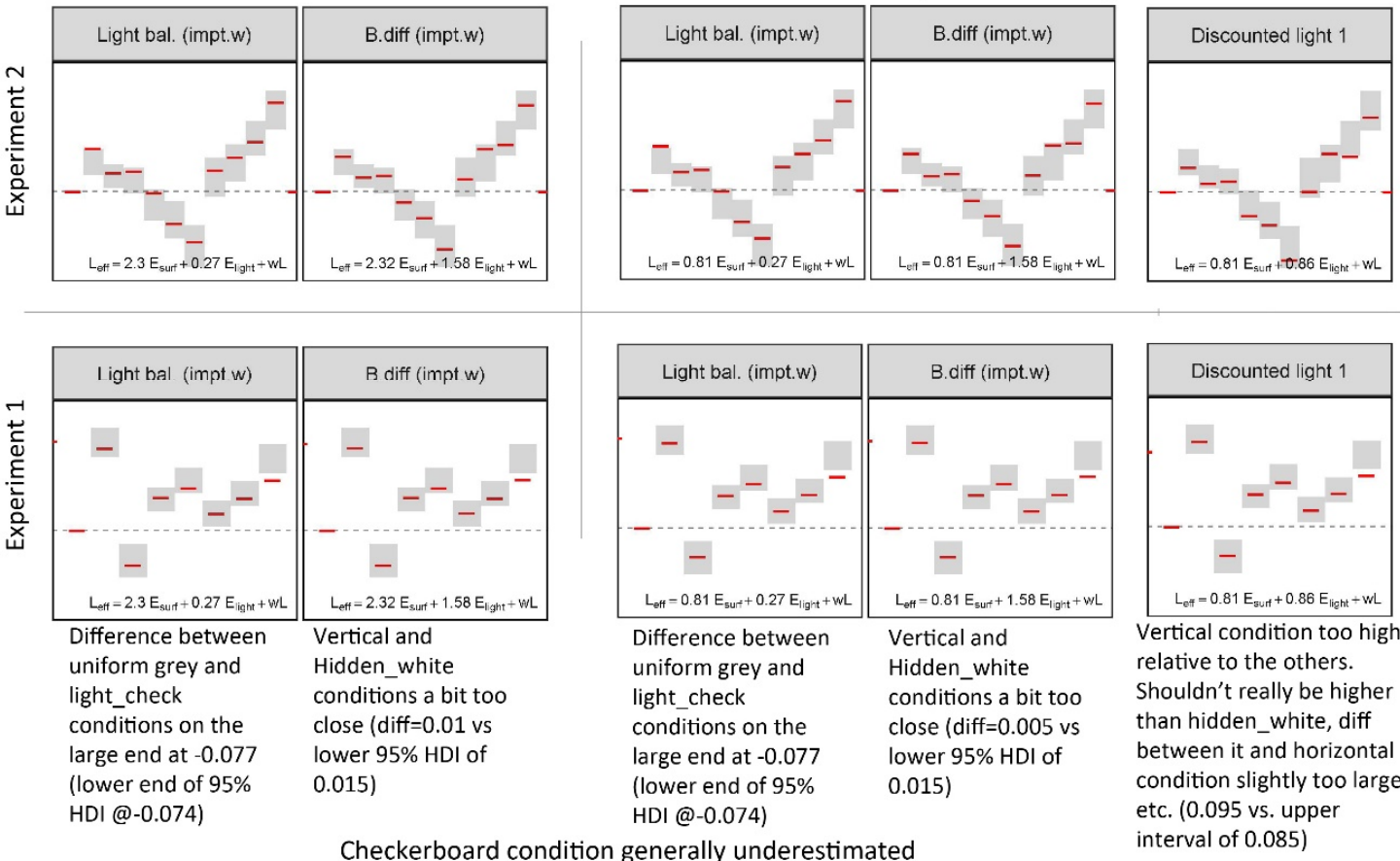

Figure 6.34 Model predictions against observed results in Experiments 1 and 2 for the five "most successful" models highlighted in Figure 6.33. Red bars show predictions, grey boxes are the estimates from the main statistical model(s) $(95 \% \mathrm{HDIs})$. Surface effect models described at the top. 
That the best performing light source effect models here were either the simple light balance or brightness difference ones is slightly disappointing, as they are arguably theoretically the weakest. The issue here is that they do not really provide an explanation for how the light source is affecting the brightness — the model just says that larger differences between light source and surface luminance/brightness can be related to decreases in spatial brightness. The "brightness difference + averaged" model makes much more sense, for example, as it takes the brightness difference and, similar to the Haubner equation, says that the brightness of the light source reduces the apparent brightness of the surfaces. It then uses that to compute a new average luminance which we can take as the "effect". Unfortunately, it performed badly, having a tendency to dramatically overestimate the brightness of the uniform grey condition in Experiment 2.

This exploration is not, it must be said, remotely exhaustive, despite the number of models tested. We can easily think of more possibilities, building off what we have already tried. Could we not try a version 2 of the light balance models, using the surface luminance as calculated from the surface effects model? What if we tried combining the glare based position weight functions with the wall/floor/ceiling weights? What if we tried to combine multiple surface or light effects models into a single model? There is no shortage of ideas we could test. The question we must ask at this point is whether or not there would be any value in this. If we did manage to create more "successful" models, would we believe them to be true?

As in our discussion of Experiment 1, we argue that the answer to this question is again "No". Consider all the models from previous studies that failed on our data (Section 3). Consider all the models explored in this chapter that succeeded on our first experiment's data, but not on the second. The only reasonable conclusion to draw from this is that a model "working" on some experimental data should not be treated as providing compelling evidence that it will generalise beyond that data. Certainly, evidence from two experiments covering 19 different conditions is more compelling than a single experiment with $\sim 8$ conditions. However, we must also note that these conditions do not remotely cover the entire range of possible light distributions — indeed, they are very unlike typical "real" spaces - and their foundations rest on a bed of sand. If the process illustrated here of attempting to design these models from "theory" demonstrated anything, it is that we simply do not know what assumptions we should be making for any model. Our position weighting functions, despite their clear importance to our results, are essentially rough guesswork $^{78}$ and are unsupported by any solid empirical data on position and spatial brightness. Questions around the things like applying the Area Rule (Gilchrist, 2006)

\footnotetext{
${ }^{78}$ Building them off studies of glare position indices and gaze direction in rooms may be better than nothing, but it is hard to say they provide strong evidence for using them in this way.
} 
(Section 6.2.1) rest on untested assumptions about how factors like lightness may relate to spatial brightness. Without the data to specifically test these ideas, and describe the patterns that need to be modelled, any attempt at model design is fundamentally limited. There are too many possibilities to practically explore, and even if they work we do not know if we were making sound assumptions to begin with.

Adding to this is the issue of uncertainty in our measurements of the light distribution. As discussed in Appendix A.3, the High-Dynamic-Range luminance maps used to measure the light distribution have a non-trivial degree of uncertainty. Scattering in the lens can result in significant errors, primarily in the form of the dark regions having their luminance overestimated. While this did not seem to be a significant problem with the first experiment's conditions, it was in the second. The Vertical condition, for example, with its black surfaces directly adjacent to the light source had some areas in the luminance maps overestimated by almost an order of magnitude when compared against spot luminance measurements. While we attempted to correct for that, it must be acknowledged that there is currently no established method for correcting HDR images for this, and our method involved a significant amount of manual interpolation and should be considered rough. For many studies these issues are not as problematic as the above description may sound. The general effect is related to the overall uniformity of a given condition. The most nonuniform conditions see the largest changes, while more uniform conditions may see no real change at all (Figure 6.35). As long as ones conditions are substantially different in uniformity, the overall pattern of results — which conditions are more or less uniform should not be expected to change. In this light, a good correlation on uncorrected measurements is likely to be maintained when they are corrected. The main effect is likely to be a slight shift in the steepness of the slope of the brightness/uniformity relationship in such analysis. 


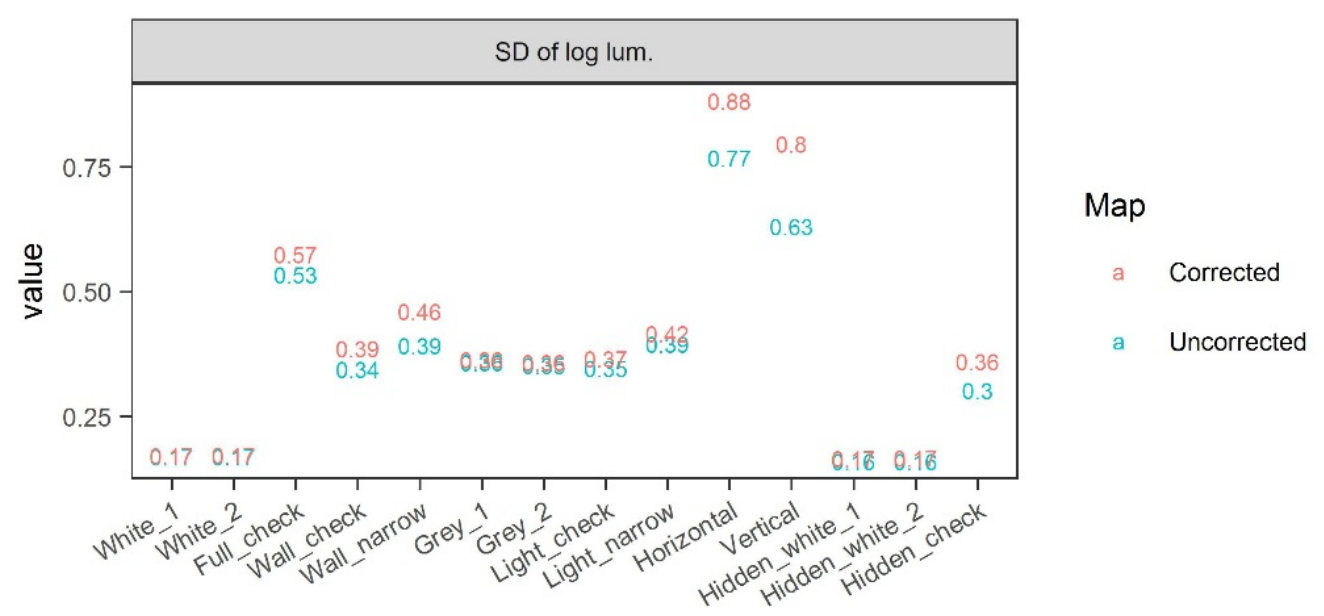

Figure 6.35 Example of the effects of scattering correction on measured uniformity (for more metrics, see Appendix A.3.3.2)

Where this does matter, however, is when we are attempting to make very precise mathematical predictions - such as we have been trying to do in this chapter. If we compare model fits using the "corrected" luminance maps to the uncorrected ones, we see that while they are strongly correlated, there may also be significant differences (Figure 6.36). Broadly, while any "clearly wrong" models can be safely assumed to be wrong, ones that are "possibly good" (i.e. all of our best performing models) are more questionable. This may be illustrated by the way that different models get identified as being potentially good if we use the uncorrected luminance maps (Figure 6.38). Due to this, when attempting to precisely fit any models to this data we must accept a certain degree of uncertainty (see an example of the potential error in Figure 6.37). For these reasons, we treat the apparently successful models with scepticism. 


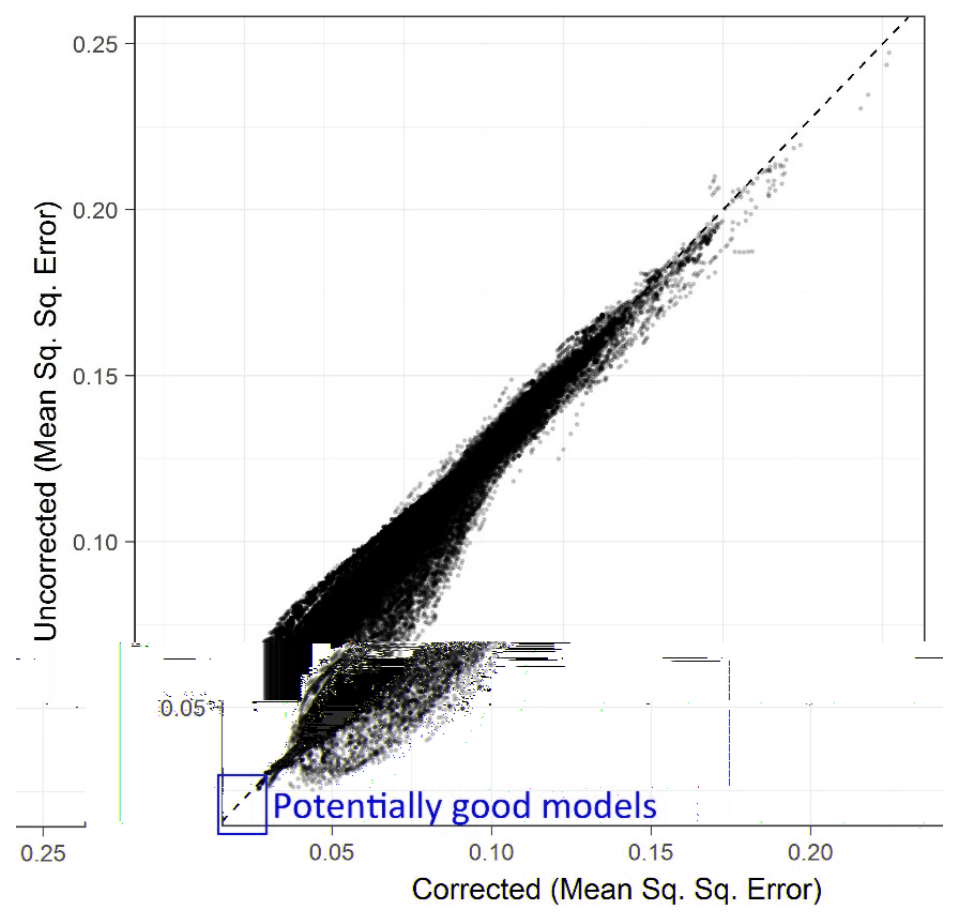

Figure 6.36 Comparison of model fits using corrected and uncorrected luminance maps for the Experiment 2 conditions. The filter we used in the earlier discussions (e.g. Figure 6.33) to identify models worth looking at was mean squared squared error $<0.03$, which is highlighted on the figure.

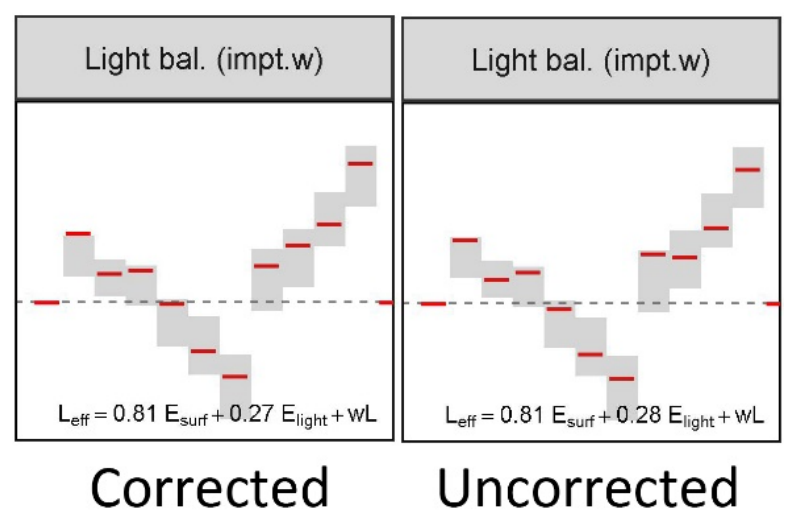

Figure 6.37 Example comparison of one of the "good" models in Figure 6.34 using the corrected and uncorrected luminance maps. This may provide an illustration of the potential error from the uncertainty in our luminance maps. 


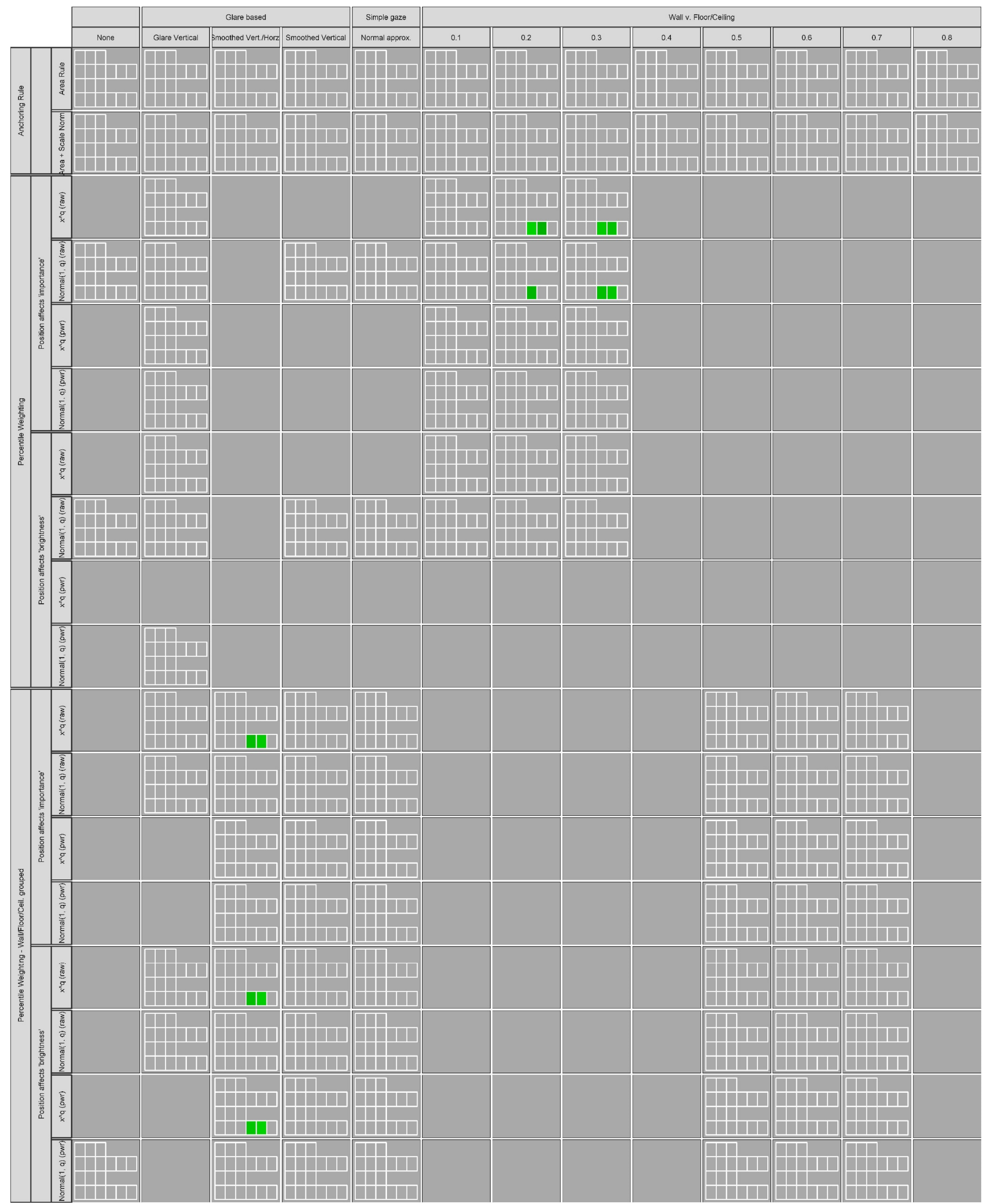

Figure 6.38 Success of different models in predicting both the results of Experiment 2 AND the results of Experiment 1 using the uncorrected luminance maps to describe the light distributions of Experiment 2. Compare to Figure 6.33. 



\subsection{Discussion: More questions than answers}

We concluded our exploration of potential models above by noting that, ultimately, no matter how successful any of our models was at fitting our experimental data, there would still be no reason to believe that they were "true". The track record of models generated by experiments like these is poor (Chapter 4), and overall should mostly give one reasons to be sceptical that any proposed model would be able to reliably predict the brightness of spaces that differ from those the model was built on. With that in mind, a good question to ask is what the point of this chapter was. If our experiments could never produce a sound model, why did we even try?

The value, we suggest, lies more in the questions raised by the exercise. As the process of attempting to design models based off what we "know" showed (Section 6.2), any attempt in this regard currently rests on foundations of sand. All our models are built on top of an array of assumptions that have not been tested in any meaningful way. Our exploration is thus less about identifying "the answer"79, but rather an illustration of the questions that we need to answer if we are to identify the answer.

The questions can be big and obvious: do we need to weight by position? Do people mostly take their cues of the "surface effects" from the highest luminance regions (such as in our percentile weighting model)? Do people discount the light source? Should we be treating the walls, floor, and ceiling as distinct grouped entities?

The questions can be subtle, involving small - yet significant - decisions in the mathematical operations: if we weight by position, when should we apply the weightings (our importance vs. brightness weighting distinction (Figure 6.7)) ? Should we be using raw luminances, or should we transform them first using something like a power function?

There are also many more questions that we have not even begun to examine in our tests here, as we lack the data required. We have not examined the effects of pattern. The 'duel'-process model does not explain the effect of pattern observed by Kato and Sekiguchi (2005) — spreading the light sources out more should generally create a more uniform surface illumination, which in our model should make the space appear slightly darker if anything, not brighter. They proposed smoothing the luminance distribution over $\sim 100^{\circ}$ in order to account for this. While such a model would appear to conflict with the effects of our checkerboard conditions (as smoothing to such a degree effectively removes most of their non-uniformity), our separation of light source and surface effects may provide an avenue for reconciliation. Perhaps it is another example of how surface patterns and light sources affect spatial brightness differently. Investigations into how such pattern effects

\footnotetext{
${ }^{79}$ Of course, we should also not forget that our analysis was still able to reject a number of possibilities
} 
work when constructed using surface reflectances instead of light sources could be of great value. For example, we could examine checkerboard conditions with different check sizes to see if they differ in brightness. Currently, we have no real idea how such factors affect spatial brightness.

Adding to this, there are many different "patterns" that can make up light distributions, many of which are not accounted for by common metrics. Does skewness matter? Do gradients have different effects from sharp edges? What about the other questions about what elements of the distribution we need to be modelling? Should we be treating the effects of illumination as the same as reflectance (we have here in order to explain Tiller and Veitch's (1995) results, but it has not been tested)? Does adaptation level affect the effects of light distribution? Working out what elements need to be accounted for in any model of the light distribution will require careful testing of each of these questions.

Some of these questions end up completely changing the framing of the model. We speak here vaguely of "surface effects" and "light source effects" because that is really the only way to generically encapsulate all the ways that one could describe the different models under our overarching 'duel'-process model theory. We initially framed the light source effects in terms of the "light balance", but the idea, for example, that people discount the light source is really quite distinct from that. Similarly, we framed surface effects in terms of "non-uniform surface patterns", but the idea that people are biased towards the brighter surfaces in making their judgements is not really the same thing, even if they are related. Indeed, while we currently favour the surface effects + light source effects framework, we cannot rule out the existence of a completely different framing that would also predict our results while not having to make that distinction.

This is worth noting, because the core limitations of the second experiment lie in its design. It was conceived as a response to the existing literature, as laid out in Section 1.3. The hypothesises were framed in terms of process, of "how the light distribution was produced" because the focus was on comparing the qualitative differences between the light distributions in different studies, and trying to determine which of them could explain the divergent results we had observed. The conditions were designed primarily from the perspective of non-uniformity because that was the dominant framework in the literature for explaining light distribution effects on spatial brightness. Hence, they were focused on ruling out most uniformity metrics as possible explanations for our results. The key comparisons were also designed to be as qualitatively similar as possible - compare, for instance, the Wall_narrow and Light_narrow conditions in the reflectance and light source sets - which limits the ability to produce position or pattern based alternative explanations. With most of the non-uniform conditions having their changes primarily around the walls, it is difficult to use position as an explanation for their different effects. Indeed, if anything 
weighting the walls more heavily would be reducing the brightness of the reflectance conditions, as the addition of the dark checks to the walls serves to lower their overall luminance in that band.

That being said, there are differences between the light source and reflectance conditions beyond their respective labels. A notable difference is that of skew. In order to maximise the uniformity differences, the reflectance conditions were designed using a white base, while the light source conditions were designed using a grey base. The downside with this is that it means that the reflectance conditions were produced by adding dark areas, while the light source conditions were produced by making the brightest areas smaller. If we compare the Wall_narrow and Light_narrow conditions then, the Wall_narrow condition is mostly light with a small area of darkness, while the Light_narrow condition is mostly relatively dark, with a small area of light - they have an opposite skew. Whether or not this can actually provide a solid explanation for the observed pattern is less clear. While we could potentially say that "Wall_narrow is brighter because it is positively skewed, while Light_narrow is darker because it is negatively skewed", the Full_checkerboard condition is even brighter but has more dark areas than the Wall_narrow condition. On a similar note, the argument could be made that most of the light source-based distribution changes in the literature would be expected to result in negatively skewed distributions (as the easiest way to make the luminance distribution more non-uniform is to reduce the area of the light source). However, looking at simple skewness in terms of the ratio of the median to the mean in our experiments shows that has problems as an explanation. If we look at our first experiment's data, the general trend is that the more negatively skewed conditions appear brighter (Figure 6.39). This makes it difficult to then attempt to explain the second experiments results by saying the light source conditions were darker because they were more negatively skewed.

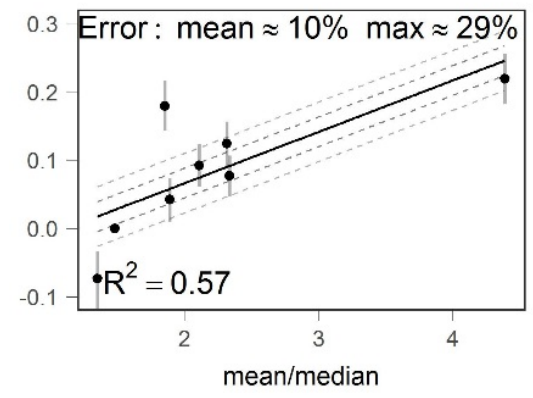

Figure 6.39 Skewness against effective light level in the results of Experiment 1. Mean > median means that the majority of the space is below the mean luminance - it is negatively skewed.

Using skewness on its own thus seems unlikely to work as a model. However, we cannot rule out the possibility of a more complex model of the light distribution that makes use of such differences to explain our results without referring to light sources and surfaces. 
A useful experiment here could be to explore "inverse" checkerboards using a uniform black base, or attempting to produce reflectance conditions more similar to the Light_narrow condition with the light source hidden (Figure 6.40).

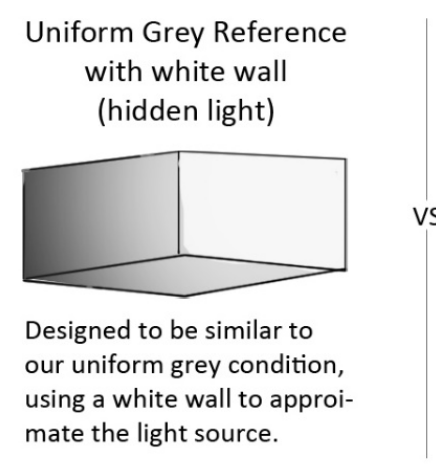

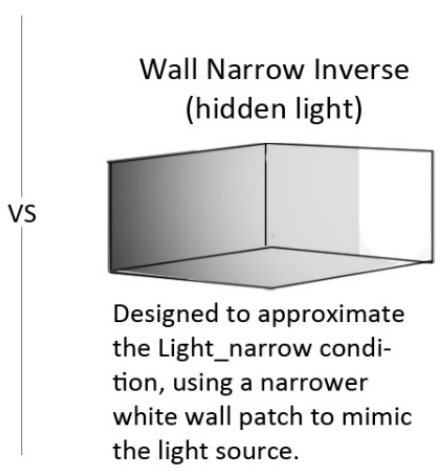

Figure 6.40 Concept for potential test of question of the different skew between our Wall_narrow and Light_narrow conditions, focusing on mimicking the Light_narrow/Uniform grey comparison using surface reflectances and no visible light source. A significantly darker grey would likely be needed to produce a strong enough contrast for easily measurable effects.

Returning to the issue of the second experiment's framing and resulting limitations, the basic issue is that it was designed to determine which of the possible qualitative differences between studies could be manipulated in order to produce diverging trends in the uniformity/brightness relationship. It was not designed to test any of the more precise questions that were brought up during our model exploration. The hypotheses were also fairly vague - a problem that was highlighted at the start of this chapter when our results pushed us to ask the question of what we actually meant by "producing non-uniformity by changing the light sources". The proposed refinement — to describe it as the effect of the "light balance" - is arguably the best framing, as the difference between the light source(s) and surfaces should be the most salient uniformity factor affected by the light source changes in most experiments. However, we may also note that it is not the only way the light sources could be changed to produce non-uniformity. Differences within or between the light sources may also be notable (particularly in the case of Kirsch (2014), where the light distributions were produced by LED panels covering the walls), as well as simply different arrangements of the light sources (though we would classify this as an effect of pattern here, and it would not change uniformity on most common metrics). The flexibility evident in how we define our hypothesis, and which carries on into the specification of the 'duel'-process model (as shown by the many ways in which we could potentially describe each of the processes), may reasonably be criticised as limiting the strength of the evidence (Gelman and Loken, 2013). Different results in terms of the observed difference between the Uniform White and Uniform Grey conditions might not have affected the main hypothesis test, but it would have affected the refinements suggested in this chapter. The 
'duel'-process model should be treated as a hypothesis, and we should be careful not to overstate the evidence for it.

We may also note that the potential challenges to the Modes of Appearance hypothesis in terms of results that do not necessarily fit it perfectly (Section 4.1.5) have not gone away. While Okajima and Fujimoto's (2008) results can potentially be explained through the interaction of the effects in the 'duel'-process model, and how surface effects can potentially overcome light source effects to produce a net increase in brightness (as shown by our Vertical and Horizontal conditions), the others are not so easy. Aya et al.'s (2015a, 2015b) conditions in particular pose a challenge, as their non-uniform distributions were produced through both changes to the light sources and light balance, and changes to the illumination distribution. It is unclear how the relative strengths of those elements compare to their strengths in our experiment, and examining that could be a useful test of our 'duel'process model. If their surface illumination differences were relatively weak compared to our ones, while their light balance differences were relatively strong, then their results (saying that non-uniform distributions were darker) would be consistent with the model. However, if the opposite was true then it would cast doubt on the way we have framed our 'duel'-process model. One possibility that this could raise is the question of the perception of light sources and luminosity. Could sufficiently bright spots of illumination be seen as luminous, and thus be treated effectively as "light sources" in the inference of spatial brightness?

Much more research is needed if we are to work out how to model the effects of light distribution, and the questions raised here may provide a blueprint to guide experiments. The effects of position appear critical. In our model attempts, for example, we found it very difficult to fit our results without using some form of weighting function that emphasised the centre or walls. However, we lack any systematic measurements of how location affects spatial brightness. Indeed, if experiments showed that location did not have significant effects, it would rule out the vast majority of our models, and suggest a need for significant rethinking. The next priority, we suggest, should be to attempt to understand surface light distributions. This is because (if our theory is correct) we can test those while avoiding confounding from light source effects, while the opposite is not true. We suggest a focus on examining the effects of pattern (for example: what happens if we look at checkerboards of different resolutions? do gradients have different effects?) and skewness. Skewed distributions would be highly relevant to the idea that the brightest areas are most influential, and the potential effects of "area" as observed in light ness studies and expressed in the Area Rule (Gilchrist, 2006). Manipulating surface distributions in that manner may thus be an ideal way to test those concepts. Once a useful model of surface effects has been built up, it would then be a good idea to examine whether or not illumination and 
reflectance should be conflated or treated separately. This, and more, is what is needed if we are to devise a useful model of spatial brightness. 


\section{Conclusions}

The general goal of the thesis was the practical measurement of the effects of light distribution on assessments of spatial brightness for the purpose of a) better describing the practical significance of light distribution effects (as most experiments had ignored the question), and b) facilitating the development of better quantitative models of light distribution and spatial brightness. Within this general concept, the ultimate focus of the thesis was the interrogation of disagreements in the light distribution/spatial brightness literature - in particular, on the apparent effect of uniformity.

The key points made by the two experiments may be summarised as follows:

- Basic observations and implications drawn from all experiments

- Effects of light distribution on spatial brightness could be substantial. Effects equivalent to a difference of $20 \%$ or more were observed repeatedly, and the largest differences were potentially equivalent to as much as a $100 \%$ increase in the overall light level.

- Contrary to the expectations of most past research, we repeatedly observed spaces with "non-uniform" light distributions appear brighter than "uniform" ones.

- Existing models of the effects of uniformity and spatial brightness do not predict these differences, suggesting they may not be reliable in practice.

- Inferences drawn (primarily) from the second experiment

○ "Uniformity of the luminance distribution" fails to predict observed patterns of spatial brightness. Results indicate both significant "uniform = brighter" and "non-uniform = brighter" effects. Continuing to attempt to predict observed light distribution effects using simple uniformity metrics appears unlikely to be productive, and is not recommended. 
- Evidence suggests that the best explanation we currently have for the disagreements between studies with regard to the effects of uniformity are apparent "Modes of Appearance" effects. When we create non-uniform patterns of illumination or surface reflectance we increase spatial brightness. When we create non-uniformity through the placement of visible light sources we decrease spatial brightness. It is thus suggested that we need to move beyond mere luminance and account for the qualitative modes of appearance of room elements, distinguishing between light sources and surfaces.

- Exploratory analysis and hypothesis generation for future work

- It is hypothesised that we can model the effects of light distribution on spatial brightness through a 'duel'-process model where the overall effect is the sum of two opposing processes: the effects of elements perceived as light sources, and the effects of elements perceived as illuminated surfaces.

- Such a model may, however, be implemented in a wide range of ways and with a wide range of assumptions, as explored in Chapter 6. Currently we lack the knowledge to identify the assumptions we should be making, and so we cannot make a mathematical model that could be used to make trustworthy quantifiable predictions.

These three "levels" of finding range from the basic observation of robust and repeated effects (shown repeatedly across multiple conditions, multiple experiments, and multiple methods), to post-hoc exploratory hypotheses. The level of confidence we should have in these findings similarly varies. The results of the second experiment, for instance, are based on both the repeated observation of an increase in brightness with non-uniform patterns of surface reflectance as well as a decrease in brightness when we try to make similar changes using the light sources. The light source effects have not been repeated to the same degree as the reflectance effects. However, the light source conditions and their observed effects are consistent with the work of Kato et al., and may be read as an independent replication of their (Kato and Hara, 2011; Kato and Sekiguchi, 2005; Kimura et al., 2004) observations. At the same time, while we might be confident in these basic observations we should be more cautious about the attempts to draw inferences about how we can or cannot model them. It is difficult to definitively rule out alternative models that have not yet been considered, and readers may reasonably be cautious about the leap required to suggest that it is unlikely that any simple luminance uniformity metric could ever work as a generalised spatial brightness model. Similarly, a challenge for comparison of the light source and reflectance conditions in Experiment 2 is that it is not really possible to generate light distributions that are perfectly identical beyond the Modes of Appearance being used. This 
is due to both the physical differences in how surface patterns and light sources work to produce light distributions, and also because if we did genuinely make them identical observers may be unable to accurately identify the Modes of Appearance being used. This makes it difficult to completely eliminate the possibility of alternative explanations that do not make specific reference to "light sources" or "surfaces".

We may also note some limitations with regards to the demonstration of large light distribution effects. The experimental effects found were much larger than the 5-10\% effect found by the previous practical estimate of such effects (Tiller and Veitch, 1995), and this emphasises the potential importance of work in the field. However, the conditions in these experiments were abstract distributions specifically designed to produce large effects. Effects in many "real" spaces may be substantially less. The reduction of the size of the effects in our second experiment compared to the first illustrates how significant changes to the light distribution may not necessarily produce significant changes to spatial brightness.

While acknowledging these caveats, however, we should also not understate the significance of these results for the field. Indeed, the most basic and reliable findings - the significant "non-uniform = brighter" effects — are in many ways the most important here. The literature had mostly concluded that non-uniformity makes spaces appear darker, and from there had focused on developing uniformity metrics with strong correlations in experiments. However, the direction of our observed effects, and the apparent failure of existing models to predict these results, challenges all of that. It suggests significant flaws with our prior understanding and a need to rethink the field's approach to the light distribution problem. Continuing to focus on identifying and refining simple uniformity metrics with good correlations seems to have questionable value. Indeed, if, as the findings of Experiment 2 suggest, increasing non-uniformity may make spaces a) appear darker, b) appear brighter, or possibly even c) not change at all, then arguably it does not even make sense to frame matters in terms of "the effect of uniformity on spatial brightness". We certainly should not assume it to be a reliable predictor of spatial brightness.

\subsection{Implications for lighting design}

The magnitude of our effects raise the possibility of worthwhile lighting energy savings by providing more efficient ways to create the brightness appearance that we desire in a space - though this may require a change in how we design lighting, for example moving towards Cuttle's suggested focus on "Perceived Adequacy of Illumination" (2013). They also highlight how light distribution decisions could have significant unintended effects on brightness if we lack a good understanding of how light distribution affects perception. The apparent need to differentiate between light sources and surfaces highlights the importance of going beyond mere luminance and considering the difference in how different elements 
actually appear, supporting Cuttle (2004). Going further, if the proposed 'duel'-process model holds true it would suggest that hiding the light source(s) (or luminous elements) can dramatically increase the brightness of a room by removing their negative perceptual effect. It also suggests that it is possible to counteract the effects of one process through the use of the other. Thus, while adjustments to uniformity (perhaps with the goal of producing visual interest (Loe et al., 2000)) may significantly affect overall brightness, the designer that understands these processes may have significant opportunity to adjust the brightness effect to their needs. Depending on the balance of light source and surface effects, brightness may be increased, decreased, or held constant at a desired level.

Philosophically, this understanding of the effects of light distribution ties in well to the more sophisticated uses of light discussed by lighting designers like Russell (2008) and Cuttle (2013). The framework of "uniformity of the luminance distribution has a simple relationship to spatial brightness" and "uniform lighting = brighter" fits neatly into a design paradigm in which the main goal is to uniformly illuminate the workplane. In contrast, the ideas here push towards a design attitude that is concerned more with the qualitative appearance of elements, how lighting and surface patterns and texture interact, and the opportunities that can be produced by the clever use of illumination diversity to create visual emphasis and interest.

\subsection{Implications for research}

The failure of the prior models may also prompt us to reflect upon the methods commonly used in the field of spatial brightness research, and the evidence that they provide (Chapter 4). Experimental studies examining -2-12 different conditions that systematically vary in some fashion can provide a) good evidence that light distribution affects spatial brightness, b) good evidence that it can affect spatial brightness significantly, and c) illustrate patterns that can be used to generate hypothesises. These are all valuable contributions. However, as has been illustrated throughout this thesis, they are often very bad at providing evidence for specific models. Indeed, we were able to readily produce 131 different models for our first experiment's results - all as well supported as any in the existing literature. The vast majority of them then failed when we attempted to extend them to our second experiment's data. One good correlation says very little. Future work should give more consideration to model assumptions if we are to produce anything robust.

Perhaps even more important, from a research perspective, is the apparent success of the Modes of Appearance hypothesis. Its success, and the failure of the tested alternative hypotheses (adaptation level, orientation, mere light source visibility), provide direction to future work and highlight the light distribution factors that we should be particularly concerned about. It suggests it is important to consider how one is producing the studied 
light distributions, and suggests a need to move beyond mere luminance. Technical advances such as HDR luminance mapping have provided us with great tools to measure and describe light distributions. However, we should be careful not to get stuck seeing the world through the eyes of our measurement tool. People are not luminance meters. The photometer is, as Waldram (1969) noted, a very poor eye.

That being said, we should be careful not to overreach here. While the Modes of Appearance hypothesis does seem to offer the potential for a framework that could unify the different observations in the literature, it is not necessarily perfect (Section 6.4). The 'duel'-process model is the result of post-hoc theorising and exploration, and should be treated as such. Nevertheless, we suggest that as we move beyond the framework of uniformity in our examination of light distribution effects (as it seems we should), that these hypotheses could provide a useful framework to direct future research. At the very least, we have strong reasons to consider that light sources and surfaces may have significantly different effects on spatial brightness and that this should be factored in to our research and experimental design.

We began this thesis with a question: what, in quantifiable terms, is the effect of light distribution on spatial brightness? Despite our advances, a comprehensive answer and the ability to actually predict the effect of an arbitrary light distribution is still a long way off. Many questions still need to be answered (Section 6.4). However, it is clear that the relationship, whatever it is, is not a simple correlation with a convenient measure of the uniformity of the luminance distribution. Our results have challenged not just the dominant framework in the literature (that of uniformity), but perhaps the most fundamental assumption in the literature: the assumption that spatial brightness is merely a function of the luminance distribution, and that the qualitative aspects of room appearance as described by the Modes of Appearance framework are not important. As we move forward then, we must consider not only the patterns we have observed, but also the research implications. It is well established that light distribution affects spatial brightness. The challenge that faces us now is the development of a robust model, and the need to carefully ensure that we know what assumptions we should be making. 


\section{References}

Akashi, Y., Tanabe, Y., Akashi, I., Mukai, K., 2000. Effect of sparkling luminous elements on the overall brightness impression: A pilot study. Light. Res. Technol. 32, 19-26. https://doi.org/10.1177/096032710003200103

Arend, L.E., Goldstein, R., 1990. Lightness and brightness over spatial illumination gradients. J. Opt. Soc. Am. A 7, 1929-1936.

Arend, L.E., Spehar, B., 1993a. Lightness, brightness, and brightness contrast: 1. Illuminance variation. Percept. Psychophys. 54, 446-456. https://doi.org/10.3758/BF03211767

Arend, L.E., Spehar, B., 1993b. Lightness, brightness, and brightness contrast: 2. Reflectance variation. Percept. Psychophys. 54, 457-468. https://doi.org/10.3758/BF03211768

Aya, K., Yamaguchi, H., Kato, M., Hara, N., Miki, Y., Yoshizawa, N., 2015a. The Spatial Brightness in the Non-uniform Illuminated Space, in: Summary of the Annual Meeting of the Architectural Institute of Japan. Architectural Institute of Japan, pp. 419-422.

Aya, K., Yoshizawa, N., Yamaguchi, H., Hara, N., Kato, M., Miki, Y., 2015b. The relationship between the brightness of overall space and the brightness in the specific visual field in the non-uniform illuminated space, in: Proceedings of 28th CIE Session 2015. CIE, Manchester, UK, pp. 1466-1470.

Bauer, B., 2009. Does Stevens's Power Law for Brightness Extend to Perceptual Brightness Averaging? Psychol. Rec. 59, 171-185.

Bean, A.R., 1978. Lighting of occupants and objects within an interior. Light. Res. Technol. 10, 146-149. https://doi.org/10.1177/096032717801000303

Bean, A.R., 1977. Impression of brightness of objects and interiors. Light. Res. Technol. 9, 103-106. https://doi.org/10.1177/096032717700900205

Bean, A.R., Hopkins, A.G., 1980. Task and background lighting. Light. Res. Technol. 12, 135-139. https://doi.org/10.1177/096032718001200303

Beck, J., 1961. Judgments of surface illumination and lightness. J. Exp. Psychol. 61, 368375. http://dx.doi.org/10.1037/h0044229

Beck, J., 1959. Stimulus correlates for the judged illumination of a surface. J. Exp. Psychol. 58, 267-274. http://dx.doi.org/10.1037/h0045132

Bernecker, C.A., Mier, J.M., 1985. The Effect of Source Luminance on the Perception of Environment Brightness. J. Illum. Eng. Soc. 15, 253-271. https://doi.org/10.1080/00994480.1985.10748647

Betancourt, M., 2017a. Robust Statistical Workflow with RStan [WWW Document]. URL https://betanalpha.github.io/assets/case_studies/rstan_workflow.html (accessed 3.25.19). 
Betancourt, M., 2017b. Diagnosing Biased Inference with Divergences [WWW Document]. URL https://mc-stan.org/users/documentation/casestudies/divergences_and_bias.html (accessed 8.27.19).

Blakeslee, B., Reetz, D., McCourt, M.E., 2008. Coming to terms with lightness and brightness: Effects of stimulus configuration and instructions on brightness and lightness judgments. J. Vis. 8, 3. https://doi.org/10.1167/8.11.3

Bodmann, H.W., Haubner, P., Marsden, A.M., 1979. A unified relationship between brightness and luminance, in: CIE Proceedings 19th Session. Kyoto, pp. 99-102.

Bodmann, H.W., Toison, M.L., 1994. Predicted brightness-luminance phenomena. Light. Res. Technol. 26, 135-143. https://doi.org/10.1177/096032719402600302

Boyce, P., 2014. Human Factors in Lighting, Third Edition. CRC Press.

Boyce, P. r., 1996. Illuminance Selection Based on Visual Performance-and other Fairy Stories. J. Illum. Eng. Soc. 25, 41-49. https://doi.org/10.1080/00994480.1996.10748146

Boyce, P.R., 2003. Human Factors in Lighting, 2nd ed. ed. Taylor \& Francis, London ; New York.

Boyce, P.R., 2001. Discussion of previously published papers Comment 1 on 'Effect of thermal conditions and light source type on visual comfort appraisal' by $\mathrm{C}$ Laurentin, V Berrutto and M Fontoynont. Light. Res. Technol. 33, 137-137. https://doi.org/10.1177/136578280103300213

Boyce, P.R., 1985. Movement under emergency lighting: the effect of illuminance. Light. Res. Technol. 17, 51-71. https://doi.org/10.1177/14771535850170020401

Boyce, P.R., 1979. Users' attitudes to some types of local lighting. Light. Res. Technol. 11, 158-164. https://doi.org/10.1177/14771535790110030501

Boyce, P.R., 1977. Investigations of the subjective balance between illuminance and lamp colour properties. Light. Res. Technol. 9, 11-24. https://doi.org/10.1177/096032717700900102

Boyce, P.R., 1976. Illuminance, lamp type and performance on a colour discrimination task. Light. Res. Technol. 8, 195-199. https://doi.org/10.1177/14771535760080040401

Boyce, P.R., 1974. Illuminance, difficulty, complexity and visual performance. Light. Res. Technol. 6, 222-226. https://doi.org/10.1177/096032717400600406

Boyce, P.R., 1973. Age, illuminance, visual performance and preference. Light. Res. Technol. 5, 125-144. https://doi.org/10.1177/096032717300500301

Boyce, P.R., 1970. The influence of illumination level on prolonged work performance. Light. Res. Technol. 2, 74-94. https://doi.org/10.1177/14771535700020020801

Boyce, P.R., Hunter, C.M., Inclan, C., 2003. Overhead Glare and Visual Discomfort. J. Illum. Eng. Soc. 32, 73-88. https://doi.org/10.1080/00994480.2003.10748406

Boyce, P.R., Veitch, J.A., Newsham, G.R., Jones, C.C., Heerwagen, J., Myer, M., Hunter, C.M., 2006. Lighting quality and office work: two field simulation experiments. Light. Res. Technol. 38, 191-223. https://doi.org/10.1191/1365782806lrt161oa

Bullough, J., Rea, M.S., 1996. Lighting for neonatal intensive care units: Some critical information for design. Light. Res. Technol. 28, 189-198. https://doi.org/10.1177/14771535960280040201 
Butler, D.L., Biner, P.M., 1987. Preferred Lighting Levels: Variability among Settings, Behaviors, and Individuals. Environ. Behav. 19, 695-721. https://doi.org/10.1177/0013916587196003

Carpenter, B., Gelman, A., Hoffman, M.D., Lee, D., Goodrich, B., Betancourt, M., Brubaker, M., Guo, J., Li, P., Riddell, A., 2017. Stan: A Probabilistic Programming Language. J. Stat. Softw. 76, 1-32. https://doi.org/10.18637/jss.v076.i01

Cauwerts, C., 2013. Influence of presentation modes on visual perceptions of daylit spaces. Université catholique de Louvain, Louvain-la-Neuve.

Chacón, J., Castellanos, M.Á., Serrano-Pedraza, I., 2015. Characterizing visual asymmetries in contrast perception using shaded stimuli. J. Vis. 15, 11-11. https://doi.org/10.1167/15.16.11

Charness, N., Dijkstra, K., 1999. Age, Luminance, and Print Legibility in Homes, Offices, and Public Places. Hum. Factors J. Hum. Factors Ergon. Soc. 41, 173-193. https://doi.org/10.1518/001872099779591204

[CIE] Commission Internationale de l'Éclairage, 2016. ILV: International lighting vocabulary, 2nd edition (No. CIE DIS 017/E:2016). CIE, Vienna, Austria.

[CIE] Commission Internationale de l'Éclairage, 2014. Guidance Towards Best Practice in Psychophysical Procedures Used When Measuring Relative Spatial Brightness (CIE Report 212:2014). CIE, Vienna, Austria.

Cohen, J., Cohen, P., West, S.G., Aiken, L.S., 2003. Applied Multiple Regression/Correlation Analysis for the Behavioral Sciences. Lawrence Erlbaum Associates. Inc.

Collins, B.L., Fisher, W., Gillette, G., Marans, R.W., 1990. Second-Level Post-Occupancy Evaluation Analysis. J. Illum. Eng. Soc. 19, 21-44. https://doi.org/10.1080/00994480.1990.10747961

Cumming, G., 2014. The New Statistics Why and How. Psychol. Sci. 25, 7-29. https://doi.org/10.1177/0956797613504966

Cumming, G., Finch, S., 2005. Inference by eye: confidence intervals and how to read pictures of data. Am. Psychol. 60, 170-180. https://doi.org/10.1037/0003066X.60.2.170

Cundall, G.P., 1972. Value for money-interior lighting. Light. Res. Technol. 4, 223235. https://doi.org/10.1177/096032717200400404

Cuttle, C., 2013. A new direction for general lighting practice. Light. Res. Technol. 45, 22-39. https://doi.org/10.1177/1477153512469201

Cuttle, C., 2010. Towards the third stage of the lighting profession. Light. Res. Technol. 42, 73-93. https://doi.org/10.1177/1477153509104013

Cuttle, C., 2004. Brightness, lightness, and providing 'a preconceived appearance to the interior.' Light. Res. Technol. 36, 201-214. https://doi.org/10.1191/1365782804li115oa

de Vries, H.J.A., Heynderickx, I.E.J., de Kort, Y.A.W., de Ruyter, B., 2015. Wall illumination - beyond room appraisal, in: Proceedings of 28th CIE Session 2015. CIE, Manchester, UK, pp. 284-290.

Diamond, A.L., 1955. Foveal simultaneous contrast as a function of inducing-field area. J. Exp. Psychol. 50, 144-152. http://dx.doi.org/10.1037/h0045936 
Dongen, N.N.N. van, Doorn, J.B. van, Gronau, Q.F., Ravenzwaaij, D. van, Hoekstra, R., Haucke, M.N., Lakens, D., Hennig, C., Morey, R.D., Homer, S., Gelman, A., Sprenger, J., Wagenmakers, E.-J., 2019. Multiple Perspectives on Inference for Two Simple Statistical Scenarios. Am. Stat. 73, 328-339. https://doi.org/10.1080/00031305.2019.1565553

Duff, J., Antonutto, G., Torres, S., 2015a. On the calculation and measurement of mean room surface exitance. Light. Res. Technol. 1477153515593579. https://doi.org/10.1177/1477153515593579

Duff, J., Kelly, K., Cuttle, C., 2015b. Spatial brightness, horizontal illuminance and mean room surface exitance in a lighting booth. Light. Res. Technol. 1477153515597733. https://doi.org/10.1177/1477153515597733

Duff, J., Kelly, K., Cuttle, C., 2015c. Perceived adequacy of illumination, spatial brightness, horizontal illuminance and mean room surface exitance in a small office. Light. Res. Technol. 1477153515599189. https://doi.org/10.1177/1477153515599189

Eaton, J.W., Bateman, D., Hauberg, S., Wehbring, R., 2019. GNU Octave version 5.1.0 manual: a high-level interactive language for numerical computations [WWW Document]. URL https://www.gnu.org/software/octave/doc/v5.1.0/ (accessed 7.29.19).

Escuyer, S., Fontoynont, M., 2001. Lighting controls: a field study of office workers' reactions. Light. Res. Technol. 33, 77-94. https://doi.org/10.1177/136578280103300202

Fagot, R.F., Stewart, M.R., 1969. Individual half-judgment brightness functions. Percept. Psychophys. 5, 165-170. https://doi.org/10.3758/BF03209551

Fischer, D., 1973. Comparison of some European interior lighting recommendations. Light. Res. Technol. 5, 186-190. https://doi.org/10.1177/096032717300500402

Flynn, J.E., 1977. A study of subjective responses to low energy and nonuniform lighting systems. Light. Des. Appl. 7, 6-15.

Flynn, J.E., Spencer, T.J., Martyniuk, O., Hendrick, C., 1973. Interim Study of Procedures for Investigating the Effect of Light on Impression and Behavior. J. Illum. Eng. Soc. 3, 87-94. https://doi.org/10.1080/00994480.1973.10732231

Fotios, S., Atli, D., 2012. Comparing Judgments of Visual Clarity and Spatial Brightness through an Analysis of Studies Using the Category Rating Procedure. LEUKOS 8, 261-281. https://doi.org/10.1582/LEUKOS.2012.08.04.002

Fotios, S., Atli, D., Cheal, C., Hara, N., 2014. Lamp spectrum and spatial brightness at photopic levels: Investigating prediction using S/P ratio and gamut area. Light. Res. Technol. 1477153514542295. https://doi.org/10.1177/1477153514542295

Fotios, S., Atli, D., Cheal, C., Houser, K., Logadóttir, Á., 2015. Lamp spectrum and spatial brightness at photopic levels: A basis for developing a metric. Light. Res. Technol. 47, 1477153513503170. https://doi.org/10.1177/1477153513503170

Fotios, S., Gado, T., 2005. A comparison of visual objectives used in side-by-side matching tests. Light. Res. Technol. 37, 117-130.

https://doi.org/10.1191/1365782805li133oa 
Fotios, S., Levermore, G.J., 1997. Perception of electric light sources of different colour properties. Light. Res. Technol. 29, 161-171. https://doi.org/10.1177/14771535970290030701

Fotios, S.A., 2001. An error in brightness matching associated with the application of dimming. Light. Res. Technol. 33, 223-229. https://doi.org/10.1177/136578280103300405

Fotios, S.A., Cheal, C., 2011. Brightness matching with visual fields of different types. Light. Res. Technol. 43, 73-85. https://doi.org/10.1177/1477153510369478

Fotios, S.A., Cheal, C., 2010. Stimulus range bias explains the outcome of preferredilluminance adjustments. Light. Res. Technol. 42, 433-447. https://doi.org/10.1177/1477153509356018

Fotios, S.A., Cheal, C., 2008. The effect of a stimulus frequency bias in side-by-side brightness ranking tests. Light. Res. Technol. 40, 43-54. http://dx.doi.org/10.1177/1477153507082001

Fotios, S.A., Cheal, C., 2007a. Lighting for subsidiary streets: investigation of lamps of different SPD. Part 2-Brightness. Light. Res. Technol. 39, 233-249. https://doi.org/10.1177/1477153507080805

Fotios, S.A., Cheal, C., 2007b. Evidence for response contraction bias in side-by-side matching tasks. Light. Res. Technol. 39, 159-169. https://doi.org/10.1177/1365782807076490

Fotios, S.A., Houser, K.W., 2013. Using Forced Choice Discrimination to Measure the Perceptual Response to Light of Different Characteristics. LEUKOS 9, 245-259. https://doi.org/10.1582/LEUKOS.2013.09.04.002

Fotios, S.A., Houser, K.W., Cheal, C., 2008. Counterbalancing Needed to Avoid Bias in Side-By-Side Brightness Matching Tasks. LEUKOS 4, 207-223. https://doi.org/10.1582/LEUKOS.2008.04.04.001

Fotios, S.A., Levermore, G.J., 1995. Visual perception under tungsten lamps with enhanced blue spectrum. Light. Res. Technol. 27, 173-179. https://doi.org/10.1177/14771535950270040801

Frick, R.W., 1998. A better stopping rule for conventional statistical tests. Behav. Res. Methods Instrum. Comput. 30, 690-697. https://doi.org/10.3758/BF03209488

Furnham, A., Boo, H.C., 2011. A literature review of the anchoring effect. J. Socio-Econ. 40, 35-42. https://doi.org/10.1016/j.socec.2010.10.008

Gelman, A., Carlin, J.B., Stern, H.S., Dunson, D.B., Vehtari, A., Rubin, D.B., 2013. Bayesian Data Analysis, 3 edition. ed. Chapman and Hall/CRC, Boca Raton.

Gelman, A., Hennig, C., 2017. Beyond subjective and objective in statistics. J. R. Stat. Soc. Ser. A Stat. Soc. 180, 967-1033. https://doi.org/10.1111/rssa.12276

Gelman, A., Lee, D., Guo, J., 2015. Stan: A Probabilistic Programming Language for Bayesian Inference and Optimization. J. Educ. Behav. Stat. 40, 530-543. https://doi.org/10.3102/1076998615606113

Gelman, A., Loken, E., 2013. The garden of forking paths: Why multiple comparisons can be a problem, even when there is no "fishing expedition" or "p-hacking" and the research hypothesis was posited ahead of time. 
Gilchrist, A.L., 2006. Seeing black and white, Oxford psychology series. Oxford University Press, Oxford ; New York.

Greenstein, V.C., Hood, D.C., 1981. Variations in brightness at two retinal locations. Vision Res. 21, 885-891. https://doi.org/10.1016/0042-6989(81)90189-9

Hirning, M.B., 2014. The application of luminance mapping to discomfort glare : a modified glare index for green buildings (Thesis). Queensland University of Technology.

Hopkinson, R.G., Collins, J.B., 1970. The Ergonomics of Lighting. Macdonald \& Co. (Publishers) Ltd, London.

Houser, K.W., Tiller, D.K., Bernecker, C.A., Mistrick, R.G., 2002. The subjective response to linear fluorescent direct/indirect lighting systems. Light. Res. Technol. 34, 243-260. https://doi.org/10.1191/1365782802li039oa

Houser, K.W., Tiller, D.K., Hu, X., 2003. Prototype demonstration of vision-tuned fluorescent lamps. Final report for California Energy Commission.

Hsieh, M., 2012. The energy-saving effect and prediction method under various illuminance distribution types. Build. Environ. 58, 145-151. https://doi.org/10.1016/j.buildenv.2012.07.001

Inanici, M.N., 2006. Evaluation of high dynamic range photography as a luminance data acquisition system. Light. Res. Technol. 38, 123-134. https://doi.org/10.1191/1365782806li164oa

Ishida, T., Ogiuchi, Y., 2002. Psychological Determinants of Brightness of a Space Perceived Strength of Light Source and Amount of Light in the Space. J. Light Vis. Environ. 26, 29-35.

Ishida, T., Ogiuchi, Y., 1999. Evaluation of Brightness of a Space Using Reference Matching Method. J. Illum. Eng. Inst. Jpn. 83, 295-305.

Iwai, W., Saito, Y., Sumi, S., Sakaguti, T., 2001. Sensation of Brightness for a Living Room with Downlights. J. Light Vis. Environ. 25, 1_66-1_72. https://doi.org/10.2150/jlve.25.1_66

Jakubiec, J.A., Van Den Wymelenberg, K., Inanici, M., Mahić, A., 2016. Accurate Measurement of Daylit Interior Scenes using High Dynamic Range Photography, in: Proceedings of CIE 2016 "Lighting Quality and Energy Efficiency." CIE, Melbourne, Australia, pp. 42-52.

Jones, L.A., 1953. The Concept of Color, in: The Science of Color. Crowell, New York, pp. 45-68.

Jones, L.A., 1943. The concept of color. J. Opt. Soc. Am. 33, 544-554.

Judd, D.B., 1961. A five-attribute system of describing visual appearance. American Society for Testing Materials, Philadelphia.

Julian, W.G., 1987. Lighting: an urgent case for a major research effort in architectural science. Build. Environ., A Special Issue in Honour of Henry J. Cowan, A.O. 22, 155-161. https://doi.org/10.1016/0360-1323(87)90003-5

Juslén, H.T., Wouters, M., Tenner, A.D., 2005. Preferred task-lighting levels in an industrial work area without daylight. Light. Res. Technol. 37, 219-231. https://doi.org/10.1191/1365782805li138oa 
Kardos, L., 1934. Ding und Schatten. Eine experimentelle Untersuchung über die Grundlagen des Farbensehens. [Thing and shadow. An experimental investigation on the basis of color vision.]. Z. Für Psychol. Physiol. Sinnesorgane Abt 1 Z. Für Psychol. Suppl. 23, 184.

Kato, M., Aya, K., Yamaguchi, H., Yoshizawa, N., Hara, N., Miki, Y., 2016. Evaluation Method of Spatial Brightness by Directional Diffusivity and Mean Luminance, in: Proceedings of CIE 2016 "Lighting Quality and Energy Efficiency." CIE, Melbourne, Australia, pp. 664-668.

Kato, M., Hara, N., 2011. Influence that luminance, size, and position of luminous surface give to "Impression of brightness of a space" [in Japanese]. Summ. Tech. Pap.

Annu. Meet. Archit. Inst. Jpn. 2011, 475-476.

Kato, M., Sekiguchi, K., 2005. "Impression of Brightness of a Space" Judged by Information from the Entire Space. J. Light Vis. Environ. 29, 123-134. https://doi.org/10.2150/jlve.29.123

Katz, D., 1935. The World Of Colour. Kegan Paul, Trench, Trubner \& Co., Ltd., London.

Kim, W., Kim, J.T., 2011. A Position Index Formula for Evaluation of Glare Source in the Visual Field. Indoor Built Environ. 20, 47-53. https://doi.org/10.1177/1420326X10389267

Kim, W., Kim, J.T., 2010. A distribution chart of glare sensation over the whole visual field. Build. Environ. 45, 922-928. https://doi.org/10.1016/j.buildenv.2009.09.013

Kimura, M., Kato, M., Sekiguchi, K., 2004. Spatial Brightness seen from Layout and Area of Window. Presented at the Proceedings of Annual Conference of The Illuminating Engineering Institute of Japan Proceedings of 2004 Annual Conference of The Illuminating Engineering Institute of Japan, The Illuminating Engineering Institute of Japan, pp. 222-223. https://doi.org/10.11515/ieijac.37.0.117.0

Kingdom, F.A., Prins, N., 2016. Psychophysics: A Practical Introduction, 2nd ed. Elsevier. https://doi.org/10.1016/C2012-0-01278-1

Kingdom, F.A.A., 2011. Lightness, brightness and transparency: A quarter century of new ideas, captivating demonstrations and unrelenting controversy. Vision Res., Vision Research 50th Anniversary Issue: Part 1 51, 652-673. https://doi.org/10.1016/j.visres.2010.09.012

Kingdom, F.A.A., 2008. Perceiving light versus material. Vision Res., Vision Research Reviews 48, 2090-2105. https://doi.org/10.1016/j.visres.2008.03.020

Kirsch, R., 2014. Lighting Quality and Energy Efficiency in Office Spaces (Ph.D. Thesis). Technical University Berlin, Berlin.

Ko, B., Koga, T., Lu, B., Hirate, K., Mizuno, M., Suzuki, N., 2013. Brightness of a Space in Terms of Variation of Luminance. J. Illum. Eng. Inst. Jpn. 97, 429-435. https://doi.org/10.2150/jieij.97.429

Kobayashi, S., Nakamura, Y., Inui, M., 1998. Impressions of Overall Brightness in a NonUniformly Illuminated Space. J. Light Vis. Environ. 22, 1_34-1_41. https://doi.org/10.2150/jlve.22.1_34 
Kozaki, A., 1973. Perception of lightness and brightness of achromatic surface color and impression of illumination. Jpn. Psychol. Res. 15, 194-203.

Kruschke, J.K., 2015. Doing Bayesian Data Analysis, Second Edition: A Tutorial with R, JAGS, and Stan, 2nd ed. Academic Press / Elsevier.

Kruschke, J.K., 2013. Bayesian estimation supersedes the t test. J. Exp. Psychol. Gen. 142, 573-603. https://doi.org/10.1037/a0029146

Kruschke, J.K., Liddell, T.M., 2017. Bayesian data analysis for newcomers. Psychon. Bull. Rev. 1-23. https://doi.org/10.3758/s13423-017-1272-1

Kruschke, J.K., Liddell, T.M., 2015. The Bayesian New Statistics: Two Historical Trends Converge (SSRN Scholarly Paper No. ID 2606016). Social Science Research Network, Rochester, NY.

Lakens, D., 2019. The practical alternative to the p-value is the correctly used p-value (preprint). PsyArXiv. https://doi.org/10.31234/osf.io/shm8v

Lakens, D., 2014. Performing High-Powered Studies Efficiently with Sequential Analyses (SSRN Scholarly Paper No. ID 2333729). Social Science Research Network, Rochester, NY.

Lee, M.D., Vanpaemel, W., 2017. Determining informative priors for cognitive models. Psychon. Bull. Rev. 1-14. https://doi.org/10.3758/s13423-017-1238-3

Levin, A., Fergus, R., Durand, F., Freeman, W.T., 2007. Image and Depth from a Conventional Camera with a Coded Aperture, in: ACM SIGGRAPH 2007 Papers, SIGGRAPH '07. ACM, New York, NY, USA. https://doi.org/10.1145/1275808.1276464

Lindner, H., Hübmer, K., Schlote, H.W., Röhl, F., 1989. Subjective lighting needs of the old and the pathological eye. Light. Res. Technol. 21, 1-10. https://doi.org/10.1177/096032718902100101

Loe, D.L., Mansfield, K.P., Rowlands, E., 2000. A step in quantifying the appearance of a lit scene. Light. Res. Technol. 32, 213-222. https://doi.org/10.1177/096032710003200405

Loe, D.L., Mansfield, K.P., Rowlands, E., 1994. Appearance of lit environment and its relevance in lighting design: Experimental study. Light. Res. Technol. 26, 119133. https://doi.org/10.1177/096032719402600301

Loe, D.L., Mansfield, K.P., Rowlands, E., 1991. Light patterns and their relevance to spatial appearance and the quality of the lit environment, in: Proceedings CIE 22nd Session. CIE, Melbourne, Australia, pp. 41-44.

Logadóttir, Á., Christoffersen, J., Fotios, S.A., 2011. Investigating the use of an adjustment task to set the preferred illuminance in a workplace environment. Light. Res. Technol. 43, 403-422. https://doi.org/10.1177/1477153511400971

Longmore, J., 1969. Comment on: The role of the level and diversity of horizontal illumination in an appraisal of a simple office task. Light. Res. Technol. 1, 44-45. https://doi.org/10.1177/14771535690010010801

Lu, X., Aya, K., Yamaguchi, H., Kato, M., Hara, N., Miki, Y., Yoshizawa, N., 2016. Development of spatial brightness evaluation method with luminance distribution; (part2) Spatial brightness evaluation formula in luminance non-uniformity, in: 
Summary of the Annual Meeting of the Architectural Institute of Japan.

Architectural Institute of Japan, pp. 553-554.

Lynes, J.A., 1983. Vision, in: Cayless, M.A., Marsden, A.M. (Eds.), Lamps and Lighting: A Manual of Lamps and Lighting. Edward Arnold, London, pp. 23-43.

Lynes, J.A., 1971. Lightness, Colour and Constancy in Lighting Design. Light. Res. Technol. 3, 24-42. https://doi.org/10.1177/147715357100300110

Lynes, J.A., 1970. Cylindrical or scalar illumination? Light. Res. Technol. 2, 265-266. https://doi.org/10.1177/14771535700020040701

Magister, B.M.O., Colombo, E.M., 2001. Inner contrast and perceptual quality in tasks with video display units. Light. Res. Technol. 33, 145-159. https://doi.org/10.1177/136578280103300302

Mahdavi, A., Eissa, H., 2002. Subjective Evaluation of Architectural Lighting via Computationally Rendered Images. J. Illum. Eng. Soc. 31, 11-20. https://doi.org/10.1080/00994480.2002.10748388

Mansfield, K.P., 2016. Personal communication.

Marks, L.E., Stevens, J.C., 1966. Individual brightness functions. Percept. Psychophys. 1, 17-24. https://doi.org/10.3758/BF03207815

Marsden, A.M., 1970. Brightness-luminance relationships in an interior. Light. Res. Technol. 2, 10-16. https://doi.org/10.1177/14771535700020010401

Martin, S.R., Williams, D.R., 2017. Outgrowing the Procrustean Bed of Normality: The Utility of Bayesian Modeling for Asymmetrical Data Analysis. https://doi.org/10.31234/osf.io/26m49

Mayo, D.G., 2018. Statistical Inference as Severe Testing: How to Get Beyond the Statistics Wars, in: Statistical Inference as Severe Testing: How to Get Beyond the Statistics Wars. Cambridge University Press, pp. i-i.

Mayo, D.G., Spanos, A., 2010. Error and Inference: Recent Exchanges on Experimental Reasoning, Reliability, and the Objectivity and Rationality of Science. Cambridge University Press.

McCourt, M.E., Blakeslee, B., Padmanabhan, G., 2013. Lighting direction and visual field modulate perceived intensity of illumination. Percept. Sci. 4, 983. https://doi.org/10.3389/fpsyg.2013.00983

McElreath, R., 2016. Statistical rethinking: a Bayesian course with examples in R and Stan, Texts in statistical science. CRC Press/Taylor \& Francis Group, Boca Raton.

McGuiness, P.J., Boyce, P.R., 1984. The effect of illuminance on the performance of domestic kitchen work by two age groups. Light. Res. Technol. 16, 131-136. https://doi.org/10.1177/14771535840160030601

Mills, E., Borg, N., 1999. Trends in Recommended Illuminance Levels: An International Comparison. J. Illum. Eng. Soc. 28, 155-163. https://doi.org/10.1080/00994480.1999.10748262

Moore, T., Carter, D.J., Slater, A.I., 2003. A qualitative study of occupant controlled office lighting. Light. Res. Technol. 35, 297-314.

https://doi.org/10.1191/1365782803li090oa 
Moore, T., Carter, D.J., Slater, A.I., 2002a. A field study of occupant controlled lighting in offices. Light. Res. Technol. 34, 191-202. https://doi.org/10.1191/1365782802lt047oa

Moore, T., Carter, D.J., Slater, A.I., 2002b. User attitudes toward occupant controlled office lighting. Light. Res. Technol. 34, 207-216. https://doi.org/10.1191/1365782802lt048oa

Morey, R.D., Hoekstra, R., Rouder, J.N., Lee, M.D., Wagenmakers, E.-J., 2015. The fallacy of placing confidence in confidence intervals. Psychon. Bull. Rev. 1-21. https://doi.org/10.3758/s13423-015-0947-8

Newsham, G.R., Cetegen, D., Veitch, J.A., Whitehead, L., 2010. Comparing Lighting Quality Evaluations of Real Scenes with Those from High Dynamic Range and Conventional Images. ACM Trans Appl Percept 7, 13:1-13:26. https://doi.org/10.1145/1670671.1670677

Newsham, G.R., Marchand, R.G., Veitch, J.A., 2004. Preferred surface luminances in offices, by evolution (No. NRCC-46976). NRC Institute for Research in Construction, Ottawa, ON.

Newsham, G.R., Richardson, C., Blanchet, C., Veitch, J.A., 2005. Lighting quality research using rendered images of offices. Light. Res. Technol. 37, 93-112. https://doi.org/10.1191/1365782805li132oa

Noguchi, K., Masuda, N., 1971. Brightness Changes in a Complex Field with Changing Illumination. Jpn. Psychol. Res. 13, 60-69. https://doi.org/10.4992/psycholres 1954.13.60

Nosek, B.A., Ebersole, C.R., DeHaven, A.C., Mellor, D.T., 2018. The preregistration revolution. Proc. Natl. Acad. Sci. 115, 2600-2606. https://doi.org/10.1073/pnas.1708274114

Oi, N., Mansfield, K.P., 2015. Lighting quality: possibility of luminance distribution as its determinant, in: Proceedings of 28th CIE Session 2015. CIE, Manchester, UK, pp. 1111-1120.

Okajima, K., Fujimoto, S., 2008. Effect of Spatial-Frequency Distribution on Brightness Perception in Light Environment. J Illum Engng Inst Jpn 92, 77-82.

Osaka, N., 1980. Brightness exponent as a function of retinal eccentricity in the peripheral visual field: Effects of dark and light adaptation. Percept. Psychophys. 27, 519-523. https://doi.org/10.3758/BF03198679

Perry, M.J., Campbell, F.W., Rothwell, S.E., 1987. A physiological phenomenon and its implications for lighting design. Light. Res. Technol. 19, 1-5. https://doi.org/10.1177/096032718701900101

Pöppel, E., Harvey Jr, L.O., 1973. Light-difference threshold and subjective brightness in the periphery of the visual field. Psychol. Forsch. 36, 145-161. https://doi.org/10.1007/BF00424967

Popper, K.R., 1963. Conjectures and Refutations: The Growth of Scientific Knowledge. Routledge.

Poulton, E.C., 1989. Bias in Quantifying Judgements. Taylor \& Francis.

Poulton, E.C., 1979. Models for biases in judging sensory magnitude. Psychol. Bull. 86, 777-803. http://dx.doi.org/10.1037/0033-2909.86.4.777 
Poulton, E.C., 1977. Quantitative subjective assessments are almost always biased, sometimes com... Br. J. Psychol. 68, 409.

R Core Team, 2016. R: A Language and Environment for Statistical Computing. R Foundation for Statistical Computing, Vienna, Austria.

Rea, M., Bullough, J., Brons, J., 2017. Parking lot lighting based upon predictions of scene brightness and personal safety. Light. Res. Technol. 49, 293-304. https://doi.org/10.1177/1477153515603758

Rea, M.S., 2014. The lumen seen in a new light: Making distinctions between light, lighting and neuroscience. Light. Res. Technol. 1477153514527599. https://doi.org/10.1177/1477153514527599

Rea, M.S., Boyce, P.R., Ouellette, M.J., 1987. On time to see. Light. Res. Technol. 19, 101-103. https://doi.org/10.1177/096032718701900403

Rea, M.S., Mou, X., Bullough, J.D., 2015. Scene brightness of illuminated interiors. Light. Res. Technol. 1477153515581412. https://doi.org/10.1177/1477153515581412

Rea, M.S., Ouellette, M.J., 1991. Relative visual performance: A basis for application. Light. Res. Technol. 23, 135-144. https://doi.org/10.1177/096032719102300301

Rea, M.S., Ouellette, M.J., 1988. Visual performance using reaction times. Light. Res. Technol. 20, 139-153. https://doi.org/10.1177/096032718802000401

Rockcastle, S.F., Chamilothori, K., Andersen, M., 2017. An Experiment in Virtual Reality to Measure Daylight-Driven Interest in Rendered Architectural Scenes, in: Proceedings of Building Simulation 2017. Presented at the Building Simulation 2017, San Francisco, California, USA.

Rouder, J.N., Speckman, P.L., Sun, D., Morey, R.D., Iverson, G., 2009. Bayesian t tests for accepting and rejecting the null hypothesis. Psychon. Bull. Rev. 16, 225-237. https://doi.org/10.3758/PBR.16.2.225

Roufs, J. a. J., 1978. Light as a true visual quantity : principles of measurement.

Royer, M.P., Houser, K.W., 2012. Spatial Brightness Perception of Trichromatic Stimuli. LEUKOS 9, 89-108. https://doi.org/10.1582/LEUKOS.2012.09.02.002

Rudd, M.E., 2013. Edge integration in achromatic color perception and the lightnessdarkness asymmetry. J. Vis. 13, 18. https://doi.org/10.1167/13.14.18

Russell, S., 2008. The Architecture of Light. Conceptnine, La Jolla, CA.

Saunders, J.E., 1969. The role of the level and diversity of horizontal illumination in an appraisal of a simple office task. Light. Res. Technol. 1, 37-46. https://doi.org/10.1177/14771535690010010801

Schirillo, J.A., 2013. We infer light in space. Psychon. Bull. Rev. 20, 905-15.

Simmons, J.P., Nelson, L.D., Simonsohn, U., 2011. False-Positive Psychology: Undisclosed Flexibility in Data Collection and Analysis Allows Presenting Anything as Significant. Psychol. Sci. 22, 1359-1366.

https://doi.org/10.1177/0956797611417632

Soranzo, A., Gilchrist, A., 2019. Layer and framework theories of lightness. Atten. Percept. Psychophys. https://doi.org/10.3758/s13414-019-01736-1

Stan Development Team, 2018. RStan: the R interface to Stan. 
Standards Association of Australia, Standards New Zealand, 2006. NZS 1680.1: Interior and workplace lighting, Australian/New Zealand standard. Standards Australia ; Standards New Zealand, Sydney, N.S.W. : Wellington, N.Z.

Stevens, J.C., Stevens, S.S., 1963. Brightness function: Effects of adaptation. J. Opt. Soc. Am. 53, 375-385. http://dx.doi.org/10.1364/JOSA.53.000375

Stevens, S.S., 1966. Duration, luminance, and the brightness exponent. Percept. Psychophys. 1, 96-100. https://doi.org/10.3758/BF03210035

Stevens, S.S., 1961. To Honor Fechner and Repeal His Law. Science, New Series 133, 80 86.

Stevens, S.S., 1960. The psychophysics of sensory function. Am. Sci. 48, 226-253.

Strasburger, H., Rentschler, I., Jüttner, M., 2011. Peripheral vision and pattern recognition: A review. J. Vis. 11, 13-13. https://doi.org/10.1167/11.5.13

Sullivan, J.T., Donn, M.R., 2017. Measuring the Effect of Light Distribution on Spatial Brightness, in: Proceedings of CIE 2017 "Smarter Lighting for Better Life." CIE, Jeju, South Korea.

Sullivan, J.T., Donn, M.R., 2016. Light Distribution and Spatial Brightness: Relative Importance of the Walls, Ceiling, and Floor, in: Proceedings of CIE 2016 "Lighting Quality and Energy Efficiency." CIE, Melbourne, Australia, pp. 59-69.

Teghtsoonian, M., Teghtsoonian, R., 1971. How repeatable are Stevens's power law exponents for individual subjects? Percept. Psychophys. 10, 147-149. https://doi.org/10.3758/BF03205774

Teghtsoonian, R., 1973. Range Effects in Psychophysical Scaling and a Revision of Stevens' Law. Am. J. Psychol. 86, 3-27. https://doi.org/10.2307/1421845

Teghtsoonian, R., 1971. On the exponents in Stevens' law and the constant in Ekman's law. Psychol. Rev. 78, 71-80. http://dx.doi.org/10.1037/h0030300

Teller, D.Y., Pereverzeva, M., Civan, A.L., 2003. Adult brightness vs. luminance as models of infant photometry: Variability, biasability, and spectral characteristics for the two age groups favor the luminance model. J. Vis. 3, 2. https://doi.org/10.1167/3.5.2

Thornton, W.A., Chen, E., 1978. What is Visual Clarity? J. Illum. Eng. Soc. 7, 85-94. https://doi.org/10.1080/00994480.1978.10747827

Thouless, R.H., 1936. Critical Notice. Br. J. Psychol. Gen. Sect. 26, 309-313. https://doi.org/10.1111/j.2044-8295.1936.tb00799.x

Tiller, D.K., 1990. Toward a Deeper Understanding of Psychological Aspects of Lighting. J. Illum. Eng. Soc. 19, 59-65. https://doi.org/10.1080/00994480.1990.10747963

Tiller, D.K., Veitch, J.A., 1995. Perceived room brightness: Pilot study on the effect of luminance distribution. Light. Res. Technol. 27, 93-101. https://doi.org/10.1177/14771535950270020401

Tiller, D.K., Veitch, J.A., Pasini, I.C., 1995. The effects of luminance distribution on perceived room brightness, in: Proceedings of the Commission Internationale de l'Éclairage 23rd Session. CIE, New Delhi, India, pp. 144-147.

Tregenza, P.R., Romaya, S.M., Dawe, S.P., Heap, L.J., Tuck, B., 1974. Consistency and variation in preferences for office lighting. Light. Res. Technol. 6, 205-211. https://doi.org/10.1177/096032717400600403 
Uttley, J., Fotios, S., Cheal, C., 2012. Satisfaction and illuminances set with usercontrolled lighting. Archit. Sci. Rev. 1-9. https://doi.org/10.1080/00038628.2012.724380

Van Den Wymelenberg, K.G., 2012. Evaluating Human Visual Preference and Performance in an Office Environment Using Luminance-based Metrics (Ph.D.). University of Washington, United States -- Washington.

van Ooyen, M. h. f., van de Weijgert, J. a. c., Begemann, S. h. a., 1987. Preferred Luminances in Offices. J. Illum. Eng. Soc. 16, 152-156. https://doi.org/10.1080/00994480.1987.10748695

Veitch, J.A., Newsham, G.R., Mancini, S., Arsenault, C.D., 2010. Lighting and office renovation effects on employee and organizational well-being (No. NRC-IRC Research Report RR-306). NRC Institute for Research in Construction, Ottawa, ON.

Wagenmakers, E.-J., Marsman, M., Jamil, T., Ly, A., Verhagen, J., Love, J., Selker, R., Gronau, Q.F., Šmíra, M., Epskamp, S., Matzke, D., Rouder, J.N., Morey, R.D., 2017. Bayesian inference for psychology. Part I: Theoretical advantages and practical ramifications. Psychon. Bull. Rev. 1-23. https://doi.org/10.3758/s13423017-1343-3

Waldram, J.M., 1969. IES Diamond Jubilee Lecture: Light and the community Part 2 The growth of lighting. Light. Res. Technol. 1, 78-85. https://doi.org/10.1177/14771535690010020601

Ward, G., 2006. hdrgen.

Wegener, D.T., Petty, R.E., Blankenship, K.L., Detweiler-Bedell, B., 2010. Elaboration and numerical anchoring: Implications of attitude theories for consumer judgment and decision making. J. Consum. Psychol. 20, 5-16. https://doi.org/10.1016/j.jcps.2009.12.003

Yamaguchi, H., Aya, K., Lu, X., Kato, M., Hara, N., Miki, Y., Yoshizawa, N., 2016. Development of spatial brightness evaluation method with luminance distribution; (part1) Modeling of spatial brightness evaluation, in: Summary of the Annual Meeting of the Architectural Institute of Japan. Architectural Institute of Japan, pp. 551-552.

Zdravković, S., Economou, E., Gilchrist, A., 2012. Grouping illumination frameworks. J. Exp. Psychol. Hum. Percept. Perform. 38, 776-784. http://dx.doi.org/10.1037/a0026335

Zdravković, S., Economou, E., Gilchrist, A., 2006. Lightness of an object under two illumination levels. Perception 35, 1185 - 1201. https://doi.org/10.1068/p5446

Zhang, Y., Julian, W.G., 2012. A predictive tool for brightness design? Light. Art Sci. Int. Des. 32, 28-35.

Zihl, J., Lissy, P., Pöppel, E., 1980. Brightness perception in the visual field. Psychol. Res. 41, 297-304. https://doi.org/10.1007/BF00308875 


\title{
9 Appendix
}

\author{
Online supplement can be found at
}

https://osf.io/gjx6k/?view_only=d3650b970a894907b71fde7a21158693 



\section{Appendix A Light measurement}

The overall light level in the experimental spaces was described using vertical illuminance at the eye of a hypothetical participant, while the light distribution was measured using High-Dynamic-Range luminance mapping following Inanici (2006). In addition to this, measurements of the Spectral Power Distribution (SPD) were taken in order to account for its possible effect on spatial brightness.

In the following sections we outline the measurement methodologies and investigations into the uncertainty of our descriptions of the lighting in our experimental conditions. To summarise:

- Repeated vertical illuminance measurements showed variance of $\sim \pm 5 \%$. To address this, the spaces were measured 5 times on different days (10 times using two meters in Experiment 2), and the results averaged to provide the overall estimate of the light level seen by participants.

- The effect of SPD on brightness was estimated using Rea et al.'s (2015) model. Differences between conditions were within $-1.5 \%$ for most key comparisons. A shift in the colour of the light was observed at very low light levels, and this was measured and accounted for by using a fitted polynomial function to adjust the "SPD effects" at low matches.

- With regards to the light distributions:

- Checks of the reliability of the measured uniformity metrics showed an uncertainty of $\pm 1 \%$ (LG-G4 smartphone) and $\pm 2.5 \%$ (Nikon D200 DSLR camera) in repeated measurements of the same scene.

- Vignetting was measured following Van Den Wymelenberg (2012) to produce a correction function. Due to observed asymmetry, this was done across eight cardinal directions to produce a 2-D map of the vignetting. Checks indicated uncertainty in the measurements, however "correcting" these had no significant effect on resulting uniformity metrics, suggesting such errors were not significant.

- We observed systematic underestimation of darker regions in the HDR luminance maps. Inanici (2006) explained such effects as being due to scattering in the lens, however our measurements showed the potential for far greater error than they observed - up to an order of magnitude in some Experiment 2 conditions. We devised a method using simple contour maps 
of the light distributions displayed on a screen to attempt to roughly measure the effects and produce a rough correction filter. The Experiment 1 conditions seemed to have minimal error in this regard, and "correcting" them had little effect on uniformity metrics. The Experiment 2 conditions were significantly more affected, with many metrics increasing by between $0--25 \%$ (or up to a factor of 3 for the very sensitive $\max / \mathrm{min}$ ratio). The uncertainty in our measurements of these effects and our correction should be noted. The main effect of the error is that conditions have their nonuniformity underestimated proportional to its magnitude. The overall pattern, and thus observed trends, do not change (which is good for older studies which have not accounted for this). It is primarily a concern when one is attempting to devise precise models that make precise predictions. Additionally, it may indicate significant issues for studies that have calibrated their luminance maps using spot luminance measurements.

\section{A.1 Uncertainty in measurements of the overall light level}

Initially the light level was measured using the mean luminance measured from calibrated HDR luminance maps (which can be seen in our initial conference papers (Sullivan and Donn, 2016)). However, concerns developed about the accuracy of spot luminance measurements and calibration of the HDR measurements due to the problems with scattering (A.3). Due to this, we switched to vertical illuminance.

\section{A.1.1 Experiment 1 conditions}

Measurements were taken with a Minolta T-1H illuminance meter placed at roughly where the eye level of a person viewing the model space would be. While repeated measurements at any one time indicated that they could be taken reliably, measurements taken on different days indicated the potential for significant $( \pm 5 \%)$ error.

Based on this, it was decided that the only reasonable option was to measure and calibrate the average light levels of the conditions using the average of multiple measurements. The first set of measurements (A) were taken with the light level measured in the diffusing box set to 250 lux. The second set were taken with the light levels set to produce a vertical illuminance of 2.75 lux - the reference light level used in Experiment 1. The later sets, being taken with the idea of measuring error, were taken with the shutters fully open, on the grounds that higher light levels would have less potential meter error (i.e. 0.1 lux error is significant at 2 lux, but much less so at, say 5 lux). 

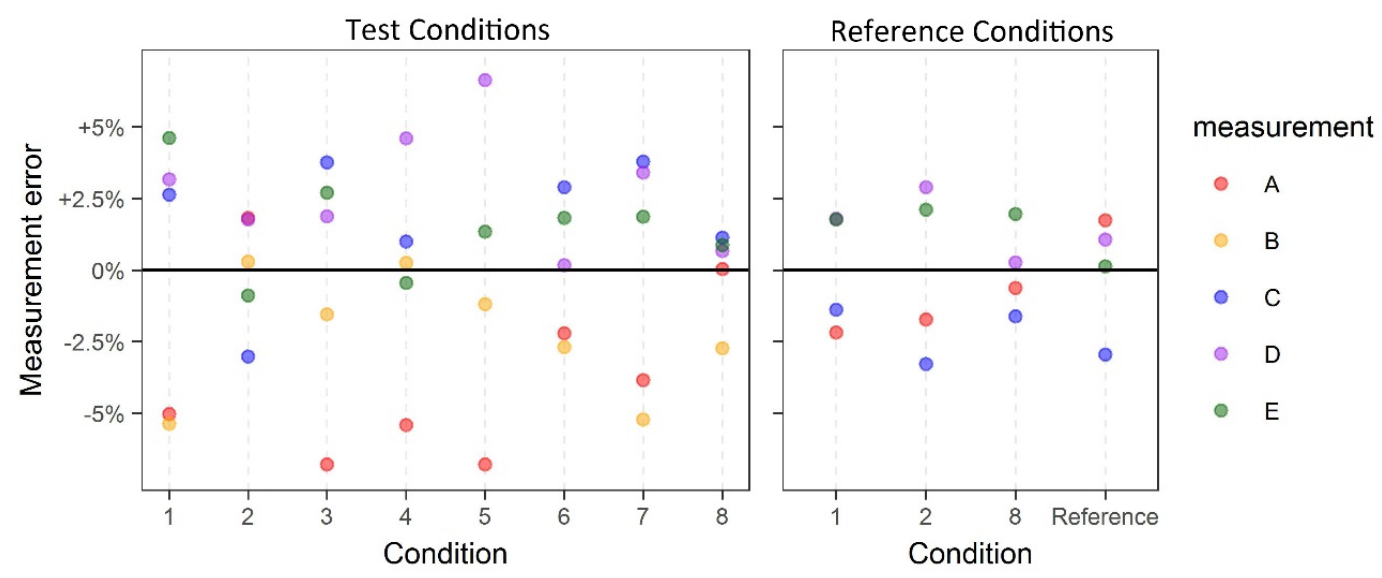

Figure 9.1 Measurement "error" in measurements of vertical illuminance for Experiment 1. Five sets of measurements labelled A-E were taken. Values plotted are normalised against the mean.

\section{A.1.2 Experiment 2 conditions}

In the second experiment, the conditions were again measured five times over the course of the experiment using a Minolta T1 illuminance meter. As a result of the later acquisition of a Minolta CL-70F CRI illuminance meter to measure SPD, five more sets of measurements were taken ${ }^{80}$. These measurements were all taken at the maximum light level for consistency. Comparisons between the meters indicated that the older T1 tended about $2 \%$ lower than the CL-70F when the sensors were placed in the same position on a desk. However when the meters were used to measure the light levels in the experimental conditions, again placing them at the expected position of the eye, the T1 tended to give readings $-5 \%$ higher than the $\mathrm{CL}-70 \mathrm{~F}$. The best explanation is that differences in dimensions were resulting in small differences in sensor position.

As neither meter position is inherently "more correct" than the other, the measurements were all averaged to form the final calibration factors used.

\footnotetext{
${ }^{80}$ We did not attempt to measure the calibration factor for the Experiment 1 conditions again with the new meter as at that point the experimental apparatus had been rearranged for Experiment 2. Trying to return it to the state that the first experiment was originally in — in particular getting the position of the light meter in the diffusing box exactly right — would have introduced unavoidable error, and made comparing the resulting calibration factors between the meters pointless.
} 


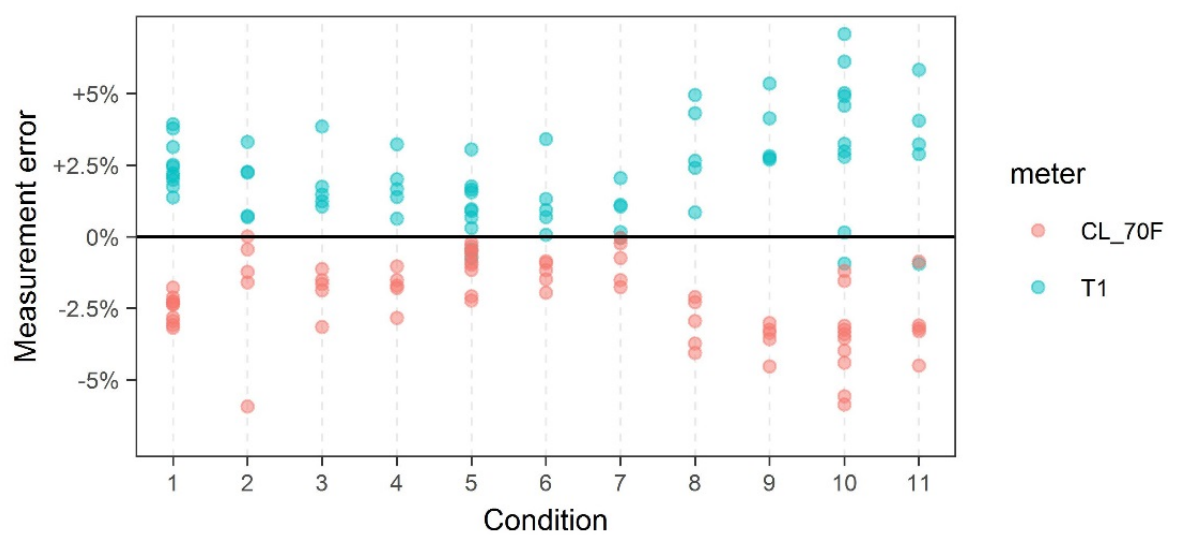

Figure 9.2 Measurement "error" in measurements of vertical illuminance for the Test conditions on the right side in Experiment 2. Five sets of measurements were taken with both a Minolta T1 illuminance meter, and a newer Minolta CL-70F illuminance meter. Values plotted are normalised against the mean.

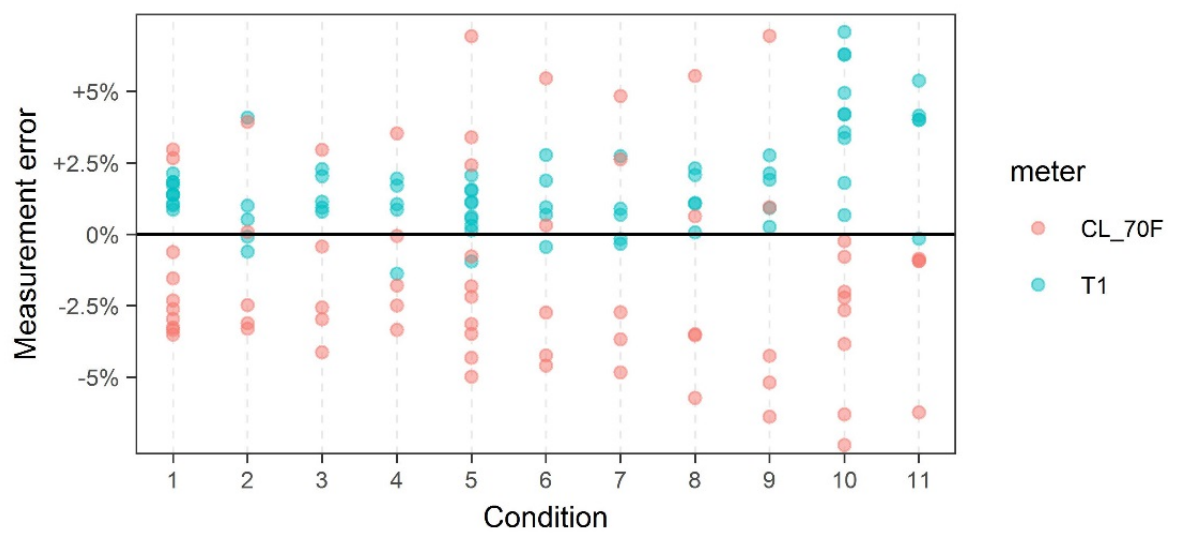

Figure 9.3 Measurement "error" in measurements of vertical illuminance for the Reference conditions on the left side in Experiment 2. Five sets of measurements were taken with both a Minolta T1 illuminance meter, and a newer Minolta CL-70F illuminance meter. Values plotted are normalised against the mean.

Additional measurement checks were carried out after the experiment was complete to double check that a) the calibration factor stayed consistent as the shutters moved, and b) that the reference light levels set were producing the precise vertical illuminances that they were intended to be.

The shutter checks indicated a subtle shift in calibration factor as the light levels were adjusted, with the calibration factor being perhaps $2 \%$ higher when the shutters were closed than would be expected from our measurements with them fully open (Figure 9.4). The overall effect of this is minor - most matches are somewhere in the middle, and thus shift by around $1 \%$, and since they are all shifting the same way the effect on the differences is 
limited. Nevertheless, to account for it we fit a simple linear function to the data in Figure 9.4, and used that to estimate an adjustment factor to apply to all the matches.

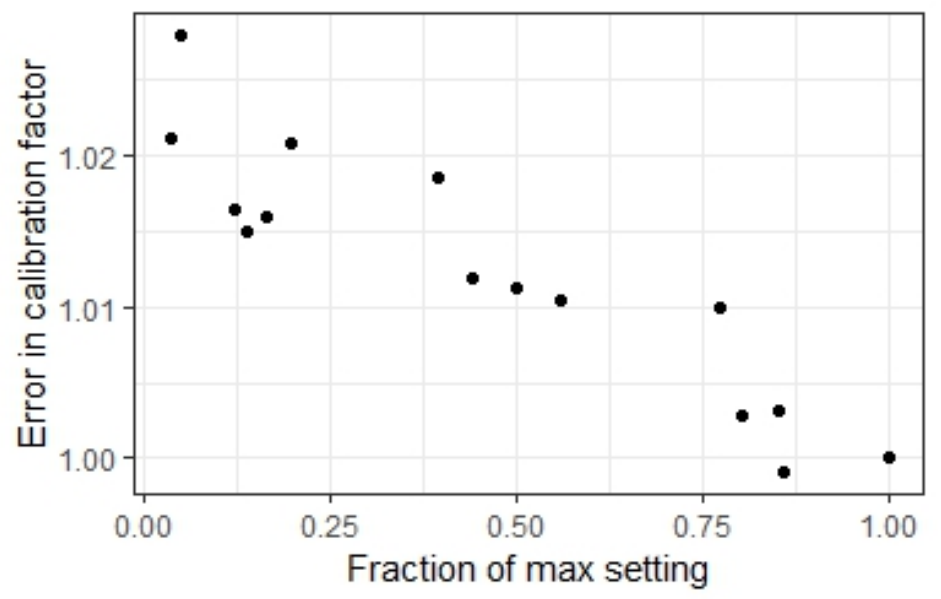

Figure 9.4 Shift in test condition calibration factor observed as a result of closing shutters in experiment 2. Measurements taking using Uniform White and Uniform Grey conditions. Each point is an average of readings taken for each of the different numbers of lamps (2-6).

Measurements of the vertical illuminance of the reference conditions taken at their different settings similarly showed them to be slightly higher than expected (with a few exceptions that may just be measurement error) (Figure 9.5). Reference light levels were adjusted to these values to check the effect on results (see sensitivity analysis in Experiment 2).

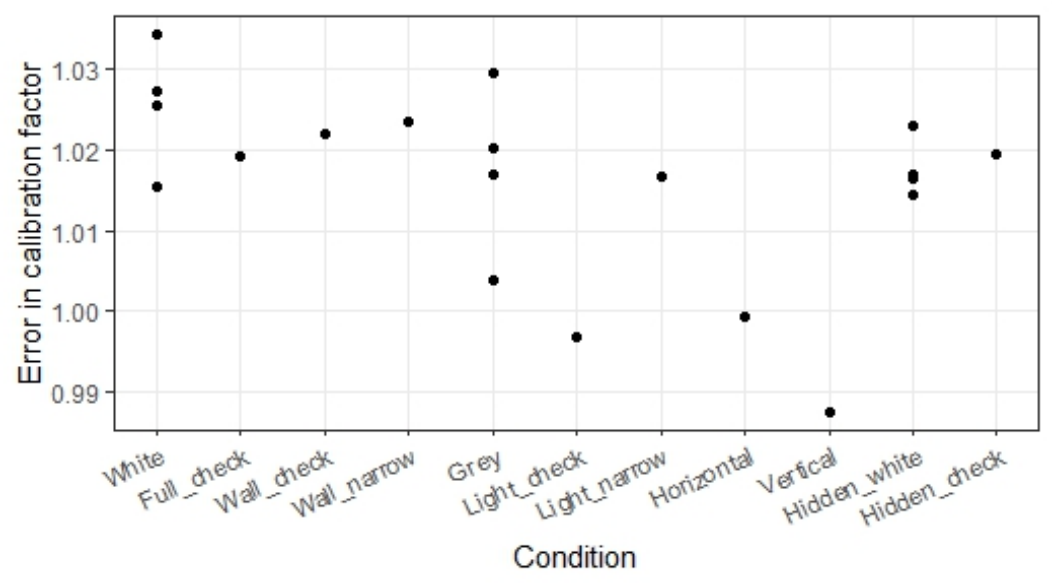

Figure 9.5 Error between calibration estimates and later measurement checks for reference conditions at the reference light levels. Measurements are the average of two measurement sessions, with measurements taken three times with each meter and averaged in each session. 


\section{A.2 SPD measurement and effects}

During construction of the spaces for the second experiment, visible differences in light colour were observed between the uniform white condition and others, with the uniform white condition appearing noticeably bluer than other conditions. Measurements of the spectral reflectance of the surfaces indicated that this was due to the plain white paper reflecting significantly more blue light than black and grey ink (Figure 9.8). This was not noticeable in the first experiment because there all the conditions were either mid-grey or a combination of black and white, which tended to average out to the same light colour as the grey. Given that light colour is known to have potentially significant effects on spatial brightness (Fotios et al., 2015), this was a concern.

Acquisition of a Minolta CL-70F CRI illuminance meter allowed the overall spectral power distribution (SPD) of the light viewed by the participants to be measured for the different conditions. The potential effect on brightness was estimated using the provisional model of spectral sensitivity for scene brightness presented by Rea et al. (Rea et al., 2015), which estimates the "brightness illuminance" by applying a different luminous efficiency function based off the combination of three luminous efficiency functions: a) the standard photopic luminous efficiency function $(V(\lambda))$, b) that of the short-wavelength cone fundamental $(S(\lambda))$, and c) that of the intrinsically photosensitive retinal ganglion cells (ipRGCs) $(\operatorname{Mel}(\lambda))($ Rea et al., 2015). The equation for this is (Rea et al., 2015):

$$
B_{v}(\lambda)=V(\lambda)+g_{v} S(\lambda)+0.5 \operatorname{Mel}(\lambda)
$$

where $g_{v}$ is estimated according to the vertical illuminance at the eyes $\left(E_{v}\right)$ :

$$
g_{v}=0.264 \ln \left(E_{v}\right)+1.093
$$

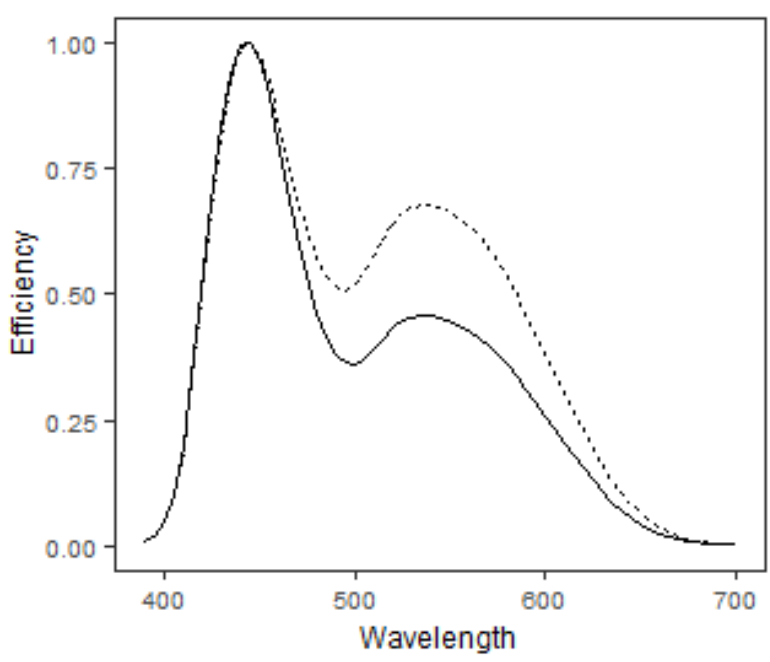

Illuminance

- 60 Lux

… 2.75 Lux

Figure 9.6 The brightness efficiency functions estimated for the average reference light levels in our two experiments according to Eq. 9.1. 
By calculating the effective brightness illuminance of the different conditions, normalised to the same light level, we can estimate the effect of the light spectrum on their apparent brightness. For example, if the estimated brightness illuminance of condition $\mathrm{B}$ is $14 \%$ higher than that of condition $\mathrm{A}$ at the same overall luminance, then we can say that the effect of the difference in colour of light is making condition B appear brighter. These estimates can then be included in our statistical models to control for the differences in light spectrum between conditions.

This is not, of course, necessarily perfect. The model of Rea et al. may be wrong in some way. However, it is the best estimate that can be provided at the moment. Older metrics based off colorimetry have been found to be flawed (Fotios et al., 2014; Royer and Houser, 2012). While it has been suggested that combinations of different metrics such as gamut area and scotopic/photopic ratio could potentially provide better predictions of brightness effects (Fotios et al., 2014), it is not clear how we could use them to make quantitative estimates of the size of the effects. In contrast, the Rea et al. model has the virtue of taking into account the whole light spectrum rather than being based on a colorimetric simplification, has been tested at both normal indoor and night time light levels (Rea et al., 2017, 2015), and provides a clear means for us to estimate the practical effect of the SPD on the effective light level.

One point worth noting, however, is that any errors in the model would need to be substantial to have a significant effect. The efficiency function being slightly out does not significantly affect the estimated differences between the conditions, as they were designed to be very close to each other in SPD. We briefly examined this by adjusting the relative contribution of the $S(\lambda)$ function to vary the resulting brightness function. As can be seen, even what may look like reasonably substantial changes to the function (Figure 9.7) generally only shift the estimated effect by around $<1 \%$, perhaps $1.5 \%$ for the largest differences (Table 9.1). 


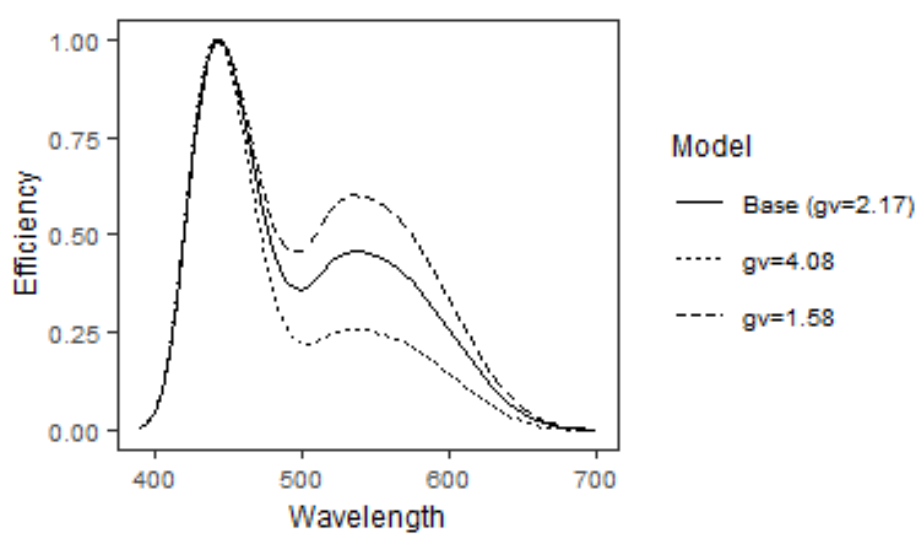

Figure 9.7 Efficiency function variations produced by varying $g_{v}$ to adjust the contribution of the $S(\lambda)$ function.

Table 9.1 Sensitivity of the estimated effect of SPD according to one set of measurements to the specification of the efficiency function (Figure 9.7).

\begin{tabular}{lrrr} 
Condition & \multicolumn{1}{c}{ Base } & $\mathbf{g}_{\mathbf{v}}=\mathbf{4 . 0 8}$ & $\mathbf{g}_{\mathbf{v}}=\mathbf{1 . 5 8}$ \\
\hline Uniform White 1 & - & - & - \\
Uniform White 2 & $99.9 \%$ & $99.8 \%$ & $99.9 \%$ \\
Full_check & $101.5 \%$ & $102.2 \%$ & $101.2 \%$ \\
Wall_check & $101.1 \%$ & $101.5 \%$ & $100.9 \%$ \\
Wall_narrow & $101.1 \%$ & $101.5 \%$ & $100.9 \%$ \\
Uniform Grey 1 & $103.9 \%$ & $105.3 \%$ & $103.2 \%$ \\
Uniform Grey 2 & $104.0 \%$ & $105.4 \%$ & $103.3 \%$ \\
Light_check & $103.4 \%$ & $104.7 \%$ & $102.8 \%$ \\
Light_narrow & $103.7 \%$ & $105.0 \%$ & $103.0 \%$ \\
Horizontal & $104.1 \%$ & $105.6 \%$ & $103.5 \%$ \\
Vertical & $103.8 \%$ & $105.2 \%$ & $103.2 \%$ \\
Hidden_white 1 & $98.6 \%$ & $97.8 \%$ & $98.9 \%$ \\
Hidden_white 2 & $98.5 \%$ & $97.7 \%$ & $98.9 \%$ \\
Hidden_check & $99.6 \%$ & $99.2 \%$ & $99.7 \%$
\end{tabular}




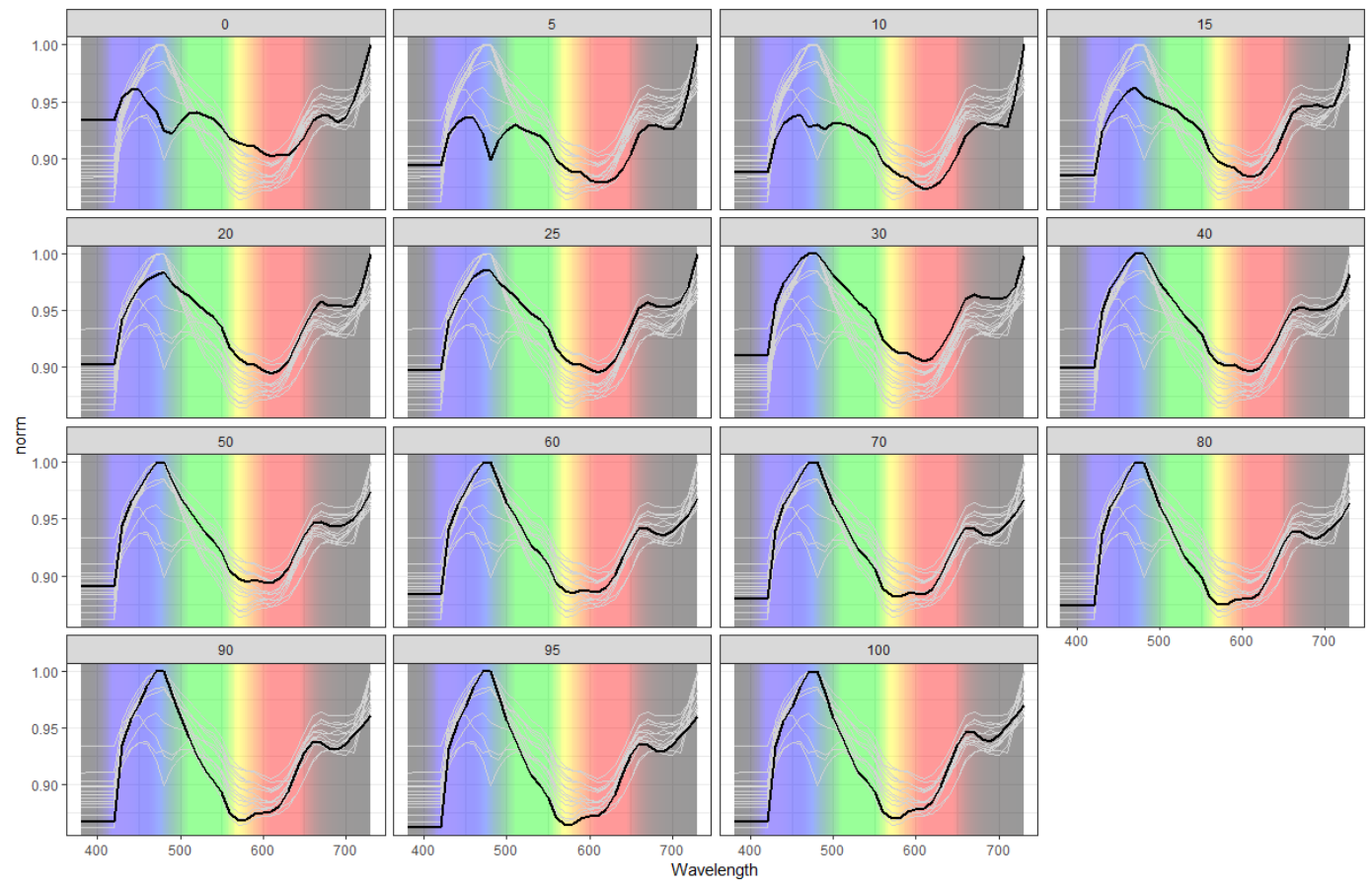

Figure 9.8 Illustration of how the normalised spectral reflectance of the surfaces changes as we progress from black ink ( 0 , top left) to plain white paper (100, bottom right). Labels on graphs are the $L^{*}$ values of the grey as set in Adobe Photoshop before printing.

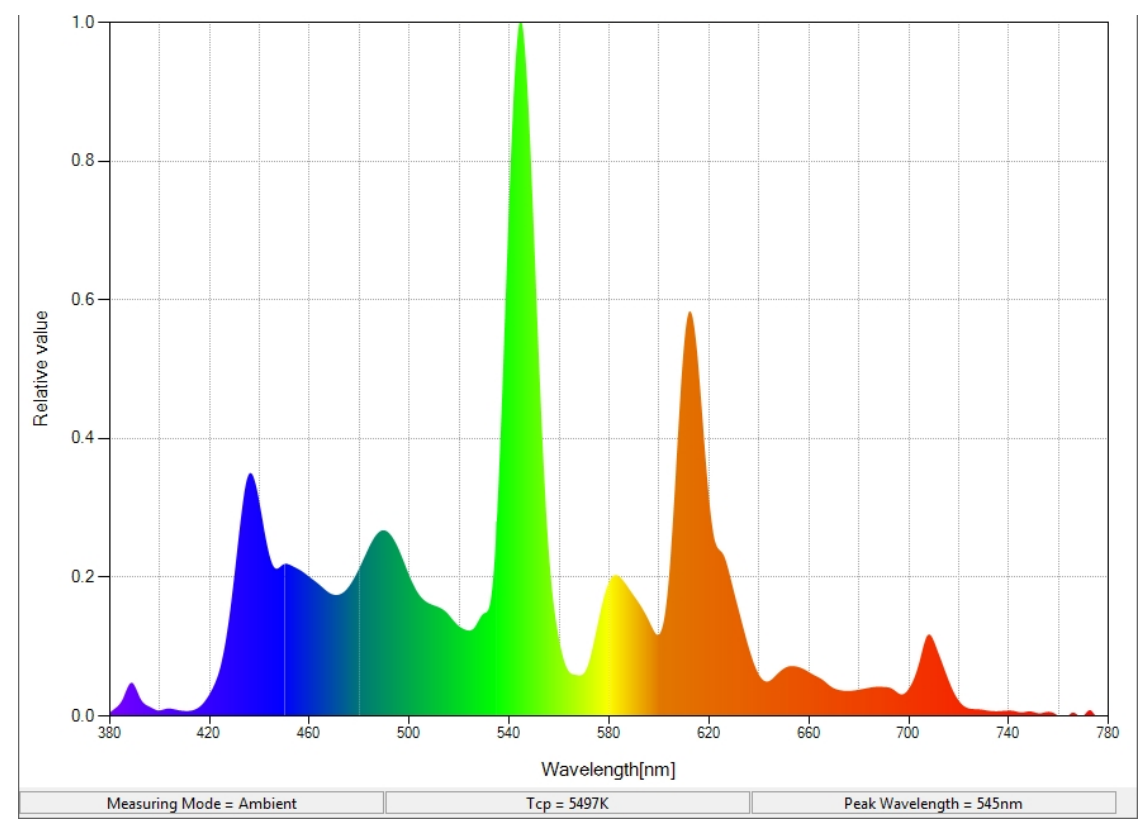

Figure 9.9 Example of SPD measurement for the Uniform White condition in Experiment 2. For more, see online supplement. 


\section{A.2.1 Experiment 1 conditions}

Measurements of illuminance and light spectrum were taken with the meter placed at the expected eye level of a participant looking into the spaces. Two rounds of measurements were taken of the test conditions on the left side. First, measurements were taken three times at each of the three light levels used in the Magnitude Estimation experiment. Second, for the brightness matching experiment, each condition was measured five times at different shutter settings ranging from fully open to as dark as could be measured. Note that because of the very low light levels of the set up in the first experiment, the whole range of possible light levels could not be measured, as the CL-70F meter could not go below 1 lux. In order to maximise the range of shutter settings that could be measured here, the number of lamps was increased from two to six for this set of measurements. When calculating the spectral effects using the Rea et al. (2015) model, the adaptation level was assumed to be that of the reference condition -2.75 lux.

The differences between conditions are minor, around 1\%, with the largest difference being the $6^{\text {th }}$ condition which appears to have around $1.5 \%$ less light than the baseline uniform grey condition.

Table 9.2 Effect of SPD on apparent light level of Experiment 1 test conditions relative to the baseline uniform grey condition. Columns show the averages of the measurements at the ME settings, the measurements across progressive shutter settings, and all the measurements together.

\begin{tabular}{|l|l|l|l|l|}
\hline & Conditions & ME & Prog. & Overall \\
\hline 1 & Uniform grey (null) & - & - & - \\
\hline 2 & White wall & $-0.9 \%$ & $-1.3 \%$ & $-1 \%$ \\
\hline 3 & Black wall & $-0.9 \%$ & $-0.5 \%$ & $-0.8 \%$ \\
\hline 4 & Black floor/white ceiling & $-1.2 \%$ & $-1.1 \%$ & $-1.1 \%$ \\
\hline 5 & White floor/black ceiling & $-1.7 \%$ & $-0.9 \%$ & $-1.4 \%$ \\
\hline 6 & Dark grey floor/light grey ceiling & $-1.4 \%$ & $-1.6 \%$ & $-1.5 \%$ \\
\hline 7 & Dark grey ceiling/light grey floor & $-1.4 \%$ & $-1.3 \%$ & $-1.4 \%$ \\
\hline 8 & Checkerboard & $+1 \%$ & $-0.1 \%$ & $+0.6 \%$ \\
\hline
\end{tabular}

As noted, it was observed that at low light levels the measured colour of the light became warmer, making it appear darker according to the Rea et al. model. Why this is unclear, though it may be worth noting here that the meter cannot work below 1 lux and stops reporting most colour metrics around 5 lux.

Regardless, to account for this we plot the relative decline in effective illuminance (according to the Rea et al. model) against the fraction of maximum possible illuminance for each condition (calculated as a rough proxy of shutter position). To estimate the potential SPD effect for individual matches, we generated a polynomial function relating 1- 
fraction to relative illuminance (Figure 9.10). This was then used to adjust the base effective illuminance estimate for each match.

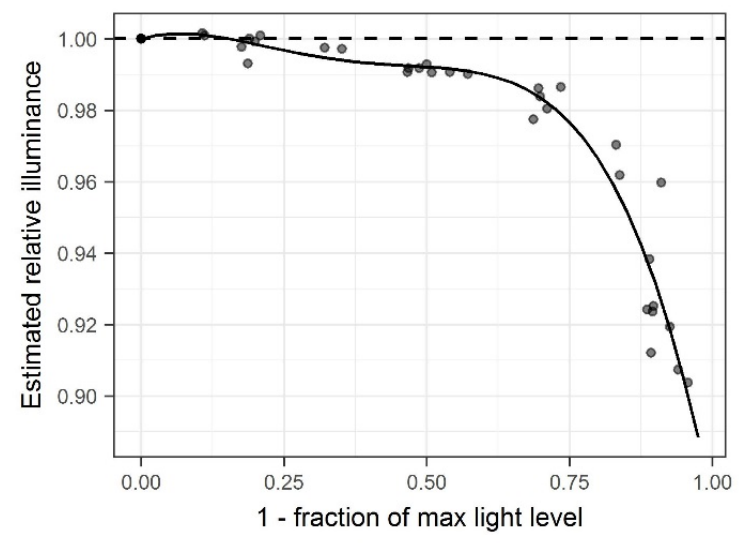

Figure 9.10 Decline in effective light level according to Rea et al. model as a function of the light level setting. When the shutters are closed, the meter measures the light as warmer, effectively reducing the light level people observe by as much as $10 \%$. The fitted line is a polynomial function used to estimate the effect for individual matches: $y=-0.7538 x^{4}+1.0293 x^{3}-0.4533 x^{2}+0.0478 x+1$

Overall, the effect is minor (Figure 9.11). The adjustment factor is about $-1 \%$ on average for 5 of the conditions, so no meaningful change to the differences between them, while the White/Black wall and checkerboard conditions shift by about $2 \%$ more.

Compared to just using the average estimate for each condition, applying the adjustment only increases the effects by $1-2 \%$. Compared to not adjusting at all, we see increases of perhaps $2-5 \%$ depending on how large the effect was. The White Wall condition, for example, goes from an effective luminance of $-+58 \%$ to $+63 \%$, which does not really change our conclusions.

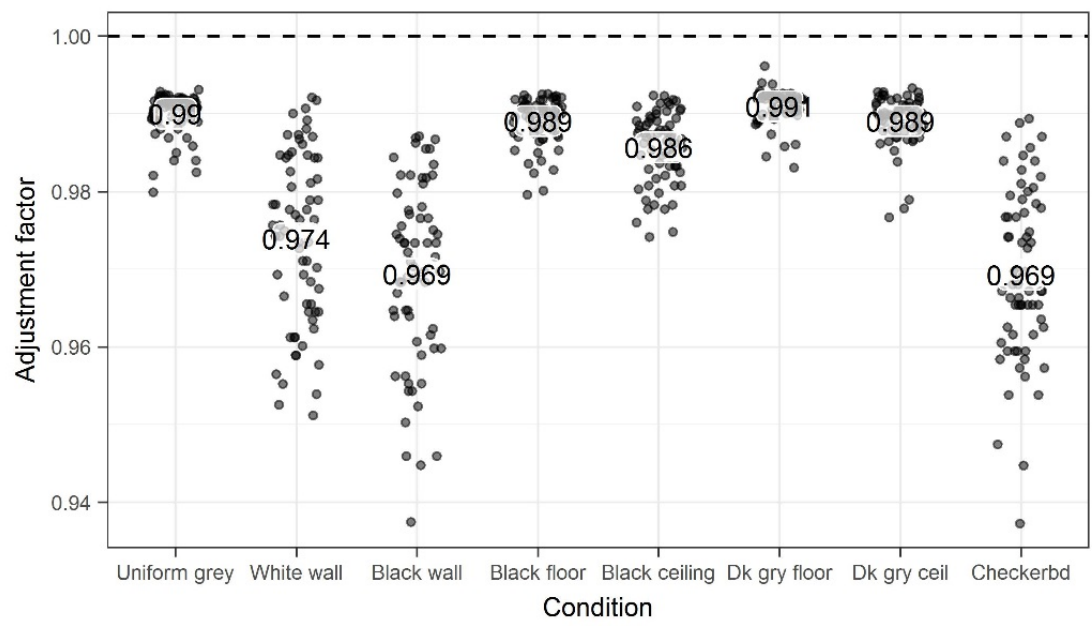

Figure 9.11 Adjustment factors estimated for matches made in Experiment 1. Labels show the average adjustment for each condition. 


\section{A.2.2 Experiment 2 conditions}

As before, SPD was measured using a Minolta CL-70F illuminance meter placed at the rough position of the participants' eyes. The effects of SPD were estimated using the Rea et al. (2015) model assuming an adaptation level of 60 lux (the average in the second experiment) and included in our statistical model. Five sets of measurements were taken and averaged. Variation between measurements was relatively low (in comparison to illuminance), being around $\pm 0.5 \%$ for the test conditions, and $\pm 1 \%$ for the reference.
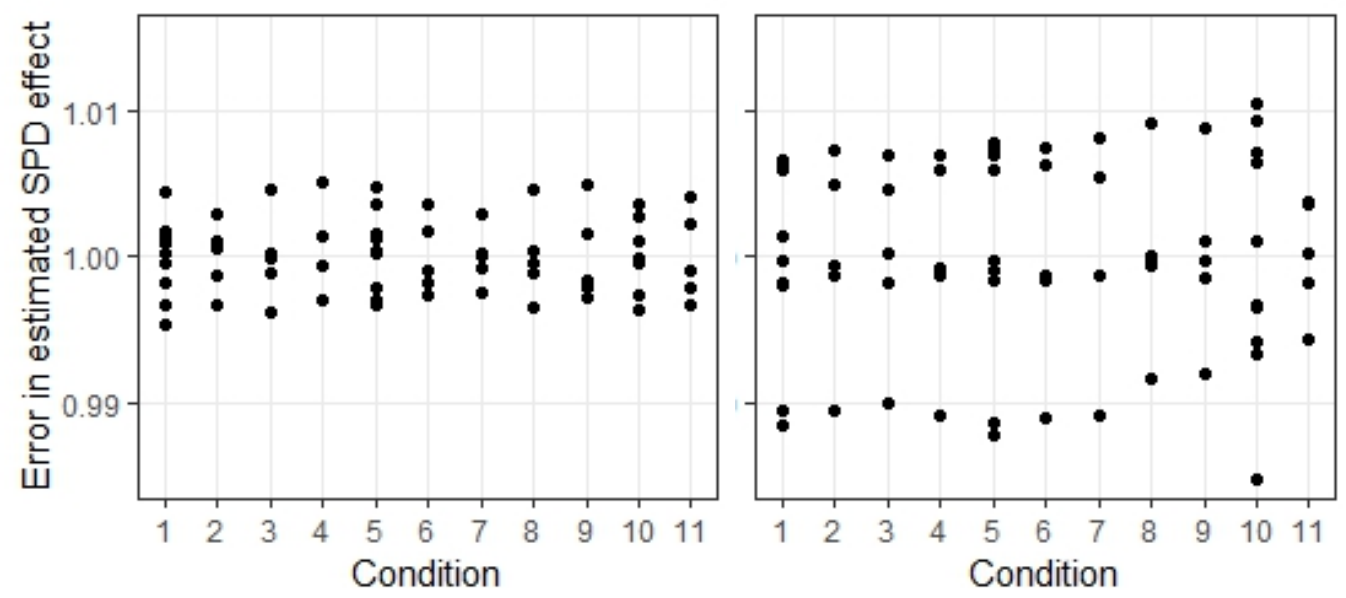

Figure 9.12 Variation in estimated SPD effects across the five different measurement sets. Left: Test conditions. Right: Reference conditions.

Measurements taken after the experiment was concluded suggested that at low light settings the lamp spectrum could become warmer, and thus effectively darker (Figure 9.13). A most light levels the effects are unnoticeable, with measurements mostly fluctuating within $\pm 1 \%$ - well within normal measurement error. Only below $-20 \%$ of the maximum light level do we start to see measurable falloff, and even then it really only starts to become significant below $\sim 10 \%$. 


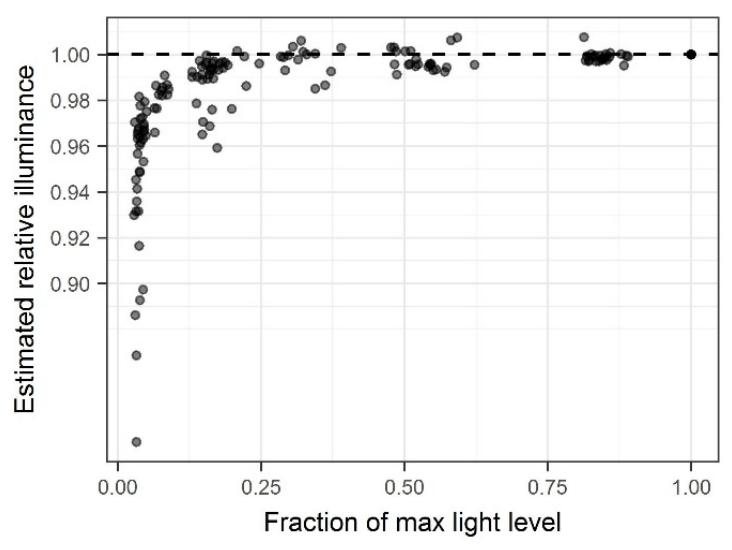

Figure 9.13 Falloff of relative illuminance according to Rea et al. model as a function of the shutter position, here measured as a fraction of the maximum light level able to be set for a given condition. Measurements were taken at multiple shutter settings for each condition.

If we look at the distribution of brightness matches here in the second experiment, we can see that the vast majority of them should be unaffected, as they are not low enough (Figure 9.14). The one condition that seems to have a reasonable number of matches that could be affected is the uniform white condition, which had a number of relatively low light level matches in its null condition trials. Hence, this could possibly affect the estimates of the reference and adjustment range biases. To estimate the effects here for individual matches, we fit a polynomial function to estimate an adjustment factor for the SPD effect of matches that were less than $20 \%$ of the maximum light level (Figure 9.15). The result of this is that number of matches have their SPD effect adjusted down by around $1 \%$, which has minimal effect on the overall results (Figure 9.16). The limited range of matches that are potentially affected, and the generally small size of the error for most of them, means that, overall, the potential impact of this is limited. 

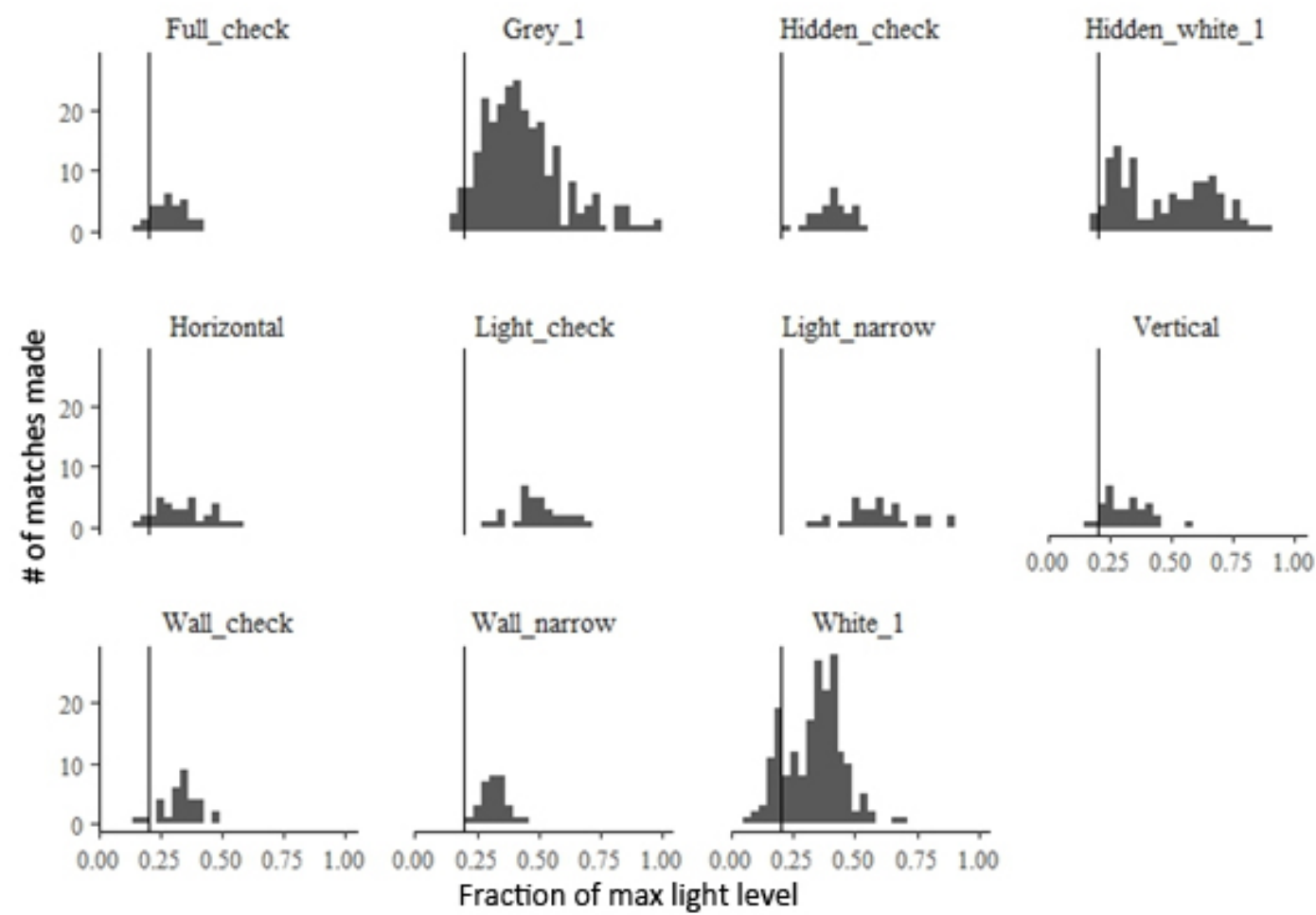

Figure 9.14 Histograms of brightness matches made for each condition according to the estimated fraction of the maximum light level for each given condition with whatever number of lamps it was using. The vertical line shows how many matches were below $20 \%$ of the maximum light level.

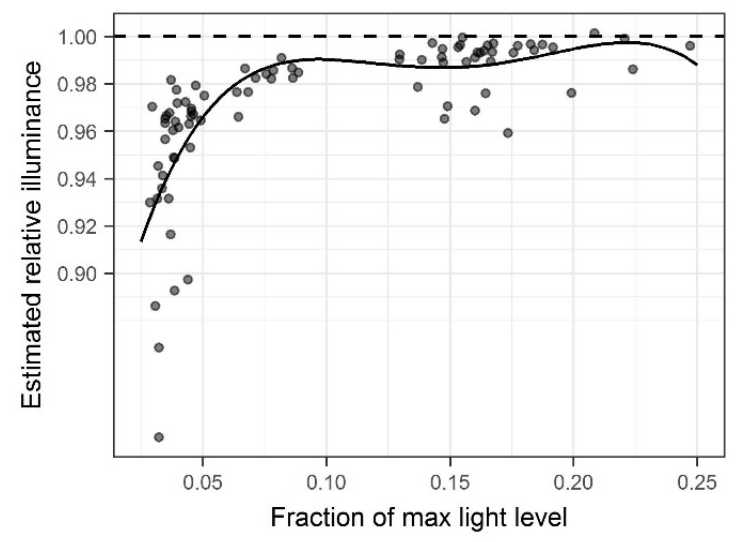

Figure 9.15 Polynomial used to roughly estimate the needed adjustment to the SPD effects for low matches 


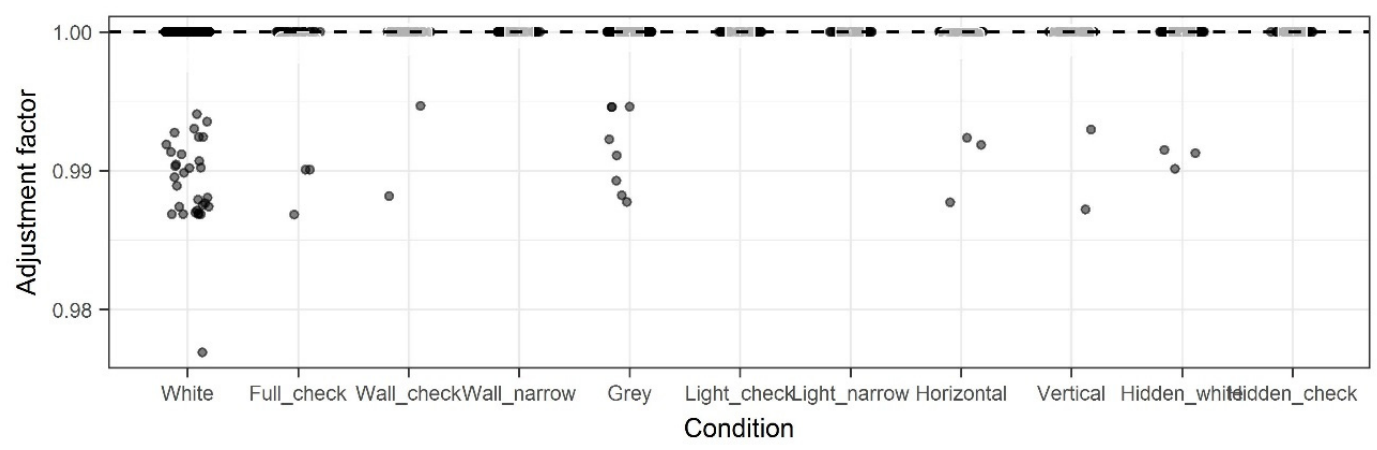

Figure 9.16 Plot of the adjustment factors applied to "correct" the SPD effects for the different matches made by participants. Each point is a brightness match made for a given test condition.

\section{A.3 Measuring the light distribution: HDR luminance mapping}

The light distribution was measured using High-Dynamic-Range (HDR) photography produce detailed maps of the entire luminance distribution of the spaces (Figure 9.17) (Hirning, 2014; Inanici, 2006; Van Den Wymelenberg, 2012). Such maps have significantly better resolution that, for example, a grid of spot luminance measurements, and can be used to calculate any luminance-based metric desired - uniformity ratios, standard deviation, metrics weighted by visual angle, and any of the spatial brightness models discussed in this thesis.

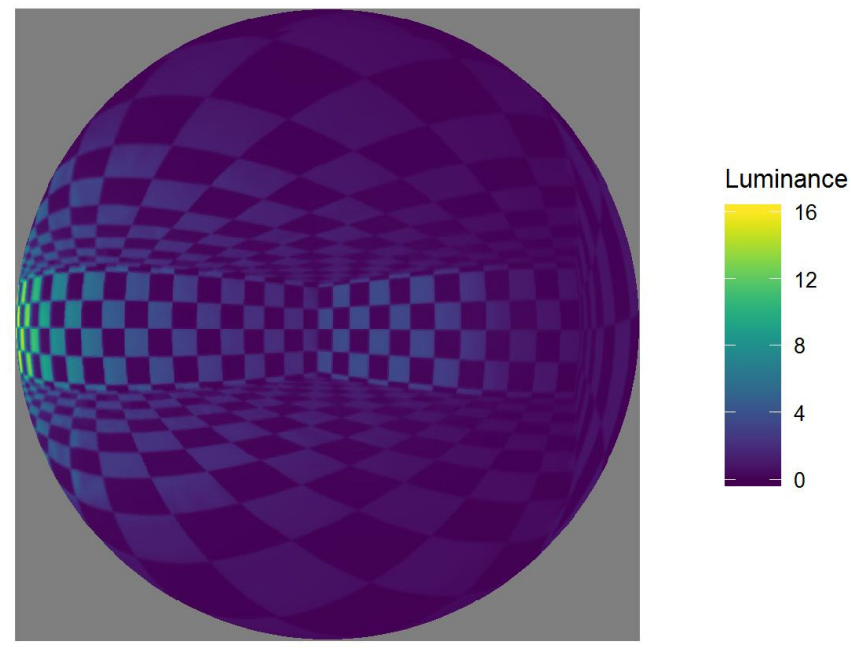

Figure 9.17 Example of a luminance map made from an HDR image using a $180^{\circ}$ fisheye lens

The basic process of creating such a luminance map is, in theory, relatively straightforward (Van Den Wymelenberg, 2012):

1. A scene is photographed at multiple exposure levels ranging from very dark to very bright. In this thesis we generally used 6-9 exposure levels. The 
recommended way to ensure that a good range is captured is that the lowest exposure should have no RGB values above 200, while the highest should have none below 20 .

2. The individual images are combined into a single HDR image using a program like hdrgen (Ward, 2006). This requires the response function of the camera, which can also be worked out by hdrgen from a set of images. For consistency, it is generally recommended that a single response function be generated from a scene that covers a wide range of light levels, and then reused for all HDR images from that camera (Van Den Wymelenberg, 2012).

3. The HDR image can now be converted into a luminance map using Radiance's pvalue command, and analysis of it can now commence.

The complications lie in making sure that the luminance maps are "accurate" and that sources of error such as vignetting are corrected for.

This section describes the accuracy of the HDR luminance maps, and the processes used for error correction. The four key areas that are discussed here are the reliability of the measurements made, vignetting correction, error due to light scattering in the lens. Calibration of the maps' overall luminance, when needed to compare them against spot measurements, was carried out using vertical illuminance. For our assessments of the light distribution and uniformity, however, such calibration was unnecessary as we were only interested in the relative luminance variation across space. In order to focus purely on the differences in light distribution, the luminance maps were all normalised to the same mean luminance before calculating uniformity metrics ${ }^{81}$.

The camera used to measure the light distributions in this thesis was an LG-G4 H815 smartphone with a Pixeet Panorama $180^{\circ}$ fisheye lens attachment. Initially, a Nikon D200 DSLR camera possessed by the School of Architecture was used with an AF Fisheye Nikkor $10.5 \mathrm{~mm}$ lens. However, it proved to be less reliable than the phone, and more importantly its "full-frame" fisheye lens cropped out substantial portions of the scene. Hence, in order to better capture the full $180^{\circ}$ field of view, the smartphone was used instead ${ }^{82}$.

\section{A.3.1 Measurement Reliability}

In order to test the basic reliability of the cameras as a measurement instrument, they were set up with a simple uniform condition (Figure 9.18), and 20 sets of photographs were taken in a row without changing anything.

\footnotetext{
${ }^{81}$ Without normalisation, for example, the standard deviation is higher at higher light levels.

${ }^{82}$ For those interested in also using a smartphone: note that in our experience trying different lenses many cheap fisheye lens attachments only provided a $\sim 140^{\circ}$ field of view in practice.
} 


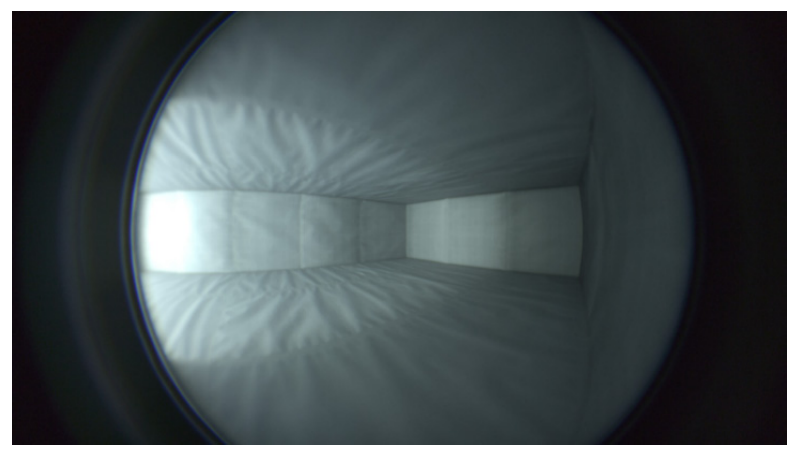

Figure 9.18 The condition used to measure the cameras' reliability. Vertical illumination was roughly 5 lux as we were using the conditions used in the first experiment in this thesis.

These HDR images were then processed as previously described, calibrated using spot luminance measurements, and standard luminance metrics were calculated.

As can be seen (Figure 9.19, Figure 9.20), the resulting measurements are not perfectly consistent. For the Nikon D200, variation was typically $\pm 2.5 \%$ at the best settings. The LG-G4 was significantly better, with variation of around $\pm 1 \%$.

Note that the maximum, and even more the minimum, were significantly more prone to noise than other metrics (especially at higher ISO settings on the D200), with variation of $\pm 5 \%$, or even over $\pm 10 \%$ being observed. This is not surprising given their dependence on the brightest/darkest single pixel in the whole image. The minimum in particular seems to be unavoidably noisy, at least in relative terms. In absolute terms the minimum would tend to be very small compared to the rest of the image, and $10 \%$ error in it would be expected to have no meaningful effect on metrics such as the mean. However, if one is calculating metrics such as the maximum/minimum ratio, then such error can significant. 

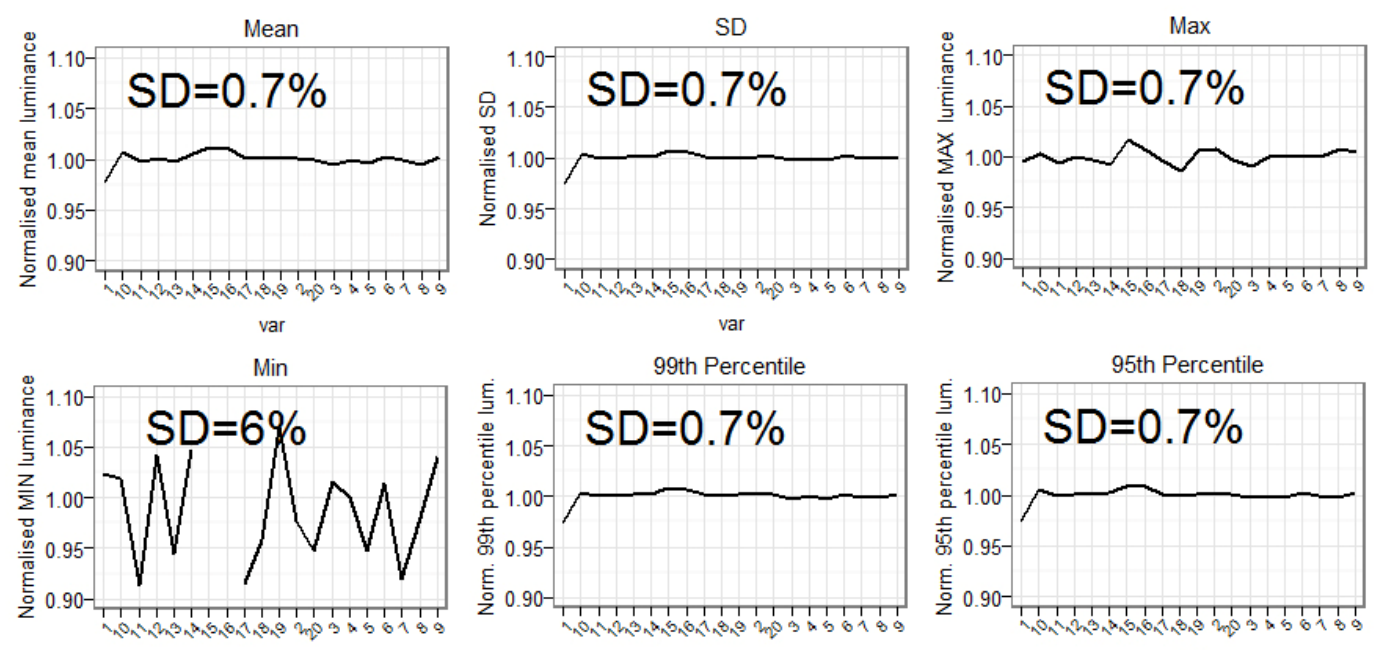

var

var

var

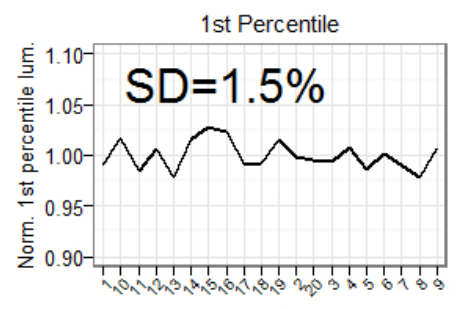

var

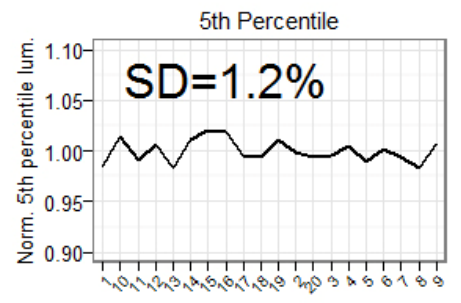

var

Figure 9.19 Variation in standard luminance metrics between 20 different photographs of the same scene using the LG-G4 camera at ISO 600. Values are normalised to the mean measurement of each metric to show the relative variation.

This highlights the poor robustness of the maximum and minimum as measurements. If one is interested in calculating metrics such as the $\mathrm{max} / \mathrm{min}$ ratio, it may be better to, instead, calculate the ratio of the $99^{\text {th }} / 1^{\text {st }}$ percentiles, which is considerable more reliable. Alternatively, multiple measurements could be taken and averaged.

Overall, outside of calculating metrics based off the minimum luminance, the LG-G4 shows excellent reliability, with $\pm 1 \%$ being a perfectly normal measurement error for light meters, and not significant. The $\pm 2.5 \%$ error of Nikon D200 is somewhat more concerning. It is, it should be noticed, still significantly smaller than both the likely size of a Just-Noticeable-Difference, as well as the statistical error we would expect in most experiments $^{83}$. In that sense, one could argue that it is acceptable, as we should not expect such errors to be perceptible, and should be expecting any predictions or estimates to have significantly more error than that regardless. That being said, if one was concerned then we could address it by simply taking multiple measurements and averaging them.

${ }^{83}$ Standard deviations in spatial brightness studies commonly range from about $8-20 \%$ 

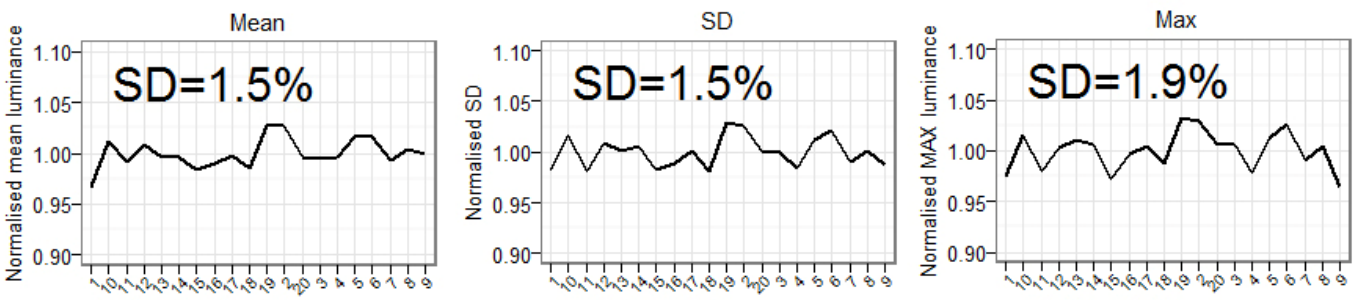

var

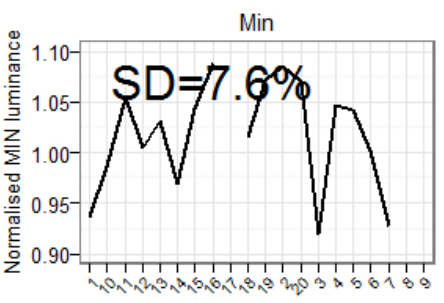

var

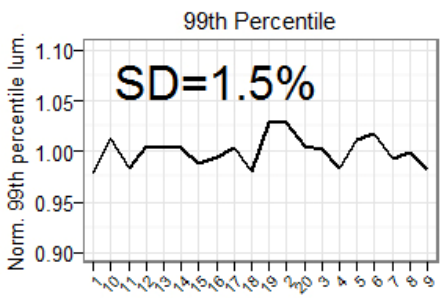

var

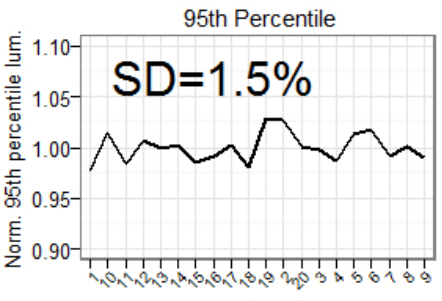

var

var

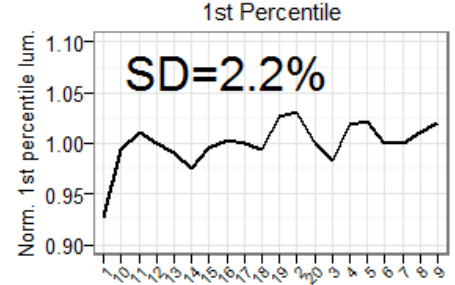

var

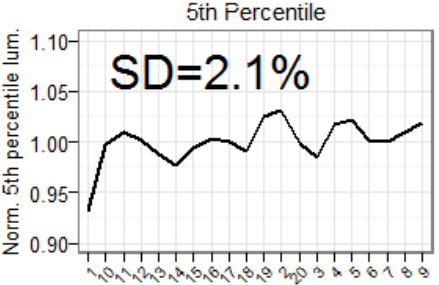

var

Figure 9.20 Variation in standard luminance metrics between 20 different photographs of the same scene using the Nikon D200 camera at F2.8 and ISO200. These settings are shown because they were the ones that provided the highest reliability. Higher ISO and F-stop settings were less reliable - though the difference between ISO200 and 400 was minor. Values are normalised to the mean measurement of each metric to show the relative variation.

Calibration to spot luminance measurements, it should be noted, does not fix the problems here. If one is relying on spot luminance measurements to get the mean luminance of the HDR image correct, one may be disappointed. Calibration against vertical illuminance of course removes any HDR error in the mean (though not the other metrics), as that is what is being calibrated ${ }^{84}$.

\footnotetext{
${ }^{84}$ Though the error in the calibration measurements themselves will remain an unavoidable issue.
} 


\section{A.3.2 Vignetting}

Vignetting was initially measured following Van Den Wymelenberg (2012). The camera was set up viewing a target placed on the wall, and then rotated through $\sim 10^{\circ}$ increments along the horizontal (Figure 9.21). By measuring the relative luminance of the target at different angles the vignetting effect could be estimated.
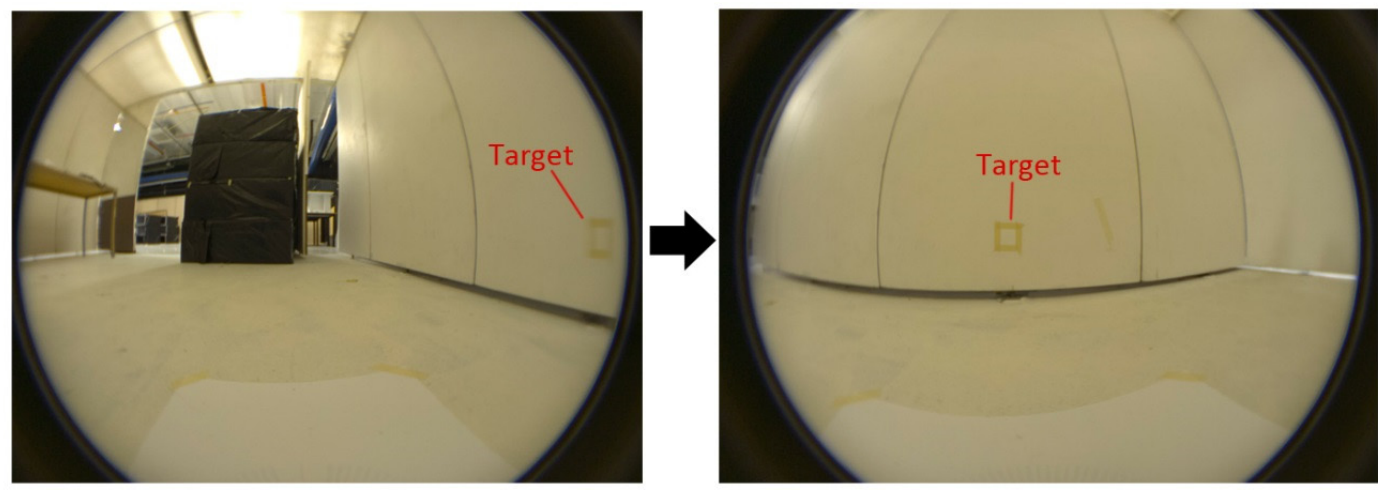

Figure 9.21 Illustration of the scene used for measuring the vignetting effect at different angles.

Unexpectedly, examination of the measurements indicated that the effects were asymmetrical. In order to measure this, the vignetting was measured again, this time with the camera rotated around the line of sight in order to measure the vignetting effect along different axes (Figure 9.22).

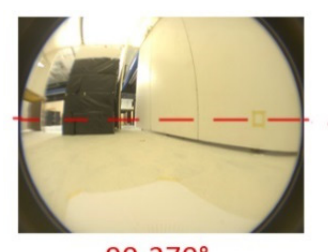

$90-270^{\circ}$

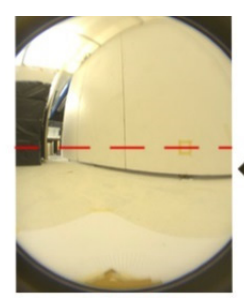

$0-180^{\circ}$

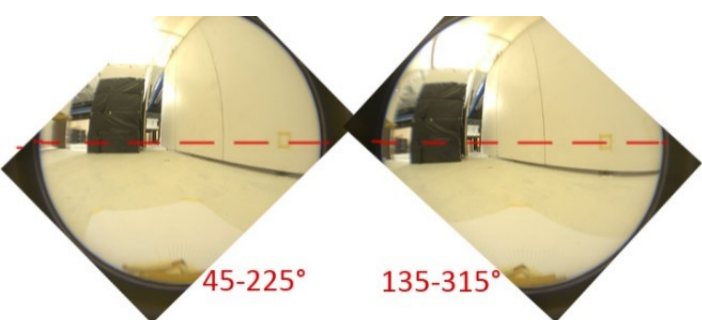

$135-315^{\circ}$

Figure 9.22 Different angles the vignetting was measured along

The results were mapped, and vignetting functions for each azimuthal angle were calculated (Figure 9.23). To create a full map, these functions were then used in $\mathrm{R}$ to define the vignetting effect along each of the principle axes. To fill in the spaces between the axes, we interpolated between the functions based on angular distance. For example, a point that was $30^{\circ}$ from the centre, and had an azimuthal angle of $67.5^{\circ}$ would be estimated as the average of what the $45^{\circ}$ function says the effect should be, and what the $90^{\circ}$ function says the effect should be. 
$315^{\circ}$

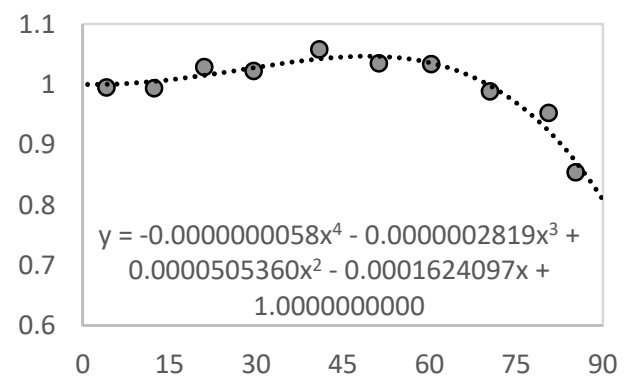

$270^{\circ}$

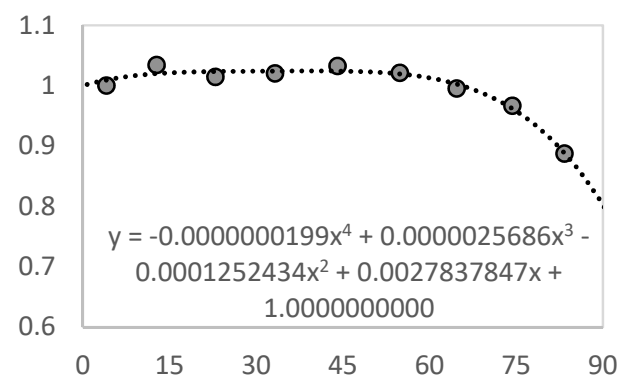

$225^{\circ}$

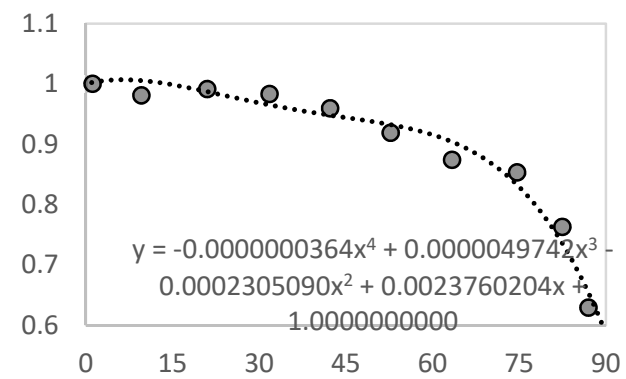

$180^{\circ}$

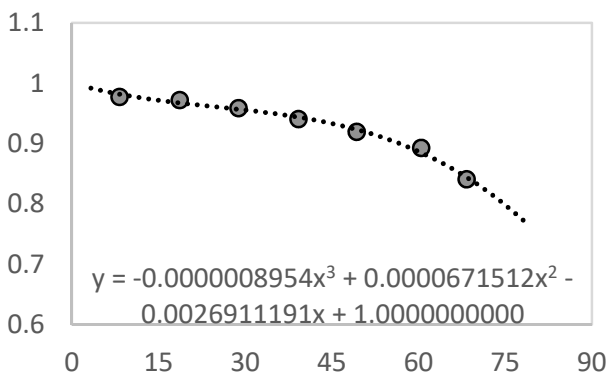

$0^{\circ}$

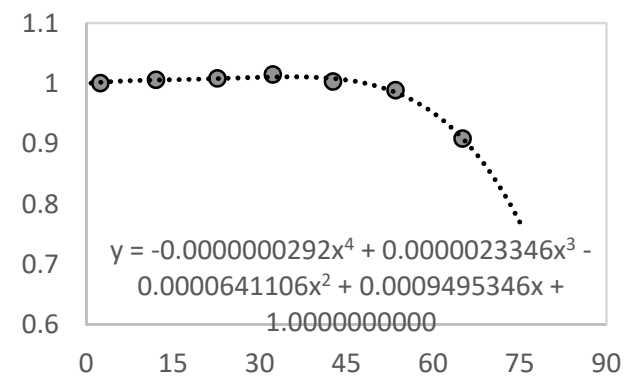

$45^{\circ}$

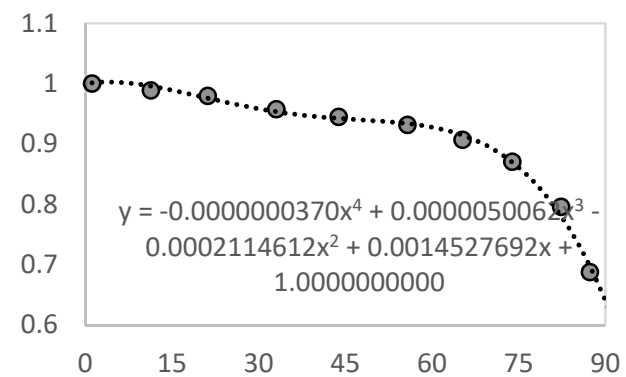

$90^{\circ}$

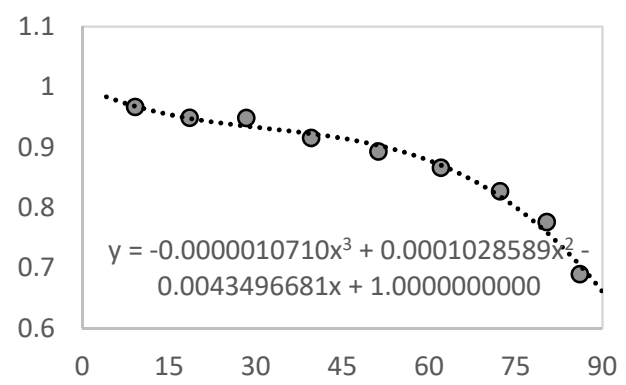

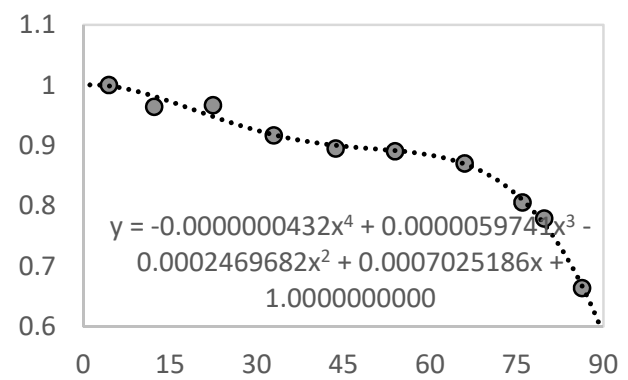

Figure 9.23 Measured vignetting effects and functions for different angles using the LG-G4 camera with fisheye lens attachment. $X$-axis is angle from the centre, $y$-axis is the ratio of the measured luminance at that angle to the measured luminance when it's at the centre. 
This map was output as a matrix that was then used to produce an HDR image of the vignetting filter using Octave (Eaton et al., 2019) (Figure 9.24). This can then be used to correct the HDR images using Radiance's pcomb command. For example, if the filter says that due to vignetting, the measured value at a point will be $90 \%$ of what it "should" be, we can correct it by dividing the value at that point by 0.9 .

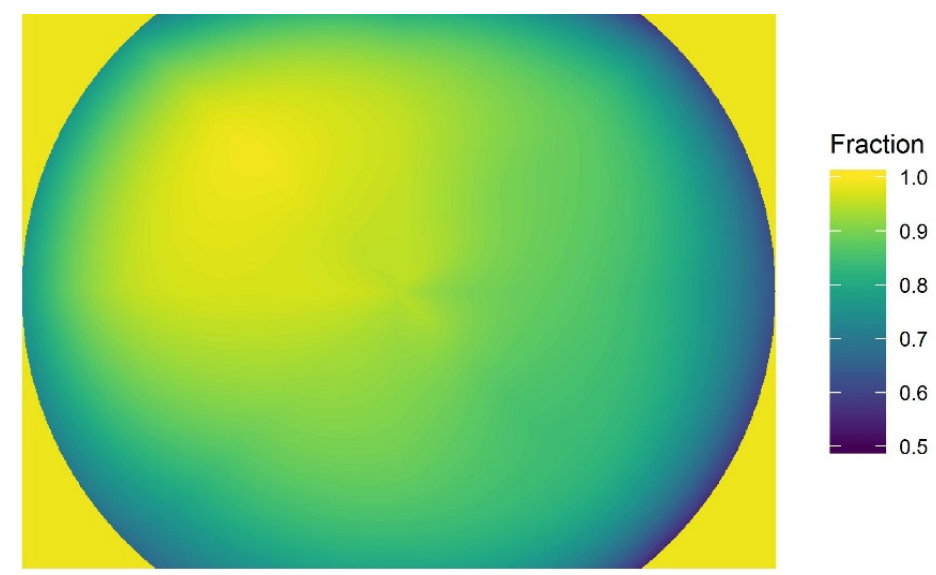

Figure 9.24 The resulting vignetting filter to "correct" the HDR images.

This was then cross-checked through an alternative approach. Instead of going through the whole process of photographing the target at different angles, the camera was simply rotated radially in $45^{\circ}$ increments. This allowed us to compare what slices of the same scene looked like through different lens angles. The asymmetry is, again, quite visible (Figure 9.26).
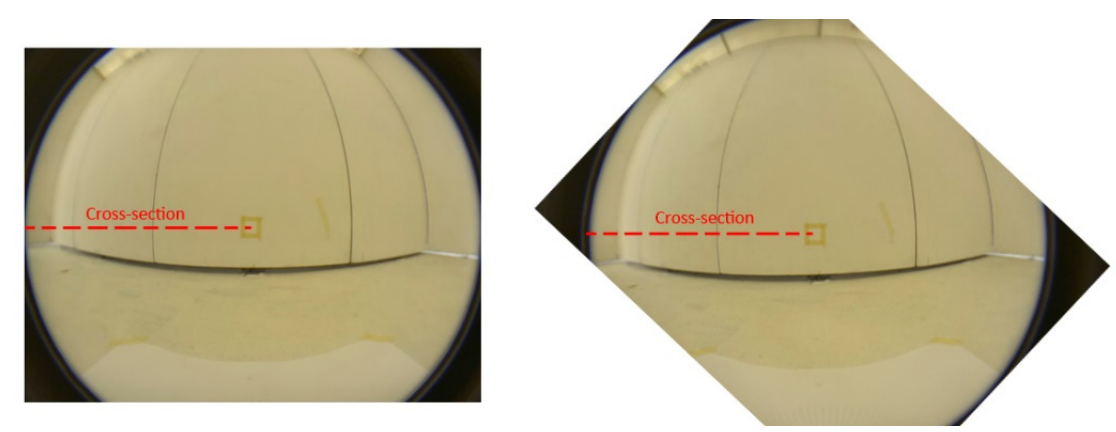

Figure 9.25 Illustration of the selection of a cross-section viewed through different angles, this one being the one plotted in the left plot in Figure 9.26 below. 

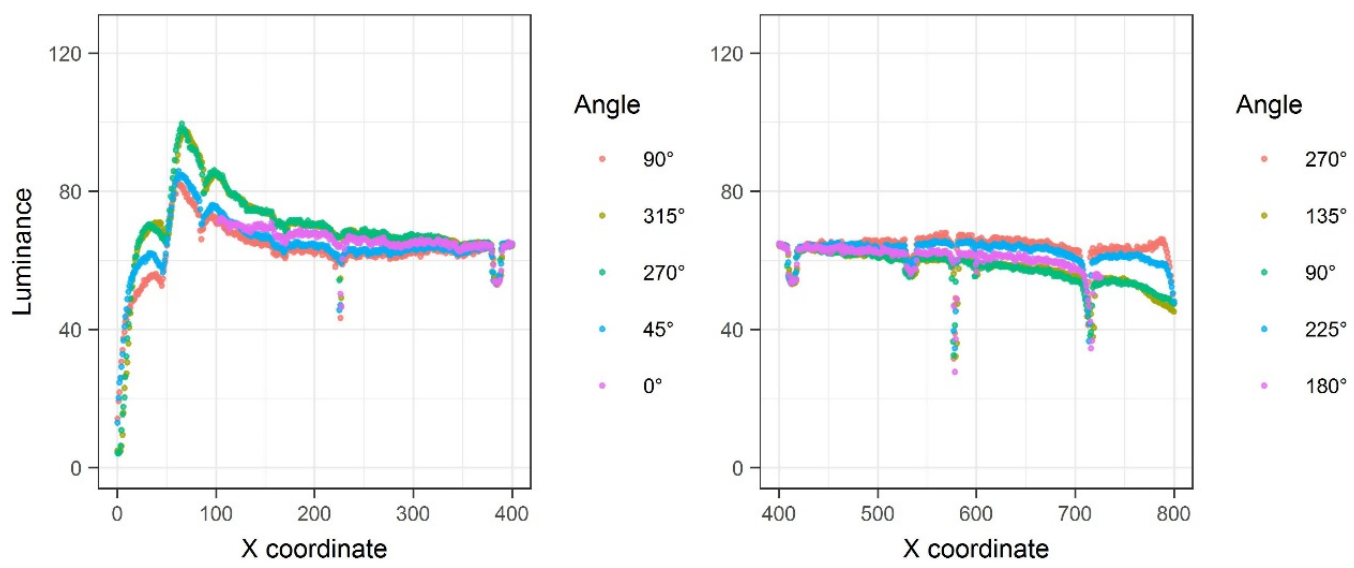

Figure 9.26 Comparison of cross-sections measured through different lens angles. Note the way that the observed luminances change significantly when viewed through different parts of the lens. Left plot is the left cross-section, right plot is the opposite right cross-section (different parts of the scene move through different parts of the lens).

Importantly, we can use the ratios of these cross-sections to check our vignetting function (Figure 9.27). The principle here is that the ratio of the measured luminances at different angles should equal the ratio of their respective vignetting functions. While this was roughly true for the $270^{\circ}, 315^{\circ}, 90^{\circ}, 45^{\circ}$, and $135^{\circ}$ functions, with error within $-2.5 \%$, there were potentially significant discrepancies with the $0^{\circ}, 180^{\circ}$, and $225^{\circ}$ functions (especially $225^{\circ}$ ).
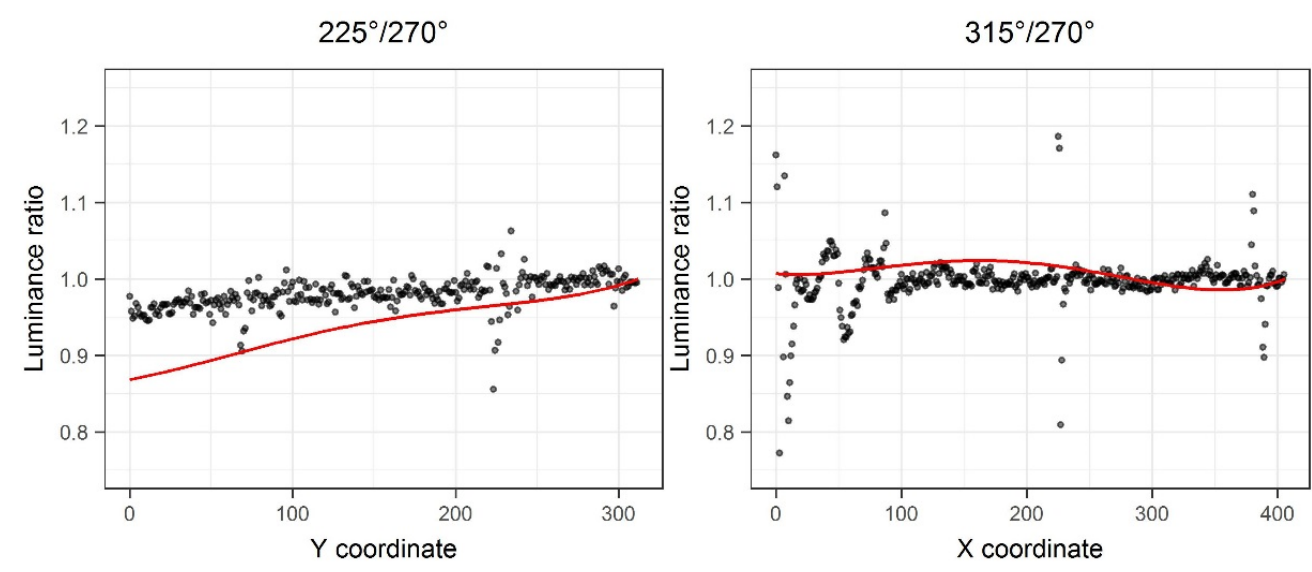

Figure 9.27 Selected comparisons of cross-sections at different angles. Points are the ratios of measured luminances in the maps at, say, $225^{\circ}$ and $270^{\circ}$. The red line shows the ratio that is predicted by our previously measured vignetting function. Ideally, these should line up. On the left we have the example of the $225^{\circ}$ angle, which seems to have the largest discrepancies. On the right, we have the comparison of $315^{\circ}$ and $270^{\circ}$, which aligns well with only some small differences of $<2 \%$.

To attempt to "fix" this, replacement functions for these angles were generated in terms of the combined $\left[270^{\circ}+315^{\circ}\right]$ function (these angles were merged because the measurements suggested their functions were practically identical, and combining their 
measurements could reduce noise in the estimate of the function). These new functions were:

$$
\begin{gathered}
y_{270+315}=-0.000000000539090 x^{5}+0.000000100289670 x^{4}-0.000007097409458 x^{3} \\
+0.000202579127311 x^{2}-0.000930098361881 x+1 \\
y_{180}=\frac{y_{270+315}(x)}{0.0017 x+1} \\
y_{225}=\frac{y_{270+315}(x)}{0.0006 x+1} \\
y_{0}=\frac{y_{270+315}(x)}{0.0000000571 x^{4}-0.0000059324 x^{3}+0.0001877776 x^{2}-0.0007800865 x+1}
\end{gathered}
$$

These were then used to create a new vignetting correction filter (Figure 9.28).

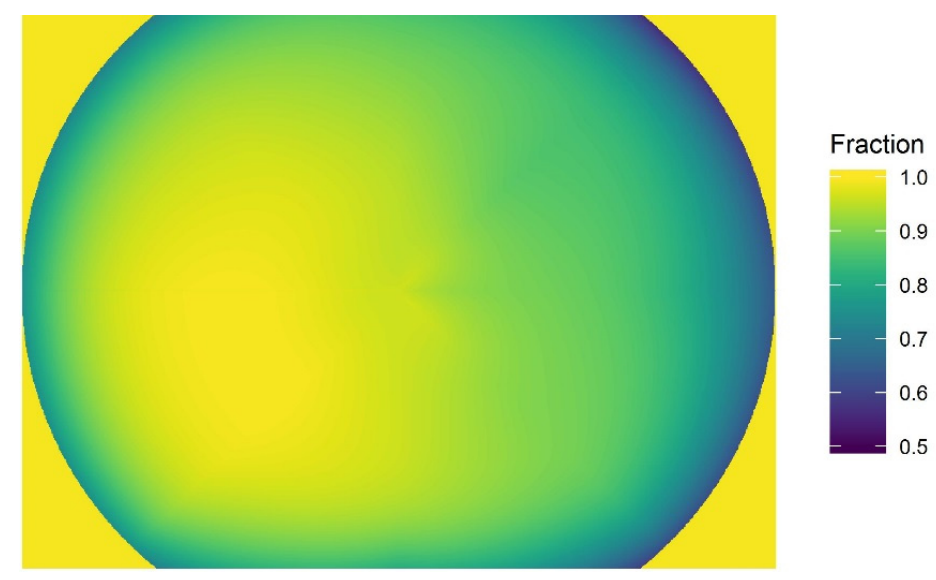

Figure 9.28 The second vignetting map used to "correct" the HDR images.

Comparing our two vignetting filters in practice, however, suggests that for most purposes the potential errors suggested by our cross-checking are not significant. Commonly used uniformity metrics, for example, are not meaningfully affected by our choice of which filter to use (Figure 9.29, Figure 9.30). Neither does there appear to be any meaningful difference between them when we attempt to check our luminance maps against spot measurements (see supplementary information).

It is not entirely clear exactly what was causing these discrepancies between the measurements. The simplest explanation would be that subtle differences in camera angle and tilt can in some situations cause notable $(-10 \%)$ correlated differences in observed luminance - an issue we also see with spot luminance measurements (see A.3.3). Regardless, as the uncertain error here did not appear to meaningfully affect our measurements of the overall light distribution, the decision was made to acknowledge it 
and continue to use the current vignetting filters rather than attempting to create a "perfect" filter.

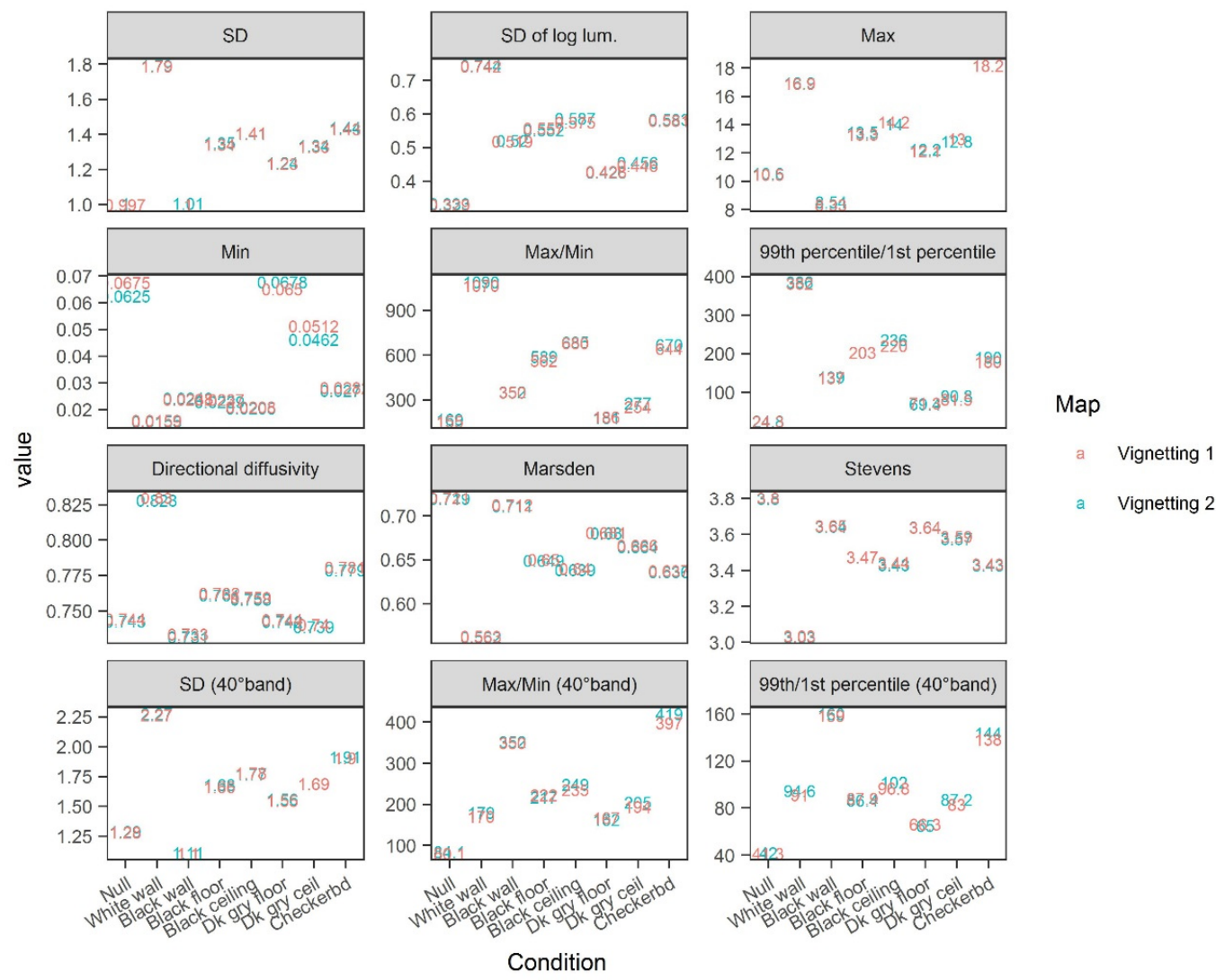

Figure 9.29 Uniformity and brightness metrics for Experiment 1 conditions calculated using different vignetting filters. 


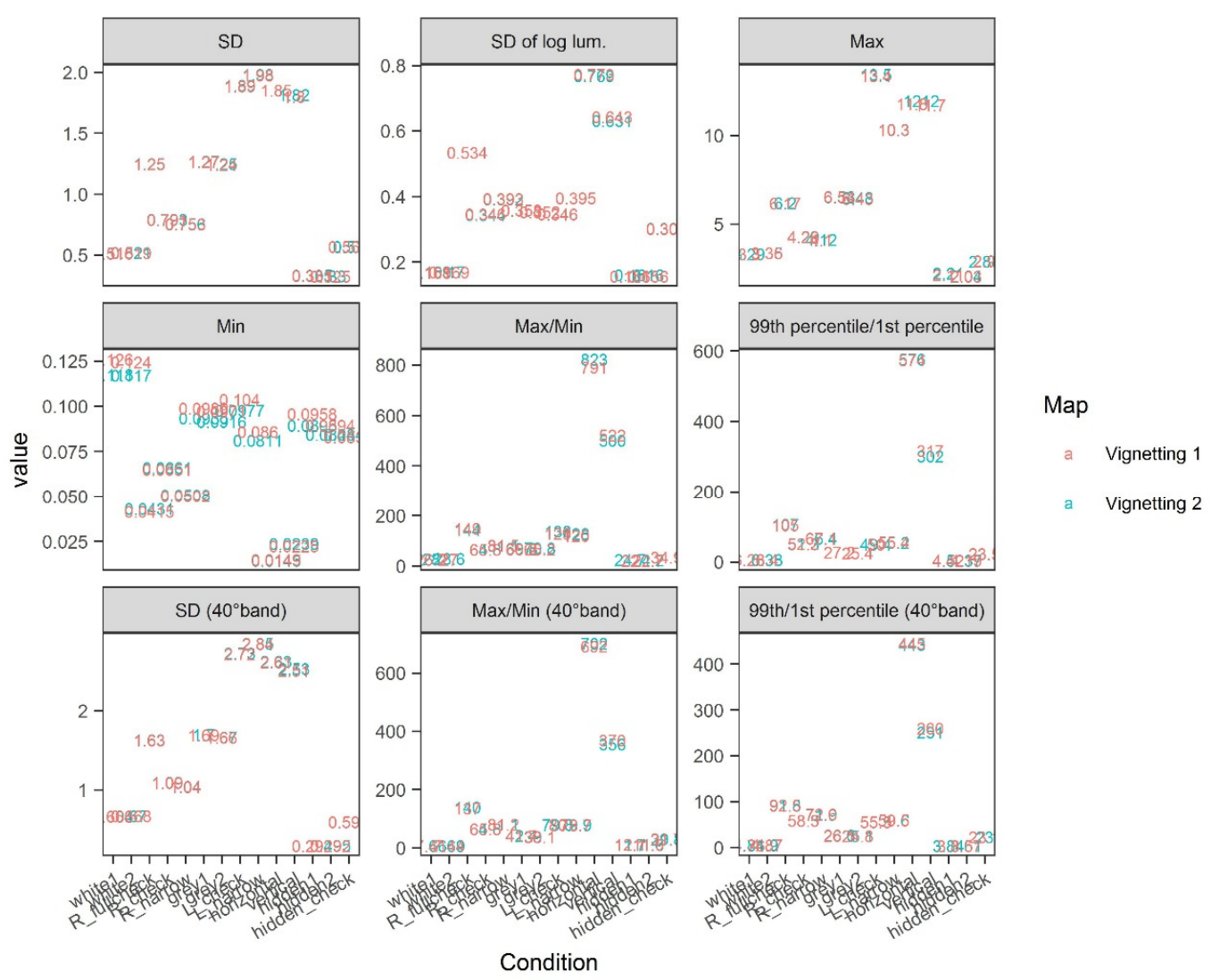

Figure 9.30 Uniformity and brightness metrics for Experiment 2 conditions calculated using different vignetting filters.

\section{A.3.3 Scattering}

During checks of the accuracy of the HDR luminance maps, it was observed that in high contrast scenes such as a checkerboard, there was a problem of overestimation of dark areas, and underestimation of light areas. As shown below, the effect could be significant, with the black checks in this case being overestimated by around $15 \%$. This was a problem both on the Nikon D200 and the LG-G4. 

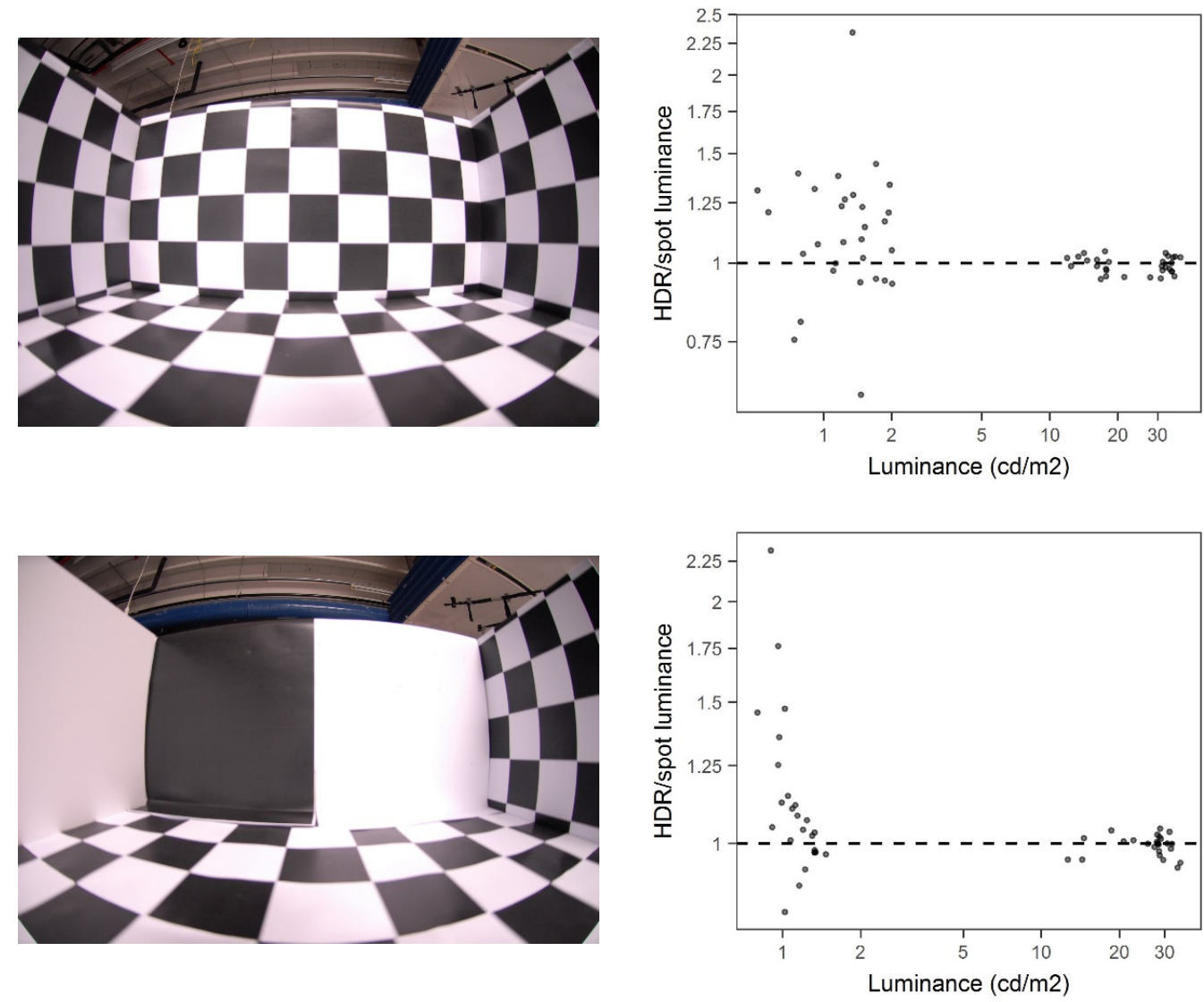

Figure 9.31 Comparison of spot measurements of high contrast scenes to measurements from an HDR luminance map using a Nikon D200 camera with fisheye lens attachment. Measurements were of the checks on the main "wall" facing the camera. The darkest measurements - of the black surfaces - are substantially noisier due to the glossiness of the surface, but show a clear trend towards being overestimated by the camera relative to the luminance meter.

The best explanation for this seems to be lens scattering. Inanici (2006) noted how the optical structure of the camera lens resulted in a degree of light scattering, such that a point light source that should theoretically just cover 1 pixel in the image would be spread out over multiple. They noted that it could be one of the factors contributing to the $-10 \%$ overestimation of the dark targets in their tests, but argued that point spread had only a limited range, and would likely be minor in most practical applications.

Unfortunately, our results suggest potentially larger effects than were identified there. Moreover, further investigation suggested that the effects could potentially propagate over at least half the width of an image (Figure 9.32).

To remove the uncertainty caused by the uncertainty of the spot luminance measurements, and get more detailed plots of the effects, we moved to setting up simple black and white scenes with movable components. The basic theory here is that by looking at how the measured luminances in the black and white parts change when exposed to the 
scattering of the other luminance, we could measure the effect of scattering as shown below (Figure 9.32).

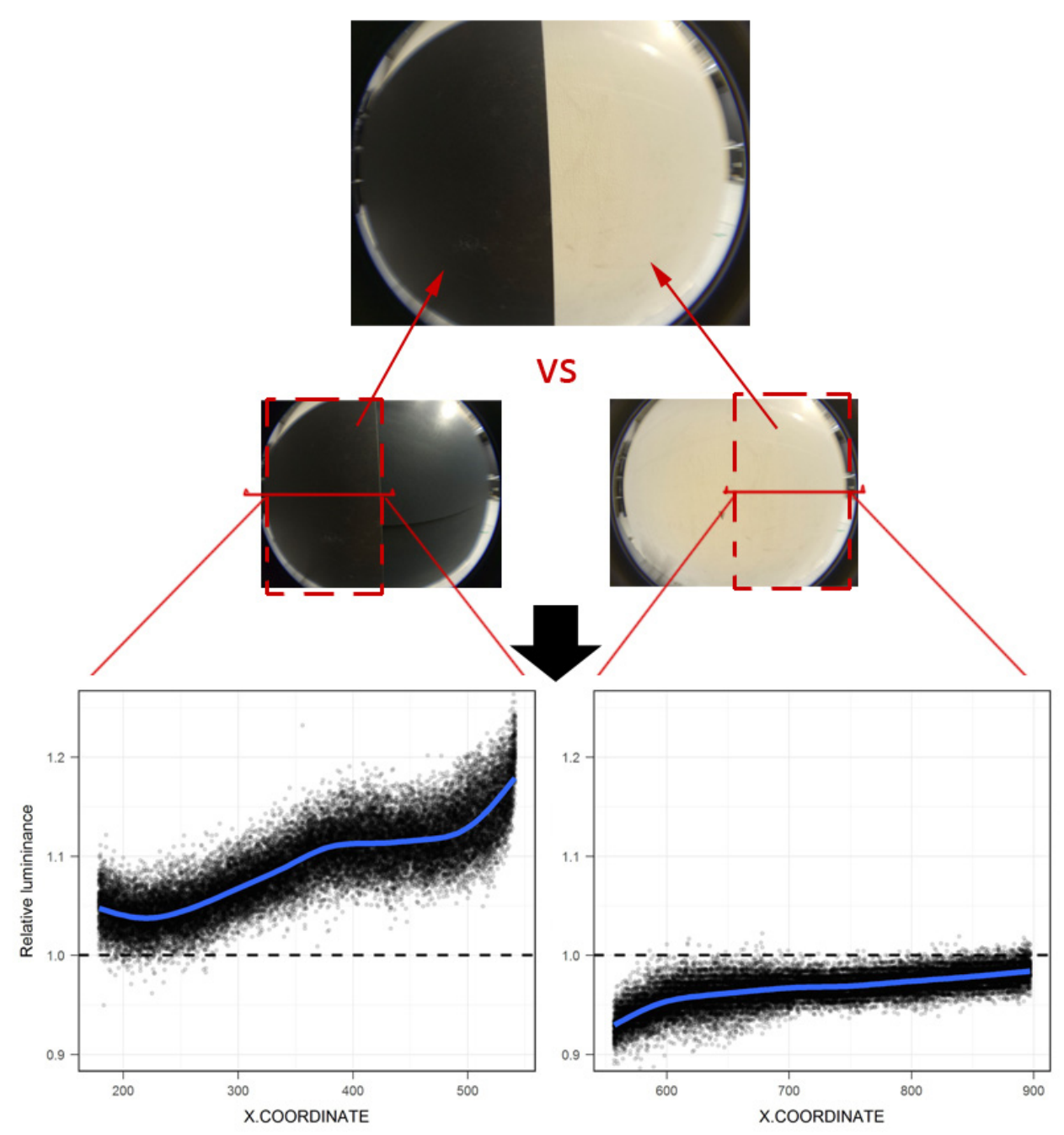

Figure 9.32 Measurement of scattering effects in a simple two-part scene using only the HDR luminance maps from an LG-G4 smartphone camera with fisheye lens attached. Theoretically, when photographing a uniform scene there should be minimal/less effects from scattering. When half the field is covered by a white surface the black surface would be expected to be overestimated due to the effects of scattering. By comparing the change in luminance from the uniform scene to the half-black scene, we can measure the effect that scattering from the adjacent white surface had on our measurements of the black surface, and vice versa.

With regards to the practical implications of this, the aggregate result of this error would be a compression of the overall contrast of the image. The resulting measurements would thus appear more uniform than they should be - with the effect being greater the higher the contrast. For a study specifically looking at the effects of light distribution using deliberately high contrast scenes, such systematic and potentially significant error is problematic. While not a problem for simply establishing that some spaces are more 
uniform than others, or for looking at basic correlations, it is a problem if one wants to develop models relating uniformity to appearance. Thus, attempts were made to try to work out a way of correcting the images to the "correct" uniformity.

It should be noted is that taking spot measurements to check for the errors in the experimental conditions is not particularly practical. As noted, they have questionable accuracy for something like this, and to do it right one would have to measure the entire space. This was very difficult for most of our conditions due to a combination of a) trying to accurately point a meter at invisible spots on a blank wall, and b) the geometry of the model openings limiting what the luminance meter could be pointed at.

The initial attempts focused on trying to determine if there was a way to measure some kind of "scattering correction function" that could be applied similar to how vignetting correction works. The first method attempted to approximate the effects of scattering by applying successive Gaussian blurs to the images. The idea here was that the effect of further blurring the image and reducing its contrast could be combined with the measurements of scattering and used to predict it. However, despite appearing promising on the initial tests, when we attempted to apply it to multiple scenes (specifically checkerboards with different resolutions), it fell apart and was unable to predict the effects well. The "best" models could create nonsensical results when applied to other scenes, for example suggesting that the luminances in the scene should range from $-6,000$ to 60,000 $\mathrm{cd} / \mathrm{m}^{2}$, which was clearly impossible.

With that failure, the image processing literature was looked to for guidance. Attempts to apply sharpening algorithms (e.g. Levin et al., 2007) to remove the effects of scattering also failed. This was due to two reasons: 1 ) because the algorithms (perhaps unsurprisingly) tended to focus on the edges in the image and not on the broader scattering effects we have identified, and 2) because while the parameters of the algorithm could be tuned to provide an acceptable correction to one scene, those same parameters would not work on another. Given that the only way to work out what the "correct" parameters are is to know what the correct luminances are, this is clearly not practical as a means of correcting shots of experimental scenes where we don't know what the correct distribution is.

Following these failures, an alternative approach was devised. Instead of attempting to create a scattering correction function that could be generally applied to the camera, we would instead attempt to measure the scattering effects for each of our scenes using HDR images.

The idea here was based upon the way we had previously measured the effects of scattering by comparing the measured luminances of a region under the effects of scattering to the measurements of a uniform scene of the same luminance. 
To allow such a comparison to be made for the experimental scenes, their luminance maps were binned using $\mathrm{R}$. This created effective contour maps, with regions set to a specific luminance (Figure 9.33). The bands covered $\sim 0.33 \log \mathrm{cd} / \mathrm{m}^{2}$, with testing indicating that differences of magnitudes less than that (i.e. within the contours) should cause minimal scattering effects.

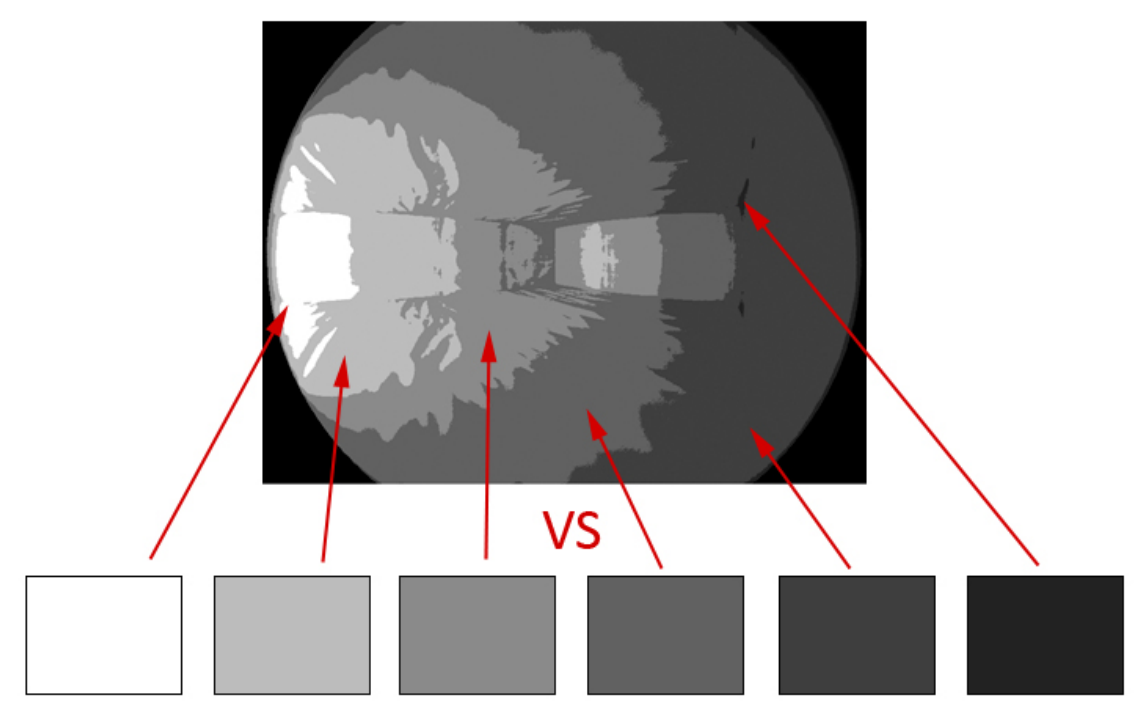

Figure 9.33 Example of a scene that has been "binned" to create a contour map of the scene. The measured luminances of the contours are then compared against measurements of uniform fields of the same greys.

The contours, with their defined luminances, can then have the measurements of them compared to uniform images of the same luminance in order to measure the scattering effects. This was done by displaying them on a large OLED TV screen and placing the camera close to the screen such that it filled as much of the field of view as possible.

To reduce noise in the difference-map a 3-pixel radius median blur was applied to the image (Figure 9.34). This was to make it easier to read, as direct pixel-to-pixel comparisons between even the same images will inevitably show significant noise that is not indicative of any larger trend. 

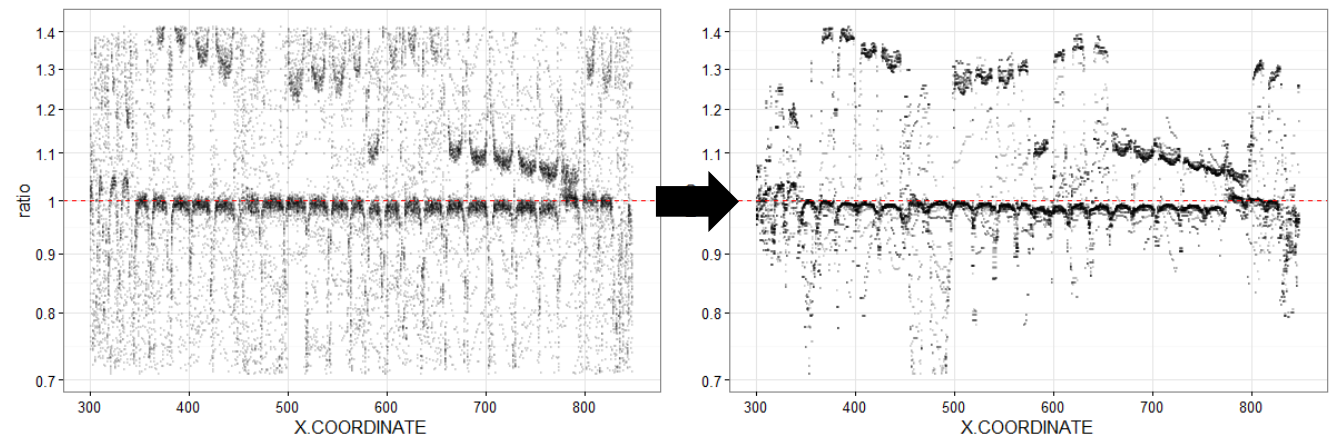

Figure 9.34 Illustration of the use of smoothing to reduce noise in the pixel-by-pixel luminance map comparison (in this case, a cross-section through an image of a checkerboard pattern). The left image shows the initial readings, where the luminance of every pixel in one image is divided by that of the corresponding pixel in the other. The right shows the pattern after smoothing.

The change-maps were then saved as images with a scale such that $\operatorname{RGB}(100,100,100)$ represents $100 \%$ (i.e. no-change), and each 1 point change on the scale represented a $1 \%$ change in luminance. These maps could then be read in an image editing tool like Adobe Photoshop and used as a guide in order to paint a scattering effect map over the original HDR images. They were then applied in the same manner as the vignetting correction filters to correct the HDR images using Radiance.
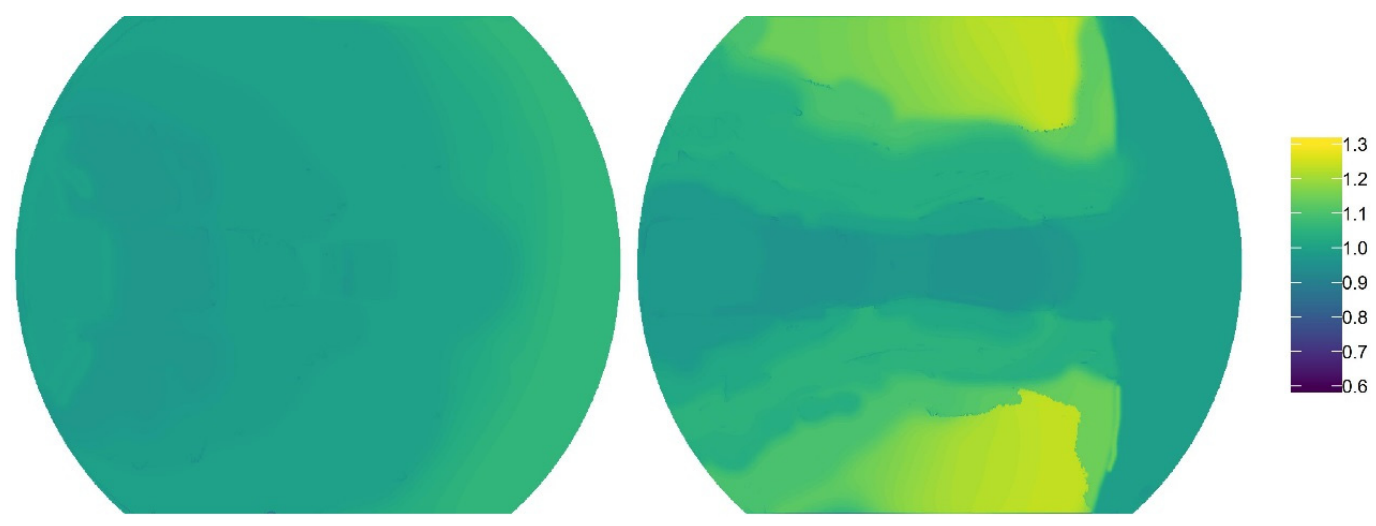

Figure 9.35 Examples of scattering effect filters created for the Experiment 1 conditions. The left condition is the uniform grey one, which had minimal contrast. The right is the White Wall condition, which had more significant contrast and so correspondingly more scattering.

The idea here is that the contour maps presented on the screen should be approximating the actual luminance distribution of the scenes they're portraying. Hence, measurements of them can be used as a rough proxy for measurements of the actual scenes, and any observed scattering effects should be similar to that that the photos of the actual scenes would be suffering from.

There are, however, several issues that reduce their accuracy. Firstly, they are rough contour maps. This can be used to get a rough estimate of the possible scattering effects across the image, but some degree of interpolation across regions has to be done in order to 
match the gradients of the real scenes. Secondly, the scenes are not precisely the same size. Displayed on a screen they cannot fill the whole $180^{\circ}$ field in the same way as a $3 \mathrm{D}$ environment. This reduction in angular area would be expected to exaggerate scattering effects to some degree. Thirdly, the display will be somewhat distorted, as the camera is now taking a fisheye-lens photo of a fisheye-lens image displayed on a flat screen. Fourthly, as the image we are using to measure the scattering is itself based on a measurements suffering from scattering, the contrast in the image will be somewhat lower than what it is in the actual scene, thus reducing the scattering effect we measure. That being said, given that the shift caused by scattering is much smaller than the difference in luminance that produces it, this change may not necessarily have a substantial effect.

In terms of the effect on analysis and conclusions in our study and others, what should be noted is that effects are relative to how non-uniform the condition is. Effectively, scattering compresses the apparent uniformity range and differences between conditions. However, it generally does not alter the overall pattern. Conditions that are highly nonuniform will still be described as highly non-uniform. Conditions that are uniform will still be described as uniform. Thus, while the error and our crude correction method are a source of uncertainty, it should not significantly affect the appearance of a trend. Models that fit badly will fit badly regardless of the uncertainty in the correction. The error here is mostly a concern if one is trying to devise a model to make very precise predictions. While the lack of consideration of scattering in past studies in the literature is an issue, it is unlikely to change their conclusions about general trends. Future work though should take it into consideration, and attempt to account for it. If we are to develop mathematical models to predict the effects of the light distribution on spatial brightness, it will be necessary to develop ways to reliably account for the effects of scattering. The crude approach we have devised here should be treated only as a stopgap measure.

It is, however, potentially a more serious issue for studies that tried to calibrate their luminance maps using spot luminance measurements, and then used them to measure mean luminance. As we have demonstrated here, the effects of scattering can cause significant errors in calibration if one is calibrating to specific points. Illuminance meters are thus preferred as a means of measuring the overall light level. It seems safer to relegate HDR luminance mapping to being a means of measuring the light distribution, and not the mean luminance ${ }^{85}$.

\footnotetext{
${ }^{85}$ A caveat: our focus was on simple artificially lit scenes with the luminaire itself not directly visible. Much of the research into HDR luminance mapping has focused on its application for daylighting, where the issue of actually capturing the entire dynamic range in the image (e.g. Jakubiec et al., 2016) may overshadow these problems.
} 


\section{A.3.3.1 Experiment 1 conditions}

Overall, scattering in Experiment 1 appears to be relatively minor compared to in Experiment 2, and does not substantially affect our measurements of the light distribution. Examination of our estimates of the scattering suggests that it can, in some regions, shift measured luminances by up to $30 \%$ (Figure 9.36). However, the overall effect of this is limited (Figure 9.37). Aggregate measures of uniformity such as the standard deviation are virtually unaffected - though they become more affected if the luminances are log transformed. This is due to the fact that the largest relative changes occur to the lowest luminances, which means that they are very small changes in absolute terms. Uniformity ratios that place great emphasis on those values may also be more affected — though their sensitivity to such things is also part of why they are questionable as metrics.

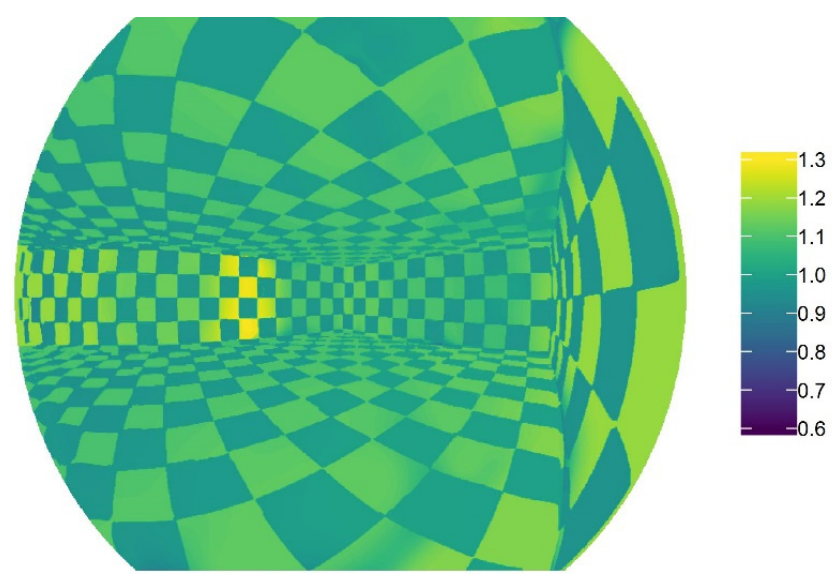

Figure 9.36 Example of scattering effects for checkerboard condition. Note the effects approaching $+30 \%$ in some regions.

Spot luminance checks similarly show no sign of significant systematic error that could be attributed to scattering, and indicate that at least between the areas that were measured, the relative differences in luminance were being captured reasonably well (Figure 9.38) 

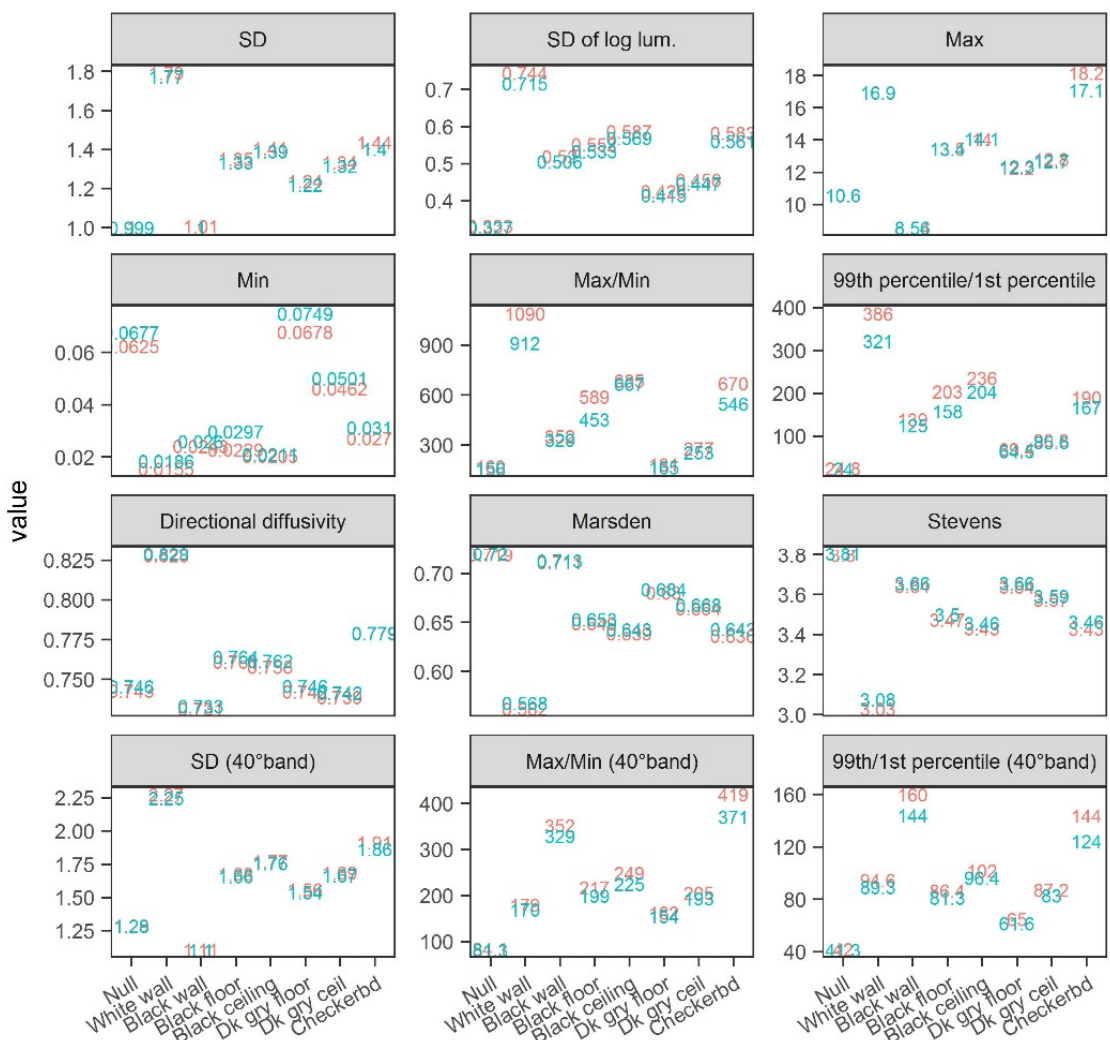

Map
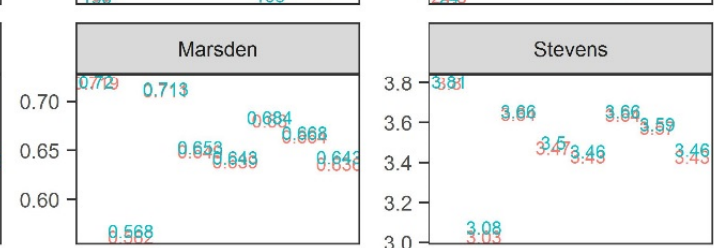

Corrected

a Uncorrected
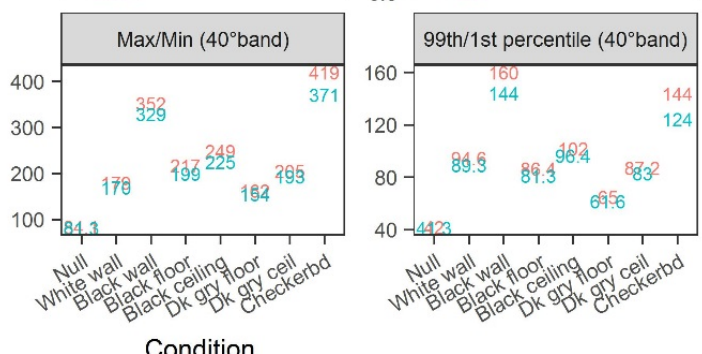

Figure 9.37 Effect of scattering correction on assorted uniformity metrics for Experiment 1 conditions. 


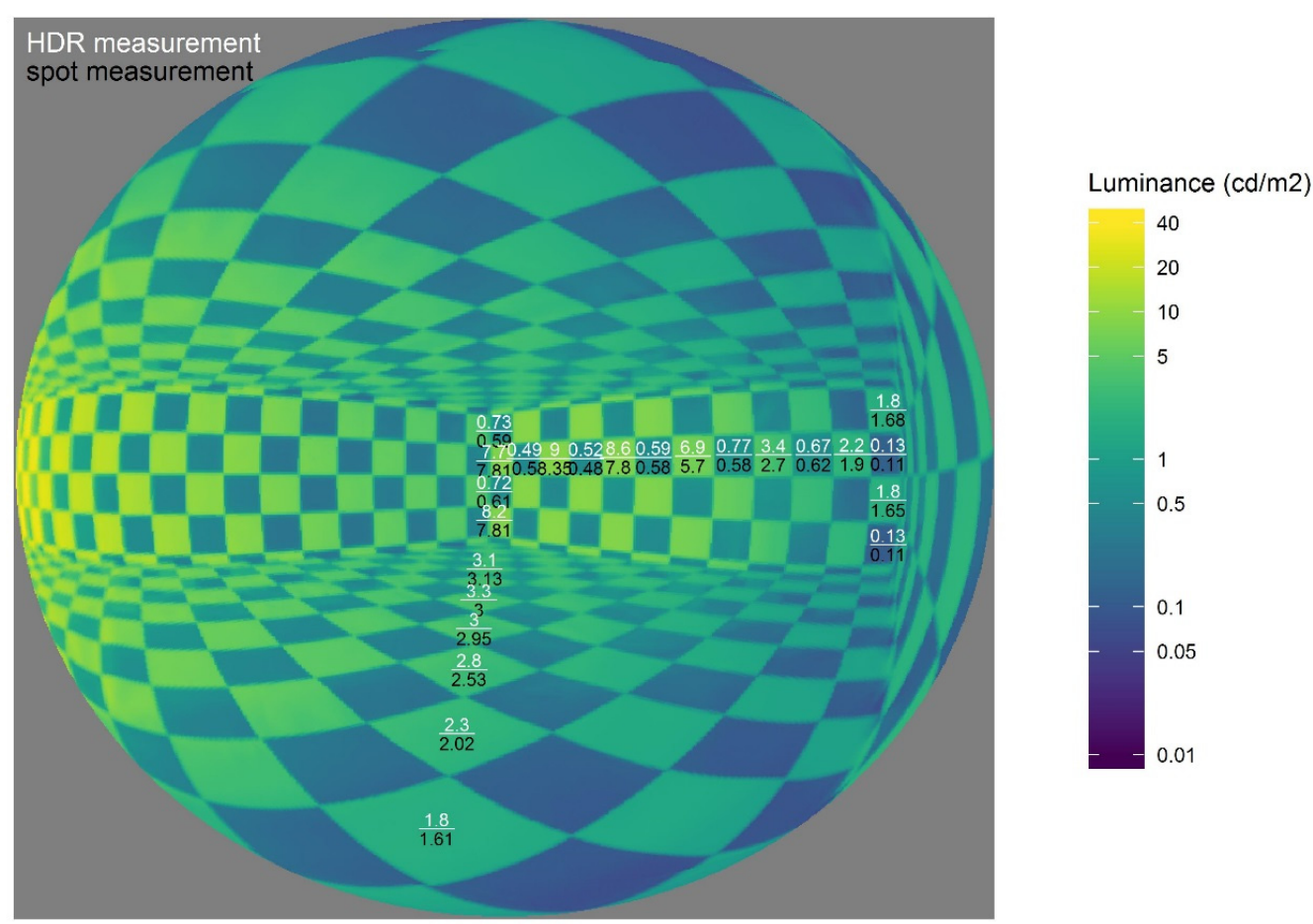

Figure 9.38 Comparison of spot luminance checks for the Checkerboard condition of the first experiment.
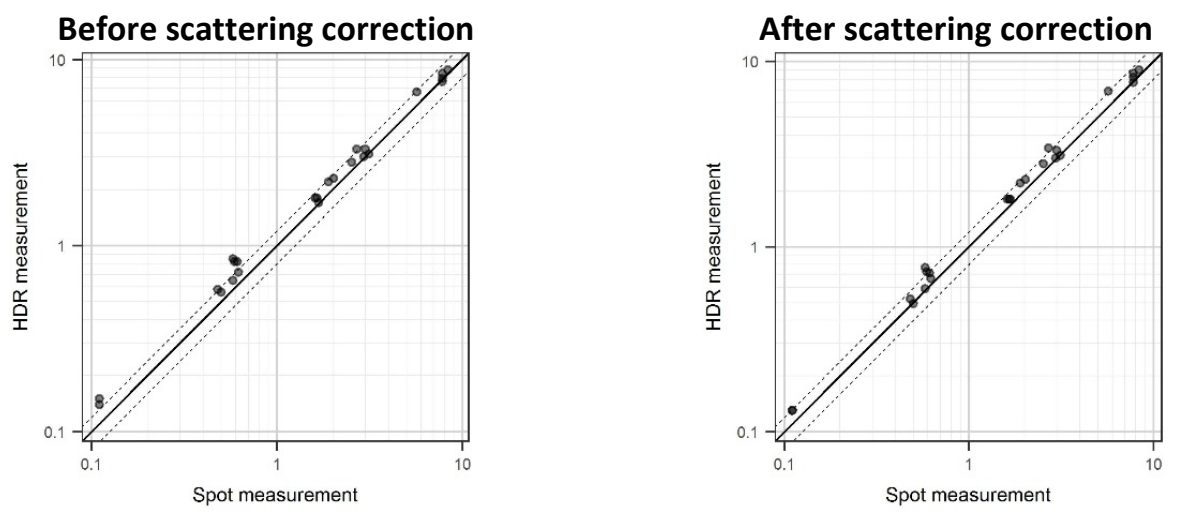

Figure 9.39 Comparison of HDR measurements against spot measurements for the checkerboard condition of Experiment 1 . The diagonal line shows the theoretical 1 to 1 fit that they should be along if the measurements were perfectly aligned. Dashed lines to either side show $\pm 20 \%$ as a reasonable error range given the uncertainty in spot luminance readings. An indication of scattering effects can be seen if the line formed by the measurements has a slope that diverges from 1. After applying scattering correction, the lower luminance black checks are darkened in the luminance map, resulting in the readings aligning better with the slope of 1 . 
Figure 9.40 Other luminance maps. The next four pages show the corrected luminance maps and spot measurements of the other seven conditions. Note that the "spot" measurements were taken as the average of multiple readings running down the corner of the back wall as a visual guide. Uncorrected maps can be found in the online supplement.

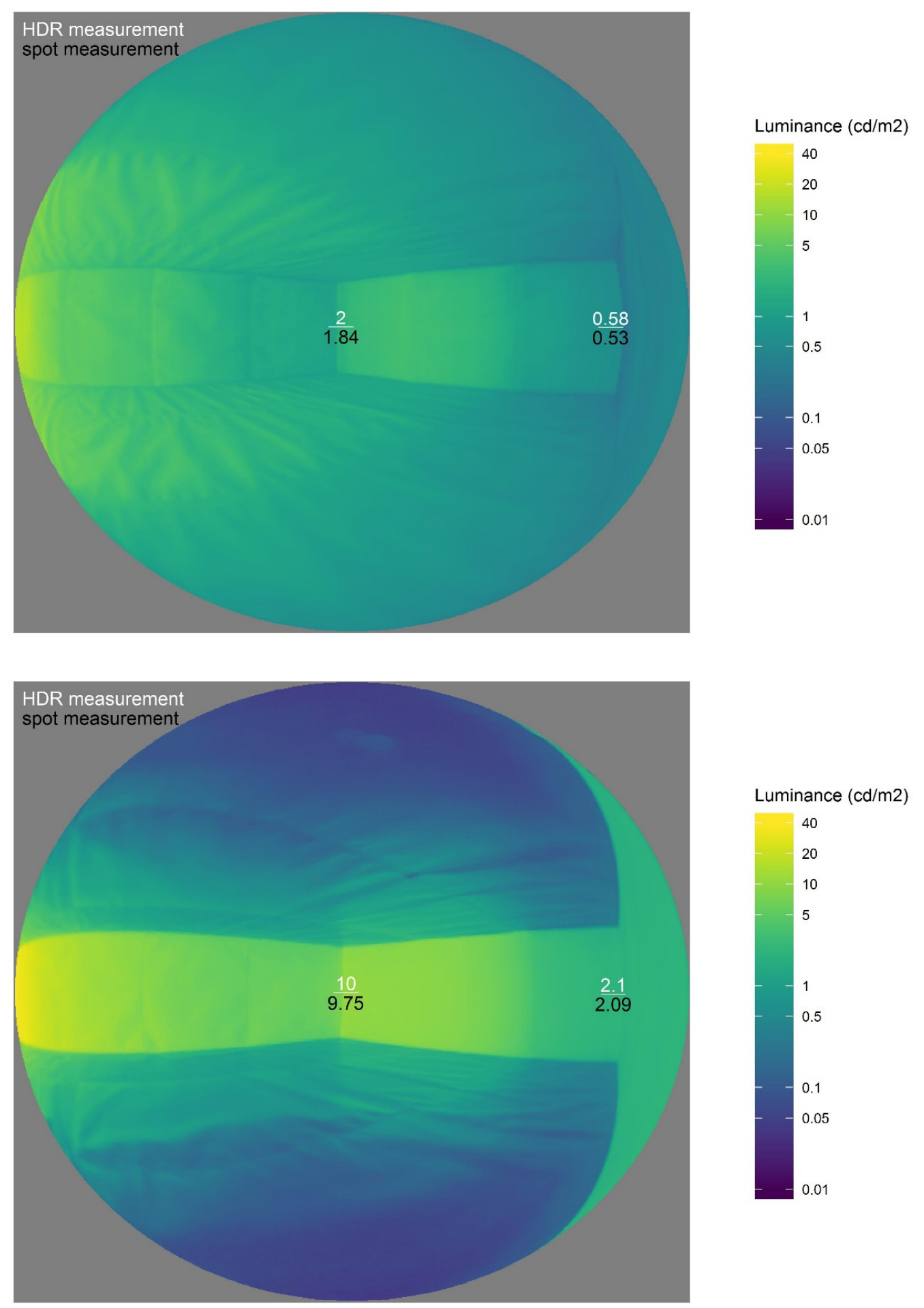




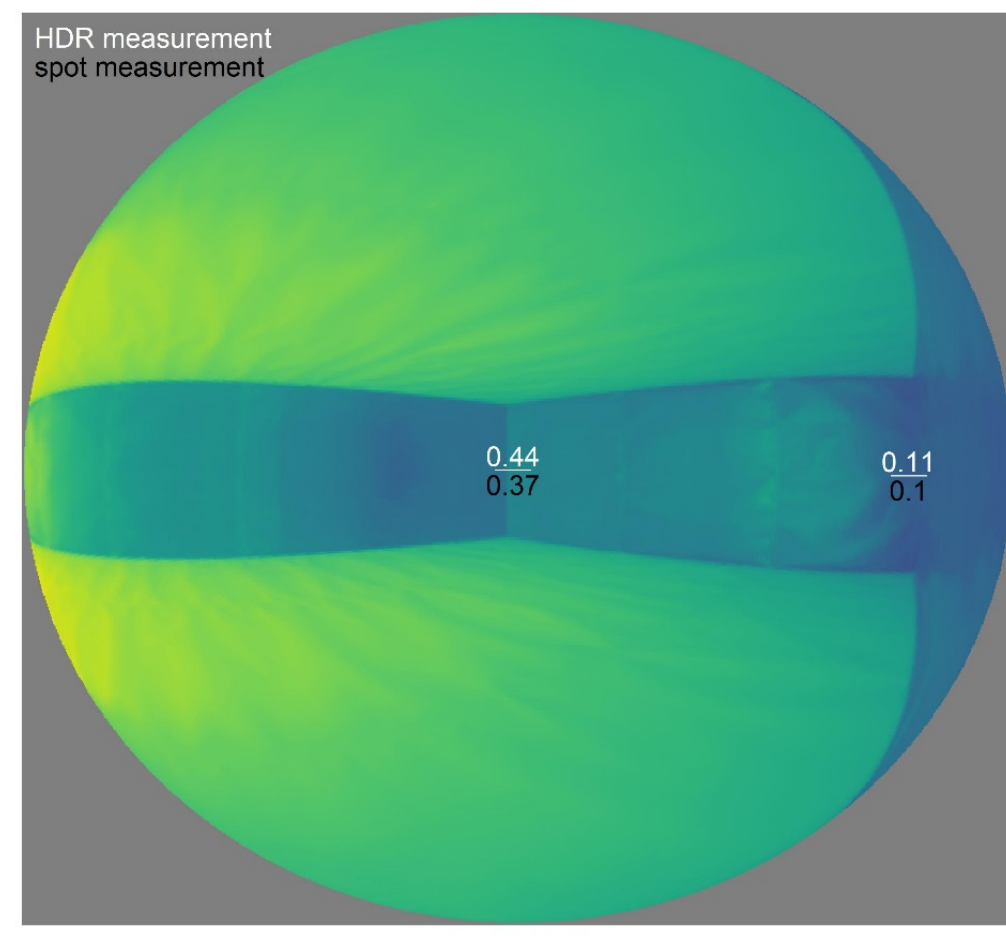

\section{Luminance $(\mathrm{cd} / \mathrm{m} 2)$}
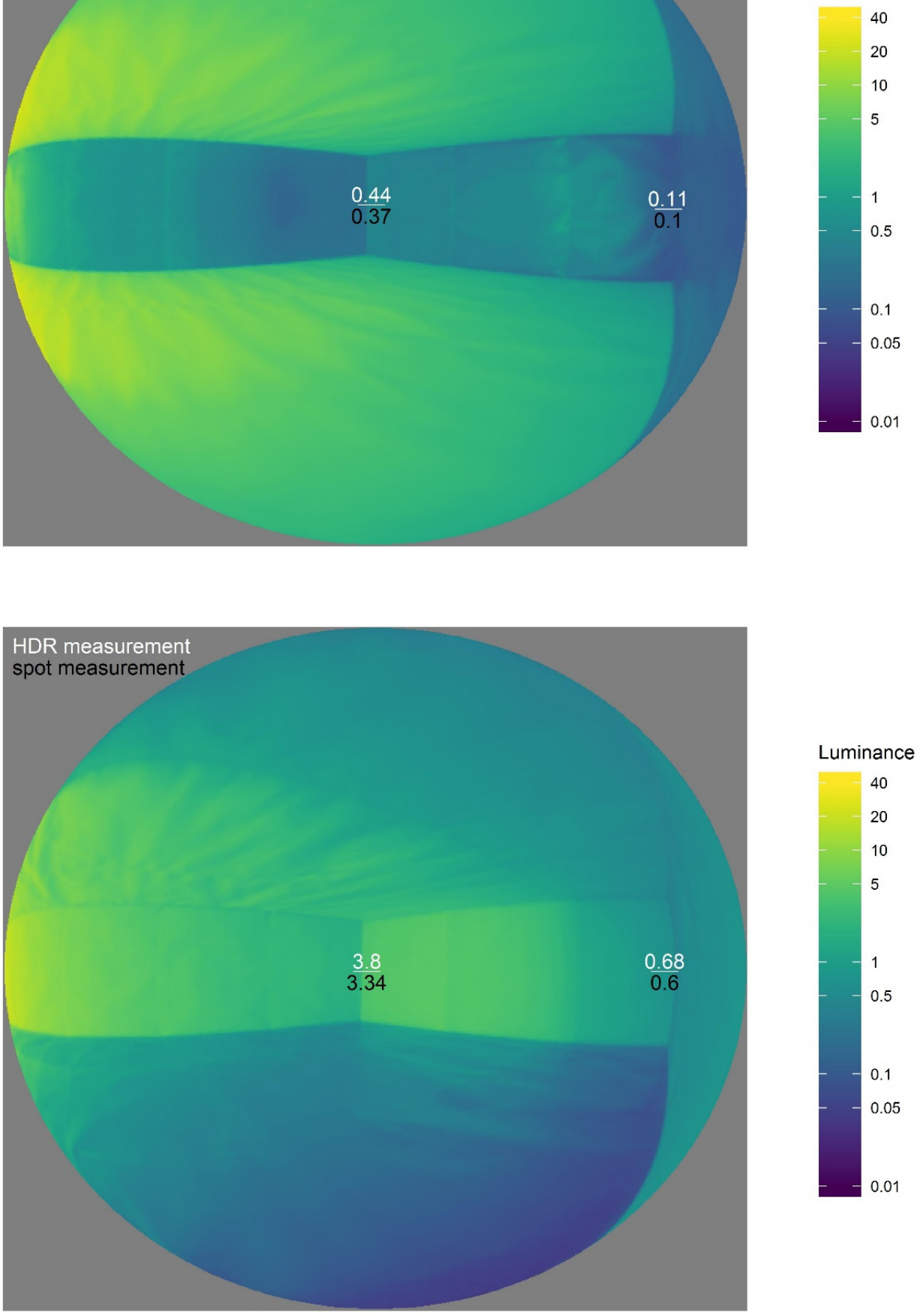

Luminance $(\mathrm{cd} / \mathrm{m} 2)$

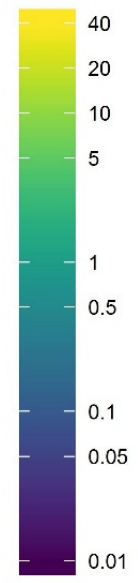




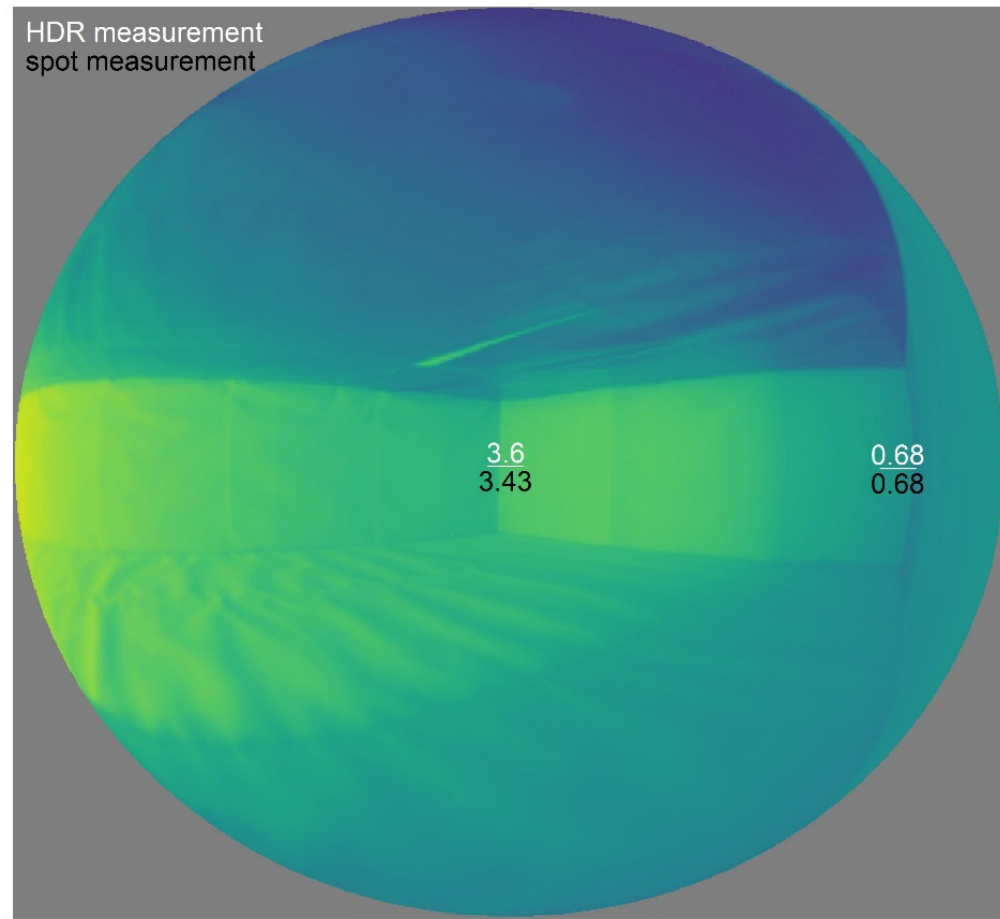

Luminance $(\mathrm{cd} / \mathrm{m} 2)$

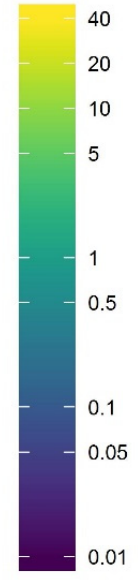

HDR measurement

spot measurement 


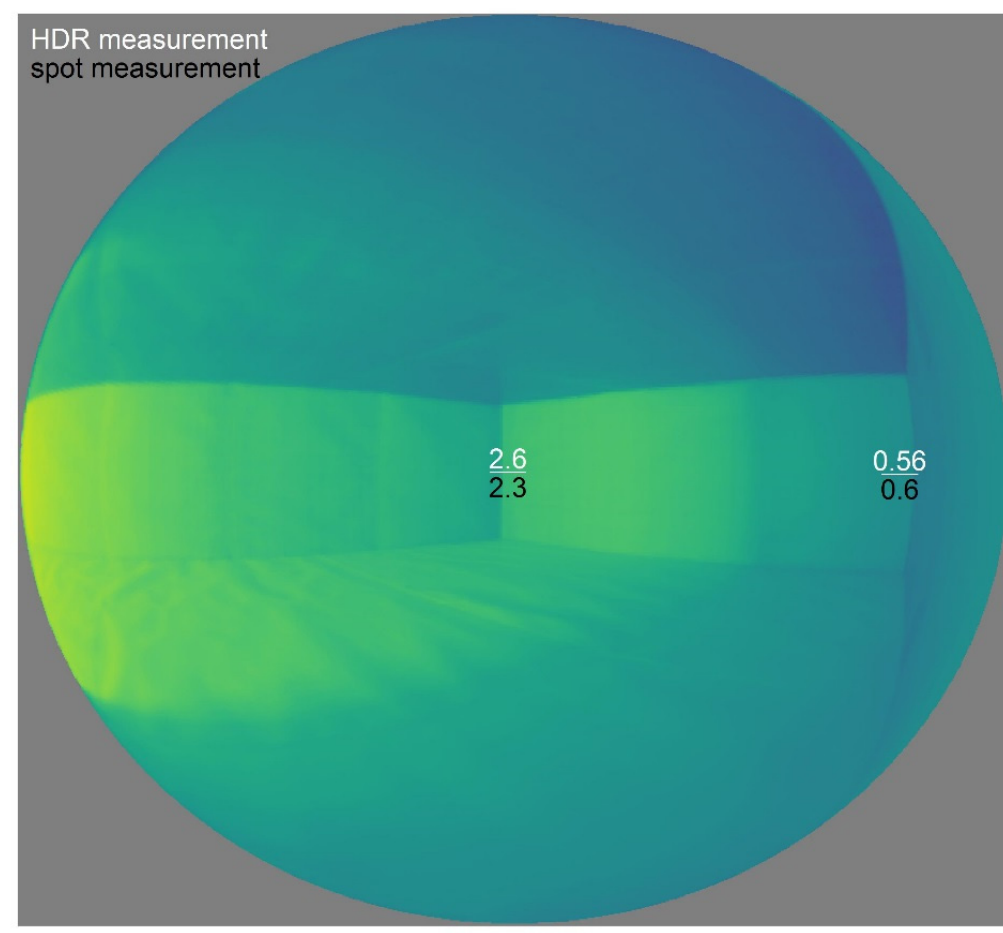


Table 9.3 Comparison of HDR measurements against spot measurements for other Experiment 1 conditions. The diagonal line shows the theoretical 1 to 1 fit that they should be along if the measurements were perfectly aligned. Dashed lines to either side show $\pm 20 \%$ as a reasonable error range given the uncertainty in spot luminance readings.

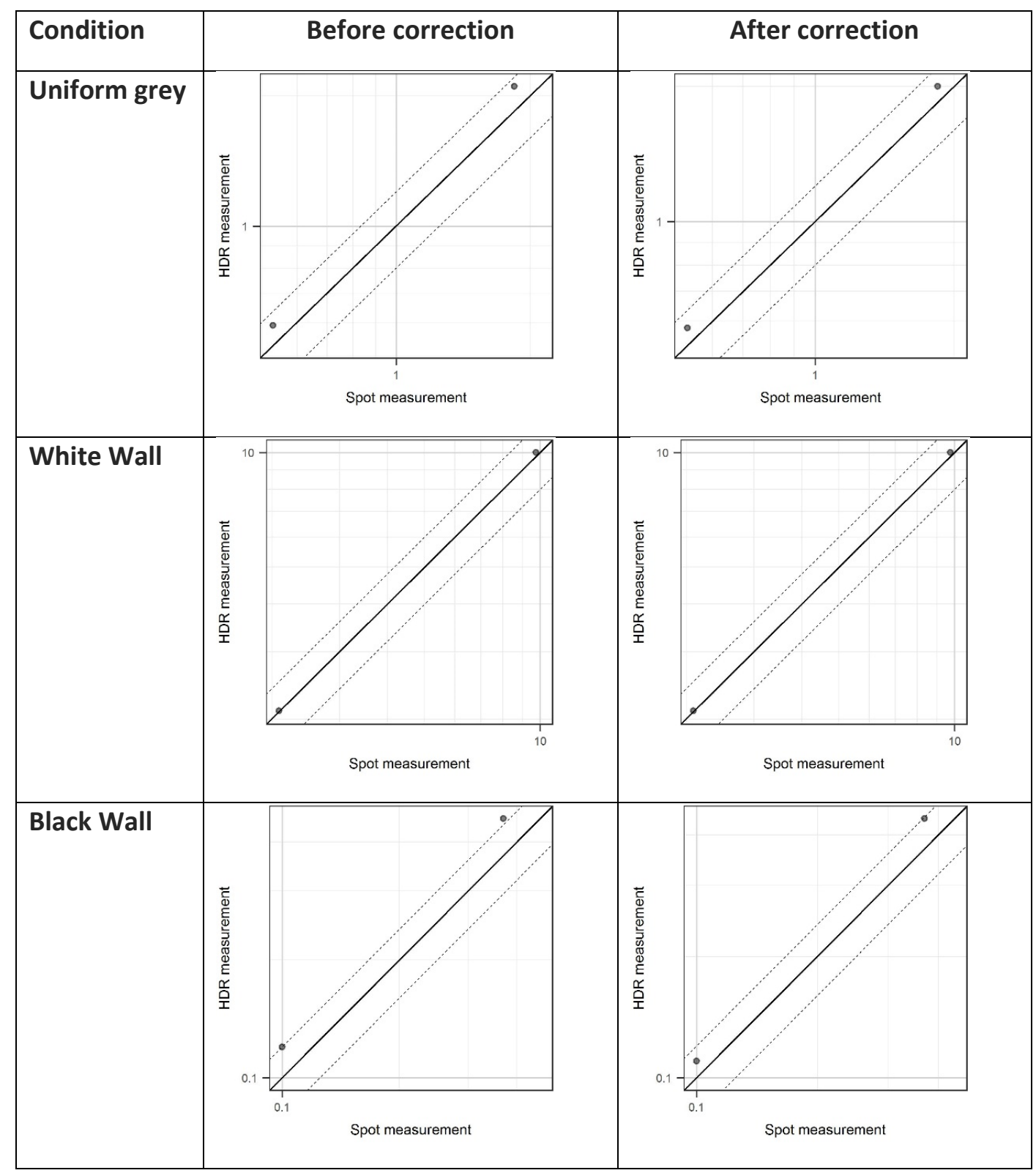




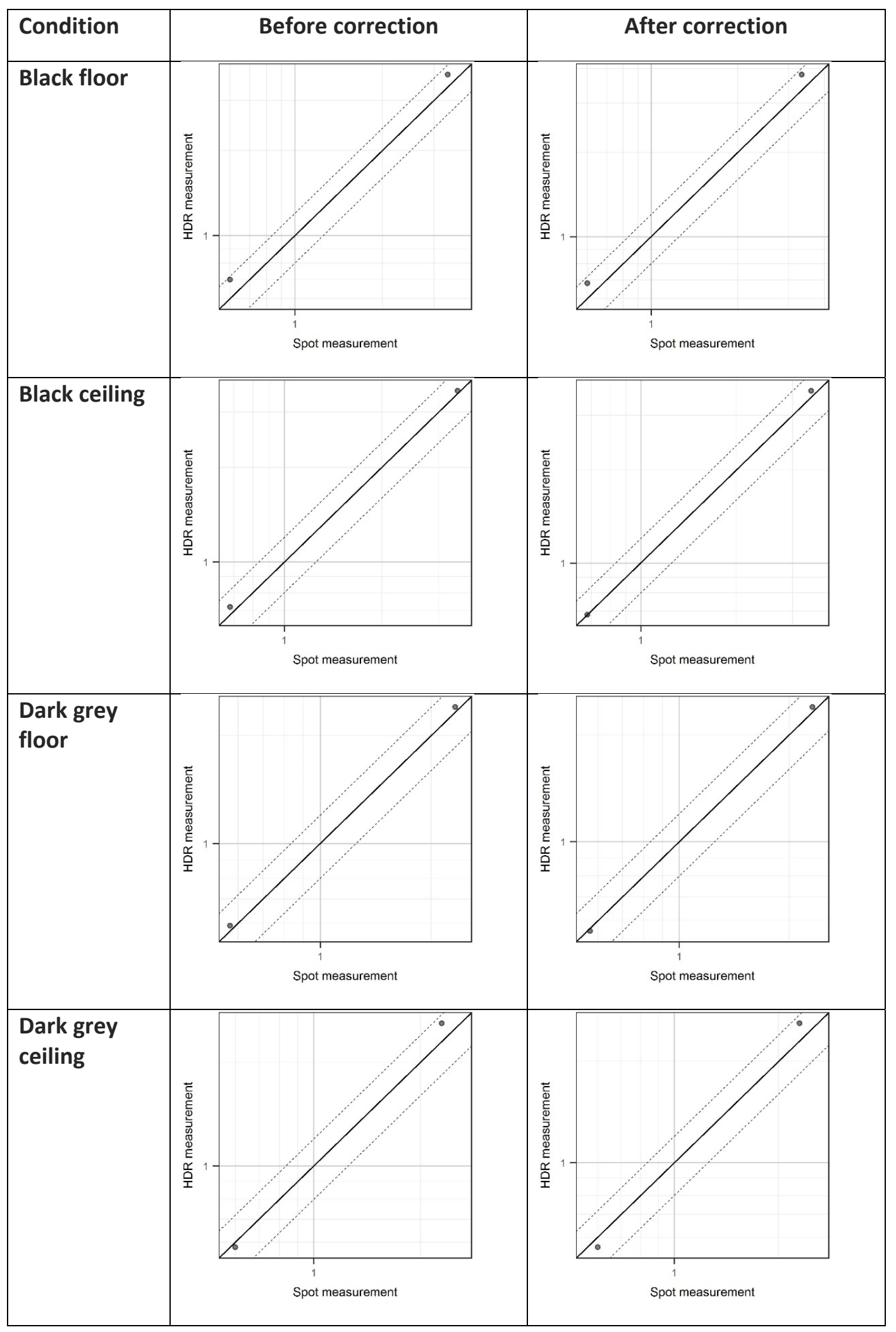




\section{A.3.3.2 Experiment 2 conditions}

The conditions in the second experiment showed significantly greater signs of scattering-related errors. The vertical condition in particular, likely due to the close proximity to its darkest regions to the light source, had very large overestimations of the black surfaces. In some cases, by an order of magnitude (Figure 9.41, Figure 9.42).

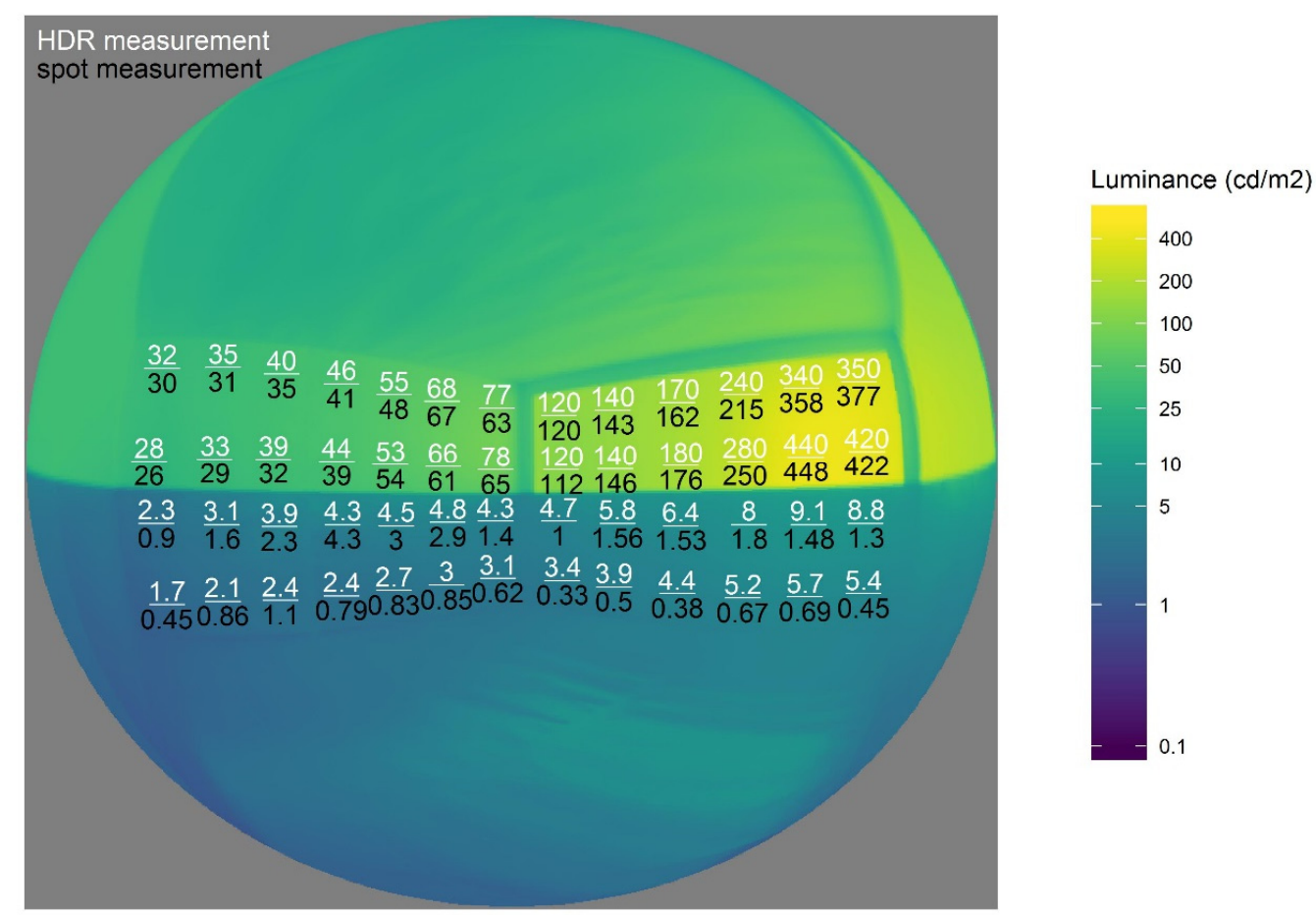

Figure 9.41 Uncorrected luminance map of the Vertical condition in Experiment 2 compared against spot luminance measurements.

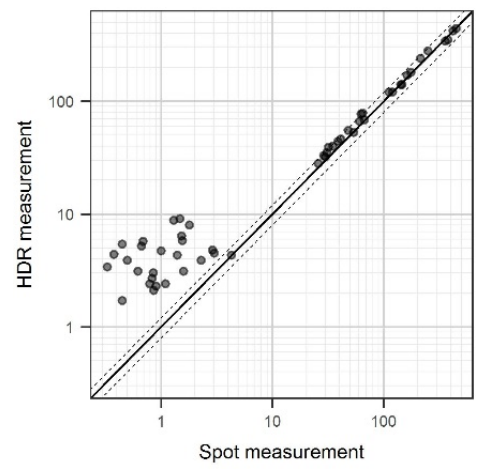

Figure 9.42 Comparison of HDR measurements to spot luminance measurements in the vertical condition of Experiment 2. Note how the HDR measurements significantly overestimate the darker regions. 
The first attempt at correction worked for some conditions (generally the ones with relatively low error like in Experiment 1), but failed to completely fix others. Looking at the example of the Vertical condition again, we see that while the HDR measurements are much closer, they are still substantially out (Figure 9.43). To address this, the correction process was rerun but with different multipliers applied to the adjustment (Table 9.4). For example, the adjustment of the Vertical condition (\#9) was increased by a factor of five. This produced maps that were, at least on average, much more consistently aligned with the spot measurements (Figure 9.45).

Table 9.4 Multipliers applied to the scattering adjustments of each of the different conditions

\begin{tabular}{|c|c|c|c|c|c|c|c|c|c|c|}
\hline $\mathbf{1}$ & $\mathbf{2}$ & $\mathbf{3}$ & $\mathbf{4}$ & $\mathbf{5}$ & $\mathbf{6}$ & $\mathbf{7}$ & $\mathbf{8}$ & $\mathbf{9}$ & $\mathbf{1 0}$ & $\mathbf{1 1}$ \\
\hline $\mathrm{x} 1$ & $\mathrm{x} 1$ & $\mathrm{x} 2$ & $\mathrm{x} 3$ & $\mathrm{x} 1$ & $\mathrm{x} 2$ & $\mathrm{x} 3$ & $\mathrm{x} 5$ & $\mathrm{x} 5$ & $\mathrm{x} 1$ & $\mathrm{x} 2$ \\
\hline
\end{tabular}

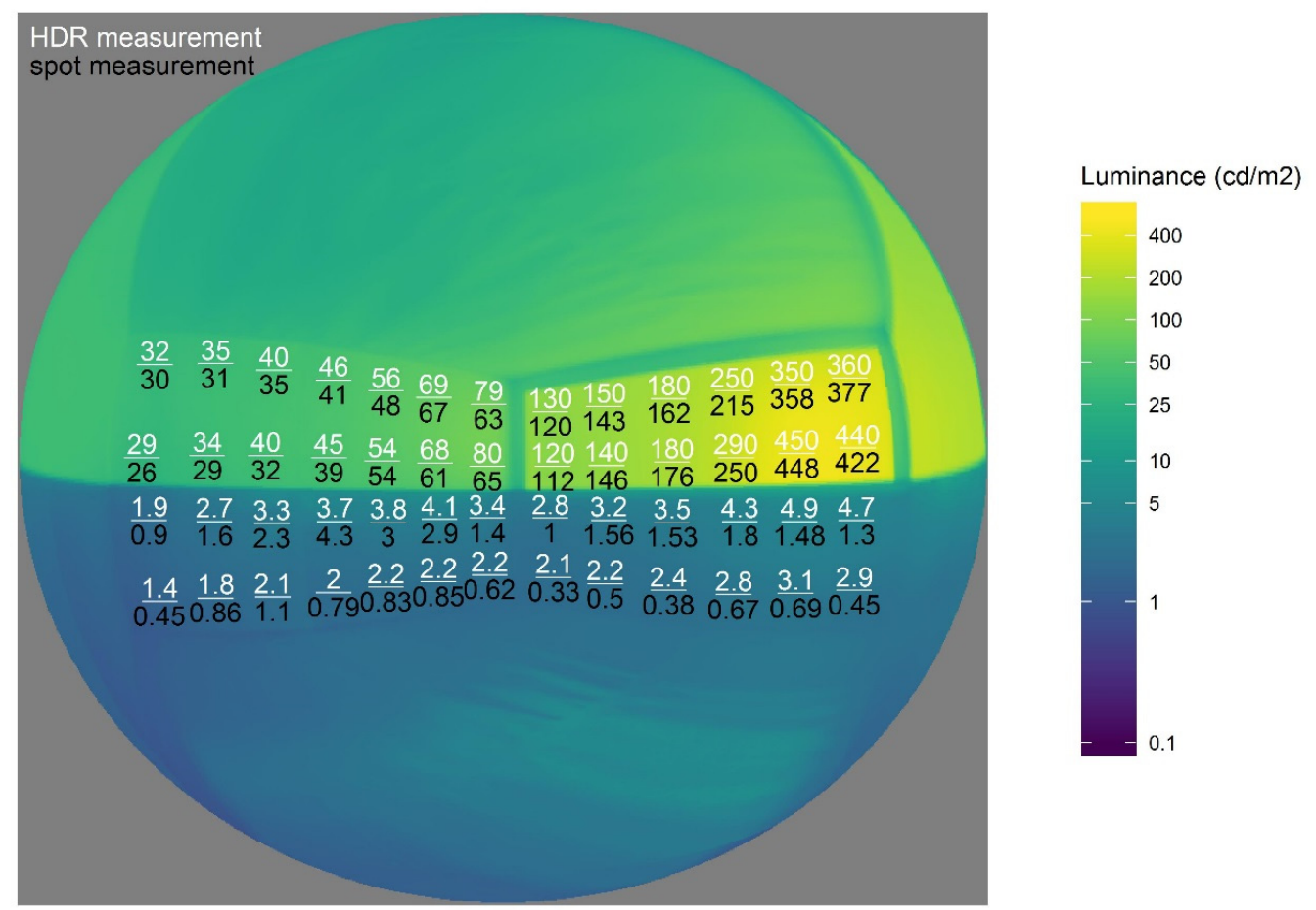

Figure 9.43 Luminance map of the Vertical condition in Experiment 2 compared against spot luminance measurements after the initial scattering correction attempt. 


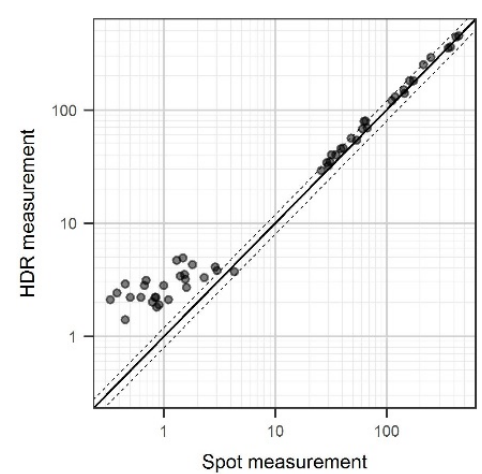

Figure 9.44 Comparison of HDR measurements to spot luminance measurements in the vertical condition of Experiment 2 after the initial scattering correction attempt. Note how the HDR measurements still significantly overestimate the darker regions.

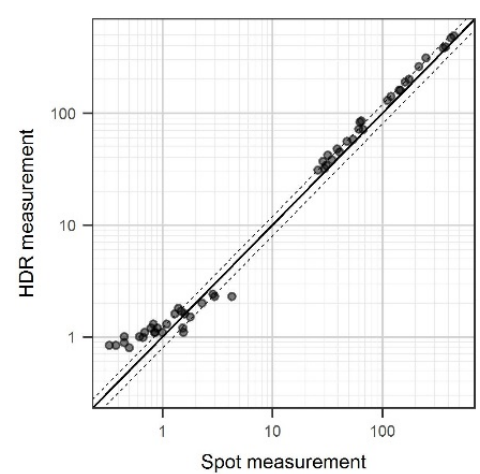

Figure 9.45 Comparison of HDR measurements to spot luminance measurements in the vertical condition of Experiment 2 after the adjusted scattering correction attempt. While there is still significant uncertainty in the lower measurements, they are on average reasonably in line.

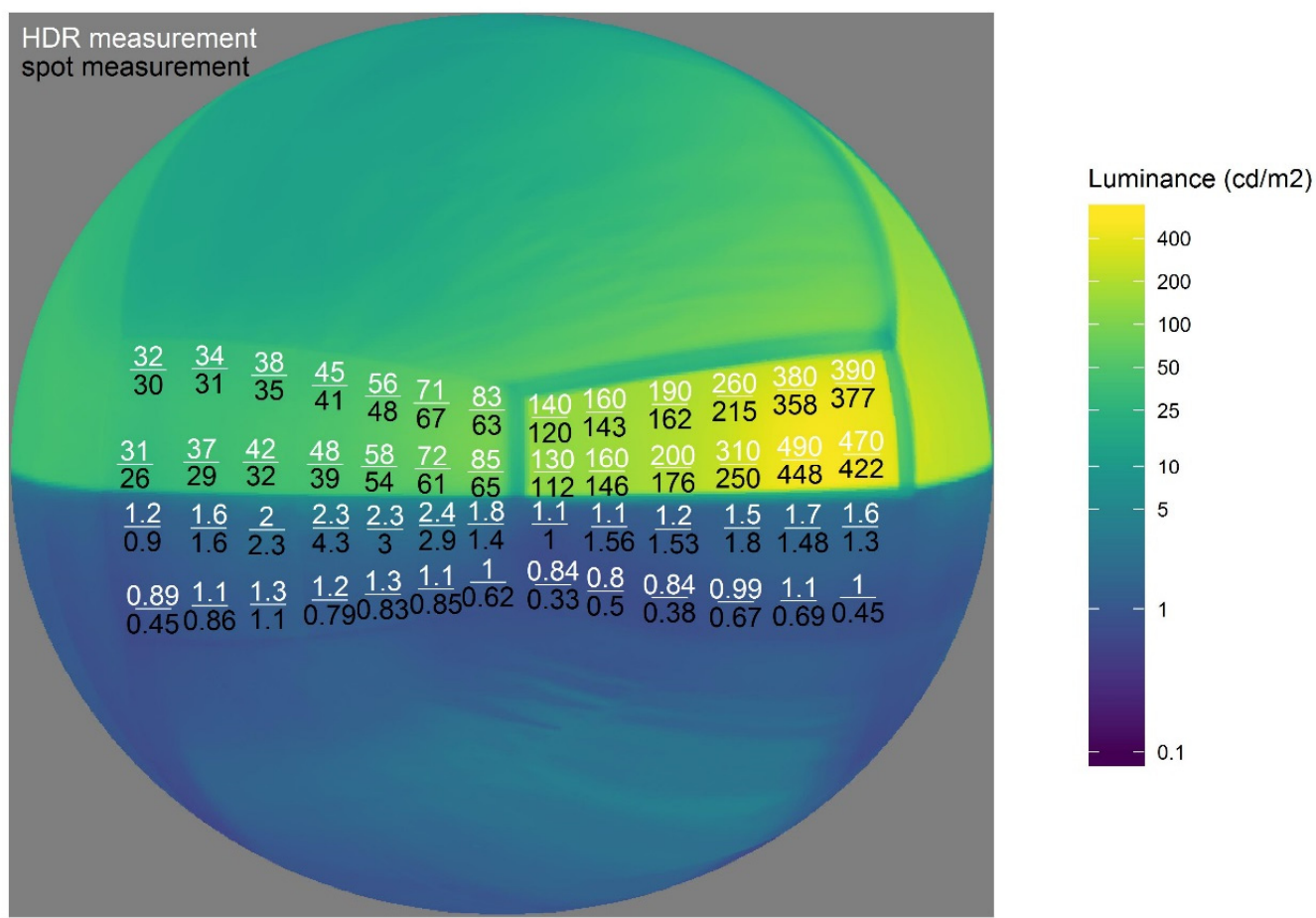

Figure 9.46 Luminance map of the Vertical condition in Experiment 2 compared against spot luminance measurements after the adjusted scattering correction attempt.

This highlights a basic flaw in the correction method: if the scattering errors are sufficiently large then the HDR luminance map will substantially underestimate the differences between areas. For example, some measurements here were out by almost an 
order of magnitude. When we bin these maps to create the contour map, those regions will thus be placed in contours two or three levels higher than they should be, and when we measure the scattering it will greatly underestimate the effect.

In practical terms, the effect on common uniformity metrics is mixed (Figure 9.47). Effects are more noticeable than in the first experiment, however aggregate measures like the SD continue to be minimally impacted. The max/min uniformity ratio is dramatically impacted, however the basic pattern remains the same.

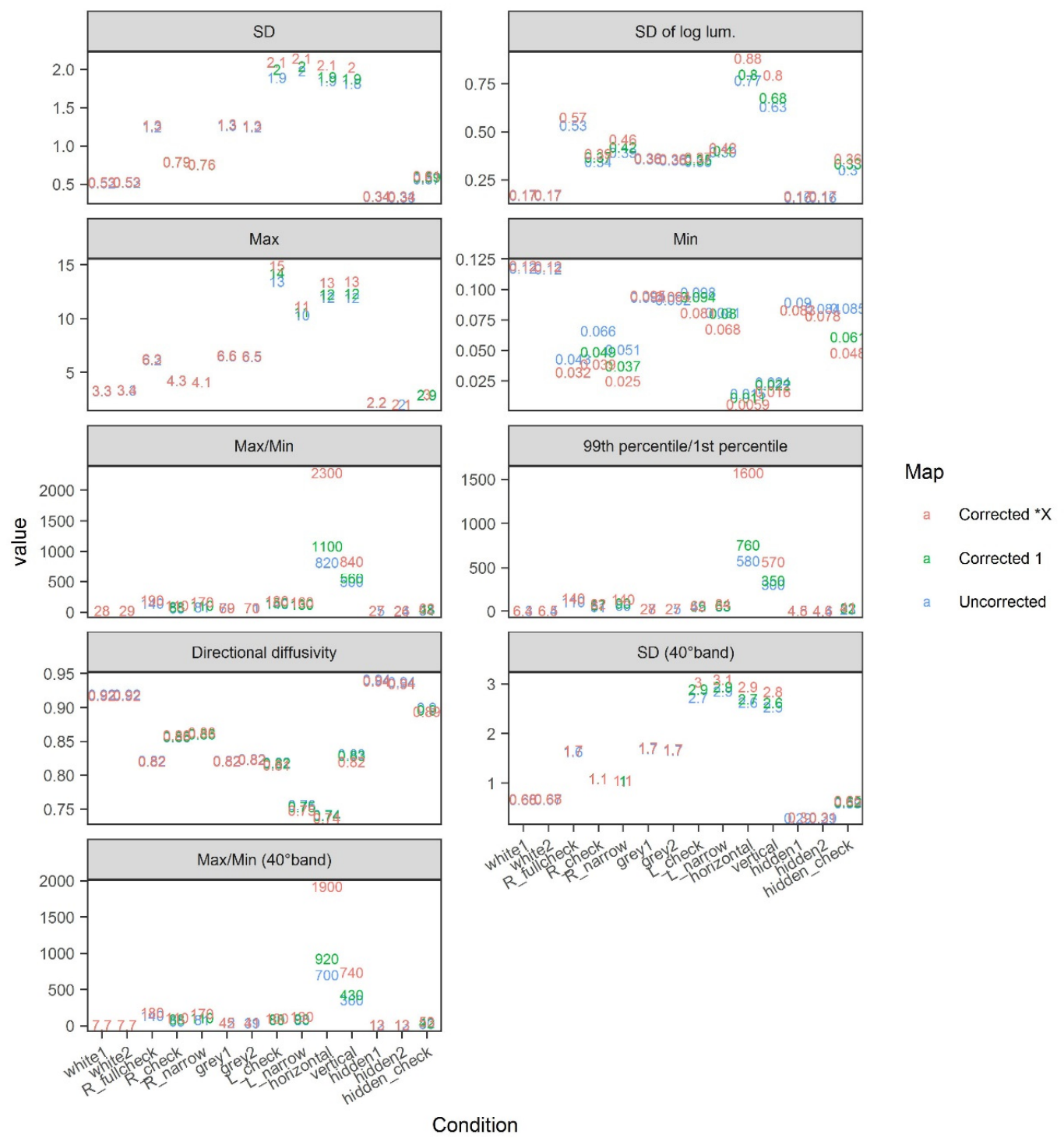

Figure 9.47 Effect of scattering correction on common uniformity metrics for Experiment 2 
Figure 9.48 Luminance maps. The next six pages show the corrected luminance maps and spot measurements of the Experiment 2 conditions. Uncorrected maps can be found in the online supplement.

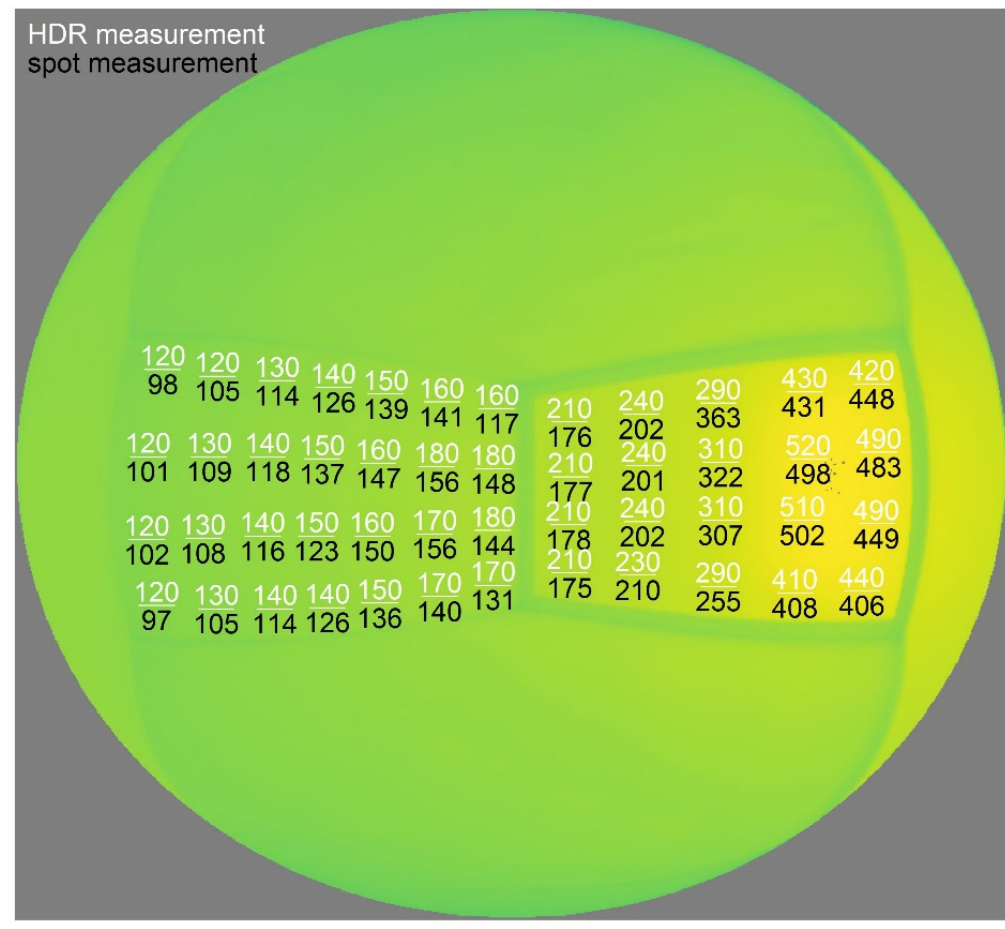

Luminance $(\mathrm{cd} / \mathrm{m} 2)$
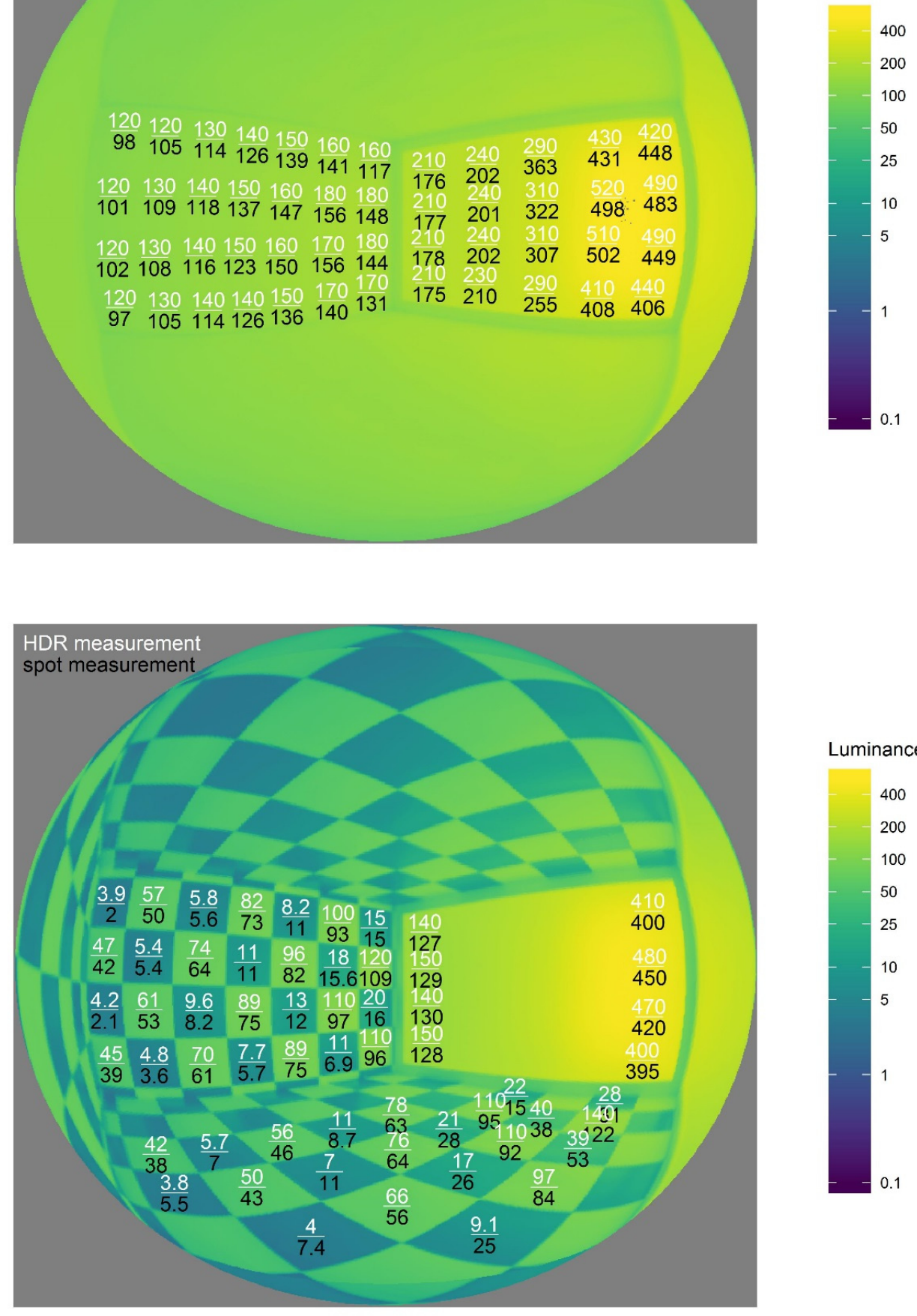

Luminance $(\mathrm{cd} / \mathrm{m} 2)$

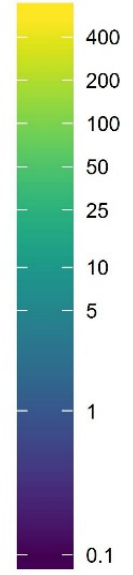




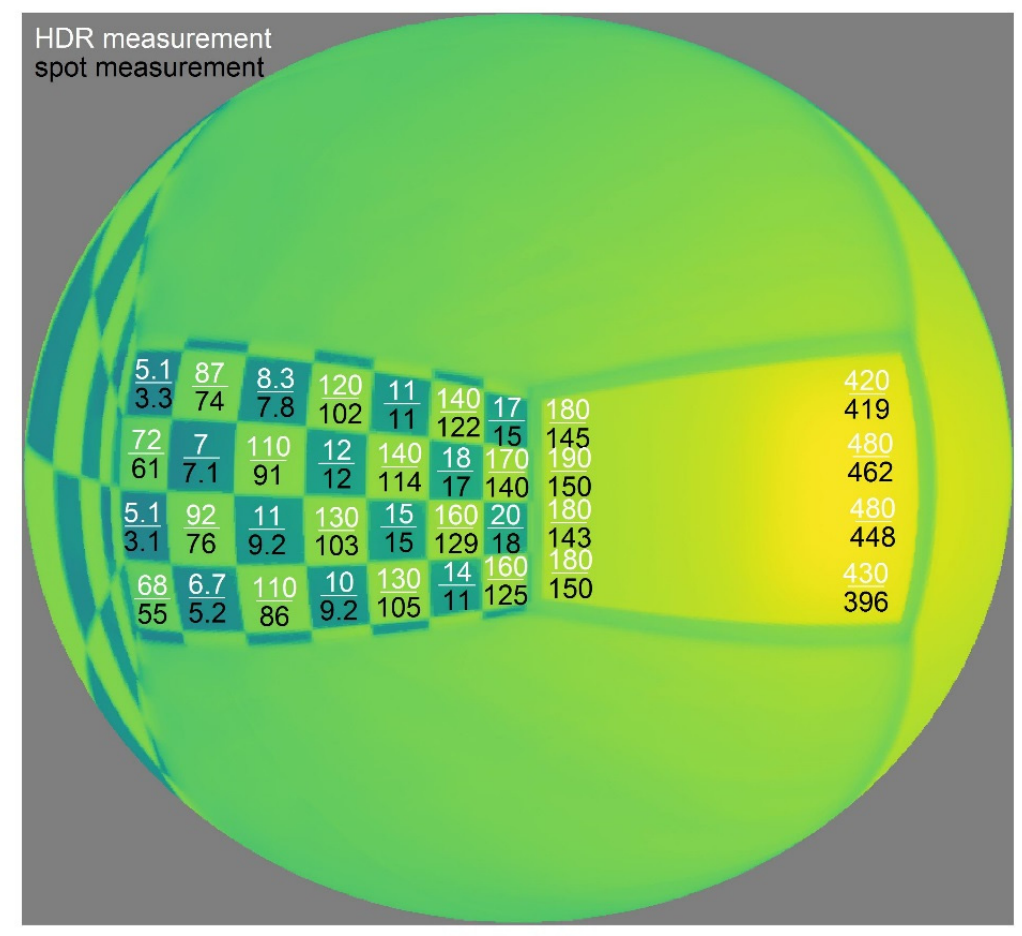

Luminance $(\mathrm{cd} / \mathrm{m} 2)$
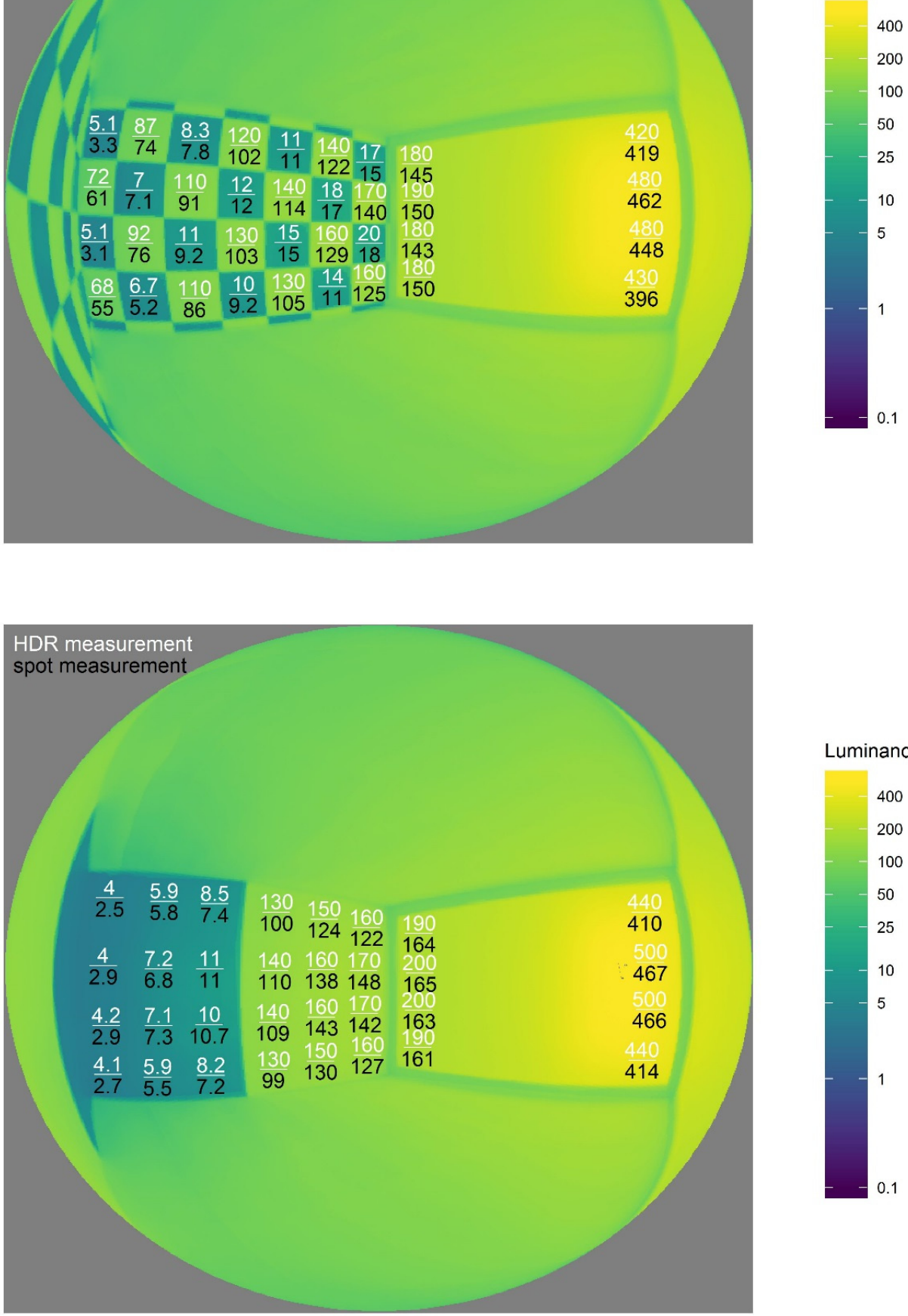

Luminance $(\mathrm{cd} / \mathrm{m} 2)$

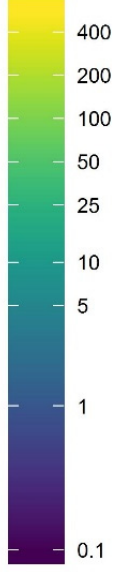




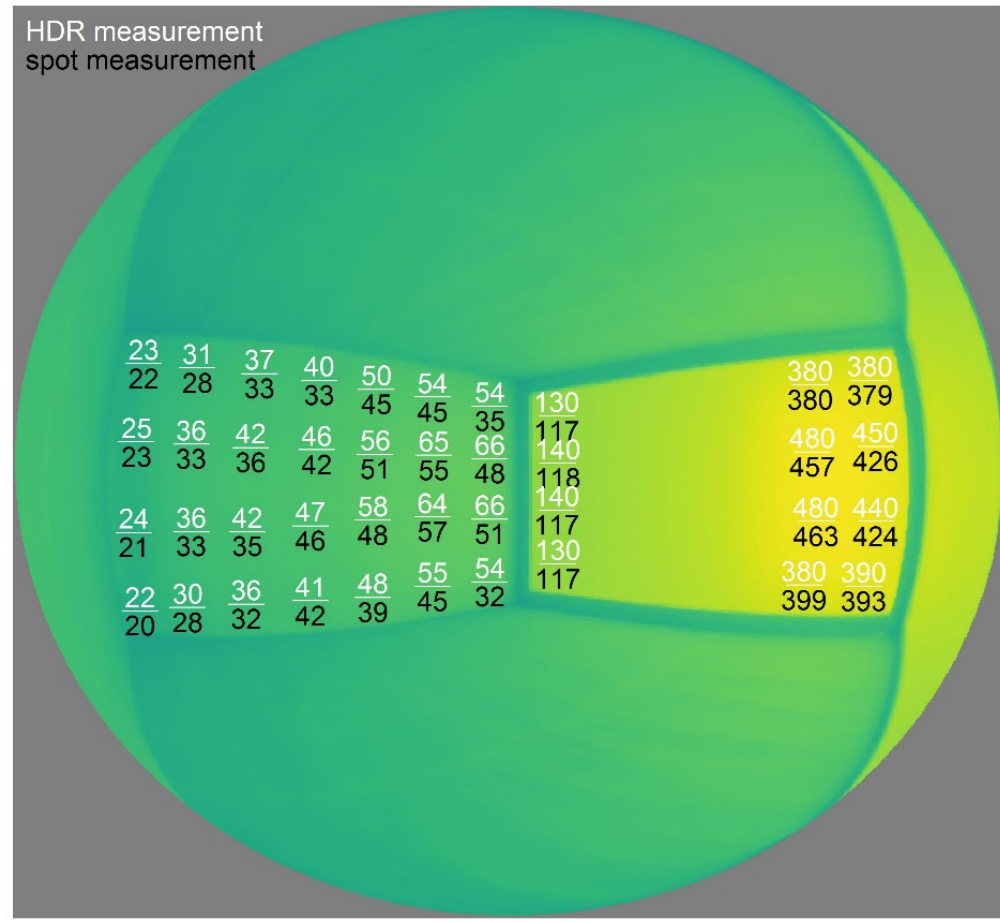

Luminance $(\mathrm{cd} / \mathrm{m} 2)$

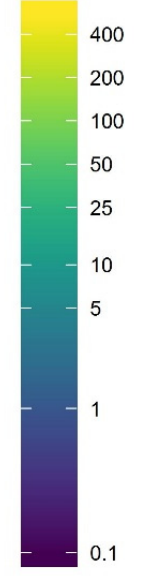

HDR measurement spot measurement

$\overline{9.4}$

12

$\frac{12}{11}$

$\frac{12}{11}$

11

$\frac{11}{7.8}$

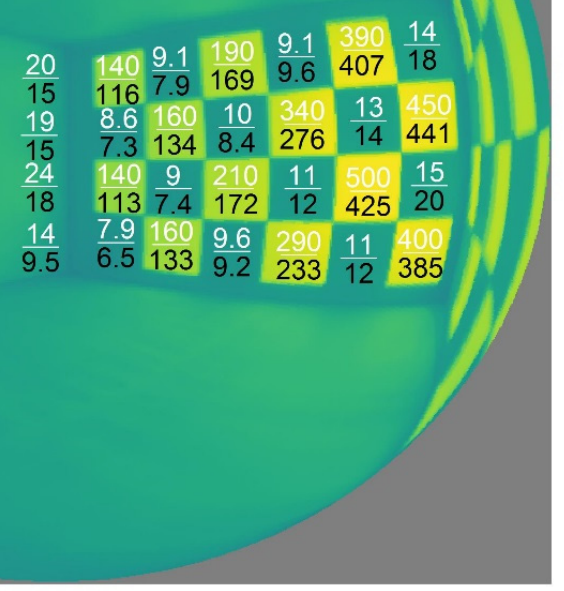

Luminance $(\mathrm{cd} / \mathrm{m} 2)$

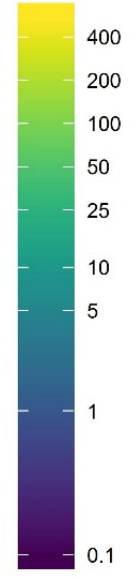




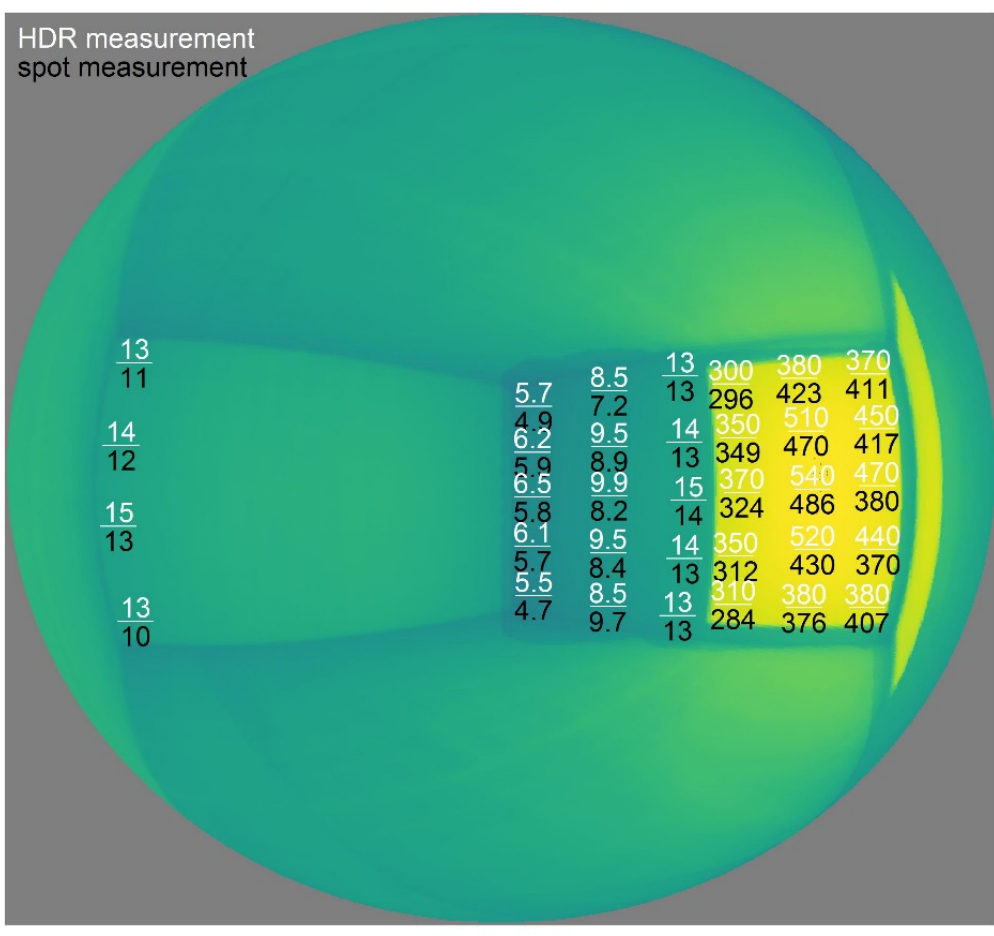

Luminance (cd/m2)
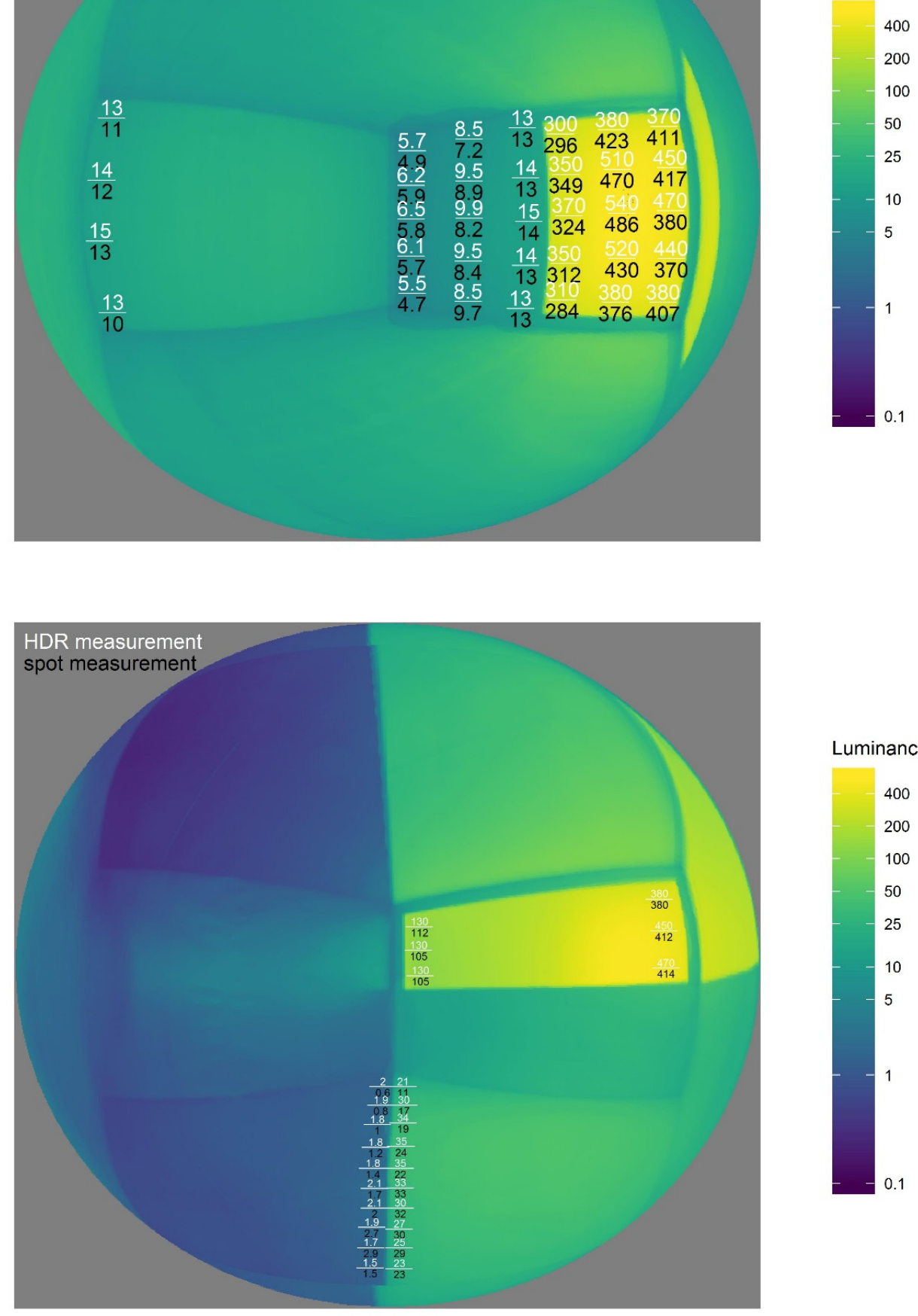

Luminance $(\mathrm{cd} / \mathrm{m} 2)$

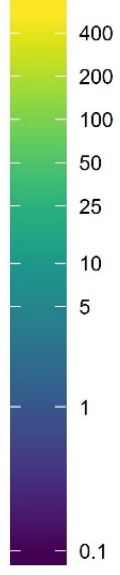




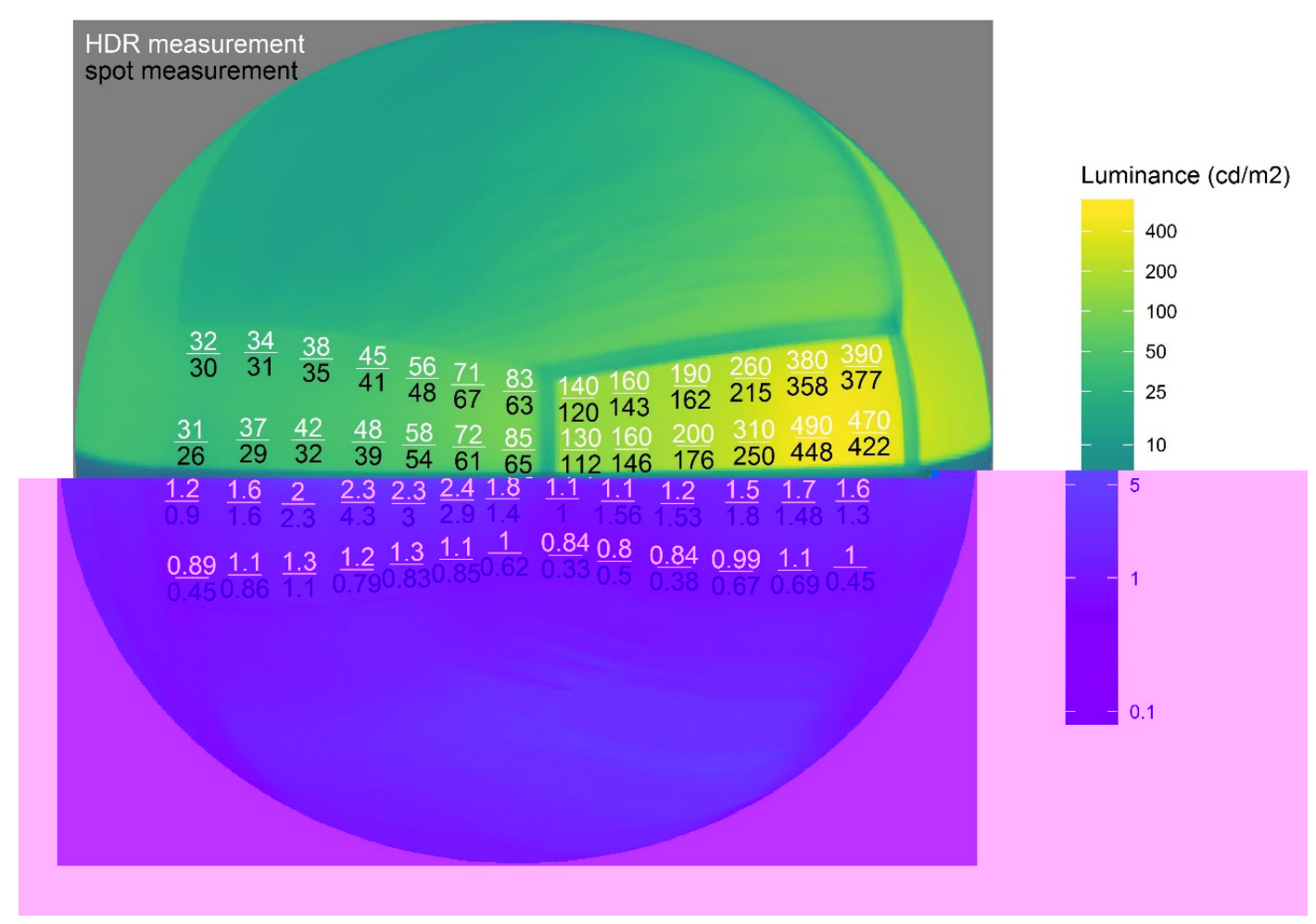

HDR measurement spot measurement
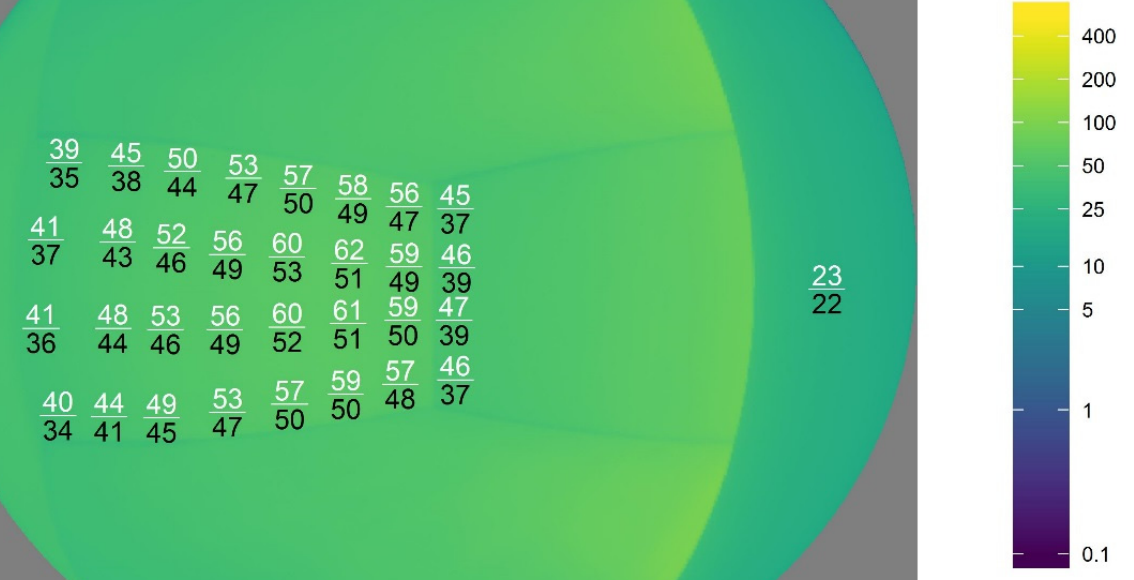


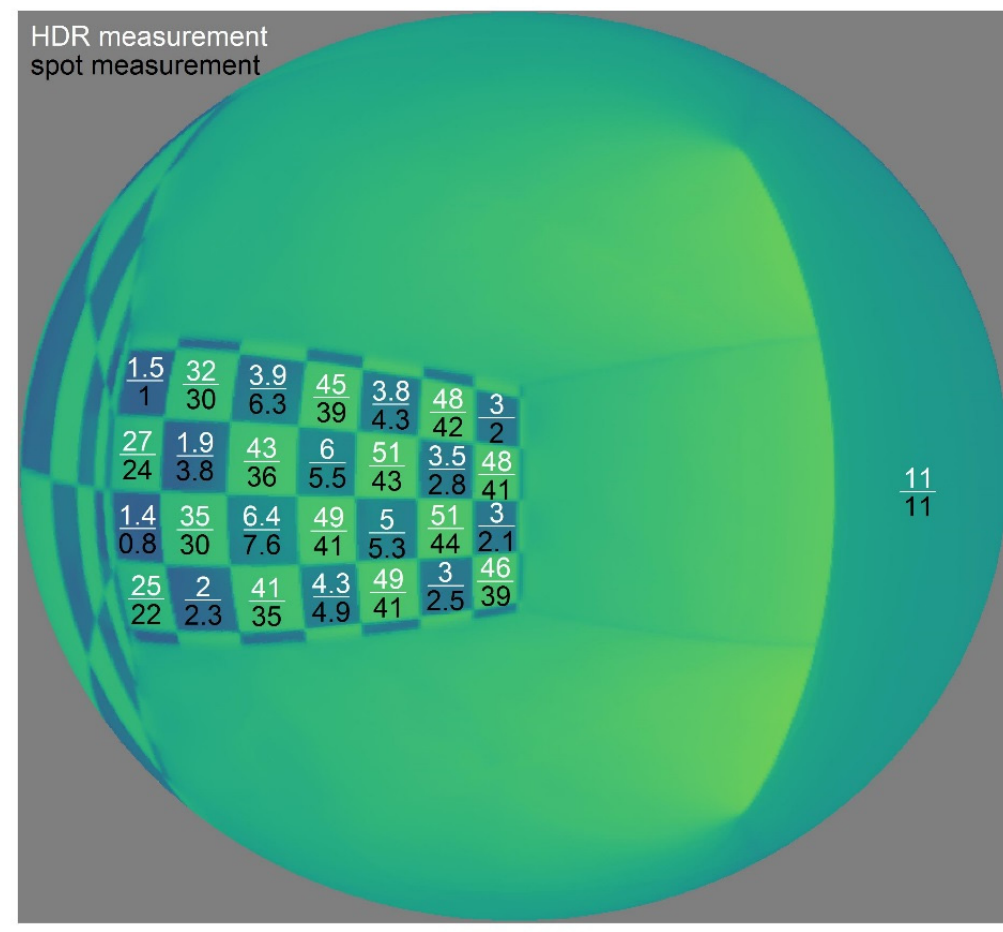

Luminance (cd/m2)

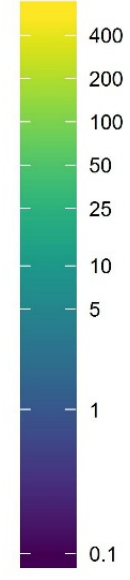


Table 9.5 Comparison of HDR measurements against spot measurements for Experiment 2 conditions. The diagonal line shows the theoretical 1 to 1 fit that they should be along if the measurements were perfectly aligned. Dashed lines to either side show $\pm 20 \%$ as a reasonable error range given the uncertainty in spot luminance readings.

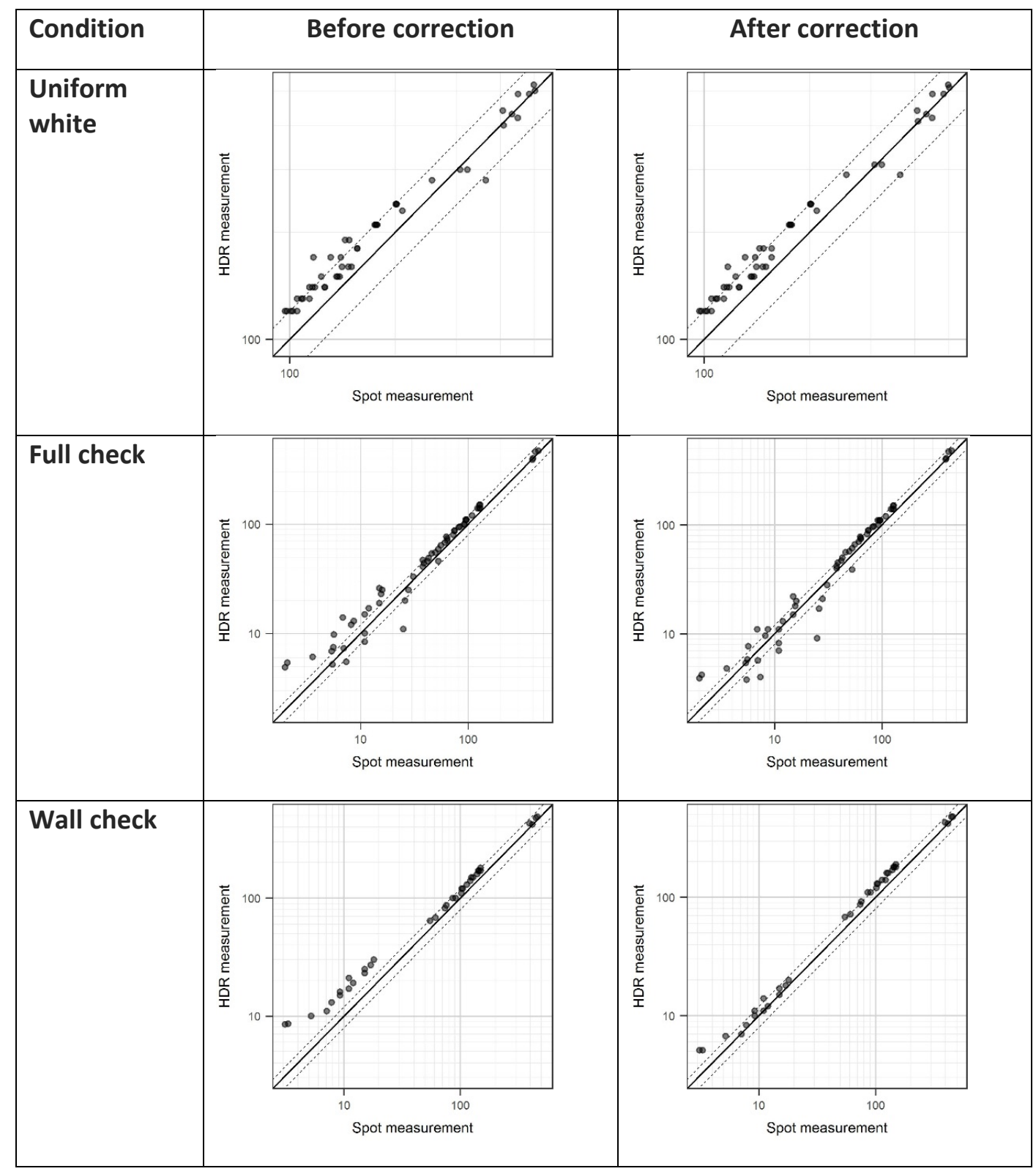




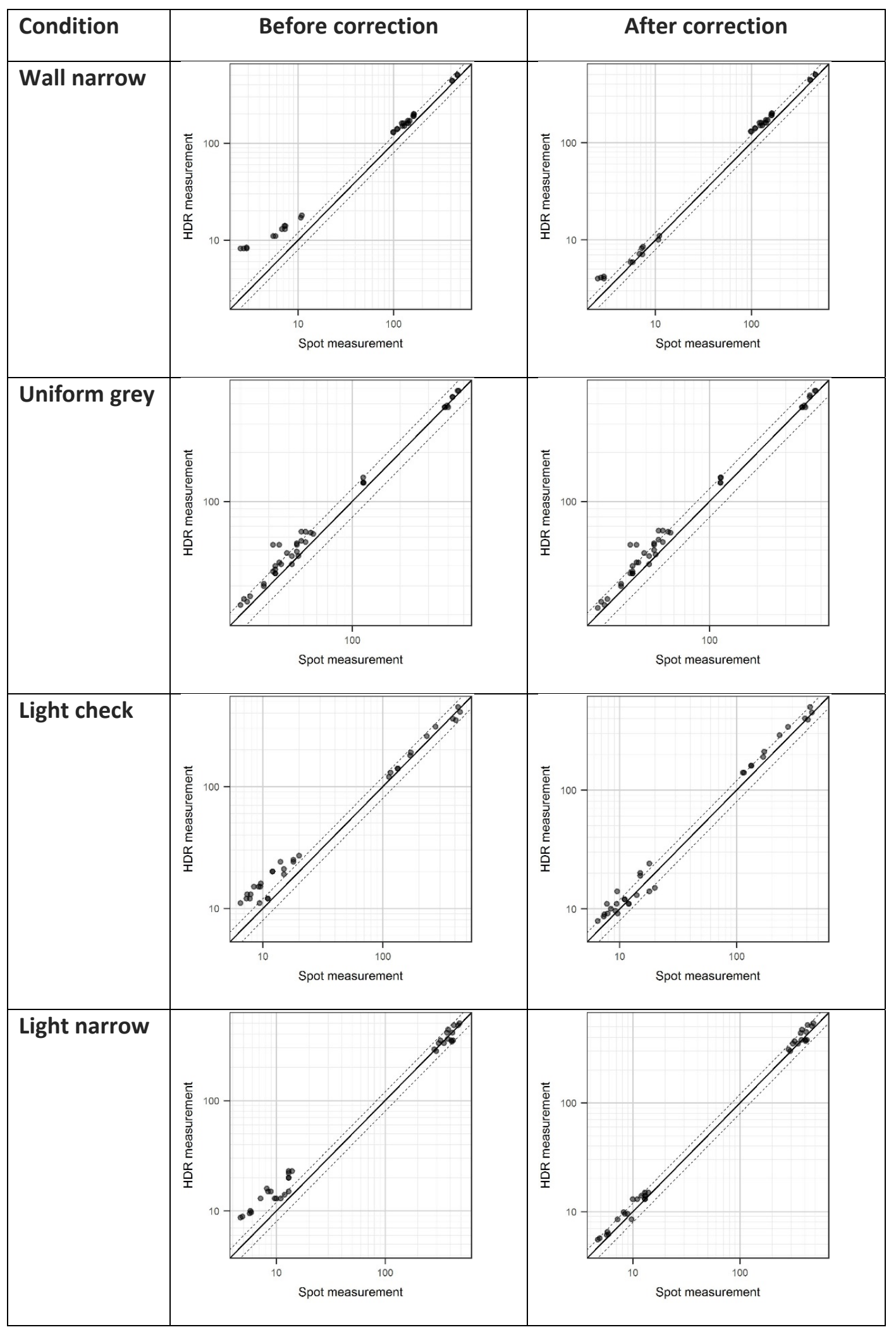




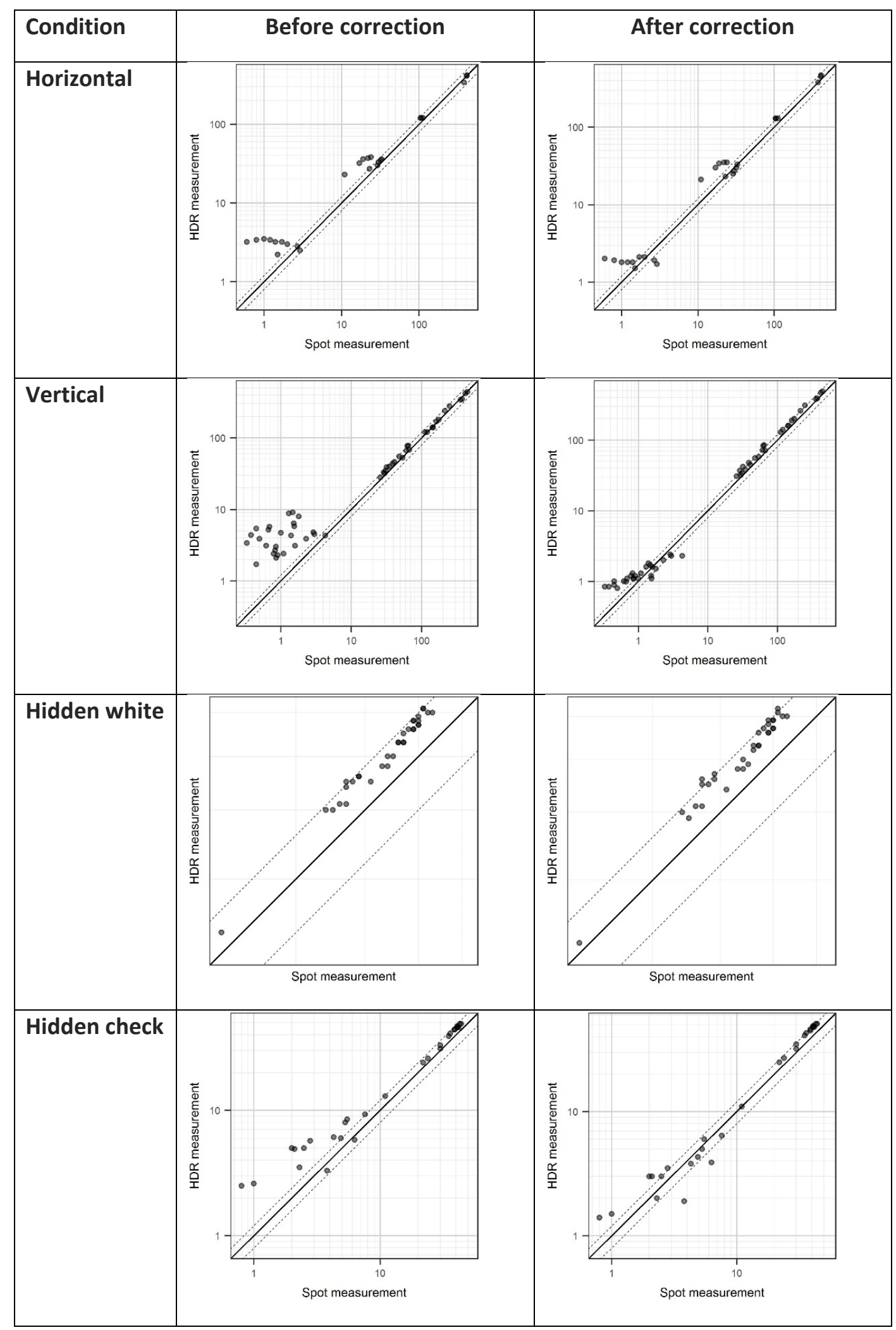




\section{A.3.4 Luminance mapping workflow}

1) Take an exposure bracketed sequence of photos, making sure that the sequence covers the full range following common practice (highest has no RGB values $<20$, lowest has none above 200)

a. Camera settings: ISO100, shutter speed varied

b. For each scene, both horizontal and vertical orientated shots are taken, in order to capture the full $180^{\circ}$ field. These are later combined into one when processed in $\mathrm{R}$.

2) Take the raw images, and convert them into jpg format using Adobe Bridge

a. Black, brightness, and contrast are not enhanced (set to 0 , change from defaults)

b. White balance set to florescent, with colour temperature set to an appropriate value depending on the light source $(5500 \mathrm{~K}$ for "daylight" CFLs in conditions, default $3800 \mathrm{~K}$ for images in lighting lab under more normal florescent light)

c. Important thing is settings are consistent across images being compared.

3) Jpgs are converted to .hdr format using Hdrgen on an Ubuntu virtual machine. Hdrgen is batch run using shell scripts dynamically generated with $\mathrm{R}$

4) Hdr images are reduced in size using Radiance's $p$ filt command in order to expedite later data processing (following Van Den Wymelenberg, 2012)(resulting image size $624 \times 1112$, fisheye diameter -815 pixels wide)

5) Images are cropped down to the fisheye using Radiance's pcompos command [crop_hdr.bat].

\section{C: \Radiance $\backslash$ bin $\backslash$ pcompos $-x \quad 815$-y 624 . \hdr $\backslash$ base $\ \% \sim n f \% ~ x f$} $-1590>$. \hdr $\backslash$ crop $\backslash \% \%$ nf\% $\%$ xf

6) The scattering correction (once it has been produced) is applied to the images using Radiance's pcomb command

7) The vignetting correction is applied to the images using Radiance's pcomb command

C: \Radiance \bin \pcomb -e

"ro=ri (1)/ri (2);go=gi (1)/gi (2) ; bo=bi (1)/bi (2)"

. \hdr \crop \\%\% nf\%\% xf vig2_180.hdr >

. \hdr \vignette $\ \%$ nf\%\% xf 
8) Images are now expanded to as $815 \times 815$ square in preparation for conversion to a hemispherical projection using Radiance's pcompos command

C: \Radiance $\backslash$ bin $\backslash$ pcompos $-\mathrm{x} 815$-y 815

. \hdr \vignette \\%\% nf\%\% xf $096>$. \hdr $\backslash$ expand $\backslash \% \% \sim n f \% \%$ xf

9) Necessary header information (view details) is inserted back into the images by using Notepad++'s "find/replace in file" abilities to batch replace the line "FORMAT=32-bit_rle_rgbe" with the line "FORMAT=32-bit_rle_rgbelnVIEW= -vta -vh 180 -vv 180".

10) Hdr images are adjusted from equidistant projection to hemispherical projection using Radiance's pinterp command

C: \Radiance \bin \pinterp - vf . \hdr \expand $\backslash \%$ nf\%\% xf -vth -x 815 -y 815 -ff. . hdr $\backslash$ expand $\backslash \% \%$ nf\%\% xf $1>$ . \hdr $\backslash$ hemi $\backslash \% \% \sim n f \% \%$ xf

11) A script is used to rotate the vertically oriented images by 90 degrees using Radiance's protate command

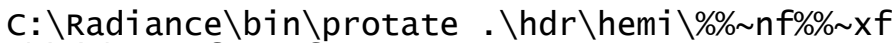
. $\backslash$ hdr $\backslash \% \% \sim \mathrm{nf} \% \sim \mathrm{xf}$

12) Per pixel RGB data is extracted from the hdr images to csv format using Radiance's pvalue command

\section{C: \Radiance \bin \pva7ue $-0-\mathrm{h}-\mathrm{H} . \backslash \mathrm{hdr} \backslash \% \% \sim \mathrm{nf} \% \% \mathrm{xf}>$} . \output $\ \% \sim n f$. CSV

13) The data is read into $R$, and luminance maps are calculated using the equation: $L=$ $179(0.2127 R+0.7151 G+0.0722 B)$ (Inanici, 2006).

14) The luminance map data is cleaned, with 0 values replaced by NA and ignored. This serves to crop out the areas outside of the fisheye.

15) Maps are normalised so that they all have the same mean luminance before calculating uniformity metrics (in order to avoid confounding on some metrics).

a. When maps are being checked against spot measurements, calibration is carried out using vertical illuminance. This is calculated by taking the mean luminance of the hemispherical map and multiplying by $\pi$ (Hirning, 2014; Van Den Wymelenberg, 2012). 


\section{A.4 Meter calibration certificates}

The LS-1 10 luminance meter was acquired in April 2015. The CL-70F illuminance meter was acquired in September 2018.

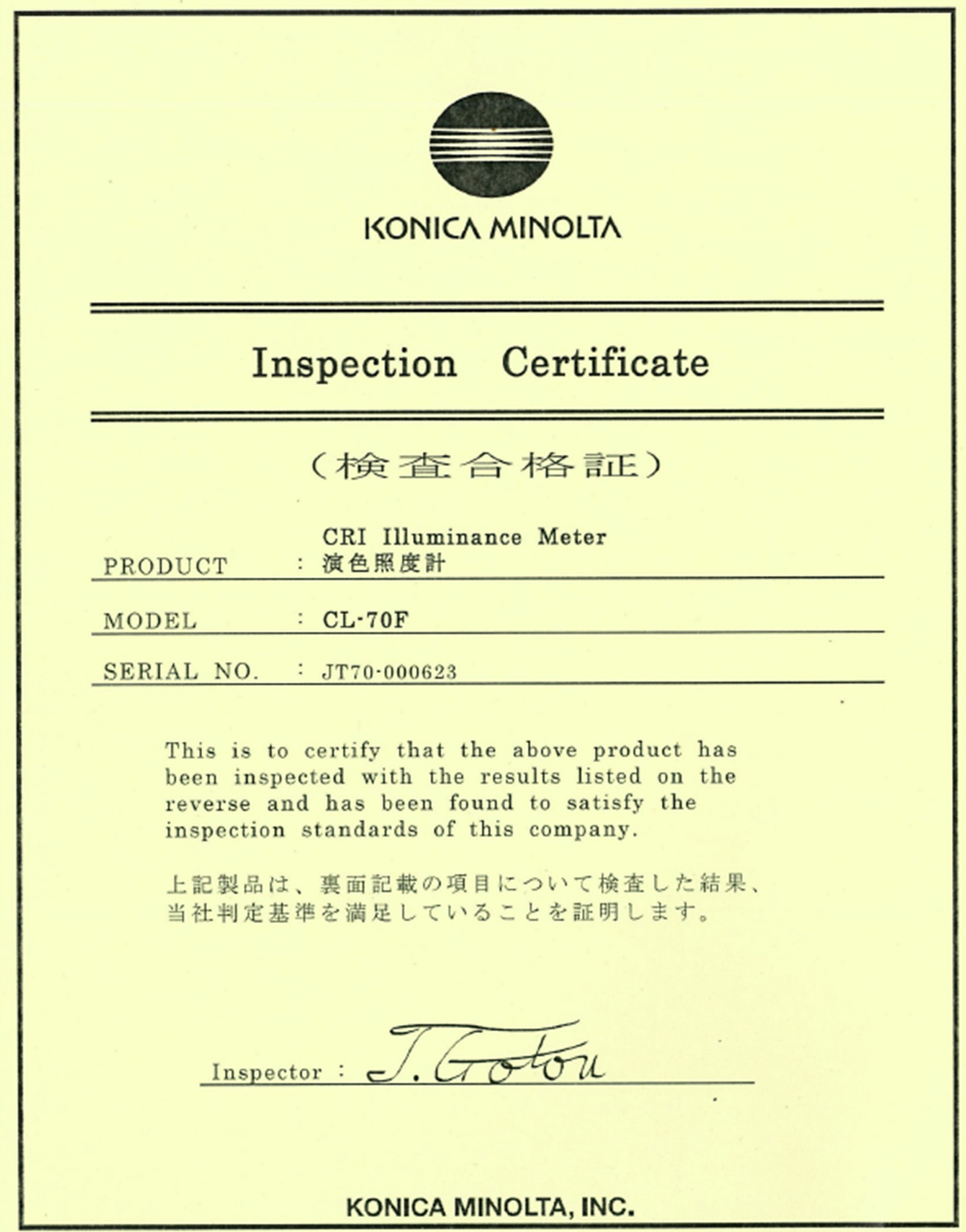

$9229 \cdot \mathrm{A} 8 \mathrm{FG} \cdot 11$ 


\begin{tabular}{|c|c|c|}
\hline \multicolumn{3}{|c|}{ ITEMS INSPECTED } \\
\hline APPEARANCE & \multicolumn{2}{|c|}{$\begin{array}{l}\text { Surface condition (scratches, stains); } \\
\text { Assembly (looseness, misalignment, gaps); Printing }\end{array}$} \\
\hline \multirow{4}{*}{ FUNCTIONS } & Display & Display panel \\
\hline & Keys \& switches & $\begin{array}{l}\text { Power button, Measuring button, Memory button, } \\
\text { Menu button, Light selection ring }\end{array}$ \\
\hline & Program & Measurement, Data display, Data setting, Data processing \\
\hline & Input/Output & USB 2.0 input/output, Sync terminal \\
\hline \multirow{3}{*}{ PERFORMANCE } & Accuracy & $\begin{array}{l}\text { Chromaticity }(\mathrm{x}, \mathrm{y}): \pm 0.003 \\
\text { Measurement conditions: Standard illuminant } \mathrm{A} .800 \mathrm{~lx}\end{array}$ \\
\hline & Linearity & $\begin{array}{l}\text { Illuminance (Ev) }: \pm 5 \% \pm 1 \text { digit of value displayed } \\
\text { Measurement conditions: Standard illuminant A.800 lx }\end{array}$ \\
\hline & Repeatability(2o) & $\begin{array}{ll}\text { Illuminance }(\mathrm{Ev}) & : 1 \%+1 \text { digit } \\
\text { Chromaticity }(\mathrm{x}, \mathrm{y}) & : 0.001 \\
\text { Measurement conditions: Standard illuminant } \mathrm{A} .800 \mathrm{~lx}\end{array}$ \\
\hline
\end{tabular}

\begin{tabular}{|c|c|c|}
\hline \multicolumn{3}{|r|}{ 検查項目 } \\
\hline 外権 & \multicolumn{2}{|c|}{ 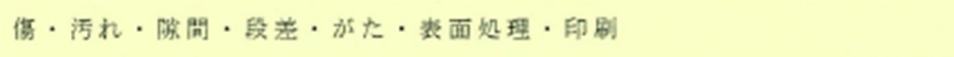 } \\
\hline \multirow{4}{*}{ 機能 } & 表示 & 表示バネル \\
\hline & キースイッチ & 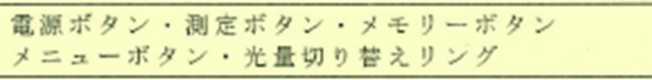 \\
\hline & プロクラム & 测定 · デー夕表示・デー夕检定・デー夕処理 \\
\hline & 入出力 & USB 2.0 伍号入出力、シンクロターミナル \\
\hline \multirow{3}{*}{ 性能 } & 础度 & $\begin{array}{l}\text { 色度 }(x, y) \quad: \quad \pm 0.003 \\
\text { 测定条件：標草イルミナントA, } 800 \quad 1 x\end{array}$ \\
\hline & 还緗性 & 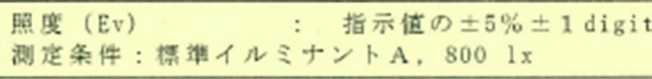 \\
\hline & 淥り返し生 $(2 \sigma)$ & 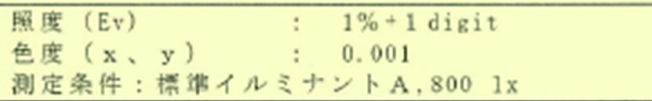 \\
\hline
\end{tabular}




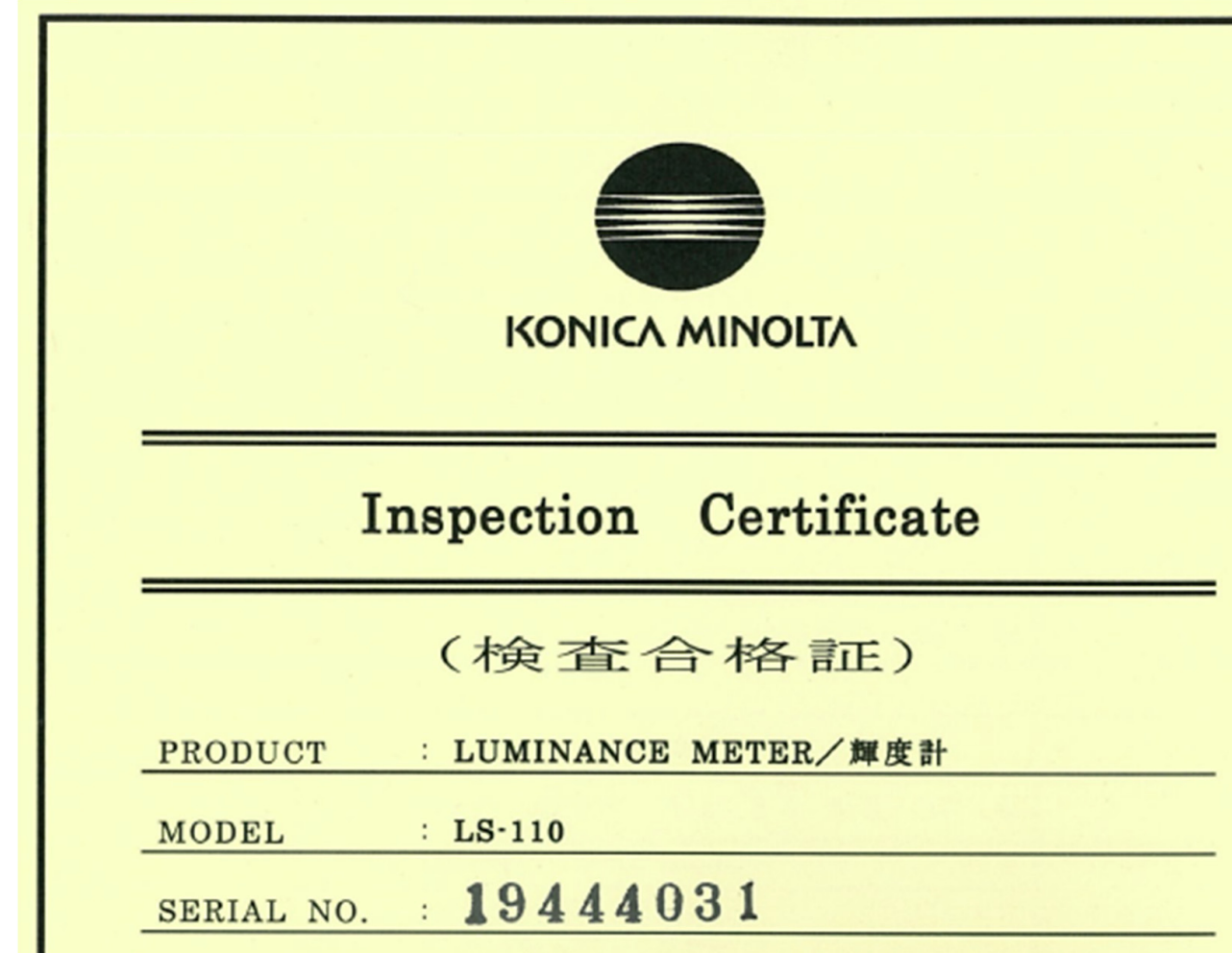

This is to certify that the above product has been inspected with the results listed on the reverse and has been found to satisfy the inspection standards of this company.

上記製品は、襄面記載の項目について検查した結果、 当社判定基淮を满足していることを証明します。

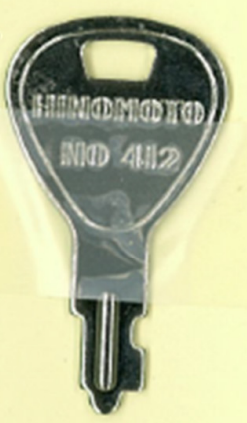

KONICA MINOLTA, INC. 


\begin{tabular}{|c|c|c|}
\hline \multicolumn{3}{|c|}{ ITEMS INSPECTED } \\
\hline APPEARANCE & \multicolumn{2}{|c|}{$\begin{array}{l}\text { Surface condition (scratches, stains); } \\
\text { Assembly (looseness, misalignment, gaps); Printing }\end{array}$} \\
\hline \multirow{4}{*}{ FUNCTIONS } & Display & External display panel, Viewfinder display \\
\hline & Finder & Eyepiece adjustment ,Focus adjustment \\
\hline & Controls & $\begin{array}{l}\text { Power switch, Calibration selector switch, } \\
\text { Measuring mode selector switch, Data control key, } \\
\text { Function key, Response speed selector switch, } \\
\text { Increase key, PEAK/CONT key }\end{array}$ \\
\hline & Input/Output & Digital output \\
\hline \multirow[t]{2}{*}{ PERFORMANCE } & Accuracy: & $\begin{array}{l}10.00 \mathrm{~cd} / \mathrm{m}^{2} \text { (or } \mathrm{fL} \text { )or more: } \\
\quad \pm 2 \% \pm 1 \text { digit of measured value } \\
\left.0.01 \text { to } 9.99 \mathrm{~cd} / \mathrm{m}^{2} \text { (or } \mathrm{fL}\right): \\
\pm 2 \% \pm 2 \text { digits of measured value }\end{array}$ \\
\hline & \multicolumn{2}{|c|}{ Measurement conditions : Illuminant $\mathrm{A}$ measured } \\
\hline
\end{tabular}

\begin{tabular}{|c|c|c|}
\hline \multicolumn{3}{|r|}{ 㭘查項目 } \\
\hline 外错 & \multicolumn{2}{|c|}{ 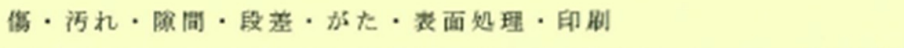 } \\
\hline \multirow{4}{*}{ 機能 } & 表示 & 外部液㫛表示 · 内部波昌表示 \\
\hline & フォインダー & 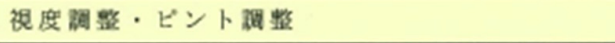 \\
\hline & キースイッチ & 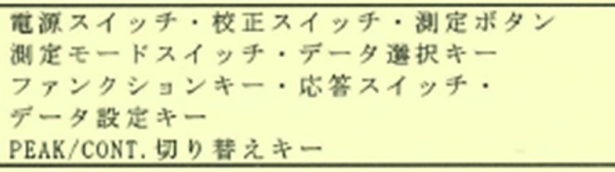 \\
\hline & 入出力 & デジタル传号出力 \\
\hline \multirow{2}{*}{ 性能 } & 泪定磪度 & $\begin{array}{ll}10.00 \mathrm{~cd} / \mathrm{a}^{2} \text { 以上 } & \text { : 测定傎の } \pm 2 \% \pm 1 \mathrm{digit} \text { 以内 } \\
0.01 \sim 9.99 \mathrm{~cd} / \mathrm{a}^{2} & \text { : 测定值の } \pm 2 \% \pm 2 \mathrm{digit} \text { 以内 } \\
\end{array}$ \\
\hline & \multicolumn{2}{|c|}{ 条件 : 標海光源A测定時 } \\
\hline
\end{tabular}

$9229 \cdot 1842 \cdot 25$ 


\section{Appendix B Experiment 1}

\section{B.1 Experiment instructions}

\section{Brightness matching experiment}

Your task here is to match the brightness of the two different spaces you see on either side. Brightness is defined here as the overall amount of light in the space.

Before each trial, you will be instructed to look into the reference space on the left. When looking in the spaces, you should adopt a viewing position such that the space fills your whole field of view, and your chin is just inside the edge of the space.

When the experimenter tells you that a trial has begun, you can then look into the test space on the right, and begin adjusting the light level using the dial by your left hand. When making your adjustments, you may look back and forth between each space as many times as you want.

While the spaces may differ in aspects such as colour, which may prevent you from matching them in visual appearance, it should not prevent you from being able to match brightness.

Once you are satisfied the two spaces are the same brightness, inform the experimenter you have completed the trial. You will then be instructed to look down and close your eyes while the spaces are changed.

You will be given some practice trials before the experiment begins. Any questions or problems you are having should be mentioned at this time.

\section{Magnitude Estimation experiment}

Your task is to assess the brightness of the different spaces you will be shown. Brightness is defined here as the overall amount of light in the space.

At the start of each trial, you will first be shown the reference space. This has a brightness of "100". You should assess the brightness of the other spaces relative to this. For example, if you think a space is twice as bright as the reference, you should say that it has a brightness of " 200 ". If you think a space is half as bright as the reference, you should say that it has a brightness of " 50 ". 
Between each trial, you will be instructed to close your eyes while the space is changed. When looking in the spaces, you should adopt a viewing position such that the space fills your whole field of view, and your chin is just inside the edge of the space.

Verbally report your best estimate of the brightness of the space to the investigator. You may look around it for as long as you need to make your judgement.

\section{B.2 Example of semi-randomised condition order}

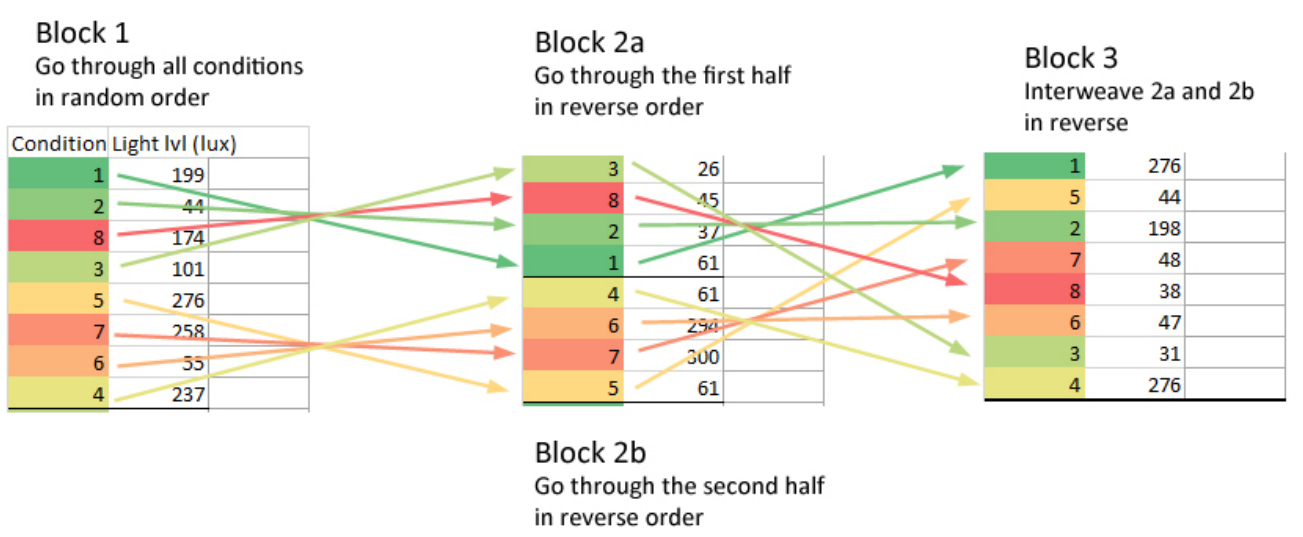

Figure 9.49 Example of the semi-randomised ordering of the conditions in Experiment 1.

\section{B.3 Measured lamp output after being turned on}

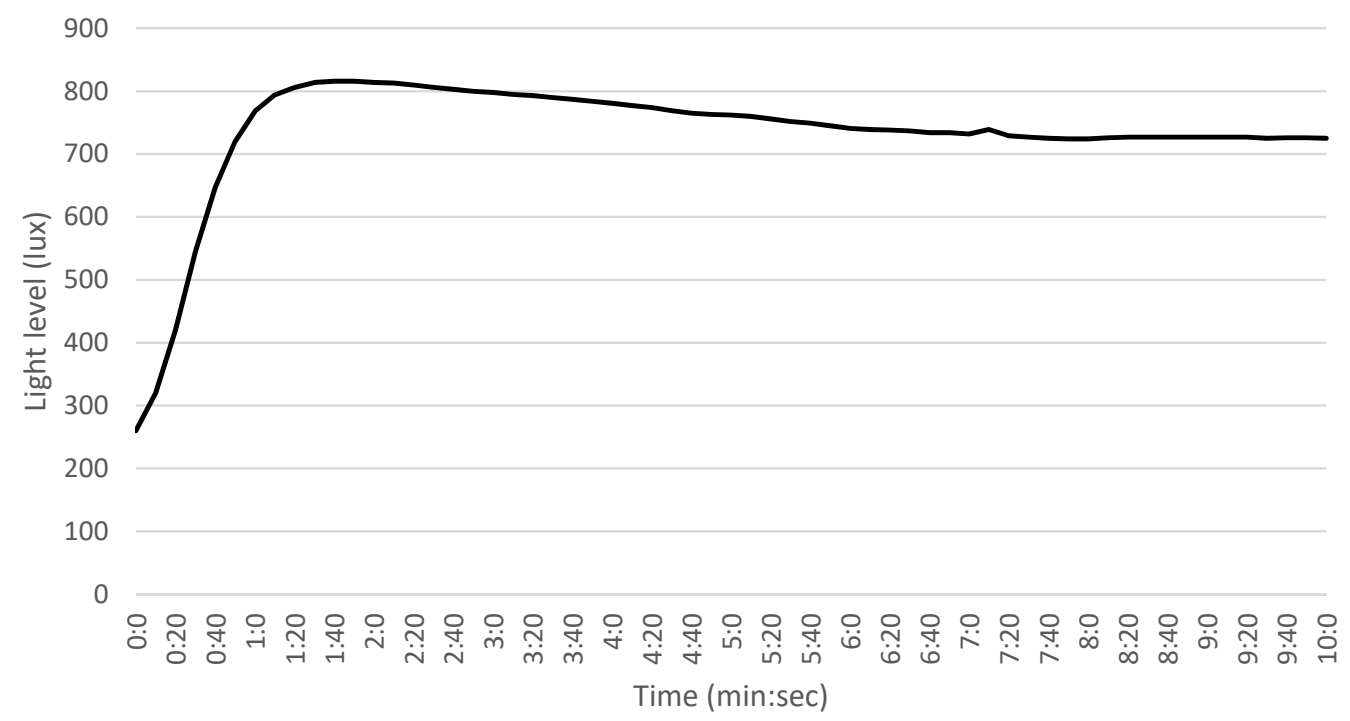

Figure 9.50 Lamp output as measured every 10 seconds for 10 minutes after being turned on 


\section{B.4 Model details}

We assume that an individual's responses to a given condition $\left(y_{\mathrm{i}}\right)$ are normally distributed with a mean describing the average match they make to the given condition $\left(\mu_{[t, r, s, p]}\right)$ and a standard deviation $\left(\sigma_{[s, p]}\right)$ describing the variation in their matches:

$$
y_{i} \sim \operatorname{normal}\left(\mu_{[t, r, s, p]}, \sigma_{[s, p]}\right)
$$

The mean match made by a subject for a given pair of conditions $\left(\mu_{[t, r, s, p]}\right)$ may vary by the individual test and reference conditions $(t, r)$, the subject $(s)$, and the pair of conditions being compared $(p)$. It is the product of the reference light level, the difference between the two conditions in apparent brightness and the various biases that may affect the match:

$$
\begin{aligned}
\mu_{[c, s, p]}=r e f & -\left(\operatorname{Eff}_{[t, s]}-\operatorname{Eff}_{[r, s]}\right)-\left(S P D_{\text {test }[t]}-S P D_{r e f[r]}\right)+\operatorname{bias}_{p o s+\operatorname{dim}}[p, s] \\
& +\operatorname{bias}_{\text {start }[t, r, p, s]}
\end{aligned}
$$

ref is the light level of the reference condition, $E f f_{[t / r, s]}$ is the effect of the condition (test or reference), SPD is the effect of SPD for that condition (pre-calculated using the Rea

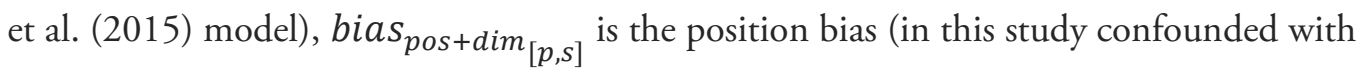

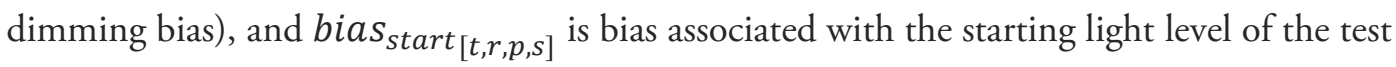
condition.

The effect of the condition(s) is assumed to vary between people, and is:

$$
E f f_{[t, s]}=E_{\mu_{[t]}}+E_{S D_{[t]}} \cdot \widetilde{E}_{[t, s]}
$$

... where $E_{\mu_{[t]}}$ is the mean effect of the condition, $E_{S D_{[t]}}$ is the between-subject variation in the effect of condition, and $\widetilde{E}_{[t, s]}$ is the parameter used for a non-centred parameterisation (Betancourt, 2017b). As some conditions might have greater variance than others, $\beta_{1 S D_{[t]}}$ is allowed to vary between conditions. However, we have no particular expectation of systematic differences here, and we could expect some similarity in the between-subject variance of one condition and the variance of another (perhaps as it would in large part be a result of the experimental design). With this in mind, we imagine the SDs for each condition as being drawn from a log-normal distribution with a mean describing the average between-subject variance $\left(E_{S D . M}\right)$, and a standard deviation describing the between-condition variation in between-subject variance $\left(E_{S D . S D}\right)^{86}$.

\footnotetext{
${ }^{86}$ We use a log-normal distribution to describe the range of between-subject standard deviations so that they are constrained to be positive. A log-normal distribution would normally be parameterised using logmean and $\log$-SD. However, this is not very intuitive to actually understand. Thus, for interpretability of the values, we parameterise it via the regular arithmetic mean and standard deviation and then convert these into the required log-mean and log-SD.
} 
Thus:

$$
\begin{gathered}
E_{\mu_{[t]}} \sim \operatorname{normal}(0,0.4) \\
E_{S D_{[t]}}=\exp \left(\log E_{S D . M}+\log E_{S D . S D} \cdot \tilde{E}_{S D[t]}\right) \\
\log E_{S D . M}=\log \left(\frac{E_{S D . M}^{2}}{\sqrt{E_{S D . S D}^{2}+E_{S D . M}^{2}}}\right) ; \log E_{S D . S D}=\sqrt{\log \left(\frac{E_{S D . S D}^{2}+E_{S D . M}^{2}}{E_{S D . M}^{2}}\right)} \\
E_{S D . M} \sim \operatorname{gamma}(4,33.3) ; E_{S D . S D} \sim \operatorname{half} \operatorname{gormal}(0,0.04) \\
\tilde{E}_{S D[c]} \sim \operatorname{normal}(0,1) \\
\tilde{E}_{[k, S]} \sim \operatorname{normal}(0,1)
\end{gathered}
$$

Position (and application of dimming) bias may vary between people, and is assumed to be constant across conditions in the main experiment. Thus:

$$
\text { bias }_{\text {pos }+\operatorname{dim}[s]}=b \beta_{0}+b \beta_{2 S D} \cdot b \tilde{\beta}_{2[s]}
$$

Where $b \beta_{0}$ is the average bias, $b \beta_{2 S D}$ describes the variation in bias between subjects, and $b \tilde{\beta}_{2[s]}$ is the non-centring parameter:

$$
\begin{gathered}
b \beta_{0} \sim \operatorname{normal}(0,0.05) \\
b \beta_{2 S D} \sim \operatorname{gamma}(4,33.3) \# \operatorname{mode}=0.09, \mathrm{sd}=0.06 \\
b \widetilde{\beta}_{2[s]} \sim \operatorname{normal}(0,1)
\end{gathered}
$$

In the counterbalanced model, we may also allow bias to vary across pairs of conditions thus:

$$
\text { bias }_{\text {position }[p, s]}=b \beta_{0}+b \beta_{1 S D} \cdot b \tilde{\beta}_{1[p]}+b \beta_{2 S D} \cdot b \tilde{\beta}_{2[s]}
$$

Where $b \beta_{1 S D}$ describes the variation in bias between different pairs of conditions and $b \tilde{\beta}_{1[p]}$ is a non-centring parameter. We suggest a half-normal prior with an SD of 0.04 here. This reflects the idea that variation of $\pm 5 \%$ or so is fairly plausible, while variation of more than $\pm 10 \%$ seems less plausible (based on the comparison of null-condition trials in Fotios and Cheal (2011) and the general scale of position bias estimates).

$$
\begin{gathered}
b \beta_{1 S D} \sim \text { half_normal }(0,0.04) \\
b \widetilde{\beta}_{1[p]} \sim \operatorname{normal}(0,1)
\end{gathered}
$$

For the start biases, we are assuming a proportional model. Thus start bias is proportional to the difference between the initial light level and the match that is to be made: 


$$
\begin{aligned}
\operatorname{bias}_{\text {start }[t, r, p, s]} & \\
& =k_{\text {start }[p, s]}\left(\operatorname{ref}-\left(E f f_{[t, s]}-E f f_{[r, s]}\right)-\left(S P D_{\text {test }[t]}-S P D_{\text {ref }[r]}\right)\right. \\
& -s t a r t)
\end{aligned}
$$

... where $k_{\text {start }[p, s]}$ is the start bias strength (gradient of bias against start level), and start is the initial light level of the test condition for that trial. The strength of the start point bias may vary between subjects and for different pairs of conditions, hence:

$$
k_{\text {start }[p, s]}=\beta_{0 . s t a r t}+\beta_{1 . s t a r t . S D} \cdot \tilde{\beta}_{1 . s t a r t[p]}+\beta_{2 . s t a r t . S D} \cdot \tilde{\beta}_{2 . s t a r t[s]}
$$

$\ldots$ where $\beta_{0 . s t a r t}$ is the average bias, $\beta_{1 . s t a r t . S D}$ describes the variation in bias between condition pairs, and $\beta_{2 . s t a r t . S D}$ is the variation between subjects:

$$
\begin{gathered}
\beta_{0 . \text { start }} \sim \operatorname{normal}(0,0.06) \\
\beta_{1 . \text { start.SD }} \sim \operatorname{half\_ normal}(0,0.1) \\
\beta_{2 . \text { start.SD }} \sim \operatorname{half\_ normal}(0,0.1) \\
\tilde{\beta}_{1 . \operatorname{start}[p]} \sim \operatorname{normal}(0,1) \\
\tilde{\beta}_{2 . \operatorname{start}[s]} \sim \operatorname{normal}(0,1)
\end{gathered}
$$

$\sigma_{[s, p]}$, the within-person standard deviation, describes the reliability or variance of the individual's matches. It may vary by both subject $(s)$ and the pair of conditions being compared $(p)$ :

$$
\begin{gathered}
\sigma_{[s, p]}=\exp \left(\sigma_{\alpha_{0}}+\sigma_{\beta_{1 S D}} \cdot \tilde{\sigma}_{\alpha_{1}[p]}+\sigma_{\beta_{2 S D}} \cdot \tilde{\sigma}_{\alpha_{2}[s]}\right) \\
\sigma_{\alpha_{0}}=\log \left(\sigma_{\beta_{0}}\right) \\
\sigma_{\beta_{0}} \sim \operatorname{gamma}(3.49,31.1) \# \operatorname{mode}=0.08, \mathrm{sd}=0.06 \\
\sigma_{\beta_{1 S D}} \sim \operatorname{half\_ normal}(0,0.4) \\
\sigma_{\beta_{2 S D}} \sim \operatorname{half\_ normal}(0,0.3) \\
\tilde{\sigma}_{\alpha_{1}[p]} \sim \operatorname{normal}(0,1) \\
\tilde{\sigma}_{\alpha_{2}[s]} \sim \operatorname{normal}(0,1)
\end{gathered}
$$

Here, $\sigma_{\beta_{0}}$ describes the mean within-subject sd, $\sigma_{\beta_{1 S D}}$ is the variation between pairs of conditions, and $\sigma_{\beta_{2 S D}}$ is the effect of subject. An exponential function is used here to ensure that the standard deviations are positive. The effects of the conditions and subjects on are thus effectively modelled as *multipliers* of the mean SD. So, for example, one condition might have an SD that is $80 \%$ of the mean SD (though expressed on the log scale). We could re-express the equation as:

$$
\sigma_{[s, p]}=\sigma_{\beta_{0}} \cdot \exp \left(\sigma_{\beta_{1 S D}} \cdot \tilde{\sigma}_{\alpha_{1}[p]}\right) \cdot \exp \left(\sigma_{\beta_{2 S D}} \cdot \tilde{\sigma}_{\alpha_{2}[s]}\right)
$$


For the magnitude estimation experiment, the model is altered as described in the main text (Section 3.5.2.2). The key parameter that is added to the model is the brightness/luminance coefficient, $k$. It may vary by the test condition and subject:

$$
\begin{gathered}
k_{[c, s]}=k_{\mu}+\beta_{1 . k . S D} \cdot \tilde{\beta}_{1 . k[c]}+\beta_{2 . k . S D} \cdot \tilde{\beta}_{2 . k[s]} \\
k_{\mu} \sim \operatorname{normal}(0.5,0.3) \\
\beta_{1 . s t a r t . S D} \sim \operatorname{half\_ normal}(0,0.2) \\
\beta_{2 . s t a r t . S D} \sim \operatorname{gamma}(2.62,23.1) \# \text { mode }=0.07, \text { sd }=0.07 \\
\tilde{\beta}_{1 . \operatorname{start}[p]} \sim \operatorname{normal}(0,1) \\
\tilde{\beta}_{2 . \operatorname{start}[s]} \sim \operatorname{normal}(0,1)
\end{gathered}
$$

\section{B.5 Model diagnostic plots}
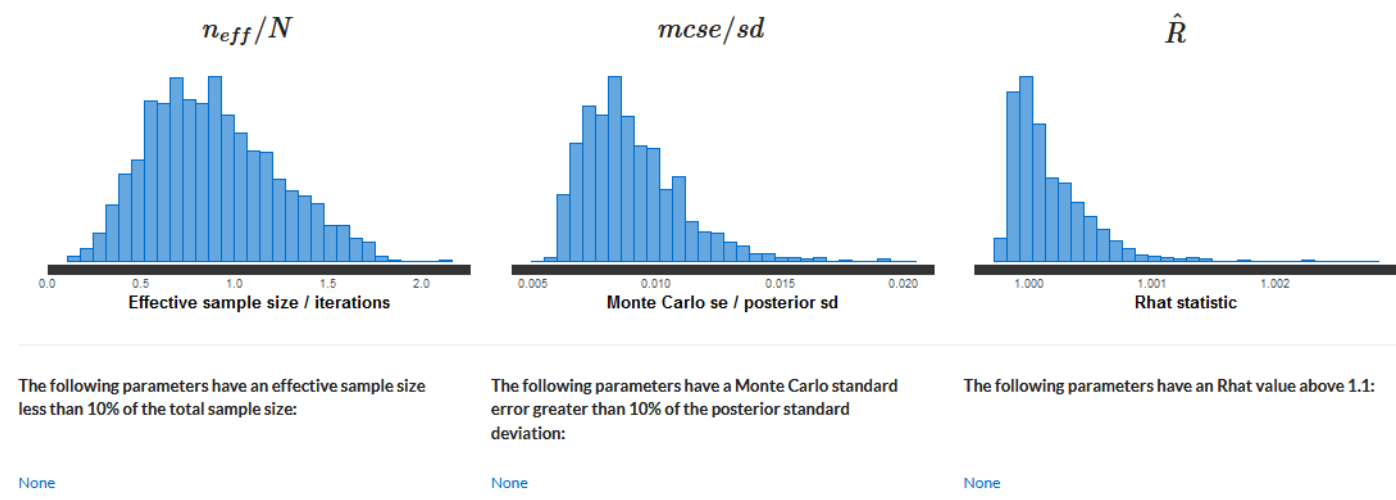

Figure 9.51 Stan model diagnostic plots produced by the ShinyStan R package. 
NUTS Energy Diagnostic
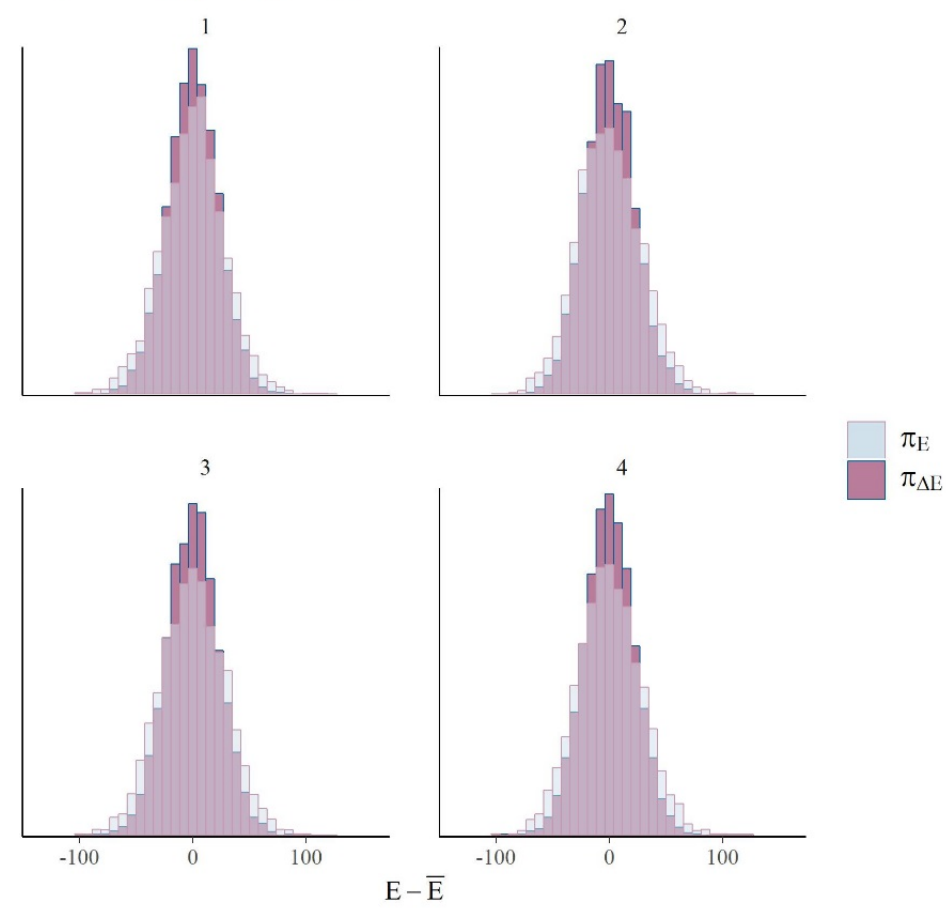

Figure 9.52 NUTS Energy Diagnostic plots produced by the bayesplot package. 
Figure 9.53 Traceplots of model parameters showing that chains are well mixed (bayesplot)
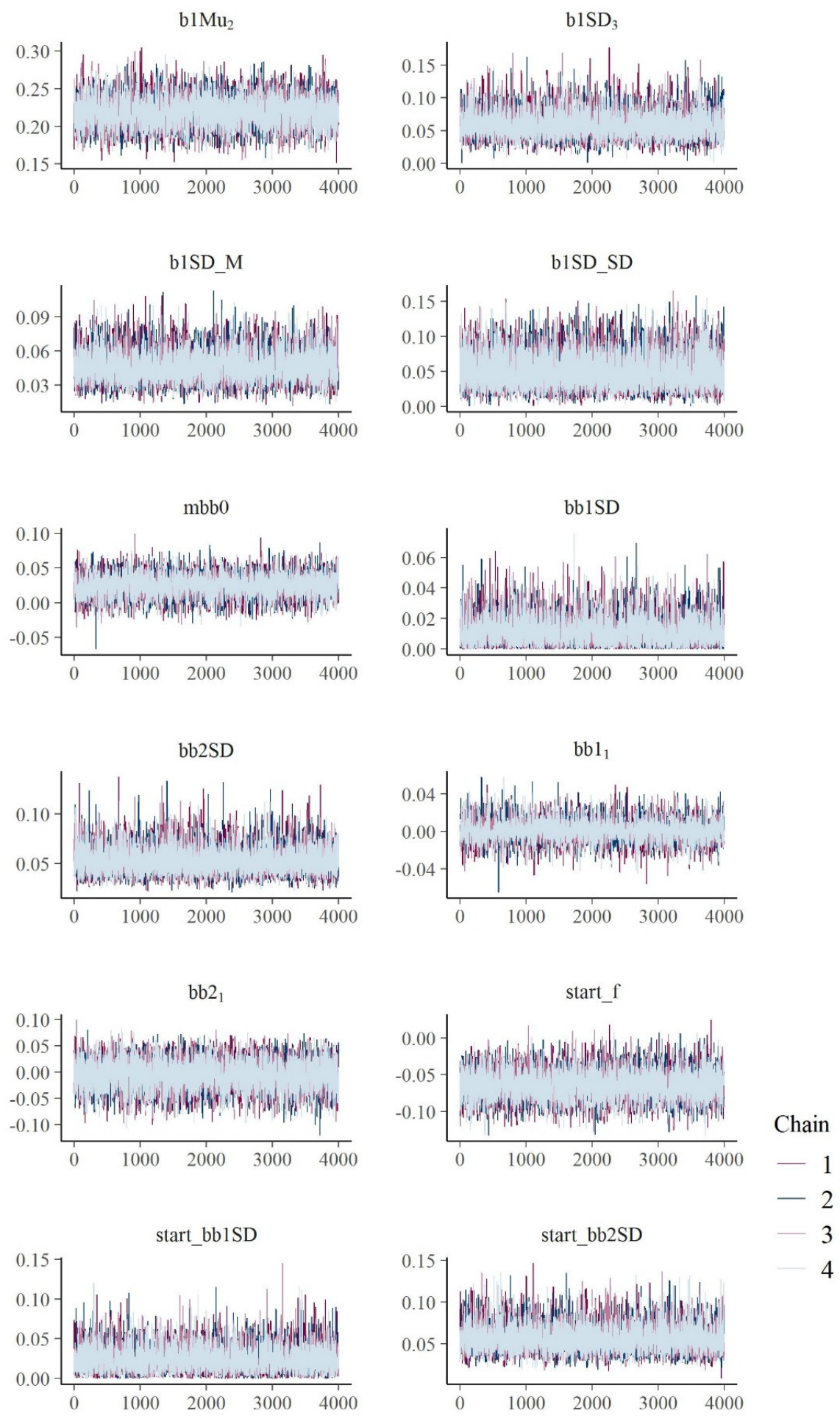

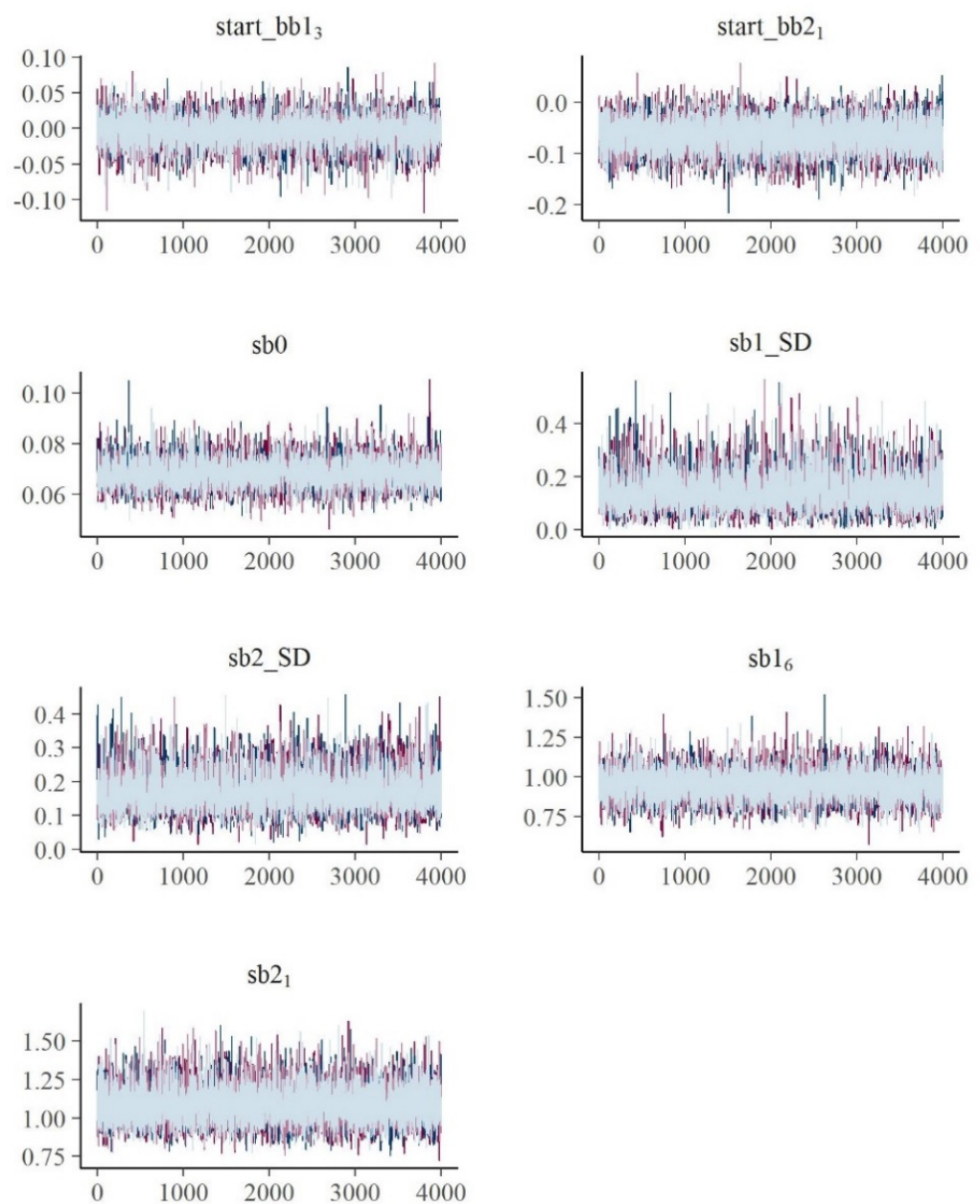


\section{B.6 Pilot study details}

The method was a brightness matching task, wherein participants were instructed to adjust the light level in a test space until it appeared to them to be as bright as a reference space (the Uniform Grey condition). Participants were given written instructions based off those described in Tiller and Veitch (1995)and Fotios and Cheal (2011) to read at the start of the test. Brightness was defined as the amount of light in the space. Participants adjusted their seat height until their eyes were levelled half way up the height of the space. They were instructed to adopt a viewing position such that their chin was just over the edge of the spaces' opening, in order to make sure that it filled their field of view. They then looked back and forth between the spaces to compare them.

The reference condition was held constant at 2.4 vertical lux in order to avoid the response contraction bias (Fotios and Cheal, 2007b) — roughly in the middle of the adjustment range in order to allow room for both high and low match settings. The starting levels were based off the amount of light measured being released into the space, and were set at 30,60,200, and 260 lux - settings designed to be spread around the light level of the reference condition. With two trials for each start setting, there were to be a total of eight trials per condition, similar to that recommended by Fotios et al. (2008). However, part way through the pilot it was found that participants were getting through the trials faster than expected, and also that the matches were being set lower than anticipated. To fill out the time, and attempt to better balance the start levels, a ninth trial starting at 15 lux was added.

Testing was spread out over three sessions on different days, each roughly 50 minutes long, in order to minimise participant fatigue. At the start of each session, participants were given several practice matches, while they spent 10 minutes adapting. This also served to ensure the lamps had time to warm up and stabilise their output in cases where the lamps had not already been turned on in advance. In each session, participants viewed each condition three times, for a total of nine matches for each condition. Test condition order was varied randomly between each session. The order within each session however was only semi-random as described in B.2.

Four people, all post-graduate students at the university, participated in the pilot. Two were male, two were female. All between 20-30 years of age. Two wore visual correction (glasses and contact lenses). 


\section{B.7 Further sensitivity analysis}

If we compare the Bayesian estimates to what we would get from calculating simple means and confidence intervals (Figure 9.54), we see that they largely line up (using the simple high/low binary start bias model as that is what the counterbalancing built into the simple mean assumes).

Pilot study (brightness matching)

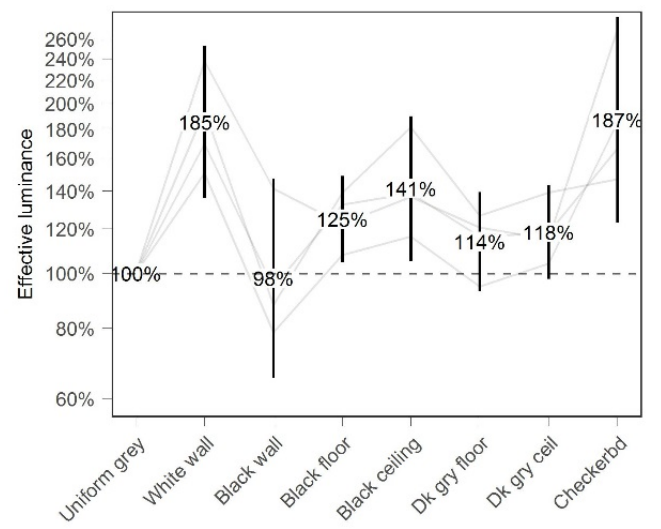

Magnitude Estimation

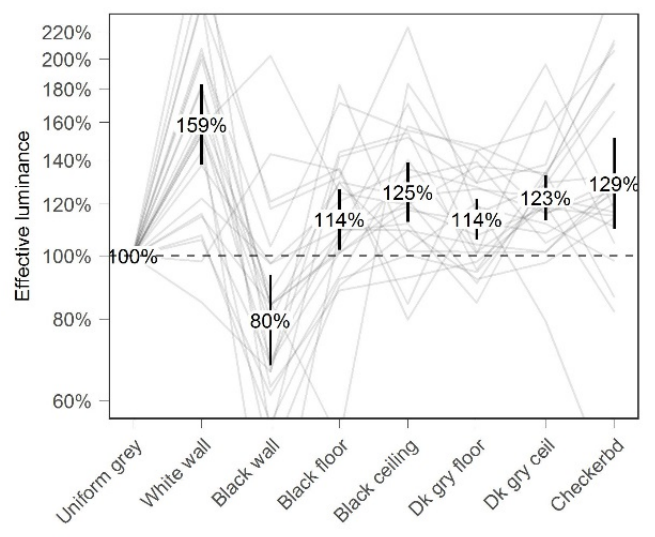

\section{Main Brightness matching w. Null}

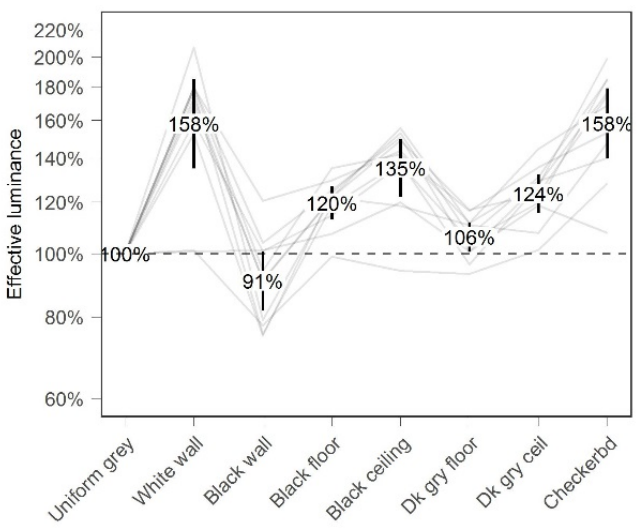

Pairwise counterbalanced matching

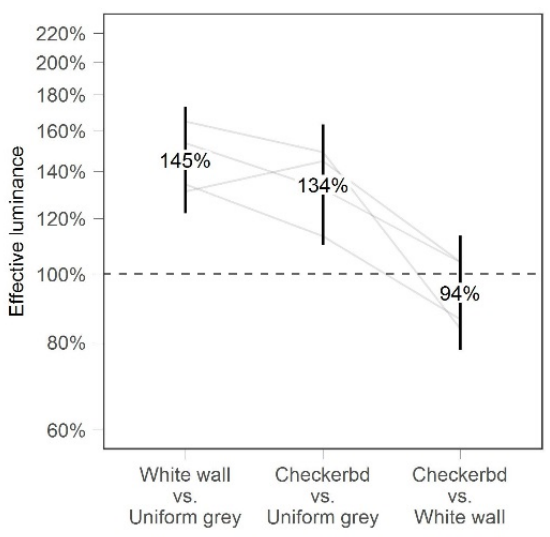

Figure 9.54 Means and 95\% confidence intervals of the effects of the experiments. Effects of conditions are normalised against the uniform grey baseline condition to control for bias. Light grey lines show individual participants' results. Brightness matches are converted to "effective luminance" simply by inverting them. SPD effects have been accounted for by simply subtracting their estimated effect (according to the Rea et al. (2015) model) on the effective light level.

Notable is that the size of the error bars varies more here. The confidence intervals are essentially noisier than the intervals in our Bayesian estimates, being wider on the conditions with the most variation in responses narrower on the conditions with the least variation in responses. This is due to the lack of partial pooling of the variances in the simple calculations. The size of the $95 \%$ intervals are largely a function of the observed variance for the group. In our Bayesian model, we described these variances using a multi- 
level structure that imposed partial-pooling on the estimates of the variances. The result of this is that the model is shrinking the estimated SDs towards the average SD. For the groups (such as the dark grey floor condition) that appear to have relatively low variance, this means that it estimates that they actually have higher variance than the raw data suggests. For the groups that appear to have relatively high variance, it does the opposite. The result of this is that the estimated uncertainty in the Bayesian model is more consistent across the conditions. In contrast, the $95 \%$ confidence intervals here are based purely off the raw SD for each condition separately. This leads them to jump more readily at groups that "appear" to have relatively high or low variance.

This, it may be argued, is an illustration of how the Bayesian analysis here is superior in some respects to what would be provided by the nominally "unbiased" confidence intervals. If we wish to get a picture of the size of the uncertainty we should have in our estimates, confidence intervals are somewhat problematic. 95\% confidence intervals are defined as being the result of a procedure that will produce intervals that overlap with the true mean $95 \%$ of the time (Cumming, 2014). The key point here is that the $95 \%$ confidence is a property of the procedure, and not any given interval. The width of the resulting confidence intervals can vary widely (Cumming, 2014). Even if all our conditions had the same underlying variance, we would expect some of them to end up with narrow intervals and others to end up with wide intervals purely from chance. If one is wanting to look at those intervals to get an understanding of how uncertain they should be about the estimates for each condition, this is clearly a problem. The appearance of intervals that indicate "low" or "high" uncertainty will themselves be in no small part the result of noise. By regularising the estimates of the variance in the Bayesian model we counter this, while still allowing the variance to vary if the data provides good evidence for it.

Another notable difference is in the point estimates of the Magnitude Estimation results. While these also show the same pattern as in our Bayesian estimates, and would lead us to the same conclusions, the average estimates would appear to diverge significantly in places. For example, while our estimate of the effect of the White Wall condition was $-+80 \%$ in our Bayesian model, it is only $-+60 \%$ from the simple mean. While the Bayesian model is much more sophisticated, and differs in many ways, we would generally not expect it to diverge this much, and it is worth probing this more closely to understand why, and to make sure there is nothing wrong with the model.

The first point that should be made here is that simply calculating the means here is a somewhat flawed process. One issue is that due to the uncertainty in measured light level, the different conditions are not (according to our best estimates) all at precisely the same luminance. A better way to estimate the effects of the conditions here is to run a simple linear regression with brightness predicted as a function of light level + condition, with 
conditions defined using dummy variables (0/1). If we do this (and also adjust the light level to account for the SPD effects), then our estimates shift from the simple means - the effect of the White Wall condition for instance is estimated to be around $+65 \%$ (Table 9.6). This is closer to our Bayesian estimate, but still with significant differences for a couple of conditions.

The first thing to do is to simplify our Bayesian model, and cut it out all of our complex multi-level variance parameters to get to a model that is basically the same as the simple linear regression. If we do this, we get results that are within $1 \%$ of those from our simple linear regression, suggesting that it there is no need to be concerned about the basic process. Working through the individual parameters in the model, the key element appears to be the between-person variance in standard deviation. If we remove this parameter from the model, and make the assumption that everyone has the same level of reliability in their magnitude estimates, then we get almost exactly the same results as our simple linear regression.

Table 9.6 Comparison of condition effects estimated by different models (relative to condition 1).

\begin{tabular}{|l|l|l|l|l|l|}
\cline { 2 - 6 } \multicolumn{1}{c|}{} & $\begin{array}{l}\text { Simple } \\
\text { means }\end{array}$ & $\begin{array}{l}\text { Simple } \\
\text { linear } \\
\text { model }\end{array}$ & $\begin{array}{l}\text { Bayesian } \\
\text { model }\end{array}$ & $\begin{array}{l}\text { Removing } \\
\text { subject } \\
\text { var. in SD }\end{array}$ & $\begin{array}{l}\text { Simple } \\
\text { Bayes } \\
\text { model }\end{array}$ \\
\hline 1 Uniform Grey & - & - & - & $-17 \%$ & $-16 \%$ \\
\hline 2 White Wall & $59 \%$ & $65 \%$ & $78 \%$ & $65 \%$ & $66 \%$ \\
\hline 3 Black Wall & $-20 \%$ & $-16 \%$ & $-20 \%$ & $-17 \%$ & $21 \%$ \\
\hline 4 Black floor & $14 \%$ & $20 \%$ & $19 \%$ & $19 \%$ & $30 \%$ \\
\hline 5 Black Ceiling & $25 \%$ & $29 \%$ & $30 \%$ & $27 \%$ & $17 \%$ \\
\hline 6 Dk gry floor & $14 \%$ & $16 \%$ & $16 \%$ & $16 \%$ & $23 \%$ \\
\hline 7 Dk gry ceiling & $23 \%$ & $22 \%$ & $23 \%$ & $23 \%$ & $33 \%$ \\
\hline 8 Checkerboard & $29 \%$ & $32 \%$ & $41 \%$ & $33 \%$ & \\
\hline
\end{tabular}

To understand this better, let us examine the estimated effect of the White Wall condition for individuals according to the different models (Figure 9.55, Figure 9.56). If the SD is not allowed to vary between people, the model infers greater variation between them in the estimates of the underlying effects of conditions. When we allow that SD may vary between people, these estimates become tighter, as the model explains some of the observed differences in people's means by some of them being less reliable than others. In particular, we can see that some of the particularly low estimates (such as subject 10) lose that effect and stop dragging the overall mean down as much because they appear to have more uncertain responses than other participants (Figure 9.57). 


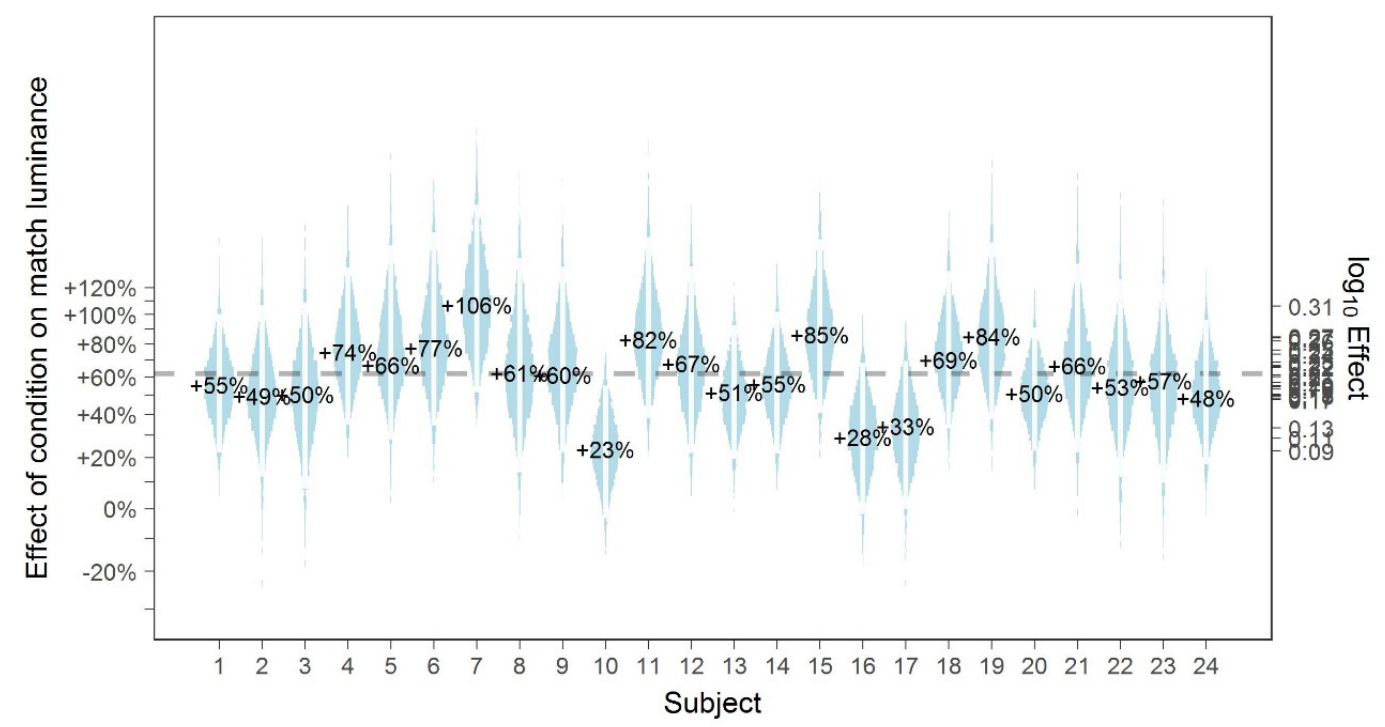

Figure 9.55 Effect of White Wall condition for individual participants estimated using a model with no between-subject variation in SD

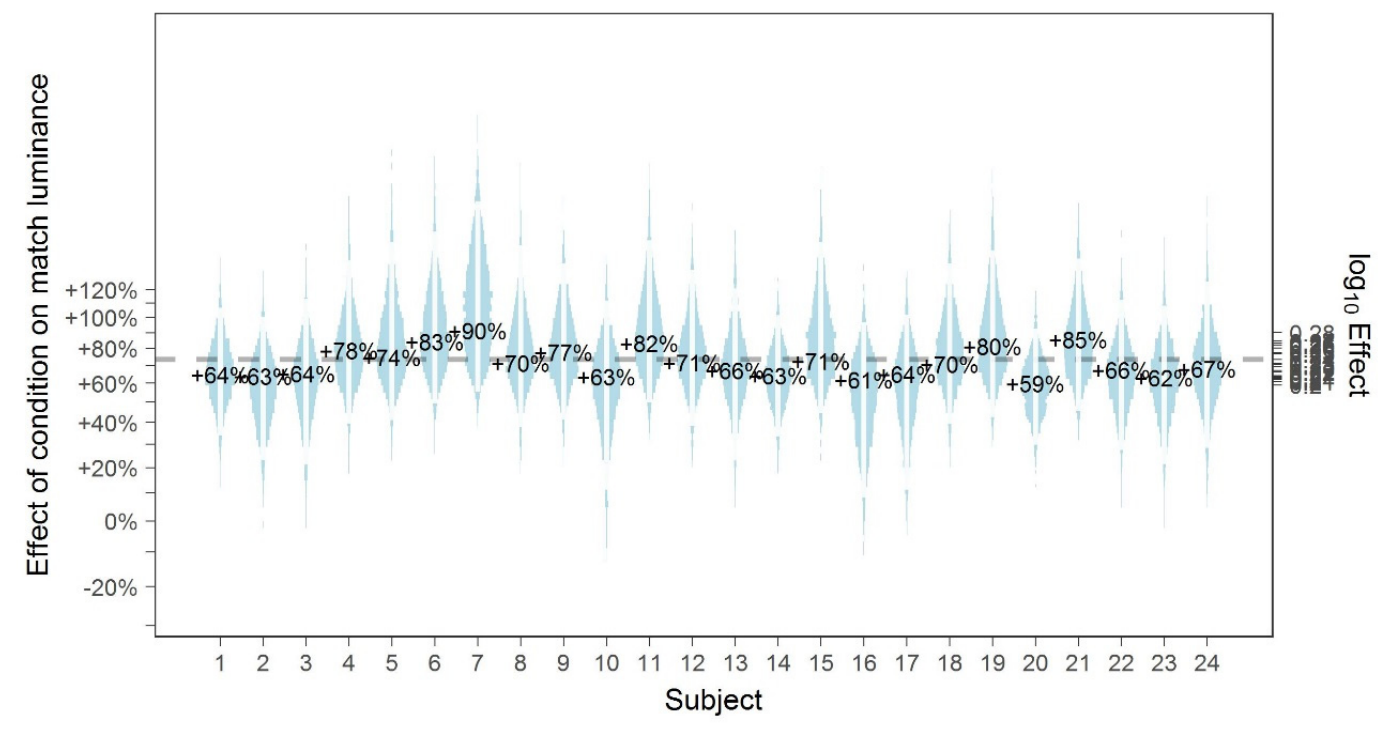

Figure 9.56 Effect of White Wall condition for individual participants estimated using the full model allowing SD to vary between people

To assume that everyone is the same would, we would argue, be a rather implausible and unjustified assumption to make. Moreover, our experimental data consistently shows significant variation between people in terms of effects, biases, and variance. On these grounds we are comfortable assuming that our Bayesian model is, indeed, providing 
reasonable estimates that are likely "better" than what other simpler statistical estimates may suggest.

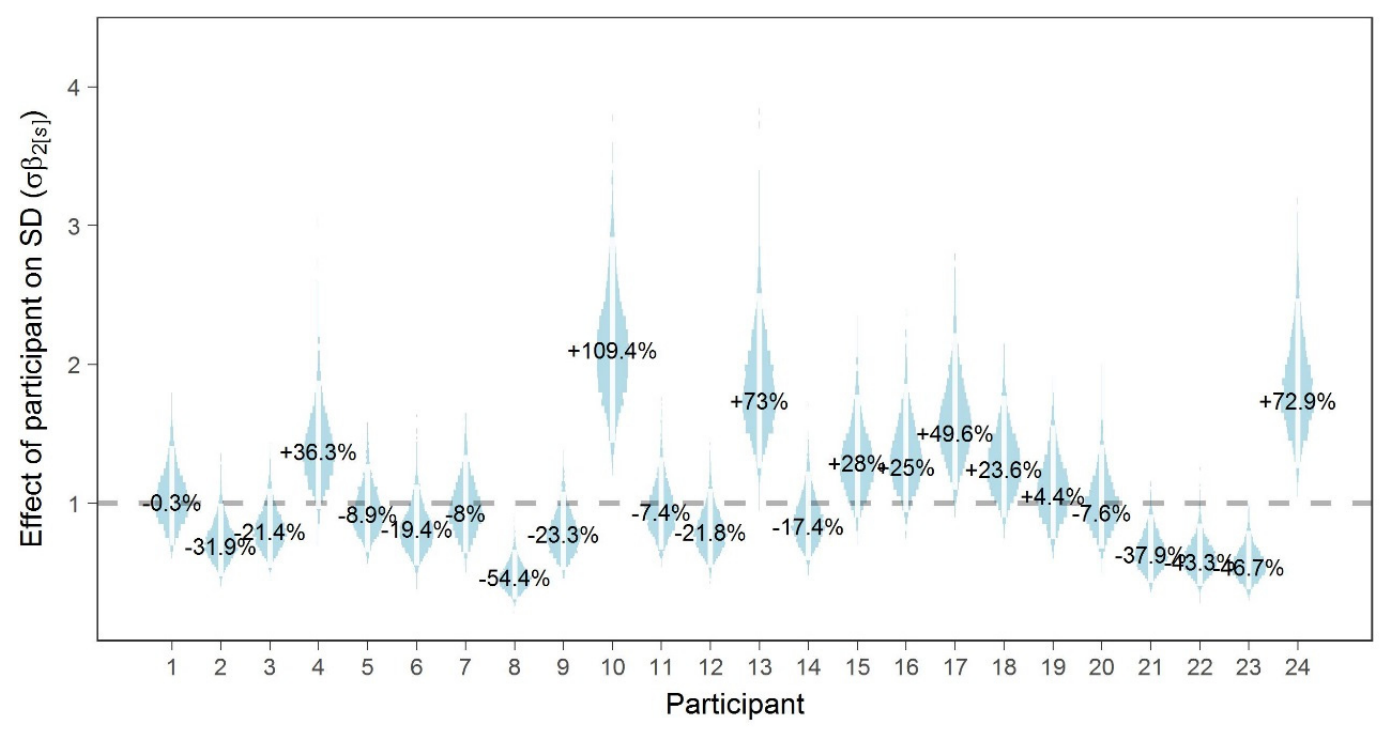

Figure 9.57 Estimated effect of participant on standard deviation. An effect of, for example, $+100 \%$ would mean that that person is estimated as having an SD roughly twice as large as the average.

\section{B.8 Other observations}

We can also examine other parameters in the model such as the estimates of the biases and variances. We will focus here on the estimates from our overall model using all the experiment 1 data (for other model variants, see online supplement).

\section{Biases}

Estimates of position bias in the model are ultimately uncertain, though in the range that we would expect from the literature $( - \pm 10 \%)$ (Figure 9.58, Figure 9.59). They might be substantial, they might be insignificant. 


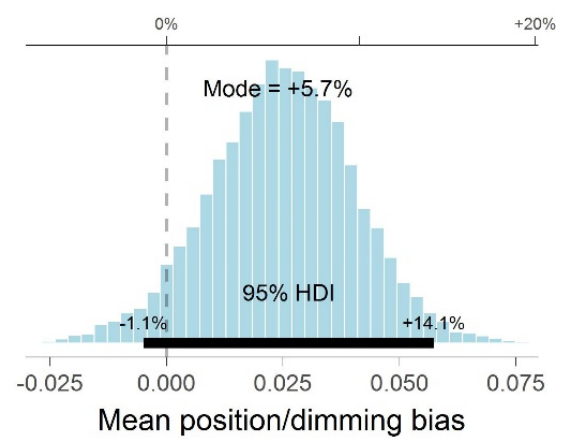

Figure 9.58 Position+dimming bias for brightness matching experiments

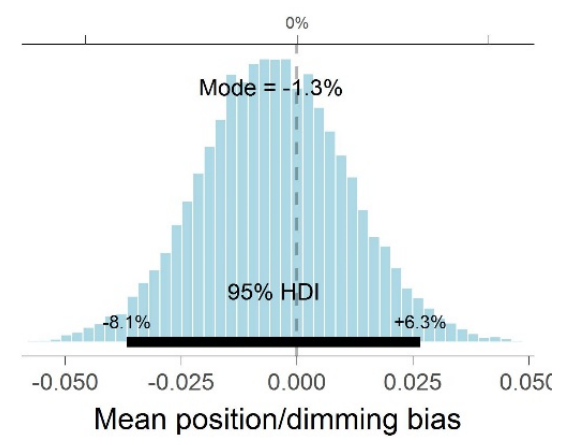

Figure 9.59 Position bias in Magnitude Estimation experiment

Unsurprisingly, we see evidence for significant variation in bias between different people. If we look at estimates for individual participants (Figure 9.62), the data suggests that these differences can be very substantial, with differences of $- \pm 10 \%$ or so not being uncommon. Indeed, the data of one participant (\#15) from the counterbalanced experiment suggested a bias on the order of $-30 \%$ higher than the mean. Considering that the mean bias is somewhere around $0-10 \%$, this is quite remarkable. A standard deviation of around 0.06 on the log scale is perfectly in line with what is often seen in the literature for spatial brightness matching, so this level of variance does not seem excessive.

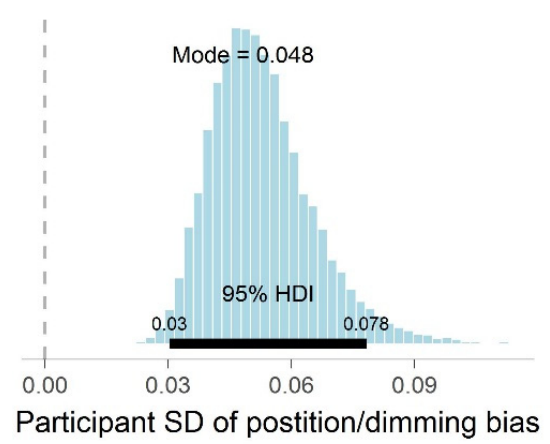

Figure 9.60 SD of between-person variation in position+dimming bias for brightness matching experiments

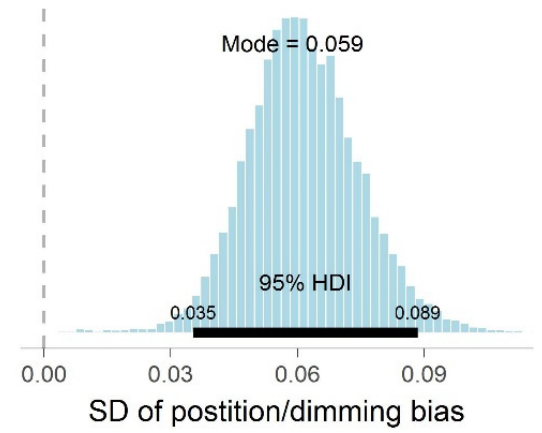

Figure 9.61 SD of between-person variation in position bias in Magnitude Estimation experiment

Whether or not these apparent differences between people are stable, and would replicate if the same people were tested again at a later date is a question that has not, to our knowledge, been tested. 


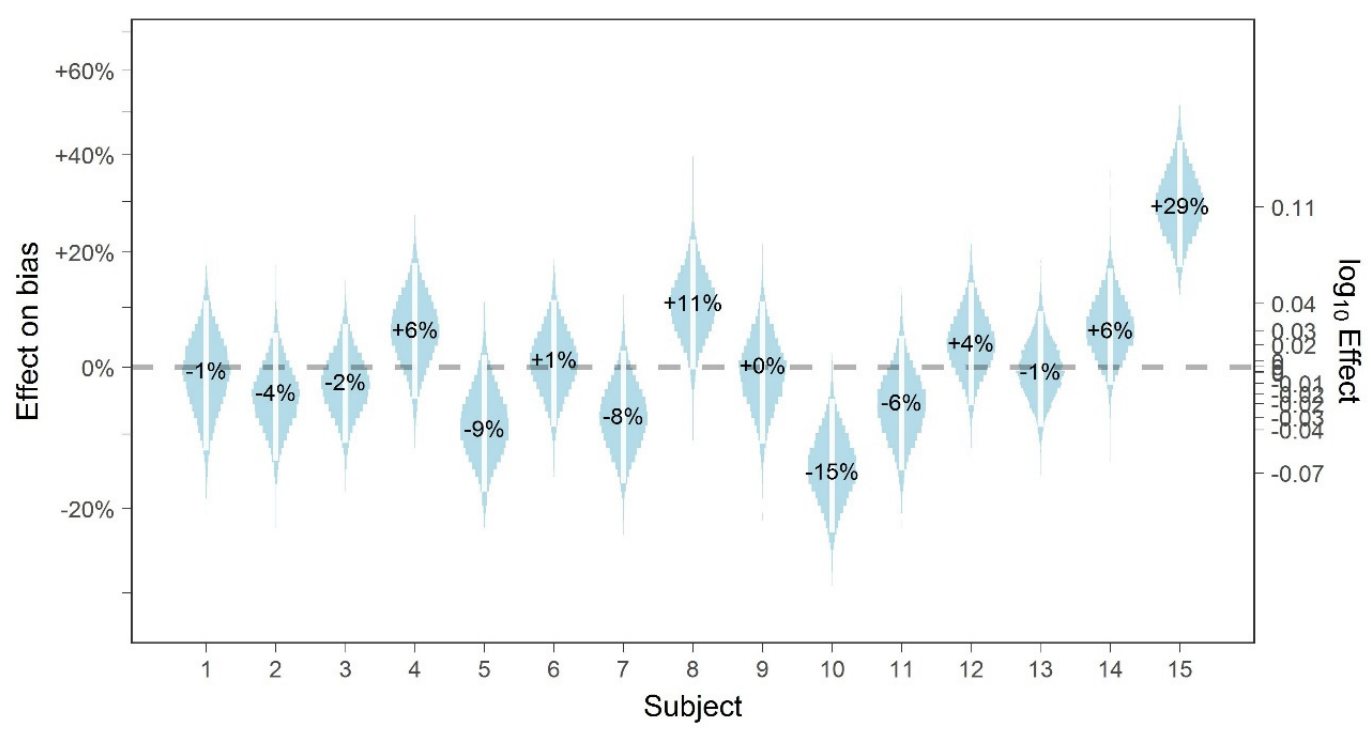

Figure 9.62 Estimates of individual participants' effects on position bias in brightness matching experiments

Regarding possible variation between conditions, the evidence is generally limited. The estimate of the SD has shrunk towards zero from our prior (a half-normal with an SD of 0.04 ), but our counterbalanced experiment was very small and could not really say much about the subject - as can be seen in the broad and not particularly informative individual estimates. Overall, we should remain equivocal on the subject.
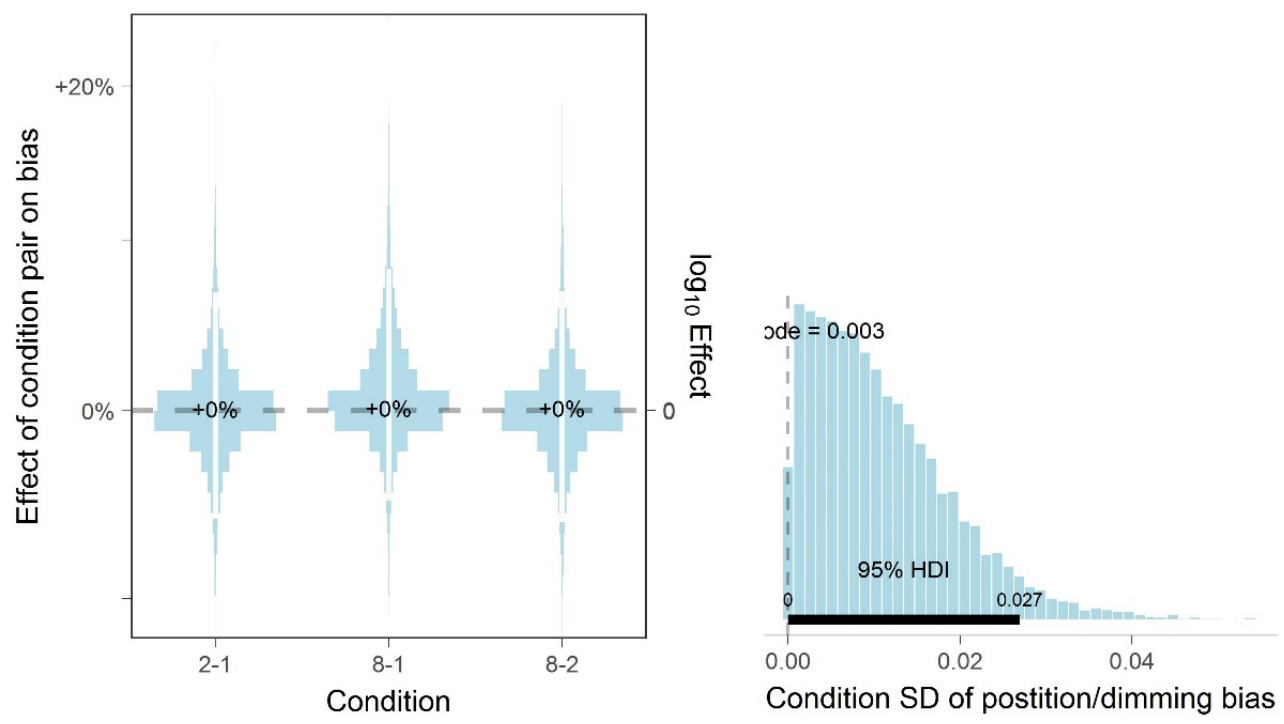

Figure 9.63 Estimated between condition variance in position bias. Left: estimates of effect of individual pairs of conditions in counterbalanced experiment on bias. Right: Estimated standard deviation 
The data more strongly supports the presence of start-point bias - and this is true regardless of whether or not you subscribe to the bias being a linear function of the difference between match setting and initial light level, or it being a binary high/low function. If a linear function, the data suggests a slope of about 0.025 to 0.1 (Figure 9.64). So, for example, if your start point was half of your match setting, that would be a difference of $\sim 0.3$ on the $\log 10$ scale. $\sim 0.3^{*} 0.06=0.018=\sim 4 \%$, so you would expect such a lower set point to lead to matches (for that condition) being underestimated by around $4 \%$ on average. Alternatively, under a binary model of the bias the data suggests that our high set point matches were over/underestimated by $-2-9 \%$ depending on the set point.

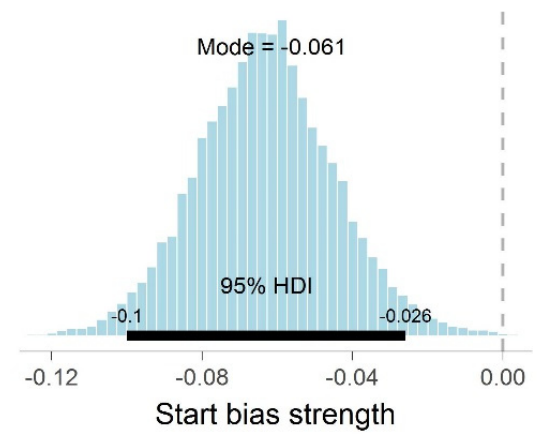

Figure 9.64 Start point bias under a linear model

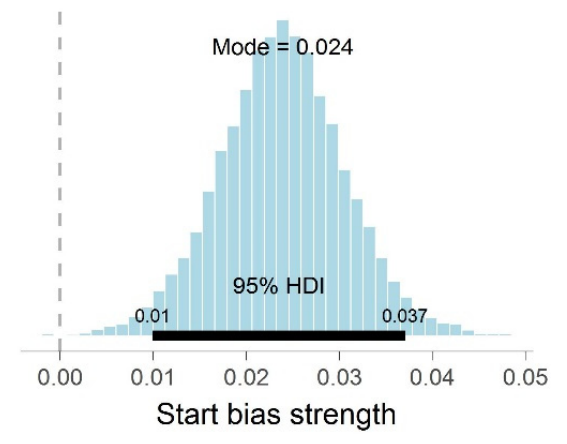

Figure 9.65 Start point bias under a binary high/low model

Again, we see solid evidence for between-person variation in start point bias (Figure 9.67), but much less evidence for significant between-condition variation (Figure 9.66). The estimates are fairly uncertain. While we might say the evidence points at betweencondition variance probably not being large, we would need a lot more data to say anything with confidence.

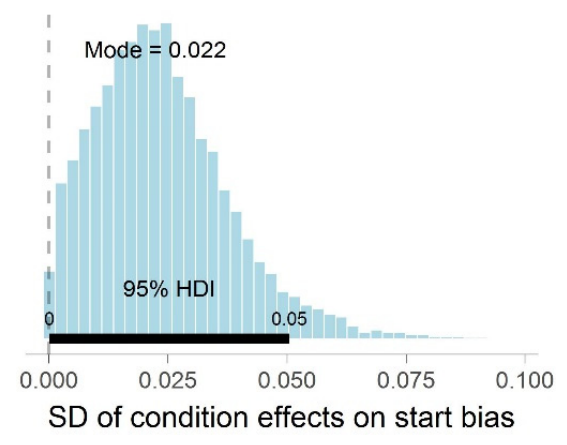

Figure 9.66 SD of between-condition variation in start point bias for brightness matching experiments (linear model)

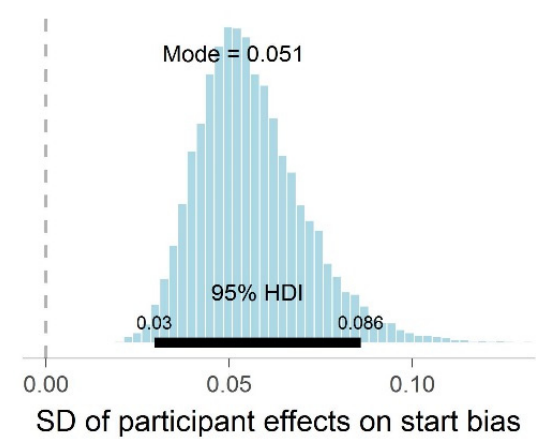

Figure 9.67 SD of between-person variation in start point bias for brightness matching experiments (linear model) 


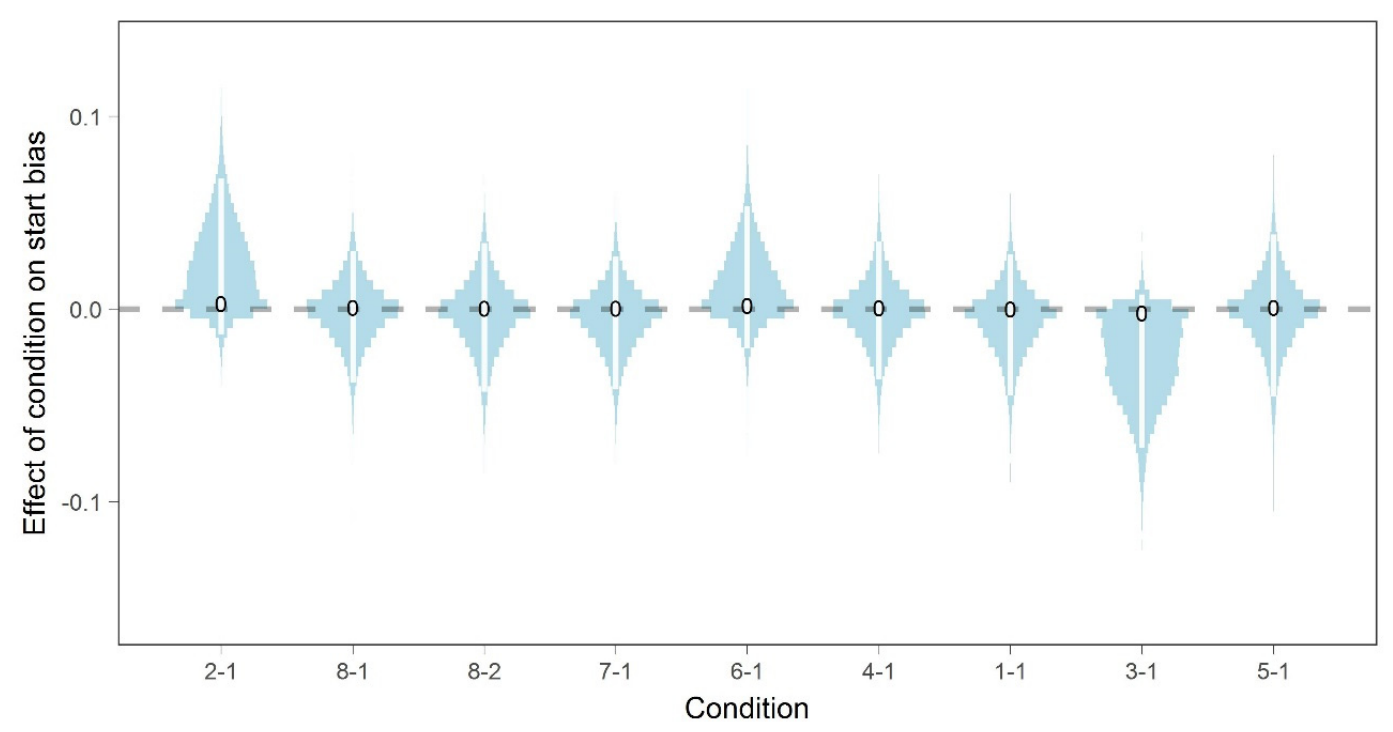

Figure 9.68 Estimated differences in start point bias between pairs of conditions (linear model)

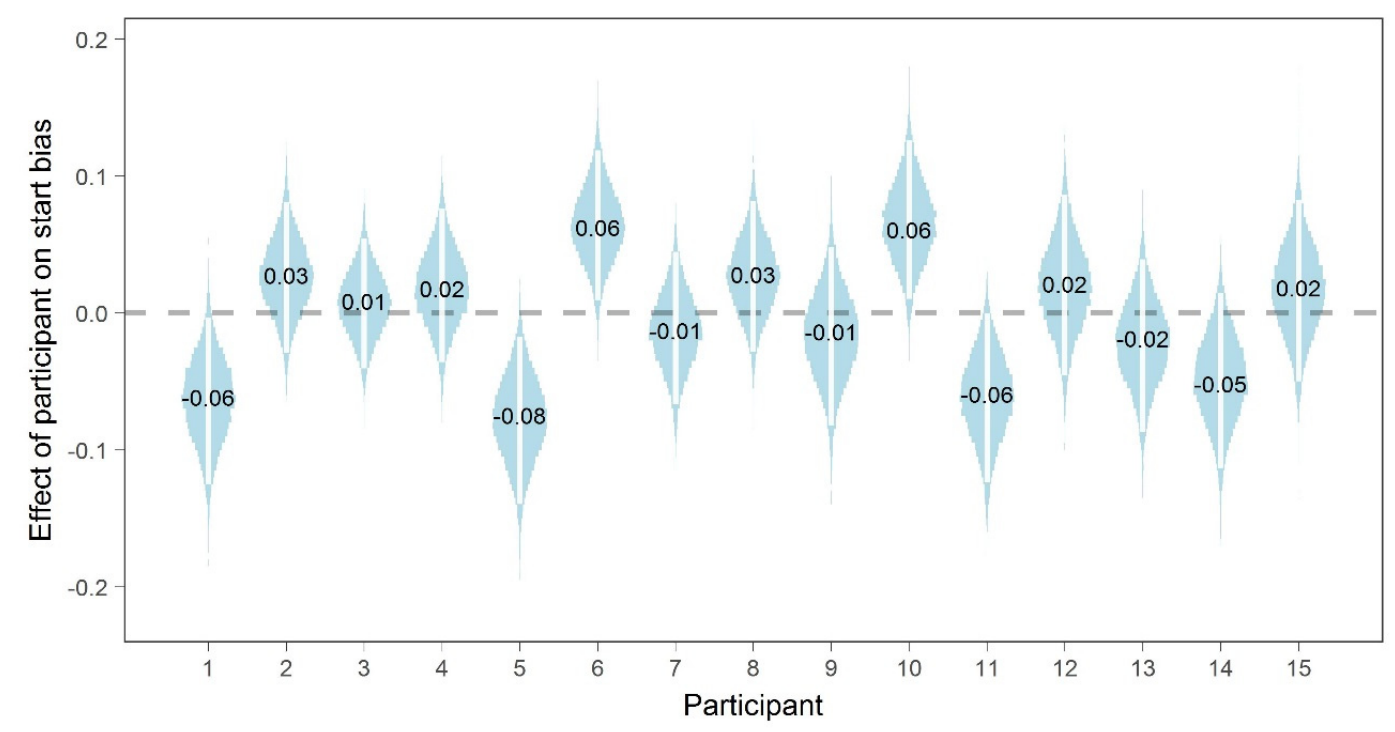

Figure 9.69 Estimated differences between participants in start point bias (linear model)

\section{Variation in condition effects}

The effects of the conditions can also vary significantly between people. The average SD here is within the typical range scene for brightness matching studies in the literature (Figure 9.70, compare back to Figure 3.17), though on the lower end — similar to, for example, Fotios and Cheal (2011). We also note here that the data suggests that there is a degree of variation between the conditions (Figure 9.71, Figure 9.73). 


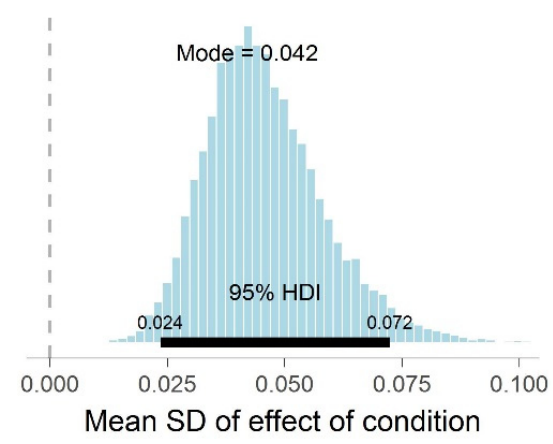

Figure 9.70 Mean SD of effect of condition in brightness matching experiments

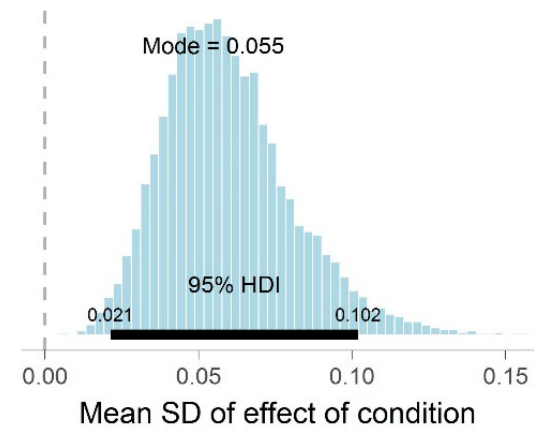

Figure 9.72 Mean SD of effect of condition in magnitude estimation experiment

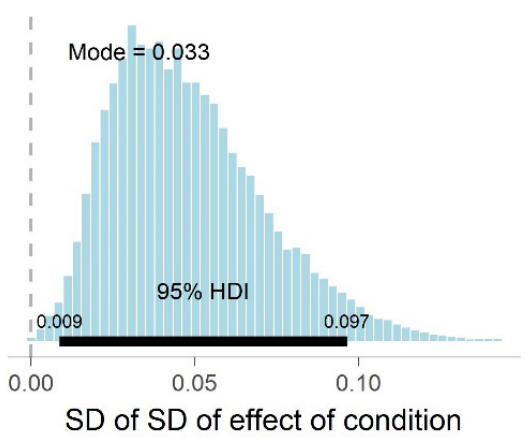

Figure 9.71 SD of SD of effect of condition in brightness matching experiments

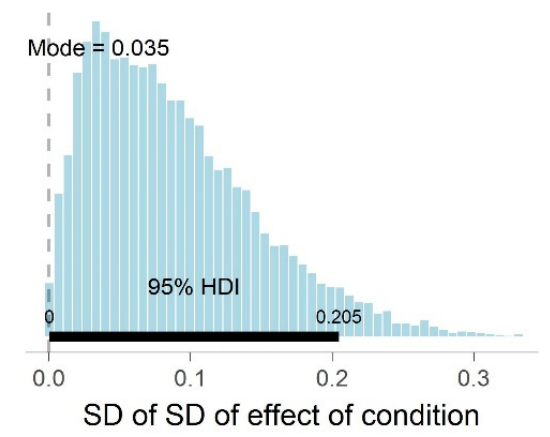

Figure 9.73 SD of SD of effect of condition in magnitude estimation experiment

The White Wall, Black Wall, and Checkerboard conditions seem to have much more variance between people than do the other conditions, and this pattern is seen in both the brightness matching and magnitude estimation results (though in the case of the ME results there is substantial uncertainty in the estimates, and the ranges of plausible values overlap substantially between conditions) (Figure 9.74, Figure 9.75). It is not clear why such a pattern would be observed. The White Wall and Checkerboard conditions could, perhaps, point to a pattern where larger effects see more scope for disagreement between people, but the Black Wall condition is not substantially larger in effect than the floor and ceiling conditions. Another possibility perhaps is the idea that the similarity of the floor and ceiling conditions with each other meant that people had more "practice" with those conditions and so became more consistent. However, when we look at the estimates of within-person reliability we do not see a similar pattern (Figure 9.85), so that hypothesis does not really seem to be supported by the data. 


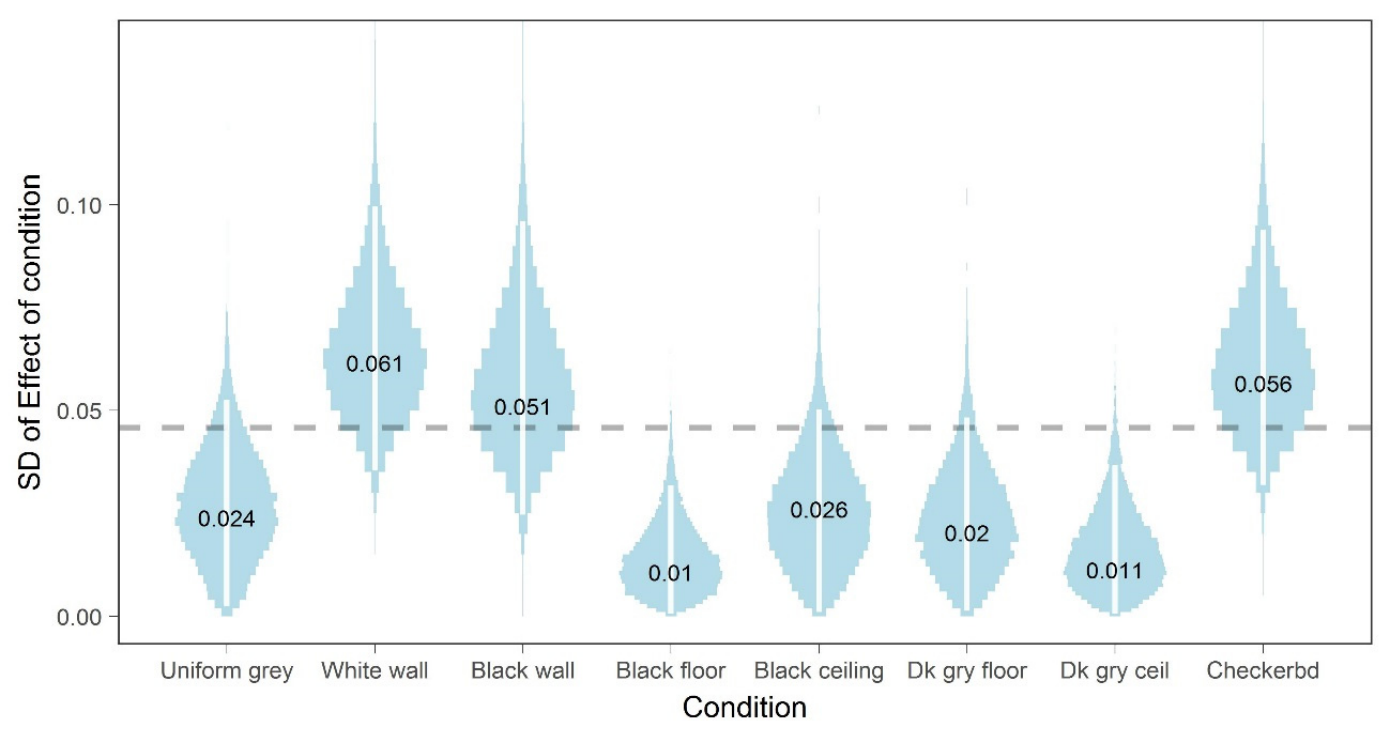

Figure 9.74 Standard deviations of effects of individual conditions in brightness matching experiments

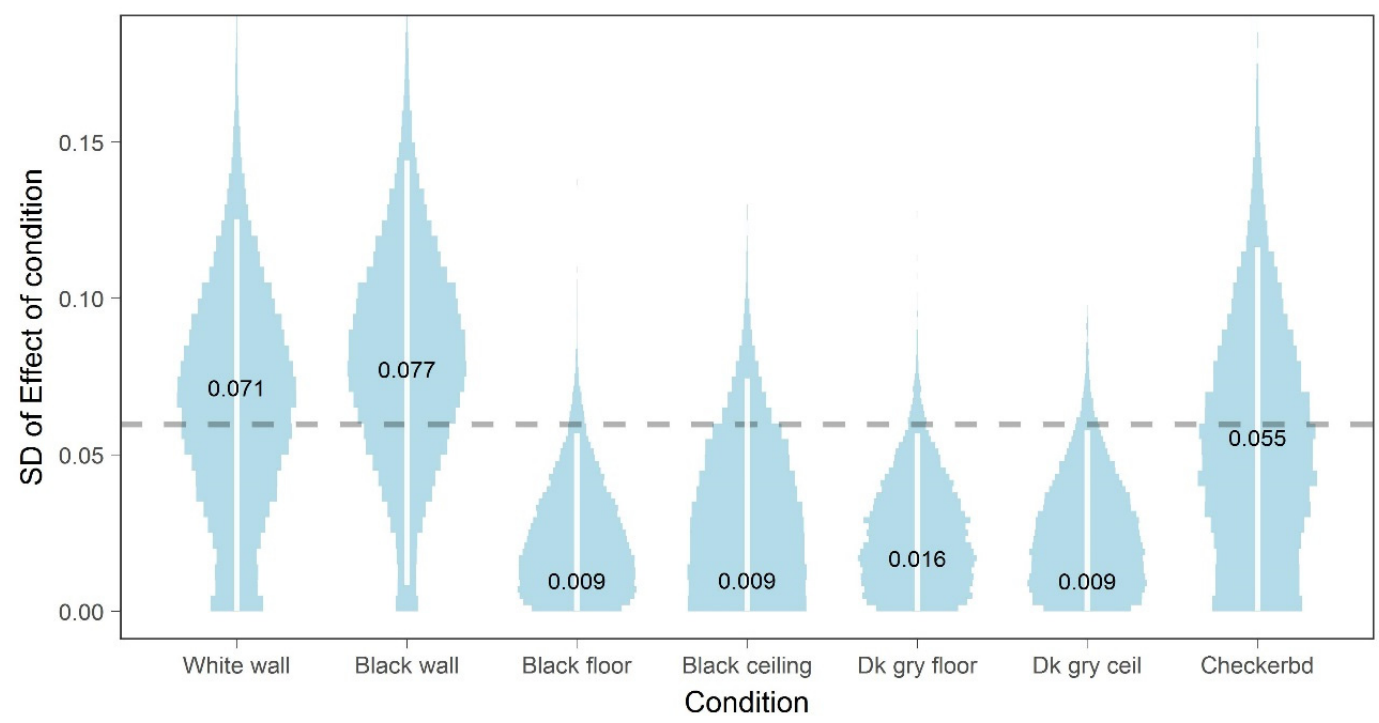

Figure 9.75 Standard deviations of effects of individual conditions in magnitude estimation experiment

To get a feeling for what this variation means, we can look at what the model suggests the condition effects for each individual participant is (Figure 9.76). For the three high variance conditions there appears to be substantial disagreement between people on how bright or dark they are. One question this brings up is whether or not these apparent differences between people actually reflect stable underlying differences in perception between them. Teghtsoonian and Teghtsoonian (1971), for example, found that a significant amount of the variation in the slope of the brightness/luminance relationship did not necessarily reflect reliable differences between people, as the individual responses 
changed significantly when people were tested again after a month. Whether or not this is true for these light distribution effects is an open question, and one that may merit further research. Overall, these results should serve as a reminder of the fact that people are not all the same, and that the average may not always well predict individuals'. 


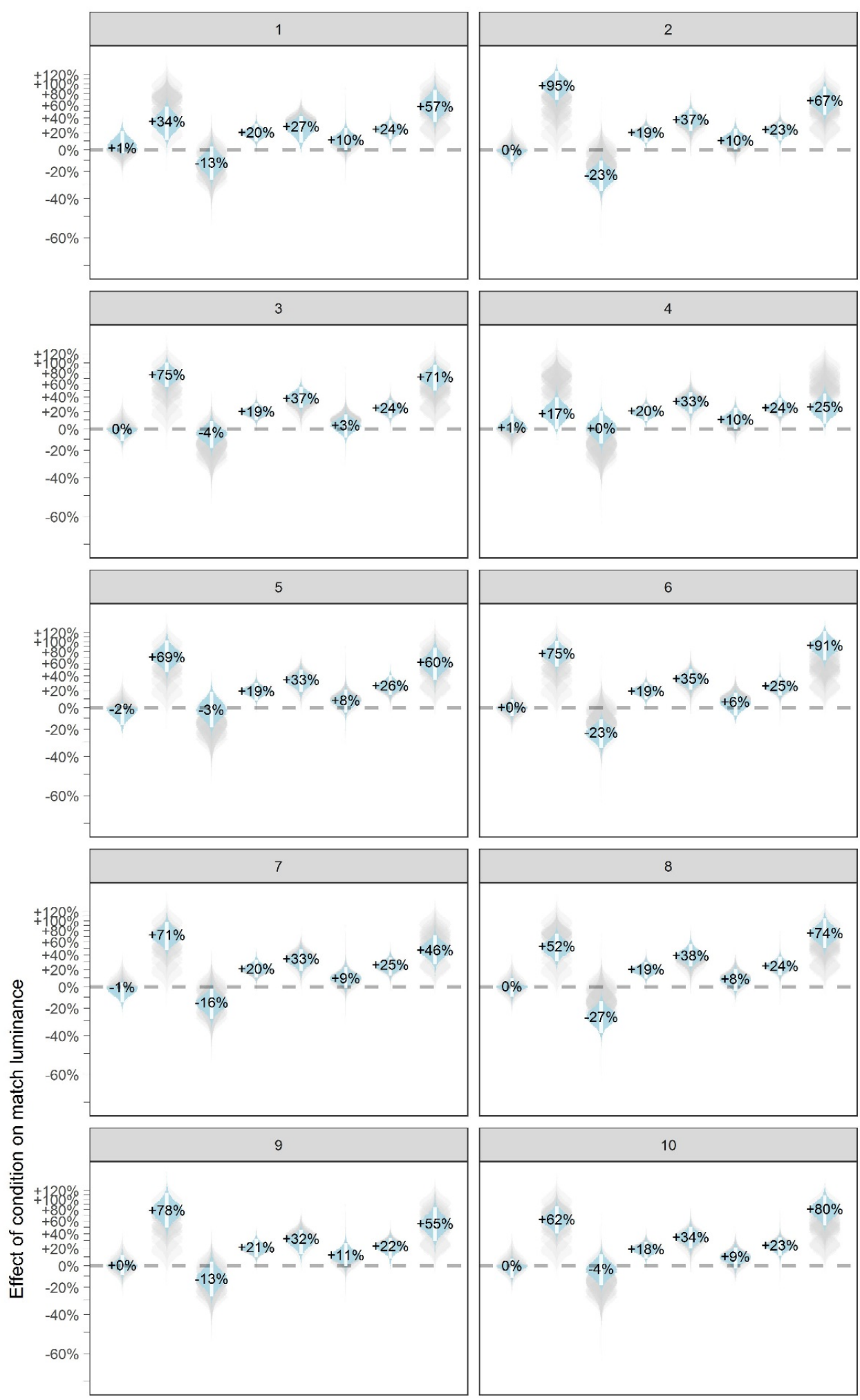

Figure 9.76 Estimates of the effects of the conditions for a selection of individual participants (\#110 of the brightness matching experiment). Light grey "shadows" show the distributions of the other participants. 


\section{Within-person variance/reliability}

As can be readily seen in the raw data, people's individual matches/brightness estimates have considerable uncertainty and unreliability. The estimated standard deviations for this are within expected bounds for this kind of study (Figure 9.77, Figure 9.78) (see the discussion on Priors for a summary of past precedent).

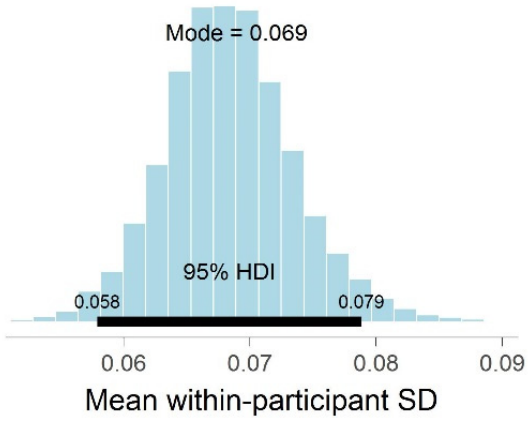

Figure 9.77 Mean within-person SD of brightness matches

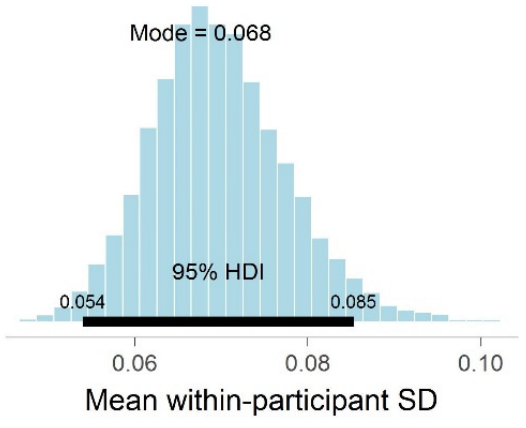

Figure 9.78 Mean within-person SD of magnitude estimates

Looking at the possible variation between conditions and people, we again see significant variation between people, with some individuals being significantly more or less reliable than others. Variation between people appears to be higher for Magnitude Estimation, and this is likely just a reflection of the different methodology and the fact that the error here is in terms of brightness rather than match luminance. It is very apparent in the Magnitude Estimation data that different people use the numbers in very different ways, with one person, for example, only using numbers between around 90-120, while another was using numbers all the way up to 300 to estimate brightness. The differences we see between people here are likely a reflection of that.

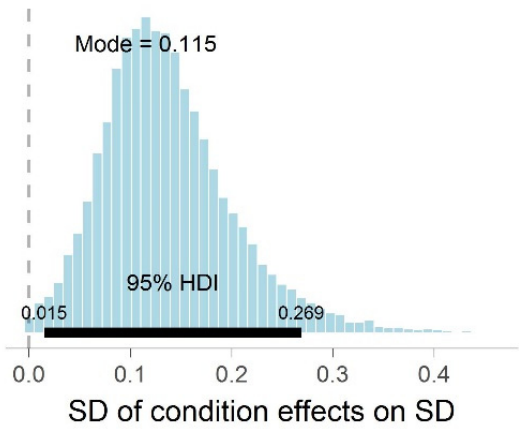

Figure 9.79 SD of condition variance in withinperson SD of brightness matches

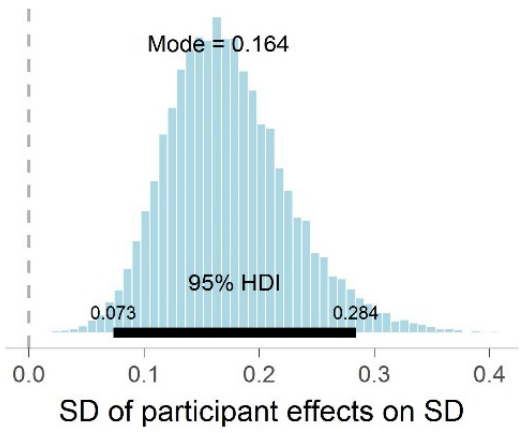

Figure 9.80 SD of between-person variance in within-person SD of brightness matches 


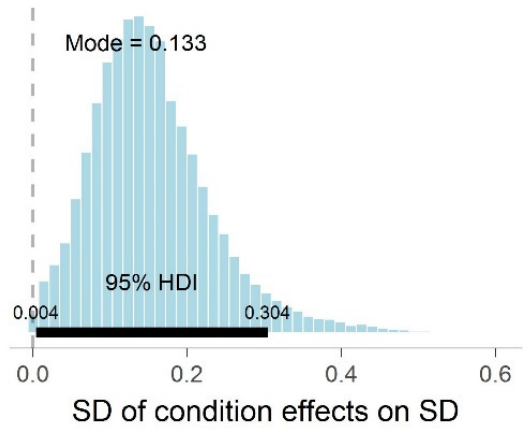

Figure 9.81 SD of condition variance in withinperson SD of Magnitude Estimates

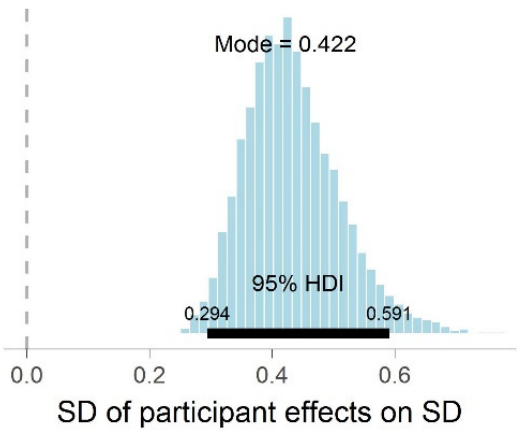

Figure 9.82 SD of between-person variance in within-person SD of Magnitude Estimates

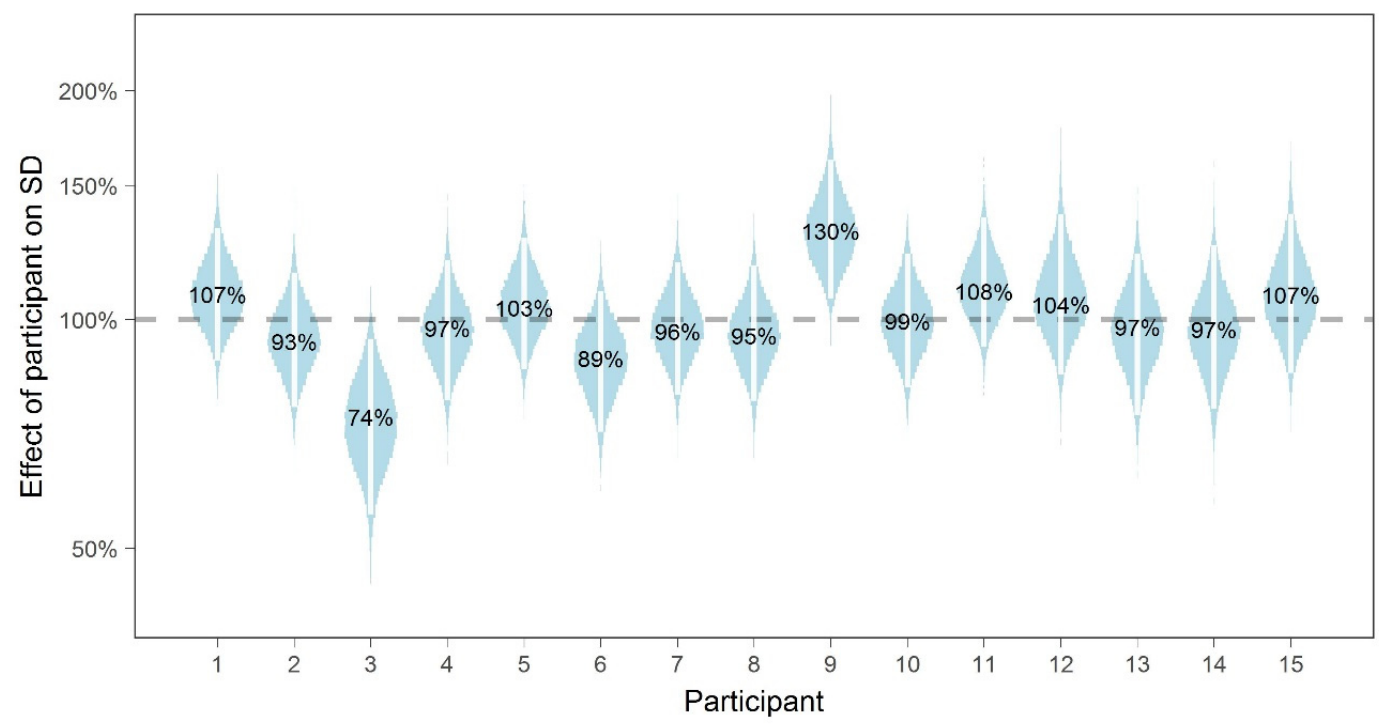

Figure 9.83 Differences between individual participants' in within-person SD for brightness matches. Note here that the model describes their effects as relative to the average SD, so, for example, participant 9 is estimated to have an SD around 30\% larger than the average in the experiment. 


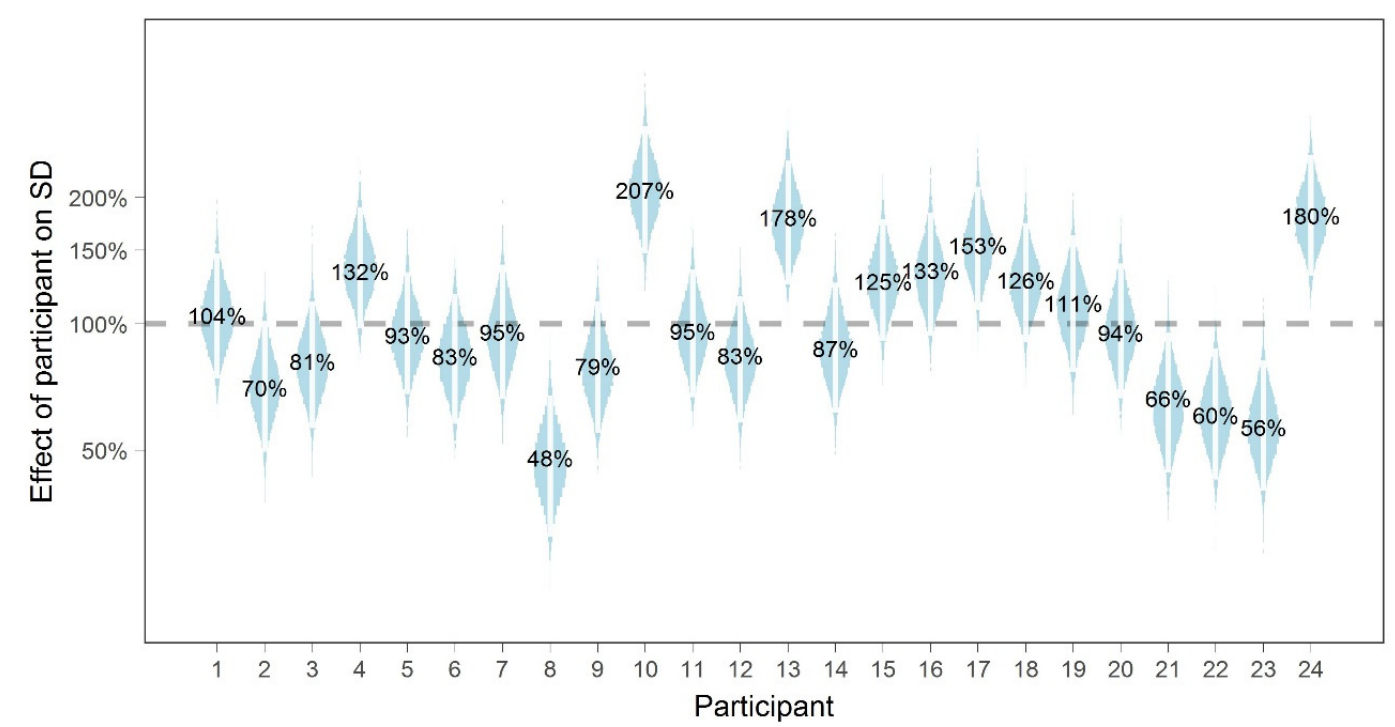

Figure 9.84 Differences between individual participants' in within-person SD for Magnitude Estimates. Note here that the model describes their effects as relative to the average SD, so, for example, participant 10 is estimated to have an SD around twice the average in the experiment.

The data also suggests that there may be variation in uncertainty of matches and estimates between the different pairs of conditions, with some comparisons potentially being more or less difficult than others. That being said, the variation does appear to be significantly less than the variation between people, and it may not be substantial.

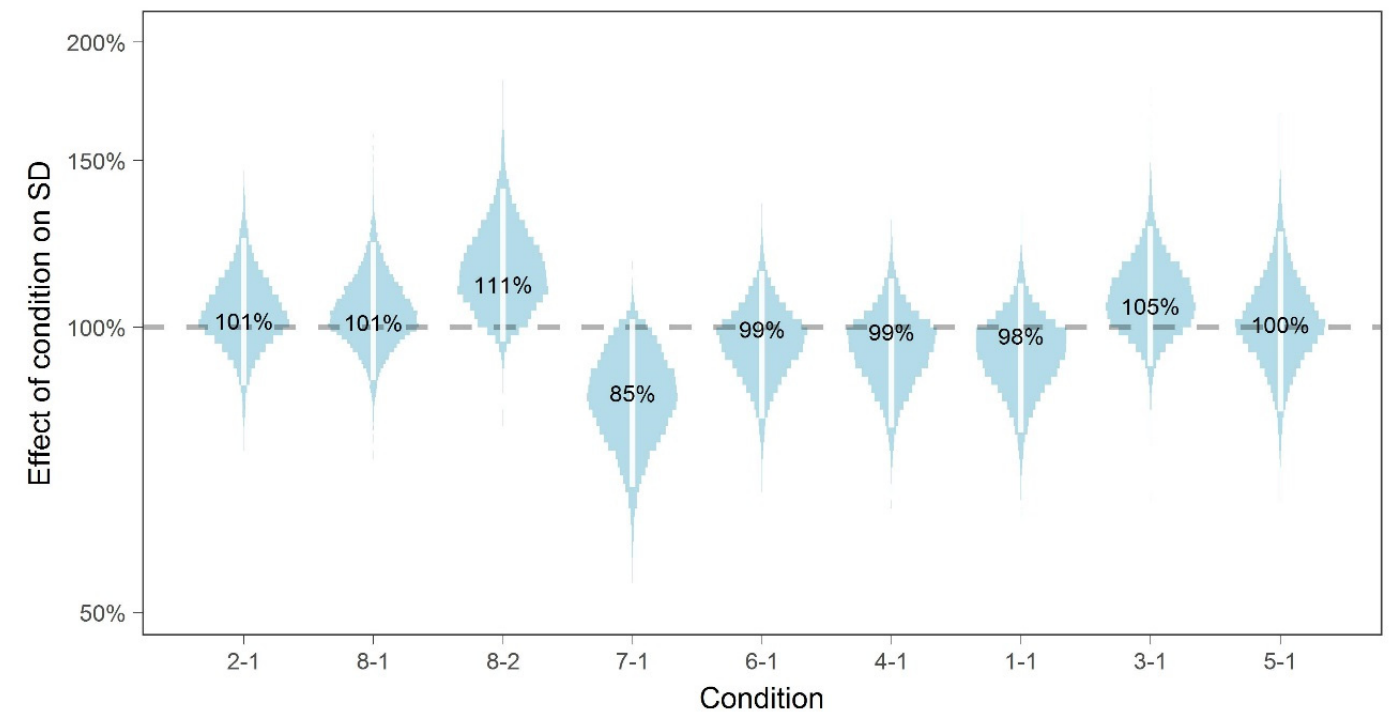

Figure 9.85 Differences in within-person SD of brightness matches between pairs of conditions 


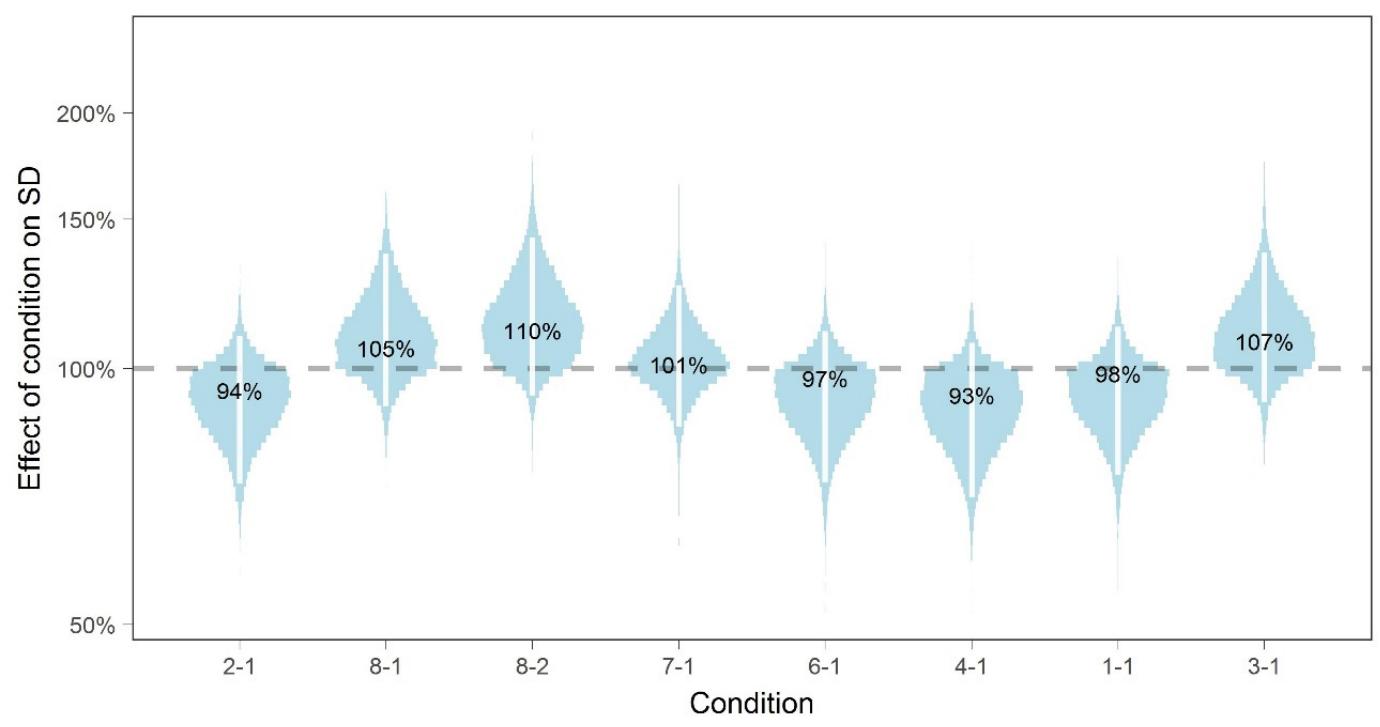

Figure 9.86 Differences in within-person SD of magnitude estimates between pairs of conditions

\section{Brightness/luminance relationship}

The slope of the brightness/luminance relationship as estimated here is somewhere around 0.5-0.6, similar to that in Rea et al.'s (2015) results and within the expected range. Again, we see substantial variation between people, which is unsurprising - though the variation here is larger than what we would have expected from the simpler studies of target brightness (which had SDs in the 0.05 to 0.1 range). There is also again little evidence in favour of significant variation between conditions here, though estimates are uncertain.

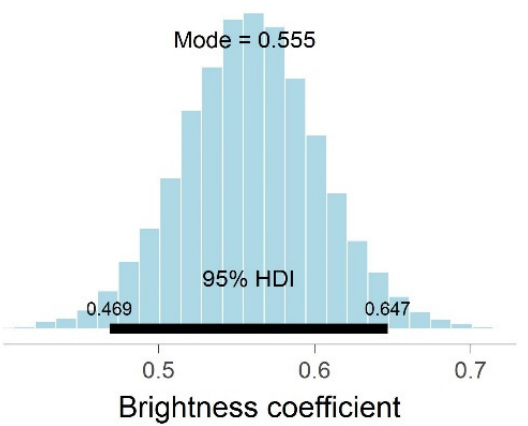

Figure 9.87 Slope of the brightness/luminance relationship in the magnitude estimation experiment 


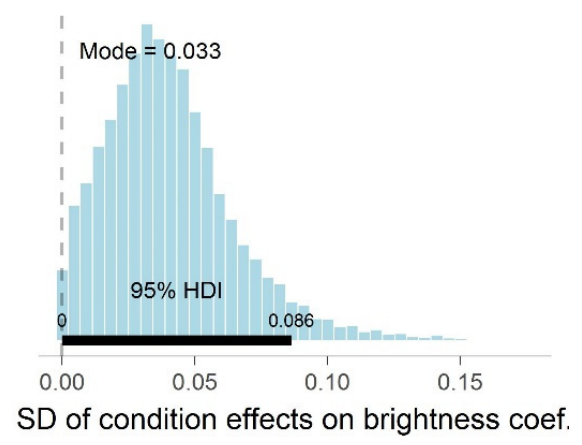

Figure 9.88 SD of variation in slope of brightness/luminance relationship between conditions

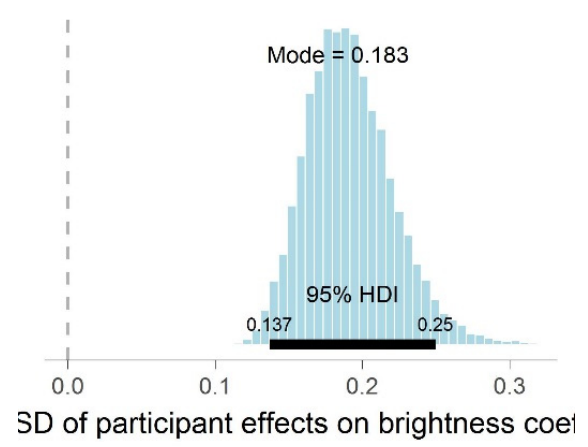

Figure 9.89 SD of variation in slope of brightness/luminance relationship between people

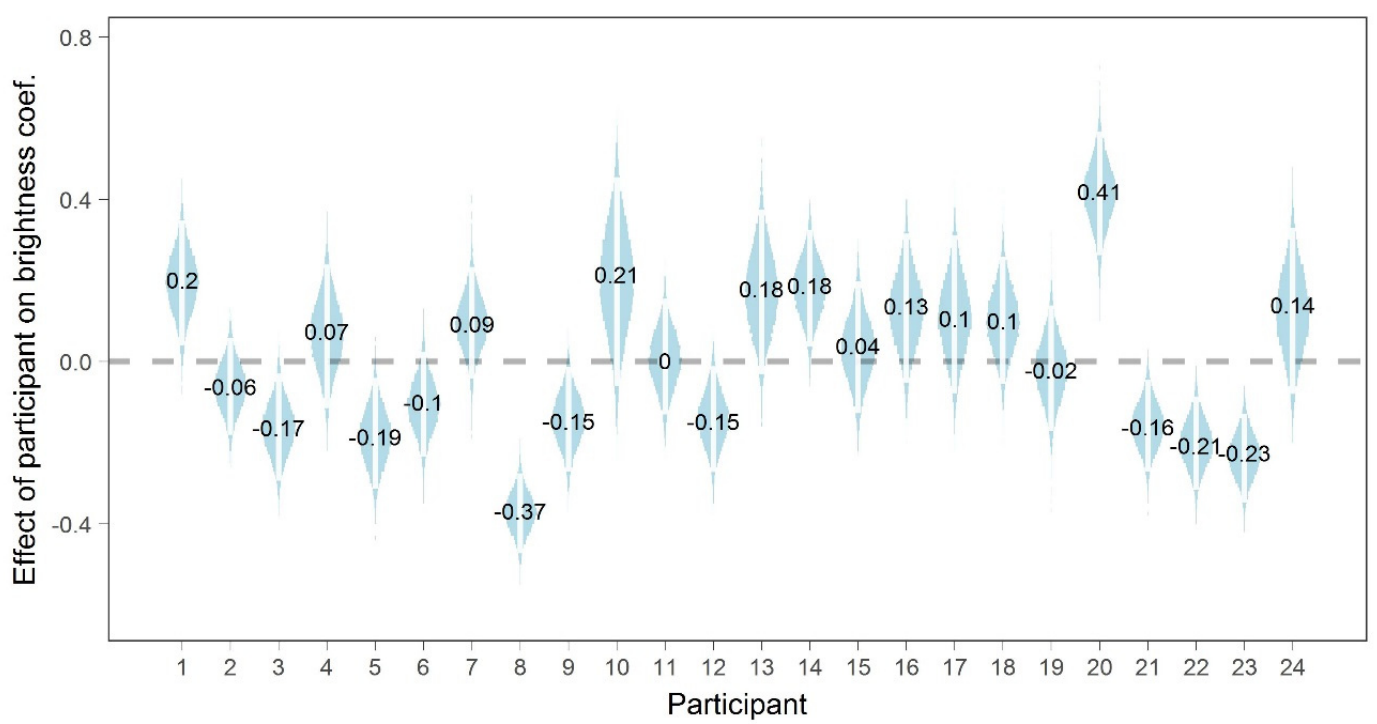

Figure 9.90 Differences in the slope of the brightness/luminance relationship between people 


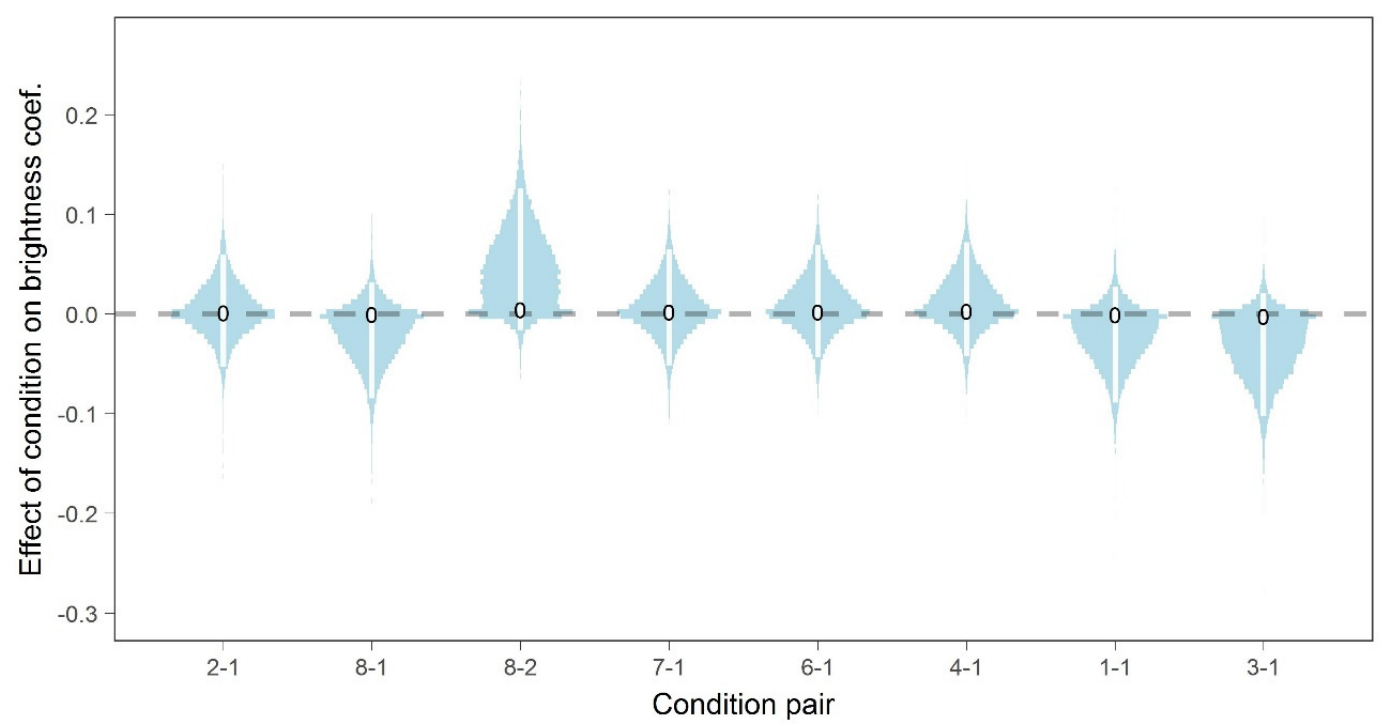

Figure 9.91 Differences in the slope of the brightness/luminance relationship between conditions 


\section{Appendix C Implications of simple models of bias control in brightness matching experiments}

The following chapter examines the mathematical logic of simple models of bias in brightness matching experiments. Its main purpose, in role of the context of the thesis, was to determine whether or not we should be completely counterbalancing in Experiment 2 (Answer: yes). It also provides the background and logic as to why we are modelling the biases the way we do in our analysis, as well as illustrating the limitations of counterbalancing and why simply averaging counterbalanced trials may not deal with all bias.

In summary, we extend previous discussions of bias in brightness matching experiments with the following key points:

- Counterbalancing is preferred to null condition trials because it is more robust. Null condition trials fail if bias is not a constant across different conditions. Counterbalancing is robust to this at least as long as a) the variance is between the pairs of conditions being compared rather than the individual conditions, or $b$ ) the bias is symmetrical. Even when it fails, it may still mitigate the bias far better than null trials.

- Counterbalancing is not perfect. For constant biases it fails under the conditions described above. For proportional biases, it will leave a residual bias dependent upon the size of the difference between the conditions.

○ "Symmetry" cannot be measured, and so if bias does vary between individual conditions it may present unavoidable source of uncertainty. The only way to avoid this would be a strong theoretical argument in favour of specific assumptions.

- The residual bias from proportional biases can be manually corrected for if one can measure the strength of the biases. Alternatively, rather than multiple manual corrections that do not account for the uncertainty in the estimates of the bias strength, it may be better to simply fit the whole model at once in a tool like Stan (Gelman et al., 2015). 
- We may add adjustment range bias as another possible bias that should be considered. Previous discussions of response contraction bias may also be consistent with proportional adjustment range bias. These biases may be confounded in many studies. Similarly, observations of conservative adjustment bias may just be the result of the residual bias left after counterbalancing proportional biases.

\section{C.1 Introduction}

It is recommended that all spatial brightness matching studies use counterbalancing and/or null condition trials to measure and remove the various biases that have been identified (Fotios et al., 2015, 2008). Counterbalancing in particular is favoured. Fotios et al. (2008, p. 221) argue that "Null condition data can be used to correct non-null condition data which did not employ sufficient counterbalancing, but this should be considered a last resort: the preferred approach is to employ counterbalancing and use null condition data as a back-up validation." However, this preference is not explained.

In a null-condition trial two otherwise identical conditions are compared. In theory people should set them to the same light level for equal brightness, because they are the same. Discrepancies from unity indicate the presence of unwanted bias. This is how many of the above biases were identified. In this way, bias can be measured and, if it is present, can be subtracted from one's results. Counterbalancing involves applying the bias equally to both conditions being compared, and then averaging them to remove the bias. For example, for position bias you would do one trial with the test condition on the left, then another with it on the right. With bias being applied to both conditions, averaging the two trials should cancel it out.

Counterbalancing, however, can create practical difficulties. Allowing the participant to be able to adjust either condition can make the experimental setup more complicated, as can the need to change both sides. In some cases - especially with light distribution presenting a given condition on both the left and right sides may require multiple versions of the condition to be created. Perhaps most importantly, needing to change both conditions in every trial rather than just one can significantly increase the time required for the experiment, and the burden on the participants.

The question that motivated this chapter is to what extent the recommendation for counterbalancing over null condition trials is justified. After-all, in theory, if null condition trials can be used to accurately measure the bias then they should also work perfectly fine as controls for that bias on their own — and would be easier. 
To answer this, we build upon previous discussions of the topic by examining the mathematical logic of the identified biases, and how the proposed means of bias control actually work. Looking at the issue of bias control in brightness matching experiments from this angle brought to light a number of important issues. In particular, that our theorised model of how the bias affects people's perception is critical, and that neither null condition trials nor counterbalancing can necessarily be assumed to eliminate bias. Both depend on certain assumptions to hold, and a number of plausible models of different biases can cause them to fail. In some cases these errors can be corrected for, in others, the bias cannot easily be dealt with. With some biases such as response contraction it is even possible that counterbalancing could induce the bias instead of removing it.

\section{C.2 Fundamentals: the basic brightness matching experiment}

To ground our discussion, we start with the basic model of a hypothetical unbiased brightness matching experiment (Figure 9.92).

Imagine that we are comparing two different "conditions" that have the same mean luminance (Figure 9.92a). These could be spaces with different light distributions as in this study, or perhaps spaces with different colours of light. However, due to the effect of this difference, a person viewing the two conditions will see them as having different brightnesses - they will "appear" to have different mean luminances. In this example, we assume the "Test" condition appears brighter than the "Reference" condition (Figure 9.92b).

Now, in the matching task, we have the participant adjust the light level of the Test condition so that it appears to them to have the same overall light level/brightness as the Reference condition (Figure 9.92c). Since (in this case) the Test appears brighter, this requires them to lower its light level. The result of this is that when the two conditions appear to have the same light level to the participant, the Test will physically be at a lower light level than the Reference (Figure 9.92d). 
What we measure (L)

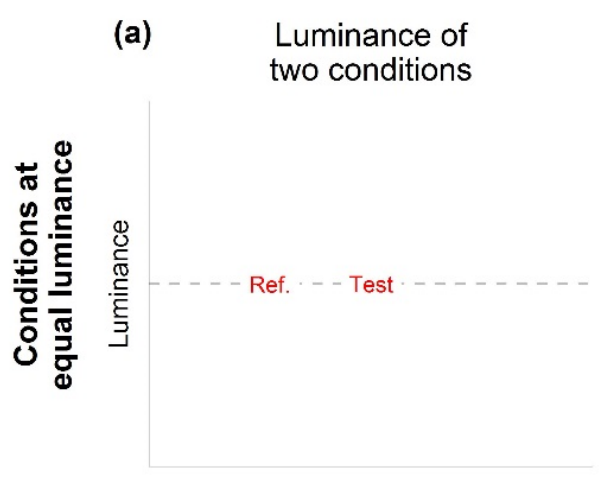

(d)

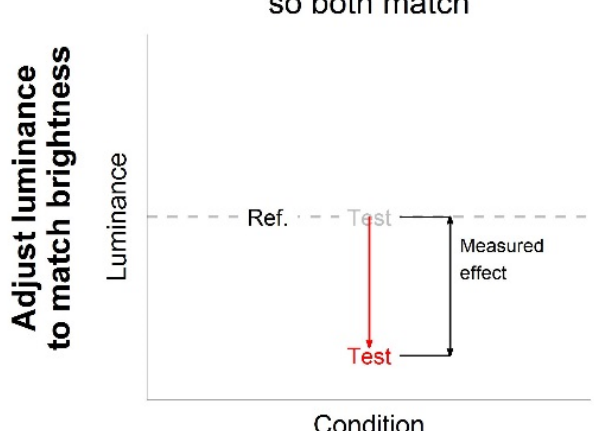

What the participant sees $\left(L^{\mathrm{ap}}\right)$

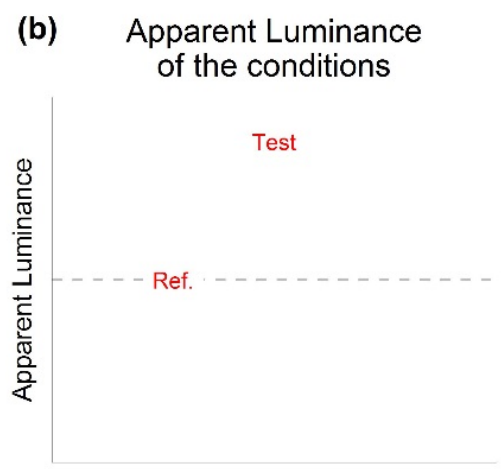

(c) Apparent adjustment

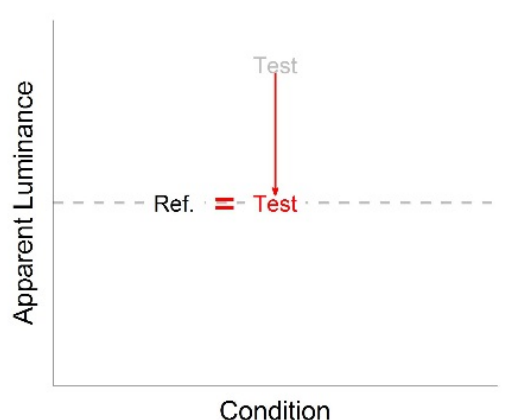

Figure 9.92 Illustration of how the basic brightness matching model works. The top panels show what the person may initially see. The bottom panels then show how they make a brightness match. If we compare two conditions that differ on some factor that affects their apparent brightness then we can measure the effect of this factor by looking at the difference in mean luminance when both have been adjusted to match in apparent luminance (brightness).

Mathematically then, an unbiased brightness match will be set to the reference light level minus the difference in apparent light level between conditions ${ }^{87}$ :

$$
\text { match }=r e f-\left(E f f_{\text {test }}-E f f_{\text {ref }}\right)
$$

Where $E f f$ is the effect of a condition (test or reference) on the apparent light level in the space, ref is the reference light level, and match is the resulting match light level set by the viewer for the test condition.

${ }^{87}$ Throughout this paper we will work in logarithmic units because it makes the math simpler. If we were to work in ratios, we would have to deal with the fact that a) they are asymmetric (a $50 \%$ decrease is equivalent to a $100 \%$ increase in the opposite direction), and b) we would have to use geometric means, all of which would make it much harder to follow. On a log scale, however, if the test condition appears to have 0.1 units more light then we know that to make a brightness match we should either a) set the test to be -0.1 units lower than the reference, or b) set the reference to be 0.1 units higher than the test. Similarly, effects can all simply be summed rather than multiplied. The same equation on the regular ratio scale would be match $=\frac{\text { ref }}{\frac{E f_{\text {test }}}{E f_{\text {ref }}}} *$ bias $_{\text {total }}$, which is rather less readable. 
The typical observed effect $\left(o b s_{\text {eff }}\right)$ in an experiment is measured as the difference between the match made and the reference light level:

$$
\begin{aligned}
o b s_{\text {eff }} & =\text { match }- \text { ref } \\
& =r e f-\left(E f f_{\text {test }}-E f f_{\text {ref }}\right)-r e f \\
& =-\left(E f f_{\text {test }}-E f f_{\text {ref }}\right)
\end{aligned}
$$

If the test condition appears brighter than the reference, then match $<$ ref, and the test will require less light than the reference condition for equal brightness. If the test condition is darker than the reference then the opposite is true, and it will require more light.

The introduction of bias changes the equation to:

$$
\text { match }=\text { ref }-\left(E f f_{\text {test }}-E f f_{\text {ref }}\right)+\text { bias }_{\text {total }}
$$

The result of this is that a match will be either under or overestimated depending on the direction of the bias, as illustrated by the example of position bias below (Figure 9.93).

\section{What we measure $(L) \quad$ What the participant sees $\left(L^{a p}\right)$}
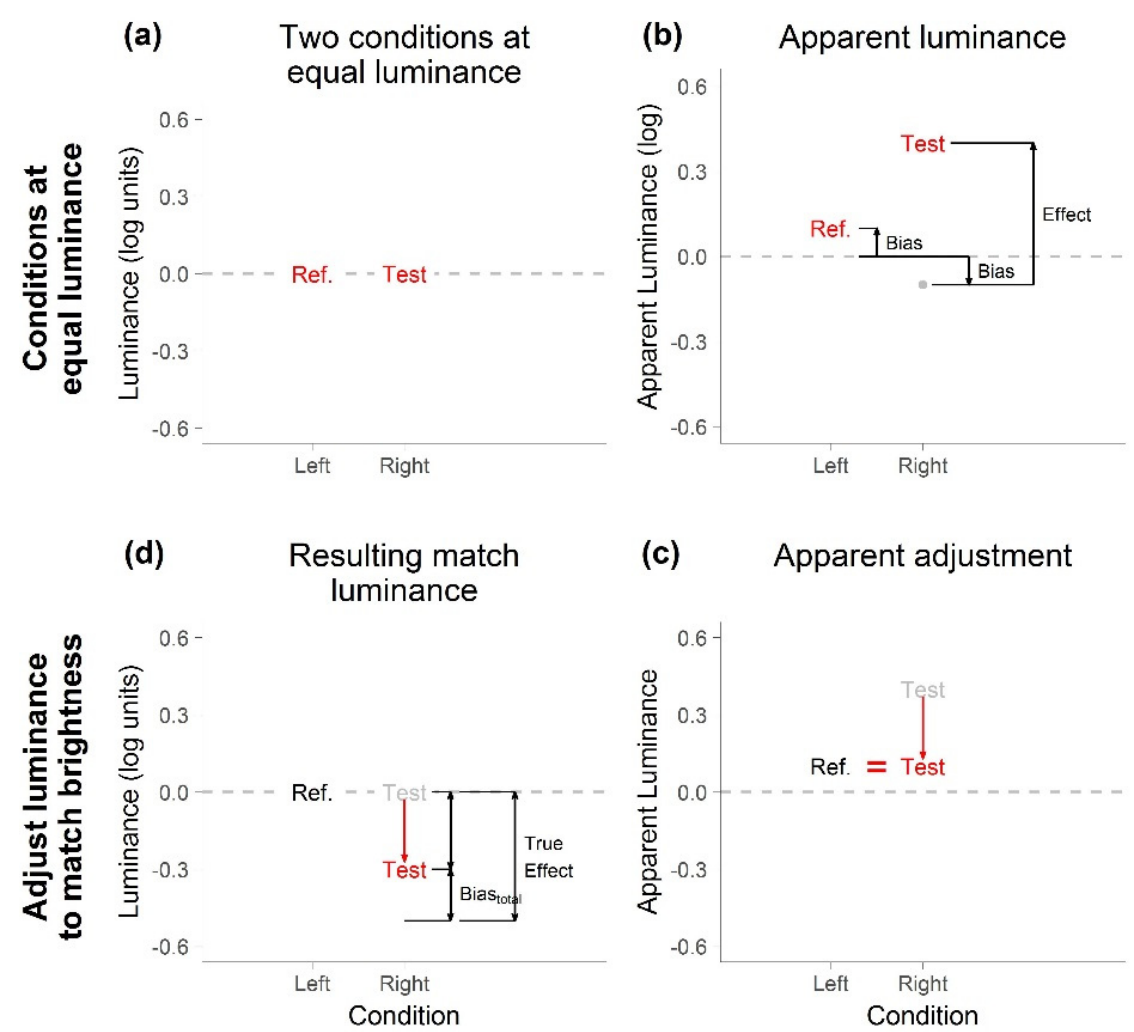

Figure 9.93 Illustration of how the basic position bias model works when we are comparing two different conditions. In this example, bias causes the reference condition on the left appears brighter and the test condition on the right to appear darker. The resulting difference that is measured is the sum of the true effect of the conditions and the total bias. 
To account for this bias, null condition trials or counterbalancing can be used. The idea behind null condition trials is straightforward. In a null condition trial both conditions are identical, hence the observed effect is simply equal to the bias:

$$
\begin{aligned}
o b s_{\text {eff }} & =r e f-\left(E f f_{\text {test }}-E f f_{\text {test }}\right)+\text { bias }_{\text {total }}-\text { ref } \\
& =\text { ref }-0+\text { bias }_{\text {total }}-r e f \\
& =\text { bias }_{\text {total }}
\end{aligned}
$$

In this way, the bias can be measured. By subtracting the null from the observed test result you should get the true unbiased difference between the test and reference conditions:

$$
\begin{aligned}
o b s_{\text {eff }}-o b s_{\text {null }} & =-\left(E f f_{\text {test }}-E f f_{\text {ref }}\right)+\text { bias }_{\text {total }}-\text { bias }_{\text {total }} \\
& =-\left(E f f_{\text {test }}-E f f_{\text {ref }}\right)
\end{aligned}
$$

This requires a critical assumption: that the bias in the null condition trial and the bias in the test are the same. If this is not true, if bias is not homogeneous across conditions, then a residual bias equal to the difference between the two biases will remain.

Counterbalancing is more involved. Somewhat simplistically, if you carry out two trials, swapping which condition you apply the bias to, then in one trial the observed effect will overestimated by the bias, and in the other it will be underestimated (Figure 9.94). 
What we measure (L)

(a)

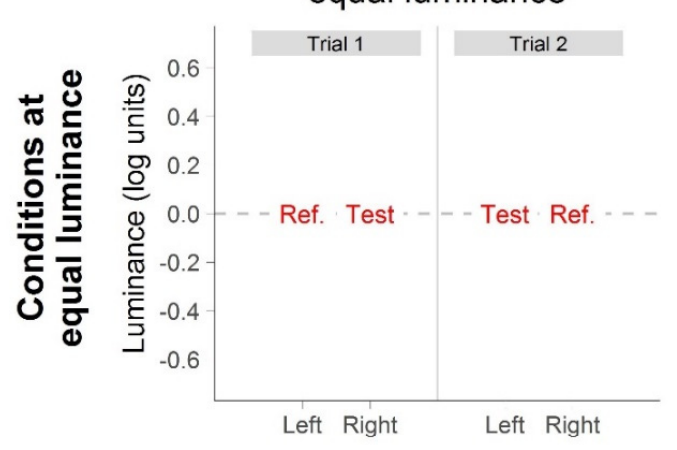

(d) Resulting match luminance

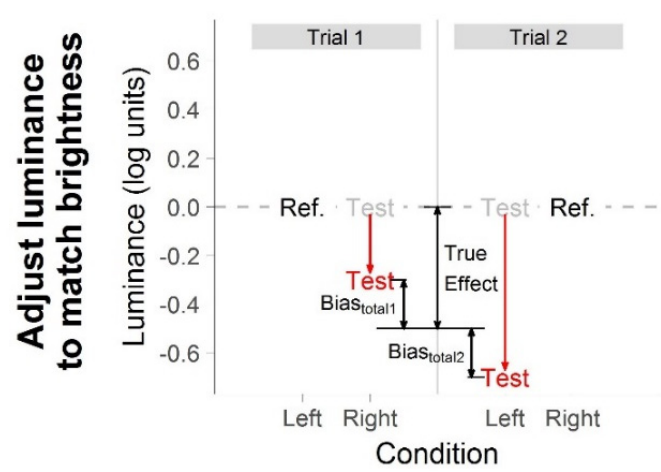

What the participant sees $\left(\mathrm{L}^{\mathrm{ap}}\right)$

(b) Apparent luminance

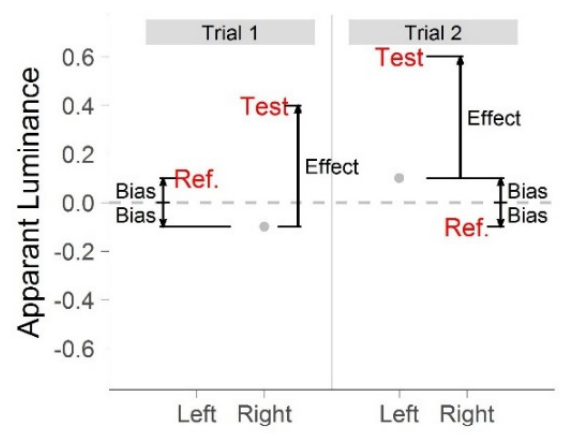

(c) Apparent adjustment

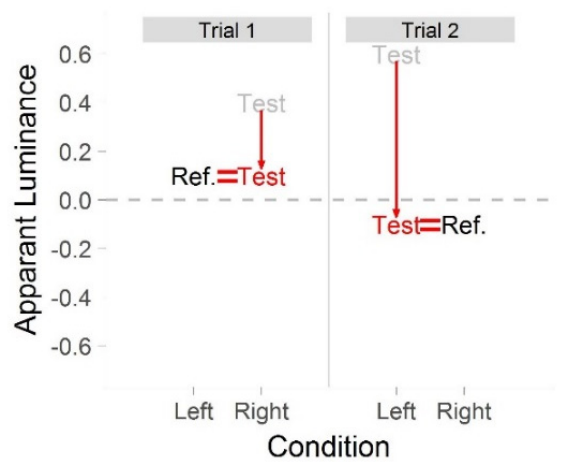

Figure 9.94 Illustration of the use of counterbalancing to address simple position bias in the comparison of two different conditions. Two trials must be carried out. In the first trial in this example, the Test condition is on the right. In the second, the positions are swapped and the Test is on the left. By averaging the results of the two trials, the true effect of the Test condition (relative to the Reference) can be extracted (d).

The degree of bias in a given match is described by the "total bias" (bias total). This is the aggregate effect of all the biases acting upon the conditions in the trial. At the most basic level, we will define this as:

$$
\text { bias }_{\text {total }}=\text { bias }_{\text {ref }}-\text { bias }_{\text {test }}
$$

If the reference condition has a bias making it appear brighter by 0.1 units, and the test condition has a bias making it appear darker by 0.1 units, then the resulting match will be biased by 0.2 units $(0.1--0.1=0.2)$.

In a simple counterbalanced experiment we have two trials, with the bias swapping around. To take our previous example, this means that in one trial the total bias would be 
$0.1--0.1=0.2$, and in the other it would be $-0.1-0.1=-0.2$. In other words, bias $_{\text {trial1 }}=-$ bias $_{\text {trial2 }}$.

Thus, averaging the two trials results in the bias cancelling itself out:

$$
\begin{aligned}
o b s_{\text {eff } 1} & =-\left(E f f_{\text {test }}-E f f_{\text {ref }}\right)+\text { bias }_{\text {total }} \\
o b s_{\text {eff } 2} & =-\left(E f f_{\text {test }}-E f f_{\text {ref }}\right)-\text { bias }_{\text {total }} \\
\frac{o b s_{e f f 1}+o b s_{e f f 2}}{2} & =\frac{-2\left(E f f_{\text {test }}-E f f_{\text {ref }}\right)+b i a s_{\text {total }}-b i a s_{\text {total }}}{2} \\
& =-\left(E f f_{\text {test }}-E f f_{\text {ref }}\right)
\end{aligned}
$$

From this we can also see that the difference between the two trials will be equal to twice the bias. Hence a counterbalanced experiment can be used to measure bias strength just like a null condition trial. The equation can also be looked at from the opposite direction - in a counterbalanced experiment you are counterbalancing which conditions are applied to which biases. Therefore, if you calculate the difference between conditions as, say, Left - Right instead of Test - Reference, then the effect of the conditions will cancel out and leave you with the strength of the bias.

At this point it may seem as though counterbalancing would have the same limitations as null condition trials - requiring the assumption that bias needs to be the same for both conditions. This is, however, not entirely true. To explain this, however, we must first discuss the different models of bias.

\section{C.3 Bias models}

How bias is calculated depends upon the model used. Different biases may be modelled in different ways. While this has not be explicitly addressed in previous work, we can identify two simple models that are implicit in the ways that the biases are discussed. These are the "constant" and "proportional" models.

\section{C.3.1 Constant models}

Constant biases consist of those biases that are the result of a binary factor (or are discussed as if they were the result of). These include positional bias, bias due to the simple application of dimming, and potentially start point bias. In side-by-side brightness matching the space can be either on the left or on the right. Its position is thus a binary factor. Similarly, a space could either be the one being dimmed or not, or start at a high light level or a low one.

The constant models are perhaps overly simplistic in some cases. Whether or not start point bias should be treated as a constant - that the bias is merely a function of whether or 
not it is high or low and that how high or how low does not matter is questionable. Such a model is, however, implicit in studies that simply report counterbalancing high and low start points without reference to the actual light levels. Even when the light levels are reported, if they are not evenly spaced around the match light levels then this could be said to similarly imply a belief that the exact magnitude of the initial light levels is not of particular importance, and that the counterbalancing will work regardless.

So, how does bias control work for constant biases?

As noted before, null condition trials have the limitation that they are only valid if we can assume that bias strength is homogeneous. If bias strength varies between conditions then we cannot assume that the bias measured in the null trial is the same as that in the other trials.

So, if we are worried about heterogeneity of bias, what can we do? Would counterbalancing be better? Intuitively, one might suspect not, on the grounds that counterbalancing involves applying the bias evenly to both conditions to cancel its effects out. Hence, if the bias was different for each condition then one might expect counterbalancing to also fail.

Fortunately, this is not the case. There are two reasons for this. The first is that there are multiple ways in which "bias being constant across conditions" could be interpreted in a brightness matching experiment:

1. Bias is a factor of the individual conditions. For example, a checkerboard condition might have a different level of positional bias than a simple uniform grey condition.

2. Bias is a factor of the comparison being made. For example, we could suggest that comparing two very different conditions is harder than comparing two identical conditions, and that peoples' responses are more affected by bias when they're more uncertain.

Both of these ways of thinking about the assumption would cause null condition trials to fail. However, only the first could be a problem in a counterbalanced experiment. If bias is a product of the pair of conditions being compared, then within any set of counterbalanced experiments for a pair of conditions the bias will the same. Trial 1 compares condition A against condition B. Trial 2 compares condition B against condition A. In both trials the pair of conditions is the same, and thus the bias would be the same (at least, if it works this way).

Even if bias varies as a function of the individual conditions it is not necessarily a problem. When we look at Figure 9.94, we can see that what actually matters is the total 
bias in each trial. As long as this is of equal magnitude and opposite sign, then the counterbalancing will work.

For example, let us consider position bias. Let us say that in trial 1 , the test condition is on the right, and in trial 2 it is on the left. Therefore:

$$
\begin{aligned}
& \text { bias }_{\text {total1 }}=\text { bias }_{\text {ref,left }}-\text { bias }_{\text {test,right }} \\
& \text { bias }_{\text {total2 }}=\text { bias }_{\text {ref,right }}-\text { bias }_{\text {test,left }}
\end{aligned}
$$

If bias is the same for both the reference and test conditions then bias $_{\text {test }}=$ bias $_{\text {ref }}$ and so we could simplify this to:

$$
\begin{gathered}
\text { bias }_{\text {total1 }}=\text { bias }_{\text {left }}-\text { bias }_{\text {right }} \\
\text { bias }_{\text {total2 }}=\text { bias }_{\text {right }}-\text { bias }_{\text {left }} \\
\therefore \text { bias }_{\text {total1 }}=- \text { bias }_{\text {total2 }}
\end{gathered}
$$

And so when the trials are averaged the biases will cancel out, as we know. But what about if the biases are different for each individual condition? If bias test, left $\neq$ bias $_{\text {ref, left }}$ ?

The answer is that counterbalancing will still work if we can assume that bias is symmetrical. By this, we mean that the bias between, for example, left and right is evenly distributed between the two conditions, such that bias left $=-$ bias $_{\text {right }}$. This is what we see in Figure 9.94.

If this is the case, then the equations become:

$$
\begin{gathered}
\text { bias }_{\text {total1 }}=\text { bias }_{\text {ref }}+\text { bias }_{\text {test }} \\
\text { bias }_{\text {total2 }}=- \text { bias }_{\text {ref }}-\text { bias }_{\text {test }} \\
\therefore \text { bias }_{\text {total1 }}=- \text { bias }_{\text {total2 }}
\end{gathered}
$$

And so again the total bias can cancel out between the trials.

However, symmetry is not something that can simply be assumed to be true. The studies that identify the existence of biases using null condition trials only tell us that there is a difference in apparent brightness between two experimental factors - for example that the condition on the left tends to require less light than the one on the right for equal brightness, or that the condition being adjusted is set to a lower level than the one set to a fixed light level. However, mathematically, we can describe this difference in many different ways. We could split the difference symmetrically between the conditions, but we could also put all the bias on the left or the right, or anywhere in between (Figure 9.95). 

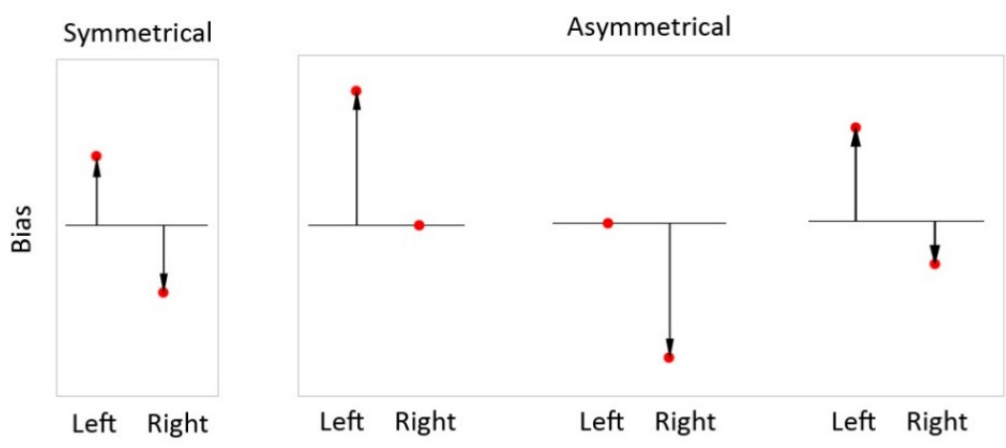

Figure 9.95 Illustration of the concept of symmetry. Observations of bias simple tell us that there is, for example, an apparent difference between the left and the right. However, the elements producing this difference can be broken down between the two sides in many different ways.

If the bias varies between the conditions and is asymmetrical then counterbalancing will fail to eliminate it. One will be left with a residual bias equal to the average of the biases in the trials:

$$
\frac{\text { bias }_{\text {total1 }}+\text { bias }_{\text {total2 }}}{2}=\frac{\text { bias }_{\text {ref,left }}-\text { bias }_{\text {test,right }}+\text { bias }_{\text {ref,right }}-\text { bias }_{\text {test,left }}}{2}
$$

As a note here: We have used the example of position bias here due to its simplicity, but bias due to the application of dimming (conservative adjustment) works the same way albeit with a twist required to the calculations. The critical element is that to counterbalance the application of dimming one needs to consistently calculate TestReference, however, the match in the second trial will be the opposite of this. If trial one had the test condition dimmed to match the reference:

$$
\text { match }_{1}=r e f-\left(E f f_{\text {test }}-E f f_{\text {ref }}\right)+\text { bias }_{\text {dim }}
$$

Then when we swap the conditions around and dim the reference, the match will be:

$$
\text { match }_{2}=\operatorname{ref}-\left(E f f_{\text {ref }}-E f f_{\text {test }}\right)+\text { bias }_{\text {dim }}
$$

Averaging these two directly would actually counterbalance the main effects, and instead tell us the bias. To counterbalance the bias, we instead need to consistently calculate our observed effects in terms of Test-Reference:

$$
\begin{aligned}
o b s_{\text {eff } 1} & =\text { match }_{1}-r e f=r e f-\left(E f f_{\text {test }}-E f f_{\text {ref }}\right)+b i a s_{\text {dim }}-r e f \\
& =-\left(E f f_{\text {test }}-E f f_{\text {ref }}\right)+\text { bias }_{\text {dim }} \\
o b s_{e f f 2} & =r e f-\text { match }_{1}=r e f-\left(r e f-\left(E f f_{\text {test }}-E f f_{\text {ref }}\right)+b i a s_{\text {dim }}\right) \\
& =\left(E f f_{\text {ref }}-E f f_{\text {test }}\right)-\text { bias }_{\text {dim }} \\
& =-\left(E f f_{\text {test }}-E f f_{\text {ref }}\right)-\text { bias }_{\text {dim }}
\end{aligned}
$$

Thus, when we add $o b s_{e f f 1}+o b s_{e f f 2}$ the bias will be eliminated leaving us with the effect of the conditions. 
These assumptions, and how they affect the success of bias control, are summarised below:

Table 9.7 Relationship of constant bias model assumptions to effectiveness of control methods

\begin{tabular}{|c|c|c|c|c|}
\hline \multicolumn{3}{|c|}{ Assumptions } & \multicolumn{2}{|c|}{ Control method } \\
\hline Homogeneous? & $\begin{array}{l}\text { Varies } \\
\text { between... }\end{array}$ & Symmetrical? & $\begin{array}{l}\text { Null condition } \\
\text { trial }\end{array}$ & Counterbalancing \\
\hline Yes & & & Works & Works \\
\hline \multirow[t]{4}{*}{ No } & \multirow{2}{*}{$\begin{array}{l}\text { Pairs of } \\
\text { conditions }\end{array}$} & Yes & Fails & Works \\
\hline & & No & Fails & Works \\
\hline & \multirow{2}{*}{$\begin{array}{l}\text { Individual } \\
\text { conditions }\end{array}$} & Yes & Fails & Works \\
\hline & & No & Fails & Fails \\
\hline
\end{tabular}

Even in the case of failure here though counterbalancing is still superior to the null condition trial. When the null condition trial fails, one will be left with a residual bias equal to the difference between the bias in the null condition trial and the bias in the test condition trial. With counterbalancing, however, it is reduced by a factor dependent upon the degree of asymmetry.

To quantify the effect of this, let us define a symmetry factor, $s$, that measures the proportion of the total bias that is on the left. Hence, if the total bias (for a given condition) is the difference between the bias on the left and the bias on the right, then the left bias is a fraction of this and the right bias is the remainder pointing in the opposite direction. Thus, for the test condition:

$$
\begin{aligned}
& \text { bias }_{\text {test }}=\text { bias }_{\text {test,left }}-\text { bias }_{\text {test,right }} \\
& \text { bias }_{\text {test,left }}=s \cdot \text { bias }_{\text {test }} \\
& \text { bias }_{\text {test,right }}=-(1-s) \text { bias }_{\text {test }}
\end{aligned}
$$

Using the symmetry factor $s$, we may substitute the left and right biases in our previous equations as follows:

$$
\begin{aligned}
\text { bias }_{\text {total1 }} & =s \cdot \text { bias }_{\text {ref }}--(1-s) \text { bias }_{\text {test }} \\
\text { bias }_{\text {total2 }} & =-(1-s) \text { bias }_{\text {ref }}-s \cdot \text { bias }_{\text {test }}
\end{aligned}
$$

Accordingly, we may calculate the residual bias as:

$$
\begin{aligned}
\text { bias }_{\text {residual }} & =\frac{s \cdot \text { bias }_{\text {ref }}-(1-s) \text { bias }_{\text {ref }}+(1-s) \text { bias }_{\text {test }}-s \cdot \text { bias }_{\text {test }}}{2} \\
& =(s-0.5) \text { bias }_{\text {ref }}+(0.5-s) \text { bias }_{\text {test }} \\
& =(s-0.5)\left(\text { bias }_{\text {ref }}-\text { bias }_{\text {test }}\right)
\end{aligned}
$$

In other words, if $100 \%$ of the bias is on one side then the residual bias will be half (10.5 ) of the difference between the bias of the test and reference conditions. If $75 \%$ of the bias is on one side, then the residual bias will be $25 \%(0.75-0.5)$ of the difference. Thus, it 
is mitigated compared to using a null condition trial, where the residual bias would be $100 \%$ of the difference. If the asymmetry is less than complete, with some on both sides/factors, then it is even further reduced. Because of this, counterbalancing is better than null condition trials for these biases even when it fails.

We may also note that position and dimming bias can be counterbalanced with a single pair of trials. To counterbalance, we just change both at the same time, for example comparing the test condition on the left and dimmed against it on the right and fixed:

$$
\begin{aligned}
& o b s_{\text {left,dim }}=\text { match }-r e f=-\left(E f f_{\text {test }}-E f f_{\text {ref }}\right)+b i a s_{\text {pos }}+b i a s_{\text {dim }} \\
& o b s_{\text {right,fix }}=\text { ref }- \text { match }=-\left(-\left(E f f_{\text {ref }}-E f f_{\text {test }}\right)+b i a s_{\text {pos }}+\text { bias }_{\text {dim }}\right) \\
& =-\left(E f f_{\text {test }}-E f f_{\text {ref }}\right)-\text { bias }_{\text {pos }}-\text { bias }_{\text {dim }} \\
& \frac{o b s_{\text {left }, \text { dim }}+o b s_{\text {right,fix }}}{2} \\
& =\frac{-2\left(E f f_{\text {test }}-E f f_{\text {ref }}\right)+\left(\text { bias }_{\text {pos }}-\text { bias }_{\text {pos }}+\text { bias }_{\text {dim }}-\text { bias }_{\text {dim }}\right)}{2} \\
& =-\left(E f f_{\text {test }}-E f f_{\text {ref }}\right)
\end{aligned}
$$

And if bias is asymmetrical and varies between conditions, the uncertainty is just the sum of the two residual biases:

$$
\left(s_{p}-0.5\right)\left(\text { bias }_{\text {pos,ref }}-\text { bias }_{\text {pos,test }}\right)+\left(s_{d}-0.5\right)\left(\text { bias }_{\text {dim,ref }}-\text { bias }_{\text {dim,test }}\right)
$$

Start point bias (if modelled as a constant bias) works slightly differently. The reason for this is that the basic counterbalancing does not involve swapping the two conditions at all. Instead, one simply counterbalances a high and a low start point for the test condition. Because of this, the bias in a high and a low trial would be:

$$
\begin{aligned}
& \text { bias }_{\text {trial1 }}=\text { bias }_{\text {test,high }} \\
& \text { bias }_{\text {trial2 }}=\text { bias }_{\text {test }, \text { low }}
\end{aligned}
$$

In this light, the question of the assumption of homogeneity is a moot point as the bias is being counterbalanced against a counterpart with exactly the same condition(s). The asymmetry equation may be used to calculate the residual bias as follows:

$$
\text { bias }_{\text {residual }}=(s-0.5) \text { bias }_{\text {total }}
$$

Where $s$ is the symmetry factor, and bias $s_{\text {total }}=$ bias $_{\text {high }}-$ bias $_{\text {low }}$.

In the worst case, if all the bias was on (say) the high trial and there was no bias on the low, then the residual bias would be $50 \%$ of the total. Given that this is the same as if the bias was symmetrical and we employed no bias control, this is not a particularly desirable result. Note here that the equation is a function of the total bias of the test condition, rather than the difference between the bias of each condition. Thus, constant start point 
bias, controlled in this way, would suffer more from failures of symmetry than the others - unless the difference between the biases of each condition is larger than the bias of the individual condition. It is, however, possible to improve upon this by counterbalancing the application of dimming as well, as shown below.

As before, the observed effect of a matching experiment may be calculated as the difference between the match light level and the reference light level:

$$
o b s_{\text {eff }}=\text { match }- \text { ref }=-\left(E f f_{\text {test }}-E f f_{\text {ref }}\right)+\text { bias }
$$

Following this, we have the observed effects of our first two trials adjusting the test to match the reference:

$$
\begin{aligned}
& o b s_{\text {eff } 1}=-\left(E f f_{\text {test }}-E f f_{\text {ref }}\right)+b i a s_{\text {test }, \text { high }} \\
& o b s_{\text {eff } 2}=-\left(E f f_{\text {test }}-E f f_{\text {ref }}\right)+b i a s_{\text {test }, \text { low }}
\end{aligned}
$$

And the observations of our next two trials adjusting the reference to match the test:

$$
\begin{aligned}
& o b s_{\text {eff } 3}=-\left(E f f_{\text {ref }}-E f f_{\text {test }}\right)+b i a s_{\text {ref }, \text { high }} \\
& o b s_{\text {eff } 4}=-\left(E f f_{\text {ref }}-E f f_{\text {test }}\right)+b i a s_{\text {ref }, \text { low }}
\end{aligned}
$$

As before, to average these trials where we have counterbalanced dimming, we need all of them to be consistent - either test - reference or reference - test. To consistently measure test-reference, we must reverse the second set of trials instead calculating ref - match, or just $-1 * o b s_{\text {eff }}$ :

$$
\begin{aligned}
& -o b s_{\text {eff } 3}=-\left(E f f_{\text {test }}-E f f_{\text {ref }}\right)-\text { bias }_{\text {ref,high }} \\
& -o b s_{\text {eff } 4}=-\left(E f f_{\text {test }}-E f f_{\text {ref }}\right)-\text { bias }_{\text {ref,low }}
\end{aligned}
$$

Thus, if we average all of these together then the biases can counterbalance:

$$
\text { bias }_{\text {average }}=\frac{\text { bias }_{\text {test }, \text { high }}+\text { bias }_{\text {test }, \text { low }}-\text { bias }_{\text {ref,high }}-\text { bias }_{\text {ref,low }}}{4}
$$

If bias is homogeneous $\left(\right.$ bias $_{\text {test }}=$ bias $_{\text {ref }}$ ) then the sum becomes:

$$
\text { bias }_{\text {high }}+\text { bias }_{\text {low }}-\text { bias }_{\text {high }}-\text { bias }_{\text {low }}=0
$$

While if the bias is symmetrical $\left(\right.$ bias $_{\text {low }}=-$ bias $\left._{\text {high }}\right)$ then it becomes:

$$
\text { bias }_{\text {test }}-\text { bias }_{\text {test }}-\text { bias }_{\text {ref }}+\text { bias }_{\text {ref }}=0
$$

So, just as with position and conservative adjustment bias, as long as either symmetry or homogeneity are true then counterbalancing will eliminate the bias. If neither are true, then the residual bias will be a function of the symmetry factor as shown before. However, we are now counterbalancing four ways, hence:

$$
\text { bias }_{\text {test }}=\text { bias }_{\text {test,high }}-\text { bias }_{\text {test,low }}
$$




$$
\begin{gathered}
\text { bias }_{\text {test }, \text { high }}=s \cdot \text { bias }_{\text {test }} \\
\text { bias }_{\text {test } \text { low }}=-(1-s) \text { bias }_{\text {test }} \\
\text { bias }_{\text {residual }}= \\
=\frac{s \cdot \text { bias }_{\text {test }}-(1-s) \text { bias }_{\text {test }}-s \cdot \text { bias }_{\text {ref }}+(1-s) \text { bias }_{\text {ref }}}{4} \\
=\frac{(2 s-1)}{4} \text { bias }_{\text {test }}+\frac{(-2 s+1)}{4} \text { bias }_{\text {ref }} \\
=\frac{2 s-1}{4}\left(\text { bias }_{\text {test }}-\text { bias }_{\text {ref }}\right)=\frac{s-0.5}{2}\left(\text { bias }_{\text {test }}-\text { bias }_{\text {ref }}\right)
\end{gathered}
$$

And so the residual bias here would, at most, only be a quarter of the difference between the biases of each condition. This is half the potential error compared to the position or conservative adjustment bias, which could only counterbalance two ways.

To conclude then: when dealing with constant biases, counterbalancing is preferable to the use of null condition trials due to its greater robustness to model assumptions. In the circumstances where it does fail, it will still mitigate the residual bias by a factor of at least two. It should be noted, however, that the symmetry factor, $s$, is unmeasurable. We can only measure, for example, the difference between left and right — not the individual left and right components. Hence, assuming potential asymmetry in, say, position bias means that we have an uncertainty of \pm 0.5 bias $_{\text {pos }}$ in our estimates of the condition effects.

\section{C.3.2 Proportional models}

Proportional biases are ones where the bias is related to a continuous scale. Examples are start point bias, response contraction bias due to varying references, and adjustment range bias.

For simplicity, we will assume that bias is a linear function of distance (probably on a log scale given how brightness perception works). Thus:

$$
\text { bias }=k * d
$$

Where $k$ is the strength of the bias (generally the slope of bias against the relevant light level), and $d$ is the measure of the distance between the current condition and whatever is serving as the bias point. This depends on the bias in question.

The idea of response contraction bias, for instance, is that people are not just matching to the current reference, but also to some idea of the average reference they are seen (Fotios et al., 2008). Thus, when they are asked to make matches their results tend to be drawn towards the average reference condition. So, for example, when the reference light level is higher than the average they will tend to set matches lower than they otherwise would. If they are matching to a reference that is set to a lower light level than average, their matches will tend to be biased upwards. 
This bias can thus be modelled as being proportional to the distance between the current reference and the average reference. Hence:

$$
d=r e f_{i}-\overline{r e f}
$$

Where $r e f_{i}$ is the light level of the current reference, and $\overline{r e f}$ is the mean reference level. However, people do not see physical light level - they see apparent light level ("brightness"). If we account for this, then we also need to account for the effects of the conditions on the apparent light level of the references. Thus:

$$
d=r e f_{\text {apparent }}-\overline{\text { refapparent }}
$$

Where the apparent reference light level would be the sum of the reference light level plus the effect of condition, and the average reference would be the average reference light level plus the weighted average effect of condition:

$$
d_{i}=\left(r e f_{i}+E f f_{r e f_{i}}\right)-\left(\overline{r e f}+\frac{\sum\left(w \cdot E f f_{r e f}\right)}{\sum w}\right)
$$

where $\overline{r e f}$ is the mean reference light level, $E f f_{\text {ref }}$ is the effect of the reference condition(s), $i$ is the index of the current condition, and $w$ is a vector of weightings based off the frequency that each condition is used as a reference.

Adjustment range bias is not one of the biases suggested in Fotios et al. (2008). However, it is a known issue in other forms of adjustment task (Logadóttir et al., 2011), and also works as an alternative explanation for the bias observed in a number of the studies discussed in Fotios et al. (2008) (discussed in C.4.1). For adjustment range bias, wherein responses are biased by the adjustment range allowed, we could model it as being proportional to the distance between the intended match and the centre of the adjustment range. Hence:

$$
d=r e f-\left(E f f_{\text {test }}-E f f_{\text {ref }}\right)-\text { centre }_{\text {test }}
$$

For bias where responses are drawn to (or possibly away from, see (Houser et al., 2003)) the initial light level of the test condition, the relevant distance would be the distance between the intended match point and the initial light level. Thus:

$$
d=r e f-\left(E f f_{\text {test }}-E f f_{\text {ref }}\right)-\text { start }
$$

The challenge of all of these proportional models is that counterbalancing does not work perfectly on them. Because the bias size is dependent on the effect of the conditions being examined, it will inherently vary between the different conditions. In the case of start point bias, the match point will almost always be closer to one start point than the other, and so the bias will be asymmetrical. This, as before, causes problems for the use of null 
condition trials and counterbalancing. We discuss each of the different biases in turn, as they work slightly differently.

\section{C.3.2.1 Response contraction due to varying reference}

The simple model of response contraction bias that is implicit in published discussions of the topic (Fotios et al., 2008) assumes two things:

a) Bias is proportional to the distance between the actual reference level and the average reference level

b) Bias is based off the physical light level

Under these assumptions the bias can, theoretically, be controlled by either null condition trials or counterbalancing the application of dimming. This is because the physical light level is a known quantity that can be held constant across conditions. Hence, for example, we can dim condition A with B at a specific reference light level, and then swap them around to counterbalance them. Assuming that it is purely a function of the reference light level, the bias would then be the same for each condition and would counterbalance out.

However, assuming it is a function of the physical light level is highly questionable. Given that the idea of the model is based on the reference people are matching to, it would seem most logical to base the model around what people are actually matching. People are not matching the physical light level. They are matching the apparent light level. This distinction has significant implications for the use of counterbalancing. Apparent brightness in spatial brightness matching studies is a product of not just the light level, but also the independent variable - generally the light distribution or spectrum. Thus, if you use multiple conditions as references (such as by counterbalancing the application of dimming) you are effectively using multiple light levels as references. Hence, you would be expected to have response contraction even your references are always set to the same light level. Moreover, this bias will not be eliminated by the use of counterbalancing.

To explain: assume we carry out two trials comparing condition A and condition B, counterbalancing the application of dimming. The equations are:

$$
\begin{aligned}
& \text { match }_{1: A B}=r e f_{B}-\left(E f f_{A}-E f f_{B}\right)+k_{r e f}\left(r e f_{B}+E f f_{B}-\overline{r e f}\right) \\
& \text { match }_{2: B A}=r e f_{A}+\left(E f f_{A}-E f f_{B}\right)+k_{r e f}\left(r e f_{A}+E f f_{A}-\overline{r e f}\right)
\end{aligned}
$$

Where $k_{r e f}$ is the bias strength factor (essentially the slope of bias against reference light level).

If we average the results, then we get: 


$$
\begin{aligned}
\text { mean } & =-\left(E f f_{A}-E f f_{B}\right)+\frac{k_{r e f}\left(r e f_{B}+E f f_{B}-\overline{r e f}\right)-k_{r e f}\left(r e f_{A}+E f f_{A}-\overline{r e f}\right)}{2} \\
& =-\left(E f f_{A}-E f f_{B}\right)+\frac{k_{r e f}\left(r e f_{B}+E f f_{B}\right)-k_{r e f}\left(r e f_{A}+E f f_{A}\right)}{2} \\
& =-\left(E f f_{A}-E f f_{B}\right)+\frac{k_{r e f}}{2}\left(r e f_{B}-r e f_{A}\right)-\frac{k_{r e f}}{2}\left(E f f_{A}-E f f_{B}\right) \\
& =-\left(1+\frac{k_{r e f}}{2}\right)\left(E f f_{A}-E f f_{B}\right)+\frac{k_{r e f}}{2}\left(r e f_{B}-r e f_{A}\right)
\end{aligned}
$$

When the reference light level is the same in both trials (as we would expect it to be) this becomes simply:

$$
\text { mean }=-\left(1+\frac{k_{r e f}}{2}\right)\left(E f f_{A}-E f f_{B}\right)
$$

In other words, the size of the residual bias after counterbalancing is proportional to the difference between the two conditions. Note that in a null condition trial there would thus be no residual bias, because the difference is 0 . This could lull one into thinking that counterbalancing dimming will remove the bias. However, it generally will not. The null trial is just a special case. Also note that the mean $\operatorname{ref}(\overline{r e f})$ fortunately cancels out, saving one from having to calculate it.

That being said, it must be acknowledged that this residual bias will generally be small. For example, at a difference between conditions of $\sim-30 \%$, the largest estimate of the error (using the data of Tiller and Veitch (1995)) would only be $-3 \%$. Not necessarily meaningless, but also likely imperceptible and ultimately small relative to the size of the main effect. Note though that it is possible to estimate $k_{\text {ref }}$ if trials are run at multiple light levels by simply calculating the change in observed effect for a pair of conditions.

Mathematically (just focusing on response contraction bias), the observed effect is:

$$
o b s_{\text {eff }}=-\left(E f f_{\text {test }}-E f f_{\text {ref }}\right)+k_{\text {ref }}\left(r e f_{i}-\overline{r e f}\right)
$$

If we look at the difference between two matches using the same conditions made at different light levels, the effects (Eff test and Eff ref $)$ will be the same, and so will cancel out:

$$
\begin{gathered}
o b s_{e f f 1}-o b s_{e f f 2}=k_{r e f}\left(r e f_{1}-r e f_{2}\right) \\
\therefore k_{r e f}=\frac{o b s_{e f f 1}-o b s_{e f f 2}}{r e f_{1}-r e f_{2}}
\end{gathered}
$$

Confounding from constant biases in this process is not a concern, as it would cancel itself out in the calculation of $k_{r f}$ just like the condition effects did. Once $k_{r e f}$ has been estimated, it can be used to correct the observed effect. 


\section{C.3.2.2 Adjustment range bias}

The adjustment range bias differs from the previous in that it is based entirely upon the adjustment range of the test condition, and so is more like start point bias. One consequence of this is that - if it exists - it would always have an effect. It would be present even if only a single condition was assessed at a single light level. The only time it would not would be if the intended match setting was also at the centre of the adjustment range.

The model here describes the bias as one in which matches tend towards the centre of the adjustment range. Thus, the bias is proportional to the distance between the desired match setting and the centre of the adjustment range.

For a single match:

$$
\text { match }=r e f-\left(E f f_{\text {test }}-E f f_{\text {ref }}\right)+k_{\text {rng }}\left(r e f-\left(E f f_{\text {test }}-E f f_{\text {ref }}\right)-\text { centre }_{\text {test }}\right)
$$

Where $k_{\text {rng }}$ is the bias strength factor, and centre test $_{\text {is }}$ the centre of the adjustment range.

The strength of the bias $\left(k_{\mathrm{rng}}\right)$ can be estimated in much the same way as with reference response contraction bias. If we match the same conditions at multiple adjustment ranges we can then measure the strength of the bias by simply looking at the difference in observed effect between the two conditions as a function of a difference in their adjustment ranges:

$$
k_{r n g}=\frac{o b s_{e f f 1}-o b s_{e f f 2}}{\text { centre }_{1}-\text { centre }_{2}}
$$

And correct the observed results using the equation ${ }^{88}$ :

$$
E f f_{\text {test }}-E f f_{\text {ref }}=\frac{o b s_{\text {test }}-k_{r n g}\left(r e f-\text { centre }_{\text {test }}\right)}{-\left(1+k_{\text {rng }}\right)}
$$

If bias strength varies significantly between conditions then it would be necessary to measure the bias strength factor for each condition by carrying every test out at multiple adjustment ranges.

As before, it should be noted that counterbalancing the application of dimming will not work to eliminate the bias. Using the same example as before, comparing A vs. B and counterbalancing application of dimming:

$$
\begin{aligned}
& \operatorname{match}_{1: A B}=\operatorname{ref}_{B}-\left(E f f_{A}-E f f_{B}\right)+k_{r n g}\left(r e f_{B}-\operatorname{diff}_{A B}-\text { centre }_{A}\right) \\
& \text { match }_{2: B A}=\operatorname{ref}_{A}+\left(E f f_{A}-E f f_{B}\right)+k_{r n g}\left(r e f_{A}+\operatorname{diff}_{A B}-\text { centre }_{B}\right)
\end{aligned}
$$

Assuming the reference level is constant $\left(r e f_{A}=r e f_{B}\right)$, then averaging gives us:

$$
\begin{aligned}
& { }^{88}{ }^{o b s_{\text {test }}}=\text { match }- \text { ref }=-\left(E f f_{\text {test }}-E f f_{\text {ref }}\right)+k\left(r e f-\left(E f f_{\text {test }}-E f f_{\text {ref }}\right)-\text { centr }_{\text {test }}\right) \\
& =-(1+k)\left(E f f_{\text {test }}-E f f_{\text {ref }}\right)+k\left(\text { ref }- \text { centre }_{\text {test }}\right)
\end{aligned}
$$


mean

$$
\begin{aligned}
& =-\left(E f f_{A}-E f f_{B}\right) \\
& +\frac{k_{r n g}\left(r e f_{B}-\left(E f f_{A}-E f f_{B}\right)-\text { centre }_{A}\right)-k_{r n g}\left(r e f_{A}+\left(E f f_{A}-E f f_{B}\right)-\text { centr }_{B}\right)}{2} \\
& \quad=-\left(1+k_{r n g}\right)\left(E f f_{A}-E f f_{B}\right)+\frac{k_{r n g}}{2}\left(\text { centre }_{B}-\text { centre }_{A}\right)
\end{aligned}
$$

If the adjustment range is the same for both conditions then the average will simplify down to $-\left(1+k_{r n g}\right)\left(E f f_{A}-E f f_{B}\right)$. When studying light distribution, however, this may often be difficult compared to keeping the reference light level or start points consistent within a set of trials.

\section{C.3.2.3 Start point bias}

Bias associated with the initial light level - the start point - can perhaps be more logically modelled as a function of the distance. Looking at the broader literature on anchoring effects, they are known to be affected by the extremity of the anchor, with more distant anchors producing larger effects (Furnham and Boo, 2011). At the same time, it must be noted that the literature also suggests that this effect is limited, and that once the anchor is outside the range of plausible answers the bias can asymptote (Furnham and Boo, 2011), or even shrink (Wegener et al., 2010). Thus, while the idea that start point bias would be dependent on how far the start point is from the match setting makes a lot of sense, it is also plausible that it could plateau once the distance is large enough.

If we assume a linear function, then using a null condition trial to account for the bias would leave a residual bias of:

$$
\text { bias }_{\text {residual }}=\text { bias }_{\text {main }}-\text { bias }_{\text {null }}=-k_{\text {start }}\left(E f f_{\text {test }}-E f f_{\text {ref }}\right)
$$

Given that the bias of the null condition trial is:

$$
\text { bias }_{\text {null }}=k_{\text {start }}(\text { ref }- \text { start })
$$

And the bias of the main trial is:

$$
\text { bias }_{\text {main }}=k_{\text {start }}\left(\text { ref }-\left(E f f_{\text {test }}-E f f_{\text {ref }}\right)-\text { start }\right)
$$

This will be an under or over-estimate depending on the alignment of start point with the effect of the test condition. If the test condition appears brighter, then it will be set to a lower light level than the reference. If the start point is low, then the bias will cause it to be set even lower ${ }^{89}$, and so it will be overestimated. If the start point is high, the opposite will occur.

\footnotetext{
${ }^{89}$ Assuming the bias is that matches are drawn towards the start point
} 
Generally, however, the start point is counterbalanced. In such a situation, a residual bias will be left if the start points are not evenly spaced around the "correct" match setting. Each bias will equal the distance from the start point to the intended match setting ${ }^{90}$ :

$$
\begin{aligned}
& \text { bias }_{\text {low }}=k_{\text {start }}\left(\text { ref }-\left(E f f_{\text {test }}-E f f_{\text {ref }}\right)-\operatorname{start}_{\text {low }}\right) \\
& \text { bias }_{\text {high }}=k_{\text {start }}\left(\text { ref }-\left(E f f_{\text {test }}-E f f_{\text {ref }}\right)-\operatorname{start}_{\text {high }}\right)
\end{aligned}
$$

Averaging the observed effects will give us:

mean

$$
\begin{aligned}
& =-\left(E f f_{\text {test }}-E f f_{\text {ref }}\right) \\
& +\frac{k_{\text {start }}\left(r e f-\left(E f f_{\text {test }}-E f f_{\text {ref }}\right)-\text { start }_{\text {high }}\right)+k_{\text {start }}\left(r e f-\left(E f f_{\text {test }}-E f f_{\text {ref }}\right)-\text { start }_{\text {low }}\right)}{2} \\
& =-\left(E f f_{\text {test }}-E f f_{\text {ref }}\right)+k_{\text {start }}\left(r e f-\left(E f f_{\text {test }}-E f f_{\text {ref }}\right)\right) \\
& \quad-\frac{k_{\text {start }}\left(\text { start }_{\text {high }}+\text { start }_{\text {low }}\right)}{2} \\
& =-\left(1+k_{\text {start }}\right)\left(E f f_{\text {test }}-E f f_{\text {ref }}\right)+k_{\text {start }}\left(r e f-\frac{\text { start }_{\text {high }}+\text { start }_{\text {low }}}{2}\right)
\end{aligned}
$$

The residual bias is both a function of the difference between conditions, and the difference between the reference light level and the centre of the start points. Note here that in this formulation, if the bias is one where the matches are drawn towards the start points, then $k_{\text {start }}$ will be negative. $\left(1+k_{\text {start }}\right)\left(E f f_{\text {test }}-E f f_{\text {ref }}\right)$ will thus be an underestimate of the difference between conditions.

As before, we if we know $k_{\text {start }}$ we can use it to correct our observed effects. If we calculate the difference between the high and low matches, the intended match $\left(\right.$ ref $\left.-\left(E f f_{\text {test }}-E f f_{\text {ref }}\right)\right)$ is the same for both, and so will cancel out (as would position and conservative adjustment bias). The difference between the high and low matches is thus equal to $k_{\text {start }}$ times the difference between the start points:

$$
\begin{aligned}
\text { match }_{\text {high }}-\text { match }_{\text {low }} & =\text { bias }_{\text {high }}-\text { bias }_{\text {low }} \\
& =k_{\text {start }}\left(\text { start }_{\text {low }}-\text { start }_{\text {high }}\right)
\end{aligned}
$$

\footnotetext{
${ }^{90} \mathrm{~A}$ reasonable question that may occur here is why, after making such a big deal out of the importance of looking at matters through the lens of apparent luminance with regards to response contraction bias we are not doing the same here. The answer is that it gives exactly the same results. Let us instead model $\mathrm{d}$ as equal to the difference between the apparent luminance of the match point (i.e. the reference) and the apparent luminance of the start point. The equation becomes:

$$
\begin{aligned}
& d=\left(r e f+E f f_{\text {ref }}\right)-\left(\text { start }+E f f_{\text {test }}\right) \\
& =r e f+\left(E f f_{\text {ref }}-E f f_{\text {test }}\right)-\text { start } \\
& =r e f-\left(E f f_{\text {test }}-E f f_{\text {ref }}\right)-\text { start }
\end{aligned}
$$

In other words, exactly the same as what we have. The same reasoning may be applied to the calculation of adjustment range bias.
} 
And so we can calculate $k_{\text {start }}$ for any pair of conditions:

$$
k_{\text {start }}=\frac{\text { match }_{\text {high }}-\text { match }_{\text {low }}}{\text { start }_{\text {low }}-\text { start }_{\text {high }}}
$$

The counterbalanced results can then be corrected for by the equation:

$$
-\left(E f f_{\text {test }}-E f f_{\text {ref }}\right)=\frac{\text { mean }_{\text {obs }}}{1+k_{\text {start }}}-k_{\text {start }}\left(\text { ref }-\frac{\text { start }_{\text {high }}+\text { start }_{\text {low }}}{2}\right)
$$

If the application of dimming has also been counterbalanced, then we would be averaging:

$$
\begin{aligned}
\text { mean }_{1}= & \text { match }- \text { ref } \\
= & -\left(1+k_{\text {start }}\right)\left(E f f_{\text {test }}-E f f_{\text {ref }}\right) \\
& \quad+k_{\text {start }}\left(\text { ref }-\frac{\text { start }_{\text {high }, \text { test }}+\text { start }_{\text {low }, \text { test }}}{2}\right) \\
\text { mean }_{2}= & \text { ref }- \text { match } \\
= & -\left(1+k_{\text {start }}\right)\left(E f f_{\text {test }}-E f f_{\text {ref }}\right) \\
& \quad-k_{\text {start }}\left(\text { ref }-\frac{\text { start }_{\text {high }, \text { ref }}+\text { start }_{\text {low }, \text { ref }}}{2}\right)
\end{aligned}
$$

Which reduces to just:

$$
o b s_{e f f}=-(1+k)\left(E f f_{\text {test }}-E f f_{\text {ref }}\right)
$$

if the start points are consistent for both conditions. Again, the residual bias is purely a function of the difference between the conditions in this case. If the start points vary, there will be a component derived from the average difference:

$$
\begin{aligned}
\text { obs }_{\text {eff }}=-(1 & +k)\left(E f f_{\text {test }}-E f f_{\text {ref }}\right) \\
& +\frac{k\left(\text { start }_{\text {high,ref }}-\text { start }_{\text {high,test }}+\text { start }_{\text {low }, \text { ref }}-\text { start }_{\text {low }, \text { test }}\right)}{2}
\end{aligned}
$$

\section{C.4 Discussion}

As shown, the success of bias control methods is dependent on the model assumptions made. We should thus ask whether or not our existing knowledge can tell us anything about assumptions such as homogeneity and symmetry. Additionally, it is worth examining the evidence for biases such as Adjustment Range bias, which has not previously been raised as a problem. 


\section{C.4.1 Adjustment range bias and confounding}

A significant issue with the proportional bias models is that they can easily be confounded with each other ${ }^{91}$. Perhaps the simplest example here is Tiller and Veitch (1995), whose data provide perhaps the clearest evidence in Fotios et al. (2008) for response contraction bias. They carried out their experiment at three different reference light levels: 300, 500, and 700 lux. Their results showed a clear trend in the bias, with matches being overestimated at the lowest light level, and progressively underestimated at the higher ones. The obvious explanation was thus a response contraction bias as a function of the reference level. However, these results are just as consistent - in fact even more so - with adjustment range or proportional start point bias. In the study, the adjustment range ran from 150-800 lux. Start points were counterbalanced, and were either the minimum or maximum of the adjustment range. If the matches were biased towards the centre of the adjustment range, then we would expect exactly the same pattern - a high bias at the lowest reference level, and progressive low biases at the higher ones. The same is also true for start point bias. If it is a linear function of distance then, as discussed above, the residual bias after counterbalancing is dependent on the difference between the reference light level and the centre of the start points - which in this case is the same as the centre of the adjustment range.

With these biases, the biases should be centred around their respective anchors. This is the centre of the adjustment range for the adjustment range and in this case start point bias, and the average reference level for response contraction. If we plot the biases though then we see that it does not really fit response contraction bias very well — it "should" be centred around the middle reference, but it isn't. However, it is almost perfectly centred around the centre of the adjustment range. Thus, it would suggest that some combination of start point and adjustment range bias may be a better explanation for these results.

\footnotetext{
${ }^{91}$ In fact, one of the points that prompted the consideration of potential adjustment range bias were the some of the additional references Fotios et al. used in their discussion of response contraction bias. They referred to the studies of Teller et al. (2003) as supporting evidence for similar effects in other research. Teller et al., however, was a bias from the test stimulus range — not the reference.
} 


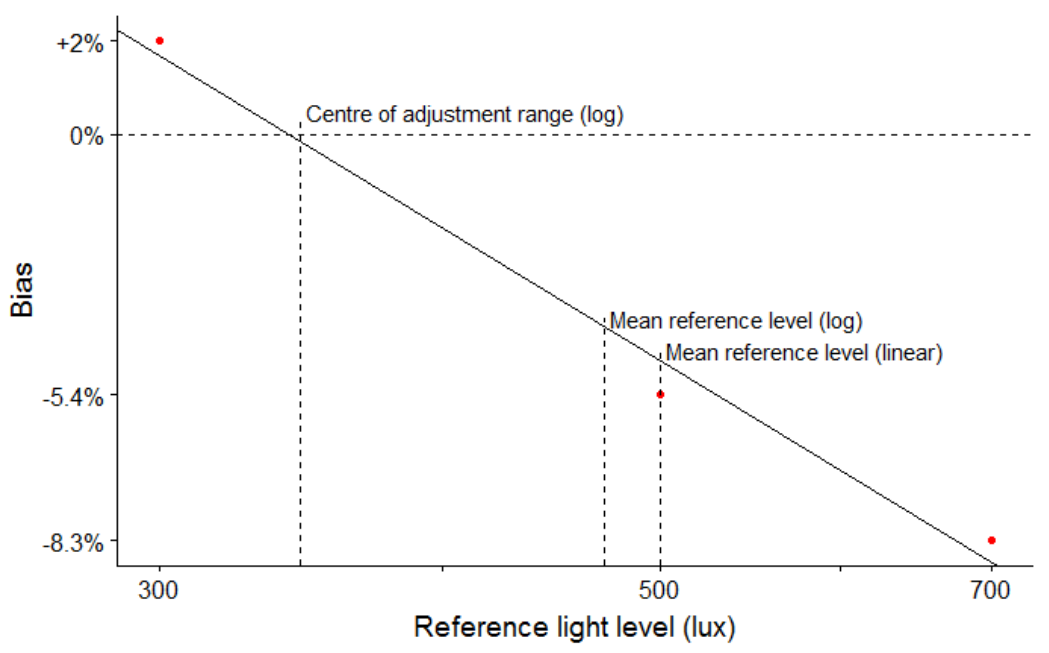

Figure 9.96 Reported Response Contraction Bias in Tiller and Veitch (1995). Response contraction bias should be centred around the average reference light level of $\sim 500$ lux. Instead, it appears to be centred around the centre of the adjustment range, as calculated on the log scale.

If we examine the other brightness matching studies discussed by Fotios et al. (2008), the same potential for confounding can be seen. Fotios and Cheal (2007b) provided evidence for response contraction bias, though unfortunately did not clearly describe either their adjustment range or their start points (describing them only as "clearly higher or lower than the reference"). In Fotios and Cheal (2007a), however, they identified their start points as "approximately two S-classes above or below the reference". For their different reference levels, this would translate to:

Table 9.8 Estimated start points in Fotios and Cheal (2007a, 2007b)

\begin{tabular}{|l|l|l|l|}
\hline Reference (lux) & Lower & Higher & Mean of log lux \\
\hline 15 & 7.5 & 33 & 15.7 \\
\hline 10 & 5 & 22 & 10.5 \\
\hline 7.5 & 3 & 15 & 6.7 \\
\hline 5 & 2 & 10 & 4.5 \\
\hline 2 & 1 & 5 & 2.2 \\
\hline 1.4 & 0.6 & 3 & 1.3 \\
\hline
\end{tabular}

This would suggest that at the very least matches were able to be made within this range. If we take 0.6-33 lux as a rough indication of the range, then the average on a log scale would be -4.4 lux. This is consistent with the overall pattern of "matches are higher at the lowest reference, lower at the highest reference". It does predict that the largest difference should be for the highest condition, and not the lowest, which is not what was observed. However, as the differences are on the order of 1-2 percentage points, this is within the level of statistical error we would expect. 
Such confounding can also provide an alternative explanation for the observations of apparent bias associated with the application of dimming — what Fotios et al. (2008) described as conservative adjustment bias. If we look at the studies providing evidence for dimming bias, we see that while it is not always clear exactly what their ranges are, the general indication is that the centre of the ranges, when calculated on the log scale, would be lower than the reference light level (often substantially so). On these grounds then, it would be consistent with our proportional models for adjustment range or start point bias for the dimmed condition to be set lower than it "should" be ${ }^{92}$. The appearance of a bias due to the application of dimming may thus just be a reflection of our proportional biases, and not exist as a constant.

Table 9.9 Summary of the adjustment range, start points, and reference light levels in studies providing evidence for bias associated with the application of dimming ${ }^{93}$.

\begin{tabular}{|c|c|}
\hline $\begin{array}{l}\text { Fotios and Levermore } \\
\text { (1995) }\end{array}$ & $\begin{array}{l}\text { - } \quad \text { Start points/range not reported } \\
\text { - } \quad \text { Figures indicate range of at least } 25-800 \text { lux for a log } \\
\text { mean } \sim 140 \text { lux. Null trials had a } 70 \% \text { filter applied, } \\
\text { which would reduce this to } \sim 98 \text { lux. } \\
\text { - } \quad \text { Reference }-200 \text { and } 400 \text { lux (reading off graphs) }\end{array}$ \\
\hline Fotios and Gado (2005) & $\begin{array}{l}\text { - Range: 3-340 lux (Veriwide lamps), 5-540 lux (warm } \\
\text { white). Log mean is }-32 \text { and }-52 \text { lux respectively. } \\
\text { - } \quad \text { Reference: } 320 \text { lux }\end{array}$ \\
\hline Houser et al. (2003) & $\begin{array}{l}\text { - Range not reported - note that in this study light } \\
\text { level was adjusted by experimenters according to } \\
\text { participant instructions. } \\
\text { - Start points } 150 / 450 \text { lux. Log mean } \sim 260 \text { lux } \\
\text { - } \quad \text { Reference: } 300 \text { lux }\end{array}$ \\
\hline
\end{tabular}

If this explanation of "conservative adjustment bias" is correct, then this would make it easier to more efficiently account for bias, as it would be unnecessary to try to control it by counterbalancing dimming. High powered research to provide a strong answer to this question could thus be of value.

\footnotetext{
${ }^{92}$ Houser et al. (2003) is somewhat anomalous as a study. Their reported bias was that the dimmed condition was set to a higher level than it should be - the opposite of the other studies, and the opposite of what we would predict from our model. Note however that not only was this bias observed in the opposite direction from what other studies suggest, but so was position and start bias. Drawing strong conclusions from it is thus difficult, and the study stands out as an outlier.

${ }^{93}$ Several other studies were discussed in Fotios (2001) as a demonstration of how a failure to control for this potential bias made their results untrustworthy. In those studies, application of dimming was confounded with the main study variables and often position, meaning they cannot tell us how large the dimming bias is on its own, and so we will not discuss them here.
} 


\section{C.4.2 Linearity}

The implementations of proportional models discussed here assumed simple linear functions. Such assumptions are common in research (see linear regression), and it makes the mathematics of the effects relatively straightforward. However, while it serves as a useful starting point for examining the implications of proportional biases, it should be noted that we cannot necessarily assume that the relationships are linear. For example, as discussed with the different possible models of start bias, it is quite plausible that the actual relationship there is one where the bias increases with distance up to a point, and then plateaus. A more accurate description may thus be some form of S-shaped function. Whether or not going to such lengths would be necessary, or whether or not we might be able to reasonably approximate such a function with either a linear function (for the nonplateau areas) or a constant (for the plateau areas) is an open question. Until more research is carried out to explore the relationships here in detail, what we can say is limited.

\section{C.4.3 Homogeneity}

Evidence for or against variation in bias strength between conditions is weak - which is unsurprising given the likely small size of any such differences. Estimates do vary significantly between studies, this is true. For dimming (measured as dimming/fixed) we have rough estimates of $-9 \%$ (Fotios and Gado, 2005), -5.5\% (Fotios, 2001), -3\% (Fotios and Cheal, 2011), and $<+1 \%$ (Houser et al., 2003). For position (left/right) we have $-13 \%$ (Thornton and Chen, 1978), -3.5\% (Boyce, 1977), -2\% (Fotios and Gado, 2005), -1\% (Fotios and Cheal, 2007a), and $+5 \%$ (Houser et al., 2003). This provides some indication that variation in bias could potentially be an issue, but it must be noted that:

a) We would expect a degree of statistical variation just from chance

b) Studies varied significantly in both stimulus and methodology

c) When constructing spaces, especially complex full scale ones, it is impossible to ensure they are completely identical (Fotios et al., 2008)

d) Small differences in sensor placement between conditions could readily produce differences of a few percent

e) Similarly, there is the potential for subtle differences in light colour — in our experiments, despite using the same lamps and setup on both sides, there was nevertheless an small unexplained difference in the colour of the light between the left and the right, equivalent to $-2 \%$ difference in light level according to the Rea et al. (2015) model.

Due to the relatively small size of the biases, even small errors can significantly shift our estimates of them. This makes it hard to be confident in the precise estimates, and difficult to compare different conditions and studies. 
These caveats aside, there are a few studies that provide us the opportunity to examine variation within them - though none specifically carried out any statistical analysis of variation in bias. Some show no indication of variation: position bias varied by only $\sim 1 \%$ in the null trials of Fotios and Cheal (2007a); dimming bias varies only by $\sim 1 \%$ in Fotios and Cheal (2011). The different null trials of Boyce (1977) show more variation, varying by up to $5 \%$, but this is within the uncertainty of that experiment (Figure 9.97). The null trials of Fotios and Cheal (2011) show even more variation (Figure 9.98), but the sample size was low ( $\mathrm{n}=6$ on the left trials, $\mathrm{n}=4$ on the right).

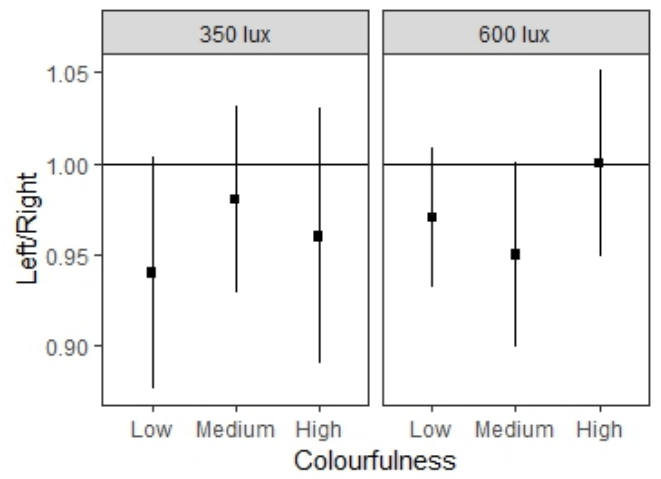

Figure 9.97 Position bias in null trials of Boyce (1977). Error bars are 95\% confidence intervals.

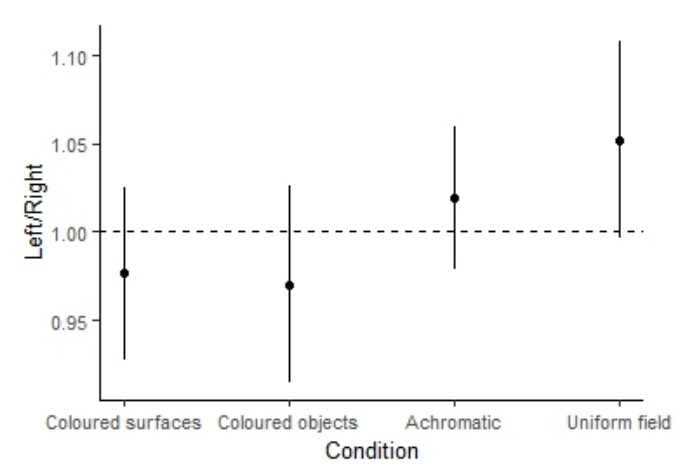

Figure 9.98 Position bias in null trials of Fotios and Cheal (2011). Error bars are 95\% confidence intervals.

For start point bias, there is a lack of useful data for within-study comparisons outside of our experiments. Comparisons between different studies show the possibility of variation in slope: the data of Houser et al. (2003) as described in Fotios et al. (2008) suggest a slope of $\sim 0.076$, while the data of Fotios and Cheal (2007a) suggest little bias, with a slope - 0.016. Similarly for response contraction bias the data of Tiller and Veitch (1995) suggests a slope of $\sim-0.12$, while the data of Fotios and Cheal (2007b) suggests a slope of $\sim-0.04$ for the null trials, and --0.08 for the main experiment. This comparison could, however, be confounded by simultaneous adjustment range and start point bias, making it difficult to draw strong conclusions. Our results, however, do suggest that there may be statistical variation in bias between conditions - though it appears to be less than variation between people (B.8, D.5).

Overall, there is reason to be concerned about the potential for heterogeneity of bias, and so counterbalancing may be preferred over null condition trials. Whether or not we should conceive of the bias as varying as a function of the pair of conditions, or as a function of the individual conditions, however, is unknown. 


\section{C.4.4 Symmetry}

The question of the assumption of symmetry is a complex one, and depends on the specific bias. For start point bias, the high and the low bias are quite distinct, and if we are assuming a constant model rather than a proportional one there is no inherent reason to consider one to be dependent on the other. It is thus entirely plausible that they may not be exactly the same magnitude.

For position and dimming bias, the question depends on our concept of the bias, and what we might consider the "neutral unbiased point". For position bias, the effect described by Fotios et al. is along the lines of "a tendency to act as if the left condition were brighter than the right". The problem is that all we have is the difference between left and right, and we could potentially describe the cause of this in a number of different ways. We could say it is because being on the left makes a space appear brighter, or because being on the right makes a space appear darker, ascribing all the bias to one or the other of the sides. We could also say that both happen at the same time to produce a more symmetrical effect. Is there any way to make a convincing case for one option?

An argument for symmetry would be that there seems to be little reason to imagine that one side or the other is seen in an "unbiased" way, and that it is just the other side that is perceived "incorrectly". Similarly, from the perspective of what we are trying to predict, we are generally not trying to predict the appearance of a "space on the left" or a "space on the right". The typical scenario we would want to predict would be a person within a space neither on the left nor the right. This would seem to align more with a point somewhere between our test spaces instead of picking a side. We could, however, also argue that this whole argument is incoherent, and that the bias is fundamentally a product of the difference between left and right, and the idea of applying part of the bias to one side or the other is fundamentally nonsensical. In this framing then, symmetry would be irrelevant, counterbalancing would work perfectly, and the idea of adding the part of the bias affecting the reference condition side to the apparent brightness of the reference condition (in the calculation of response contraction bias) would be nonsense ${ }^{94}$.

Dimming bias has similar issues. If we conceive of the bias as a tendency to act as if a space one is adjusting appears brighter than one that is fixed, then the same question how

\footnotetext{
${ }^{94}$ It could also be pointed out that there is possibly no real practical concern here. The fraction of the bias in the response contraction bias calculation may resist counterbalancing, true, but it would also be tiny. Plausible values for $\mathrm{k}_{\mathrm{ref}}$ are somewhere around -0.06. If dimming and position bias were around 10\% (a higher end estimate), then $\frac{k_{r e f}}{2}\left(\right.$ bias $_{\text {pos }}+$ bias $\left._{\text {dim }}\right)$ would be expected to be a fraction of a percent. True, if we ascribed all the observed bias in Tiller and Veitch (1995) to response contraction, then the estimate of $\mathrm{k}_{\mathrm{ref}}$ there would be --0.12 , but that is more likely to be a combination of multiple biases, and other estimates are much smaller.
} 
we distribute the bias arises. In this case, however, there are more possibilities in terms of what one might consider a reasonable baseline. "Bias" is, in effect, a function of whether or not the results are diverging from the "truth" we want to measure. If we were to argue that the typical "real world" condition we are interested in is a space with a fixed light level, then from that perspective a space "unbiased by dimming" would be one with a fixed light level. On the other hand, we might argue that people can often control the lighting in real spaces, and so the space with dimming applied might be a more appropriate reference point. Or we could say that both occur in the real world, and that the simplest thing to do is to just split the difference and imagine a point somewhere in the middle, assuming symmetry. Again, there is not an obviously correct answer here.

An alternative framing could be that the bias is just one where people have a tendency to err on the side of being too low when asked to make a brightness match. In this case, the bias would effectively all be applied to the dimmed condition, making it asymmetrical (though as this would assume zero bias on the fixed reference condition, there would be no dimming bias component in the response contraction bias calculation, which keeps things simple).

Overall, the symmetry question is problematic. It has ramifications for our ability to control certain biases, but at the same time actually measuring it is impossible, and on a theoretical level there is no obvious "right" answer. Indeed, a reasonable case can be made that the concept does not even make sense. Moreover, it only matters if bias varies between individual conditions, and the practical effect of it is only significant if there are large differences in bias strength between conditions.

\section{C.5 Practical significance of errors}

Finally, an important question to ask is how important failures to adequately control for bias are. Does it matter if one fails to account for possible asymmetry? If one fails to account for proportionality?

Overall, the largest single potential error here (Table 9.10) would be just using a null trial instead of counterbalancing - if there are large differences in bias between conditions and the position/dimming biases stack then error of $\sim 10 \%$ is plausible. This also has a simple solution that is recommended: counterbalance or measure the error via contrasts as discussed previously. That being said, if counterbalancing is not used we may note that the potential error is still limited. We could still readily believe in studies presenting evidence of large effects of $+20 \%$ or more (depending on their statistical error of course), even if we should treat their point estimates as being significantly more uncertain. 
Looking to the more complex questions, arguably the next largest potential error comes from asymmetry + individual variation in bias, producing an uncertainty of maybe $\sim \pm 3$ $5 \%$. This also happens to be unavoidable if it exists, and it is quite possible that it does not.

Failure to account for proportional biases has moderate significance (at least as long as one is counterbalancing the application of dimming). Individually, the residual from each bias after counterbalancing is likely to be minor relative to the size of the main effects, however they could readily stack up and produce a combined effect that is noticeable. The most significant one to worry about here could be adjustment range bias, as different conditions can often have significantly different ranges, adding more potential bias. Response contraction bias induced by counterbalancing, however, is probably not a major concern on its own (likely less than 1\% for most studies). 
Table 9.10 Summary of various bias control "failures" and their - rough - potential for error. Error estimates should be taken as highly approximate. We discuss the following possible "failures" under the assumption that the issue they are examining "exists" and that it has not been accounted for. In practice, of course, one of the complications is that we cannot be sure that many of these assumptions hold.

\begin{tabular}{|c|c|c|}
\hline Failure & Potential error & Notes \\
\hline $\begin{array}{l}\text { Just use null trial to measure } \\
\text { position/dimming bias. Fail to } \\
\text { account for heterogeneity }\end{array}$ & $<10 \% \ldots$ or $0 \%$ & $\begin{array}{l}\text { Residual bias equal to the difference in bias between the null trial and } \\
\text { the other trials. Differences could be as large as } 5 \% \text {, though probably } \\
\text { less. Adding both biases then means error could be as high as } 10 \% \text {, or } \\
\text { they could cancel out. }\end{array}$ \\
\hline $\begin{array}{l}\text { Counterbalance, but there is } \\
\text { asymmetry and individual } \\
\text { heterogeneity }\end{array}$ & $\pm 3-5 \%$ & $\begin{array}{l}\text { Asymmetry is impossible to measure, and all we can say is that it should } \\
\text { be between } 0-1 \text {. Residual bias is } \pm 0.5 \text { (bias }_{\text {ref }}-\text { bias }_{\text {test }} \text { ). With } \\
\text { differences up to } 5 \% \text { as before, this means up to } \pm 2.5 \% \text { for each bias, } \\
\text { and probably less. }\end{array}$ \\
\hline $\begin{array}{l}\text { Fail to account for } \\
\text { proportionality in start bias }\end{array}$ & $\begin{array}{l}\text { Difference component (if } \\
\text { dimming not counterbalanced): } \\
\sim 3 \% \text { if reference is twice the } \\
\text { average start point. } \\
\text { Relative reduction in effect: } \\
\text { If test is twice as bright as } \\
\text { condition then } \sim 3 \% \text {, if difference } \\
\text { is } \sim 10 \% \text { then } 0.4 \% . .\end{array}$ & $\begin{array}{l}\text { The residual bias of the proportional biases has two components - a } \\
\text { relative reduction in the main effect }\left(-\left(1+k_{\text {start }}\right)\left(E f f_{\text {test }}-\right.\right. \\
\left.\left.E f f_{\text {ref }}\right)\right) \text {, and a component based off differences in, in this case, the } \\
\text { start points }\left(k_{\text {start }}\left(\text { ref }-\frac{\text { start }_{\text {high }}+\text { start }_{\text {low }}}{2}\right)\right) . \mathrm{K}_{\text {start }} \text { probably around }{ }_{-} \\
0.03--0.06 \text {. } \\
\text { Size of biases thus depends on the size of the above differences. }\end{array}$ \\
\hline $\begin{array}{l}\text { Fail to account for adjustment } \\
\text { range bias }\end{array}$ & $\begin{array}{l}\text { Difference component: } \\
\sim 3 \% \text { if reference is twice the } \\
\text { centre of the adjustment range, } \\
\text { more if it is larger } \\
\text { Relative reduction in effect: } \\
\text { If test is twice as bright as } \\
\text { condition then } \sim 3 \%, \text { if difference } \\
\text { is } \sim 10 \% \text { then } \sim 0.4 \% \ldots\end{array}$ & $\begin{array}{l}\text { Effects largely work the same way as start bias above, though the } \\
\text { difference component may not necessarily be eliminated by } \\
\text { counterbalancing dimming (since the conditions may differ in their } \\
\text { adjustment ranges) } \\
\text { Can easily stack with the start bias above. }\end{array}$ \\
\hline
\end{tabular}




\begin{tabular}{|l|l|l|}
\hline Failure & Potential error & Notes \\
\hline $\begin{array}{l}\text { Fail to account for } \\
\text { counterbalancing induced } \\
\text { response contraction bias }\end{array}$ & $\begin{array}{l}\text { Relative reduction in effect: } \\
\text { If test is twice as bright as } \\
\text { condition then } \sim 1.5 \%, \text { if } \\
\text { difference is } \sim 10 \% \text { then } 0.2 \% \ldots\end{array}$ & $\begin{array}{l}\text { Difference component here generally not relevant as reference will be } \\
\text { held constant between counterbalanced conditions. } \\
\text { Effect is halved by counterbalancing dimming, so is probably smaller } \\
\text { than the other proportional biases. Largest estimate of } k_{\text {ref }}=0.12 \text { (using } \\
\text { Tiller and Veitch, 1995), but this is probably the result of multiple } \\
\text { proportional biases working together. }\end{array}$ \\
& \begin{tabular}{l} 
Can easily stack with the other proportional biases. \\
\hline
\end{tabular} \\
\hline
\end{tabular}





\section{C.6 Postscript: All-possible-pairs design as a means of bias control}

On a side note, readers may note the similarities between the adjustment range bias discussed here, and the stimulus range and stimulus frequency biases identified in Fotios and Houser (2013) for forced-choice discrimination experiments. In that paper, they proposed using an all-possible-pairs design to control these biases (though in CIE 212:2014 it was noted that the proposal needed validation). This was proposed for a very different method than the brightness matching approach used in this thesis, and indeed Fotios and Houser (2013) suggested that using other methods like brightness matching instead of forced-choice discrimination could also be a way of avoiding these biases while not having to deal with the logistical costs of all-possible-pairs. More recently, however, Fotios (pers. Comm) has suggested that those recommendations were incorrect, and that an all-possiblepairs design should be used in brightness matching experiments as well. This raises two questions:

1. Could the biases identified for forced-choice discrimination tasks apply to brightness matching tasks as well?

2. Would an all-possible-pairs design work to control these biases?

To begin: what are stimulus range and stimulus frequency biases? And how does a forced-choice discrimination task differ from the brightness matching we have been focused on?

In a forced-choice discrimination task, participants are shown two conditions or stimuli, and asked to compare them. For example, they may be asked to say which one is brightest. This choice may be "forced" in that they are not allowed to say the conditions are the same, or that they do not know - they have to say that one is brighter or darker. If this is done over a range of light levels, it is possible to compute the probability that a given condition will be seen as brighter than the other as a function of the difference in their light levels. The 50\% mark, where both conditions are equally likely to be said to be "brighter", is the Point of Subjective Equality (PSE), and is equivalent to the match point in a brightness matching task.

Stimulus Range and Stimulus Frequency biases refer to two biases that may affect how people make the "brighter" or "darker" judgements. In the Stimulus Range bias, when a test condition is shown at a range of different light levels then participants may base their judgements (at least in part) on where the test condition falls in the range - if it is relatively high they assume it is probably brighter, if relatively low then darker. In the Stimulus Frequency bias participants act as if, for example, the test condition is equally as likely to be "brighter" than the reference as it is to be "darker", and try to evenly distribute 
their responses accordingly. Of course, unless the PSE is at the centre of the stimulus range this will not be the case, and so by behaving as such their responses may be biased. These are illustrated in Figure 9.99 on the following page.

Within the context of the experiments discussed by Fotios and Houser (2013), the result of both of these biases is the same - the PSE is biased towards the centre of the stimulus range. Functionally, they are just two different explanations for the same observation in the two experiments Fotios and Houser discuss as evidence. The effect is also the same as the hypothetical "adjustment range bias" we raised earlier in the context of matching. Theoretically, the idea that participants in a matching experiment may, when uncertain, base their inferences about whether or not the test condition is brighter or darker than the reference off where the current light level falls in the adjustment range they are exploring is also plausible. If one was uncertain about whether or not the test was darker than the reference, but did know that it was currently set to a relatively low light level then guessing that it is more likely to be darker than brighter would not be unreasonable. Correspondingly, one might err on the side of caution and adjust the light level up a bit, thus shifting the match point towards the centre of the adjustment range. Thus, it would seem reasonable to suggest that a bias along these lines could potentially affect matching experiments as well.

So, if we are worried about such a bias is all-possible-pairs a solution? ${ }^{95}$

In an all-possible-pairs design, one compares all stimulus conditions to all other conditions (Figure 9.100). The problem with this is that when you have a large number of conditions, the number of possible comparisons can rapidly explode and become impractical - which is why Fotios and Houser (2013) noted that it may be better to use alternative methods in such cases.

\footnotetext{
${ }^{95}$ A sidenote: Fotios and Cheal (2008) also suggested that one could control the bias by simply centring one's stimulus range around the Point of Subjective Equality. This is formally correct, but also useless in practice. The reason it works is that if the centre of the stimulus range and the PSE are the same, then the biased result is the same as the "true" result — effectively you are biasing your results towards the unbiased answer. In most experiments though, you do not know what the correct answer is. That is why you are doing the experiment. Fotios and Cheal (2008) suggested using model predictions to identify the point of subjective equality to centre your ranges, but this is also problematic. If you are testing a model, or trying to replicate a previous study, then deliberately biasing your results towards the answer you want actively undermines the entire point of your work.
} 


\section{Stimulus Range Centring Bias}

Judgements are based off where the test stimuli lie in the range of test stimulus levels

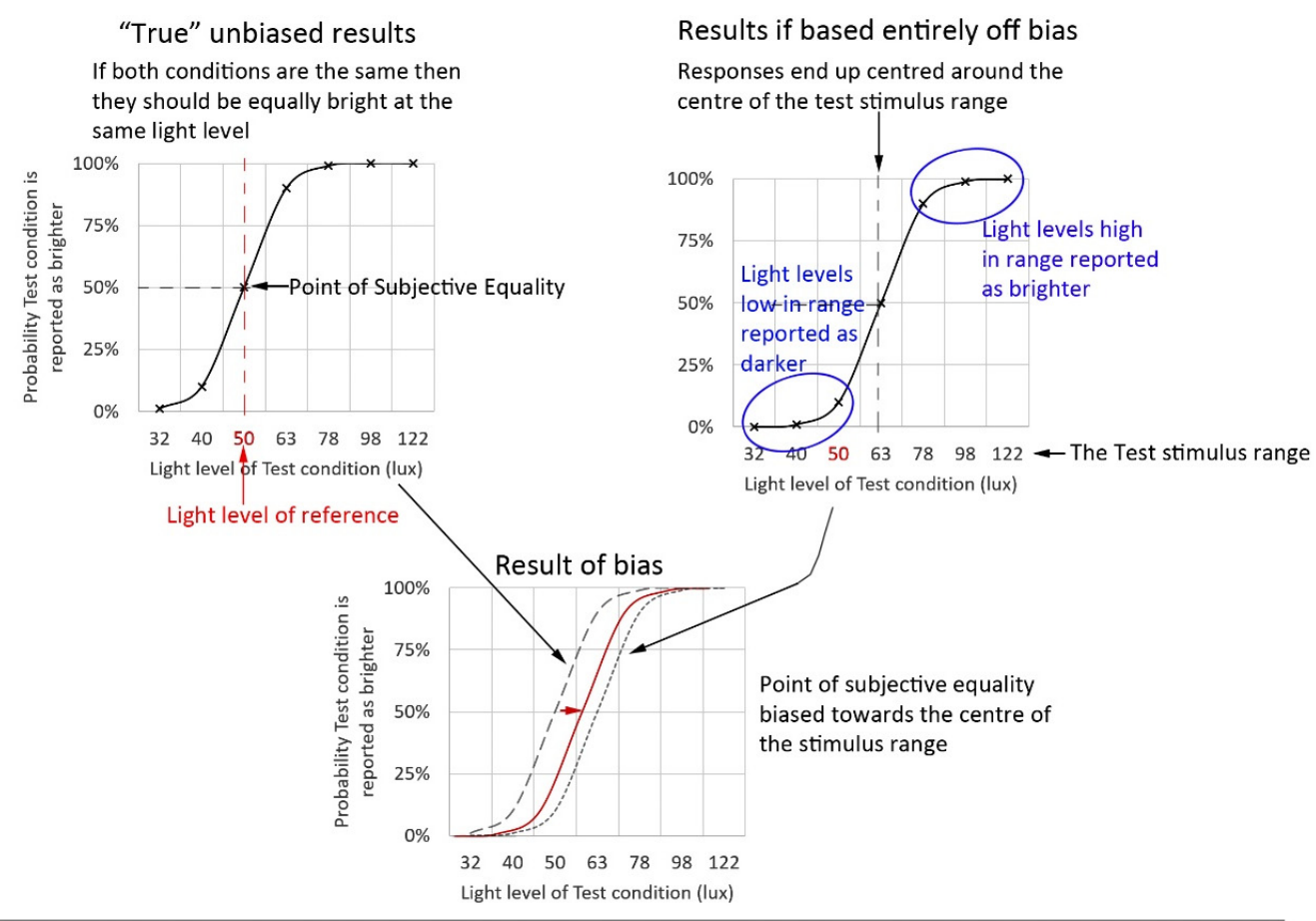

\section{Stimulus Frequency Bias}

Participants act as if all responses (e.g. brighter/darker) are equally likely to be correct

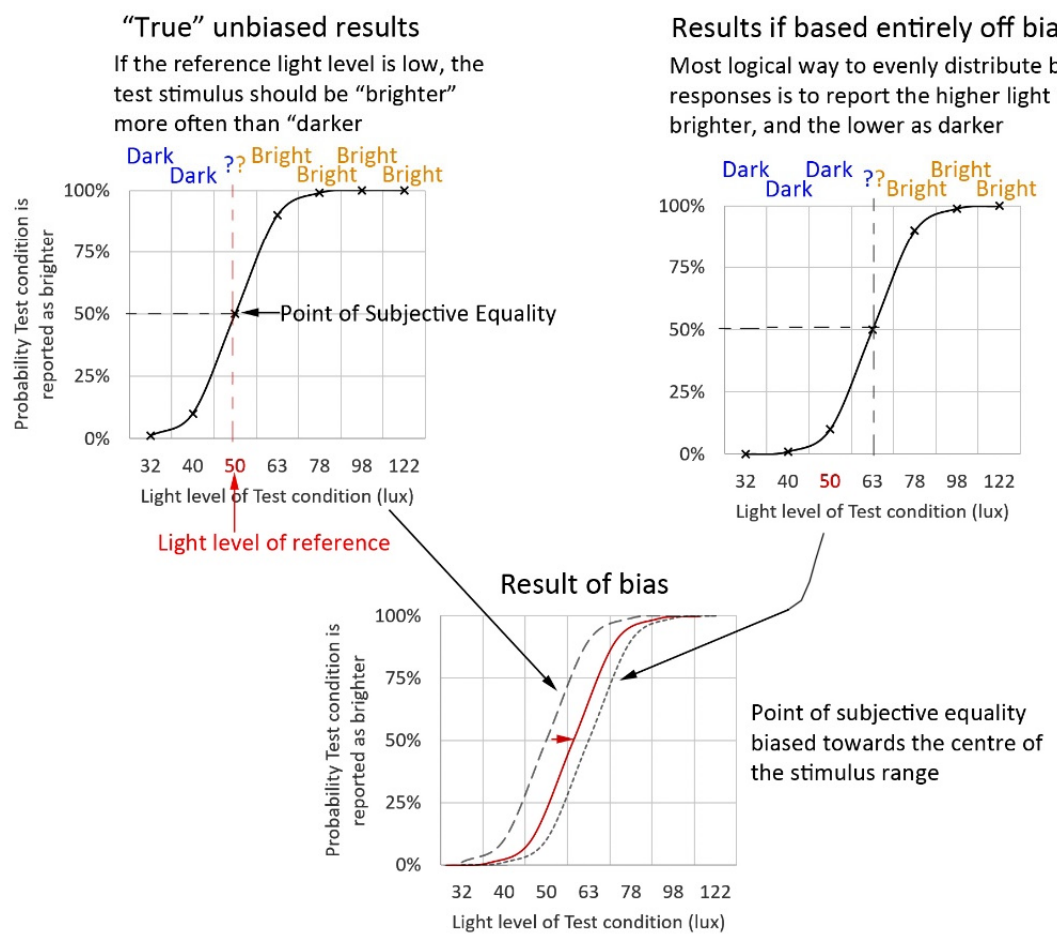

Figure 9.99 Visual explanation of Stimulus Range and Frequency Bias as applied to Brightness Discrimination tests using the simple example of a null-condition trial 


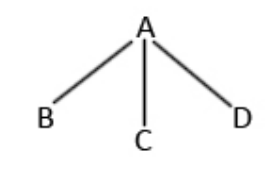

Test stimuli compared to single reference $(A)$

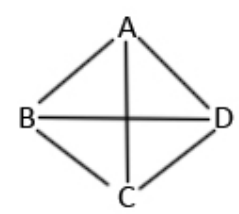

All-possible-pairs

Figure 9.100 Comparison of four stimuli (A-D) in two ways: comparing B-D against a single reference $(A)$, or comparing all possible pairs against each other

To understand the bias control, we must first understand the conditions that may create the biases. Stimulus range bias is held to need:

1) A distinct test stimulus range that an observer could theoretically use to infer the relative brightness of the test stimuli without reference to the reference stimulus

2) A constant reference stimulus (note that this is not actually proven in the references provided $^{96}$ )

While stimulus frequency bias relies on:

1) An explicit or implicit response scale for the subject to distribute their responses over

2) The responses being unevenly distributed over the scale

Participants being able to clearly differentiate test and reference conditions in order to identify stimulus ranges and apply response scales is thus a key component. Asking participants “is [Test] brighter than [Reference]?" is a bad idea, as it would directly contribute to the conditions the biases need to occur. Better is to ask "Which condition is brightest?", as was done in the example from Fotios and Cheal (2008). Note though that they still found the bias despite this. This may be because the experimental setup could produce the scale implicitly. In their experiment they examined the bias by setting up a reference condition at a constant light level and adjusting the test condition through the range of light levels. In such a situation, we might expect that even if participants are not explicitly asked "is [Test] brighter than [Reference]?", they will be able to infer that that is the question they are being asked from the context, and respond accordingly. Even if we had multiple different conditions and thus no set reference, we could envisage other ways

\footnotetext{
96 The paper Fotios and Houser (2013) used to illustrate the bias (Teller et al., 2003) used constant references, true, however this does not prove that such are required. Similarly, the discussion of the bias in Poulton (1977, pp. 412-413), from which Fotios and Houser appear to be drawing, was highlighting the scenario as one which may produce a particularly strong bias, and the study referenced showed how subjects may stop attending to a constant reference after enough time (in the example, 40 trials). Again, this does not prove that such a bias cannot occur without a constant reference.
} 
to set up fail states: for example, asking "is the left condition brighter than the right?" or "is the first condition brighter than the second?". If, for example, the correct answer was mostly "left is brightest" or "the second is brightest" then we could again have an effective unbalanced response scale that would be prone to stimulus frequency bias.

So, how does all-possible-pairs design address these problems? The proposal of allpossible-pairs in Fotios and Houser (2013) (repeated in CIE 212:2014) is not clear on this. After rejecting the earlier proposal of centring the test stimulus range around the point of subjective equality (which would ensure response frequencies are equal and balanced), they leap to all-possible-pairs, simply saying:

The results of Fotios and Cheal suggest that stimulus magnitudes in the range should be equal around that expected to be equal in brightness as the reference in order to permit an approximately equal number of brighter and dimmer responses and thus avoid a stimulus frequency bias. That approach, however, is likely to enhance the centering bias found by Teller and others. It is therefore suggested that discrimination procedures should avoid using a constant reference and should instead employ an allpossible-pairs approach, which will counter both the stimulus frequency and centering biases. (Fotios and Houser, 2013, p. 255)

The same explanation is given in CIE 212:2014. In later tables of recommendations, it is stated that: "In tests where a range of stimuli are compared against a single reference stimulus, stimulus range bias and stimulus frequency bias are expected" (CIE, 2014, p. 37). Thus, the assumption would appear to be that as long as we do not have a constant reference condition, the biases will not manifest. The immediate question this raises is: why do we need to do all possible pairs then? If all we need is to just ensure the reference is varied then we can do that without doing every single possible stimulus combination (Figure 9.101).

\begin{tabular}{|c|c|c|c|c|}
\hline \multicolumn{4}{|c|}{$\begin{array}{l}\text { All-possible-pairs design for six conditions } \\
(A->F) \text { : }\end{array}$} & $\begin{array}{l}\text { A much simpler and faster design } \\
\text { which compares all six conditions } \\
\text { while repeatedly varying pairs: }\end{array}$ \\
\hline$A$ & v. B & B & v. $F$ & $A$ v. $B$ \\
\hline A & v. C & & v. D & B v. C \\
\hline A & v. D & & v. E & C v. D \\
\hline A & v. $E$ & C & v. $F$ & $D \quad$ v. $E$ \\
\hline A & v. $F$ & & v. $E$ & $\mathrm{E} \quad$ v. $\mathrm{F}$ \\
\hline B & v. C & D & v. $F$ & F v. A \\
\hline B & v. D & & v. $F$ & \\
\hline$B$ & v. $E$ & & & \\
\hline
\end{tabular}

Figure 9.101 How all-possible-pairs is not needed to avoid having a constant reference in the comparisons you are making 
Could there be an alternative justification for all-possible-pairs? Could it have some ability to address the drivers of stimulus frequency bias beyond just varying the reference?

The initial issue that was highlighted is that stimulus frequency bias may be produced when you have unequal response frequencies. One thing all-possible-pairs does do is ensure that all the different stimuli are viewed an equal number of times ${ }^{97}$ - it ensures equal stimulus frequencies. Building on that, if we are comparing all the conditions against all other conditions then intuitively we might expect that the brightest half will be brighter than the others at least half the time, while the darker half will be darker than the others at least half the time. Could this ensure that the responses balance out?

The answer, unfortunately, is no. As illustrated in the examples below (Table 9.11, Table 9.12, Figure 9.102), if the experimental design sets up a situation in which one has a response scale that the participants could distribute their responses over (e.g. "brighter" vs "darker") then an all-possible-pairs design will do nothing to ensure that the responses will be balanced.

This is not to say that all-possible-pairs does nothing. Varying the condition comparisons may be useful to break things up and make it difficult for participants to do things like keeping track of where test stimuli fall in their light level range, or prevent people from identifying conditions as "test" and "reference" conditions and producing implicit scales ${ }^{98}$. This might disrupt the conditions that produce the biases. However, allpossible-pairs is not needed to do this, and may be highly inefficient.

Table 9.11 Illustration of how an all-possible-pairs design may not work to deal with stimulus frequency bias if there is an explicit or implicit reference condition and response scale. We compare stimuli A-D where A > B > C > D. In this setup, the correct answer is always "the test stimulus is brighter". "Brighter" and "darker" responses are not equally balanced.

\begin{tabular}{|l|l|l|l|}
\hline Test & Reference & Question & Correct Answer \\
\hline A & B & Is "A" brighter or darker? & Brighter \\
\hline A & C & Is "A" brighter or darker? & Brighter \\
\hline A & D & Is "A" brighter or darker? & Brighter \\
\hline B & C & Is "B" brighter or darker? & Brighter \\
\hline B & D & Is "B" brighter or darker? & Brighter \\
\hline C & D & Is "C" brighter or darker? & Brighter \\
\hline
\end{tabular}

\footnotetext{
${ }_{97}$ Though we may note here that even if this was needed, all-possible-pairs is not needed to do this - the simpler design shown in Figure 9.101 for example also shows each condition an equal number of times.

${ }^{98}$ Of course, one still needs to avoid creating such scales through your experimental design. Do not ask "is X brighter or darker than Y?" If you have unavoidable markers like left vs. right, then make sure the stimuli are shown equally on both sides so that that cannot produce the bias.
} 
Table 9.12 Illustration of how an all possible pairs design may not work to deal with stimulus frequency bias if there is an explicit or implicit reference condition and response scale. We compare stimuli A-D where A > B > C > D. In this setup, the correct answer is always " 1 is brighter". " 1 " and " 2 " responses are not equally balanced.

\begin{tabular}{|l|l|l|l|}
\hline Cond. 1 & Cond. 2 & Question & Correct Answer \\
\hline A & B & Which condition is brighter? & 1 \\
\hline A & C & Which condition is brighter? & 1 \\
\hline A & D & Which condition is brighter? & 1 \\
\hline B & C & Which condition is brighter? & 1 \\
\hline B & D & Which condition is brighter? & 1 \\
\hline C & D & Which condition is brighter? & 1 \\
\hline
\end{tabular}

So, returning to the question of whether or not we should be thinking about using an all-possible-pairs design in the context of brightness matching experiments we now have our answer: no. If we assume that just varying the reference eliminates the biases then we can do that by just counterbalancing the application of dimming. If we do not assume that will work, and look at the other conditions that drive the biases, then we see that allpossible-pairs cannot do anything about them. In the context of a brightness matching task, wherein one is adjusting a test condition to match a provided reference, the subject will unavoidably be faced with a clear test stimulus range and reference condition. All-possiblepairs will not change that. If we are concerned about a bias based off the test stimulus adjustment range then we already have a solution: carry out matches at multiple adjustment ranges in order to measure the strength of the bias and control for it. 
Setup:

Five levels of A vs. 5 levels

of $B$ in all-possible-pairs

design.

A > B at equal light level.

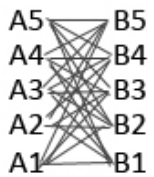

Implicit question: is A brighter or darker than B?

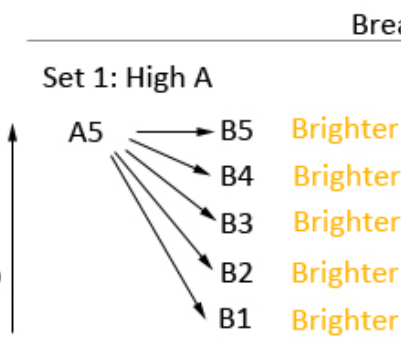

Set 2: Low A

Set 3: high-mid A

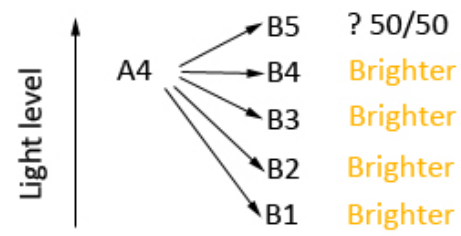

Set 4: low-mid A

A2

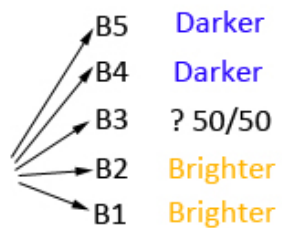

Set 5: Mid A

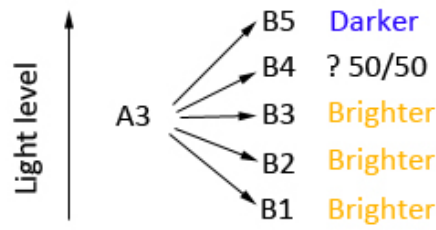

Figure 9.102 More complex illustration of an all-possible-pairs design where one has two conditions compared at multiple light levels. In such a case, the recommended path is to treat each light level as a distinct condition that must be accounted for in the all-possible-pairs design. Again, we see that should an implicit response scale arise (e.g. is A brighter or darker than B?) then the allpossible-pairs design would not ensure that both brighter and darker responses were balanced.

To conclude: while some form of stimulus range centring bias is plausible in the context of brightness matching, an all-possible-pairs design would be unnecessary and ineffectual at controlling it. It is not recommended. 


\section{Appendix D Experiment 2}

\section{D.1 Example of trial order}

Table 9.13 Example of sheet used to describe and record the trials and matches made in a session. Note that while trials are randomised, the number of lamps is reduced in sequence, constraining the randomisation.

\begin{tabular}{|c|c|c|c|c|c|}
\hline \#lamps & Ref & Ref_condition & Test_condition & Start & Match \\
\hline 6 & 189 & Grey_1 & Horizontal & 635 & \\
\hline 6 & 292 & Hidden_white_1 & Hidden_check & 780 & \\
\hline 6 & 288 & Hidden_white_2 & Hidden_white_1 & 367 & \\
\hline 6 & 189 & Grey_1 & Vertical & 644 & \\
\hline 6 & 189 & Grey_1 & Light_check & 732 & \\
\hline 5 & 157 & White_1 & Full_check & 650 & \\
\hline 4 & 189 & Grey_1 & Light_narrow & 57 & \\
\hline 3 & 355 & Horizontal & Grey_1 & 34 & \\
\hline 3 & 157 & White_1 & Wall_narrow & 21 & \\
\hline 3 & 380 & Grey_2 & Grey_1 & 412 & \\
\hline 3 & 380 & Grey_2 & Grey_1 & 34 & \\
\hline 3 & 361 & Vertical & Grey_1 & 34 & \\
\hline 3 & 288 & Hidden_white_2 & Hidden_white_1 & 367 & \\
\hline 3 & 157 & White_1 & Wall_check & 23 & \\
\hline 3 & 133 & Grey_2 & Grey_1 & 34 & \\
\hline 3 & 133 & Grey_2 & Grey_1 & 412 & \\
\hline 2 & 201 & Wall_check & White_1 & 17 & \\
\hline 2 & 190 & Wall_narrow & White_1 & 348 & \\
\hline 2 & 397 & Light_check & Grey_1 & 34 & \\
\hline 2 & 278 & Full_check & White_1 & 348 & \\
\hline 2 & 68 & White_2 & White_1 & 17 & \\
\hline 2 & 201 & Wall_check & White_1 & 348 & \\
\hline 2 & 227 & Grey_1 & White_1 & 348 & \\
\hline 2 & 306 & Light_narrow & Grey_1 & 34 & \\
\hline 2 & 397 & Light_check & Grey_1 & 343 & \\
\hline 2 & 195 & White_2 & White_1 & 348 & \\
\hline
\end{tabular}




\section{D.2 Model details}

We assume that an individual's responses to a given condition $\left(y_{\mathrm{i}}\right)$ are normally distributed with a mean describing the average match they make to the given condition $\left(\mu_{[t, r, s, p]}\right)$ and a standard deviation $\left(\sigma_{[s, p]}\right)$ describing the variation in their matches:

$$
y_{i} \sim \operatorname{normal}\left(\mu_{[t, r, s, p]}, \sigma_{[s, p]}\right)
$$

The mean match made by a subject for a given pair of conditions $\left(\mu_{[t, r, s, p]}\right)$ may vary by the individual test and reference conditions $(t, r)$, the subject $(s)$, and the pair of conditions being compared $(p)$. It is assumed to be the product of the reference light level, the difference between the two conditions in apparent brightness and the various biases that may affect the match:

$$
\begin{gathered}
\mu_{[t, r, s, p]}=\operatorname{ref}-\left(\operatorname{Eff}_{[t, s]}-\operatorname{Eff}_{[r, s]}\right)-\left(S P D_{\text {test }[t]}-S P D_{\text {ref }[r]}\right) \\
+\operatorname{bias}_{\text {pos }+\operatorname{dim}_{[p, s]}}+\operatorname{bias}_{\text {start }[p, s]}+\operatorname{bias}_{\text {ref }[s]}+\operatorname{bias}_{\text {rng }[s]}
\end{gathered}
$$

$r e f$ is the light level of the reference condition, $E f f_{[t / r, s]}$ is the effect of the condition (test or reference), SPD is the effect of SPD for that condition (pre-calculated using the Rea

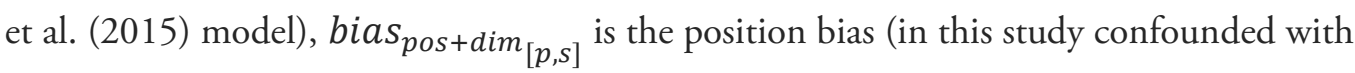
dimming bias), $\operatorname{bias}_{\left.\operatorname{start}_{[} p, s\right]}$ is bias associated with the starting light level of the test

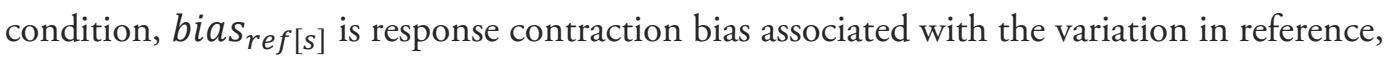
and bias $_{r n g[s]}$ is bias associated with the adjustment range of the test condition.

Most of these factors are the same as in B.4, so will not be repeated here. The new elements are the response contraction bias and the adjustment range bias, which are assumed to be proportional biases similar to start point bias.

Response contraction bias due to different reference conditions is assumed to be proportional to the difference between the apparent light level of the current reference and the average reference:

$$
\operatorname{bias}_{r e f[r, s]}=k_{r e f[s]}\left(r e f+E f f_{[r, s]}+S P D_{r e f[r]}-\overline{r e f_{[s]}}\right)
$$

Where $k_{r e f[s]}$ is the bias strength (slope of bias against reference light level), and $\overline{r e f_{[s]}}$ is the mean apparent reference light level, and is calculated as:

$$
\overline{r e f_{[s]}}=\overline{r e f_{l}}+\frac{w_{[r]} \cdot E f f_{[r, s]} \cdot S P D_{r e f[r]}}{\sum w_{[r]}}
$$

Where $\overline{r e f}$ is the average reference light level, and $w_{[r]}$ is a vector of weightings based off the frequency that each condition is used as a reference. Essentially, it is the sum of the average reference light level and the average effect of the reference condition(s). 
The strength of the bias may vary between people, but is assumed to be homogenous across conditions as we have only measured it for the null trials, and not the other conditions:

$$
\begin{gathered}
k_{r e f[s]}=\beta_{0 . r e f}+\beta_{2 . r e f . S D} \cdot \tilde{\beta}_{2 . r e f[s]} \\
\beta_{0 . r e f} \sim \operatorname{Normal}(-0.06,0.1) \\
\beta_{2 . r e f . S D} \sim \operatorname{half\_ normal}(0,0.1) \\
\tilde{\beta}_{2 . r e f[s]} \sim \operatorname{Normal}(0,1)
\end{gathered}
$$

Bias due to different ranges is assumed to be proportional to the difference between the centre of the adjustment range (centre $\left.e_{[t\}}\right)$ and the intended match:

$$
\begin{aligned}
& \text { bias }_{r n g[t, r, s]}=k_{\text {range }[s]}\left(r e f-\left(E f f_{[t, s]}-E f f_{[r, s]}\right)-\left(S P D_{\text {test }[t]}-S P D_{\text {ref }[r]}\right)\right. \\
& \left.-\operatorname{centr} e_{[t]}\right) \\
& k_{\text {range }[s]}=\beta_{\text {0.range }}+\beta_{2 . \text { range.SD }} \cdot \tilde{\beta}_{2 . \text { range }[s]} \\
& \beta_{\text {0.range }} \sim \operatorname{Normal}(0,0.06) \\
& \beta_{\text {2.range.SD }} \sim \text { half_normal }(0,0.1) \\
& \tilde{\beta}_{2 . r a n g e[s]} \sim \operatorname{Normal}(0,1)
\end{aligned}
$$




\section{D.3 Model diagnostic plots}

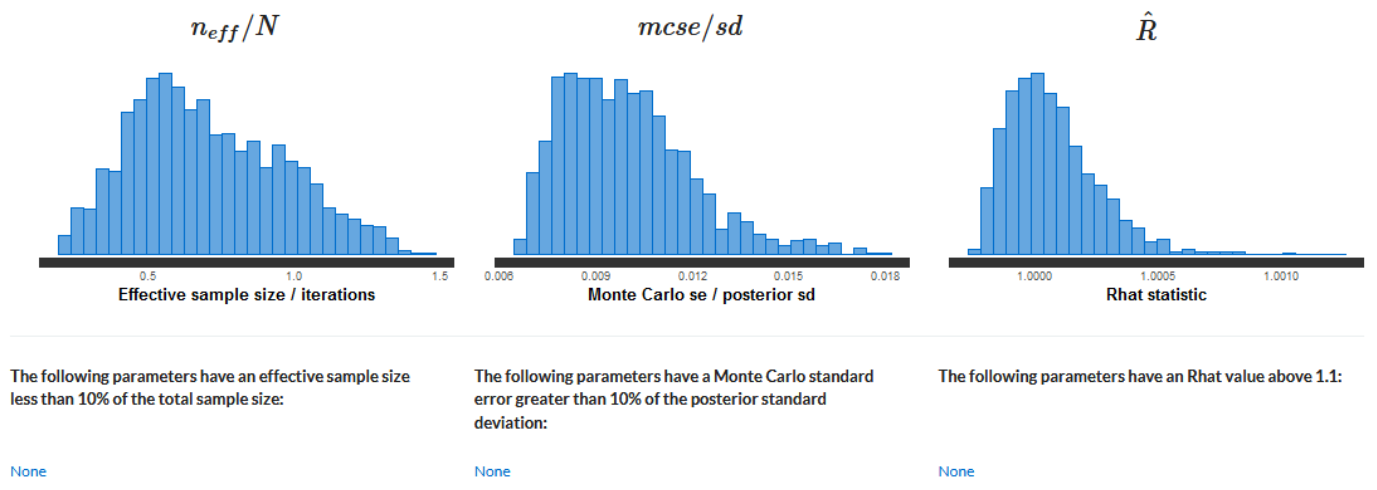

Figure 9.103 Stan model diagnostic plots produced by the ShinyStan R package.

NUTS Energy Diagnostic
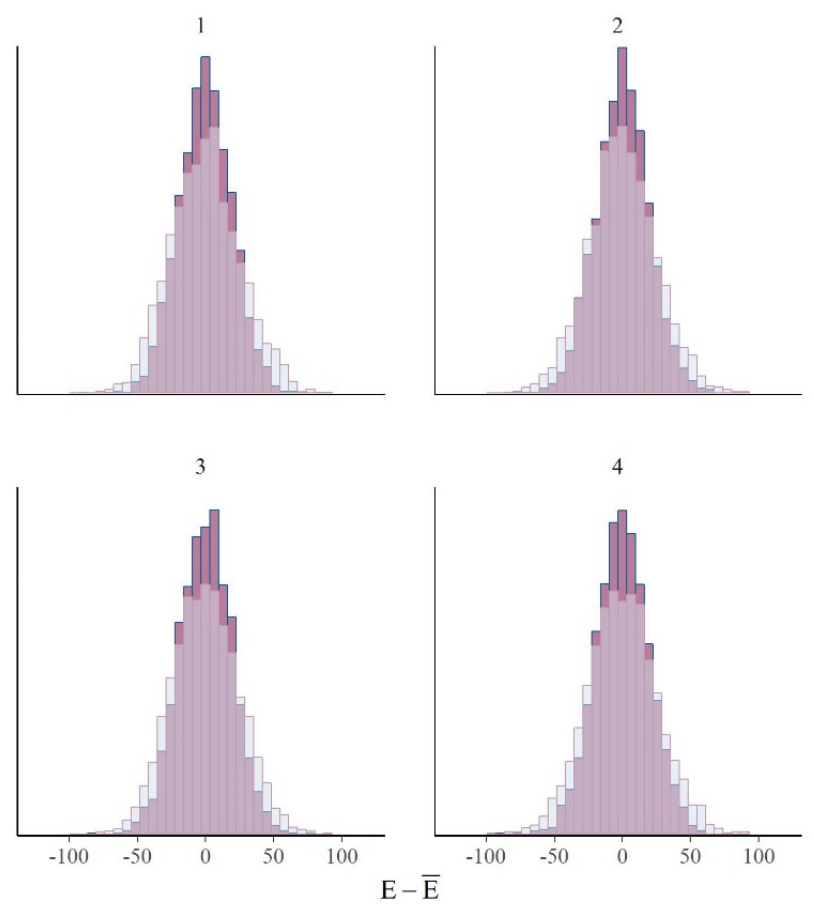

Figure 9.104 NUTS Energy Diagnostic plots produced by the bayesplot package. 
Figure 9.105 Traceplots of model parameters showing that chains are well mixed (bayesplot)
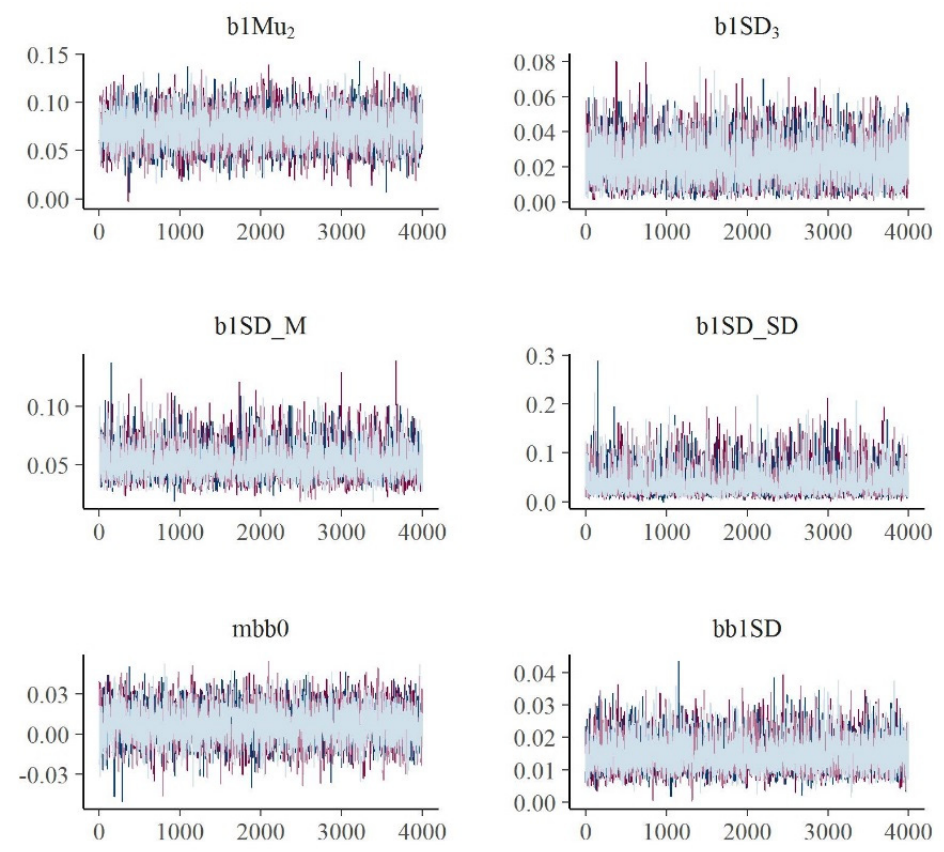

$\mathrm{bb} 2 \mathrm{SD}$

$\mathrm{bbl}_{1}$
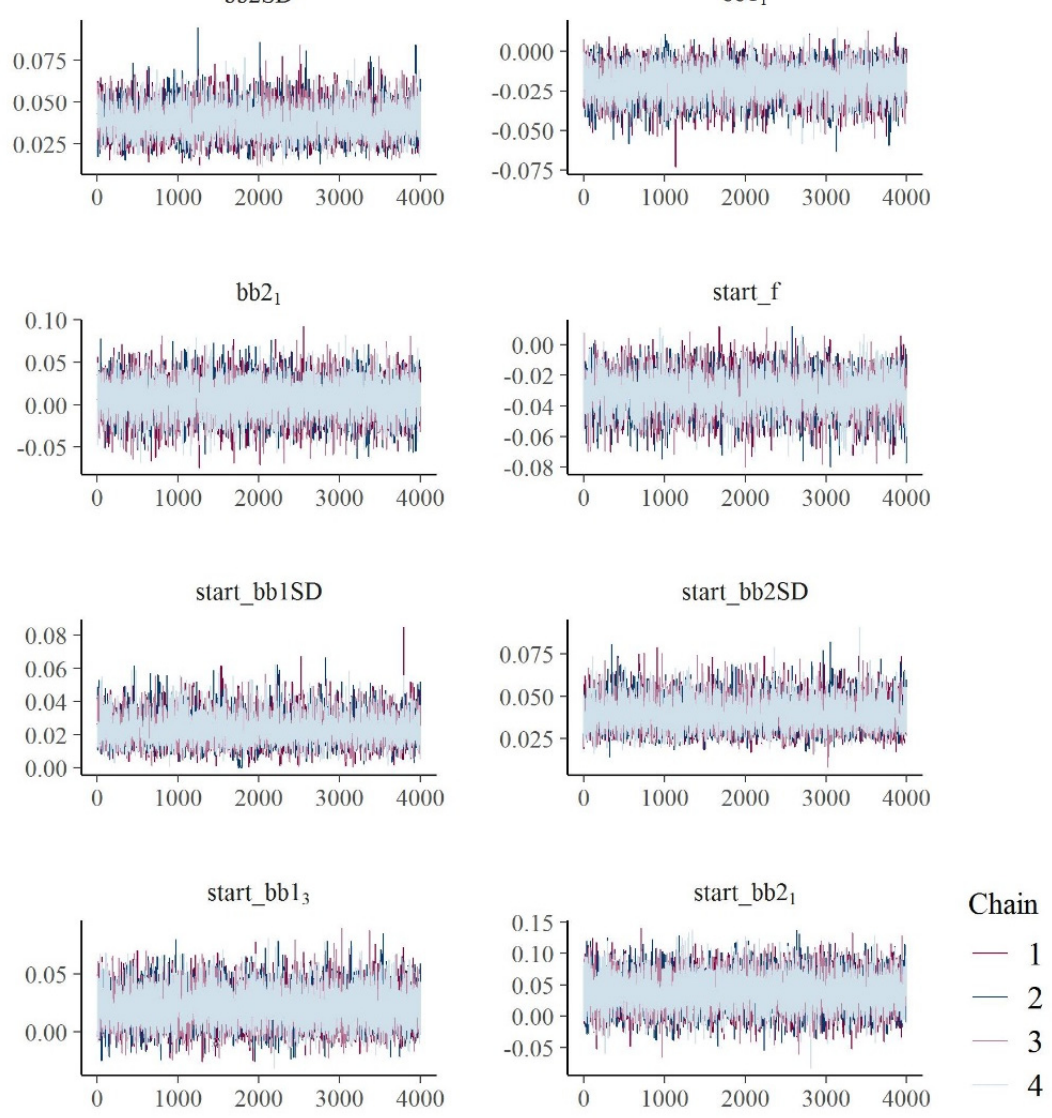

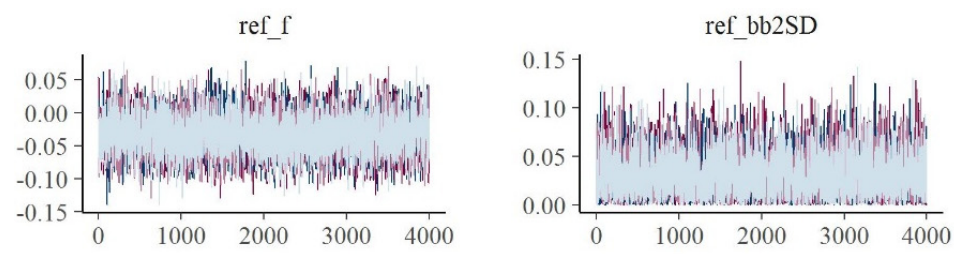

ref_bb2
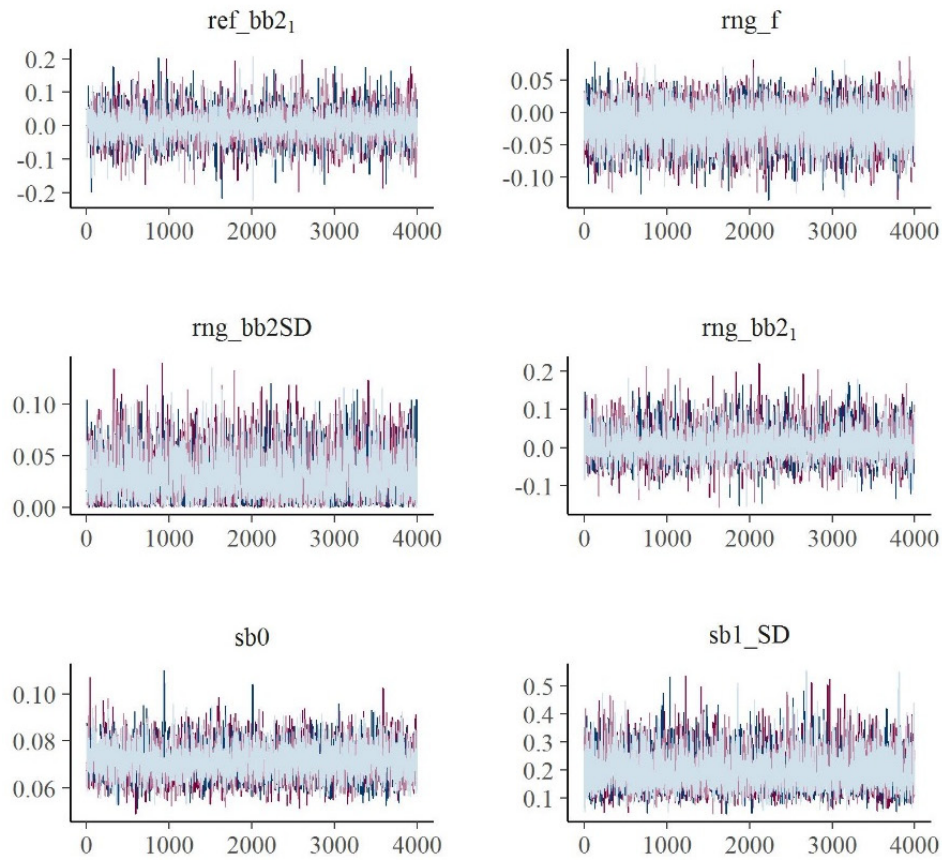

sb2_SD

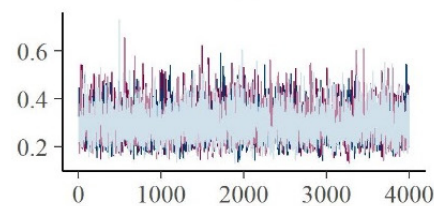

$\mathrm{sbl}_{6}$
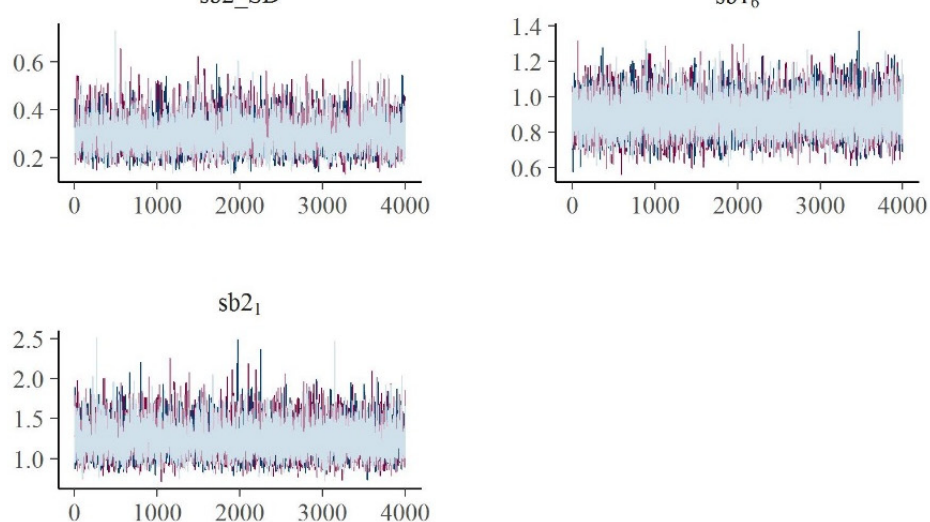


\section{D.4 Simple summary statistics}

Note that the simple means and confidence intervals calculated here have had the effects of SPD accounted for, but do not account for any of the proportional biases.
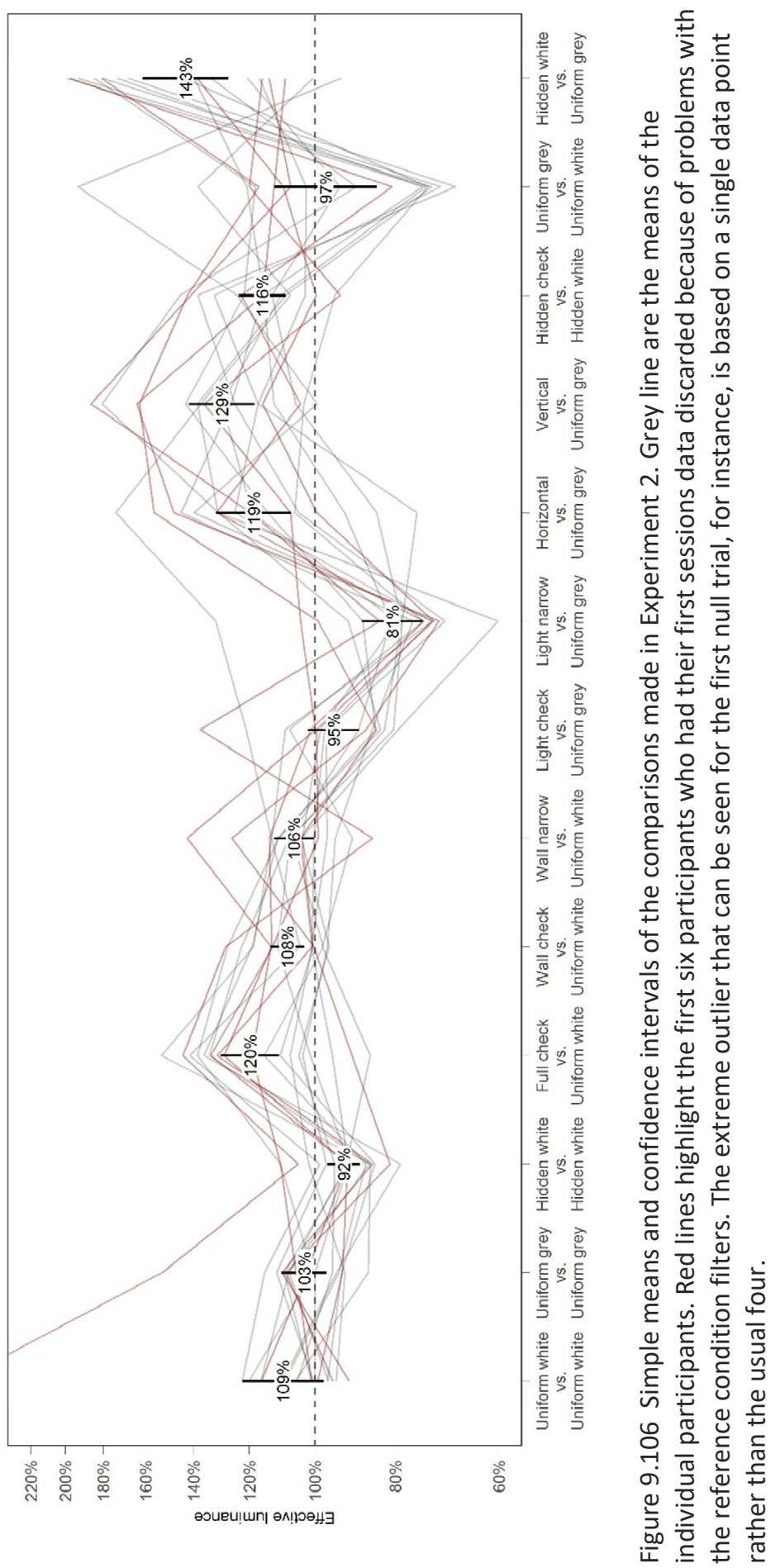


\section{D.5 Other observations}

The following are estimates of the other parameters in the primary statistical model. For other model versions, see online supplement.

\section{Between-person variance in condition effects}

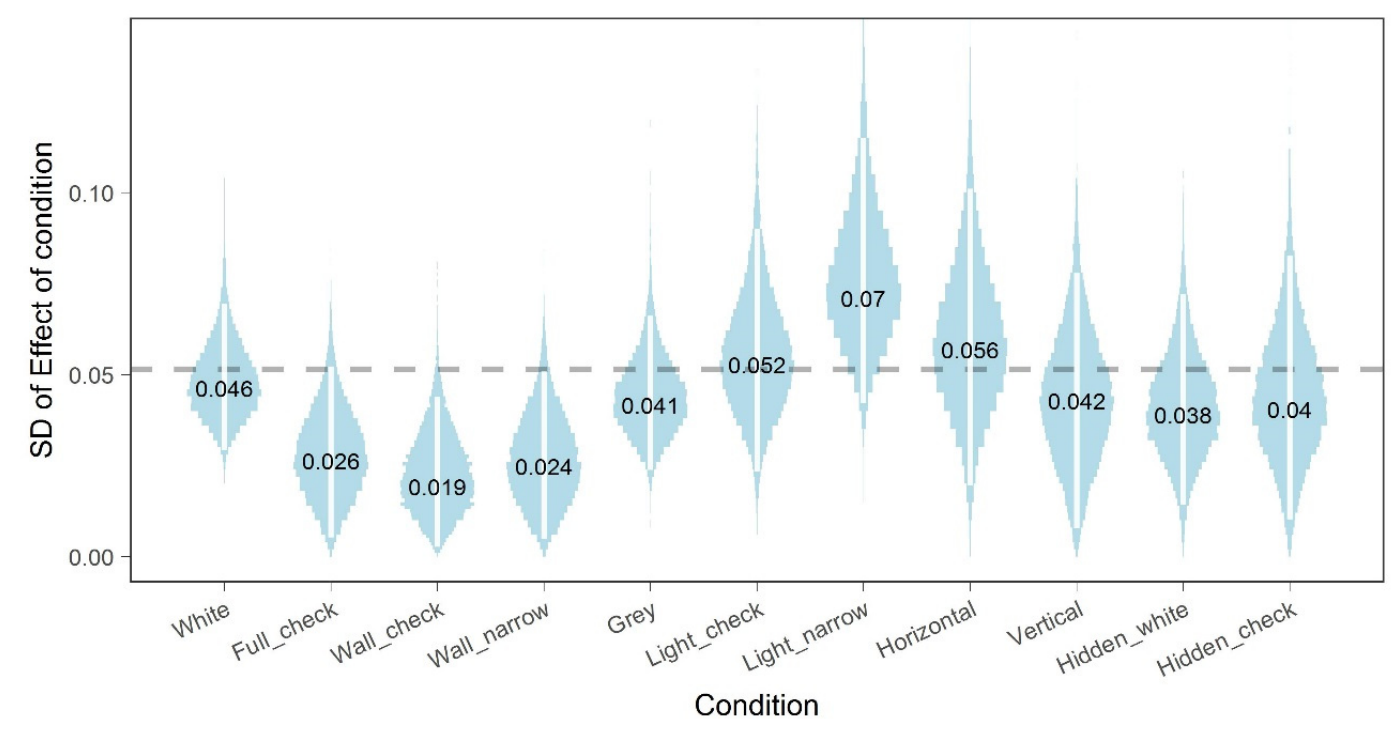

Figure 9.107 Between-person standard deviations for the effect of each individual condition

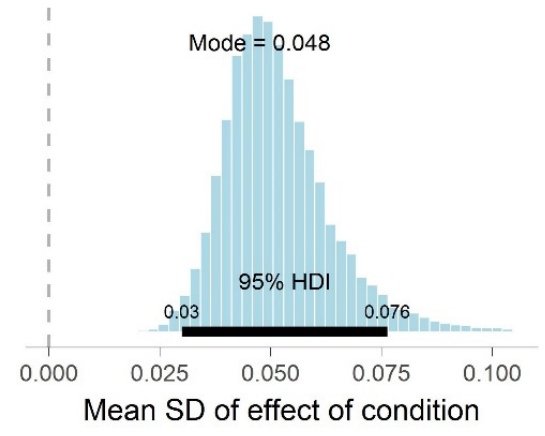

Figure 9.108 Mean between-person SD

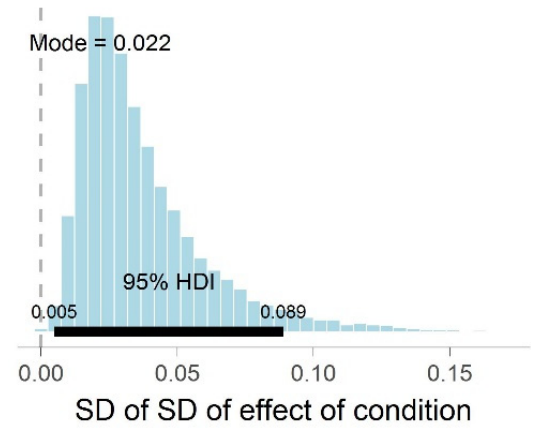

Figure 9.109 Between-condition SD of between-person SD 
Figure 9.110 (Next two pages): Estimated condition effects for individual participants.

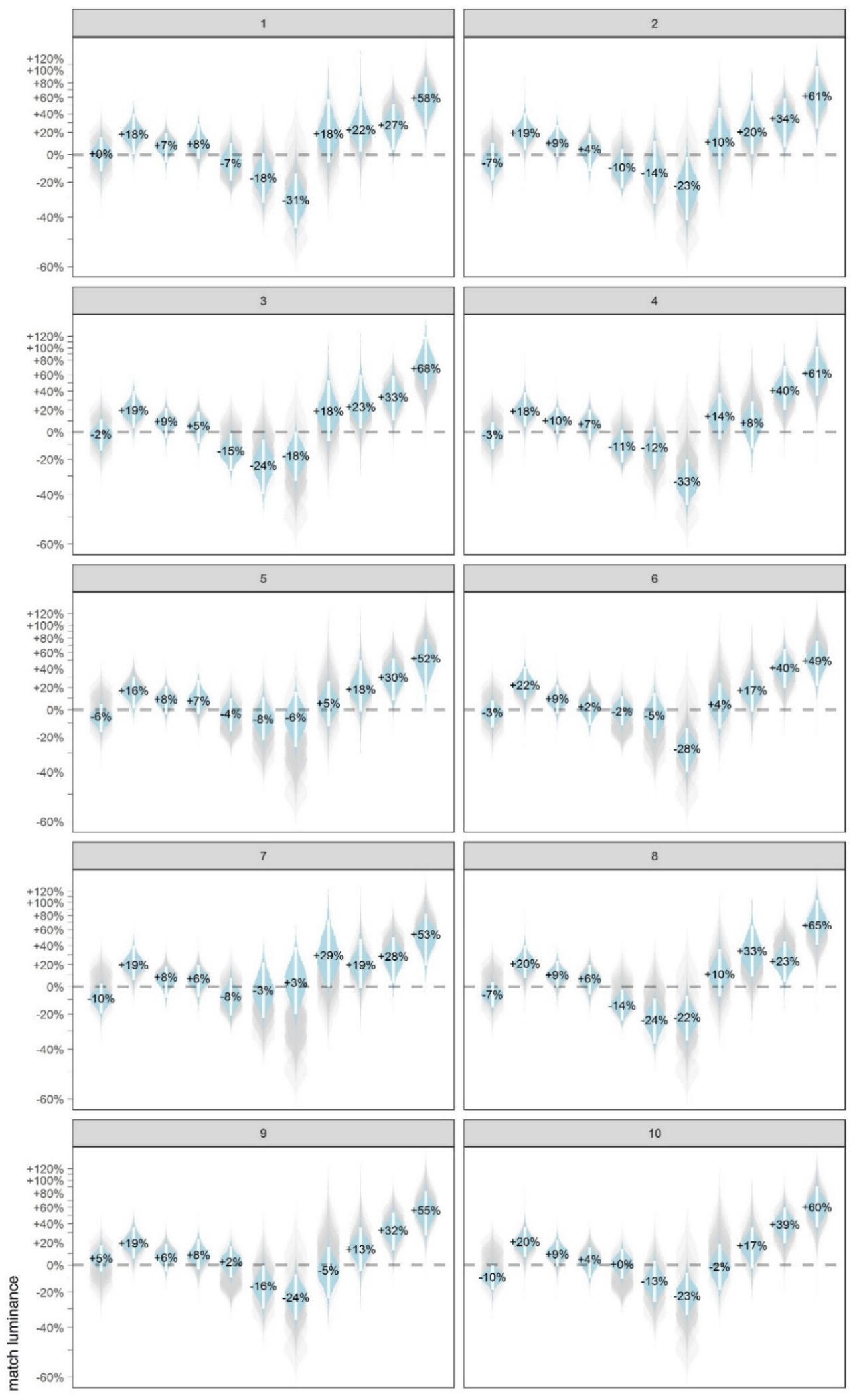



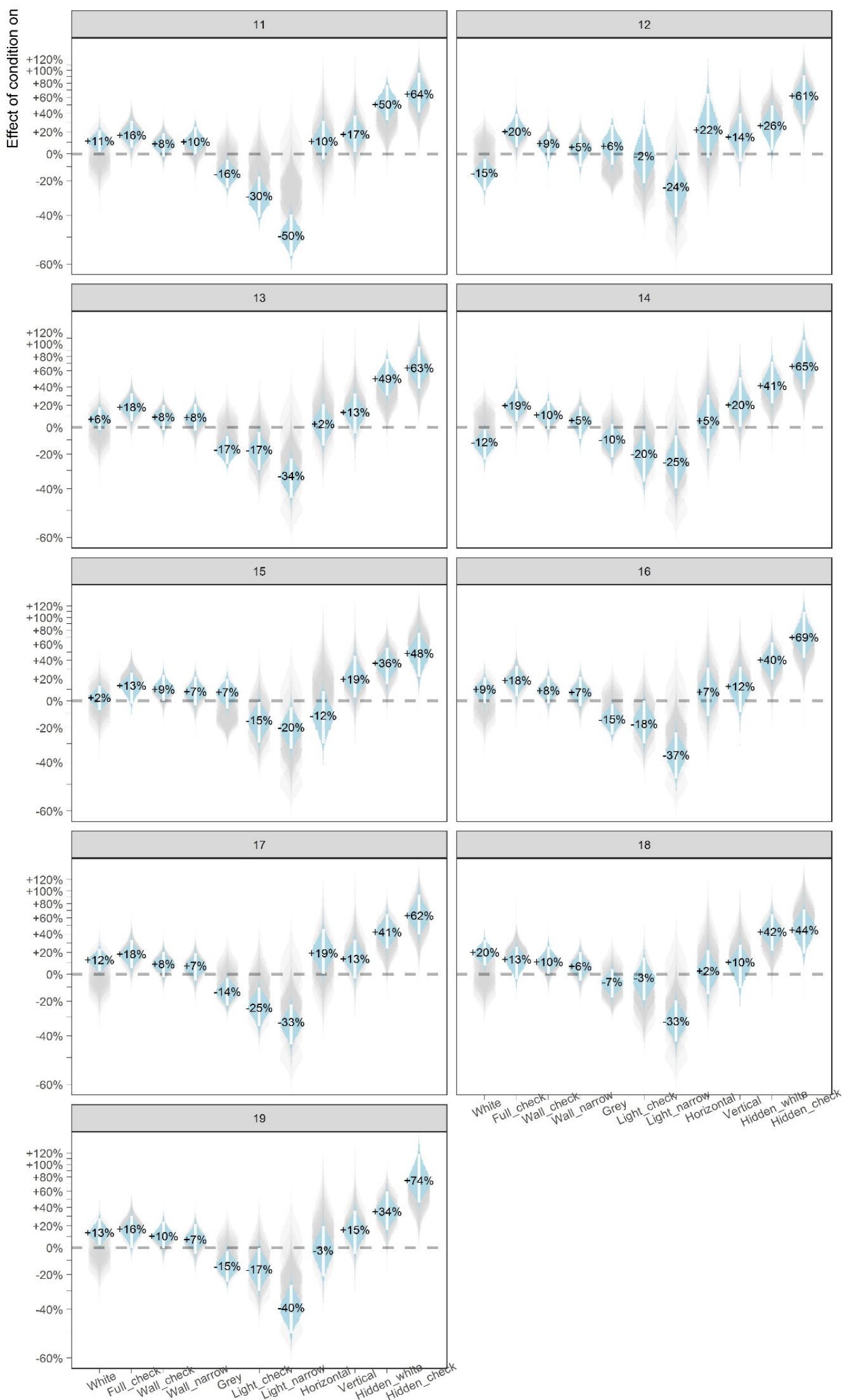

Condition 


\section{Within-person variance}

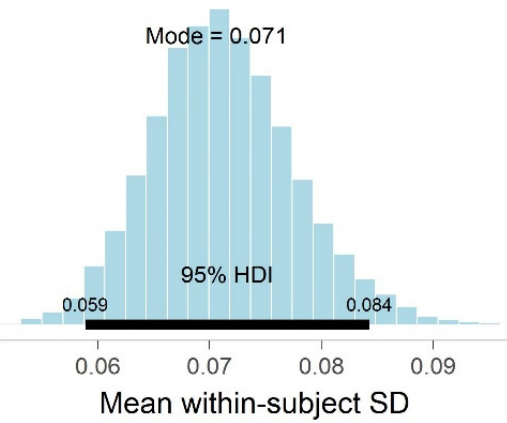

Figure 9.111 Mean within-person standard deviation

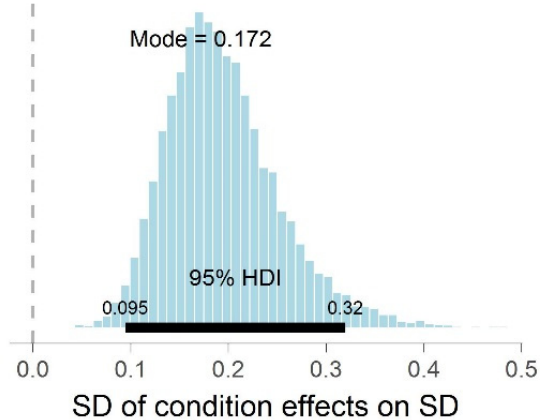

Figure 9.112 SD of variation between conditions in SD

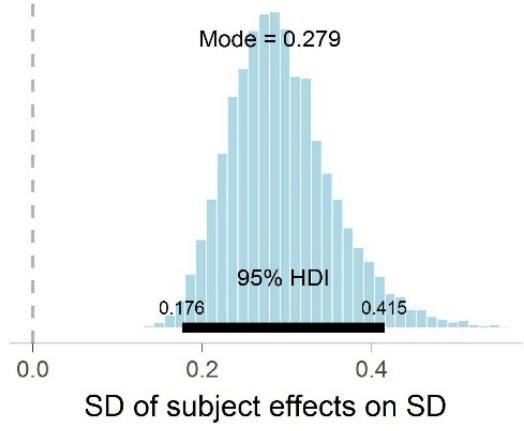

Figure 9.113 SD of variation between people in SD

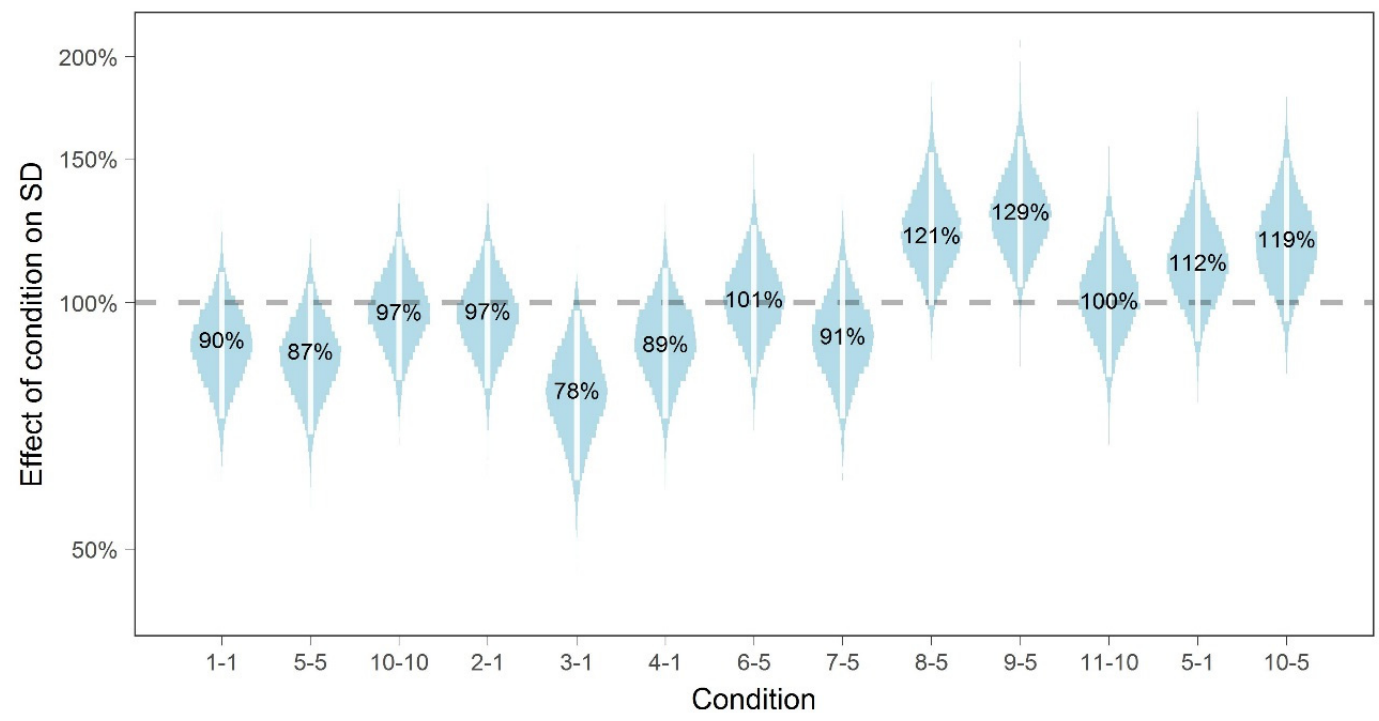

Figure 9.114 Estimated differences between pairs of conditions in within-person SD (read as multipliers of the mean described in Figure 9.111) 


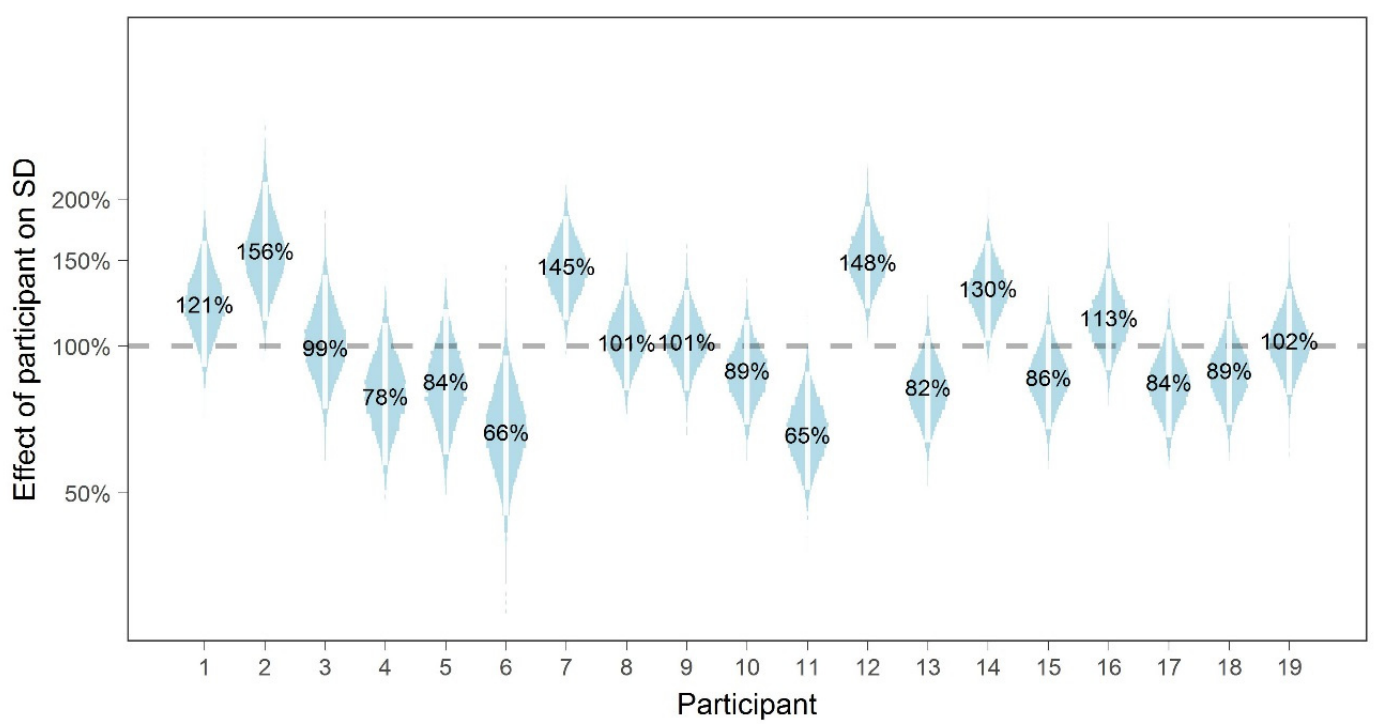

Figure 9.115 Estimated differences between participants in within-person SD (read as multipliers of the mean described in Figure 9.111)

\section{Position+dimming bias}

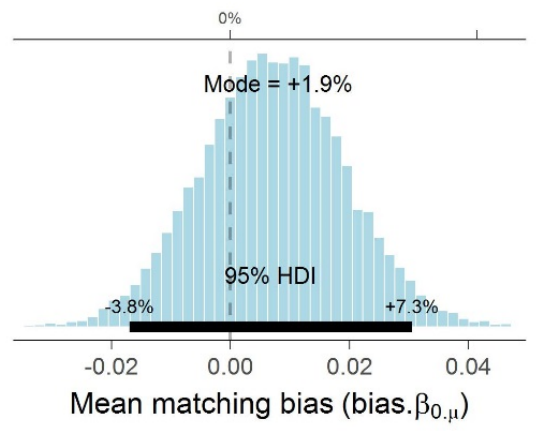

Figure 9.116 Mean position+dimming bias

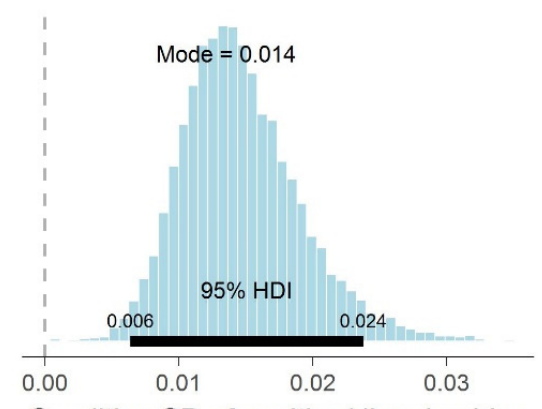

Condition SD of position/dimming bias

Figure 9.117 SD of variation in position+dimming bias between pairs of conditions

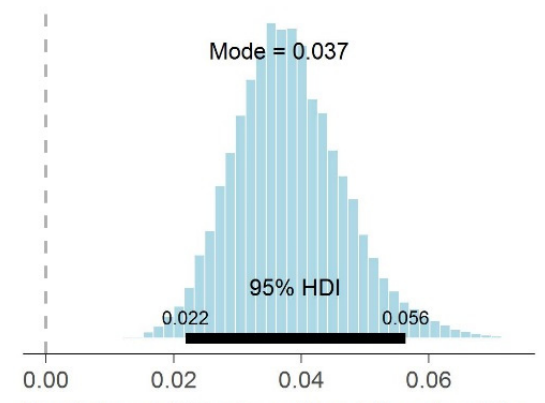

Participant SD of position/dimming bias

Figure 9.118 SD of variation in position+dimming bias between people 


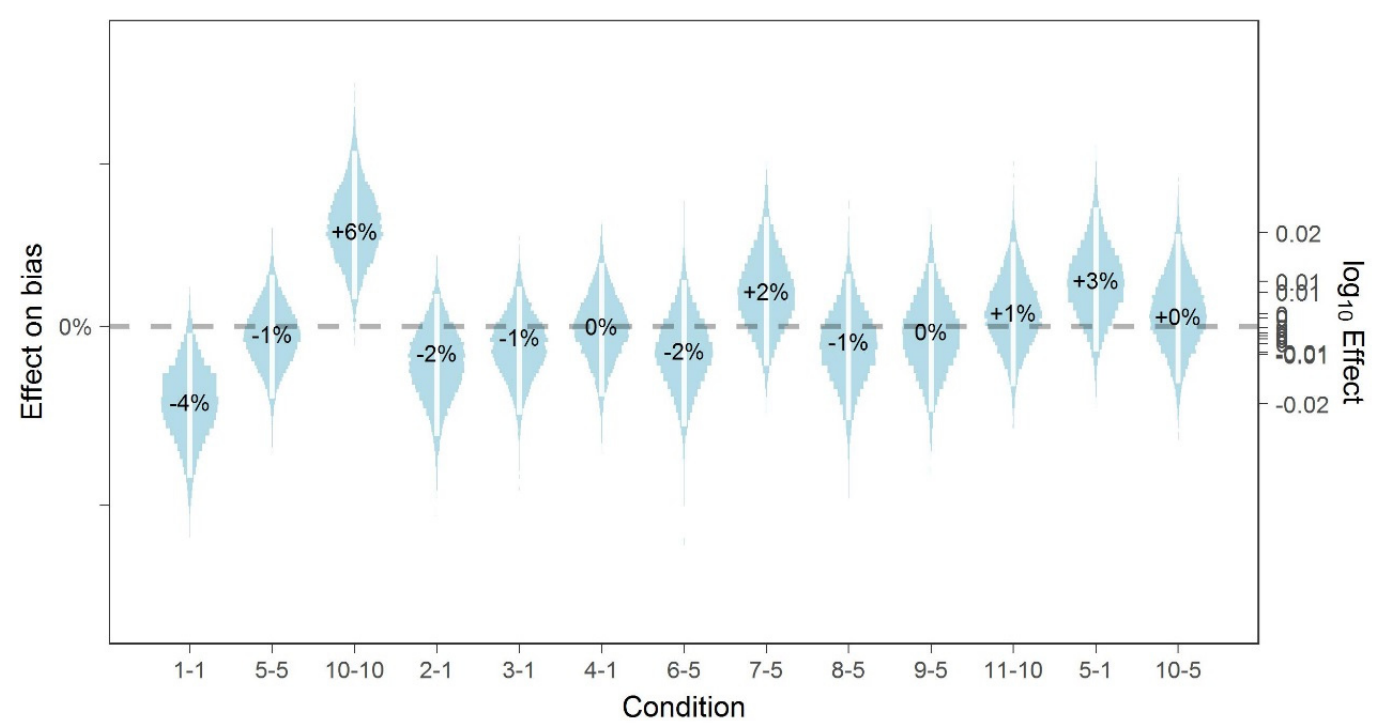

Figure 9.119 Differences in position+dimming bias for pairs of conditions

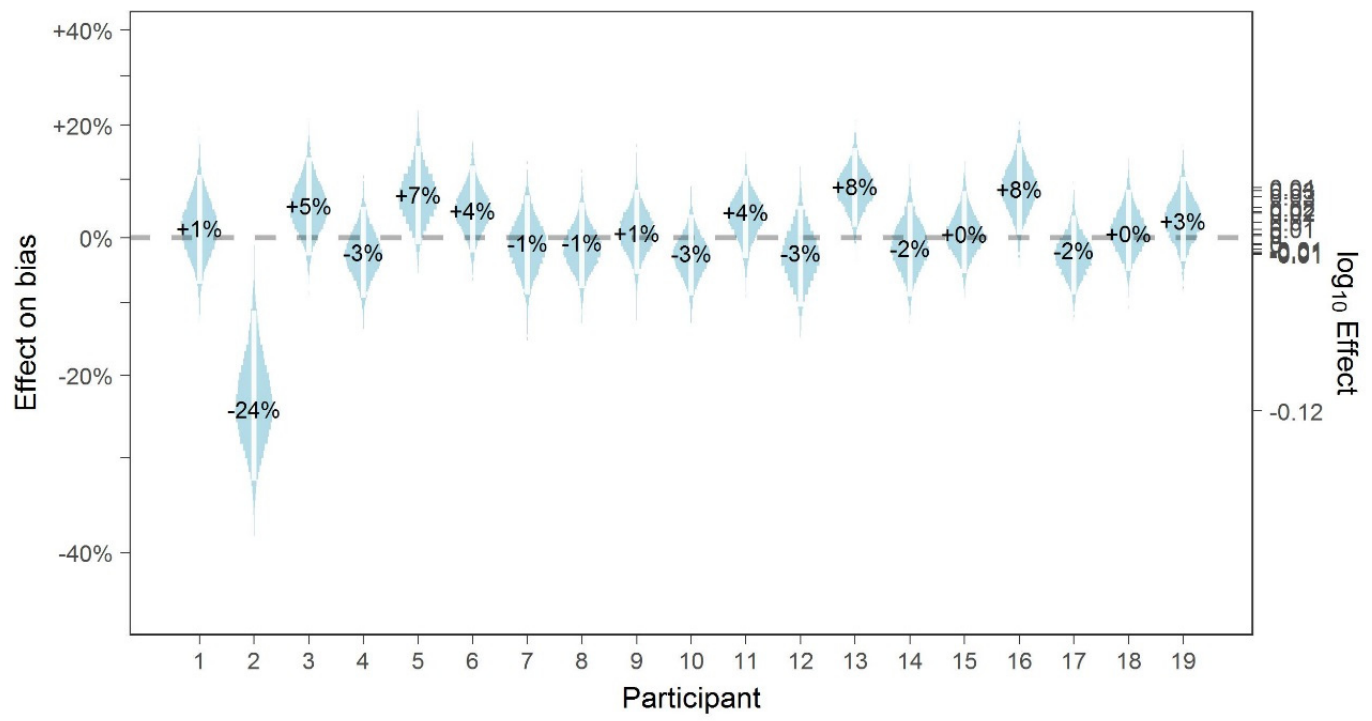

Figure 9.120 Differences in position+dimming bias for participants 


\section{Start point bias}

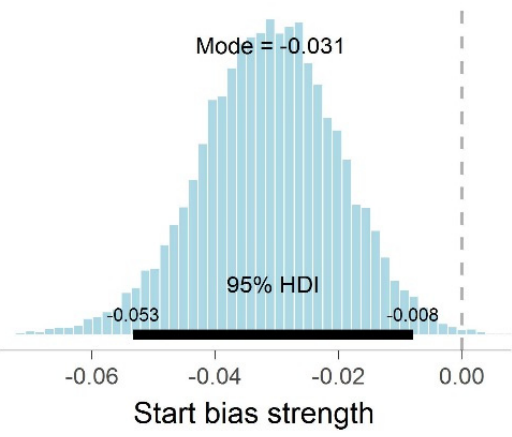

Figure 9.121 Mean start bias strength

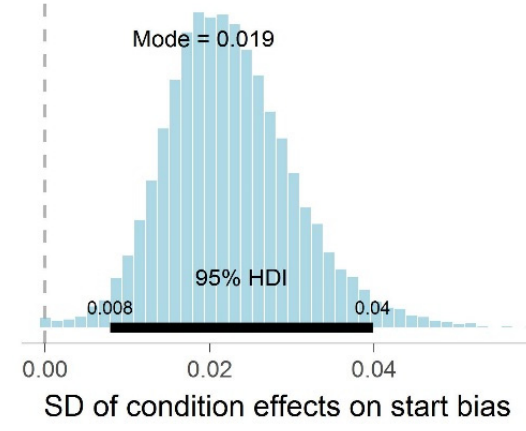

Figure 9.122 SD of variation in start bias between pairs of conditions

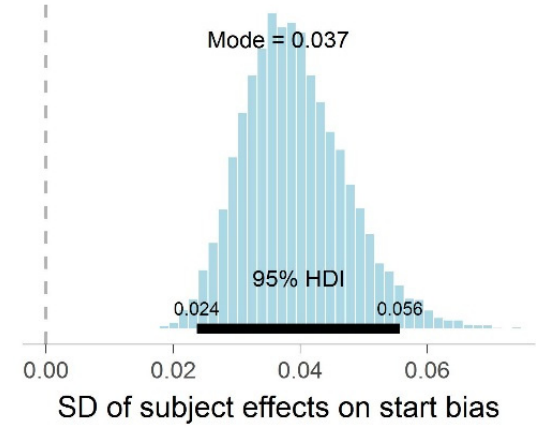

Figure 9.123 SD of variation in start bias between people

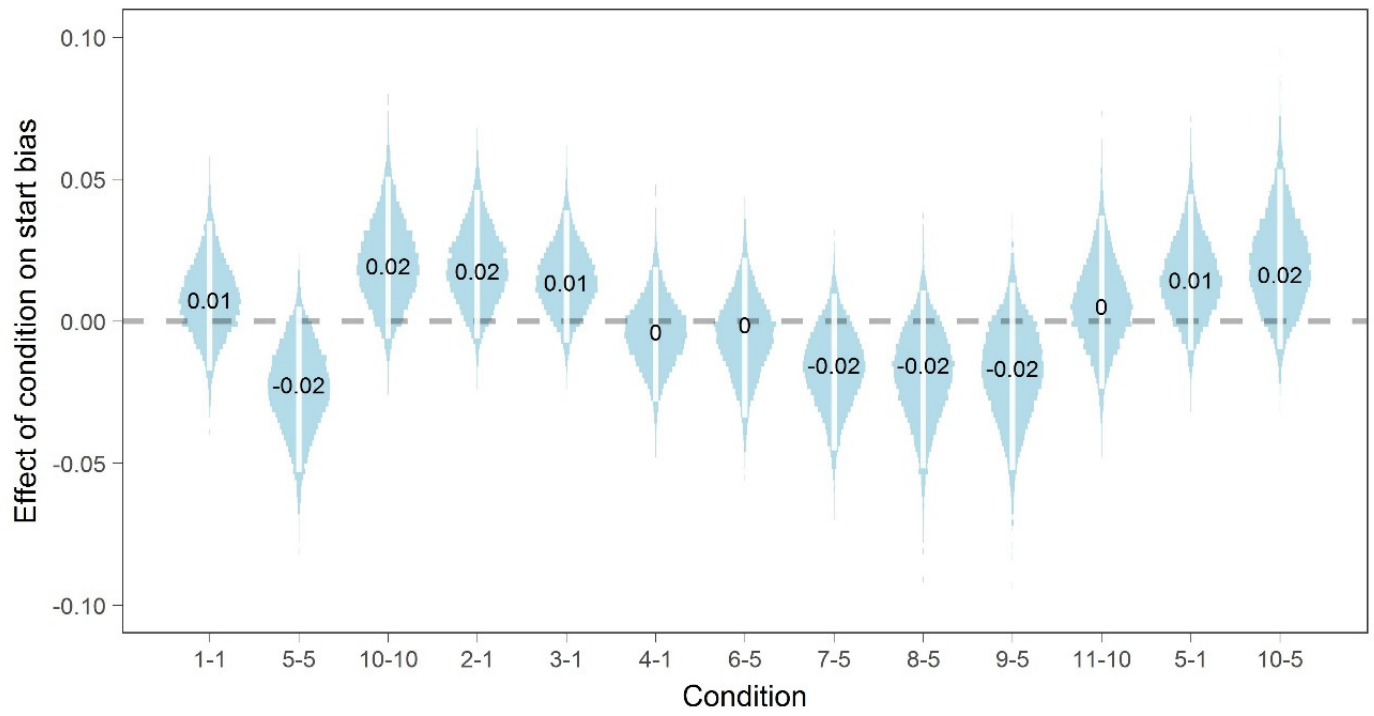

Figure 9.124 Differences in start bias between pairs of conditions 


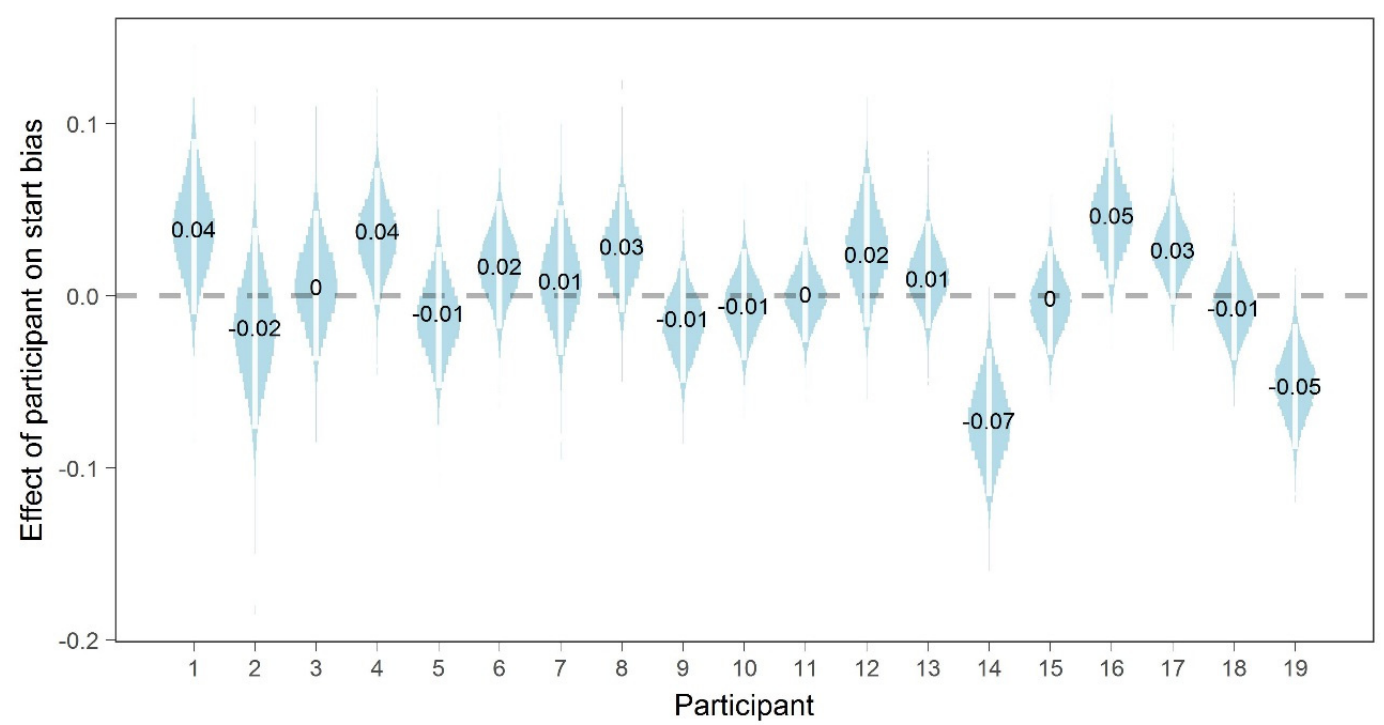

Figure 9.125 Differences in start bias between participants 


\section{Reference response contraction bias}

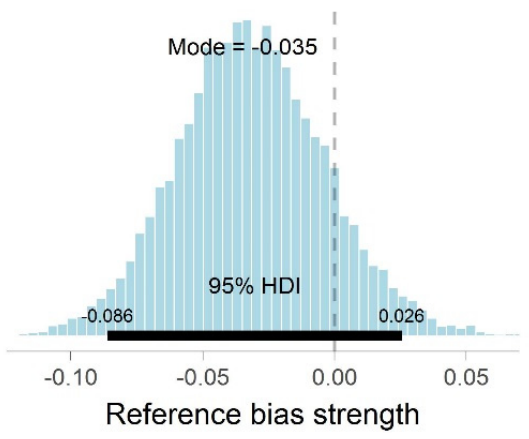

Figure 9.126 Mean response contraction bias

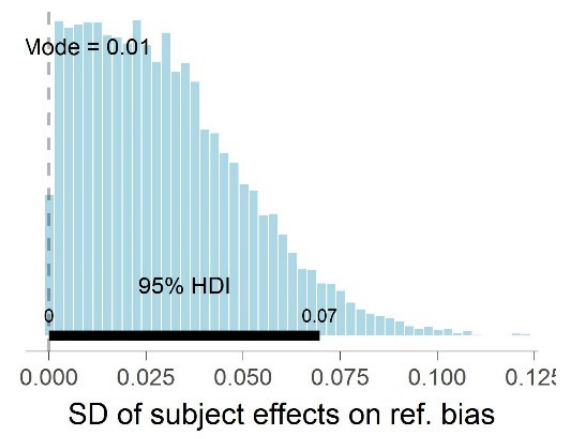

Figure 9.127 SD of variation in response contraction bias between people

Note there is clearly not enough data to accurately measure the variation between people here.

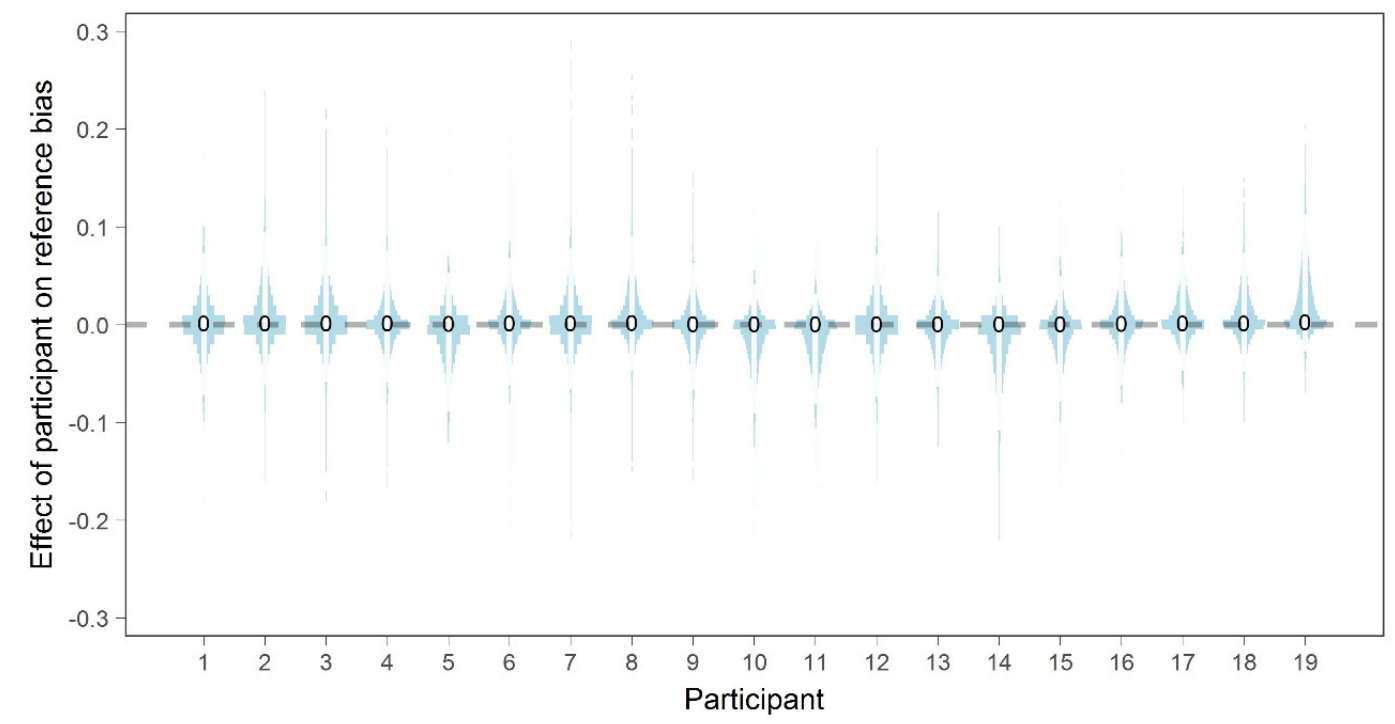

Figure 9.128 Differences in response contraction bias between participants 


\section{Adjustment range bias}

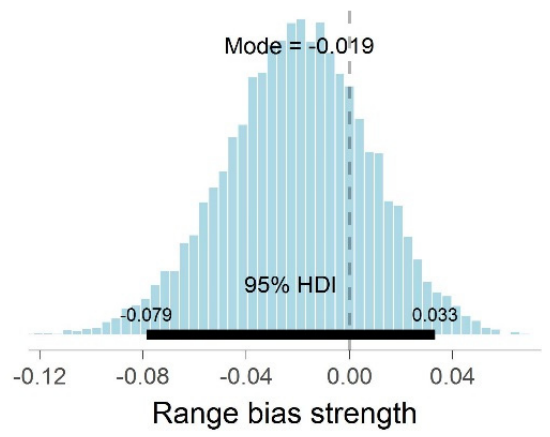

Figure 9.129 Mean adjustment range bias

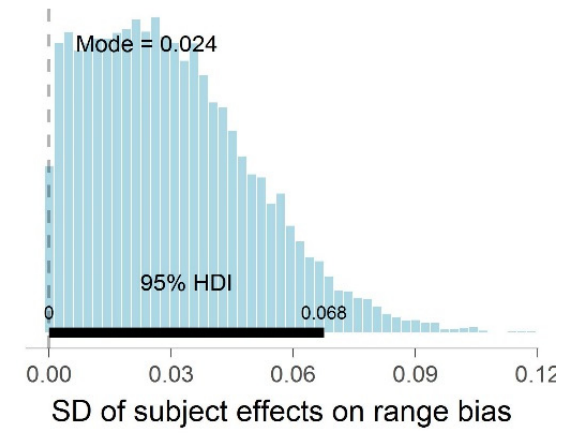

Figure 9.130 SD of variation in adjustment range bias between people

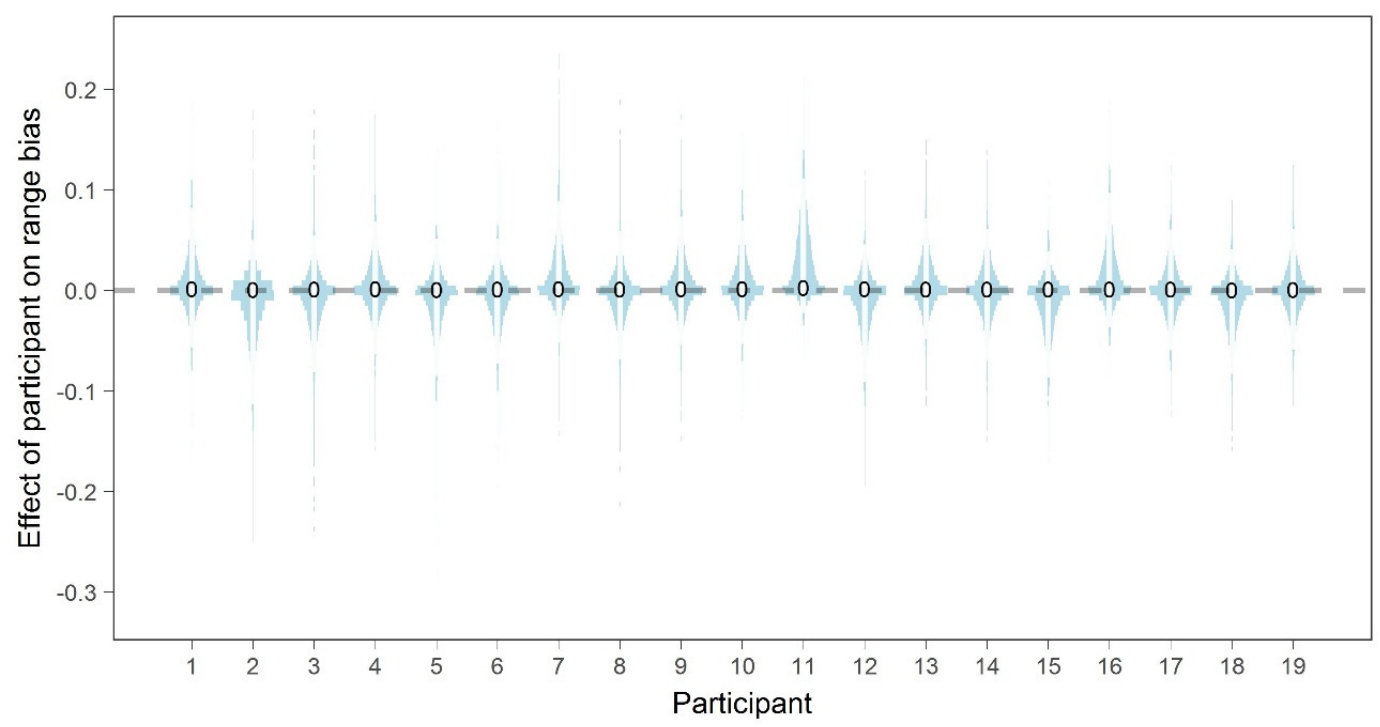

Figure 9.131 Differences in adjustment range bias between participants 